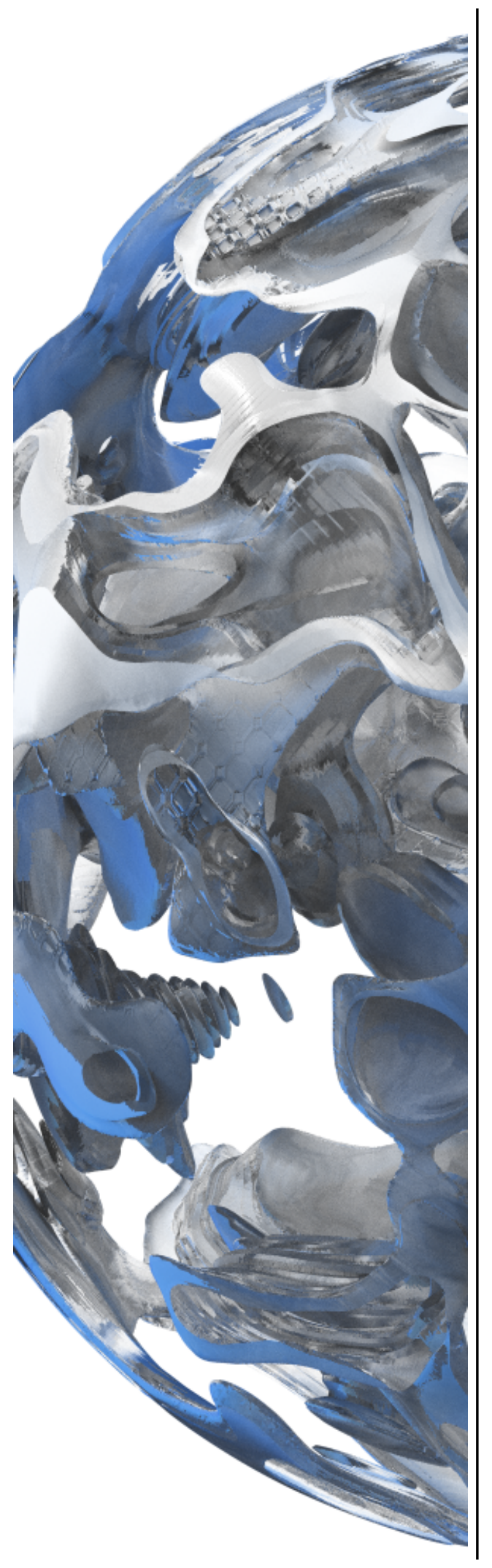

\title{
The VTK-m User's Guide
}

\author{
VTK-m version 1.7
}

Kenneth Moreland

With contributions from:

Vicente Bolea, Hank Childs, Nickolas Davis, Mark Kim, James Kress, Matthew Letter, Li-Ta Lo, Robert Maynard, Sujin Philip, David Pugmire, Nick Thompson, Allison Vacanti, Abhishek Yenpure, and the VTK-m community

December 28, 2021

http://m.vtk.org http://kitware.com 
All product names mentioned herein are the trademarks of their respective owners.

This document is available under a Creative Commons Attribution 4.0 International license available at http://creativecommons.org/licenses/by/4.0/.

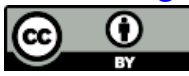

This project has been funded in whole or in part with Federal funds from the Department of Energy, including from Oak Ridge National Laboratory, Los Alamos National Laboratory, and Sandia National Laboratories.

This manuscript has been authored in part by UT-Battelle, LLC, under contract DE-AC05-00OR22725 with the US Department of Energy (DOE). The US government retains and the publisher, by accepting the article for publication, acknowledges that the US government retains a nonexclusive, paid-up, irrevocable, worldwide license to publish or reproduce the published form of this manuscript, or allow others to do so, for US government purposes.

Sandia National Laboratories is a multimission laboratory managed and operated by National Technology and Engineering Solutions of Sandia LLC, a wholly owned subsidiary of Honeywell International Inc. for the U.S. Department of Energy's National Nuclear Security Administration under contract DE-NA0003525.

Moreland, K. (2021). The VTK-m User's Guide, Tech report ORNL/TM-2021/2346, Oak Ridge National Laboratory. 


\section{CONTRIBUTORS}

This book includes contributions from the VTK-m community including the VTK-m development team and the user community. We would like to thank the following people for their significant contributions to this text:

Vicente Bolea, Nickolas Davis, Matthew Letter, and Nick Thompson for their help keeping the user's guide up to date with the VTK-m source code.

Sujin Philip, Robert Maynard, James Kress, Abhishek Yenpure, Mark Kim, and Hank Childs for their descriptions of numerous filters.

Allison Vacanti for her documentation of several VTK-m features in Sections 26.11 and 26.12 and select filters.

David Pugmire for his documentation of partitioned data sets (Section 7.5) and select filters.

Abhishek Yenpure and Li-Ta Lo for their documentation of locator structures (Chapter 30).

Li-Ta Lo for his documentation of random array handles and particle density filters.

James Kress for his documentation on VTK-m's testing classes.

\section{ABOUT THE COVER}

The cover image is a visualization of the temperature field computed by the Nek5000 thermal hydraulics simulator. In the simulation twin inlets pump air into a box with a temperature difference between the 2 inlets. The visualization is provided by Matthew Larsen at Lawrence Livermore National Laboratory.

The interior cover image represents seismic wave propagation through the Earth. The visualization is provided by Matthew Larsen at Lawrence Livermore National Laboratory.

The cover design was done by Steve Jordan.

Join the VTK-m Community at http://m.vtk.org 

I Getting Started $\quad 1$

1 Introduction $\quad 3$

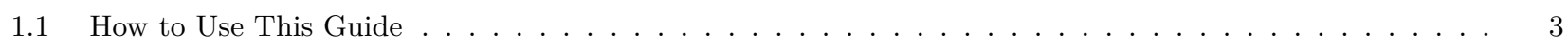

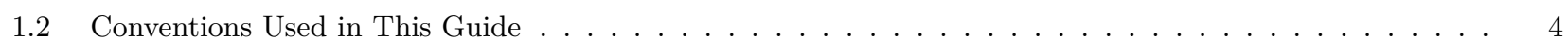

2 Build and Install VTK-m $r$

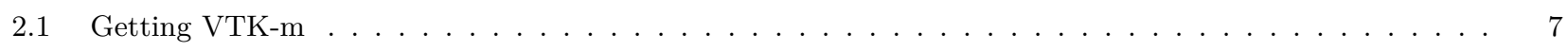

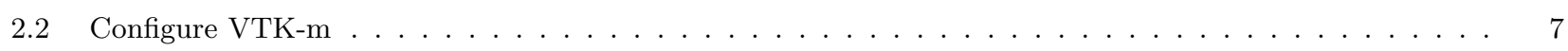

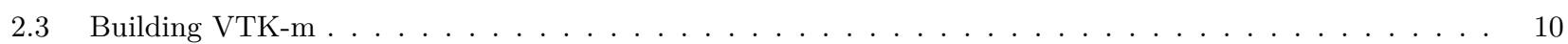

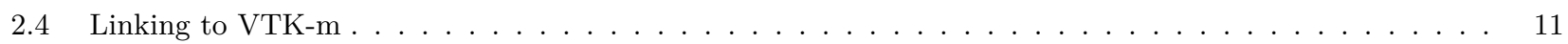

3 Quick Start $r$

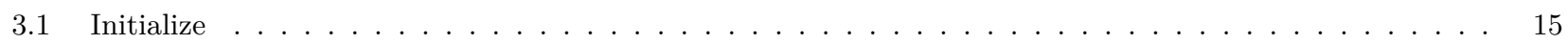

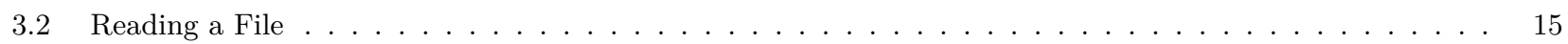

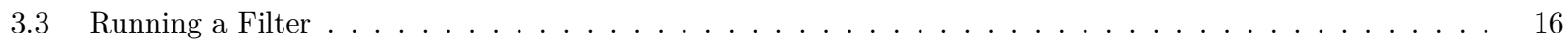

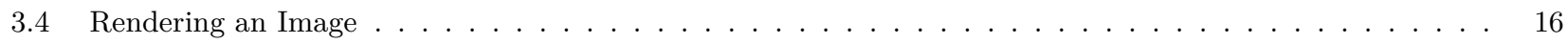

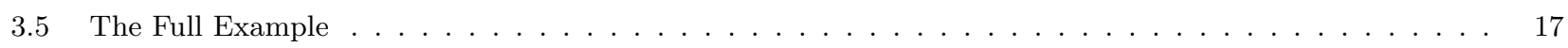

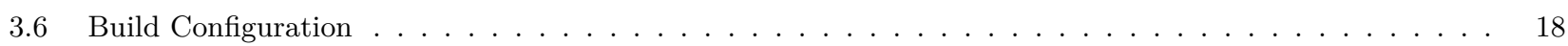

II Using VTK-m $r$

4 Base Types $r$

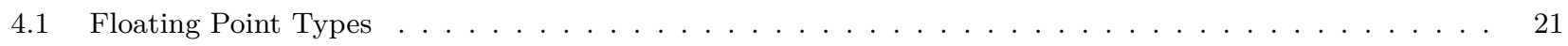

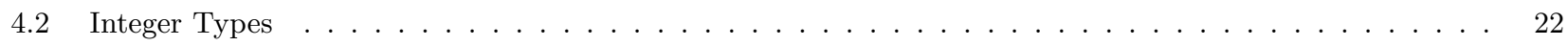

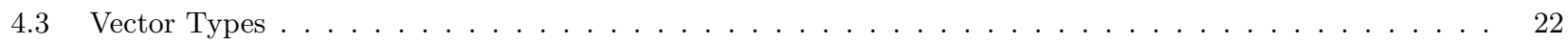

$\begin{array}{llr}5 & \text { VTK-m Version } & 25\end{array}$ 
6 Initialization

7 Data Sets 29

7.1 Building Data Sets . . . . . . . . . . . . . . . . . . . . . . . . . . 29

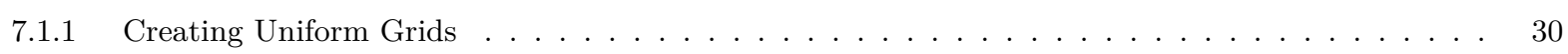

7.1 .2 Creating Rectilinear Grids . . . . . . . . . . . . . . . . . . . . . . 30

7.1 .3 Creating Explicit Meshes . . . . . . . . . . . . . . . . . . . . . . . . . . . . . . . .

7.1 .4 Add Fields . . . . . . . . . . . . . . . . . . . . . . . . . . . . . . . . . . . . . . . . .

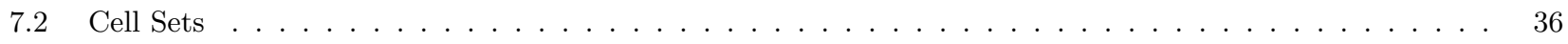

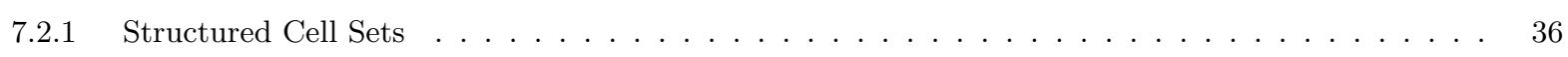

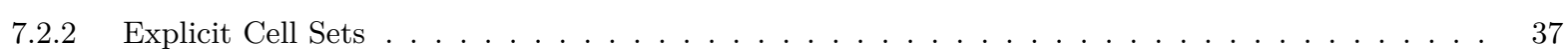

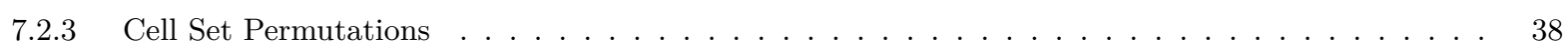

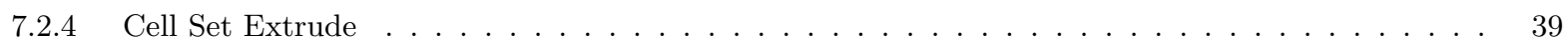

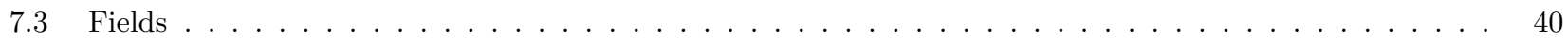

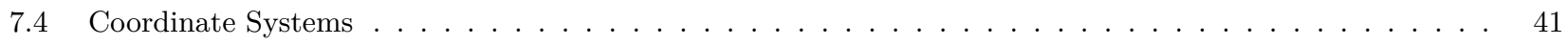

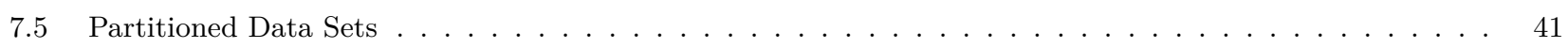

8 File I/O 43

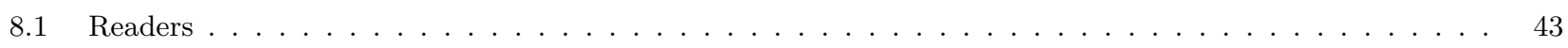

8.1 Legacy VTK File Reader . . . . . . . . . . . . . . . . . . . . . . . . . . . . 43

8.1 .2 Image Readers . . . . . . . . . . . . . . . . . . . . . . . . . . . . . . . . . . . . . .

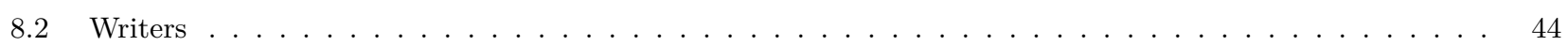

8.2.1 Legacy VTK File Writer . . . . . . . . . . . . . . . . . . . . . 45

8.2 .2 Image Writers . . . . . . . . . . . . . . . . . . . . . . . . . . . . . . . . . . . . . .

9 Running Filters 47

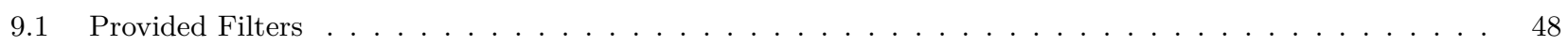

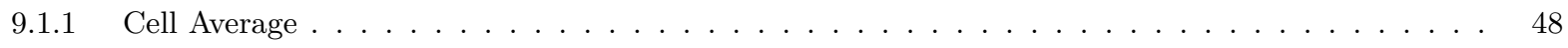

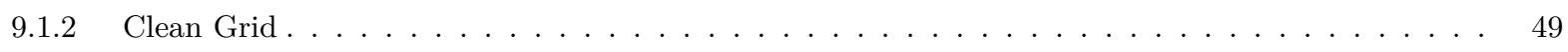

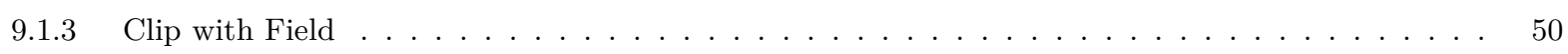

9.1 Clip with Implicit Function . . . . . . . . . . . . . . . . . . . . . . . . . . . . . . .

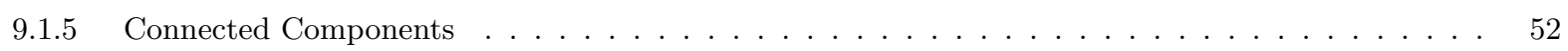

Cell Connectivity . . . . . . . . . . . . . . . . . . . . . . . . . . . 52

Image Field . . . . . . . . . . . . . . . . . . . . . . . . . . . . . . . 52

9.1.6 Coordinate System Transforms . . . . . . . . . . . . . . . . . . . . . . . . . . . . . .

Cylindrical Coordinate System Transform . . . . . . . . . . . . . . . . . . . . . . 53

Spherical Coordinate System Transform . . . . . . . . . . . . . . . . . . . . . . . . . 54

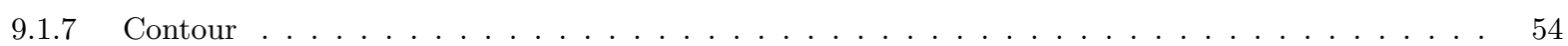




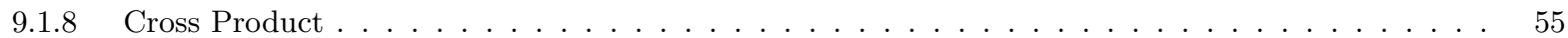

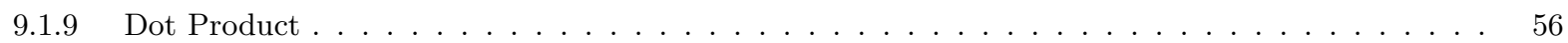

$9.1 .10 \quad$ External Faces $\ldots \ldots \ldots \ldots \ldots \ldots \ldots \ldots \ldots \ldots$

9.1 .11 Extract Structured . . . . . . . . . . . . . . . . . . . . . . . . 57

9.1 .12 Field to Colors $\ldots \ldots \ldots \ldots \ldots \ldots \ldots \ldots \ldots \ldots$

9.1 .13 Ghost Cell Classification . . . . . . . . . . . . . . . . . . . . . . . . . . . . . . . 60

9.1 .14 Ghost Cell Removal _. . . . . . . . . . . . . . . . . . . . . . . . . . . . . . 60

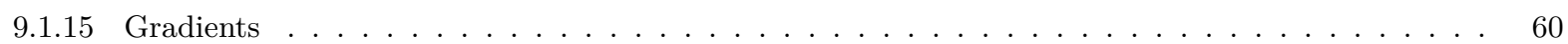

9.1 .16 Histogram . . . . . . . . . . . . . . . . . . . . . . . . . . 62

9.1 .17 Lagrangian Coherent Structures . . . . . . . . . . . . . . . . . . . . . . . . . 62

9.1 .18 Mesh Quality Metrics _. . . . . . . . . . . . . . . . . . . . 63

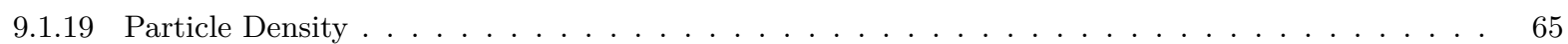

Nearest Grid Point . . . . . . . . . . . . . . . . . . . . . . . . 66

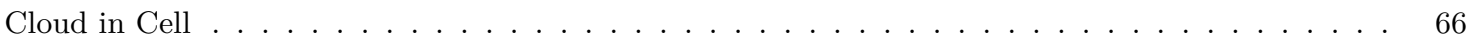

9.1 .20 Point Average . . . . . . . . . . . . . . . . . . . . . . . . 66

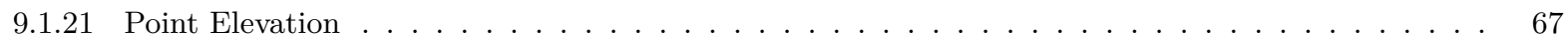

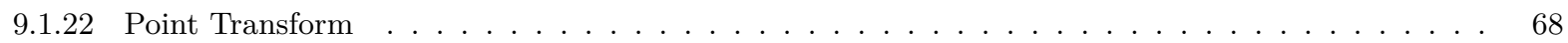

9.1 .23 Stream Tracing . . . . . . . . . . . . . . . . . . . . . . . . . . . . . . 69

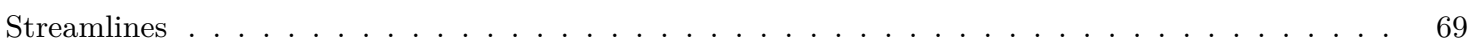

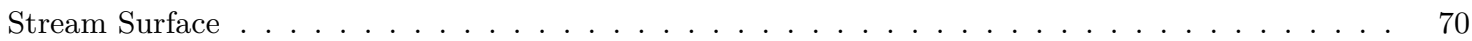

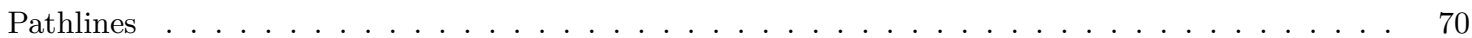

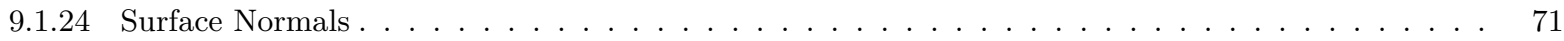

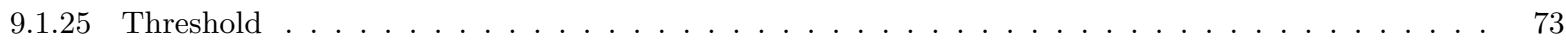

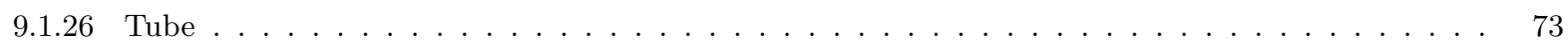

9.1 .27 Vector Magnitude . . . . . . . . . . . . . . . . . . . . . . . . . . . . . 74

9.1 .28 Vertex Clustering . . . . . . . . . . . . . . . . . . . . . . . . 74

9.1 .29 Warp Scalar . . . . . . . . . . . . . . . . . . . . . . . . 75

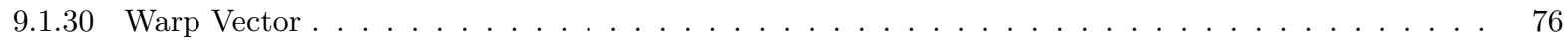

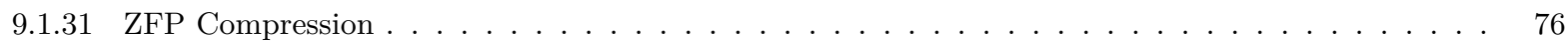

$9.2 \quad$ Advanced Field Management . . . . . . . . . . . . . . . . . . . . . . . . . . 77

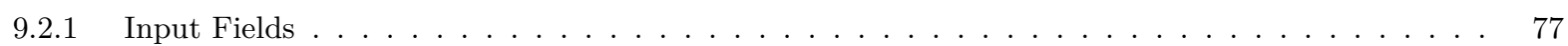

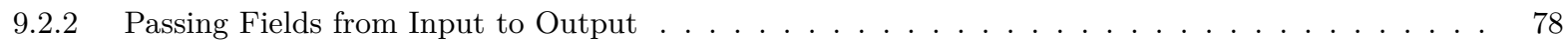

10 Rendering $\quad 81$

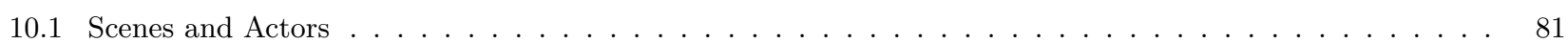

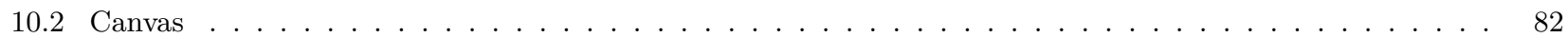

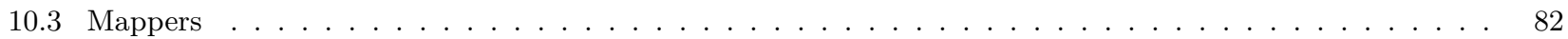




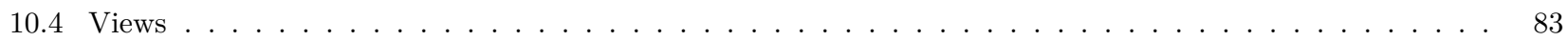

10.5 Changing Rendering Modes . . . . . . . . . . . . . . . . . . . . . . . . . . 84

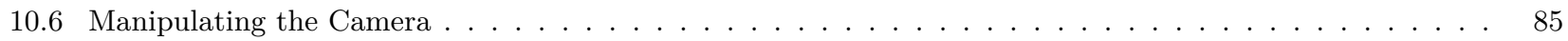

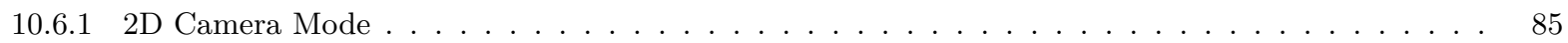

View Range . . . . . . . . . . . . . . . . . . . . . . . . . . . . . 85

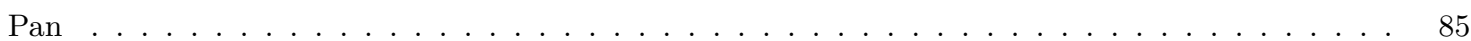

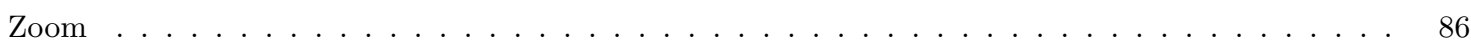

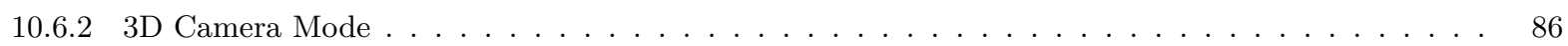

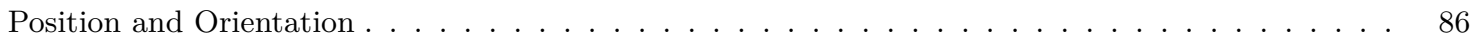

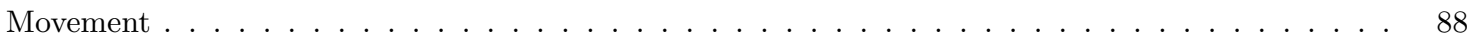

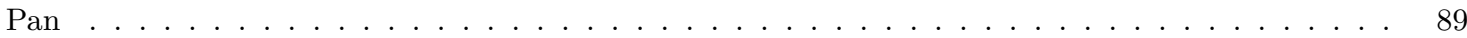

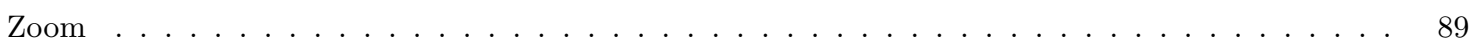

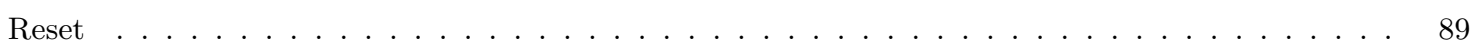

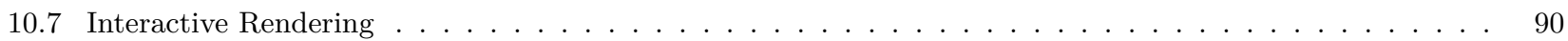

10.7 .1 Rendering Into a GUI $\ldots \ldots \ldots \ldots \ldots \ldots \ldots$

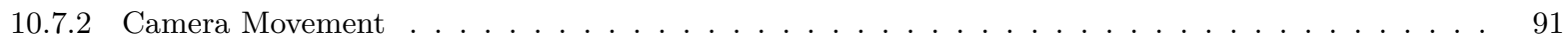

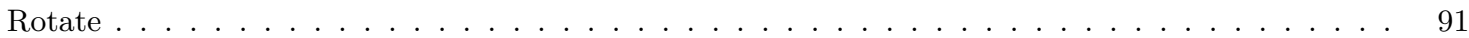

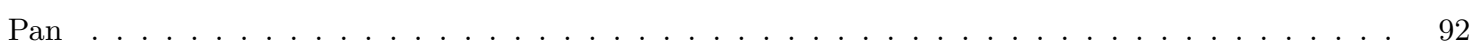

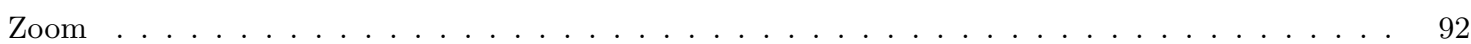

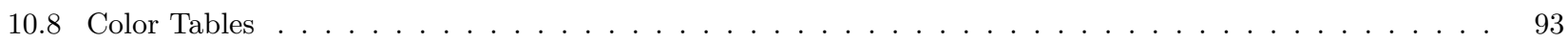

11 Error Handling $\quad 95$

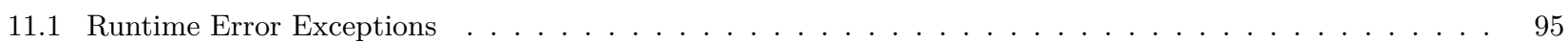

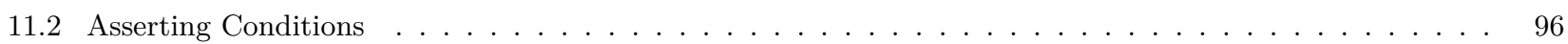

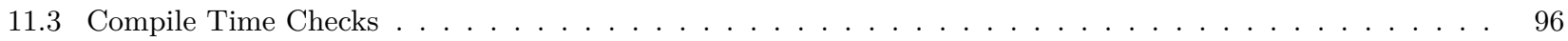

12 Managing Devices $\quad 99$

12.1 Device Adapter Tag . . . . . . . . . . . . . . . . . . . . . . . . . . . . . 99

12.2 Device Adapter Id . . . . . . . . . . . . . . . . . . . . . . . . . . . . . 100

12.3 Runtime Device Tracker . . . . . . . . . . . . . . . . . . . . . . . . . . . . . . . . . . 101

13 Timers $\quad 103$

14 Implicit Functions $r$

14.1 Provided Implicit Functions . . . . . . . . . . . . . . . . . . . . . . . 105

$14.1 .1 \quad$ Plane . . . . . . . . . . . . . . . . . . . . . . . . . . . 105

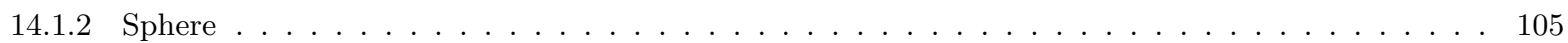

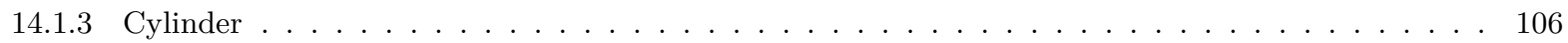




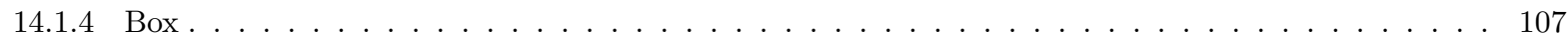

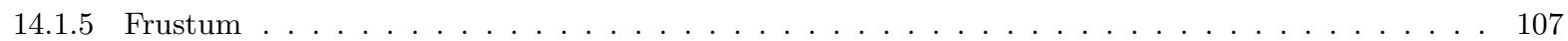

14.2 General Implicit Functions ～. . . . . . . . . . . . . . . . . . . . . . 107

$\begin{array}{lr}\text { III Developing Algorithms } & 109\end{array}$

15 General Approach $r 11$

15.1 Package Structure . . . . . . . . . . . . . . . . . . . . . . . . . . . . . . . 112

15.2 Function and Method Environment Modifiers . . . . . . . . . . . . . . . . . . . . . . . . 113

16 Basic Array Handles $r \begin{array}{ll}115\end{array}$

16.1 Creating Array Handles . . . . . . . . . . . . . . . . . . . . . . 116

16.2 Deep Array Copies . . . . . . . . . . . . . . . . . . . . . . . . . . . . . . . . . . . 118

16.3 The Hidden Second Template Parameter ～. . . . . . . . . . . . . . . . . . . . . . . . . . 118

16.4 Mutability . . . . . . . . . . . . . . . . . . . . . . . . . . . . . . 119

17 Simple Worklets $r \begin{aligned} & 121\end{aligned}$

17.1 Control Signature . . . . . . . . . . . . . . . . . . . . . . . . . . . . . . . . . 122

17.2 Execution Signature . . . . . . . . . . . . . . . . . . . . . . . . . . . . 122

17.3 Input Domain . . . . . . . . . . . . . . . . . . . . . . . . . . . . . . . . . 123

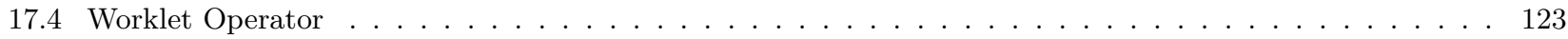

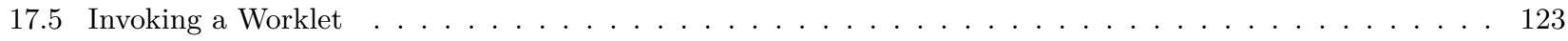

17.6 Preview of More Complex Worklets . . . . . . . . . . . . . . . . . . . . . . . . . . . . . 124

18 Basic Filter Implementation $\quad 125$

$\begin{array}{llr}\text { IV Advanced Development } & 129\end{array}$

19 Advanced Types $r$

19.1 Single Number Types . . . . . . . . . . . . . . . . . . . . . . . . . . . . . . . . 131

19.2 Vector Types . . . . . . . . . . . . . . . . . . . . . . . . . . . . . . . . 131

19.3 Range . . . . . . . . . . . . . . . . . . . . . . . . . . . . . . . . . . . . . . 135

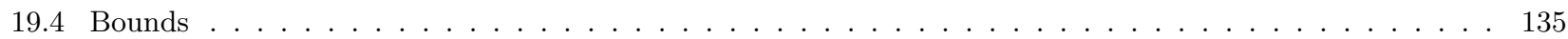

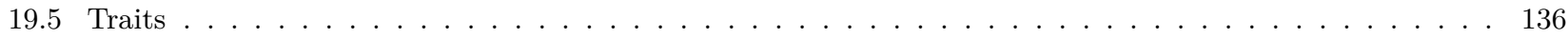

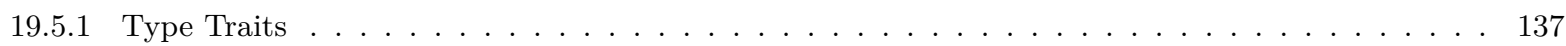

19.5.2 Vector Traits . . . . . . . . . . . . . . . . . . . . . . . . 138

19.6 List Templates . . . . . . . . . . . . . . . . . . . . . . . . . . . . . . 141

19.6.1 Building Lists . . . . . . . . . . . . . . . . . . . . . . . . . . . 141 
19.6 .2 Type Lists . . . . . . . . . . . . . . . . . . . . . . . . . . . . 141

19.6 .3 Querying Lists . . . . . . . . . . . . . . . . . . . . . . . . . 143

19.6 .4 Operating on Lists . . . . . . . . . . . . . . . . . . . . . . . . . . . 144

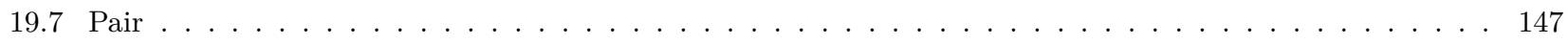

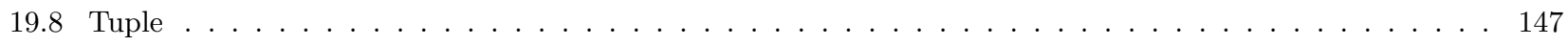

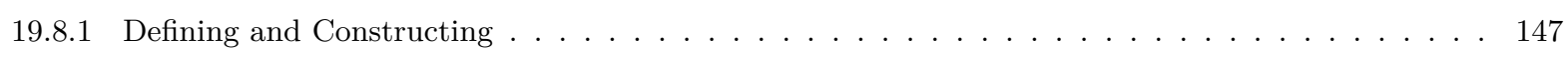

19.8 Querying . . . . . . . . . . . . . . . . . . . . . 147

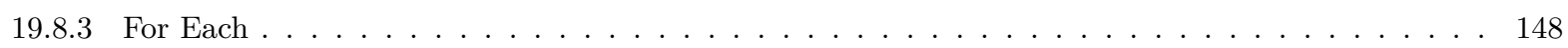

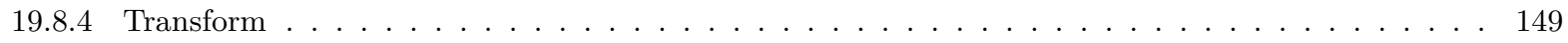

19.8 .5 Apply . . . . . . . . . . . . . . . . . . . . . . . . . . . . . 149

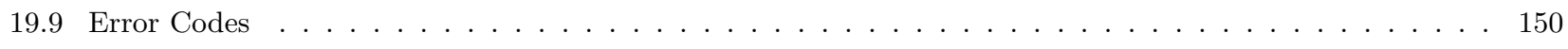

20 Logging $r$

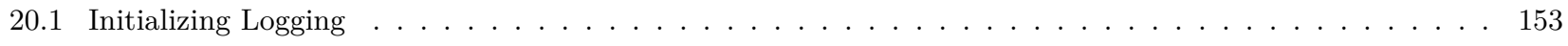

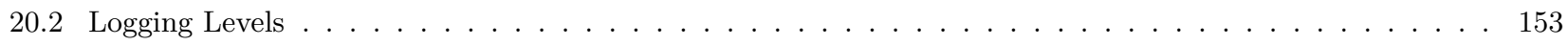

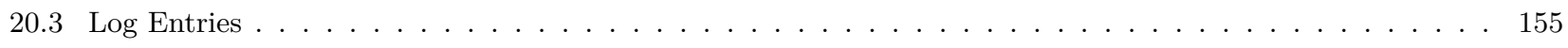

$20.3 .1 \quad$ Basic $\log$ Entries . . . . . . . . . . . . . . . . . . . . . 155

20.3 .2 Conditional $\log$ Entries $\ldots \ldots \ldots \ldots \ldots \ldots \ldots \ldots \ldots$

$20.3 .3 \quad$ Scoped $\log$ Entries $\ldots \ldots \ldots \ldots \ldots \ldots \ldots \ldots$

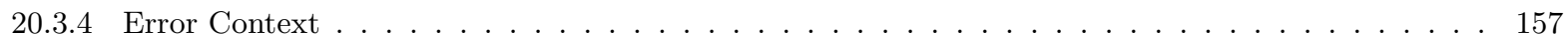

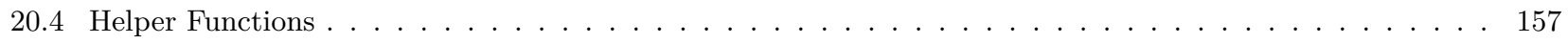

21 Worklet Type Reference $r$

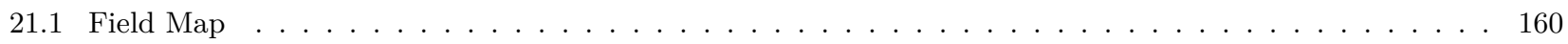

21.2 Topology Map . . . . . . . . . . . . . . . . . . . . . . . . . 162

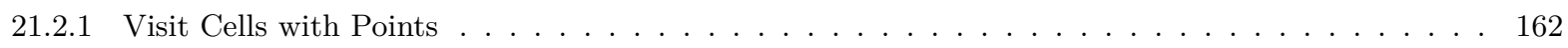

21.2 .2 Visit Points with Cells _ . . . . . . . . . . . . . . . . . . . . 165

21.2 .3 General Topology Maps . . . . . . . . . . . . . . . . . . . . . . 168

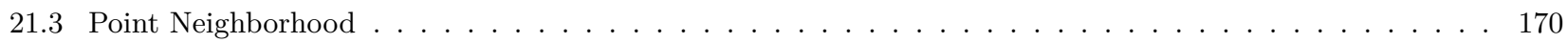

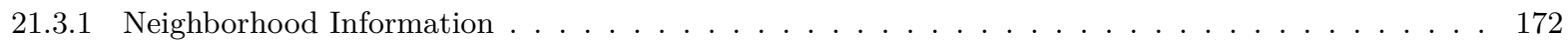

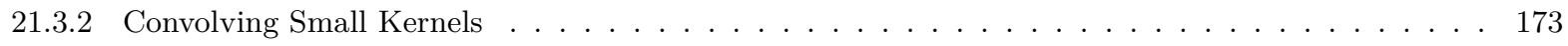

21.4 Reduce by Key . . . . . . . . . . . . . . . . . . . . . . . . . . . . . . . . . . 174

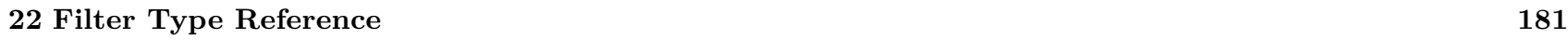

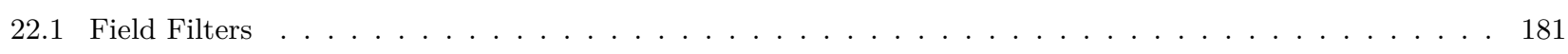

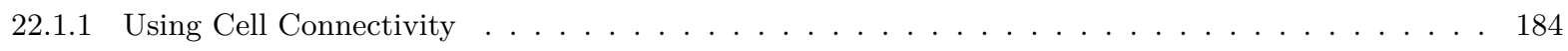

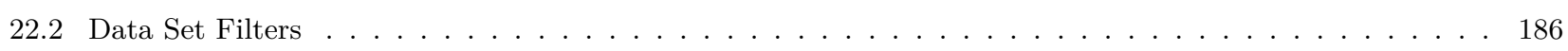

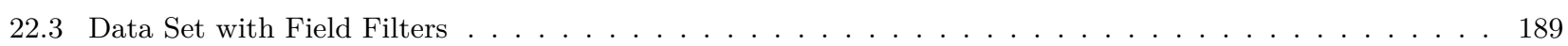


22.4 Applying Policies . . . . . . . . . . . . . . . . . . . . . . . . . . . . 192

22.4.1 Getting Field Data . . . . . . . . . . . . . . . . . . . . . . . . . . . . . 193

22.4 .2 Getting Cell Sets . . . . . . . . . . . . . . . . . . . . . . . . . . 193

23 Worklet Error Handling $\quad 195$

$\begin{array}{lr}24 \text { Math } & 197\end{array}$

24.1 Basic Math . . . . . . . . . . . . . . . . . . . . . . . . . . . . . . 197

24.2 Vector Analysis . . . . . . . . . . . . . . . . . . . . . . . . . . 200

24.3 Matrices . . . . . . . . . . . . . . . . . . . . . . . . . . . . . . . 201

24.4 Newton's Method . . . . . . . . . . . . . . . . . . . . . . . . . . . 202

25 Working with Cells $\quad 205$

25.1 Cell Shape Tags and Ids . . . . . . . . . . . . . . . . . . . . . . . . . . . 205

25.1.1 Converting Between Tags and Identifiers . . . . . . . . . . . . . . . . . . . . . . . . 205

25.1 .2 Cell Traits . . . . . . . . . . . . . . . . . . . . . . . . . . . . . . . 207

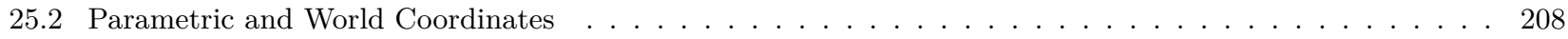

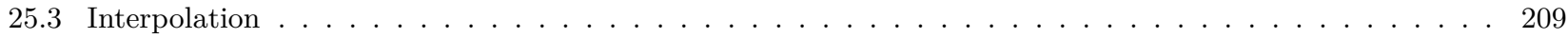

25.4 Derivatives . . . . . . . . . . . . . . . . . . . . . . . . . . . . . . 209

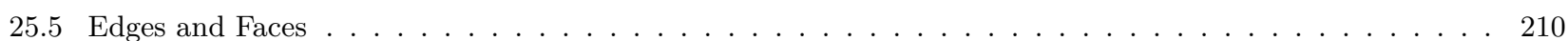

26 Fancy Array Handles $\quad 215$

26.1 Constant Arrays . . . . . . . . . . . . . . . . . . . . . . . . . . . . . 215

26.2 ArrayHandleView . . . . . . . . . . . . . . . . . . . . . . . . . . 216

26.3 Uniform Random Bits Array . . . . . . . . . . . . . . . . . . . . . . . . . . . . . . . . . . . 216

26.4 Counting Arrays . . . . . . . . . . . . . . . . . . . . . . . . . . . 217

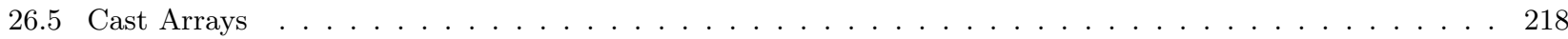

26.6 Discard Arrays . . . . . . . . . . . . . . . . . . . . . . . . . . . . . . . . . . . . . . 219

26.7 Permuted Arrays . . . . . . . . . . . . . . . . . . . . . . . . . . . . . . . . . 219

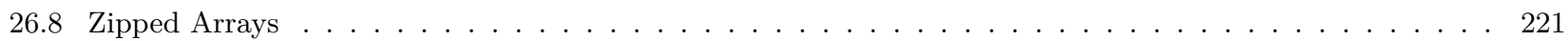

26.9 Coordinate System Arrays ～. . . . . . . . . . . . . . . . . . . . . . . . . . . . . 221

26.10 Composite Vector Arrays ． . . . . . . . . . . . . . . . . . . . . . . . . . . . . 223

26.11 Extract Component Arrays . . . . . . . . . . . . . . . . . . . . . . . . . . . . . . . . . . . . . . . . 224

26.12 Swizzle Arrays . . . . . . . . . . . . . . . . . . . . . . . . . . . . . . 225

26.13 Grouped Vector Arrays ． . . . . . . . . . . . . . . . . . . . . . . . . . . 225

27 Accessing and Allocating Array Handles $r$

27.1 Array Portals . . . . . . . . . . . . . . . . . . . . . . . . . . . . . . . . . . . . . . . 229

27.2 Allocating and Populating Array Handles . . . . . . . . . . . . . . . . . . . . . . . . . . 232 
27.3 Compute Array Range . . . . . . . . . . . . . . . . . . . . . . . . . . . . . 233

27.4 Interface to Execution Environment . . . . . . . . . . . . . . . . . . . . . . . 233

28 Global Arrays and Topology 237

28.1 Whole Arrays . . . . . . . . . . . . . . . . . . . . . . . . . . . . . . . . . . . . . . . . . . . . . . .

28.2 Atomic Arrays . . . . . . . . . . . . . . . . . . . . . . . . . . . 239

28.3 Whole Cell Sets . . . . . . . . . . . . . . . . . . . . . . . . . . . . 241

29 Execution Objects 245

30 Locators 249

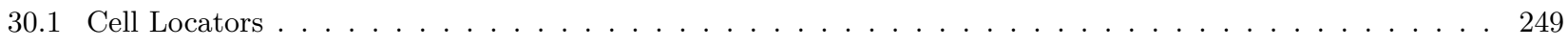

30.1 Building a Cell Locator . . . . . . . . . . . . . . . . . . . . . . . 250

30.1.2 Using Cell Locators in a Worklet . . . . . . . . . . . . . . . . . . . . . . 250

30.2 Point Locators . . . . . . . . . . . . . . . . . . . . . . . . . . . . . . 252

30.2.1 Building Point Locators . . . . . . . . . . . . . . . . . . . . . . . 252

30.2.2 Using Point Locators in a Worklet . . . . . . . . . . . . . . . . . . . . . 253

31 Worklet Input Output Semantics 255

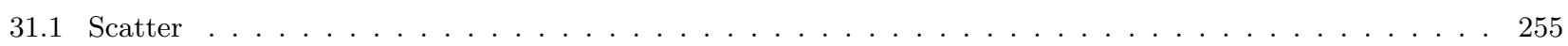

32 Generating Cell Sets 261

32.1 Single Cell Type . . . . . . . . . . . . . . . . . . . . . . . . . . . . . . . . . . . . . . . . . . . . . . .

32.2 Combining Like Elements . . . . . . . . . . . . . . . . . . . . . . . . . . . . 264

32.3 Faster Combining Like Elements with Hashes . . . . . . . . . . . . . . . . . . . . . . . . . . . . . 269

32.4 Variable Cell Types . . . . . . . . . . . . . . . . . . . . . . . . . . 277

33 Unknown Array Handles 283

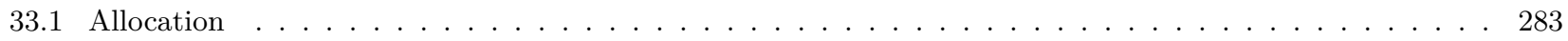

33.2 Casting to Known Types . . . . . . . . . . . . . . . . . . . . . . . . . 285

33.3 Casting to a List of Potential Types . . . . . . . . . . . . . . . . . . . . . . . 287

33.4 Accessing Truly Unknown Arrays . . . . . . . . . . . . . . . . . . . . . . . . 289

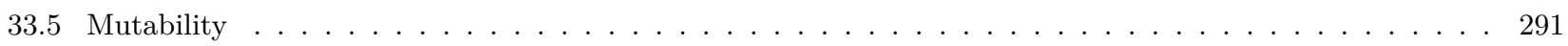

34 Device Algorithms 293

34.1 BitFieldToUnorderedSet . . . . . . . . . . . . . . . . . . . . . . . . 293

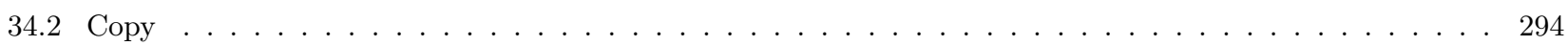

34.3 Copylf . . . . . . . . . . . . . . . . . . . . . . . . . . . 294

34.4 CopySubRange . . . . . . . . . . . . . . . . . . . . . . . . . . 295

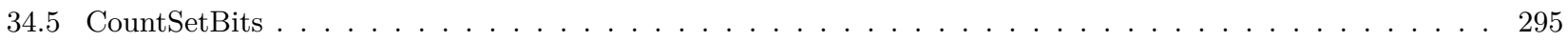




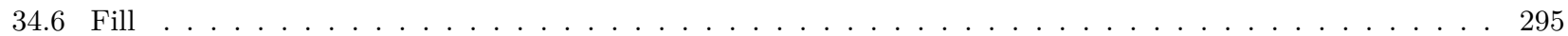

34.7 LowerBounds . . . . . . . . . . . . . . . . . . . . . . . . . . . 296

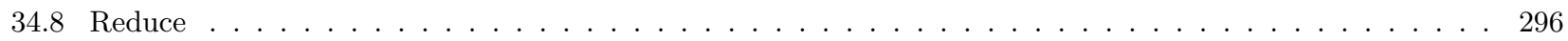

34.9 ReduceByKey . . . . . . . . . . . . . . . . . . . . . . . . . . 297

34.10 ScanInclusive . . . . . . . . . . . . . . . . . . . . . . . . . . 297

34.11 ScanInclusiveByKey . . . . . . . . . . . . . . . . . . . . . . . . . 298

34.12 ScanExclusive . . . . . . . . . . . . . . . . . . . . . . . . . 298

34.13 ScanExclusiveByKey . . . . . . . . . . . . . . . . . . . . . . . 299

34.14 ScanExtended . . . . . . . . . . . . . . . . . . . . . . . . . 299

34.15 Schedule . . . . . . . . . . . . . . . . . . . . . . . . . . 300

34.16 Sort . . . . . . . . . . . . . . . . . . . . . . . . . . 300

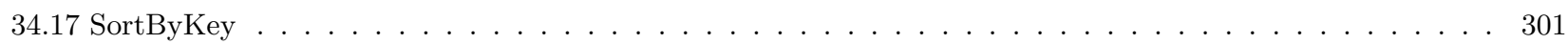

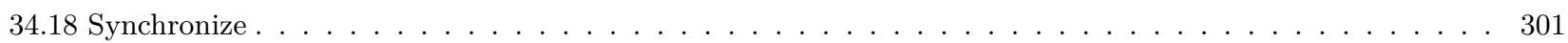

34.19 Transform . . . . . . . . . . . . . . . . . . . . . . . . . . 301

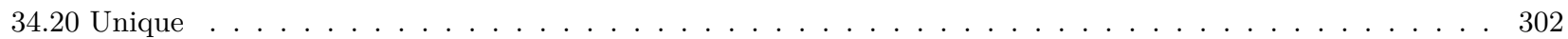

34.21 UpperBounds . . . . . . . . . . . . . . . . . . . . . . . . . . . 302

34.22 Specifying the Device Adapter . . . . . . . . . . . . . . . . . . . . . 303

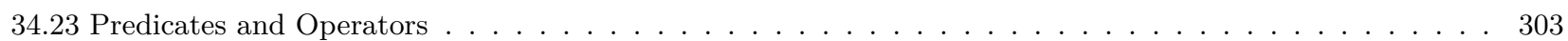

34.23 .1 Unary Predicates . . . . . . . . . . . . . . . . . . . . . . . . . . . . . . . . . . . . . . . .

34.23 .2 Binary Predicates . . . . . . . . . . . . . . . . . . . . . . . 304

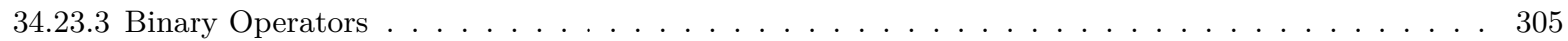

34.23.4 Creating Custom Comparators . . . . . . . . . . . . . . . . . 306

35 Custom Array Storage 307

35.1 Implicit Array Handles . . . . . . . . . . . . . . . . . . . . . . . . . . . 308

35.2 Transformed Arrays . . . . . . . . . . . . . . . . . . . . . . . . . 310

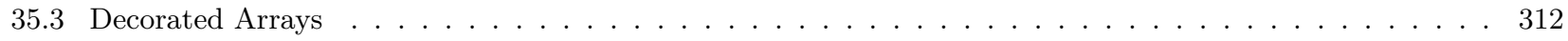

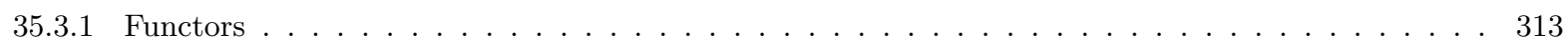

35.3 .2 Interface . . . . . . . . . . . . . . . . . . . . . . . . . . . . 314

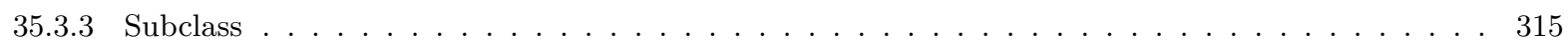

35.4 Derived Storage . . . . . . . . . . . . . . . . . . . . . . . . . 316

35.4.1 Array Portal . . . . . . . . . . . . . . . . . . . . . . . . . . . . . . . . . . .

35.4 .2 Storage . . . . . . . . . . . . . . . . . . . . . . . . . . . . . . . . . . . . . . .

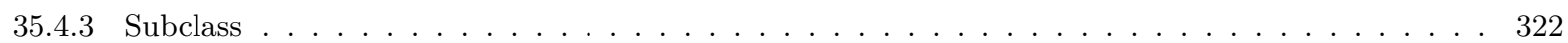

35.5 Adapting Data Structures . . . . . . . . . . . . . . . . . . . . . . . . . . 323

35.5.1 Buffer Objects . . . . . . . . . . . . . . . . . . . . . . . . . . . . . . . . . . . . . . . .

35.5.2 Array Portal . . . . . . . . . . . . . . . . . . . . . . . 325 


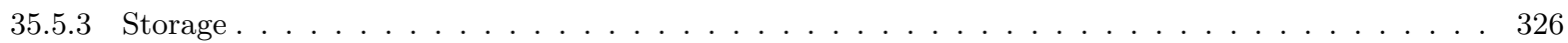

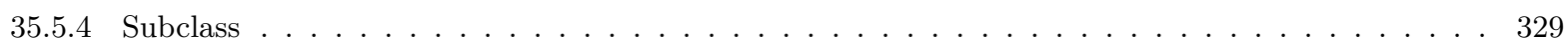

36 Regression Testing $\quad 331$

36.1 Running Regression Testing . . . . . . . . . . . . . . . . . . . . . . . . . . 331

36.1 .1 Regression Testing Using ctest . . . . . . . . . . . . . . . . . . . . . . 331

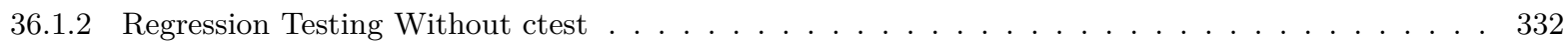

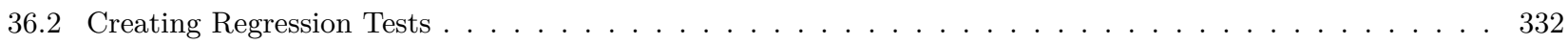

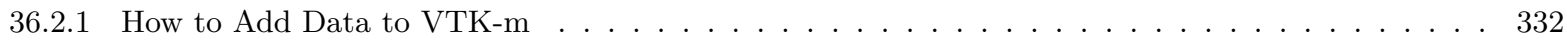

V Core Development $\quad 333$

$\begin{array}{ll}37 \text { Try Execute } & 335\end{array}$

38 Implementing Device Adapters $\quad 337$

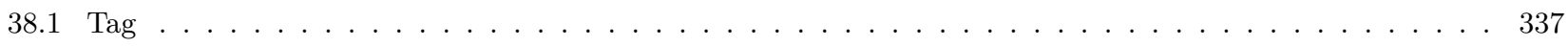

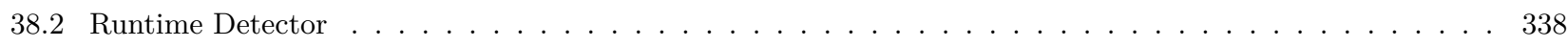

38.3 Memory Manager . . . . . . . . . . . . . . . . . . . . . . . . . . . . . 339

38.4 Runtime Device Configuration . . . . . . . . . . . . . . . . . . . . . . . . 341

38.5 Virtual Object Transfer . . . . . . . . . . . . . . . . . . . . . . . . . . . . . . . . . 344

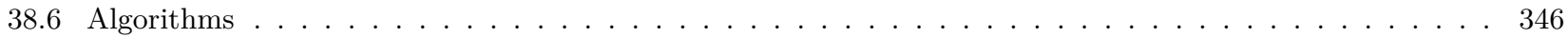

38.7 Timer Implementation $\ldots \ldots \ldots \ldots \ldots \ldots \ldots \ldots \ldots$

39 Function Interface Objects $\quad 353$

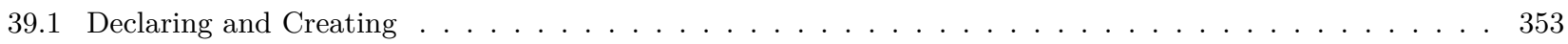

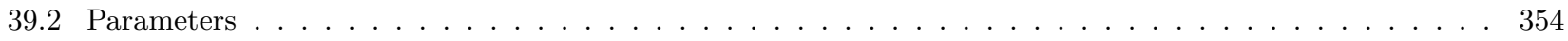

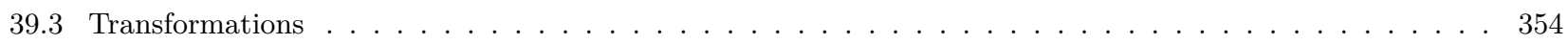

40 Worklet Arguments $\quad 357$

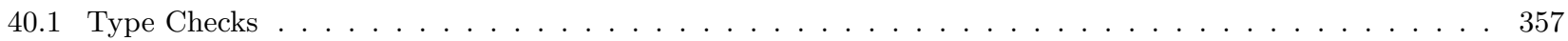

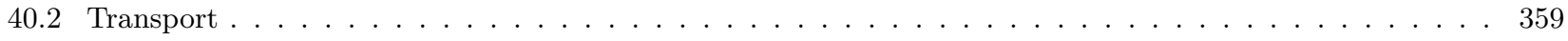

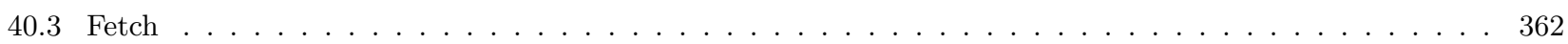

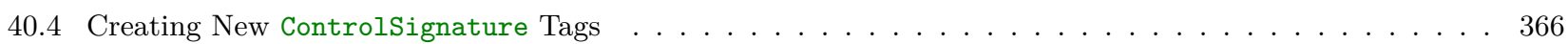

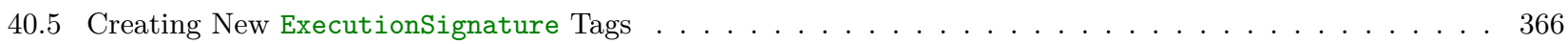

41 New Worklet Types $\quad 369$

41.1 Motivating Example . . . . . . . . . . . . . . . . . . . . . . . . . . . . . 369

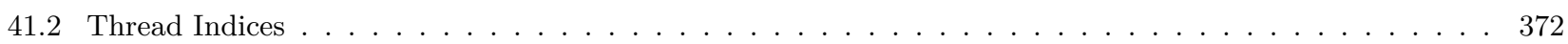

41.3 Signature Tags . . . . . . . . . . . . . . . . . . . . . . . . . . . . . 374 
41.4 Worklet Superclass . . . . . . . . . . . . . . . . . . . . . . . . . . . 377

41.5 Dispatcher . . . . . . . . . . . . . . . . . . . . . . . . . . . . . . 379

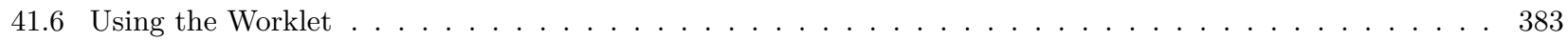

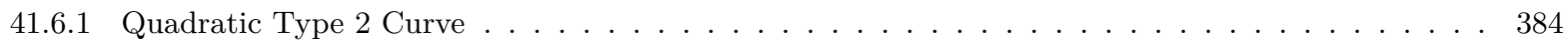

41.6 .2 Tree Fractal . . . . . . . . . . . . . . . . . . . . . . . . . . 385

41.6 .3 Dragon Fractal . . . . . . . . . . . . . . . . . . . . . . . . . . . 387

41.6.4 Hilbert Curve . . . . . . . . . . . . . . . . . . . . . . . . . . . . . . . . . . . . . . . . . . . . . 389

VI Appendix $\quad 393$

$\begin{array}{lr}\text { Index } & 395\end{array}$ 



\section{LIST OF FIGURES}

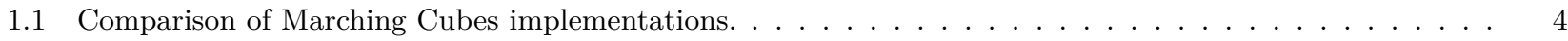

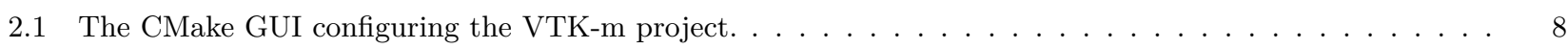

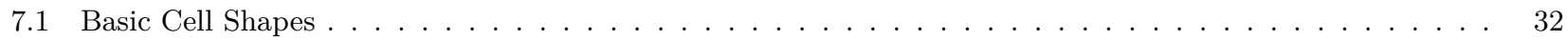

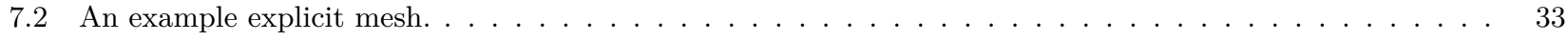

7.3 The relationship between a cell shape and its topological elements (points, edges, and faces). . . . . . . 36

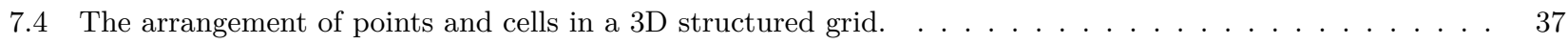

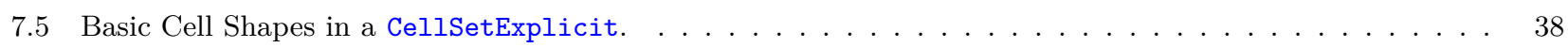

7.6 Example of cells in a CellSetExplicit and the arrays that define them. . . . . . . . . . . . . . 39

7.7 An example of an extruded wedge from XZ-plane coordinates. Six wedges are extracted from three XZ-plane

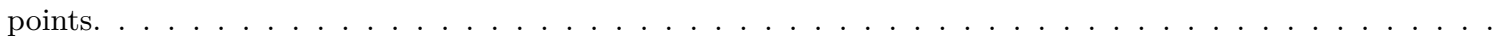

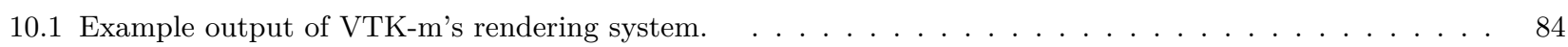

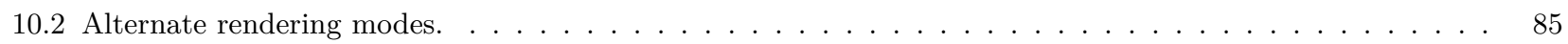

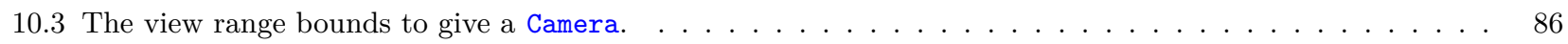

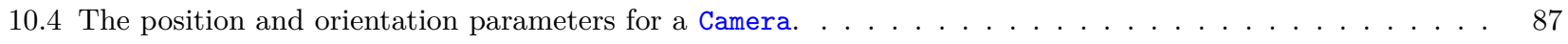

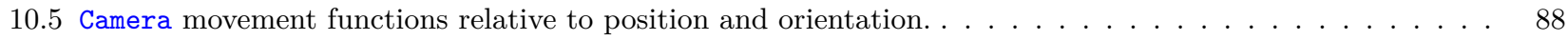

14.1 Visual Representation of an Implicit Plane. The red dot and arrow represent the origin and normal of the plane, respectively. For demonstrative purposes the plane as shown with limited area, but in actuality the

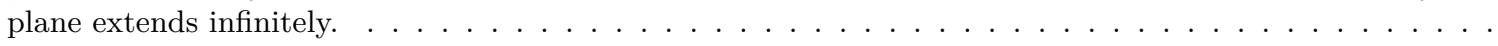

14.2 Visual Representation of an Implicit Sphere. The red dot represents the center of the sphere. The radius is the length of any line (like the blue one shown here) that extends from the center in any direction to the surface.

14.3 Visual Representation of an Implicit Cylinder. The red dot represents the center value, and the red arrow represents the vector that points in the direction of the axis. The radius is the length of any line (like the blue one shown here) that extends perpendicular from the axis to the surface.

14.4 Visual Representation of an Implicit Box. The red dots represent the minimum and maximum points. . . 
14.5 Visual Representation of an Implicit Frustum. The red dots and arrows represent the points and normals defining each enclosing plane. The blue dots represent the 8 vertices, which can also be used to define the

15.1 Diagram of the VTK-m framework. . . . . . . . . . . . . . . . . . . . . . . . 112

15.2 VTK-m package hierarchy. . . . . . . . . . . . . . . . . . . . . . . 113

21.1 The collection of values for a reduce by key worklet. . . . . . . . . . . . . . . . . . . . . . 175

25.1 Basic Cell Shapes . . . . . . . . . . . . . . . . . . . . . . . . . . . 206

25.2 The constituent elements (points, edges, and faces) of cells. . . . . . . . . . . . . . . . . . . 210

28.1 The angles incident around a point in a mesh. . . . . . . . . . . . . . . . . . . . . . . . . 242

32.1 Duplicate lines from extracted edges. . . . . . . . . . . . . . . . . . . . . . . . 265

35.1 Array handles, storage objects, and the underlying data source. . . . . . . . . . . . . . . . . 307

41.1 Basic shape for the Koch Snowflake. . . . . . . . . . . . . . . . . . . . . . . . 370

41.2 The Koch Snowflake after multiple iterations. . . . . . . . . . . . . . . . . . . . . . . . . . 370

41.3 Parametric coordinates for the Koch Snowflake shape. . . . . . . . . . . . . . . . . . . . . . 370

41.4 Applying the line fractal transform for the Koch Snowflake. . . . . . . . . . . . . . . . . . . . 371

41.5 The quadratic type 2 curve fractal. . . . . . . . . . . . . . . . . . . . . . . . 384

41.6 The tree fractal. . . . . . . . . . . . . . . . . . . . . . . . . . . . . . 385

41.7 The first four iterations of the dragon fractal. . . . . . . . . . . . . . . . . . . . . . . 387

41.8 The dragon fractal after 12 iterations. . . . . . . . . . . . . . . . . . . . . . . . . . 388

41.9 Hilbert curve fractal. . . . . . . . . . . . . . . . . . . . . . . . . 390 


\section{LIST OF EXAMPLES}

2.1 Running CMake on downloaded VTK-m source (Unix commands). . . . . . . . . . . . . . . . . 8

2.2 Using make to build VTK-m. . . . . . . . . . . . . . . . . . . . . . . . . . . 10

2.3 Loading VTK-m configuration from an external CMake project. . . . . . . . . . . . . . . 11

2.4 Linking VTK-m code into an external program. . . . . . . . . . . . . . . . . . . . . 11

2.5 Using an optional component of VTK-m. . . . . . . . . . . . . . . . . . . . . 13

3.1 Initializing VTK-m. . . . . . . . . . . . . . . . . . . . . . . . . . . . . . . . . .

3.2 Reading data from a VTK legacy file. . . . . . . . . . . . . . . . . . . . . . . . 16

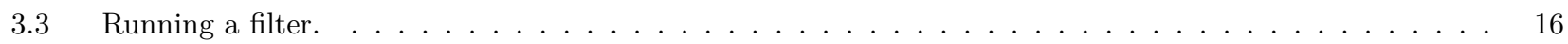

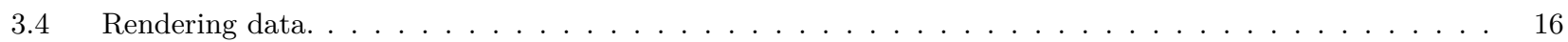

3.5 Simple example of using VTK-m. . . . . . . . . . . . . . . . . . . . . . . . 17

3.6 CMakeLists.txt to build a program using VTK-m. . . . . . . . . . . . . . . . . . . . . 18

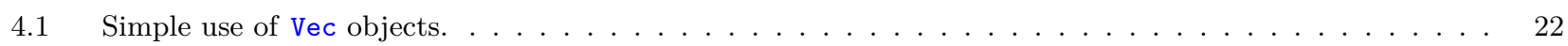

6.1 Calling Initialize. . . . . . . . . . . . . . . . . . . . . . . . 28

7.1 Creating a uniform grid. . . . . . . . . . . . . . . . . . . . . . . . . . . . . . .

7.2 Creating a uniform grid with custom origin and spacing. . . . . . . . . . . . . . . . 30

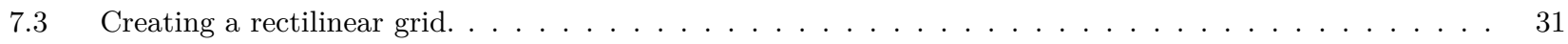

7.4 Creating an explicit mesh with DataSetBuilderExplicit. . . . . . . . . . . . . . 32

7.5 Creating an explicit mesh with DataSetBuilderExplicitIterative. . . . . . . . . . . . . . 34

7.6 Adding fields to a DataSet. . . . . . . . . . . . . . . . . . . . . . . . . 35

7.7 Subsampling a data set with CellsetPermutation. . . . . . . . . . . . . . . . . 39

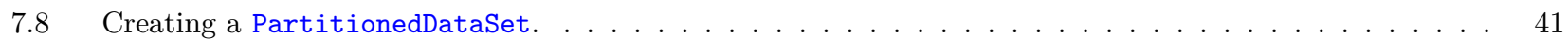

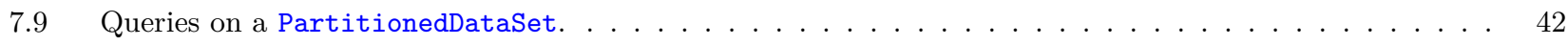

7.10 Applying a filter to multi block data. . . . . . . . . . . . . . . . . . . . . . . . . 42

8.1 Reading a legacy VTK file. . . . . . . . . . . . . . . . . . . . . . . . . 43

8.2 Reading an image from a PNG file. . . . . . . . . . . . . . . . . . . . . . . . . . 44

8.3 Reading an image from a PNM file. . . . . . . . . . . . . . . . . . . . . . . 44 
8.4 Writing a legacy VTK file. . . . . . . . . . . . . . . . . . . . . . . . . . . 45

8.5 Writing an image to a PNG file. . . . . . . . . . . . . . . . . . . . . . . 45

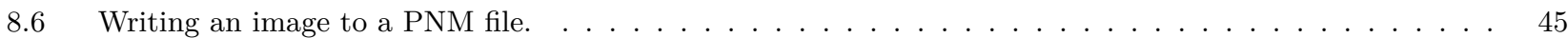

9.1 Using PointElevation, which is a field filter. . . . . . . . . . . . . . . . . . . . 4 47

9.2 Using ClipWithField. . . . . . . . . . . . . . . . . . . . . . . . 50

9.3 Using ClipWithImplicitFunction. . . . . . . . . . . . . . . . . . . . 51

9.4 Using Contour, which is a data set with field filter. . . . . . . . . . . . . . . . . . 55

9.5 Using Streamline, which is a data set with field filter. . . . . . . . . . . . . . . . . . . 69

9.6 Using StreamSurface, which is a data set with field filter. . . . . . . . . . . . . . . . . . . 70

9.7 Using Pathline, which is a data set with field filter. . . . . . . . . . . . . . . . . . . 71

9.8 Using Tube, which is a data set with field filter. . . . . . . . . . . . . . . . . . . 74

9.9 Using VertexClustering. . . . . . . . . . . . . . . . . . . . . 75

9.10 Setting a field's active filter with an association. . . . . . . . . . . . . . . . . . . 78

9.11 Turning off the passing of all fields when executing a filter. . . . . . . . . . . . . . . . . . . . 78

9.12 Setting one field to pass by name. . . . . . . . . . . . . . . . . . . . . . . 79

9.13 Using a list of fields for a filter to pass. . . . . . . . . . . . . . . . . . . . . . 79

9.14 Excluding a list of fields for a filter to pass. . . . . . . . . . . . . . . . . . . . 79

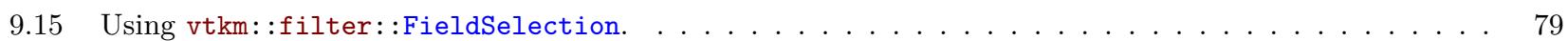

9.16 Selecting one field and its association for a filter to pass. . . . . . . . . . . . . . . . . . . 79

9.17 Selecting a list of fields and their associations for a filter to pass. . . . . . . . . . . . . . . . . . 79

10.1 Creating an Actor and adding it to a Scene. . . . . . . . . . . . . . . . . . 81

10.2 Creating a canvas for rendering. . . . . . . . . . . . . . . . . . . . . . . . . . . . .

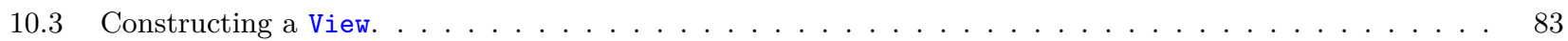

10.4 Changing the background and foreground colors of a View. . . . . . . . . . . . . . . . . 83

10.5 Using Canvas : :Paint in a display callback. . . . . . . . . . . . . . . . . . . . . 83

10.6 Saving the result of a render as an image file. . . . . . . . . . . . . . . . . . . . 84

10.7 Creating a mapper for a wireframe representation. . . . . . . . . . . . . . . . . . . . 84

10.8 Creating a mapper for point representation. . . . . . . . . . . . . . . . . . . . 84

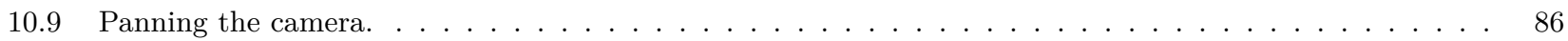

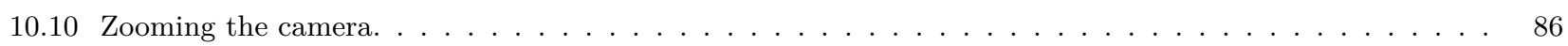

10.11 Directly setting vtkm: :rendering: :Camera position and orientation. . . . . . . . . . . . . . . . 87

10.12 Moving the camera around the look at point. . . . . . . . . . . . . . . . . . . 88

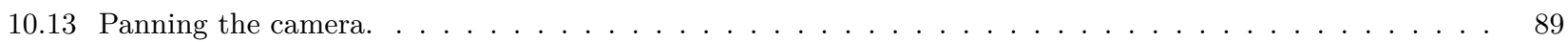

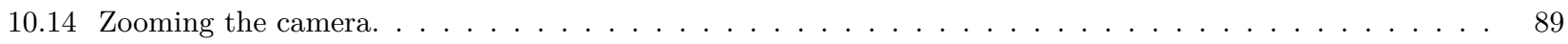

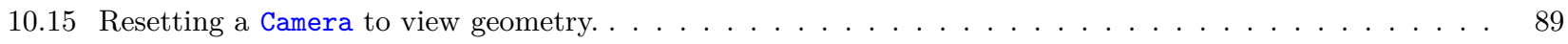

10.16 Resetting a Camera to be axis aligned. . . . . . . . . . . . . . . . . . . . . . . 90

10.17 Rendering a View and pasting the result to an active OpenGL context. . . . . . . . . . . . . . . . 99 
10.18 Interactive rotations through mouse dragging with Camera: :TrackballRotate. . . . . . . . . . . 91

10.19 Pan the view based on mouse movements. . . . . . . . . . . . . . . . . . . . . . . . 992

10.20 Zoom the view based on mouse movements. . . . . . . . . . . . . . . . . . . . . 93

10.21 Specifying a ColorTable for an Actor. . . . . . . . . . . . . . . . . . . . . . . 93

11.1 Simple error reporting. . . . . . . . . . . . . . . . . . . . . . . . . . . 95

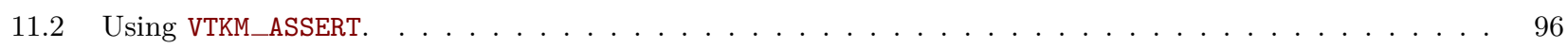

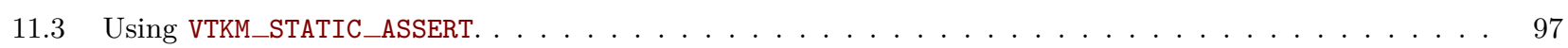

12.1 Specifying a device using a device adapter tag. . . . . . . . . . . . . . . . . . . . . 100

12.2 Restricting which devices VTK-m uses per thread. . . . . . . . . . . . . . . . . . . . . 102

12.3 Disabling a device with RuntimeDeviceTracker. . . . . . . . . . . . . . . . . . 102

13.1 Using vtkm: cont: Timer. . . . . . . . . . . . . . . . . . . . . . . . . . . . . .

14.1 Passing an implicit function to a filter. . . . . . . . . . . . . . . . . . . . 107

15.1 Usage of an environment modifier macro on a function. . . . . . . . . . . . . . . . . . . . . 114

15.2 Suppressing warnings about functions from mixed environments. . . . . . . . . . . . . . . . . . . . 114

16.1 Creating an ArrayHandle for output data . . . . . . . . . . . . . . . . . . . . . . 116

16.2 Creating an ArrayHandle from initially specified values. . . . . . . . . . . . . . . . . . . 116

16.3 Creating a typed ArrayHandle from initially specified values. . . . . . . . . . . . . . . . 116

16.4 Creating an ArrayHandle that points to a provided $\mathrm{C}$ array. . . . . . . . . . . . . . . . . . . 117

16.5 Creating an ArrayHandle that points to a provided std: : vector. . . . . . . . . . . . . . . . 117

16.6 Invalidating an ArrayHandle by letting the source std: : vector leave scope. . . . . . . . . . . . . . 117

16.7 Deep copy ArrayHandles of the same type. . . . . . . . . . . . . . . . . . . . 118

16.8 Using ArrayCopy. . . . . . . . . . . . . . . . . . . . . . . . . . . 118

16.9 Declaration of the vtkm: : cont : : ArrayHandle templated class. . . . . . . . . . . . . 118

16.10 Templating a function on an ArrayHandle's parameters . . . . . . . . . . . . . . . . . . . . . . 119

16.11 A template parameter that should be an ArrayHandle. . . . . . . . . . . . . . . . . . . . . 119

17.1 A simple worklet. . . . . . . . . . . . . . . . . . . . . . . . . . . . . .

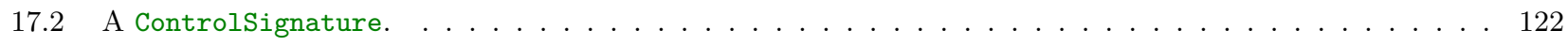

17.3 An ExecutionSignature. . . . . . . . . . . . . . . . . . . . . . . . . . . . . . . . . . . .

17.4 An InputDomain declaration. . . . . . . . . . . . . . . . . . . . . . . . 123

17.5 An overloaded parenthesis operator of a worklet. . . . . . . . . . . . . . . . . . 123

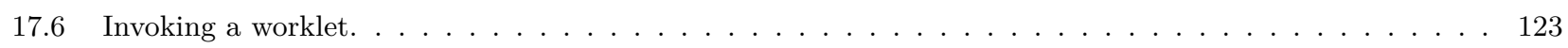

17.7 A more complex worklet. . . . . . . . . . . . . . . . . . . . . . 124

18.1 Header declaration for a simple filter. . . . . . . . . . . . . . . . . . . . . 125

18.2 Constructor for a simple filter. . . . . . . . . . . . . . . . . . . . . . . . . . . . . . . . . . . . .

18.3 Implementation of DoExecute for a simple filter. . . . . . . . . . . . . . . . . . . . . . 126

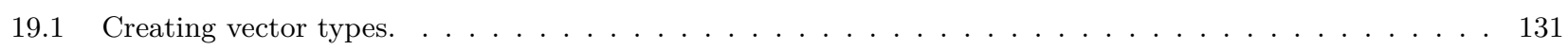

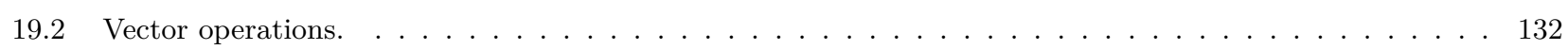




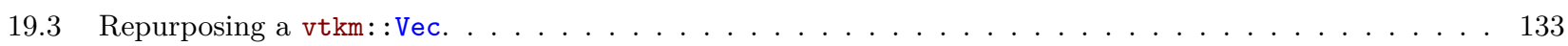

19.4 Using vtkm: :VecCConst with a constant array. . . . . . . . . . . . . . . . . . . 133

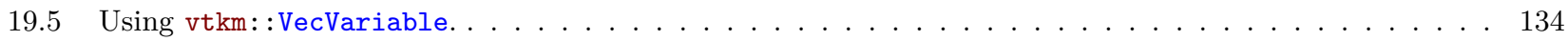

19.6 Using vtkm: :Range. . . . . . . . . . . . . . . . . . . . . . . . 135

19.7 Using vtkm: :Bounds. . . . . . . . . . . . . . . . . . . . . . . 136

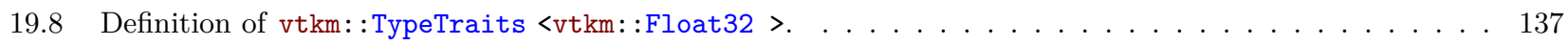

19.9 Using TypeTraits for a generic remainder. . . . . . . . . . . . . . . . . . . . . . 137

19.10 Definition of vtkm: :VecTraits <vtkm: :Id3 > . . . . . . . . . . . . . . . . . 139

19.11 Using VecTraits for less functors. . . . . . . . . . . . . . . . . . . . . . . . 140

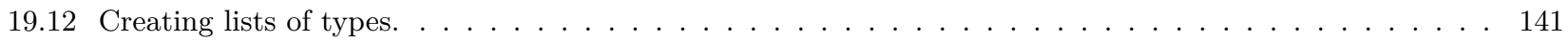

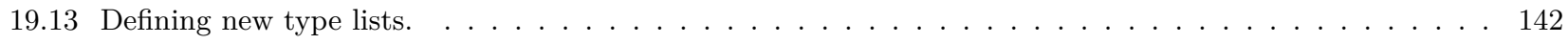

19.14 Checking that a template parameter is a valid List. . . . . . . . . . . . . . . . . . 143

19.15 Getting the size of a List. . . . . . . . . . . . . . . . . . . . . . . . . 143

19.16 Determining if a List contains a particular type. . . . . . . . . . . . . . . . . . . . . . 143

19.17 Using indices with List. . . . . . . . . . . . . . . . . . . . . . . . . 143

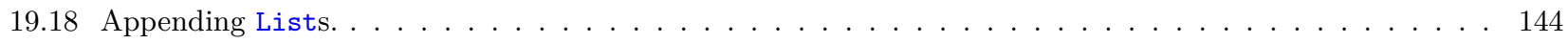

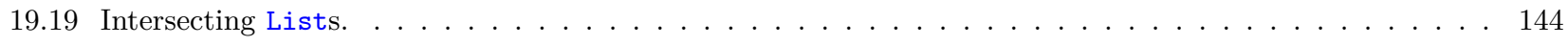

19.20 Applying a List to another template. . . . . . . . . . . . . . . . . . . . . . . . . . . . . . . . .

19.21 Transforming a List using a custom template. . . . . . . . . . . . . . . . . . . . . . . 145

19.22 Removing items from a List. . . . . . . . . . . . . . . . . . . . . . . . . . 145

19.23 Creating the cross product of 2 Lists. . . . . . . . . . . . . . . . . . . . . . . 145

19.24 Converting dynamic types to static types with ListForEach. . . . . . . . . . . . . . . . . . . . 146

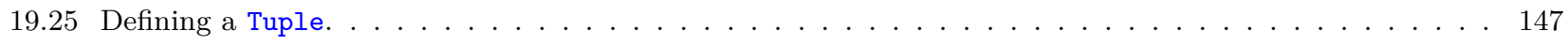

19.26 Initializing values in a Tuple. . . . . . . . . . . . . . . . . . . . . . . . . . . . . . 147

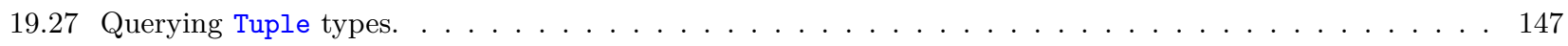

19.28 Retrieving values from a Tuple. . . . . . . . . . . . . . . . . . . . . . . . 148

19.29 Using Tuple: :ForEach to check the contents. . . . . . . . . . . . . . . . . . . . . . . . 148

19.30 Using Tuple: :ForEach to aggregate. . . . . . . . . . . . . . . . . . . . . . 148

19.31 Transforming a Tuple. . . . . . . . . . . . . . . . . . . . . . . . . . . . 149

19.32 Applying a Tuple as arguments to a function. . . . . . . . . . . . . . . . . . . . . . . 149

19.33 Using extra arguments with Tuple: : Apply. . . . . . . . . . . . . . . . . . . . . 150

19.34 Checking an ErrorCode and reporting errors in a worklet. . . . . . . . . . . . . . . . . . . . 151

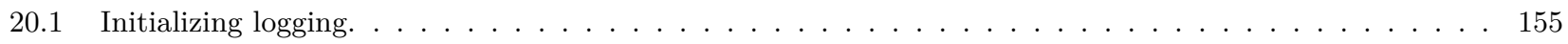

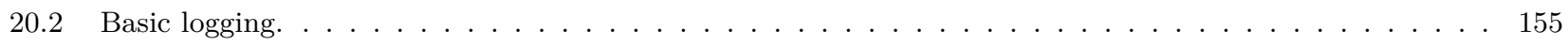

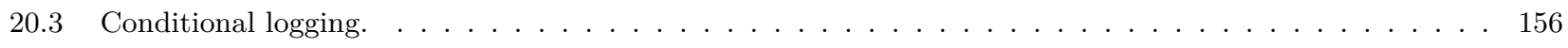

20.4 Scoped logging. . . . . . . . . . . . . . . . . . . . . . . . . . . 156

20.5 Scoped logging in a function. . . . . . . . . . . . . . . . . . . . . . . . . . . . . . . . . . . . . 
20.6 Providing an error context for logging. . . . . . . . . . . . . . . . . . . . . . . 157

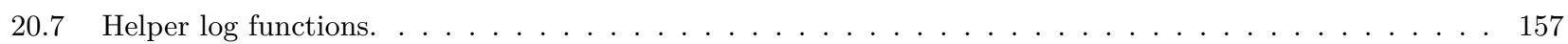

21.1 Implementation and use of a field map worklet. . . . . . . . . . . . . . . . . . . . 161

21.2 Leveraging field maps and field maps for general processing. . . . . . . . . . . . . . . . . . . . 162

21.3 Implementation and use of a visit cells with points worklet. . . . . . . . . . . . . . . . . . 165

21.4 Implementation and use of a visit points with cells worklet. . . . . . . . . . . . . . . . . . . 167

21.5 Retrieve neighborhood field value. . . . . . . . . . . . . . . . . . . . . . . . . 172

21.6 Iterating over the valid portion of a neighborhood. . . . . . . . . . . . . . . . . . . 173

21.7 Implementation and use of a point neighborhood worklet. . . . . . . . . . . . . . . . . . 173

21.8 A helper class to manage histogram bins. . . . . . . . . . . . . . . . . . . . . . . 177

21.9 A simple map worklet to identify histogram bins, which will be used as keys. . . . . . . . . . . 177

21.10 Creating a vtkm: :worklet: Keys object. . . . . . . . . . . . . . . . . 178

21.11 A reduce by key worklet to write histogram bin counts. . . . . . . . . . . . . . . . . . . 178

21.12 A worklet that averages all values with a common key. . . . . . . . . . . . . . . . . . . . . 179

21.13 Using a reduce by key worklet to average values falling into the same bin. . . . . . . . . . . . . . . 179

22.1 Header declaration for a field filter. . . . . . . . . . . . . . . . . . . . . . . 182

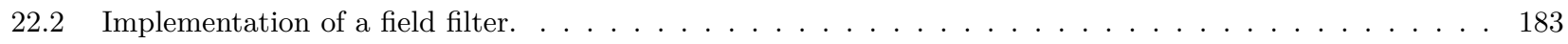

22.3 Header declaration for a field filter using cell topology. . . . . . . . . . . . . . . . . . . . . . . 184

22.4 Implementation of a field filter using cell topology. . . . . . . . . . . . . . . . . . . . . . . . . . 185

22.5 Header declaration for a data set filter. . . . . . . . . . . . . . . . . . . . . . . . 187

22.6 Implementation of the DoExecute method of a data set filter. . . . . . . . . . . . . . . . . . . 188

22.7 Implementation of the DoMapField method of a data set filter. . . . . . . . . . . . . . . . . . 188

22.8 Header declaration for a data set with field filter. . . . . . . . . . . . . . . . . . . . . 190

22.9 Implementation of the DoExecute method of a data set with field filter. . . . . . . . . . . . . . . . 191

22.10 Implementation of the DoMapField method of a data set with field filter. . . . . . . . . . . . . . . . . 192

22.11 Using a policy to get the ArrayHandle out of a Field. . . . . . . . . . . . . . . . . . . . 193

22.12 Using a policy to get the Cellset from a DataSet. . . . . . . . . . . . . . . . . . . . . . 194

22.13 Using a policy to get on structured cell sets. . . . . . . . . . . . . . . . . . . . . 194

23.1 Raising an error in the execution environment. . . . . . . . . . . . . . . . . . . 195

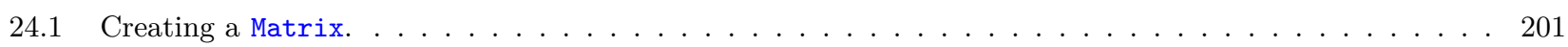

24.2 Using NewtonsMethod to solve a small system of nonlinear equations. . . . . . . . . . . . . . . . . . 203

25.1 Using CellShapeIdToTag. . . . . . . . . . . . . . . . . . . . . . 206

25.2 Using CellTraits to implement a polygon normal estimator. . . . . . . . . . . . . . . . . . . 207

25.3 Interpolating field values to a cell's center. . . . . . . . . . . . . . . . . . . 209

25.4 Computing the derivative of the field at cell centers. . . . . . . . . . . . . . . . . . . 210

25.5 Using cell edge functions. . . . . . . . . . . . . . . . . . . . . . . . . 211

25.6 Using cell face functions. . . . . . . . . . . . . . . . . . . . . . . . . . . . . . 212 
26.1 Using ArrayHandleConstant. . . . . . . . . . . . . . . . . . . . . . . . . . . . 215

26.2 Using make_ArrayHandleConstant. . . . . . . . . . . . . . . . . . . . . . . . 216

26.3 Using ArrayHandleView. . . . . . . . . . . . . . . . . . . . . . . . . . . 216

26.4 Using make_ArrayHandleView. . . . . . . . . . . . . . . . . . . . . . . . 216

26.5 Using ArrayHandleRandomUniformBits. . . . . . . . . . . . . . . . . . . . . . 216

26.6 ArrayHandleRandomUniformBits is functional . . . . . . . . . . . . . . . . . . . . . 217

26.7 Independent ArrayHandleRandomUniformBits. . . . . . . . . . . . . . . . . . . . . . . . . . . 217

26.8 Using ArrayHandleIndex. . . . . . . . . . . . . . . . . . . . . . . . . . . . . 217

26.9 Using ArrayHandleCounting. . . . . . . . . . . . . . . . . . . . . . . . . . . . . . 217

26.10 Using make_ArrayHandleCounting. . . . . . . . . . . . . . . . . . . . . . . . . . 218

26.11 Counting backwards with ArrayHandleCounting. . . . . . . . . . . . . . . . . . . 218

26.12 Using ArrayHandleCounting with vtkm: :Vec objects. . . . . . . . . . . . . . . . . . 218

26.13 Using ArrayHandleCast. . . . . . . . . . . . . . . . . . . . . . . . . . . . 218

26.14 Using make_ArrayHandleCast. . . . . . . . . . . . . . . . . . . . . . . . . . . . . 219

26.15 Using ArrayHandleDiscard. . . . . . . . . . . . . . . . . . . . . . . . . . . . . 219

26.16 Using ArrayHandlePermutation. . . . . . . . . . . . . . . . . . . . . . . . . . 219

26.17 Using make_ArrayHandlePermutation. . . . . . . . . . . . . . . . . . . . . . 220

26.18 Using ArrayHandleZip. . . . . . . . . . . . . . . . . . . . . . . . . . . . . . . . 221

26.19 Using make_ArrayHandleZip. . . . . . . . . . . . . . . . . . . . . . . . . . . . 221

26.20 Using ArrayHandleUniformPointCoordinates. . . . . . . . . . . . . . . . . . . . . . . . . . 222

26.21 Using a ArrayHandleCartesianProduct. . . . . . . . . . . . . . . . . . . . . . . 222

26.22 Using make_ArrayHandleCartesianProduct. . . . . . . . . . . . . . . . . . . . . . 223

26.23 Using ArrayHandleCompositeVector. . . . . . . . . . . . . . . . . . . . . 223

26.24 Using make_ArrayHandleCompositeVector. . . . . . . . . . . . . . . . . . . . . . . 224

26.25 Extracting components of Vecs in an array with ArrayHandleExtractComponent. . . . . . . . . . . . . . 224

26.26 Using make_ArrayHandleExtractComponent. . . . . . . . . . . . . . . . . . . . . . . 225

26.27 Swizzling components of Vecs in an array with ArrayHandleSwizzle. . . . . . . . . . . . . . . . . 225

26.28 Using make_ArrayHandleSwizzle. . . . . . . . . . . . . . . . . . . . . . . . . . 225

26.29 Using ArrayHandleGroupVec. . . . . . . . . . . . . . . . . . . . . . . . 226

26.30 Using make_ArrayHandleGroupVec. . . . . . . . . . . . . . . . . . . . . . . . . 226

26.31 Using ArrayHandleGroupVecVariable. . . . . . . . . . . . . . . . . . . . . . . . . . . 226

26.32 Using MakeArrayHandleGroupVecVariable. . . . . . . . . . . . . . . . . . . . . . . 227

27.1 A simple array portal implementation. . . . . . . . . . . . . . . . . . . . . . . . 229

27.2 Using ArrayPortalToIterators. . . . . . . . . . . . . . . . . . . . . . . 230

27.3 Using ArrayPortalToIteratorBegin and ArrayPortalToIteratorEnd. . . . . . . . . . . . . . . 231

27.4 Using portals from an ArrayHandle. . . . . . . . . . . . . . . . . . . . . . . . . 231

27.5 Allocating an ArrayHandle. . . . . . . . . . . . . . . . . . . . . . . . . . . 232 
27.6 Resizing an ArrayHandle. . . . . . . . . . . . . . . . . . . . . . . . . 232

27.7 Populating a newly allocated ArrayHandle. . . . . . . . . . . . . . . . . . . . . . . . . . 232

27.8 Using ArrayRangeCompute. . . . . . . . . . . . . . . . . . . . . . . 233

27.9 Using an execution array portal from an ArrayHandle. . . . . . . . . . . . . . . . . . . . . 234

28.1 Using WholeArrayIn to access a lookup table in a worklet. . . . . . . . . . . . . . . . . 238

28.2 Using AtomicArrayInOut to count histogram bins in a worklet. . . . . . . . . . . . . . . . . 240

28.3 Using WholeCellSetIn to sum the angles around each point. . . . . . . . . . . . . . . . . . . . . . 242

29.1 Using ExecObject to access a lookup table in a worklet. . . . . . . . . . . . . . . . . . . . 245

30.1 Constructing a Celllocator. . . . . . . . . . . . . . . . . . . . . 250

30.2 Using a Celllocator in a worklet. . . . . . . . . . . . . . . . . . . . 251

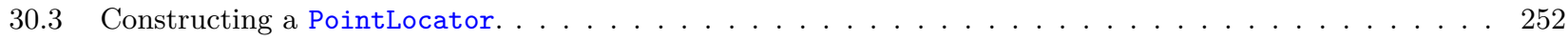

30.4 Using a PointLocator in a worklet. . . . . . . . . . . . . . . . . . . . . . . . . . . . . . . . .

31.1 Declaration of a scatter type in a worklet. . . . . . . . . . . . . . . . . . . . 256

31.2 Invoking with a custom scatter. . . . . . . . . . . . . . . . . . . . . . . 256

31.3 Using ScatterUniform. . . . . . . . . . . . . . . . . . . . . . 257

31.4 Using ScatterCounting. . . . . . . . . . . . . . . . . . . . . . 257

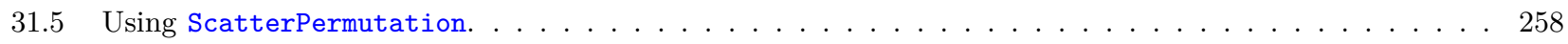

32.1 A simple worklet to count the number of edges on each cell. . . . . . . . . . . . . . . . . 261

32.2 A worklet to generate indices for line cells. . . . . . . . . . . . . . . . . . . . . . . 262

32.3 Invoking worklets to extract edges from a cell set. . . . . . . . . . . . . . . . . . . . 263

32.4 Converting cell fields using a simple permutation. . . . . . . . . . . . . . . . . . . . . . 264

32.5 A simple worklet to count the number of edges on each cell. . . . . . . . . . . . . . . 265

32.6 Worklet generating canonical edge identifiers. . . . . . . . . . . . . . . . . . 266

32.7 A worklet to generate indices for line cells from combined edges. . . . . . . . . . . . . . . . . 266

32.8 Invoking worklets to extract unique edges from a cell set. . . . . . . . . . . . . . . . . . . 268

32.9 Converting cell fields that average collected values. . . . . . . . . . . . . . . . . . . . . 269

32.10 A simple worklet to count the number of edges on each cell. . . . . . . . . . . . . . . . . . . 270

32.11 Worklet generating hash values. . . . . . . . . . . . . . . . . . . . . 270

32.12 Worklet to resolve hash collisions occurring on edge identifiers. . . . . . . . . . . . . . . . . . 271

32.13 A worklet to generate indices for line cells from combined edges and potential collisions. . . . . . . . . 273

32.14 Invoking worklets to extract unique edges from a cell set using hash values. . . . . . . . . . . . . . . . 274

32.15 A worklet to average values with the same key, resolving for collisions. . . . . . . . . . . . . . . 275

32.16 Invoking the worklet to process cell fields, resolving for collisions. . . . . . . . . . . . . . . . . . . 276

32.17 A worklet to count the points in the final cells of extracted faces . . . . . . . . . . . . . . . 277

32.18 Converting counts of connectivity groups to offsets for ArrayHandleGroupVecVariable. . . . . . . . . . . 278

32.19 A worklet to generate indices for polygon cells of different sizes from combined edges and potential collisions.278

32.20 Invoking worklets to extract unique faces from a cell set. . . . . . . . . . . . . . . . . . . . . . 279 
33.1 Creating an UnknownArrayHandle. . . . . . . . . . . . . . . . . . . . . . . . . 283

33.2 Checking the size of an ArrayHandle and resizing it. . . . . . . . . . . . . . . . . . . . . . . . 284

33.3 Creating a new instance of an unknown array handle. . . . . . . . . . . . . . . . . . . . . . 284

33.4 Creating a new basic instance of an unknown array handle. . . . . . . . . . . . . . . . . . . . 284

33.5 Creating a new array instance with floating point values. . . . . . . . . . . . . . . . . . . 284

33.6 Retrieving an array of a known type from UnknownArrayHandle. . . . . . . . . . . . . . . . . . . 285

33.7 Alternate form for retrieving an array of a known type from UnknownArrayHandle. . . . . . . . . . . . . 285

33.8 Getting a cast array handle from an ArrayHandleCast. . . . . . . . . . . . . . . . . . . . . . 285

33.9 Querying whether a given ArrayHandle can be retrieved from an UnknownArrayHandle. . . . . . . . . . . 285

33.10 Deep copy arrays of unknown types. . . . . . . . . . . . . . . . . . . . . . . . . 286

33.11 Using ArrayCopyShallowIfPossible to get an unknown array as a particular type. . . . . . . . . . . 286

33.12 Operating on an UnknownArrayHandle with CastAndCallForTypes. . . . . . . . . . . . . . . . . . . 287

33.13 Using UncertainArrayHandle to cast and call a functor. . . . . . . . . . . . . . . . . . . . . . . . 288

33.14 Resetting the types of an UnknownArrayHandle. . . . . . . . . . . . . . . . . . . . . . . . . 288

33.15 Extracting the first component of every value in an UnknownArrayHandle. . . . . . . . . . . . . . . . . 289

33.16 Checking the base component type in an UnknownArrayHandle. . . . . . . . . . . . . . . . . . . . 289

33.17 Extracting each component from an UnknownArrayHandle. . . . . . . . . . . . . . . . . . . 290

33.18 Extracting all components from an UnknownArrayHandle at once. . . . . . . . . . . . . . . . . . . . 290

33.19 Calling a functor for nearly any type of array stored in an UnknownArrayHandle. . . . . . . . . . . . . . 291

33.20 Using a const UnknownArrayHandle for a function output. . . . . . . . . . . . . . . . . . . . . . . . 291

33.21 Passing an ArrayHandle as an output UnknownArrayHandle. . . . . . . . . . . . . . . . . . . . . . . 291

34.1 Using the BitFieldToUnorderedSet algorithm. . . . . . . . . . . . . . . . . . . 293

34.2 Using the Copy algorithm. . . . . . . . . . . . . . . . . . . . . . . . . . . 294

34.3 Using the CopyIf algorithm. . . . . . . . . . . . . . . . . . . . . . . . . . . . . 294

34.4 Using the CopySubRange algorithm. . . . . . . . . . . . . . . . . . . . . . . . . . . 295

34.5 Using the CountSetBits algorithm. . . . . . . . . . . . . . . . . . . . . 295

34.6 Using the Fill algorithm. . . . . . . . . . . . . . . . . . . . . . . . . . . . . 295

34.7 Using the LowerBounds algorithm. . . . . . . . . . . . . . . . . . . . . . . . . . . 296

34.8 Using the Reduce algorithm. . . . . . . . . . . . . . . . . . . . . . . . . . 296

34.9 Using the ReduceByKey algorithm. . . . . . . . . . . . . . . . . . . . . . . . . . . 297

34.10 Using the ScanInclusive algorithm. . . . . . . . . . . . . . . . . . . . . . . . . . . 297

34.11 Using the ScanInclusiveByKey algorithm. . . . . . . . . . . . . . . . . . . . . . . . 298

34.12 Using the ScanExclusive algorithm. . . . . . . . . . . . . . . . . . . . . . . 298

34.13 Using ScanExclusiveByKey algorithm. . . . . . . . . . . . . . . . . . . . . . . . 299

34.14 Using ScanExtended algorithm. . . . . . . . . . . . . . . . . . . . . . . 300

34.15 Using the Sort algorithm. . . . . . . . . . . . . . . . . . . . . . . . . . . 300

34.16 Using the SortByKey algorithm. . . . . . . . . . . . . . . . . . . . . . . . . . 301 
34.17 Using the Transform algorithm. . . . . . . . . . . . . . . . . . . . . . . . . . 301

34.18 Using the Unique algorithm. . . . . . . . . . . . . . . . . . . . . . . . . . . . . 302

34.19 Using the UpperBounds algorithm. . . . . . . . . . . . . . . . . . . . . . . . . . 302

34.20 Using the DeviceAdapter with vtkm: : cont: : Algorithm. . . . . . . . . . . . . . 303

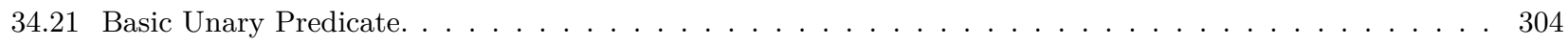

34.22 Basic Binary Predicate. . . . . . . . . . . . . . . . . . . . . . . . 304

34.23 Basic Binary Operator. . . . . . . . . . . . . . . . . . . . . . . . 305

34.24 Custom Unary Predicate Implementation. . . . . . . . . . . . . . . . . . . . . . . . . . 306

34.25 Custom Unary Predicate Usage. . . . . . . . . . . . . . . . . . . . . . . . 306

35.1 Declaration of the vtkm: : cont: : ArrayHandle templated class (again). . . . . . . . . . . . . 307

35.2 Specifying the storage type for an ArrayHandle. . . . . . . . . . . . . . . . . . 308

35.3 Functor that doubles an index. . . . . . . . . . . . . . . . . . . . . . . . . . . . . . . .

35.4 Declaring a ArrayHandleImplicit. . . . . . . . . . . . . . . . . . . . 309

35.5 Using make_ArrayHandleImplicit. . . . . . . . . . . . . . . . . . . . . . . 309

35.6 Custom implicit array handle for even numbers. . . . . . . . . . . . . . . . . . . . . . . . 310

35.7 Functor to scale and bias a value. . . . . . . . . . . . . . . . . . . . . . 310

35.8 Using make_ArrayHandleTransform. . . . . . . . . . . . . . . . . . . . . . . 311

35.9 Custom transform array handle for scale and bias. . . . . . . . . . . . . . . . . . . . . . . 311

35.10 Functor that interlaces two array portals. . . . . . . . . . . . . . . . . . . . . 313

35.11 Inverse functor for writing data to interlaced array portals. . . . . . . . . . . . . . . . . . 313

35.12 Decorator implementation class for interleaving ArrayHandles. . . . . . . . . . . . . . . . . . . . . 314

35.13 Custom decorator array handle for interleaving arrays. . . . . . . . . . . . . . . . . . 315

35.14 Derived array portal for concatenated arrays. . . . . . . . . . . . . . . . . . . . 317

35.15 Prototype for vtkm: : cont: : internal: :Storage. . . . . . . . . . . . . . . . . . 318

35.16 Storage for derived container of interlaced arrays. . . . . . . . . . . . . . . . . . . . . . 319

35.17 ArrayHandle for derived storage of concatenated arrays. . . . . . . . . . . . . . . . . . . . . 322

35.18 Helper function for creating a custom derived ArrayHandle. . . . . . . . . . . . . . . . . . . . . . 323

35.19 Fictitious field storage used in custom array storage examples. . . . . . . . . . . . . . . . . 323

35.20 Array portal to adapt a third-party container to VTK-m. . . . . . . . . . . . . . . . . . . . . . 326

35.21 Prototype for vtkm: : cont: : internal: :Storage. . . . . . . . . . . . . . . . . . 326

35.22 Storage to adapt a third-party container to VTK-m. . . . . . . . . . . . . . . . . . . . . 327

35.23 Array handle to adapt a third-party container to VTK-m. . . . . . . . . . . . . . . . . . . . . . . . 329

35.24 Memory handling functions to adapt a third-party data structure to ArrayHandle. . . . . . . . . . . . . 329

35.25 Using an ArrayHandle with custom container. . . . . . . . . . . . . . . . . . . . . 330

36.1 Running all regression tests (Unix commands). . . . . . . . . . . . . . . . . . . . . 331

36.2 List all available regression tests (Unix commands) . . . . . . . . . . . . . . . . . . . . 331

36.3 Running a single regression test (Unix commands) . . . . . . . . . . . . . . . . . . . . . . 332 
36.4 Running a single regression test with verbose output (Unix commands). The verbose output will first give the exact command used to run the regression test, along with detailed test progression information. 332

36.5 Running a single regression test without calling ctest (Unix commands). . . . . . . . . . . . . . . 332

36.6 Adding test data to the VTK-m repository (Unix commands) . . . . . . . . . . . . . . . . . . 332

37.1 A function to find the average value of an array in parallel. . . . . . . . . . . . . . . . . 335

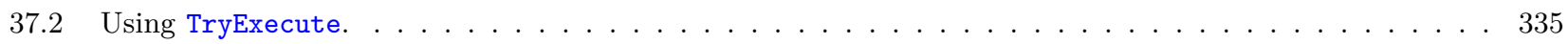

38.1 Contents of the base header for a device adapter. . . . . . . . . . . . . . . . . . . . . . 337

38.2 Implementation of a device adapter tag. . . . . . . . . . . . . . . . . . . . . . . . . . 338

38.3 Modification of DeviceAdapterListCommon in DeviceAdapterList.h . . . . . . . . . . . . . . . 338

38.4 Prototype for DeviceAdapterRuntimeDetector. . . . . . . . . . . . . . . . . . . 338

38.5 Implementation of DeviceAdapterRuntimeDetector specialization . . . . . . . . . . . . . . . 339

38.6 Prototype for DeviceAdapterMemoryManager. . . . . . . . . . . . . . . . . . . . . . 339

38.7 Specialization of DeviceAdapterMemoryManager. . . . . . . . . . . . . . . . . . . . 340

38.8 Prototype for RuntimeDeviceConfiguration. . . . . . . . . . . . . . . . . . . . . . . 341

38.9 Specialization of RuntimeDeviceConfiguration. . . . . . . . . . . . . . . . . . . . . . . . 342

38.10 Prototype for vtkm: : cont: : internal: :VirtualobjectTransfer. . . . . . . . . . . . . . . 344

38.11 Specialization of VirtualobjectTransfer. . . . . . . . . . . . . . . . . . . . . . . . . . . . . .

38.12 Minimal specialization of DeviceAdapterAlgorithm. . . . . . . . . . . . . . . . . 347

38.13 Specialization of DeviceAdapterTimerImplementation. . . . . . . . . . . . . . . . . . . 350

39.1 Declaring vtkm: :internal : FunctionInterface. . . . . . . . . . . . . . . . . . 353

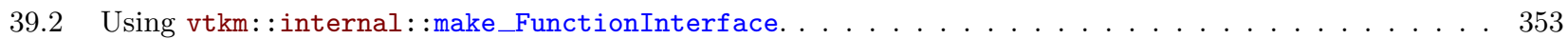

39.3 Getting the arity of a FunctionInterface. . . . . . . . . . . . . . . . . . . . . 354

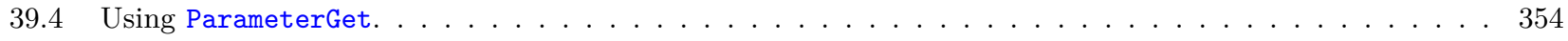

39.5 Using a static transform of function interface class. . . . . . . . . . . . . . . . . . . . . . . 354

40.1 Behavior of vtkm: : cont: :arg: :TypeCheck. . . . . . . . . . . . . . . . . . . 358

40.2 Defining a custom TypeCheck. . . . . . . . . . . . . . . . . . . . . . . . 359

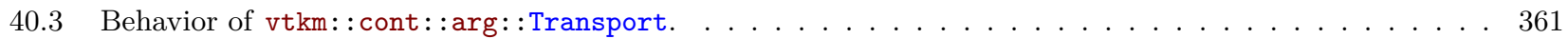

40.4 Defining a custom Transport. . . . . . . . . . . . . . . . . . . . . . . . . . . . . . . .

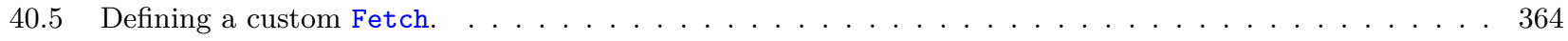

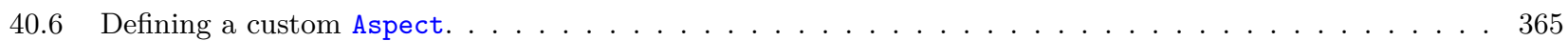

40.7 Defining a new Controlsignature tag. . . . . . . . . . . . . . . . . . . . . . . 366

40.8 Using a custom ControlSignature tag. . . . . . . . . . . . . . . . . . . . . . . 366

40.9 Defining a new ExecutionSignature tag. . . . . . . . . . . . . . . . . . . . 367

40.10 Using a custom ExecutionSignature tag. . . . . . . . . . . . . . . . . . . . . . 367

41.1 A support class for a line fractal worklet. . . . . . . . . . . . . . . . . . . . 370

41.2 Demonstration of how we want to use the line fractal worklet. . . . . . . . . . . . . . . . . . 372

41.3 Implementation of GetThreadIndices in a worklet superclass. . . . . . . . . . . . . . . . . . . . . 373 
41.4 Implementation of a thread indices class. . . . . . . . . . . . . . . . . . . . . . . . . 374

41.5 Custom ControlSignature tag for the input domain of our example worklet type. . . . . . . . . . . 375

41.6 A Fetch for an aspect that does not depend on any control argument. . . . . . . . . . . . . . . . 375

41.7 Custom ExecutionSignature tag that only relies on input domain information in the thread indices. . . 376

41.8 Output ControlSignature tag for our motivating example. . . . . . . . . . . . . . . . . 376

41.9 Implementation of Transport for the output in our motivating example. . . . . . . . . . . . . . . . . 376

41.10 Implementing a FieldIn tag. . . . . . . . . . . . . . . . . . . . . . . . . . . . 377

41.11 Superclass for a new type of worklet. . . . . . . . . . . . . . . . . . . . . . . . . . 377

41.12 Standard template arguments for a dispatcher class. . . . . . . . . . . . . . . . . . . 380

41.13 Subclassing DispatcherBase. . . . . . . . . . . . . . . . . . . . . . . 380

41.14 Typical constructor for a dispatcher. . . . . . . . . . . . . . . . . . . . . . 381

41.15 Declaration of DoInvoke of a dispatcher. . . . . . . . . . . . . . . . . . . . . . . 381

41.16 Checking the input domain tag and type. . . . . . . . . . . . . . . . . . . . . . . 381

41.17 Calling BasicInvoke from a dispatcher's DoInvoke. . . . . . . . . . . . . . . . . . . . . . . . . 382

41.18 Implementation of a dispatcher for a new type of worklet. . . . . . . . . . . . . . . . . . . . . 382

41.19 A worklet to generate a quadratic type 2 curve fractal. . . . . . . . . . . . . . . . . . . . 384

41.20 A worklet to generate a tree fractal. . . . . . . . . . . . . . . . . . . . . . . . . . . . . . . . . . .

41.21 A worklet to generate the dragon fractal. . . . . . . . . . . . . . . . . . . . . . 387

41.22 A worklet to generate the Hilbert curve. . . . . . . . . . . . . . . . . . . . . . . . . . 390 



\section{Part I}

\section{Getting Started}





\section{INTRODUCTION}

High-performance computing relies on ever finer threading. Advances in processor technology include ever greater numbers of cores, hyperthreading, accelerators with integrated blocks of cores, and special vectorized instructions, all of which require more software parallelism to achieve peak performance. Traditional visualization solutions cannot support this extreme level of concurrency. Extreme scale systems require a new programming model and a fundamental change in how we design algorithms. To address these issues we created VTK-m: the visualization toolkit for multi-/many-core architectures.

VTK-m supports a number of algorithms and the ability to design further algorithms through a top-down design with an emphasis on extreme parallelism. VTK-m also provides support for finding and building links across topologies, making it possible to perform operations that determine manifold surfaces, interpolate generated values, and find adjacencies. Although VTK-m provides a simplified high-level interface for programming, its template-based code removes the overhead of abstraction.

VTK-m simplifies the development of parallel scientific visualization algorithms by providing a framework of supporting functionality that allows developers to focus on visualization operations. Consider the listings in Figure 1.1 that compares the size of the implementation for the Marching Cubes algorithm in VTK-m with the equivalent reference implementation in the CUDA software development kit. Because VTK-m internally manages the parallel distribution of work and data, the VTK-m implementation is shorter and easier to maintain. Additionally, VTK-m provides data abstractions not provided by other libraries that make code written in VTK-m more versatile.

\subsection{How to Use This Guide}

This user's guide is organized into 5 parts to help guide novice to advanced users and to provide a convenient reference. Part I, Getting Started, provides a brief overview of using VTK-m. This part provides instructions on building VTK-m and some simple examples of using VTK-m. Users new to VTK-m are well served to read through Part I first to become acquainted with the basic concepts.

The remaining parts, which provide detailed documentation of increasing complexity, have chapters that do not need to be read in detail. Readers will likely find it useful to skip to specific topics of interest.

Part II, Using VTK-m, dives deeper into the VTK-m library. It provides much more detail on the concepts introduced in Part I and introduces new topics helpful to people who use VTK-m's existing algorithms.

Part III, Developing Algorithms, documents how to use VTK-m's framework to develop new or custom visualization algorithms. In this part we dive into the inner workings of filters and introduce the concept of a worklet, which is the base unit used to write a device-portable algorithm in VTK-m. Part III also documents many supporting functions that are helpful in implementing visualization algorithms. 


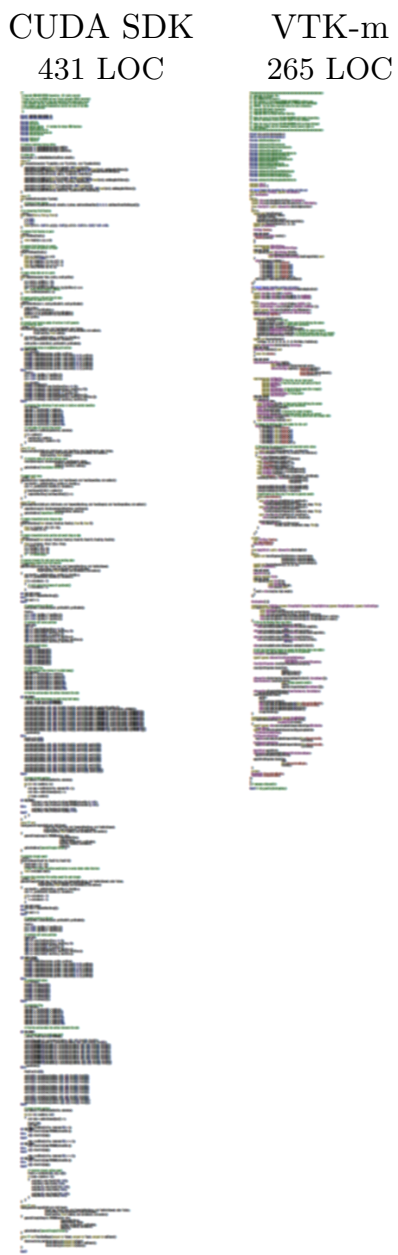

Figure 1.1: Comparison of the Marching Cubes algorithm in VTK-m and the reference implementation in the CUDA SDK. Implementations in VTK-m are simpler, shorter, more general, and easier to maintain. (Lines of code (LOC) measurements come from cloc.)

Part IV, Advanced Development, explores in more detail how VTK-m manages memory and devices. This information describes how to adapt VTK-m to custom data structures and new devices.

Part V, Core Development, exposes the inner workings of VTK-m. These concepts allow you to design new algorithmic structures not already available in VTK-m.

\subsection{Conventions Used in This Guide}

When documenting the VTK-m API, the following conventions are used.

- Filenames are printed in a sans serif font.

- $\mathrm{C}++$ code is printed in a monospace font.

- Macros and namespaces from VTK-m are printed in red. 
- Identifiers from VTK-m are printed in blue.

- Signatures, described in Chapter 17, and the tags used in them are printed in green.

This guide provides actual code samples throughout its discussions to demonstrate their use. These examples are all valid code that can be compiled and used although it is often the case that code snippets are provided. In such cases, the code must be placed in a larger context.

\section{(0) Did you know?}

\In this guide we periodically use these Did you know? boxes to provide additional information related to \& the topic at hand.

Common Errors

¿ Common Errors blocks are used to highlight some of the common problems or complications you might \& encounter when dealing with the topic of discussion. 



\section{BUILD AND INSTALL VTK-M}

Before we begin describing how to develop with VTK-m, we have a brief overview of how to build VTK-m, optionally install it on your system, and start your own programs that use VTK-m.

\subsection{Getting VTK-m}

VTK-m is an open source software product where the code is made freely available. To get the latest released version of VTK-m, go to the VTK-m releases page:

http://m.vtk.org/index.php/VTK-m_Releases

From there with your favorite browser you may download the source code from any of the recent VTK-m releases in a variety of different archive files such as zip or tar gzip.

For access to the most recent work, the VTK-m development team provides public anonymous read access to their main source code repository. The main VTK-m repository on a GitLab instance hosted at Kitware, Inc. The repository can be browsed from its project web page:

https://gitlab.kitware.com/vtk/vtk-m

We leave access to the githosted repository as an exercise for the user. Those interested in git access for the purpose of contributing to VTK-m should consult the CONTRIBUTING guidelines documented in the source code. $^{1}$

\subsection{Configure VTK-m}

VTK-m uses a cross-platform configuration tool named CMake to simplify the configuration and building across many supported platforms. CMake is available from many package distribution systems and can also be downloaded for many platforms from http://cmake.org.

Most distributions of CMake come with a convenient GUI application (cmake-gui) that allows you to browse all of the available configuration variables and run the configuration. Many distributions also come with an alternative terminal-based version (ccmake), which is helpful when accessing remote systems where creating GUI windows is difficult.

${ }^{1}$ https://gitlab.kitware.com/vtk/vtk-m/blob/master/CONTRIBUTING.md 
One helpful feature of CMake is that it allows you to establish a build directory separate from the source directory, and the VTK-m project requires that separation. Thus, when you run CMake for the first time, you want to set the build directory to a new empty directory and the source to the downloaded or cloned files. The following example shows the steps for the case where the VTK-m source is cloned from the git repository. (If you extracted files from an archive downloaded from the VTK-m web page, the instructions are the same from the second line down.)

Example 2.1: Running CMake on downloaded VTK-m source (Unix commands).
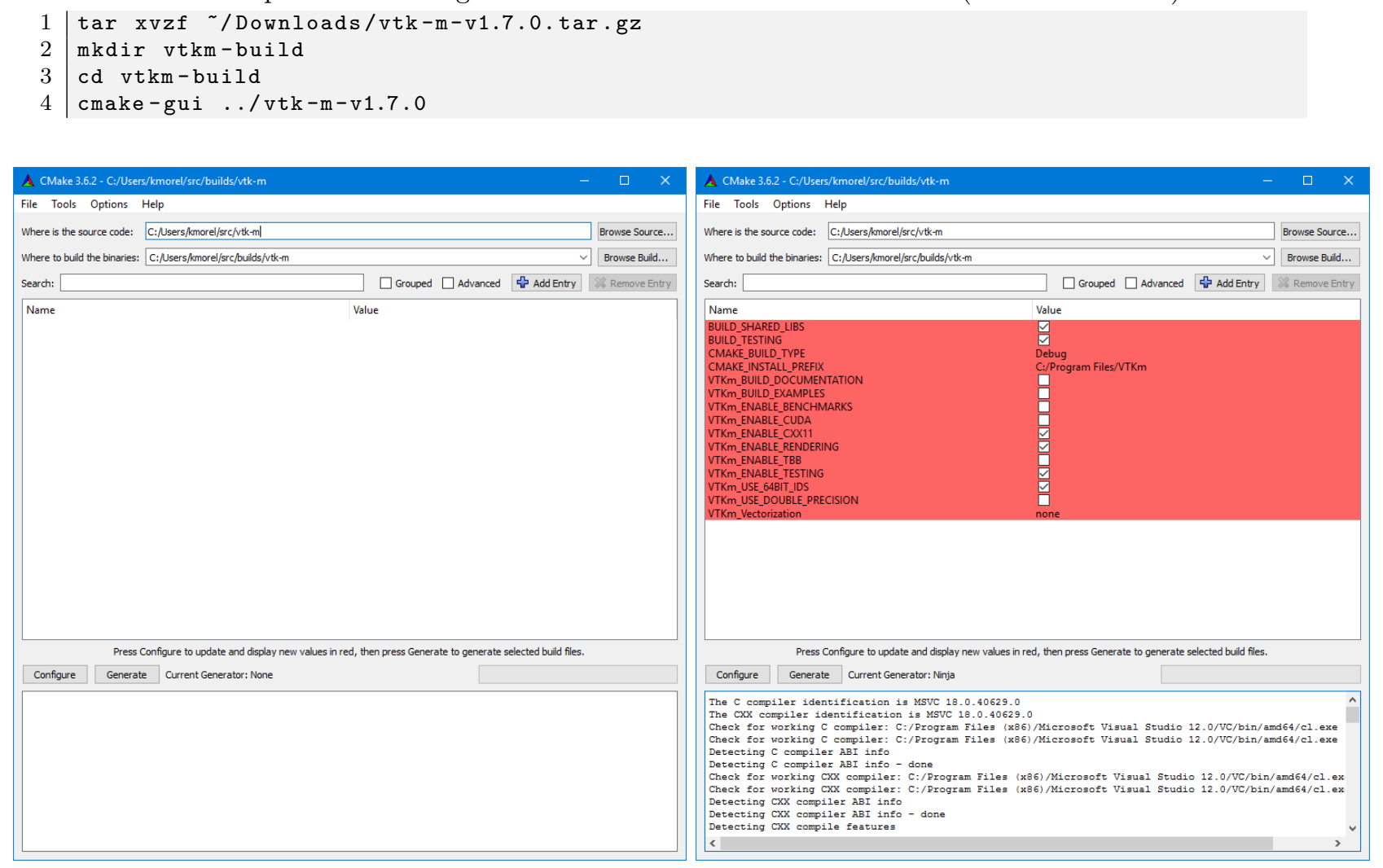

Figure 2.1: The CMake GUI configuring the VTK-m project. At left is the initial blank configuration. At right is the state after a configure pass.

The first time the CMake GUI runs, it initially comes up blank as shown at left in Figure 2.1. Verify that the source and build directories are correct (located at the top of the GUI) and then click the "Configure" button near the bottom. The first time you run configure, CMake brings up a dialog box asking what generator you want for the project. This allows you to select what build system or IDE to use (e.g. make, ninja, Visual Studio). Once you click "Finish," CMake will perform its first configuration. Don't worry if CMake gives an error about an error in this first configuration process.

\section{Sommon Broms \\ 3 Most options in CMake can be reconfigured at any time, but not the compiler and build system used. These must be set the first time configure is run and cannot be subsequently changed. If you want to change the compiler or the project file types, you will need to delete everything in the build directory and start over.}


After the first configuration, the CMake GUI will provide several configuration options as shown in Figure 2.1 on the right. You now have a chance to modify the configuration of VTK-m, which allows you to modify both the behavior of the compiled VTK-m code as well as find components on your system. Using the CMake GUI is usually an iterative process where you set configuration options and re-run "Configure." Each time you configure, CMake might find new options, which are shown in red in the GUI.

It is often the case during this iterative configuration process that configuration errors occur. This can occur after a new option is enabled but CMake does not automatically find the necessary libraries to make that feature possible. For example, to enable TBB support, you may have to first enable building TBB, configure for TBB support, and then tell CMake where the TBB include directories and libraries are.

Once you have set all desired configuration variables and resolved any CMake errors, click the "Generate" button. This will create the build files (such as makefiles or project files depending on the generator chosen at the beginning). You can then close the CMake GUI.

There are a great number of configuration parameters available when running CMake on VTK-m. The following list contains the most common configuration parameters.

BUILD_SHARED_LIBS Determines whether static or shared libraries are built.

CMAKE_BUILD_TYPE Selects groups of compiler options from categories like Debug and Release. Debug builds are, obviously, easier to debug, but they run much slower than Release builds. Use Release builds whenever releasing production software or doing performance tests.

CMAKE_INSTALL_PREFIX The root directory to place files when building the install target.

VTKm_ENABLE_EXAMPLES The VTK-m repository comes with an examples directory. This macro determines whether they are built.

VTKm_ENABLE_BENCHMARKS If on, the VTK-m build includes several benchmark programs. The benchmarks are regression tests for performance.

VTKm_ENABLE_CUDA Determines whether VTK-m is built to run on CUDA GPU devices.

VTKm_CUDA_Architecture Specifies what GPU architecture(s) to build CUDA for. The options include native, fermi, kepler, maxwell, pascal, volta, and turing.

VTKm_ENABLE_OPENMP Determines whether VTK-m is built to run on multi-core devices using OpenMP pragmas provided by the $\mathrm{C}++$ compiler.

VTKm_ENABLE_RENDERING Determines whether to build the rendering library.

VTKm_ENABLE_TBB Determines whether VTK-m is built to run on multi-core $\mathrm{x} 86$ devices using the Intel Threading Building Blocks library.

VTKm_ENABLE_LOGGING If on, the VTK-m library will output log messages to std-out/std-err. This can be particularly useful when debugging your source code or understanding what is going on in VTK-m

VTKm_ENABLE_TESTING If on, the VTK-m build includes building many test programs. The VTK-m source includes hundreds of regression tests to ensure quality during development.

VTKm_USE_64BIT_IDS If on, then VTK-m will be compiled to use 64-bit integers to index arrays and other lists. If off, then VTK-m will use 32-bit integers. 32-bit integers take less memory but could cause failures on larger data.

VTKm_USE_DOUBLE_PRECISION If on, then VTK-m will use double precision (64-bit) floating point numbers for calculations where the precision type is not otherwise specified. If off, then single precision (32-bit) floating point numbers are used. Regardless of this setting, VTK-m's templates will accept either type. 


\subsection{Building VTK-m}

Once CMake successfully configures VTK-m and generates the files for the build system, you are ready to build VTK-m. As stated earlier, CMake supports generating configuration files for several different types of build tools. Make and ninja are common build tools, but CMake also supports building project files for several different types of integrated development environments such as Microsoft Visual Studio and Apple XCode.

The VTK-m libraries and test files are compiled when the default build is invoked. For example, if Makefiles were generated, the build is invoked by calling make in the build directory. Expanding on Example 2.1

Example 2.2: Using make to build VTK-m.

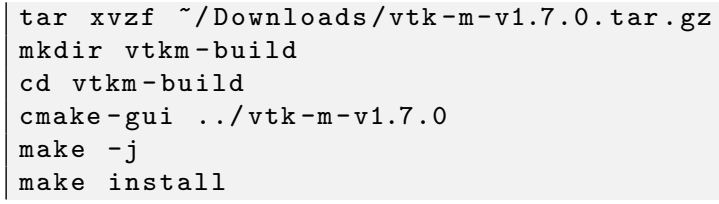

3 The Makefiles and other project files generated by CMake support parallel builds, which run multiple compile steps simultaneously. On computers that have multiple processing cores (as do almost all modern computers), this can significantly speed up the overall compile. Some build systems require a special flag to ¿ engage parallel compiles. For example, make requires the -j flag to start parallel builds as demonstrated in Example 2.2.

\section{Did you know?}

¿ Example 2.2 assumes that a make build system was generated, which is the default on most system. However, CMake supports many more build systems, which use different commands to run the build. If you are not sure what the appropriate build command is, you can run cmake --build to allow CMake to start the build using whatever build system is being used.

\section{Common Errors}

CMake allows you to switch between several types of builds including default, Debug, and Release. Programs and libraries compiled as release builds can run much faster than those from other types of builds. Thus, it is important to perform Release builds of all software released for production or where runtime is a concern. Some integrated development environments such as Microsoft Visual Studio allow you to specify the different build types within the build system. But for other build programs, like make, you have to specify the build type in the CMAKE_BUILD_TYPE CMake configuration variable, which is described in Section 2.2. 
CMake creates several build "targets" that specify the group of things to build. The default target builds all of VTK-m's libraries as well as tests, examples, and benchmarks if enabled. The test target executes each of the VTK-m regression tests and verifies they complete successfully on the system. The install target copies the subset of files required to use VTK-m to a common installation directory. The install target may need to be run as an administrator user if the installation directory is a system directory.

\section{D) Did you know?}

VTK-m contains a significant amount of regression tests. If you are not concerned with testing a build on a given system, you can turn off building the testing, benchmarks, and examples using the CMake configuration variables described in Section 2.2. This can shorten the VTK-m compile time.

\subsection{Linking to VTK-m}

Ultimately, the value of VTK-m is the ability to link it into external projects that you write. The header files and libraries installed with VTK-m are typical, and thus you can link VTK-m into a software project using any type of build system. However, VTK-m comes with several CMake configuration files that simplify linking VTK-m into another project that is also managed by CMake. Thus, the documentation in this section is specifically for finding and configuring VTK-m for CMake projects.

VTK-m can be configured from an external project using the find_package CMake function. The behavior and use of this function is well described in the CMake documentation. The first argument to find_package is the name of the package, which in this case is VTKm. CMake configures this package by looking for a file named VTKmConfig.cmake, which will be located in the lib/cmake/vtkm-1.7 directory of the install or build of VTK-m. The configurable CMake variable CMAKE_PREFIX_PATH can be set to to the build or install directory, or VTKm_DIR can be set to the directory that contains this file.

Example 2.3: Loading VTK-m configuration from an external CMake project.

1 find_package(VTKm REQUIRED)

\section{8) Did you know?}

S The CMake find_package function also supports several features not discussed here including specifying \{ a minimum or exact version of VTK-m and turning off some of the status messages. See the CMake documentation for more details.

When you load the VTK-m package in CMake, several libraries are defined. Projects building with VTK-m components should link against one or more of these libraries as appropriate, typically with the target_link_libraries command.

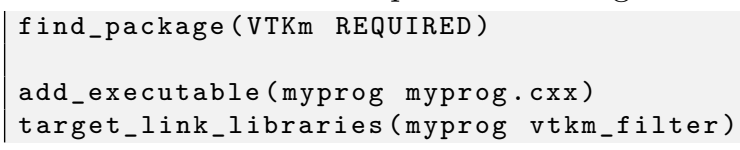

Example 2.4: Linking VTK-m code into an external program.

Several library targets are provided, but most projects will need to link in one or more of the following. 
vtkm_cont Contains the base objects used to control VTK-m. This library must always be linked in.

vtkm_filter Contains VTK-m's pre-built filters including but not limited to CellAverage, CleanGrid, Contour, ExternalFaces, and PointAverage. Applications that are looking to use VTK-m filters will need to link to this library.

vtkm_io Contains VTK-m's facilities for interacting with files. For example, reading and writing png, NetBPM, and VTK files.

vtkm_rendering Contains VTK-m's rendering components. This library is only available if VTKm_ENABLE_RENDERING is set to true.

vtkm_source Contains VTK-m's pre-built dataset generators including but not limited to Wavelet, Tangle, and Oscillator. Most applications will not need to link to this library.

\section{Did you know?}

3 The "libraries" made available in the VTK-m do more than add a library to the linker line. These libraries are actually defined as external targets that establish several compiler flags, like include file directories. Many CMake packages require you to set up other target options to compile correctly, but for VTK-m it is sufficient to simply link against the library.

\section{Common Errors}

Because the VTK-m CMake libraries do more than set the link line, correcting the link libraries can do more than fix link problems. For example, if you are getting compile errors about not finding VTK-m header files, then you probably need to link to one of VTK-m's libraries to fix the problem rather than try to add the include directories yourself.

The following is a list of all the CMake variables defined when the find_package function completes.

VTKm_FOUND Set to true if the VTK-m CMake package is successfully loaded. If find_package was not called with the REQUIRED option, then this variable should be checked before attempting to use VTK-m.

VTKm_VERSION The version number of the loaded VTK-m package. The package also sets VTKm_VERSION_MAJOR, VTKm_VERSION_MINOR, and VTKm_VERSION_PATCH to get the individual components of the version. There is also a VTKm_VERSION_FULL that is augmented with a partial git SHA to identify snapshots in between releases.

VTKm_ENABLE_CUDA Set to true if VTK-m was compiled for CUDA.

VTKm_ENABLE_OPENMP Set to true if VTK-m was compiled for OpenMP.

VTKm_ENABLE_TBB Set to true if VTK-m was compiled for TBB.

VTKm_ENABLE_RENDERING Set to true if the VTK-m rendering library was compiled.

VTKm_ENABLE_MPI Set to true if VTK-m was compiled with MPI support. 
These package variables can be used to query whether optional components are supported before they are used in your CMake configuration.

Example 2.5: Using an optional component of VTK-m.

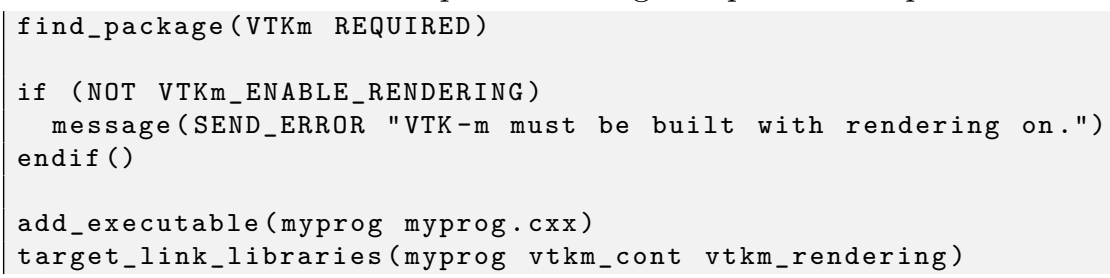





\section{QUICK START}

In this chapter we go through the steps to create a simple program that uses VTK-m. This "hello world" example presents only the bare minimum of features available. The remainder of this book documents dives into much greater detail.

We will call the example program we are building VTKmQuickStart. It will demonstrate reading data from a file, processing the data with a filter, and rendering an image of the data. Readers who are less interested in an explanation and are more interested in browsing some code can skip to Section 3.5 on page 17.

\subsection{Initialize}

The first step to using VTK-m is to initialize the library. Although initializing VTK-m is optional, it is recommend to allow VTK-m to configure devices and logging. Initialization is done by calling the vtkm: :cont: : Initialize function. The Initialize function is defined in the vtkm/cont/Initialize.h header file.

Initialize takes the argc and argv arguments that are passed to the main function of your program, find any command line arguments relevant to VTK-m, and remove them from the list to make further command line argument processing easier.

Example 3.1: Initializing VTK-m.

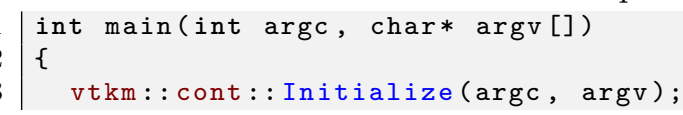

Initialize has many options to customize command line argument processing. See Chapter 6 for more details.

Did you know?

¿ Don't have access to argc and argv? No problem. You can call vtkm::cont::Initialize with no arguments.

\subsection{Reading a File}

VTK-m comes with a simple I/O library that can read and write files in VTK legacy format. These files have a ".vtk" extension. 
VTK legacy files can be read using the vtkm: : io: :VTKDataSetReader object, which is declared in the vtkm/io/VTKDataSetReader.h header file. The object is constructed with a string specifying the filename (which for this example we will get from the command line). The data is then read in by calling the VTKDataSetReader: :ReadDataSet method.

Example 3.2: Reading data from a VTK legacy file.

1 vtkm: : io: : VTKDataSetReader reader(argv[1]);

2 vtkm: : cont: : DataSet inData = reader.ReadDataSet();

The ReadDataSet method returns the data in a vtkm: : cont: :DataSet object. The structure and features of a DataSet object is described in Chapter 7. For the purposes of this quick start, we will treat DataSet as a mostly opaque object that gets passed to and from operations in VTK-m.

More information about VTK-m's file readers and writers can be found in Chapter 8.

\subsection{Running a Filter}

Algorithms in VTK-m are encapsulated in units called filters. A filter takes in a DataSet, processes it, and returns a new DataSet. The returned DataSet often, but not always, contains data inherited from the source data.

VTK-m comes with many filters, which are documented in Chapter 9. For this example, we will demonstrate the use of the vtkm: :filter: :MeshQuality filter, which is defined in the vtkm/filter/MeshQuality.h header file. The MeshQuality filter will compute for each cell in the input data will compute a quantity representing some metric of the cell's shape. Several metrics are available, and in this example we will find the area of each cell.

Like all filters, MeshQuality contains an Execute method that takes an input DataSet and produces an output DataSet. It also has several methods used to set up the parameters of the execution. Section 9.1.18 provides details on all the options of MeshQuality. Suffice it to say that in this example we instruct the filter to find the area of each cell, which it will output to a field named "area."

Example 3.3: Running a filter.

1 vtkm::filter:: MeshQuality cellArea(vtkm::filter::CellMetric::AREA);

2 vtkm: : cont: : DataSet outData = cellArea.Execute (inData);

\subsection{Rendering an Image}

Although it is possible to leverage external rendering systems, VTK-m comes with its own self-contained image rendering algorithms. These rendering classes are completely implemented with the parallel features provided by VTK-m, so using rendering in VTK-m does not require any complex library dependencies.

Even a simple rendering scene requires setting up several parameters to establish what is to be featured in the image including what data should be rendered, how that data should be represented, where objects should be placed in space, and the qualities of the image to generate. Consequently, setting up rendering in VTK-m involves many steps. Chapter 10 goes into much detail on the ways in which a rendering scene is specified. For now, we just briefly present some boilerplate to achieve a simple rendering.

Example 3.4: Rendering data.

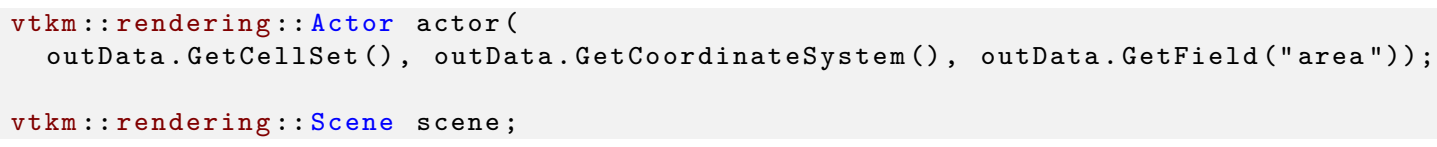




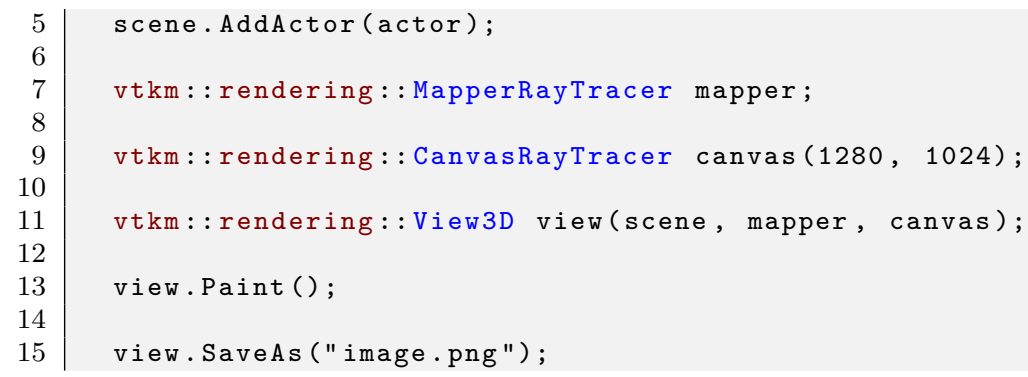

The first step in setting up a render is to create a scene. A scene comprises some number of actors, which represent some data to be rendered in some location in space. In our case we only have one DataSet to render, so we simply create a single actor and add it to a scene as shown in lines 1-5.

The second step in setting up a render is to create a view. The view comprises the aforementioned scene, a mapper, which describes how the data are to be rendered, and a canvas, which holds the image buffer and other rendering context. The view is created in line 11. The image generation is then performed by calling Paint on the view object (line 13). However, the rendering done by VTK-m's rendering classes is performed offscreen, which means that the result does not appear on your computer's monitor. The easiest way to see the image is to save it to an image file using the SaveAs method (line 15).

\subsection{The Full Example}

Putting together the examples from Sections 3.1 to 3.4, here is a complete program for reading, processing, and rendering data with VTK-m.

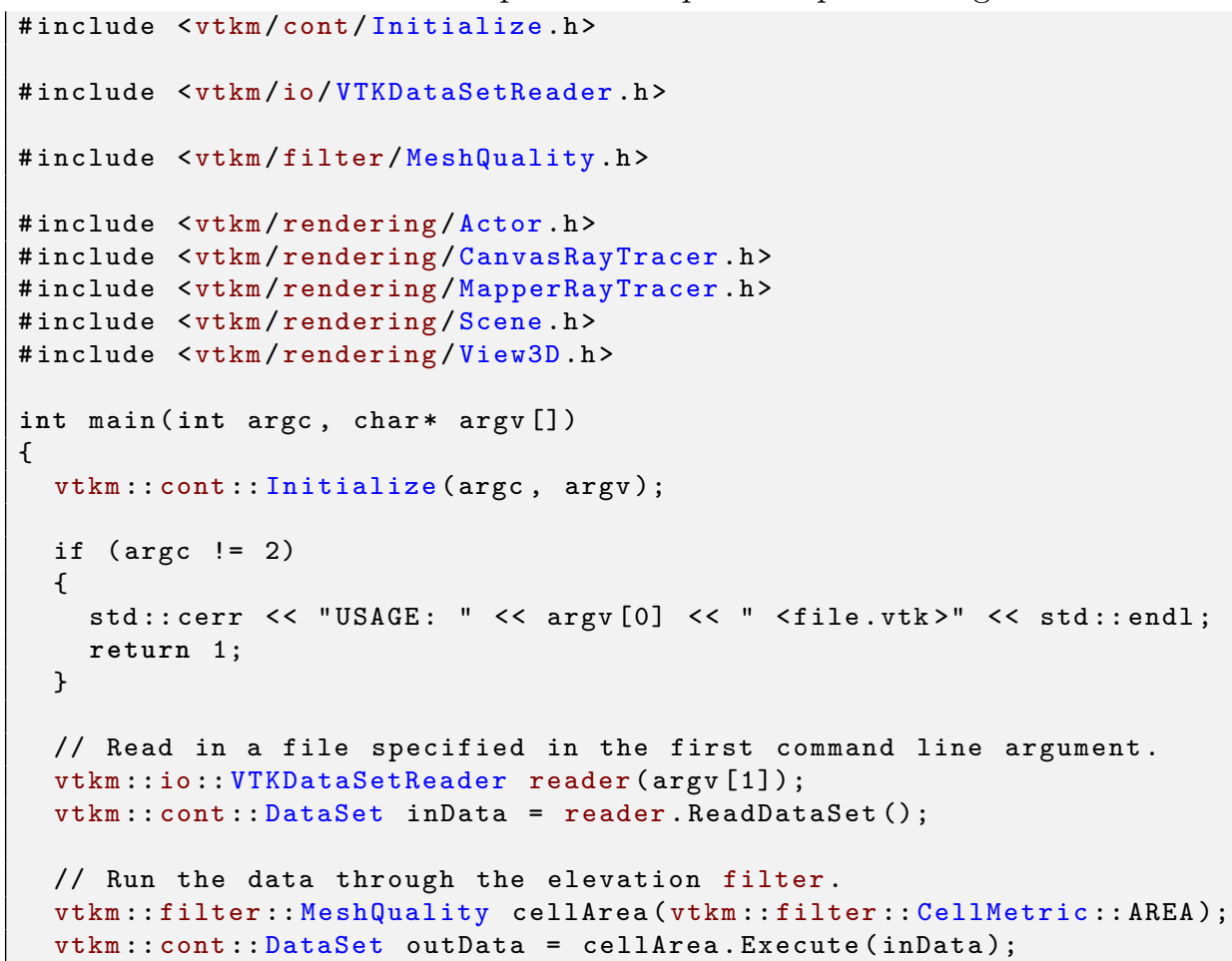




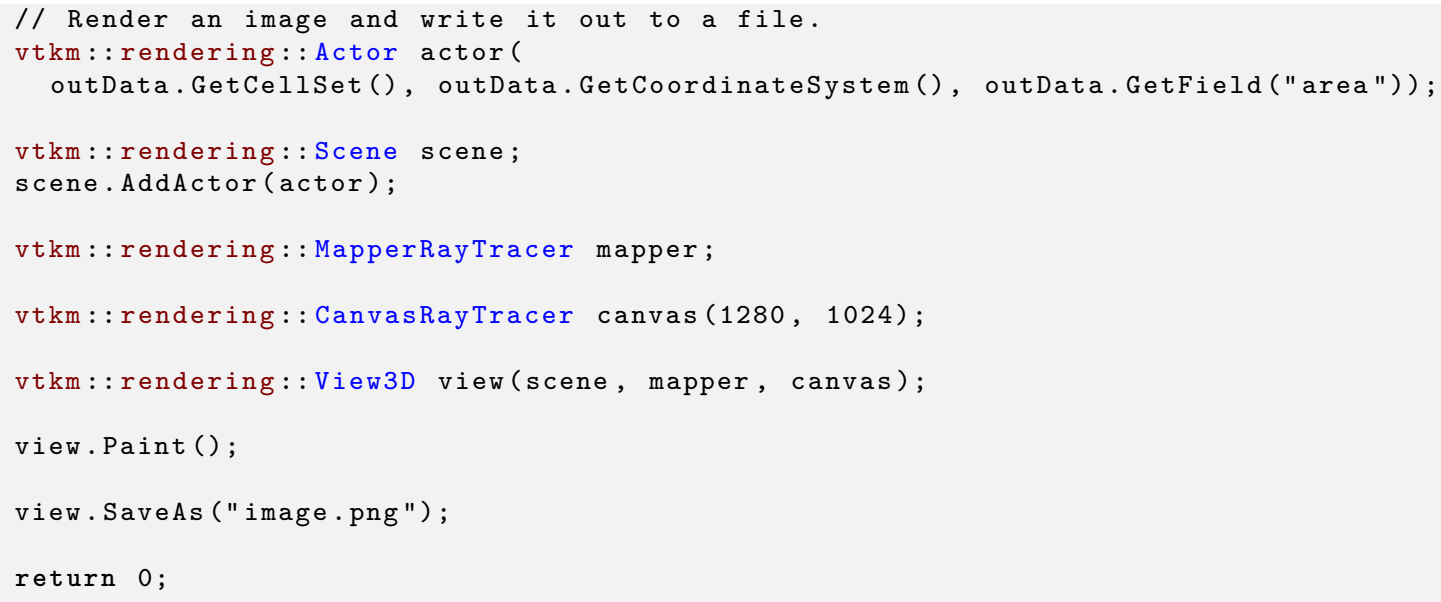

\subsection{Build Configuration}

Now that we have the program listed in Example 3.5, we still need to compile it with the appropriate compilers and flags. By far the easiest way to compile VTK-m code is to use CMake. CMake commands that can be used to link code to VTK-m are discussed in Section 2.4. The following example provides a minimal CMakeLists.txt required to build this program.

Example 3.6: CMakeLists.txt to build a program using VTK-m.

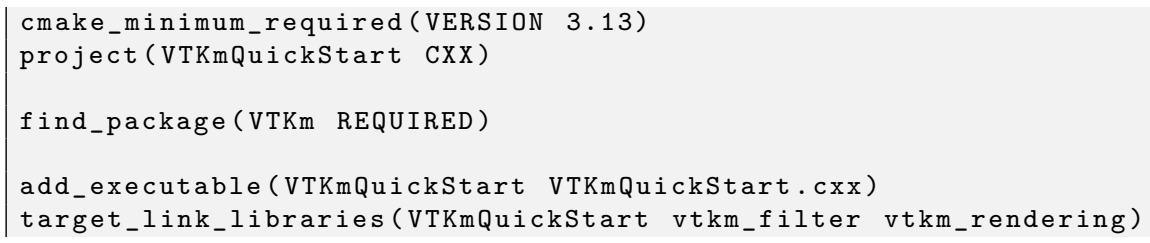

The first two lines contain boilerplate for any CMakeLists.txt file. They all should declare the minimum CMake version required (for backward compatibility) and have a project command to declare which languages are used.

The remainder of the commands find the VTK-m library, declare the program begin compiled, and link the program to the VTK-m library. These steps are described in detail in Section 2.4. 


\section{Part II}

\section{Using VTK-m}





\section{BASE TYPES}

It is common for a framework to define its own types. Even the $\mathrm{C}++$ standard template library defines its own base types like std: :size_t and std: :pair. VTK-m is no exception.

In fact VTK-m provides a great many base types. It is the general coding standard of VTK-m to not directly use the base $\mathrm{C}$ types like int and float and instead to use types declared in VTK-m. The rational is to precisely declare the representation of each variable to prevent future trouble.

Consider that you are programming something and you need to declare an integer variable. You would declare this variable as int, right? Well, maybe. In $\mathrm{C}++$, the declaration int does not simply mean "an integer." int means something much more specific than that. If you were to look up the $\mathrm{C}++11$ standard, you would find that int is an integer represented in 32 bits with a two's complement signed representation. In fact, a C++ compiler has no less than 8 standard integer types. ${ }^{1}$

So, int is nowhere near as general as the code might make it seem, and treating it as such could lead to trouble. For example, consider the MPI standard, which, back in the 1990's, implicitly selected int for its indexing needs. Fast forward to today where there is a need to reference buffers with more than 2 billion elements, but the standard is stuck with a data type that cannot represent sizes that big. ${ }^{2}$

Consequently, we feel that with VTK-m it is best to declare the intention of a variable with its declaration, which should help both prevent errors and future proof code. All the types presented in this chapter are declared in vtkm/Types.h, which is typically included either directly or indirectly by all source using VTK-m.

\subsection{Floating Point Types}

VTK-m declares 2 types to hold floating point numbers: vtkm: :Float32 and vtkm: :Float64. These, of course, represent floating point numbers with 32-bits and 64-bits of precision, respectively. These should be used when the precision of a floating point number is predetermined.

When the precision of a floating point number is not predetermined, operations usually have to be overloaded or templated to work with multiple precisions. In cases where a precision must be set, but no particular precision is specified, vtkm::FloatDefault should be used. vtkm::FloatDefault will be set to either vtkm::Float32 or vtkm::Float64 depending on whether the CMake option VTKM_USE_DOUBLE_PRECISION was set when VTK-m was compiled, as discussed in Section 2.2. Using vtkm: :FloatDefault makes it easier for users to trade off precision and speed.

\footnotetext{
${ }^{1}$ I intentionally use the phrase "no less than" for our pedantic readers. One could argue that char and bool are treated distinctly by the compiler even if their representations match either signed char or unsigned char. Furthermore, many modern $\mathrm{C}++$ compilers have extensions for less universally accepted types like 128-bit integers.

${ }^{2}$ To be fair, it is possible to represent buffers this large in MPI, but it is extraordinarily awkward to do so.
} 


\subsection{Integer Types}

The most common use of an integer in VTK-m is to index arrays. For this purpose, the vtkm: : Id type should be used. (The width of vtkm: :Id is determined by the VTKm_USE_64BIT_IDS CMake option.)

VTK-m also has a secondary index type named vtkm: IdComponent, which is smaller and typically used for indexing groups of components within a thread. For example, if you had an array of 3D points, you would use vtkm: : Id to reference each point, and you would use vtkm: : IdComponent to reference the respective $x, y$, and $z$ components.

\section{Did you know?}

The VTK-m index types, vtkm::Id and vtkm::IdComponent use signed integers. This breaks with the convention of other common index types like the $C++$ standard template library std:size_t, which use unsigned integers. Unsigned integers make sense for indices as a valid index is always 0 or greater. However, doing things like iterating in a for loop backward, representing relative indices, and representing invalid values is much easier with signed integers. Thus, VTK-m chooses to use a signed integer for indexing.

VTK-m also has types to declare an integer of a specific width and sign. The types vtkm: :Int8, vtkm::Int16, vtkm: : Int32, and vtkm: : Int64 specify signed integers of 1,2, 4, and 8 bytes, respectively. Likewise, the types vtkm: :UInt8, vtkm: :UInt16, vtkm: :UInt32, and vtkm: :UInt64 specify unsigned integers of 1, 2, 4, and 8 bytes, respectively.

\subsection{Vector Types}

Visualization algorithms also often require operations on short vectors. Arrays indexed in up to three dimensions are common. Data are often defined in 2-space and 3-space, and transformations are typically done in homogeneous coordinates of length 4 . To simplify these types of operations, VTK-m provides a collection of base types to represent these short vectors, which are collectively referred to as Vec types.

vtkm: :Vec2f, vtkm: :Vec3f, and vtkm: :Vec4f specify floating point Vecs of 2, 3, and 4 components, respectively. The precision of the floating point numbers follows that of vtkm: :FloatDefault (which, from what is said in Section 4.1, is specified by the VTKm_USE_DOUBLE_PRECISION compile option). Components of these and other Vec types can be references through the [ ] operator, much like a $\mathrm{C}$ array. Vecs also support basic arithmetic operators so that they can be used much like their scalar-value counterparts.

Example 4.1: Simple use of Vec objects.

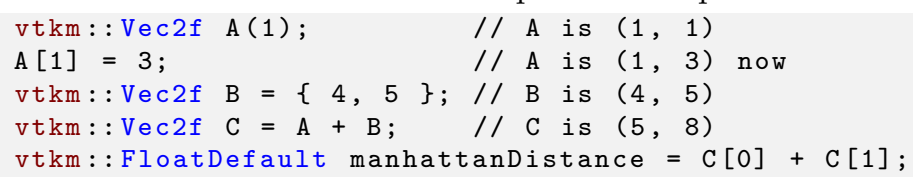

You can also specify the precision for each of these vector types by appending the bit size of each component. For example, vtkm::Vec3f_32 and vtkm::Vec3f_64 represent 3-component floating point vectors with each component being 32 bits and 64 bits respectively. Note that the precision number refers to the precision of each component, not the vector as a whole. So vtkm: :Vec3f_32 contains 3 32-bit (4-byte) floating point components, which means the entire vtkm: :Vec3f_32 requires 96 bits (12 bytes). 
To help with indexing 2-, 3-, and 4- dimensional arrays, VTK-m provides the types vtkm::Id2, vtkm::Id3, and vtkm::Id4, which are Vecs of type vtkm::Id. Likewise, VTK-m provides vtkm::IdComponent2, vtkm: :IdComponent3, and vtkm: : IdComponent4.

VTK-m also provides types for Vecs of integers of all varieties described in Section 4.2. vtkm: :Vec2i, vtkm: :Vec3i, and vtkm::Vec4i are vectors of signed integers whereas vtkm::Vec2ui, vtkm::Vec3ui, and vtkm::Vec4ui are vectors of unsigned integers. All of these sport components of a width equal to vtkm::Id. The width can be specified by appending the desired number of bits in the same way as the floating point Vecs. For example, vtkm: :Vec4ui_8 is a Vec of 4 unsigned bytes.

These types really just scratch the surface of the Vec types available in VTK-m and the things that can be done with them. See Chapter 19 for more information on Vec types and what can be done with them. 



\section{VTK-M VERSION}

As the VTK-m code evolves, changes to the interface and behavior will inevitably happen. Consequently, code that links into VTK-m might need a specific version of VTK-m or changes its behavior based on what version of VTK-m it is using. To facilitate this, VTK-m software is managed with a versioning system and advertises its version in multiple ways. As with many software products, VTK-m has three version numbers: major, minor, and patch. The major version represents significant changes in the VTK-m implementation and interface. Changes in the major version include backward incompatible changes. The minor version represents added functionality. Generally, changes in the minor version to not introduce changes to the API (although the early 1.X versions of VTK-m violate this). The patch version represents fixes provided after a release occurs. Patch versions represent minimal change and do not add features.

If you are writing a software package that is managed by CMake and load VTK-m with the find_package command as described in Section 2.4, then you can query the VTK-m version directly in the CMake configuration. When you load VTK-m with find_package, CMake sets the variables VTKm_VERSION_MAJOR, VTKm_VERSION_MINOR, and VTKm_VERSION_PATCH to the major, minor, and patch versions, respectively. Additionally, VTKm_VERSION is set to the "major.minor" version number and VTKm_VERSION_FULL is set to the "major.minor.patch" version number. If the current version of VTK-m is actually a development version that is in between releases of VTK-m, then and abbreviated SHA of the git commit is also included as part of VTKm_VERSION_FULL.

\section{Did you know?}

3 If you have a specific version of VTK-m required for your software, you can also use the version option to the find_package CMake command. The find_package command takes an optional version argument that causes the command to fail if the wrong version of the package is found.

It is also possible to query the VTK-m version directly in your code through preprocessor macros. The vtkm/Version.h header file defines the following preprocessor macros to identify the VTK-m version. VTKM_VERSION_MAJOR, VTKM_VERSION_MINOR, and VTKM_VERSION_PATCH are set to integer numbers representing the major, minor, and patch versions, respectively. Additionally, VTKM_VERSION is set to the "major.minor" version number as a string and VTKM_VERSION_FULL is set to the "major.minor.patch" version number (also as a string). If the current version of VTK-m is actually a development version that is in between releases of VTK-m, then and abbreviated SHA of the git commit is also included as part of VTKM_VERSION_FULL. 
$\sum$ Note that the CMake variables all begin with $\mathrm{VTKm}_{-}$(lowercase " $m$ ") whereas the preprocessor macros begin with VTKM _ (all uppercase). This follows the respective conventions of CMake variables and preprocessor macros.

Note that vtkm/Version.h does not include any other VTK-m header files. This gives your code a chance to load, query, and react to the VTK-m version before loading any VTK-m code proper. 
When it comes to running VTK-m code, there are a few ways in which various facilities, such as logging device connections, and device configuration parameters, can be initialized. The preferred method of initializing these features is to run the vtkm::cont: Initialize function. Although it is not strictly necessary to call Initialize, it is recommended to set up state and check for available devices.

Initialize can be called without any arguments, in which case VTK-m will be initialized with defaults. But it can also optionally take the argc and argv arguments to the main function to parse some options that control the state of VTK-m. VTK-m accepts arguments that, for example, configure the compute device to use or establish logging levels. Any arguments that are handled by VTK-m are removed from the argc/argv list so that your program can then respond to the remaining arguments.

Initialize takes an optional third argument that specifies some options on the behavior of the argument parsing. The options are specified as a bit-wise "or" of fields specified in the vtkm: : cont: : InitializeOptions enum. The available initialize options are

None Placeholder used when no options are enabled. This is the value used when the third argument to Initialize is not provided.

RequireDevice Issue an error if the device argument is not specified.

DefaultAnyDevice If no device is specified, treat it as if the user gave "--device=Any". This means that DeviceAdapterTagUndefined will never be return in the result.

AddHelp Add a help option. If "-h" or "--help" is provided, prints a usage statement. Of course, the usage statement will only print out arguments processed by VTK-m, which is why help is not given by default. A string with usage help is returned from Initialize so that the calling program can provide VTK-m's help in its own usage statement.

ErrorOnBadOption If an unknown option is encountered, the program terminates with an error. If this option is not provided, any unknown options are returned in argv. If this option is used, it is a good idea to use AddHelp as well.

ErrorOnBadArgument If an extra argument is encountered, the program terminates with an error. If this option is not provided, any unknown arguments are returned in argv.

Strict If supplied, Initialize treats its own arguments as the only ones supported by the application and provides an error if not followed exactly. This is a convenience option that is a combination of ErrorOnBadOption, ErrorOnBadArgument, and AddHelp.

As stated earlier, vtkm: :cont: : Initialize removes parsed options from the argc/argv passed to it so that the calling program can further respond to command line arguments. Additionally, Initialize returns an vtkm: : cont: : InitializeResult object that contains the following information. 
Device A vtkm: :cont: :DeviceAdapterId that represents the device specified by the command line arguments. (See Chapter 12 for details on how VTK-m represents devices.) If no device is specified in the command line options, vtkm: :cont: :DeviceAdapterTagUndefined is returned (unless the DefaultAnyDevice option is given, in which case vtkm: : cont: : DeviceAdapterTagAny is returned).

Example 6.1: Calling Initialize.

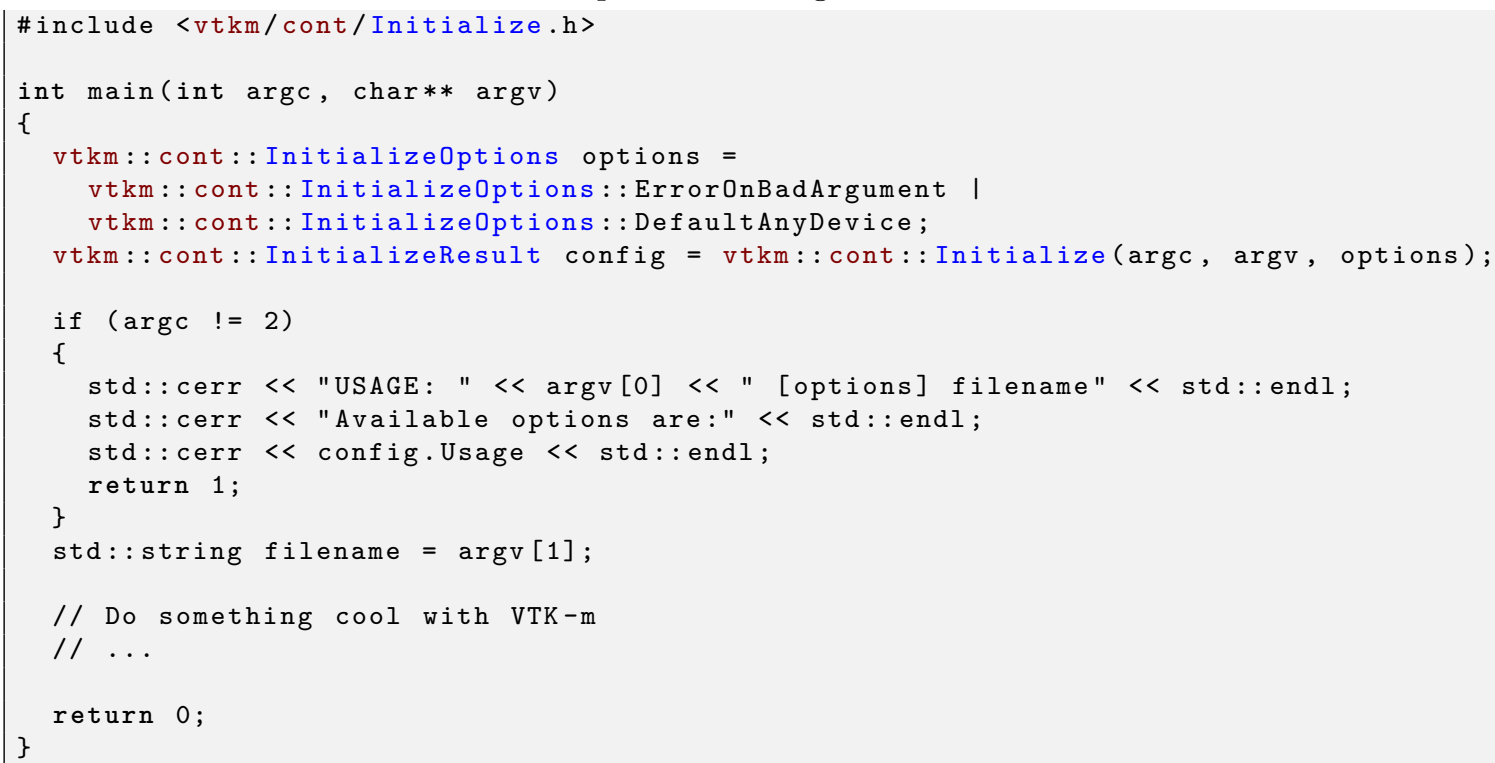




\section{DATA SETS}

A data set, implemented with the vtkm: : cont: :DataSet class, contains and manages the geometric data structures that VTK-m operates on. A data set comprises the following 3 data structures.

Cell Set A cell set describes topological connections. A cell set defines some number of points in space and how they connect to form cells, filled regions of space. A data set has exactly one cell set.

Field A field describes numerical data associated with the topological elements in a cell set. The field is represented as an array, and each entry in the field array corresponds to a topological element (point, edge, face, or cell). Together the cell set topology and discrete data values in the field provide an interpolated function throughout the volume of space covered by the data set. A cell set can have any number of fields.

Coordinate System A coordinate system is a special field that describes the physical location of the points in a data set. Although it is most common for a data set to contain a single coordinate system, VTK-m supports data sets with no coordinate system such as abstract data structures like graphs that might not have positions in a space. DataSet also supports multiple coordinate systems for data that have multiple representations for position. For example, geospatial data could simultaneously have coordinate systems defined by $3 \mathrm{D}$ position, latitude-longitude, and any number of $2 \mathrm{D}$ projections.

In addition to the base vtkm: :cont: :DataSet, VTK-m provides vtkm: :cont::PartitionedDataSet to represent data partitioned into multiple domains. A PartitionedDataSet is implemented as a collection of DataSet objects. Partitioned data sets are described later in Section 7.5.

\subsection{Building Data Sets}

Before we go into detail on the cell sets, fields, and coordinate systems that make up a data set in VTK-m, let us first discuss how to build a data set. One simple way to build a data set is to load data from a file using the vtkm: : io module. Reading files is discussed in detail in Chapter 8.

This section describes building data sets of different types using a set of classes named DataSetBuilder*, which provide a convenience layer on top of vtkm: : cont: : DataSet to make it easier to create data sets.

To simplify the introduction of DataSets, this section uses the simplest mechanisms. In many cases this involves loading data in a std: :vector and passing that to VTK-m, which usually causes the data to be copied. This is not the most efficient method to load data into VTK-m. Although it is sufficient for small 
data or data that come from a "slow" source, such as a file, it might be a bottleneck for large data generated by another library. It is possible to adapt VTK-m's DataSet to externally defined data. This is done by wrapping existing data into what is called ArrayHandle, but this is a more advanced topic that will not be addressed in this chapter. ArrayHandles are introduced in Chapter 16 and more adaptive techniques are described in later chapters.

\subsubsection{Creating Uniform Grids}

Uniform grids are meshes that have a regular array structure with points uniformly spaced parallel to the axes. Uniform grids are also sometimes called regular grids or images.

The vtkm: : cont: : DataSetBuilderUniform class can be used to easily create 2- or 3-dimensional uniform grids. DataSetBuilderUniform has several versions of a method named Create that takes the number of points in each dimension, the origin, and the spacing. The origin is the location of the first point of the data (in the lower left corner), and the spacing is the distance between points in the $\mathrm{x}, \mathrm{y}$, and $\mathrm{z}$ directions. The Create methods also take an optional name for the coordinate system and an optional name for the cell set.

The following example creates a vtkm: : cont: : DataSet containing a uniform grid of $101 \times 101 \times 26$ points.

Example 7.1: Creating a uniform grid.

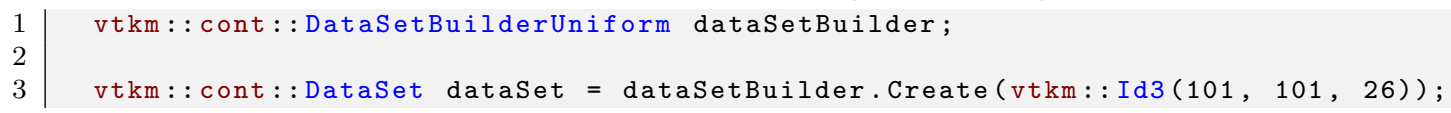

If not specified, the origin will be at the coordinates $(0,0,0)$ and the spacing will be 1 in each direction. Thus, in the previous example the width, height, and depth of the mesh in physical space will be 100, 100, and 25, respectively, and the mesh will be centered at $(50,50,12.5)$. Let us say we actually want a mesh of the same dimensions, but we want the $z$ direction to be stretched out so that the mesh will be the same size in each direction, and we want the mesh centered at the origin.

Example 7.2: Creating a uniform grid with custom origin and spacing.

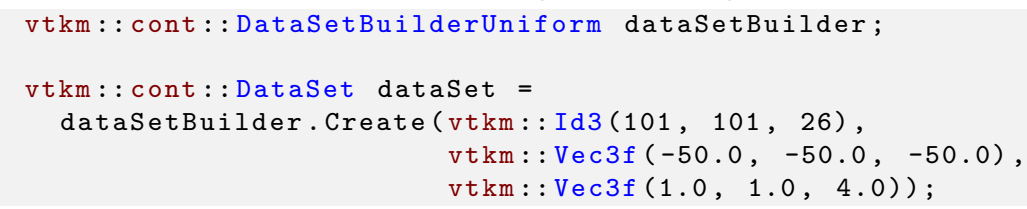

\subsubsection{Creating Rectilinear Grids}

A rectilinear grid is similar to a uniform grid except that a rectilinear grid can adjust the spacing between adjacent grid points. This allows the rectilinear grid to have tighter sampling in some areas of space, but the points are still constrained to be aligned with the axes and each other. The irregular spacing of a rectilinear grid is specified by providing a separate array each for the $\mathrm{x}, \mathrm{y}$, and $\mathrm{z}$ coordinates.

The vtkm: : cont: :DataSetBuilderRectilinear class can be used to easily create 2- or 3-dimensional rectilinear grids. DataSetBuilderRectilinear has several versions of a method named Create that takes these coordinate arrays and builds a vtkm: : cont: : DataSet out of them. The arrays can be supplied as either standard C arrays or as std: :vector objects, in which case the data in the arrays are copied into the DataSet. These arrays can also be passed as ArrayHandle objects (introduced later in this book), in which case the data are shallow copied. 
The following example creates a vtkm: : cont: : DataSet containing a rectilinear grid with $201 \times 201 \times 101$ points with different irregular spacing along each axis.

Example 7.3: Creating a rectilinear grid.

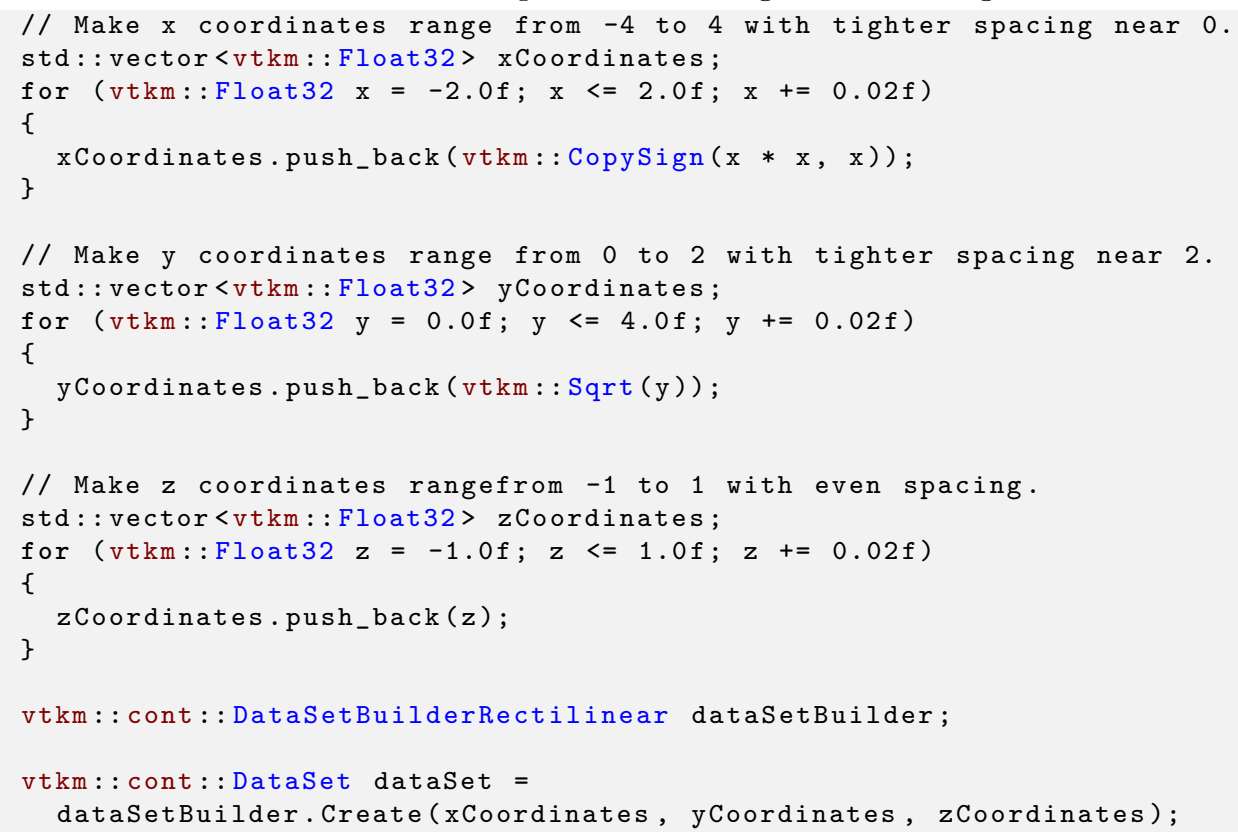

\subsubsection{Creating Explicit Meshes}

An explicit mesh is an arbitrary collection of cells with arbitrary connections. It can have multiple different types of cells. Explicit meshes are also known as unstructured grids. Explicit meshes can contain cells of different shapes. The shapes that VTK-m currently supports are listed in Figure 7.1.

The cells of an explicit mesh are defined with the following 3 arrays, which are depicted graphically in Figure 7.2 .

Shapes An array of ids identifying the shape of the cell. Each value is a vtkm: :UInt8 and should be set to one of the vtkm : : CELL_SHAPE_* constants. The shapes and their identifiers are shown in Figure 7.1. The size of this array is equal to the number of cells in the set.

Connectivity An array that lists all the points that comprise each cell. Each entry in the array is a vtkm: :Id giving the point id associated with a vertex of a cell. The points for each cell are given in a prescribed order for each shape, which is also shown in Figure 7.1. The point indices are stored consecutively from the first cell to the last.

Offsets An array of vtkm: :Id s pointing to the index in the connectivity array where the points for a particular cell starts. The size of this array is equal to the number of cells in the set plus 1 . The first entry is expected to be 0 (since the connectivity of the first cell is at the start of the connectivity array). The last entry, which does not correspond to any cell, should be the size of the connectivity array.

One important item that is missing from this list of arrays is a count of the number of indices associated with each cell. This is not explicitly represented in VTK-m's mesh structure because it can be implicitly derived from the offsets array by subtracting consecutive entries. However, it is usually the case when building an explicit 
vtkm: :CELL_SHAPE_VERTEX vtkm: :CellShapeTagVertex

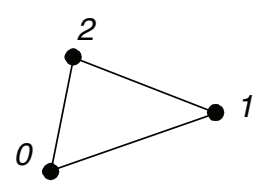

vtkm: :CELL_SHAPE_TRIANGLE vtkm: :CellShapeTagTriangle

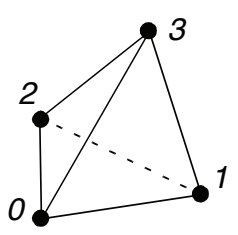

vtkm: :CELL_SHAPE_TETRA vtkm: :CellShapeTagTetra

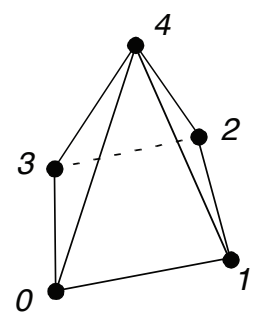

vtkm: :CELL_SHAPE_PYRAMID vtkm: :CellShapeTagPyramid

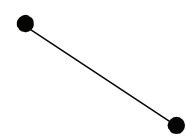

vtkm: :CELL_SHAPE_LINE

vtkm: CellShapeTagLine

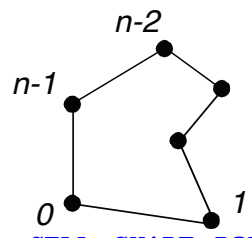

vtkm: :CELL_SHAPE_POLYGON vtkm: :CellShapeTagPolygon

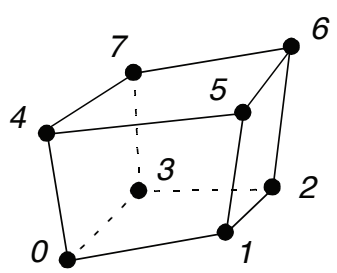

vtkm: : CELL_SHAPE_HEXAHEDRON vtkm: :CellShapeTagHexahedron

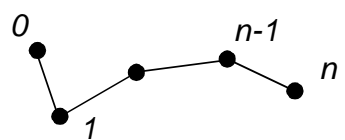

vtkm: :CELL_SHAPE_POLY_LINE

vtkm: : CellShapeTagPolyLine

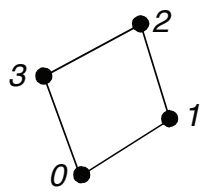

vtkm: :CELL_SHAPE_QUAD

vtkm: :CellShapeTagQuad

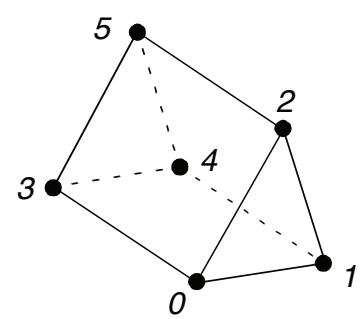

vtkm: :CELL_SHAPE_WEDGE vtkm: : CellShapeTagWedge

Figure 7.1: Basic Cell Shapes

mesh that you will have an array of these counts rather than the offsets. It is for this reason that VTK-m contains mechanisms to build an explicit data set with a "num indices" arrays rather than an offsets array.

The vtkm::cont::DataSetBuilderExplicit class can be used to create data sets with explicit meshes. DataSetBuilderExplicit has several versions of a method named Create. Generally, these methods take the shapes, number of indices, and connectivity arrays as well as an array of point coordinates. These arrays can be given in std: : vector objects, and the data are copied into the DataSet created.

The following example creates a mesh like the one shown in Figure 7.2.

Example 7.4: Creating an explicit mesh with DataSetBuilderExplicit.

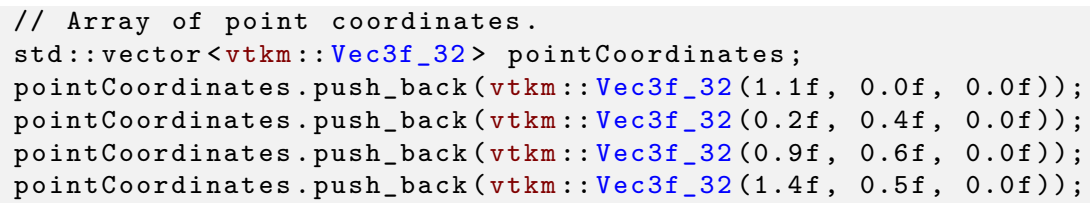




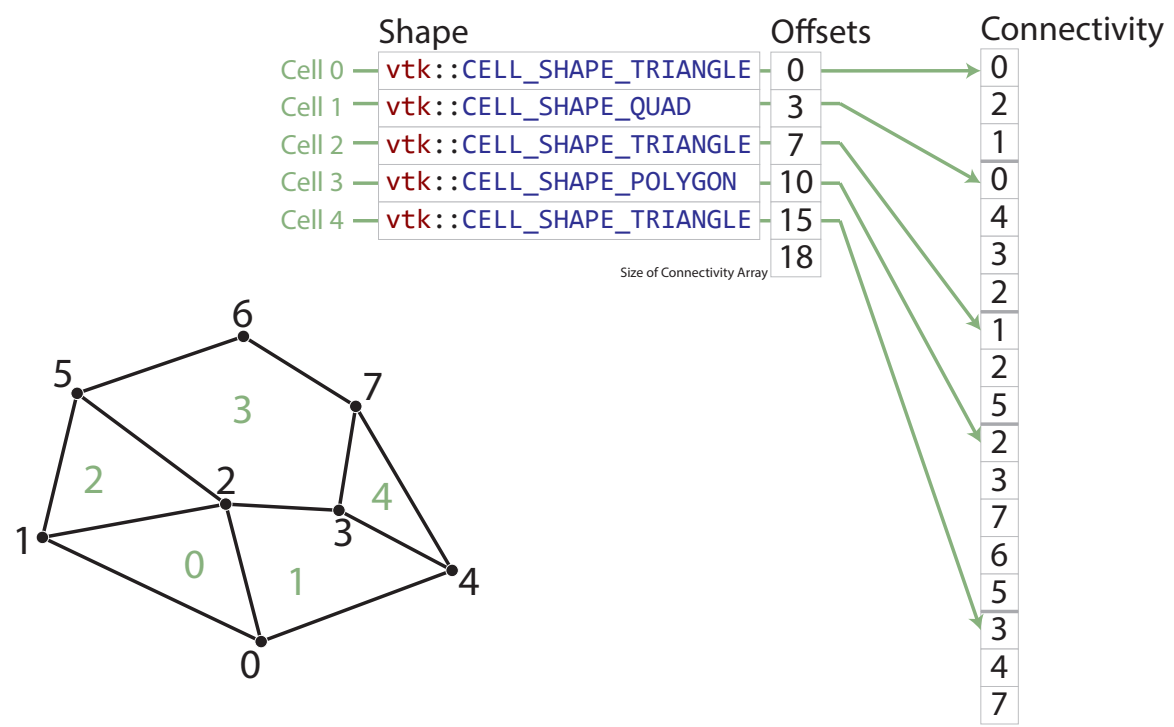

Figure 7.2: An example explicit mesh.

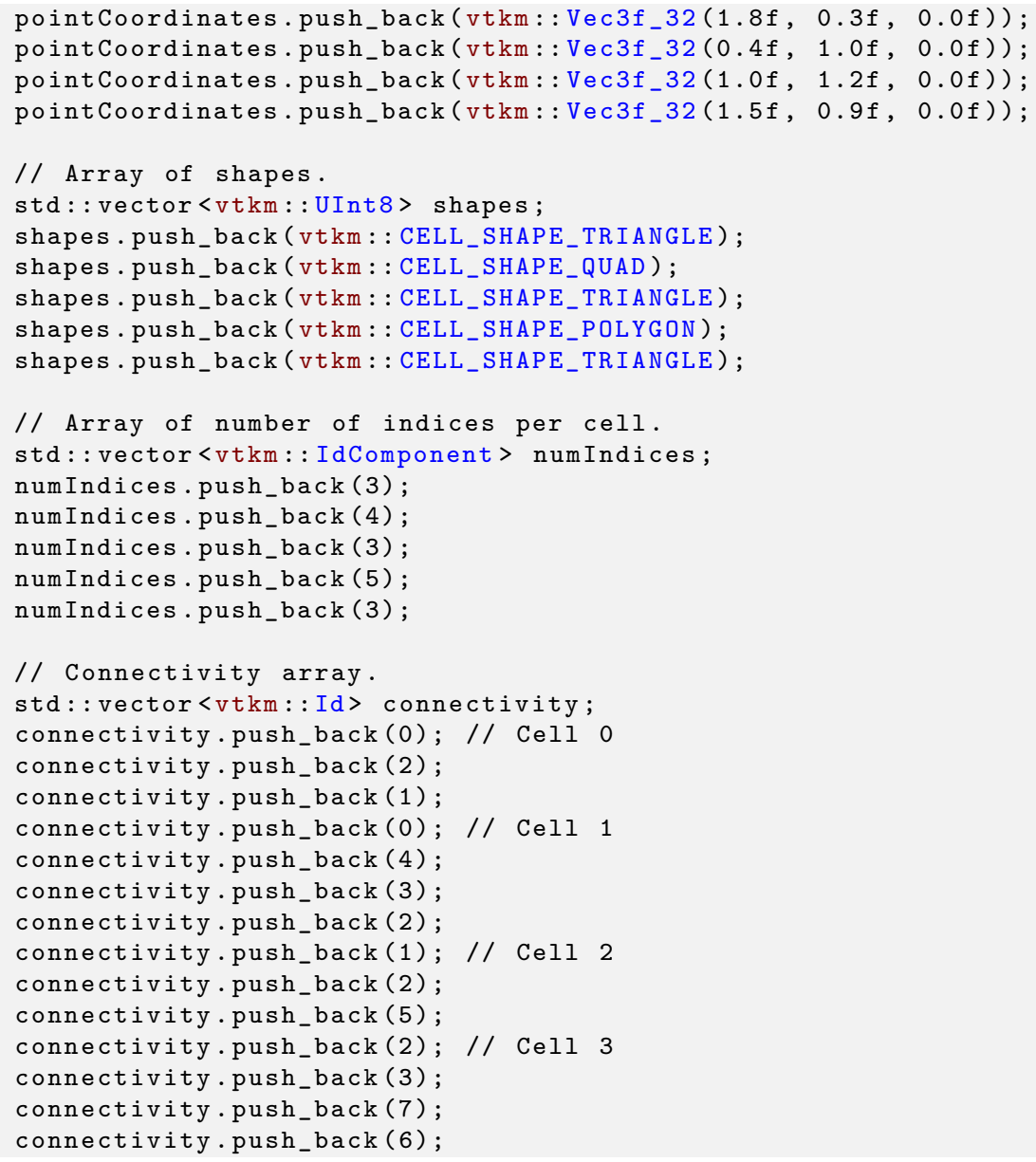




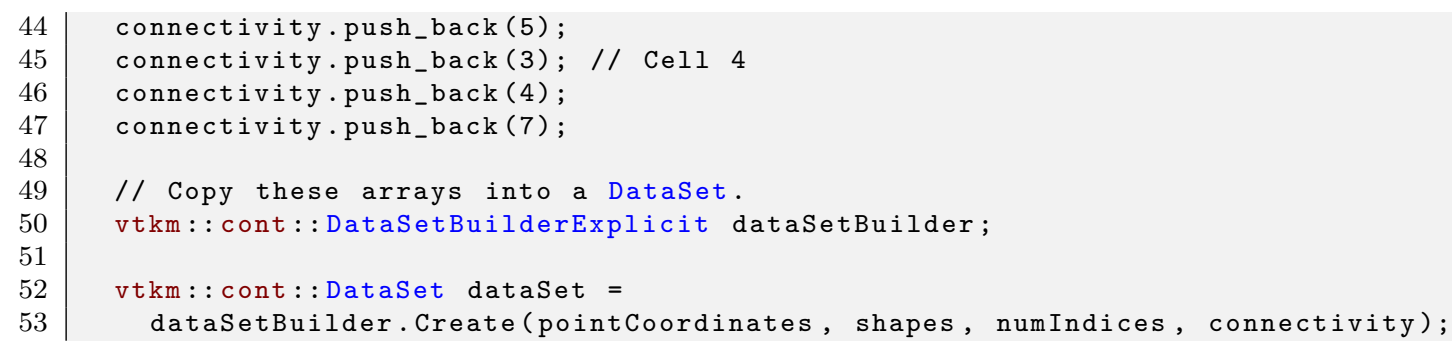

Often it is awkward to build your own arrays and then pass them to DataSetBuilderExplicit. There also exists an alternate builder class named vtkm: :cont: :DataSetBuilderExplicitIterative that allows you to specify each cell and point one at a time rather than all at once. This is done by calling one of the versions of AddPoint and one of the versions of AddCell for each point and cell, respectively. The next example also builds the mesh shown in Figure 7.2 except this time using DataSetBuilderExplicitIterative.

Example 7.5: Creating an explicit mesh with DataSetBuilderExplicitIterative.

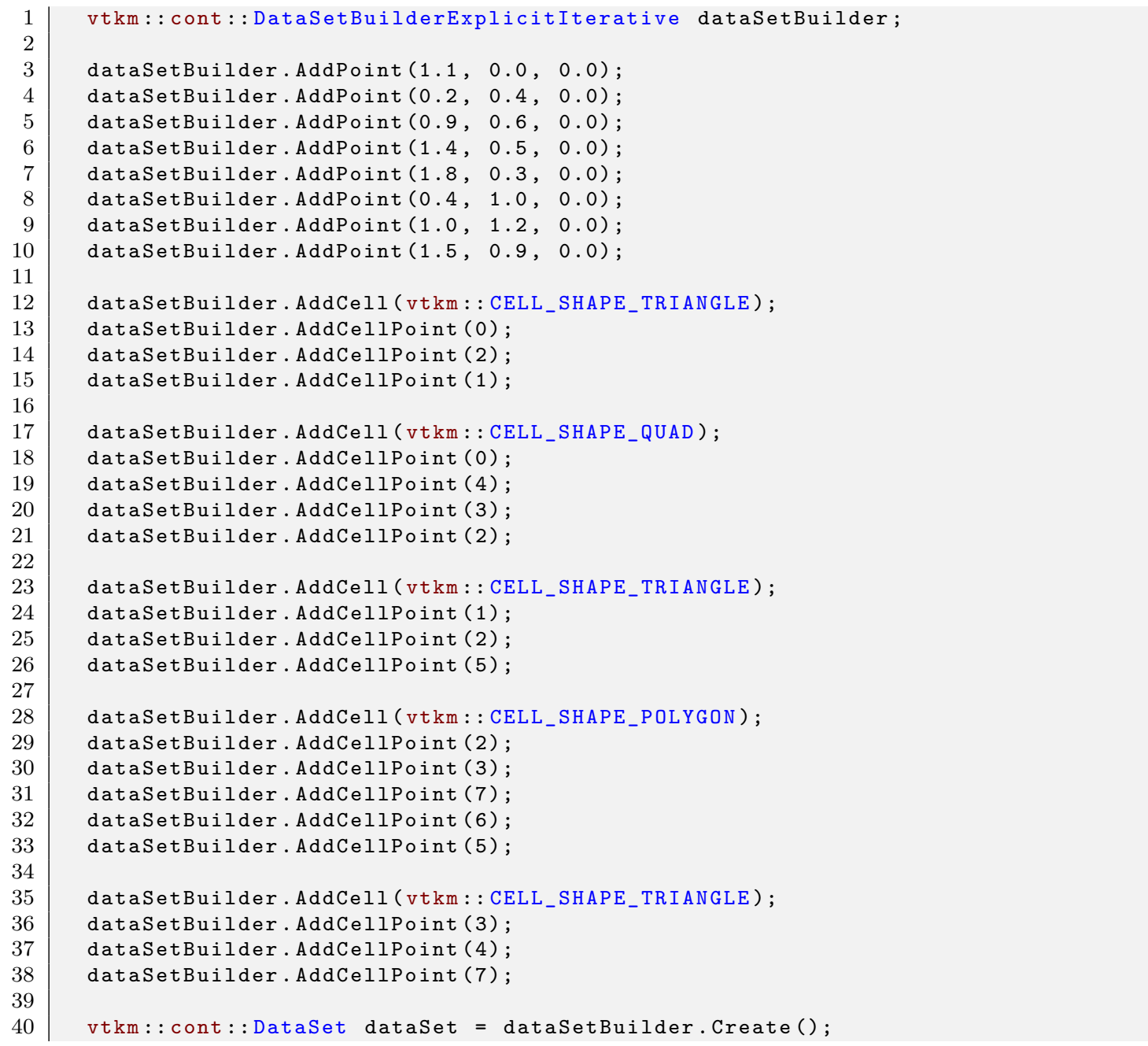




\subsubsection{Add Fields}

In addition to creating the geometric structure of a data set, it is usually important to add fields to the data. Fields describe numerical data associated with the topological elements in a cell. They often represent a physical quantity (such as temperature, mass, or volume fraction) but can also represent other information (such as indices or classifications).

The easiest way to define fields in a data set is to use the DataSet: : AddPointField and DataSet: : AddCellField methods. Each of these methods take a requisite field name and the array with with field data.

Both AddPointField and AddCellField are overloaded to accept arrays of data in different structures. Field arrays can be passed as standard $\mathrm{C}$ arrays or as std: : vectors, in which case the data are copied. Field arrays can also be passed in a ArrayHandle (introduced later in this book), in which case the data are not copied.

The following (somewhat contrived) example defines fields for a uniform grid that identify which points and cells are on the boundary of the mesh.

Example 7.6: Adding fields to a DataSet.

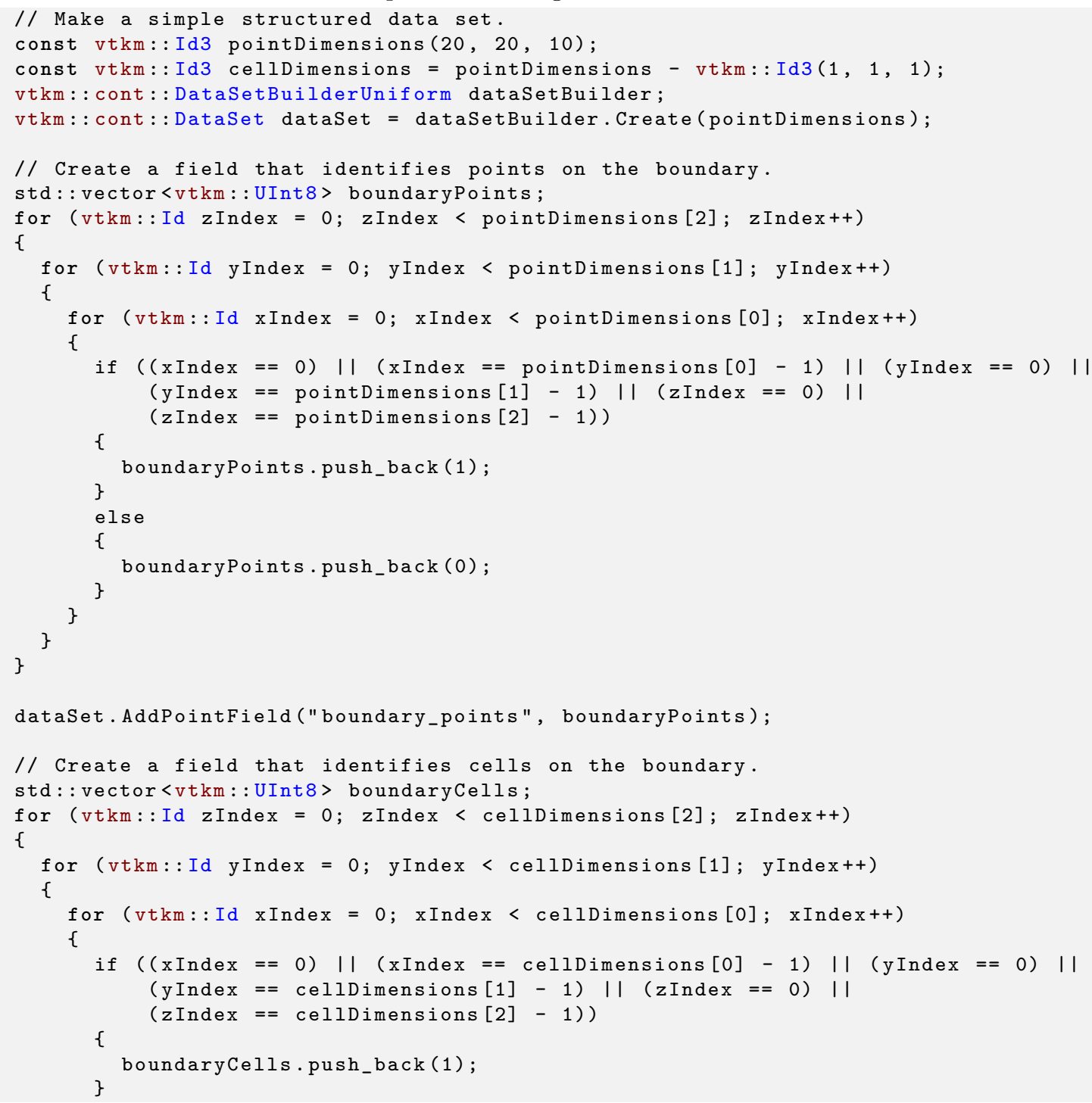




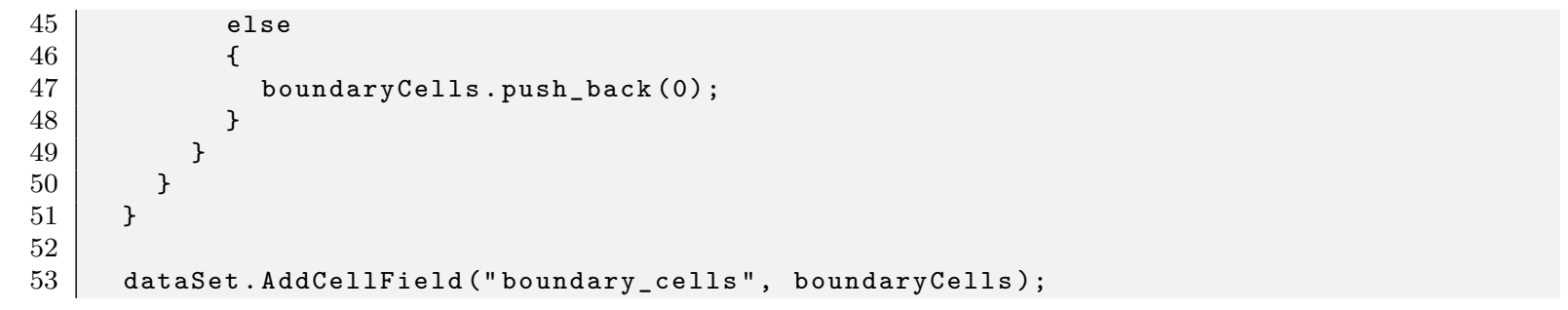

\subsection{Cell Sets}

A cell set determines the topological structure of the data in a data set. Fundamentally, any cell set is a collection of cells, which typically (but not always) represent some region in space. 3D cells are made up of points, edges, and faces. (2D cells have only points and edges, and 1D cells have only points.) Figure 7.3 shows the relationship between a cell's shape and these topological elements. The arrangement of these points, edges, and faces is defined by the shape of the cell, which prescribes a specific ordering of each. The basic cell shapes provided by VTK-m are discussed in detail in Section 25.1 starting on page 205.

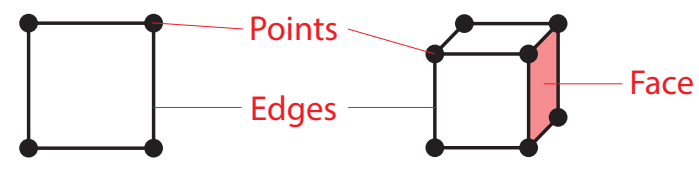

Figure 7.3: The relationship between a cell shape and its topological elements (points, edges, and faces).

There are multiple ways to express the connections of a cell set, each with different benefits and restrictions. These different cell set types are managed by different cell set classes in VTK-m. All VTK-m cell set classes inherit from vtkm: : cont: :Cellset. The two basic types of cell sets are structured and explicit, and there are several variations of these types.

\subsubsection{Structured Cell Sets}

A vtkm: : cont: :CellSetStructured defines a 1-, 2-, or 3-dimensional grid of points with lines, quadrilaterals, or hexahedra, respectively, connecting them. The topology of a CellSetStructured is specified by simply providing the dimensions, which is the number of points in the $i, j$, and $k$ directions of the grid of points. The number of points is implicitly $i \times j \times k$ and the number of cells is implicitly $(i-1) \times(j-1) \times(k-1)$ (for 3D grids). Figure 7.4 demonstrates this arrangement.

The big advantage of using vtkm: : cont: :CellSetStructured to define a cell set is that it is very space efficient because the entire topology can be defined by the three integers specifying the dimensions. Also algorithms can be optimized for CellSetStructured's regular nature. However, CellSetStructured's strictly regular grid structure also limits its applicability. A structured cell set can only be a dense grid of lines, quadrilaterals, or hexahedra. It cannot represent irregular data well.

Many data models in other software packages, such as the one for VTK, make a distinction between uniform, rectilinear, and curvilinear grids. VTK-m's cell sets do not. All three of these grid types are represented by CellSetStructured. This is because in a VTK-m data set the cell set and the coordinate system are defined independently and used interchangeably. A structured cell set with uniform point coordinates makes a uniform grid. A structured cell set with point coordinates defined irregularly along coordinate axes makes a rectilinear grid. And a structured cell set with arbitrary point coordinates makes a curvilinear grid. The point coordinates are defined by the data set's coordinate system, which is discussed in Section 7.4 starting on page 41. 


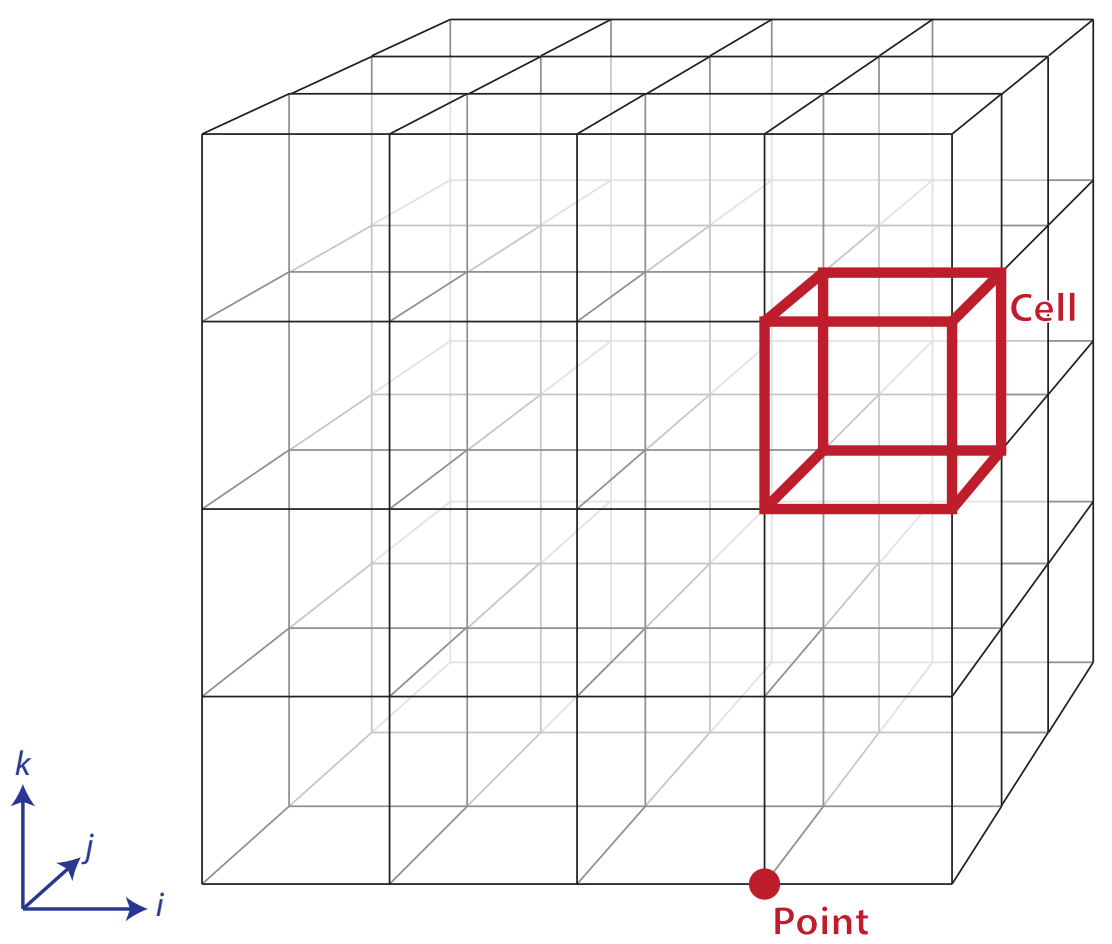

Figure 7.4: The arrangement of points and cells in a 3D structured grid.

\subsubsection{Explicit Cell Sets}

A vtkm: : cont: :CellSetExplicit defines an irregular collection of cells. The cells can be of different types and connected in arbitrary ways. The types of cell sets are listed in Figure 7.5. This is done by explicitly providing for each cell a sequence of points that defines the cell.

An explicit cell set is defined with a minimum of three arrays. The first array identifies the shape of each cell. (Identifiers for cell shapes are shown in Figure 7.5.) The second array has a sequence of point indices that make up each cell. The third array identifies an offset into the second array where the point indices for each cell is found plus an extra entry at the end set to the size of the second array. Figure 7.6 shows a simple example of an explicit cell set.

An explicit cell set can also identify the number of indices defined for each cell by subtracting consecutive entries in the offsets array. It is often the case when creating a CellSetExplicit that you have an array containing the number of indices rather than the offsets. Such an array can be converted to an offsets array that can be used with CellSetExplicit by using the vtkm: : cont: : ConvertNumComponentsToOffsets convenience function.

vtkm: : cont: :CellSetExplicit is a powerful representation for a cell set because it can represent an arbitrary collection of cells. However, because all connections must be explicitly defined, CellsetExplicit requires a significant amount of memory to represent the topology.

An important specialization of an explicit cell set is vtkm: : cont: :CellSetSingleType. CellSetSingleType is an explicit cell set constrained to contain cells that all have the same shape and all have the same number of points. So for example if you are creating a surface that you know will contain only triangles, CellSetSingleType is a good representation for these data.

Using CellSetSingleType saves memory because the array of cell shapes and the array of point counts no longer 


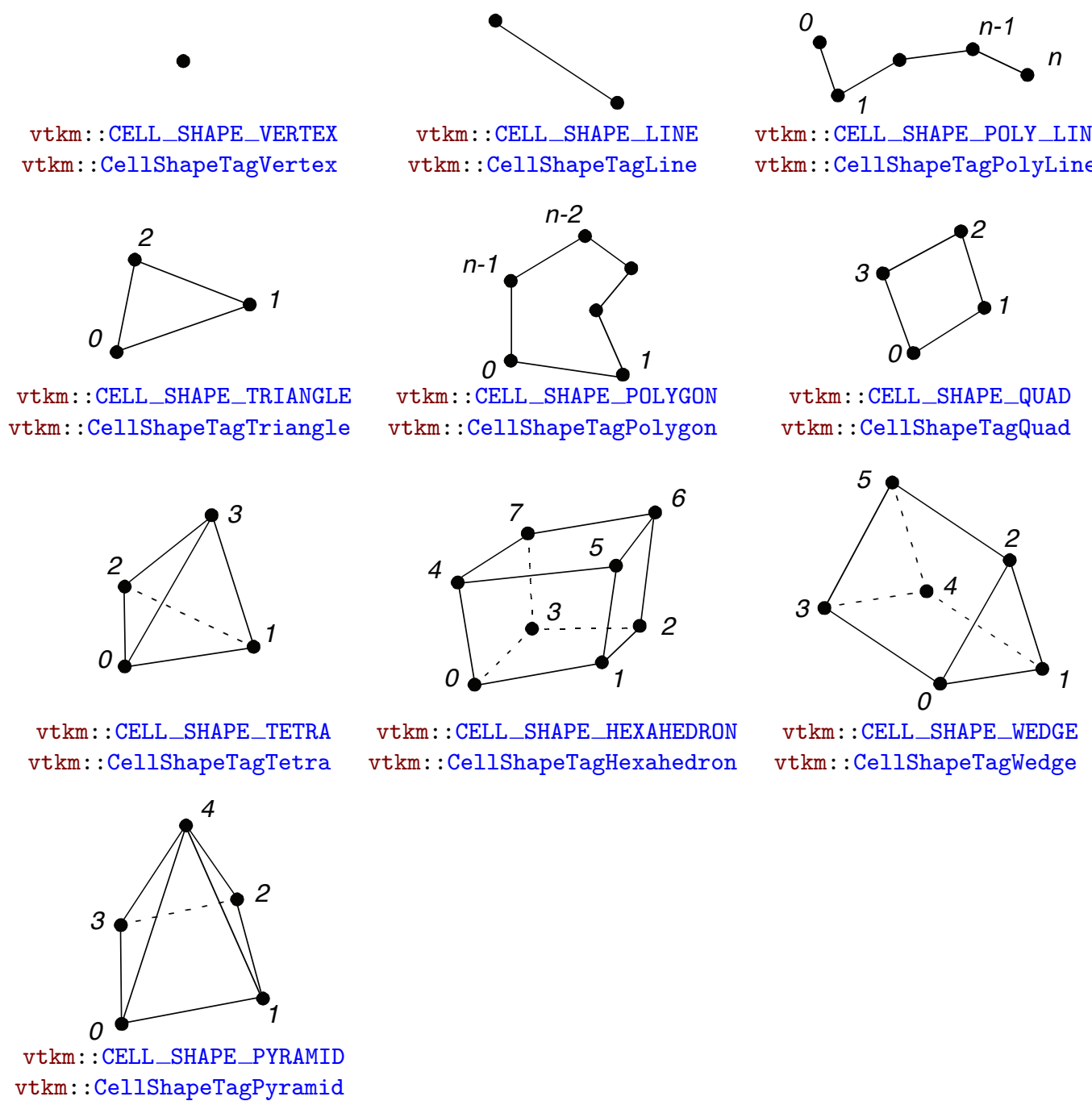

Figure 7.5: Basic Cell Shapes in a CellSetExplicit.

need to be stored. CellSetSingleType also allows VTK-m to skip some processing and other storage required for general explicit cell sets.

\subsubsection{Cell Set Permutations}

A vtkm::cont::CellSetPermutation rearranges the cells of one cell set to create another cell set. This restructuring of cells is not done by copying data to a new structure. Rather, CellSetPermutation establishes a look-up from one cell structure to another. Cells are permuted on the fly while algorithms are run.

A CellSetPermutation is established by providing a mapping array that for every cell index provides the equivalent cell index in the cell set being permuted. CellsetPermutation is most often used to mask out cells in a data set so that algorithms will skip over those cells when running. 


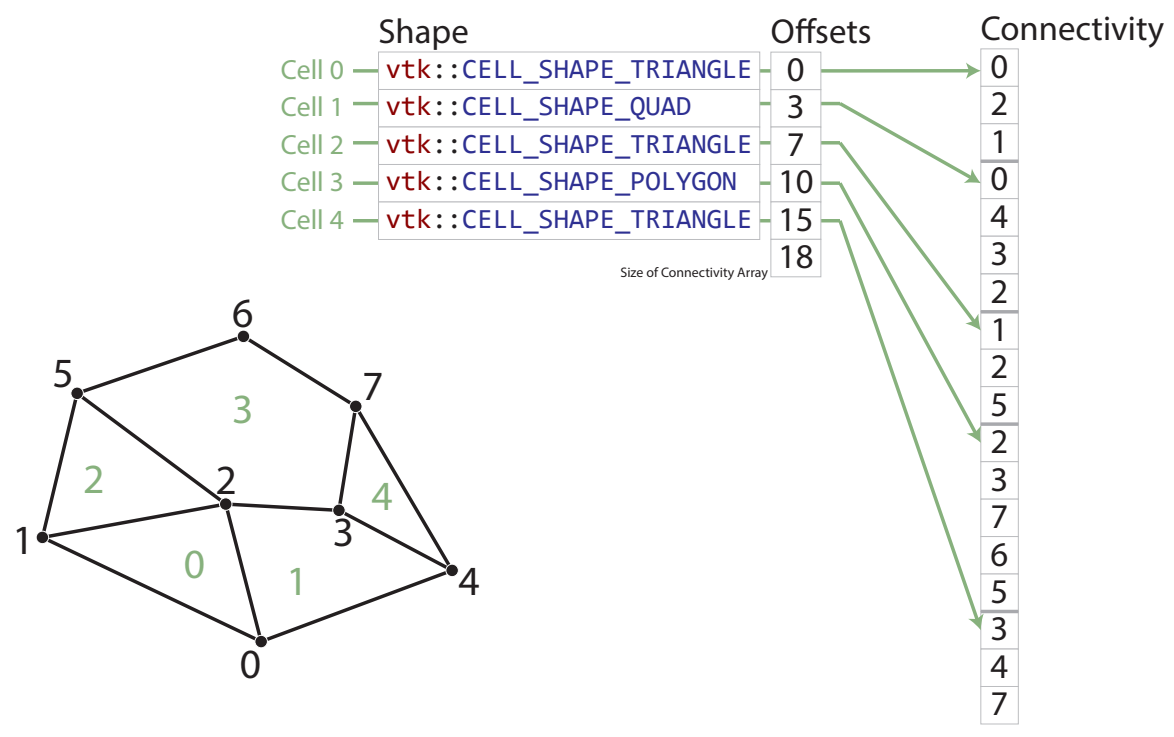

Figure 7.6: Example of cells in a CellSetExplicit and the arrays that define them.

\section{(0) Did you know?}

\Although CellSetPermutation can mask cells, it cannot mask points. All points from the original cell set are available in the permuted cell set regardless of whether they are used.

The following example uses vtkm: :cont: CellsetPermutation with a counting array to expose every tenth cell. This provides a simple way to subsample a data set.

Example 7.7: Subsampling a data set with CellSetPermutation.

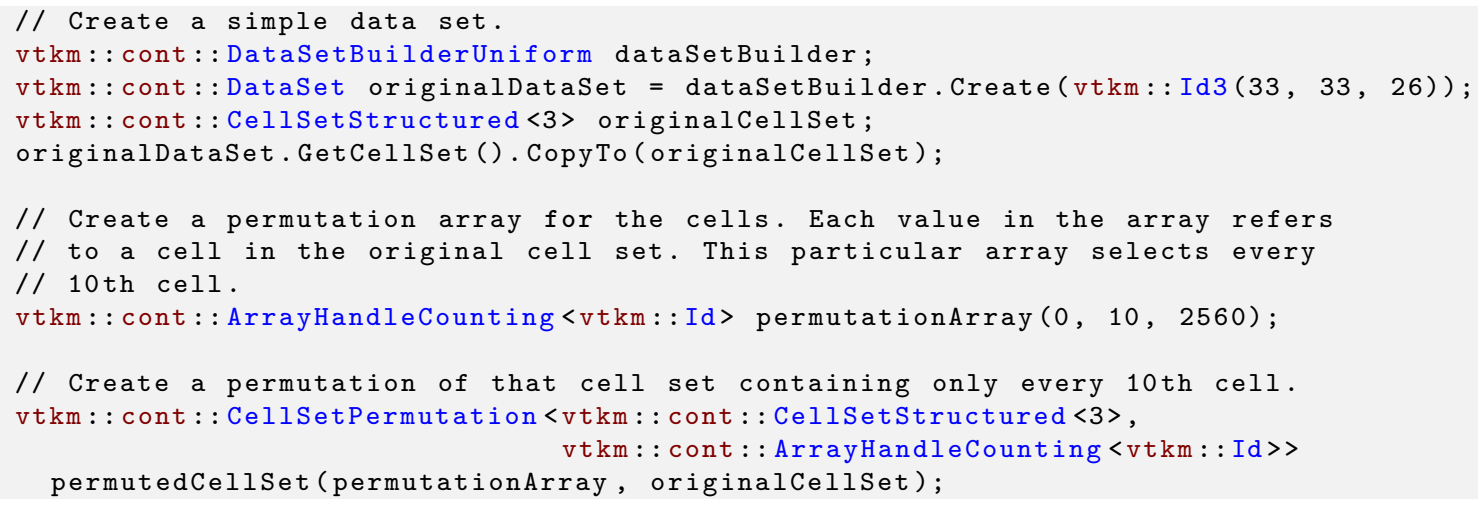

\subsubsection{Cell Set Extrude}

A vtkm: : cont: :CellSetExtrude defines a 3-dimensional extruded mesh representation from 2-dimensional coordinates in the XZ-plane. This is done by providing 2-dimensional coordinates, the number of planes to extrude along the Y-axis, and whether the resulting wedge cellset representation should be a torus or a cylinder. 


\begin{tabular}{|c|c|c|c|}
\hline$\left\{\mathbf{x}_{1}, \mathbf{z}_{1}\right\}$ & $\left\{\mathbf{x}_{2}, \mathbf{z}_{2}\right\}$ & $\left\{\mathbf{x}_{3}, \mathbf{z}_{3}\right\}$ & Wedge Count \\
\hline$\{1,0\}$ & $\{0,0\}$ & $\{1,2\}$ & 6 \\
\hline
\end{tabular}
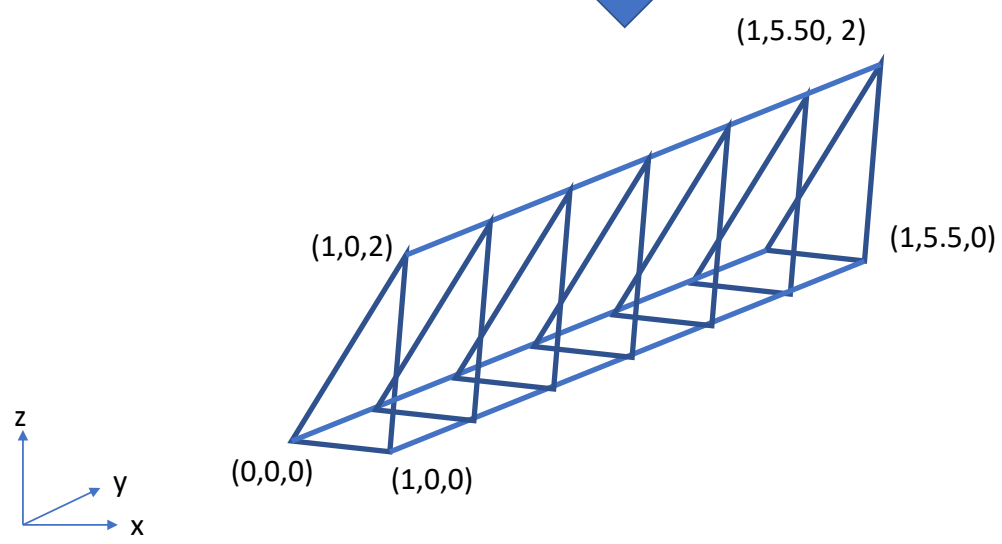

Figure 7.7: An example of an extruded wedge from XZ-plane coordinates. Six wedges are extracted from three XZ-plane points.

The extruded mesh is advantageous because it is represented on-the-fly as required, so no additional memory is required. In contrast other forms of cell sets, such as vtkm: : cont: :CellSetExplicit, need to be explicitly constructed by replicating the vertices and cells. Figure 7.7 shows an example of six wedges extruded from three 2-dimensional coordinates.

\subsection{Fields}

A field on a data set provides a value on every point in space on the mesh. Fields are often used to describe physical properties such as pressure, temperature, mass, velocity, and much more. Fields are represented in a VTK-m data set as an array where each value is associated with a particular element type of a mesh (such as points or cells). This association of field values to mesh elements and the structure of the cell set determines how the field is interpolated throughout the space of the mesh.

Fields are manged by the vtkm: :cont: :Field class. The Field object internally holds a reference to an array in a type-agnostic way. Filters and other VTK-m units will determine the type of the array and pull it out of the Field.

Field has a convenience method named GetRange that finds the range of values stored in the field array. The returned value of GetRange is an ArrayHandle containing vtkm: :Range values. The ArrayHandle will have as many values as components in the field. So, for example, calling GetRange on a scalar field will return an ArrayHandle with exactly 1 entry in it. Calling GetRange on a field of 3D vectors will return an ArrayHandle with exactly 3 entries corresponding to each of the components in the range. Details on how to get data from an ArrayHandle them is given in Chapter 27. 


\subsection{Coordinate Systems}

A coordinate system determines the location of a mesh's elements in space. The spatial location is described by providing a $3 \mathrm{D}$ vector at each point that gives the coordinates there. The point coordinates can then be interpolated throughout the mesh.

Coordinate systems are managed by the vtkm::cont::CoordinateSystem class. In actuality, a coordinate system is just a field with a special meaning, and so the CoordinateSystem class inherits from the Field class. CoordinateSystem constrains the field to be associated with points and typically has $3 \mathrm{D}$ floating point vectors for values.

In addition to all the methods provided by the Field superclass, the CoordinateSystem also provides a GetBounds convenience method that returns a vtkm: Bounds object giving the spatial bounds of the coordinate system.

It is typical for a DataSet to have one coordinate system defined, but it is possible to define multiple coordinate systems. This is helpful when there are multiple ways to express coordinates. For example, positions in geographic may be expressed as Cartesian coordinates or as latitude-longitude coordinates. Both are valid and useful in different ways.

It is also valid to have a DataSet with no coordinate system. This is useful when the structure is not rooted in physical space. For example, if the cell set is representing a graph structure, there might not be any physical space that has meaning for the graph.

\subsection{Partitioned Data Sets}

A partitioned data set, implemented with vtkm: : cont: :PartitionedDataSet, comprises a set of vtkm: : cont: :DataSet objects. The PartitionedDataSet interface allows for adding, replacing, and querying DataSets in its list with the following methods.

GetNumberOfPartitions Returns the number of partitions stored in the PartitionedDataSet.

GetPartition Returns the DataSet at a given index.

GetPartitions Returns all of the DataSets stored in the PartitionedDataSet in a std: :vector.

AppendPartition Adds a given DataSet to the end of the list of partitions.

AppendPartitions Given a list of DataSet objects, appends this list to the end of the list of partitions. This list can be given as a std: : vector or it can be an initializer list (declared in \{\} curly braces).

InsertPartition Given an index and a DataSet, places the DataSet at the given index and pushes the remaining partitions after it.

ReplacePartition Given an index and a DataSet, replaces the partition at that index with the new DataSet.

GetField Retrieves a vtkm: :cont: :Field object from the DataSet at a given index.

The following example creates a vtkm: : cont: :PartitionedDataSet containing two uniform grid data sets.

Example 7.8: Creating a PartitionedDataSet.

// Create two uniform data sets

vtkm: : cont: : DataSetBuilderUniform dataSetBuilder;

Chapter 7. Data Sets 


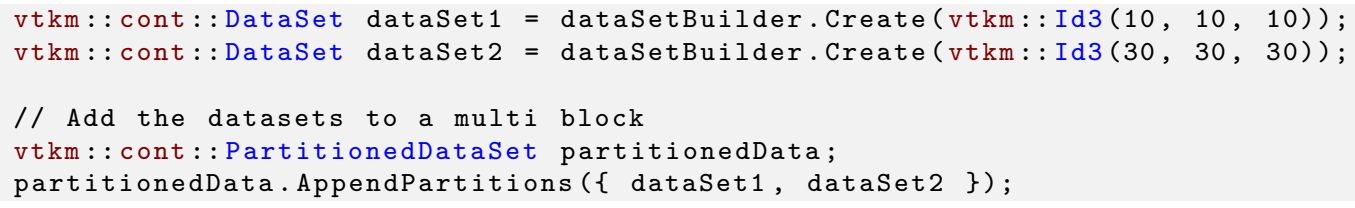

It is always possible to retrieve the independent blocks in a PartitionedDataSet, from which you can iterate and get information about the data. However, VTK-m provides several helper functions to collect metadata information about the collection as a whole.

vtkm::cont::BoundsCompute Queries the bounds of all the DataSets contained in the given PartitionedDataSet and returns a vtkm: :Bounds object encompassing the conglomerate data.

vtkm: : cont: :BoundsGlobalCompute An MPI version of BoundsCompute that also finds the bounds around the conglomerate data across all processes. All MPI processes must call this method.

vtkm::cont::FieldRangeCompute Given a PartitionedDataSet, the name of a field, and (optionally) an association of the field, returns the minimum and maximum value of that field over all the contained blocks. The result is returned in a ArrayHandle of vtkm::Range objects in the same manner as the vtkm: : cont: :Field: :GetRange method (see Section 7.3).

vtkm::cont::FieldRangeGlobalCompute An MPI version of FieldRangeCompute that also finds the field ranges over all blocks on all processes. All MPI processes must call this method.

The following example illustrates a spatial bounds query and a field range query on a vtkm: :cont: :PartitionedDataSet.

Example 7.9: Queries on a PartitionedDataSet.

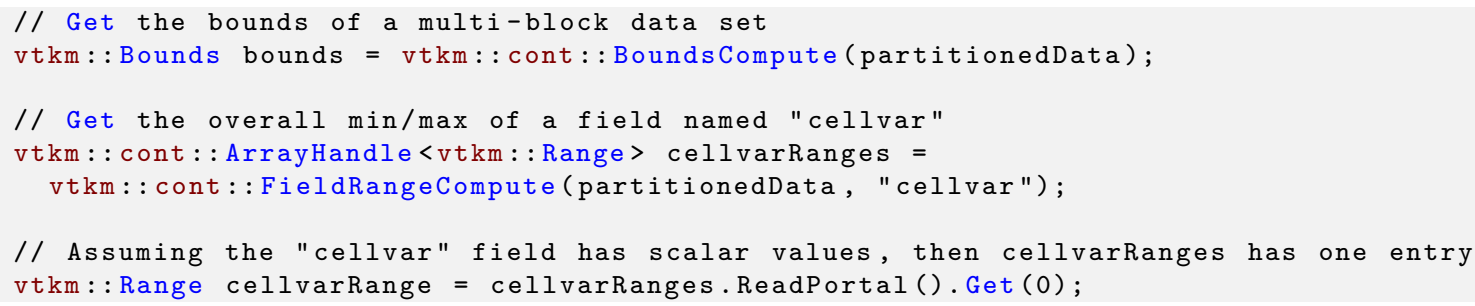

The aforementioned functions for querying a PartitionedDataSet object also work on DataSet objects. This is particularly useful with the BoundsGlobalCompute and FieldRangeGlobalCompute to manage distributed parallel objects.

Filters can be executed on PartitionedDataSet objects in a similar way they are executed on DataSet objects. In both cases, the Execute method is called on the filter giving data object as an argument.

Example 7.10: Applying a filter to multi block data.

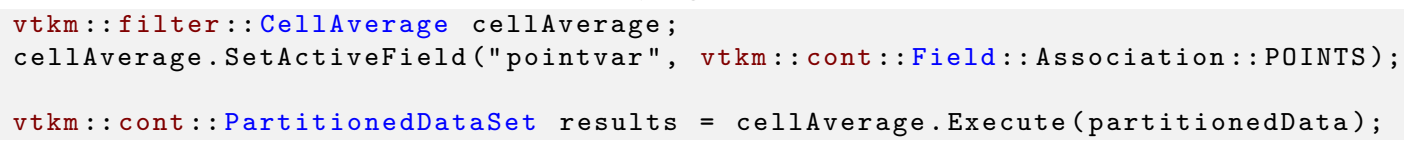




\section{FILE I/O}

Before VTK-m can be used to process data, data need to be loaded into the system. VTK-m comes with a basic file I/O package to get started developing very quickly. All the file I/O classes are declared under the vtkm: : io namespace.

\subsection{Readers}

All reader classes provided by VTK-m are located in the vtkm: :io namespace. The general interface for each reader class is to accept a filename in the constructor and to provide a ReadDataSet method to load the data from disk.

The data in the file are returned in a vtkm: : cont: :DataSet object as described in Chapter 7 , but it is sufficient to known that a DataSet can be passed around readers, writers, filters, and rendering units.

\subsubsection{Legacy VTK File Reader}

Legacy VTK files are a simple open format for storing visualization data. These files typically have a .vtk extension. Legacy VTK files are popular because they are simple to create and read and are consequently supported by a large number of tools. The format of legacy VTK files is well documented in The VTK User's Guide. ${ }^{1}$ Legacy VTK files can also be read and written with tools like ParaView and VisIt.

Legacy VTK files can be read using the vtkm::io::VTKDataSetReader class. The constructor for this class takes a string containing the filename. The ReadDataSet method reads the data from the previously indicated file and returns a vtkm: :cont: :DataSet object, which can be used with filters and rendering.

Example 8.1: Reading a legacy VTK file.

\footnotetext{
${ }^{1} \mathrm{~A}$ free excerpt describing the file format is available at http://www.vtk.org/Wiki/File:VTK-File-Formats.pdf.
} 


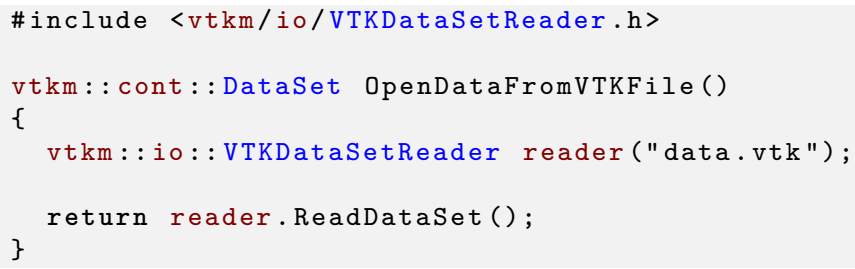

\subsubsection{Image Readers}

VTK-m provides classes to read images from some standard image formats. These readers will store the data in a vtkm: : cont: : DataSet object with the colors stored as a named point field. The colors are read as 4-component RGBA vectors for each pixel. Each component in the pixel color is stored as a 32-bit float between 0 and 1 .

Portable Network Graphics (PNG) files can be read using the vtkm: : io: : ImageReaderPNG class. A ImageReaderPNG object is constructed with the name of the file to load. The data from the file are loaded using the ReadDataSet method, which returns a DataSet object. By default, the colors are stored in a field named "colors", but the name of the field can optionally be changed using the SetPointFieldName method.

Example 8.2: Reading an image from a PNG file.

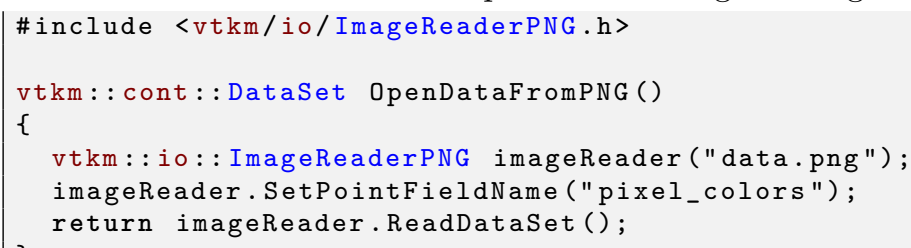

Portable anymap (PNM) files can be read using the vtkm::io: :ImageReaderPNM class. Currently, the PNM file reader only supports files using the portable pixmap (PPM) format (with magic number "P6"). These files are most commonly stored with a .ppm extension although the .pnm extension is also valid. Like for PNG files, a ImageReaderPNM is constructed with the name of the file to read from. ImageReaderPNM also uses the same ReadDataSet and optional SetPointFieldName methods.

Example 8.3: Reading an image from a PNM file.

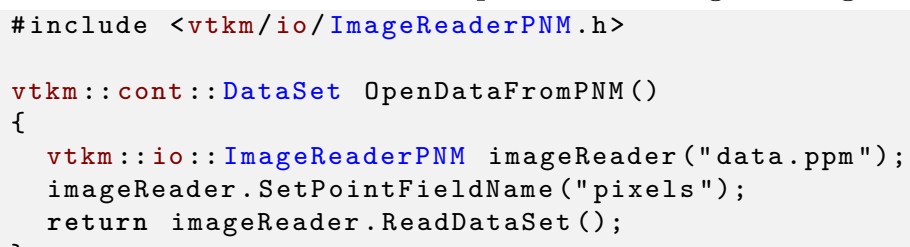

\subsection{Writers}

All writer classes provided by VTK-m are located in the vtkm: :io namespace. The general interface for each writer class is to accept a filename in the constructor and to provide a WriteDataSet method to save data to the disk. The WriteDataSet method takes a vtkm: :cont: :DataSet object as an argument, which contains the data to write to the file. 


\subsubsection{Legacy VTK File Writer}

Legacy VTK files can be written using the vtkm::io: :VTKDataSetWriter class. The constructor for this class takes a string containing the filename. The WriteDataSet method takes a vtkm: : cont: :DataSet object and writes its data to the previously indicated file.

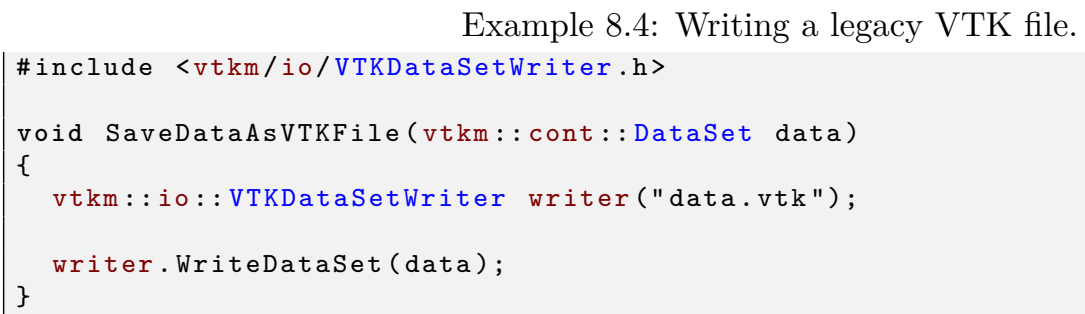

Example 8.4: Writing a legacy VTK file.

\subsubsection{Image Writers}

VTK-m provides classes to some standard image formats. These writers store data in a vtkm: : cont: :DataSet. The data must be a 2D structure with the colors stored in a point field. (See Chapter 7 for details on DataSet objects.)

Portable Network Graphics (PNG) files can be written using the vtkm::io::ImageWriterPNG class. A ImageWriterPNG object is constructed with the name of the file to save. The data are written to the file using the WriteDataSet method. This method takes a DataSet object as an argument. An optional second argument can be given to name the field containing color data to write. If the field is not given, the first point field of the appropriate type is used.

By default, PNG files are written as RGBA colors using 8-bits for each component. You can change the format written using the SetPixelDepth method. This takes an item in the ImageWriterPNG: : PixelDepth enumeration. Valid values are

PixelDepth: :PIXEL_8 8-bit RGBA values (default).

PixelDepth: :PIXEL_16 16-bit RGBA values.

Example 8.5: Writing an image to a PNG file.

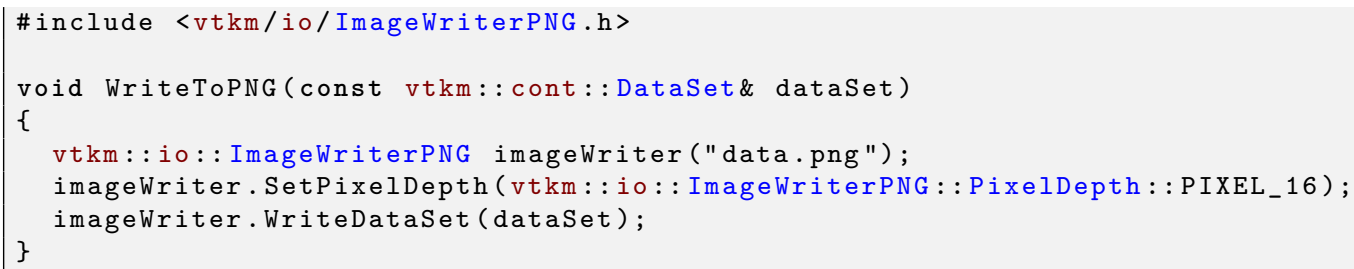

Portable anymap (PNM) files can be written using the vtkm: :io: :ImageWriterPNM class. Currently, the PNM file writer only supports files using the portable pixmap (PPM) format (with magic number "P6"). These files are most commonly stored with a .ppm extensions although the .pnm extension is also valid.

Like for PNG files, a ImageReaderPNM is constructed with the name of the file to write. ImageReaderPNM also uses the same WriteDataSet and SetPixelDepth. It also sports the same ImageReaderPNM: :PixelDepth enumeration.

Example 8.6: Writing an image to a PNM file. 
1 \#include <vtkm/io/ImageWriterPNM.h>

void WriteToPNM (const vtkm: : cont: : DataSet\& dataSet)

\{

vtkm: : io: : ImageWriterPNM imageWriter("data.ppm");

imageWriter. SetPixelDepth (vtkm: : io: : ImageWriterPNM : : PixelDepth : : PIXEL_16); \}

imageWriter. WriteDataSet (dataSet) ; 


\section{RUNNING FILTERS}

Filters are functional units that take data as input and write new data as output. Filters operate on vtkm: :cont: : DataSet objects, which are described in Chapter7.

\section{Did you know?}

The structure of filters in VTK-m is significantly simpler than their counterparts in VTK. VTK filters are arranged in a dataflow network (a.k.a. a visualization pipeline) and execution management is handled automatically. In contrast, VTK-m filters are simple imperative units, which are simply called with input data and return output data.

VTK-m comes with several filters ready for use, and in this chapter we will give a brief overview of these filters. All VTK-m filters are currently defined in the vtkm: :filter namespace. We group filters based on the type of operation that they do and the shared interfaces that they have. Later Part III describes the necessary steps in creating new filters in VTK-m.

Different filters will be used in different ways, but the basic operation of all filters is to instantiate the filter class, set the state parameters on the filter object, and then call the filter's Execute method. The Execute method takes a vtkm::cont::DataSet and returns a new DataSet, which contains the modified data. The Execute method can alternately take a vtkm::cont::PartitionedDataSet object, which is a composite of DataSet objects. In this case Execute will return another PartitionedDataSet object.

The following example provides a simple demonstration of using a filter. It specifically uses the point elevation filter to estimate the air pressure at each point based on its elevation.

Example 9.1: Using PointElevation, which is a field filter.

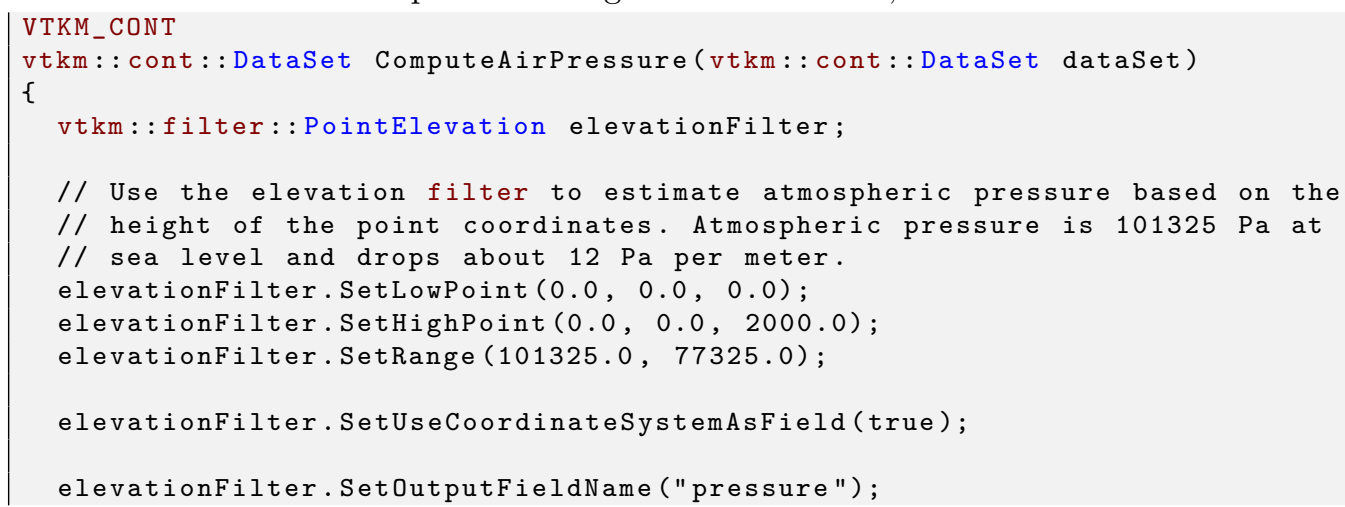




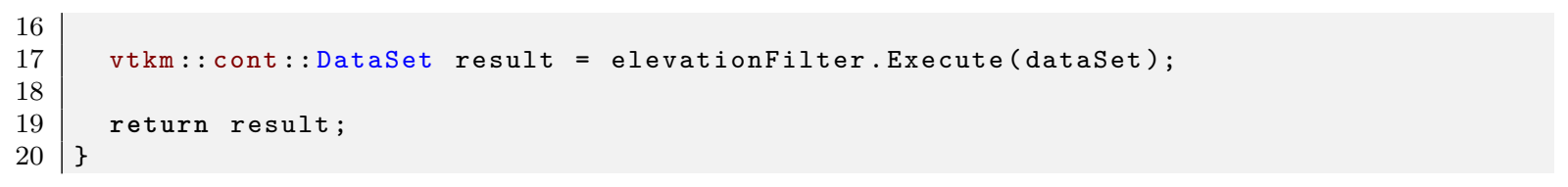

We see that this example follows the previously described procedure of constructing the filter (line 4), setting the state parameters (lines 9-15), and finally executing the filter on a DataSet (line 17).

Every vtkm: : cont: : DataSet object contains a list of fields, which describe some numerical value associated with different parts of the data set in space. Fields often represent physical properties such as temperature, pressure, or velocity. Fields are identified with string names. There are also special fields called coordinate systems that describe the location of points in space. Field are mentioned here because they are often used as input data to the filter's operation and filters often generate new fields in the output. This is the case in Example 9.1. In line 13 the coordinate system is set as the input field and in line 15 the name to use for the generated output field is selected.

\subsection{Provided Filters}

VTK-m comes with the implementation of many filters.

\subsubsection{Cell Average}

vtkm: :filter: :CellAverage is the cell average filter. It will take a data set with a collection of cells and a field defined on the points of the data set and create a new field defined on the cells. The values of this new derived field are computed by averaging the values of the input field at all the incident points. This is a simple way to convert a point field to a cell field.

The default name for the output cell field is the same name as the input point field. The name can be overridden as always using the SetOutputFieldName method.

CellAverage provides the following methods.

SetActiveField/GetActiveFieldName Specifies the name of the field to use as input.

SetUseCoordinateSystemAsField/GetUseCoordinateSystemAsField Specifies a Boolean flag that determines whether to use point coordinates as the input field. Set to false by default. When true, the values for the active field are ignored.

SetActiveCoordinateSystem/GetActiveCoordinateSystemIndex Specifies the index of which coordinate system to use as the input field. The default index is 0 , which is the first coordinate system.

SetOutputFieldName/GetOutputFieldName Specifies the name of the output field generated.

Execute Takes a data set, executes the filter on a device, and returns a data set that contains the result.

SetFieldsToPass/GetFieldsToPass Specifies which fields to pass from input to output. By default all fields are passed. See Section 9.2.2 for more details. 


\subsubsection{Clean Grid}

vtkm: :filter: :CleanGrid is a filter that converts a cell set to an explicit representation and potentially removes redundant or unused data. It does this by iterating over all cells in the data set, and for each one creating the explicit cell representation that is stored in the output. (Explicit cell sets are described in Section 7.2.2.) One benefit of using CleanGrid is that it can optionally remove unused points and combine coincident points. Another benefit is that the resulting cell set will be of a known specific type.

\section{Common Errors}

The result of vtkm::filter::CleanGrid is not necessarily smaller, memory-wise, than its input. For example, "cleaning" a data set with a structured topology will actually result in a data set that requires much more memory to store an explicit topology.

CleanGrid provides the following methods.

SetCompactPointFields/GetCompactPointFields Sets a Boolean flag that determines whether unused points are removed from the output. If true (the default), then the output data set will have a new coordinate system containing only those points being used by the cell set, and the indices of the cells will be adjusted to the new ordering of points.

SetMergePoints/GetMergePoints Sets a Boolean flag that determines whether points coincident in space are merged into a single point. If true (the default), then the output data set will have a new coordinate system containing containing only points that are unique in space, and the indices of the cells will be adjusted to the new set of points. The tolerance parameters control the proximity used for points to be considered coincident.

SetTolerance/GetTolerance Defines the tolerance used when determining whether two points are considered coincident. Because floating point parameters have limited precision, point coordinates that are essentially the same might not be bit-wise exactly the same. Thus, the CleanGrid filter has the ability to find and merge points that are close but perhaps not exact. The default tolerance is $10^{-6}$.

SetToleranceIsAbsolute/GetToleranceIsAbsolute Sets a Boolean flag that determines whether the tolerance parameter should be considered relative to the size of the data set. If false (the default), then the tolerance is multiplied by the length of the diagonal of the bounds of the data being processed. If true, then the tolerance value is used as is.

SetRemoveDegenerateCellsGetRemoveDegenerateCells Sets a Boolean flag that determines whether degenerate cells should be removed. If true (the default), then the CleanGrid filter will look for repeated points in cells and, if the repeated points cause the cell to drop dimensionality, the cell is removed. This is particularly useful when point merging is on as this operation can create degenerate cells.

SetFastMerge/GetFastMerge Sets a Boolean flag that determines whether to use a faster but less accurate method for finding coincident points. If true (the default), some corners are cut when computing coincident points. This will make the point merge step go faster but the tolerance will not be strictly followed. If false, then extra steps will be taken to ensure that all points within tolerance are merged and that only points within tolerance are merged. This flag has no effect if point merging is off.

SetActiveCoordinateSystem/GetActiveCoordinateSystemIndex Specifies the index of which coordinate system to use as when computing spatial locations in the mesh. The default index is 0 , which is the first coordinate system. 
Execute Takes a data set, executes the filter on a device, and returns a data set that contains the result.

SetFieldsToPass/GetFieldsToPass Specifies which fields to pass from input to output. By default all fields are passed. See Section 9.2.2 for more details.

\subsubsection{Clip with Field}

Clipping is an operation that removes regions from the data set based on a user-provided value or function. The vtkm::filter::ClipWithField filter takes a clip value as an argument and removes regions where a named scalar field is below (or above) that value. (A companion filter that discards a region of the data based on an implicit function is described in Section 9.1.4.)

The result of ClipWithField is a volume. If a cell has field values at its vertices that are all below the specified value, then it will be discarded entirely. Likewise, if a cell has field values at its vertices that are all above the specified value, then it will be retained in its entirety. If a cell has some vertices with field values below the specified value and some above, then the cell will be split into the portions above the value (which will be retained) and the portions below the value (which will be discarded).

This operation is sometimes called an isovolume because it extracts the volume of a mesh that is inside the iso-region of a scalar. This is in contrast to an isosurface (also known as a contour), which extracts only the surface of that iso-value. (See Section 9.1.7 for extracting an isosurface.) ClipWithField is also similar to a threshold operation, which extracts cells based on the value of field. The difference is that threshold will either keep or remove entire cells based on the field values whereas clip with carve cells that straddle the valid regions. (See section 9.1.25 for threshold extraction.)

ClipWithField provides the following methods.

SetClipValue/GetClipValue Specifies the field value for the clip operation. Regions where the active field is less than this value are clipped away from each input cell.

SetInvertClip Specifies if the result for the clip filter should be inverted. If set to false (the default), regions where the active field is less than the specified clip value are removed. If set to true, regions where the active field is more than the specified clip value are removed.

SetActiveField/GetActiveFieldName Specifies the name of the field to use as input.

SetUseCoordinateSystemAsField/GetUseCoordinateSystemAsField Specifies a Boolean flag that determines whether to use point coordinates as the input field. Set to false by default. When true, the values for the active field are ignored.

SetActiveCoordinateSystem/GetActiveCoordinateSystemIndex Specifies the index of which coordinate system to use as when computing spatial locations in the mesh. The default index is 0 , which is the first coordinate system.

Execute Takes a data set, executes the filter on a device, and returns a data set that contains the result.

SetFieldsToPass/GetFieldsToPass Specifies which fields to pass from input to output. By default all fields are passed. See Section 9.2.2 for more details.

Example 9.2: Using ClipWithField.

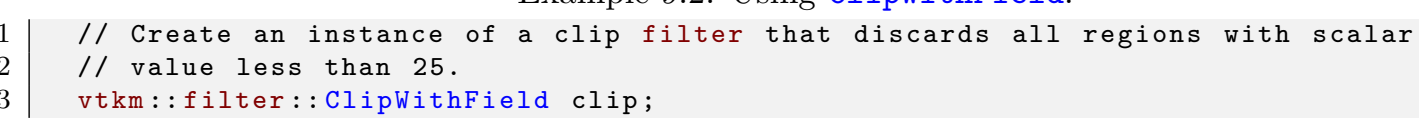




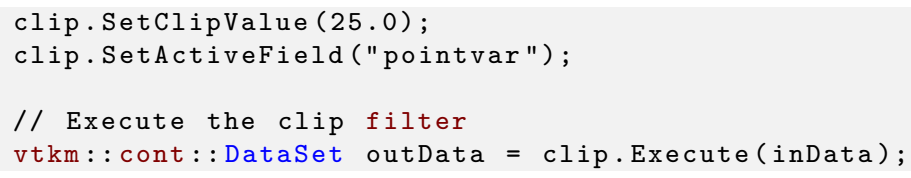

\subsubsection{Clip with Implicit Function}

Clipping is an operation that removes regions from the data set based on a user-provided value or function. The vtkm::filter::ClipWithImplicitFunction takes an implicit function as an argument. See Chapter 14 for more detail. ClipWithImplicitFunction discards regions of the original data set according to the values of the implicit function. (A companion filter that discards a region of the data based on the value of a scalar field is described in Section 9.1.3.)

The result of ClipWithImplicitFunction is a volume. If a cell has its vertices positioned all outside the implicit function, then it will be discarded entirely. Likewise, if a cell its vertices all inside the implicit function, then it will be retained in its entirety. If a cell has some vertices inside the implicit function and some outside, then the cell will be split into the portions inside (which will be retained) and the portions outside (which will be discarded).

ClipWithImplicitFunction provides the following methods.

SetImplicitFunction/GetImplicitFunction Specifies the implicit function to be used to perform the clip operation. The filter does not directly take a vtkm: ImplicitFunction but rather an ImplicitFunction wrapped inside of a vtkm: cont::ImplicitFunctionHandle. The ImplicitFunctionHandle manages the use of the virtual methods in ImplicitFunction on different devices, which may be using different memory spaces or require different processor instructions. An ImplicitFunctionHandle is easily created with the vtkm: : cont: :make_ImplicitFunctionHandle function.

SetInvertClip Specifies whether the result of the clip filter should be inverted. If set to false (the default), all regions where the implicit function is negative will be removed. If set to true, all regions where the implicit function is positive will be removed.

SetActiveCoordinateSystem/GetActiveCoordinateSystemIndex Specifies the index of which coordinate system to use as when computing spatial locations in the mesh. The default index is 0 , which is the first coordinate system.

Execute Takes a data set, executes the filter on a device, and returns a data set that contains the result.

SetFieldsToPass/GetFieldsToPass Specifies which fields to pass from input to output. By default all fields are passed. See Section 9.2.2 for more details.

In the example provided below the vtkm: : Sphere implicit function is used. This function evaluates to a negative value if points from the original dataset occur within the sphere, evaluates to 0 if the points occur on the surface of the sphere, and evaluates to a positive value if the points occur outside the sphere.

Example 9.3: Using ClipWithImplicitFunction.

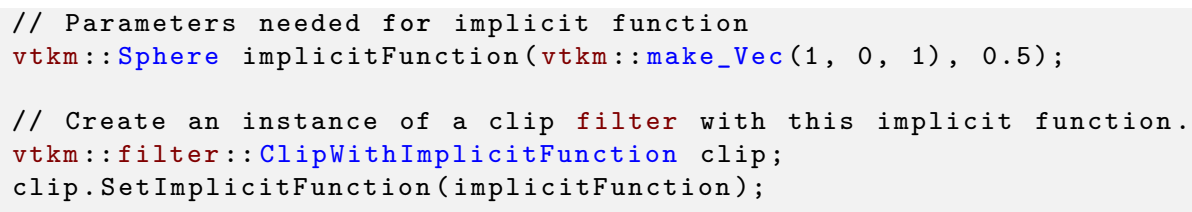




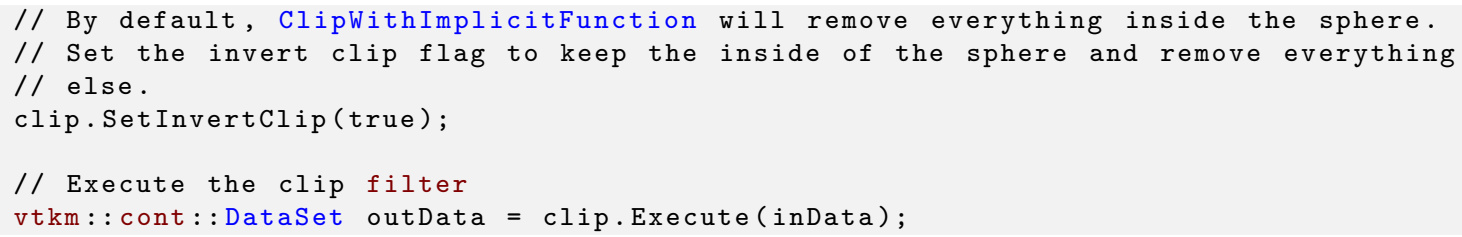

\subsubsection{Connected Components}

Connected components in a mesh are groups of mesh elements that are connected together in some way. For example, if two cells are neighbors, then they are in the same component. Likewise, a cell is also in the same component as its neighbor's neighbors as well as their neighbors and so on. Connected components help identify when features in a simulation fragment or meld.

VTK-m provides two types of connected components filters. The first filter follows topological connections to find cells that are literally connected together. The second filter takes a structured cell set and a field that classifies each cell and finds connected components where all the cells have the same field value.

\section{Cell Connectivity}

The vtkm: :filter: :CellSetConnectivity filter finds groups of cells that are connected together through their topology. Two cells are considered connected if they share an edge. CellSetConnectivity identifies some number of components and assigns each component a unique integer.

The result of the filter is a cell field of type vtkm: :Id. Each entry in the cell field will be a number that identifies to which component the cell belongs. By default, this output cell field is named "component". Although an input field can be specified, it is ignored.

CellSetConnectivity provides the following methods.

SetActiveField/GetActiveFieldName Specifies the name of the field to use as input.

SetUseCoordinateSystemAsField/GetUseCoordinateSystemAsField Specifies a Boolean flag that determines whether to use point coordinates as the input field. Set to false by default. When true, the values for the active field are ignored.

SetActiveCoordinateSystem/GetActiveCoordinateSystemIndex Specifies the index of which coordinate system to use as the input field. The default index is 0 , which is the first coordinate system.

SetOutputFieldName/GetOutputFieldName Specifies the name of the output field generated.

Execute Takes a data set, executes the filter on a device, and returns a data set that contains the result.

SetFieldsToPass/GetFieldsToPass Specifies which fields to pass from input to output. By default all fields are passed. See Section 9.2.2 for more details.

Image Field

The vtkm::filter::ImageConnectivity filter finds groups of points that have the same field value and are connected together through their topology. Any point is considered to be connected to its Moore neighborhood: 
8 neighboring points for 2D and 26 neighboring points for 3D. As the name implies, ImageConnectivity only works on data with a structured cell set. You will get an error if you use any other type of cell set.

The active field passed to the filter must be associated with the points.

The result of the filter is a point field of type vtkm: :Id. Each entry in the point field will be a number that identifies to which component the cell belongs. By default, this output point field is named "component".

ImageConnectivity provides the following methods.

SetActiveField/GetActiveFieldName Specifies the name of the field to use as input.

SetUseCoordinateSystemAsField/GetUseCoordinateSystemAsField Specifies a Boolean flag that determines whether to use point coordinates as the input field. Set to false by default. When true, the values for the active field are ignored.

SetActiveCoordinateSystem/GetActiveCoordinateSystemIndex Specifies the index of which coordinate system to use as the input field. The default index is 0 , which is the first coordinate system.

SetOutputFieldName/GetOutputFieldName Specifies the name of the output field generated.

Execute Takes a data set, executes the filter on a device, and returns a data set that contains the result.

SetFieldsToPass/GetFieldsToPass Specifies which fields to pass from input to output. By default all fields are passed. See Section 9.2.2 for more details.

\subsubsection{Coordinate System Transforms}

VTK-m provides multiple filters to translate between different coordinate systems.

\section{Cylindrical Coordinate System Transform}

vtkm: :filter: :CylindricalCoordinateSystemTransform is a coordinate system transformation filter. The filter will take a data set and transform the points of the coordinate system. By default, the filter will transform the coordinates from a Cartesian coordinate system to a cylindrical coordinate system. The order for cylindrical coordinates is $(R, \theta, Z)$

The default name for the output field is "cylindricalCoordinateSystemTransform", but that can be overridden as always using the SetOutputFieldName method.

In addition to the standard SetOutputFieldName and Execute methods, CylindricalCoordinateSystemTransform provides the following methods.

SetCartesianToCylindrical This method specifies a transformation from cartesian to cylindrical coordinates.

SetCylindricalToCartesian This method specifies a transformation from cylindrical to cartesian coordinates.

SetActiveField/GetActiveFieldName Specifies the name of the field to use as input.

SetUseCoordinateSystemAsField/GetUseCoordinateSystemAsField Specifies a Boolean flag that determines whether to use point coordinates as the input field. Set to false by default. When true, the values for the active field are ignored.

SetActiveCoordinateSystem/GetActiveCoordinateSystemIndex Specifies the index of which coordinate system to use as the input field. The default index is 0 , which is the first coordinate system. 
SetOutputFieldName/GetOutputFieldName Specifies the name of the output field generated.

Execute Takes a data set, executes the filter on a device, and returns a data set that contains the result.

SetFieldsToPass/GetFieldsToPass Specifies which fields to pass from input to output. By default all fields are passed. See Section 9.2.2 for more details.

\section{Spherical Coordinate System Transform}

vtkm: :filter: :SphericalCoordinateSystemTransform is a coordinate system transformation filter. The filter will take a data set and transform the points of the coordinate system. By default, the filter will transform the coordinates from a cartesian coordinate system to a spherical coordinate system. The order for spherical coordinates is $(R, \theta, \phi)$

The default name for the output field is "sphericalCoordinateSystemTransform", but that can be overridden as always using the SetOutputFieldName method.

In addition the standard SetOutputFieldName and Execute methods, CylindricalCoordinateSystemTransform provides the following methods.

SetCartesianToSpherical This method specifies a transformation from cartesian to spherical coordinates.

SetSphericalToCartesian This method specifies a transformation from spherical to cartesian coordinates.

SetActiveField/GetActiveFieldName Specifies the name of the field to use as input.

SetUseCoordinateSystemAsField/GetUseCoordinateSystemAsField Specifies a Boolean flag that determines whether to use point coordinates as the input field. Set to false by default. When true, the values for the active field are ignored.

SetActiveCoordinateSystem/GetActiveCoordinateSystemIndex Specifies the index of which coordinate system to use as the input field. The default index is 0 , which is the first coordinate system.

SetOutputFieldName/GetOutputFieldName Specifies the name of the output field generated.

Execute Takes a data set, executes the filter on a device, and returns a data set that contains the result.

SetFieldsToPass/GetFieldsToPass Specifies which fields to pass from input to output. By default all fields are passed. See Section 9.2.2 for more details.

\subsubsection{Contour}

Contouring is one of the most fundamental filters in scientific visualization. A contour is the locus where a field is equal to a particular value. A topographic map showing curves of various elevations often used when hiking in hilly regions is an example of contours of an elevation field in 2 dimensions. Extended to 3 dimensions, a contour gives a surface. Thus, a contour is often called an isosurface. The contouring/isosurface algorithm is implemented by vtkm: :filter: : Contour.

Contour provides the following methods.

SetIsoValue/GetIsoValue Specifies the value on which to extract the contour. The contour will be the surface where the field (provided to Execute) is equal to this value. 
SetMergeDuplicatePoints/GetMergeDuplicatePoints Specifies whether coincident points in the data set should be merged. Because the contour filter (like all filters in VTK-m) runs in parallel, parallel threads can (and often do) create duplicate versions of points. When this flag is set to true, a secondary operation will find all duplicated points and combine them together.

SetGenerateNormals/GetGenerateNormals Specifies whether to generate normal vectors for the surface. Normals are used in shading calculations during rendering and can make the surface appear more smooth. By default, the generated normals are based on the gradient of the field being contoured and can be quite expensive to compute. A faster method is available that computes the normals based on the faces of the isosurface mesh, but the normals do not look as good as the gradient based normals. Fast normals can be enabled using the flags described bellow.

SetComputeFastNormalsForStructured/GetComputeFastNormalsForStructured Specifies whether to use the fast method of normals computation for Structured data sets. This is only valid if the generate normals flag is set.

SetComputeFastNormalsForUnstructured/GetComputeFastNormalsForUnstructured Specifies whether to use the fast method of normals computation for unstructured data sets. This is only valid if the generate normals flag is set.

SetNormalArrayName/GetNormalArrayName Specifies the name used for the normals field if it is being created.

SetActiveField/GetActiveFieldName Specifies the name of the field to use as input.

SetUseCoordinateSystemAsField/GetUseCoordinateSystemAsField Specifies a Boolean flag that determines whether to use point coordinates as the input field. Set to false by default. When true, the values for the active field are ignored.

SetActiveCoordinateSystem/GetActiveCoordinateSystemIndex Specifies the index of which coordinate system to use as when computing spatial locations in the mesh. The default index is 0 , which is the first coordinate system.

Execute Takes a data set, executes the filter on a device, and returns a data set that contains the result.

SetFieldsToPass/GetFieldsToPass Specifies which fields to pass from input to output. By default all fields are passed. See Section 9.2.2 for more details.

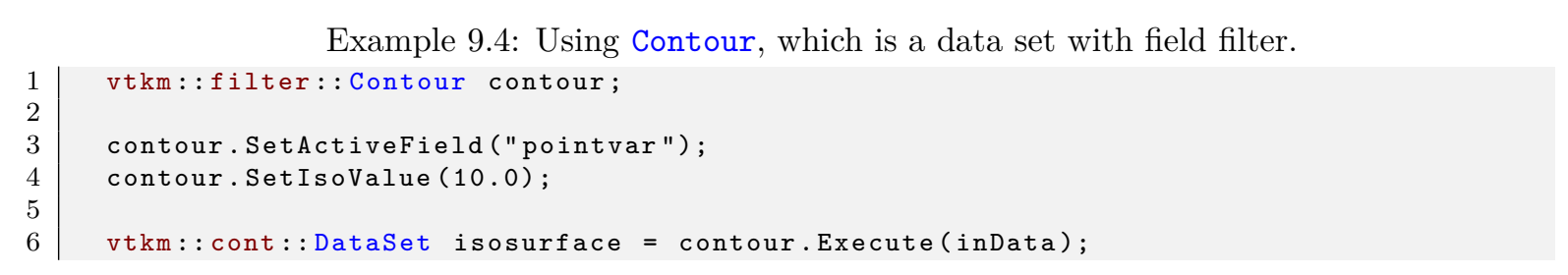

\subsubsection{Cross Product}

vtkm: filter: :CrossProduct computes the cross product of two vector fields for every element in the input data set. The cross product filter computes (PrimaryField $\times$ SecondaryField), where both the primary and secondary field are specified using methods on the CrossProduct class. The cross product computation works for both point and cell centered vector fields.

CrossProduct provides the following methods. 
SetPrimaryField/GetPrimaryFieldName Specifies the name of the field to use as input for the primary (first) value of the cross product.

SetUseCoordinateSystemAsPrimaryField/GetUseCoordinateSystemAsPrimaryField Specifies a Boolean flag that determines whether to use point coordinates as the primary input field. Set to false by default. When true, the name for the primary field is ignored.

SetPrimaryCoordinateSystem/GetPrimaryCoordinateSystemIndex Specifies the index of which coordinate system to use as the primary input field. The default index is 0 , which is the first coordinate system.

SetSecondaryField/GetSecondaryFieldName Specifies the name of the field to use as input for the secondary (second) value of the cross product.

SetUseCoordinateSystemAsSecondaryField/GetUseCoordinateSystemAsSecondaryField Specifies

Boolean flag that determines whether to use point coordinates as the secondary input field. Set to false by default. When true, the name for the secondary field is ignored.

SetSecondaryCoordinateSystem/GetSecondaryCoordinateSystemIndex Specifies the index of which coordinate system to use as the secondary input field. The default index is 0 , which is the first coordinate system.

SetOutputFieldName/GetOutputFieldName Specifies the name of the output field generated.

Execute Takes a data set, executes the filter on a device, and returns a data set that contains the result.

SetFieldsToPass/GetFieldsToPass Specifies which fields to pass from input to output. By default all fields are passed. See Section 9.2.2 for more details.

\subsubsection{Dot Product}

vtkm: :filter: :DotProduct computes the dot product of two vector fields for every element in the input data set. The dot product filter computes (PrimaryField - SecondaryField), where both the primary and secondary field are specified using methods on the DotProduct class. The dot product computation works for both point and cell centered vector fields.

DotProduct provides the following methods.

SetPrimaryField/GetPrimaryFieldName Specifies the name of the field to use as input for the primary (first) value of the dot product.

SetUseCoordinateSystemAsPrimaryField/GetUseCoordinateSystemAsPrimaryField Specifies a Boolean flag that determines whether to use point coordinates as the primary input field. Set to false by default. When true, the name for the primary field is ignored.

SetPrimaryCoordinateSystem/GetPrimaryCoordinateSystemIndex Specifies the index of which coordinate system to use as the primary input field. The default index is 0 , which is the first coordinate system.

SetSecondaryField/GetSecondaryFieldName Specifies the name of the field to use as input for the secondary (second) value of the dot product.

SetUseCoordinateSystemAsSecondaryField/GetUseCoordinateSystemAsSecondaryField Specifies a Boolean flag that determines whether to use point coordinates as the secondary input field. Set to false by default. When true, the name for the secondary field is ignored. 
SetSecondaryCoordinateSystem/GetSecondaryCoordinateSystemIndex Specifies the index of which coordinate system to use as the secondary input field. The default index is 0 , which is the first coordinate system.

SetOutputFieldName/GetOutputFieldName Specifies the name of the output field generated.

Execute Takes a data set, executes the filter on a device, and returns a data set that contains the result.

SetFieldsToPass/GetFieldsToPass Specifies which fields to pass from input to output. By default all fields are passed. See Section 9.2.2 for more details.

\subsubsection{External Faces}

vtkm: :filter::ExternalFaces is a filter that extracts all the external faces from a polyhedral data set. An external face is any face that is on the boundary of a mesh. Thus, if there is a hole in a volume, the boundary of that hole will be considered external. More formally, an external face is one that belongs to only one cell in a mesh.

ExternalFaces provides the following methods.

SetCompactPoints/GetCompactPoints Specifies whether point fields should be compacted. If on, the filter will remove from the output all points that are not used in the resulting surface. If off (the default), unused points will remain listed in the topology, but point fields and coordinate systems will be shallow-copied to the output.

SetPassPolyData/GetPassPolyData Specifies how polygonal data (polygons, lines, and vertices) will be handled. If on (the default), these cells will be passed to the output. If off, these cells will be removed from the output. (Because they have less than 3 topological dimensions, they are not considered to have any "faces.")

SetActiveCoordinateSystem/GetActiveCoordinateSystemIndex Specifies the index of which coordinate system to use as when computing spatial locations in the mesh. The default index is 0 , which is the first coordinate system.

Execute Takes a data set, executes the filter on a device, and returns a data set that contains the result.

SetFieldsToPass/GetFieldsToPass Specifies which fields to pass from input to output. By default all fields are passed. See Section 9.2.2 for more details.

\subsubsection{Extract Structured}

vtkm: :filter: :ExtractStructured is a filter that extracts a volume of interest (VOI) from a structured data set. In addition the filter is able to subsample the VOI while doing the extraction.

The output of this filter is a structured dataset. The filter treats input data of any topological dimension (i.e., point, line, plane, or volume) and can generate output data of any topological dimension.

Typical applications of this filter are to extract a slice from a volume for image processing, subsampling large volumes to reduce data size, or extracting regions of a volume with interesting data.

ExtractStructured provides the following methods. 
SetVOI/GetVOI Specifies what volume of interest (VOI) should be extracted by the filter. By default the VOI is the entire input.

SetSampleRate/GetSampleRate Specifies the sample rate of the VOI. Supports sub-sampling on a per dimension basis.

SetIncludeBoundary/GetIncludeBoundary Specifies if the VOI is inclusive or exclusive on the boundary of the VOI.

SetActiveCoordinateSystem/GetActiveCoordinateSystemIndex Specifies the index of which coordinate system to use as when computing spatial locations in the mesh. The default index is 0 , which is the first coordinate system.

Execute Takes a data set, executes the filter on a device, and returns a data set that contains the result.

SetFieldsToPass/GetFieldsToPass Specifies which fields to pass from input to output. By default all fields are passed. See Section 9.2.2 for more details.

\subsubsection{Field to Colors}

vtkm: :filter: :FieldToColors takes a field in a data set, looks up each value in a color table, and writes the resulting colors to a new field. The color to be used for each field value is specified using a vtkm: :cont: :ColorTable object. ColorTable objects are also used with VTK-m's rendering module and are described in Section 10.8.

FieldToColors has three modes it can use to select how it should treat the input field.

FieldToColors: : SCALAR Treat the field as a scalar field. It is an error to provide a field of any type that cannot be directly converted to a basic floating point number (such as a vector).

FieldToColors: :MAGNITUDE Given a vector field, take the magnitude of each field value before looking it up in the color table.

FieldToColors: : COMPONENT Select a particular component of the vectors in a field to map to colors.

Additionally, FieldToColors has different modes in which it can represent colors in its output.

FieldToColors: :RGB Output colors are represented as RGB values with each component represented by an unsigned byte. Specifically, these are vtkm::Vec3ui_8 values.

FieldToColors: :RGBA Output colors are represented as RGBA values with each component represented by an unsigned byte. Specifically, these are vtkm::Vec4ui_8 values.

FieldToColors provides the following methods.

SetColorTable/GetColorTable Specifies the vtkm::cont::ColorTable object to use to map field values to colors.

SetMappingMode/GetMappingMode Specifies the input mapping mode. The value is one of the FieldToColors: :SCALAR, FieldToColors: : MAGNITUDE, or FieldToColors: : COMPONENT selectors described previously.

SetMappingToScalar Sets the input mapping mode to scalar. Shortcut for SetMappingMode(vtkm: :filter: :FieldToColors: : SCALAR ). 
SetMappingToMagnitude Sets the input mapping mode to vector. Shortcut for SetMappingMode(vtkm::filter: :FieldToColors: :MAGNITUDE ).

SetMappingToComponent Sets the input mapping mode to component. Shortcut for SetMappingMode(vtkm: :filter: :FieldToColors: :COMPONENT ).

IsMappingScalar Returns true if the input mapping mode is scalar (FieldToColorsSCALAR).

IsMappingMagnitude Returns true if the input mapping mode is magnitude (FieldToColors: :MAGNITUDE).

IsMappingComponent Returns true if the input mapping mode is component (FieldToColors: :COMPONENT).

SetMappingComponent/GetMappingComponent Specifies the component of the vector to use in the mapping. This only has an effect if the input mapping mode is set to FieldToColors : :COMPONENT.

SetOutputMode/GetOutputMode Specifies the output representation of colors. The value is one of the FieldToColors: :RGB or FieldToColors: :RGBA selectors described previously.

SetOutputToRGB Sets the output representation to 8-bit RGB. Shortcut for SetOutputMode(vtkm: :filter: :FieldToColors : :RGB ).

SetOutputToRGBA Sets the output representation to 8-bit RGBA. Shortcut for SetOutputMode(vtkm: :filter: :FieldToColors: :RGBA ).

IsOutputRGB Returns true if the output representation is 8-bit RGB (FieldToColors: :RGB).

IsOutputRGBA Returns true if the output representation is 8-bit RGBA (FieldToColors: :RGBA).

SetNumberOfSamplingPoints/GetNumberOfSamplingPoints Specifies how many samples to use when looking up color values. The implementation of FieldToColors first builds an array of color samples to quickly look up colors for particular values. The size of this lookup array can be adjusted with this parameter. By default, an array of 256 colors is used.

SetActiveField/GetActiveFieldName Specifies the name of the field to use as input.

SetUseCoordinateSystemAsField/GetUseCoordinateSystemAsField Specifies a Boolean flag that determines whether to use point coordinates as the input field. Set to false by default. When true, the values for the active field are ignored.

SetActiveCoordinateSystem/GetActiveCoordinateSystemIndex Specifies the index of which coordinate system to use as the input field. The default index is 0 , which is the first coordinate system.

SetOutputFieldName/GetOutputFieldName Specifies the name of the output field generated.

Execute Takes a data set, executes the filter on a device, and returns a data set that contains the result.

SetFieldsToPass/GetFieldsToPass Specifies which fields to pass from input to output. By default all fields are passed. See Section 9.2.2 for more details. 


\subsubsection{Ghost Cell Classification}

vtkm: :filter::GhostCellClassify adds a cell centered field to the input data set that marks each cell as either vtkm: :CellClassification: :NORMAL or vtkm::CellClassification::GHOST. The outer layer of cells are marked as GHOST, and the remainder are marked as *CellClassificationNORMAL. This filter only supports uniform and rectilinear data sets. The default field is "vtkmGhostCells".

GhostCellClassify provides the following methods.

SetActiveCoordinateSystem/GetActiveCoordinateSystemIndex Specifies the index of which coordinate system to use as when computing spatial locations in the mesh. The default index is 0 , which is the first coordinate system.

Execute Takes a data set, executes the filter on a device, and returns a data set that contains the result.

SetFieldsToPass/GetFieldsToPass Specifies which fields to pass from input to output. By default all fields are passed. See Section 9.2.2 for more details.

\subsubsection{Ghost Cell Removal}

vtkm::filter::GhostCellRemove is a filter that is used to remove cells from a data set according to a cell centered field that is provided to the filter. The default field used for removal is "vtkmGhostCells". The field is of type vtkm: :UInt8, and represents a bit-field to classify each cell. By default, if the input is a structured data set the filter will attempt to output a structured data set. If this is not possible, an explicit data set is produced. The field specified for cell removal is not passed to the output.

GhostCellRemove provides the following methods.

RemoveAllGhost Remove all cells where the value is a ghost cell (i.e. vtkm: CellClassification: :GHOST).

RemoveByType Remove cells specified by the vtkm: :UInt8 using a bitwise "and" operation with the type field. The values in vtkm: CellClassification can be combined with a logical "or" operation to specify the type. Current values of vtkm: CellClassification include: NORMAL, GHOST, and INVALID.

SetActiveCoordinateSystem/GetActiveCoordinateSystemIndex Specifies the index of which coordinate system to use as when computing spatial locations in the mesh. The default index is 0 , which is the first coordinate system.

Execute Takes a data set, executes the filter on a device, and returns a data set that contains the result.

SetFieldsToPass/GetFieldsToPass Specifies which fields to pass from input to output. By default all fields are passed. See Section 9.2.2 for more details.

\subsubsection{Gradients}

vtkm: : filter: :Gradient computes the gradient of a point based input field for every element in the input data set. The gradient computation can either generate cell center based gradients, which are fast but less accurate, or more accurate but slower point based gradients. The default for the filter is output as cell centered gradients, but can be changed by using the SetComputePointGradient method. The default name for the output fields is "Gradients", but that can be overridden as always using the SetOutputFieldName method.

Gradient provides the following methods. 
SetComputePointGradient/GetComputePointGradient Specifies whether we are computing point or cell based gradients. The output field(s) of this filter will be point based if this is enabled.

SetComputeDivergence/GetComputeDivergence Specifies whether the divergence field will be generated. By default the name of the array will be "Divergence" but can be changed by using SetDivergenceName. The field will be a cell field unless ComputePointGradient is enabled. The input array must have 3 components in order to compute this. The default is off.

SetComputeVorticity/GetComputeVorticity Specifies whether the vorticity field will be generated. By default the name of the array will be "Vorticity" but can be changed by using SetVorticityName. The field will be a cell field unless ComputePointGradient is enabled. The input array must have 3 components in order to compute this. The default is off.

SetComputeQCriterion/GetComputeQCriterion Specifies whether the Q-Criterion field will be generated. By default the name of the array will be "QCriterion" but can be changed by using SetQCriterionName. The field will be a cell field unless ComputePointGradient is enabled. The input array must have 3 components in order to compute this. The default is off.

SetComputeGradient/GetComputeGradient Specifies whether the actual gradient field is written to the output. When processing fields that have 3 components it is desirable to compute information such as Divergence, Vorticity, or Q-Criterion without incurring the cost of also having to write out the $3 \times 3$ gradient result. The default is on.

SetColumnMajorOrdering/SetRowMajorOrdering When processing input fields that have 3 components, the output will be a a $3 \times 3$ gradient. By default VTK-m outputs all matrix like arrays in Row Major ordering (C-Ordering). The ordering can be changed when integrating with libraries like VTK or with FORTRAN codes that use Column Major ordering. The default is Row Major. This setting is only relevant for 3 component input fields when SetComputeGradient is enabled.

SetDivergenceName/GetDivergenceName Specifies the output cell normals field name. The default is "Divergence".

SetVorticityName/GetVorticityName Specifies the output Vorticity field name. The default is "Vorticity"

SetQCriterionName/GetQCriterionName Specifies the output Q-Criterion field name. The default is "QCriterion".

SetActiveField/GetActiveFieldName Specifies the name of the field to use as input.

SetUseCoordinateSystemAsField/GetUseCoordinateSystemAsField Specifies a Boolean flag that determines whether to use point coordinates as the input field. Set to false by default. When true, the values for the active field are ignored.

SetActiveCoordinateSystem/GetActiveCoordinateSystemIndex Specifies the index of which coordinate system to use as the input field. The default index is 0 , which is the first coordinate system.

SetOutputFieldName/GetOutputFieldName Specifies the name of the output field generated.

Execute Takes a data set, executes the filter on a device, and returns a data set that contains the result.

SetFieldsToPass/GetFieldsToPass Specifies which fields to pass from input to output. By default all fields are passed. See Section 9.2.2 for more details. 


\subsubsection{Histogram}

vtkm: :filter: :Histogram computes a histogram of a given field.

The default number of bins in the output histogram is 10, but that can be overridden using the SetNumberOfBins method.

The default name for the output fields is "histogram". The name can be overridden as always using the SetOutputFieldName method.

Histogram provides the following methods.

SetRange/GetRange Specifies an explicit range to use to generate the histogram. If no range is set the fields global range is used.

GetBinDelta Get the size of each bin from the last computed field.

GetComputedRange Get the computed local range of the histogram from the last computed field.

SetActiveField/GetActiveFieldName Specifies the name of the field to use as input.

SetUseCoordinateSystemAsField/GetUseCoordinateSystemAsField Specifies a Boolean flag that determines whether to use point coordinates as the input field. Set to false by default. When true, the values for the active field are ignored.

SetActiveCoordinateSystem/GetActiveCoordinateSystemIndex Specifies the index of which coordinate system to use as the input field. The default index is 0 , which is the first coordinate system.

SetOutputFieldName/GetOutputFieldName Specifies the name of the output field generated.

Execute Takes a data set, executes the filter on a device, and returns a data set that contains the result.

SetFieldsToPass/GetFieldsToPass Specifies which fields to pass from input to output. By default all fields are passed. See Section 9.2.2 for more details.

\subsubsection{Lagrangian Coherent Structures}

Lagrangian coherent structures (LCS) are distinct structures present in a flow field that have a major influence over nearby trajectories over some interval of time. Some of these structures may be sources, sinks, saddles, or vortices in the flow field. Identifying Lagrangian coherent structures is part of advanced flow analysis and is an important part of studying flow fields. These structures can be studied by calculating the finite time Lyapunov exponent (FTLE) for a flow field at various locations, usually over a regular grid encompassing the entire flow field. If the provided input dataset is structured, then by default the points in this dataset will be used as seeds for advection.

The vtkm::filter:LagrangianStructures filter is used to compute the FTLE of a flow field. LagrangianStructures has the following methods.

SetStepSize/GetStepSize Set or retrieve the step size for a single advection step for the particles used to calculate the FTLE.

SetNumberOfSteps/GetNumberOfSteps Set or retrieve the maximum number of steps a particle is allowed to traverse while calculating the FTLE field. 
SetAdvectionTime/GetAdvectionTime Set or retrieve the time interval of advection. The FTLE field is calculated over some finite time, and the advection time determines that interval of time used.

SetUseAuxiliaryGrid/GetUseAuxiliaryGrid Set or retrieve the flag to use auxiliary grids. When this flag is off (the default), then the points of the mesh representing the vector field are advected and used for computing the FTLE. However, if the mesh is too coarse, the FTLE will likely be inaccurate. Or if the mesh is unstructured the FTLE may be less efficient to compute. When this flag is on, an auxiliary grid of uniformly spaced points is used for the FTLE computation.

SetAuxiliaryGridDimensions/GetAuxiliaryGridDimensions Set or retrieve the dimensions of the auxiliary grid for FTLE calculation. Seeds for advection will be placed along the points of this auxiliary grid. This option has no effect unless the UseAuxiliaryGrid option is on.

SetUseFlowMapOutput/GetUseFlowMapOutput Set or retrieve the flag to use flow maps instead of advection. If the start and end points for FTLE calculation are known already, advection is an unnecessary step. This flag allows users to bypass advection, and instead use a precalculated flow map. By default this option is off.

SetFlowMapOutput/GetFlowMapOutput Set or retrieve the array representing the flow map output to be used for FTLE calculation.

SetOutputFieldName/GetOutputFieldName Set or retrieve the name of the output field in the dataset returned by the LagrangianStructures filter. By default, the field will be names "FTLE".

SetActiveCoordinateSystem/GetActiveCoordinateSystemIndex Specifies the index of which coordinate system to use as when computing spatial locations in the mesh. The default index is 0 , which is the first coordinate system.

Execute Takes a data set, executes the filter on a device, and returns a data set that contains the result.

SetFieldsToPass/GetFieldsToPass Specifies which fields to pass from input to output. By default all fields are passed. See Section 9.2.2 for more details.

\subsubsection{Mesh Quality Metrics}

vtkm: :filter: :MeshQuality is a filter that can calculate various metrics for evaluating mesh quality. It generates a new field as output, based on the cells of an input data set. The metrics for this filter come from the Verdict library, and full mathematical descriptions for each metric can be found in the Verdict documentation. ${ }^{1}$

The following table lists the supported mesh quality metrics. The constructor for vtkm: :filter: :MeshQuality takes an argument from the enum vtkm: :filter::CellMetric, which specifies the desired metric to calculate. The possible values for the CellMetric enum are listed in the following table. Also listed in the table is the default name for the field being created, which can be overridden using the SetOutputFieldName method. Also, because most metrics only work on select types of cells, the table specifies on which cell types each metric works using the abbreviations "Tri" for triangles, "Quad" for quadrilaterals, "Tet" for tetrahedrons, "Pyr" for pyramids, "Wed" for wedges, and "Hex" for hexahedrons.

${ }^{1}$ https://www.csimsoft.com/download?file=Documents/sand20071751.pdf 


\begin{tabular}{|c|c|c|c|}
\hline Constructor Argument & $\begin{array}{l}\text { Default } \\
\text { Output Name }\end{array}$ & $\begin{array}{l}\text { Supported } \\
\text { Cell Types }\end{array}$ & Brief Description \\
\hline CellMetric: :AREA & area & Tri, Quad & Area of a $2 \mathrm{D}$ cell \\
\hline CellMetric: :ASPECT_GAMMA & aspectGamma & Tet & $\begin{array}{l}\text { Compare root-mean-square } \\
\text { edge length to volume }\end{array}$ \\
\hline CellMetric: :ASPECT_RATIO & aspectRatio & $\begin{array}{l}\text { Tri, Quad, } \\
\text { Tet, Hex }\end{array}$ & $\begin{array}{l}\text { Ratio involving longest edge } \\
\text { and circumradius }\end{array}$ \\
\hline CellMetric: : CONDITION & condition & $\begin{array}{l}\text { Tri, Quad, } \\
\text { Tet, Hex }\end{array}$ & $\begin{array}{l}\text { Condition number of } \\
\text { weighted Jacobian matrix }\end{array}$ \\
\hline CellMetric: :DIAGONAL_RATIO & diagonalRatio & Quad, Hex & $\begin{array}{l}\text { Ratio of minimum and } \\
\text { maximum diagonals }\end{array}$ \\
\hline CellMetric: :DIMENSION & dimension & Hex & $\begin{array}{l}\text { Designed specifically for } \\
\text { Sandia's Pronto code }\end{array}$ \\
\hline CellMetric: : JACOBIAN & jacobian & $\begin{array}{l}\text { Quad, Tet, } \\
\text { Hex }\end{array}$ & $\begin{array}{l}\text { Minimum determinant of Jacobian } \\
\text { matrix, over corners and cell center }\end{array}$ \\
\hline CellMetric: :MAX_ANGLE & maxAngle & Tri, Quad & Maximum angle within cell, in degrees \\
\hline CellMetric: :MAX_DIAGONAL & maxDiagonal & Hex & Length of maximum diagonal in cell \\
\hline CellMetric: :MIN_ANGLE & minAngle & Tri, Quad & Minimum angle within cell, in degrees \\
\hline CellMetric: :MIN_DIAGONAL & minDiagonal & Hex & Length of minimum diagonal in cell \\
\hline CellMetric: :ODDY & oddy & Quad, Hex & $\begin{array}{l}\text { Maximum deviation of a metric tensor } \\
\text { from an identity matrix, over all } \\
\text { corners and cell center }\end{array}$ \\
\hline $\begin{array}{l}\text { CellMetric: :- } \\
\text { RELATIVE_SIZE_SQUARED }\end{array}$ & $\begin{array}{l}\text { relativeSize } \\
\text { Squared }\end{array}$ & $\begin{array}{l}\text { Tri, Quad, } \\
\text { Tet, Hex }\end{array}$ & $\begin{array}{l}\text { The ratio of the area/volume of } \\
\text { this cell compared to mesh average }\end{array}$ \\
\hline CellMetric: :SCALED_JACOBIAN & scaledJacobian & $\begin{array}{l}\text { Tri, Quad, } \\
\text { Tet, Hex }\end{array}$ & $\begin{array}{l}\text { Derived from the Jacobian metric, } \\
\text { with normalization involving edge length }\end{array}$ \\
\hline
\end{tabular}




\begin{tabular}{|c|c|c|c|}
\hline Constructor Argument & $\begin{array}{l}\text { Default } \\
\text { Output Name }\end{array}$ & $\begin{array}{l}\text { Supported } \\
\text { Cell Types }\end{array}$ & Brief Description \\
\hline CellMetric: : SHAPE & shape & $\begin{array}{l}\text { Tri, Quad, } \\
\text { Tet, Hex }\end{array}$ & $\begin{array}{l}\text { Varies by shape type, including } \\
\text { incorporating Jacobian or Condition } \\
\text { Consult Verdict manual }\end{array}$ \\
\hline CellMetric: :SHAPE_AND_SIZE & shapeAndSize & $\begin{array}{l}\text { Tri, Quad, } \\
\text { Tet, Hex }\end{array}$ & $\begin{array}{l}\text { Shape metric multiplied by } \\
\text { relative size squared metric }\end{array}$ \\
\hline CellMetric: : SHEAR & shear & Quad, Hex & $\begin{array}{l}\text { Min. value of Jacobian at each corner, } \\
\text { divided by length of adjacent edges }\end{array}$ \\
\hline CellMetric: :SKEW & skew & Quad, Hex & Maximum angle between principle axes \\
\hline CellMetric: :STRETCH & stretch & Quad, Hex & $\begin{array}{l}\text { Ratio of minimum edges and maximum } \\
\text { diagonal, normalized for unit cube }\end{array}$ \\
\hline CellMetric: :TAPER & taper & Quad & $\begin{array}{l}\text { Maximum ratio of cross-derivative } \\
\text { with associated principal axis }\end{array}$ \\
\hline CellMetric: : VOLUME & volume & $\begin{array}{l}\text { Tet, Pyr, } \\
\text { Wed, Hex }\end{array}$ & Volume of a 3D cell \\
\hline CellMetric: :WARPAGE & warpage & Quad & Captures angles between diagonals \\
\hline
\end{tabular}

Finally, MeshQuality provides the following methods.

SetOutputFieldName/GetOutputFieldName Specifies the name of the output field generated.

Execute Takes a data set, executes the filter on a device, and returns a data set that contains the result.

SetFieldsToPass/GetFieldsToPass Specifies which fields to pass from input to output. By default all fields are passed. See Section 9.2.2 for more details.

\subsubsection{Particle Density}

Analysis of particle-based simulation data usually requires conversion or deposition of particles' attributes, such as mass, to an overlaying mesh. This allows further identification of regions of interest based on the spatial distribution of particles attributes, for example, high density regions could be considered as clusters or halos while low density regions could be considered as bubbles or cavities in the particle data.

VTK-m provides two types of particle density filters. Since there is no specific Cellset for particles in VTK-m, both filter treat the CoordinateSystem of the input DataSet as position of particles while ignoring the details of the Cellset. Particles are infinitesimal in size with finite mass (or other scalar attributes such as charge). Both filters estimate density by imposing a regular grid as specified in the constructors. They differ in how they deposit particle attributes to the mesh. 


\section{Nearest Grid Point}

The vtkm: :filter::ParticleDensityNearestGridPoint filter groups particles in each cell and sums up the attributes, creating a cell centered density field. Once the sum of the attribute is computed for each grid cell, it is divided by the volume of the cell. Thus, the density will be computed as the units of the scalar field per the cubic units of the coordinate system. ParticleDensityNearestGridPoint provides the following methods.

SetActiveField/GetActiveFieldName Specify the particle attribute to be deposited.

SetDivideByVolume/GetDivideByVolume If you just want a sum of the attribute in each cell, turn off the DivideByVolume feature of this filter by SetDivideByVolume(false).

SetComputeNumberDensity/GetComputeNumberDensity In addition, you can also simply count the number of particles in each cell by calling SetComputeNumberDensity(true).

SetActiveCoordinateSystem/GetActiveCoordinateSystemIndex Specifies the index of which coordinate system to use as when computing spatial locations in the mesh. The default index is 0 , which is the first coordinate system.

Execute Takes a data set, executes the filter on a device, and returns a data set that contains the result.

\section{Cloud in Cell}

The vtkm: :filter: :ParticleDensityCloudInCell filter redistributes a particle's attribute to the 8 vertices of the containing cell. The filter then sums up all the contributions of particles for each vertex in the grid. This creates a point centered density field. Once the sum of the attribute is computed for each grid points, it is divided by the volume of each cell. Thus, the density will be computed as the units of the scalar field per the cubic units of the coordinate system. ParticleDensityNearestGridPoint provides the following methods.

SetActiveField/GetActiveFieldName Specify the particle attribute to be deposited.

SetDivideByVolume/GetDivideByVolume If you just want a sum of the attribute in each cell, turn off the DivideByVolume feature of this filter by SetDivideByVolume ( $f a l s e)$.

SetComputeNumberDensity/GetComputeNumberDensity In addition, you can also simply count the number of particles in each cell by calling SetComputeNumberDensity(true).

SetActiveCoordinateSystem/GetActiveCoordinateSystemIndex Specifies the index of which coordinate system to use as when computing spatial locations in the mesh. The default index is 0 , which is the first coordinate system.

Execute Takes a data set, executes the filter on a device, and returns a data set that contains the result.

\subsubsection{Point Average}

vtkm: :filter: :PointAverage is the point average filter. It will take a data set with a collection of cells and a field defined on the cells of the data set and create a new field defined on the points. The values of this new derived field are computed by averaging the values of the input field at all the incident cells. This is a simple way to convert a cell field to a point field.

The default name for the output cell field is the same name as the input point field. The name can be overridden as always using the SetOutputFieldName method.

PointAverage provides the following methods. 
SetActiveField/GetActiveFieldName Specifies the name of the field to use as input.

SetUseCoordinateSystemAsField/GetUseCoordinateSystemAsField Specifies a Boolean flag that determines whether to use point coordinates as the input field. Set to false by default. When true, the values for the active field are ignored.

SetActiveCoordinateSystem/GetActiveCoordinateSystemIndex Specifies the index of which coordinate system to use as the input field. The default index is 0 , which is the first coordinate system.

SetOutputFieldName/GetOutputFieldName Specifies the name of the output field generated.

Execute Takes a data set, executes the filter on a device, and returns a data set that contains the result.

SetFieldsToPass/GetFieldsToPass Specifies which fields to pass from input to output. By default all fields are passed. See Section 9.2.2 for more details.

\subsubsection{Point Elevation}

vtkm: :filter: :PointElevation computes the "elevation" of a field of point coordinates in space. The filter will take a data set and a field of 3 dimensional vectors and compute the distance along a line defined by a low point and a high point. Any point in the plane touching the low point and perpendicular to the line is set to the minimum range value in the elevation whereas any point in the plane touching the high point and perpendicular to the line is set to the maximum range value. All other values are interpolated linearly between these two planes. This filter is commonly used to compute the elevation of points in some direction, but can be repurposed for a variety of measures. Example 9.1 gives a demonstration of the elevation filter.

The default name for the output field is "elevation", but that can be overridden as always using the SetOutputFieldName method.

PointElevation provides the following methods.

SetLowPoint/SetHighPoint This pair of methods is used to set the low and high points, respectively, of the elevation. Each method takes three floating point numbers specifying the $x, y$, and $z$ components of the low or high point.

SetRange Sets the range of values to use for the output field. This method takes two floating point numbers specifying the low and high values, respectively.

SetActiveField/GetActiveFieldName Specifies the name of the field to use as input.

SetUseCoordinateSystemAsField/GetUseCoordinateSystemAsField Specifies a Boolean flag that determines whether to use point coordinates as the input field. Set to false by default. When true, the values for the active field are ignored.

SetActiveCoordinateSystem/GetActiveCoordinateSystemIndex Specifies the index of which coordinate system to use as the input field. The default index is 0 , which is the first coordinate system.

SetOutputFieldName/GetOutputFieldName Specifies the name of the output field generated.

Execute Takes a data set, executes the filter on a device, and returns a data set that contains the result.

SetFieldsToPass/GetFieldsToPass Specifies which fields to pass from input to output. By default all fields are passed. See Section 9.2.2 for more details. 


\subsubsection{Point Transform}

vtkm::filter::PointTransform is the point transform filter. The filter will take a data set and a field of 3 dimensional vectors and perform the specified point transform operation. Multiple point transformations can be accomplished by subsequent calls to the filter and specifying the result of the previous transform as the input field.

The default name for the output field is "transform", but that can be overridden as always using the SetOutputFieldName method. By default, produced field replaces the coordinate system.

PointTransform provides the following methods.

SetTranslation This method translates, or moves, each point in the input field by a given direction. This method takes either a three component vector of floats, or the $x, y, z$ translation values separately.

SetRotation This method is used to rotate the input field about a given axis. This method takes a single floating point number to specify the degrees of rotation and either a vector representing the rotation axis, or the $x, y, z$ axis components separately.

SetRotationX This method is used to rotate the input field about the $x$ axis. This method takes a single floating point number to specify the degrees of rotation.

SetRotationY This method is used to rotate the input field about the $y$ axis. This method takes a single floating point number to specify the degrees of rotation.

SetRotationz This method is used to rotate the input field about the $z 0$ axis. This method takes a single floating point number to specify the degrees of rotation.

SetScale This method is used to scale the input field. This method takes either a single float to scale each vector component of the field equally, or the $x, y, z$ scaling values as separate floats, or a three component vector.

SetTransform This is a generic transform method. This method takes a $4 x 4$ matrix and applies this to the input field.

SetChangeCoordinateSystem/GetChangeCoordinateSystem When this flag is on, the default, the coordinate system in the output DataSet is replaced with the transformed point coordinates .

SetActiveField/GetActiveFieldName Specifies the name of the field to use as input.

SetUseCoordinateSystemAsField/GetUseCoordinateSystemAsField Specifies a Boolean flag that determines whether to use point coordinates as the input field. Set to false by default. When true, the values for the active field are ignored.

SetActiveCoordinateSystem/GetActiveCoordinateSystemIndex Specifies the index of which coordinate system to use as the input field. The default index is 0 , which is the first coordinate system.

SetOutputFieldName/GetOutputFieldName Specifies the name of the output field generated.

Execute Takes a data set, executes the filter on a device, and returns a data set that contains the result.

SetFieldsToPass/GetFieldsToPass Specifies which fields to pass from input to output. By default all fields are passed. See Section 9.2.2 for more details. 


\subsubsection{Stream Tracing}

Stream tracing is a visualization technique used to characterize the structure of flow. The flow itself is defined by a vector field of velocities. Stream tracing works by following the path taken by the flow. There are multiple ways in which to represent flow in this manner, and consequently VTK-m contains several filters that trace streams in different ways.

\section{Streamlines}

Streamlines are a powerful technique for the visualization of flow fields. A streamline is a curve that is parallel to the velocity vector of the flow field. Individual streamlines are computed from an initial point location (seed) using a numerical method to integrate the point through the flow field.

vtkm: :filter: :Streamline provides the following methods.

SetSeeds Specifies the seed locations for the streamlines. Each seed is advected in the vector field to generate one streamline for each seed. The seeds are specified in an ArrayHandle containing vtkm::Particle objects.

SetStepSize Specifies the step size used for the numerical integrator ( $4^{\text {th }}$ order Runge-Kutta method) to integrate the seed locations through the flow field.

SetNumberOfSteps Specifies the number of integration steps to be performed on each streamline.

SetActiveField/GetActiveFieldName Specifies the name of the field to use as input.

SetUseCoordinateSystemAsField/GetUseCoordinateSystemAsField Specifies a Boolean flag that determines whether to use point coordinates as the input field. Set to false by default. When true, the values for the active field are ignored.

SetActiveCoordinateSystem/GetActiveCoordinateSystemIndex Specifies the index of which coordinate system to use as when computing spatial locations in the mesh. The default index is 0 , which is the first coordinate system.

Execute Takes a data set, executes the filter on a device, and returns a data set that contains the result.

SetFieldsToPass/GetFieldsToPass Specifies which fields to pass from input to output. By default all fields are passed. See Section 9.2.2 for more details.

Example 9.5: Using Streamline, which is a data set with field filter.

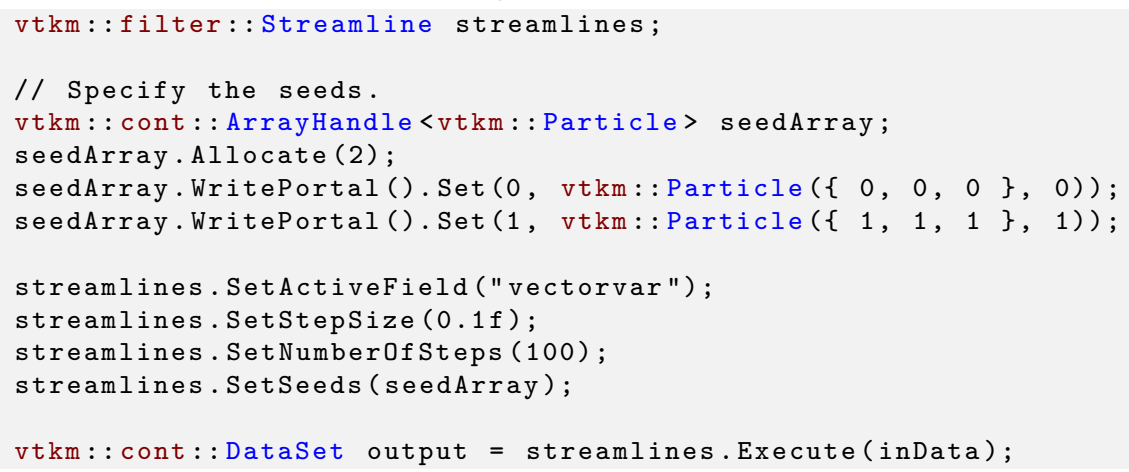




\section{Stream Surface}

A stream surface is defined as a continuous surface that is everywhere tangent to a specified vector field. vtkm: :filter: : StreamSurface computes a stream surface from a set of input points and the vector field of the input data set. The stream surface is created by creating streamlines from each input point and then connecting adjacent streamlines with a series of triangles.

vtkm: :filter: :StreamSurface provides the following methods.

SetSeeds Specifies the seed locations for the edge of the stream surface. The seeds are specified in an ArrayHandle containing vtkm: :Particle objects.

SetStepSize Specifies the step size used for the numerical integrator $\left(4^{\text {th }}\right.$ order Runge-Kutta method) to integrate the seed locations through the flow field.

SetNumberOfSteps Specifies the number of integration steps to be performed on each seed.

SetActiveField/GetActiveFieldName Specifies the name of the field to use as input.

SetUseCoordinateSystemAsField/GetUseCoordinateSystemAsField Specifies a Boolean flag that determines whether to use point coordinates as the input field. Set to false by default. When true, the values for the active field are ignored.

SetActiveCoordinateSystem/GetActiveCoordinateSystemIndex Specifies the index of which coordinate system to use as when computing spatial locations in the mesh. The default index is 0 , which is the first coordinate system.

Execute Takes a data set, executes the filter on a device, and returns a data set that contains the result.

SetFieldsToPass/GetFieldsToPass Specifies which fields to pass from input to output. By default all fields are passed. See Section 9.2.2 for more details.

Example 9.6: Using StreamSurface, which is a data set with field filter.

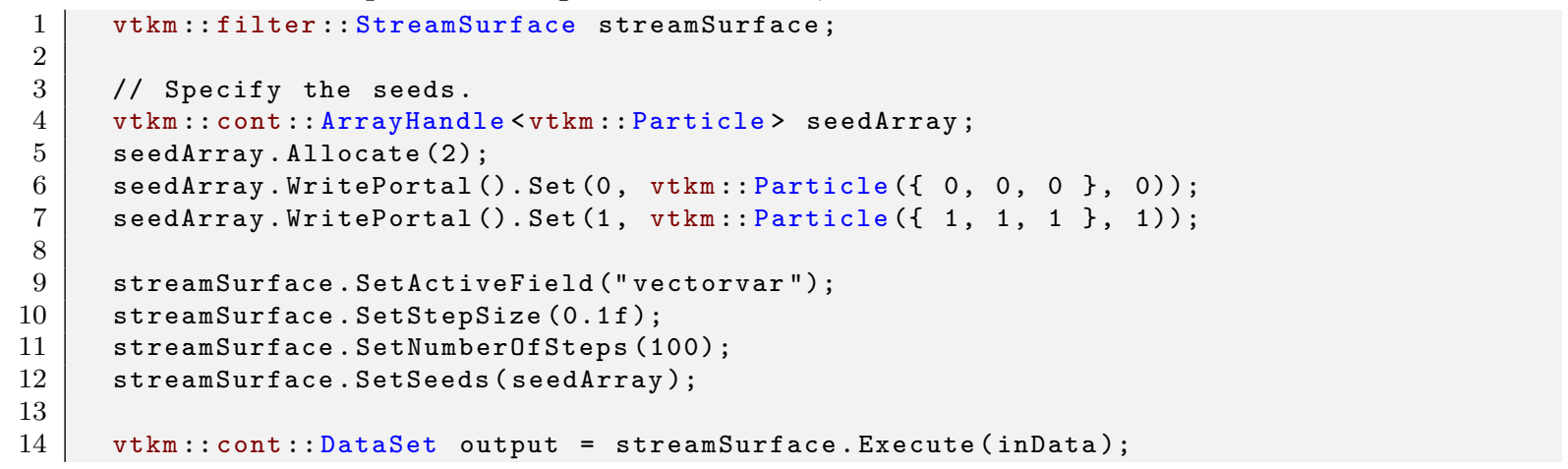

\section{Pathlines}

Pathlines are the analog to Streamlines for time varying vector fields. Individual pathlines are computed from an initial point location (seed) using a numerical method to integrate the point through the flow field. This filter requires two data sets as input. The data set passed into the filter is termed "Previous" and the "Next" data set is specified to the filter using a method.

vtkm: :filter: :Pathline provides the following methods. 
SetPreviousTime Specifies time value for the input data set.

SetNextTime Specifies time value for the next data set.

SetNextDataSet Specifies the data set for the next time step.

SetSeeds Specifies the seed locations for the pathlines. Each seed is advected in the vector field to generate one streamline for each seed. The seeds are specified in an ArrayHandle containing vtkm: :Particle objects.

SetStepSize Specifies the step size used for the numerical integrator ( $4^{\text {th }}$ order Runge-Kutta method) to integrate the seed locations through the flow field.

SetNumberOfSteps Specifies the number of integration steps to be performed on each pathline.

SetActiveField/GetActiveFieldName Specifies the name of the field to use as input.

SetUseCoordinateSystemAsField/GetUseCoordinateSystemAsField Specifies a Boolean flag that determines whether to use point coordinates as the input field. Set to false by default. When true, the values for the active field are ignored.

SetActiveCoordinateSystem/GetActiveCoordinateSystemIndex Specifies the index of which coordinate system to use as when computing spatial locations in the mesh. The default index is 0, which is the first coordinate system.

Execute Takes a data set, executes the filter on a device, and returns a data set that contains the result.

SetFieldsToPass/GetFieldsToPass Specifies which fields to pass from input to output. By default all fields are passed. See Section 9.2.2 for more details.

Example 9.7: Using Pathline, which is a data set with field filter.

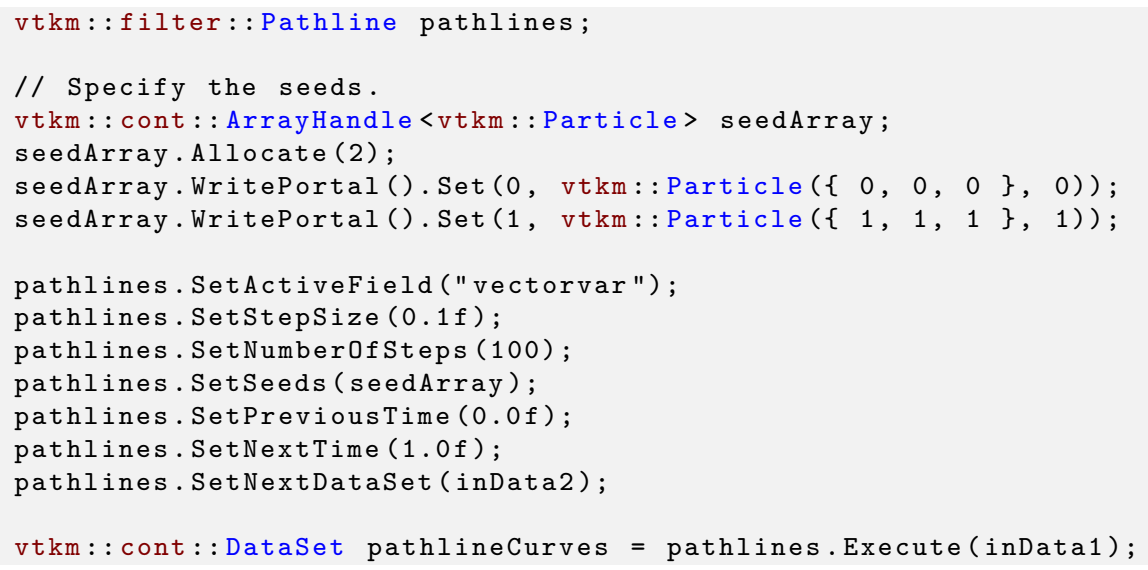

\subsubsection{Surface Normals}

vtkm: :filter: :SurfaceNormals computes the surface normals of a polygonal data set at its points and/or cells. The filter takes a data set as input and by default, uses the active coordinate system to compute the normals. Optionally, a coordinate system or a point field of $3 \mathrm{~d}$ vectors can be explicitly provided to the Execute method. The point and cell normals may be oriented to a point outside of the manifold surface by setting the auto orient normals option (SetAutoOrientNormals), or they may point inward by also setting flip normals (SetFlipNormals) to true. Triangle vertices will be wound counter-clockwise around the cell normals when the 
consistency option (SetConsistency) is enabled. For non-polygonal cells, a zeroed vector is assigned. The point normals are computed by averaging the cell normals of the incident cells of each point.

The default name for the output fields is "Normals", but that can be overridden using the SetCellNormalsName and SetPointNormalsName methods. The filter will also respect the name in SetOutputFieldName if neither of the others are set.

SurfaceNormals provides the following methods.

SetGenerateCellNormals/GetGenerateCellNormals Specifies whether the cell normals should be generated. This is off by default.

SetGeneratePointNormals/GetGeneratePointNormals Specifies whether the point normals should be generated. This is on by default.

SetNormalizeCellNormals/GetNormalizeCellNormals Specifies whether cell normals should be normalized (made unit length). This is on by default. The intended use case of this flag is for faster, approximate point normals generation by skipping the normalization of the face normals. Note that when set to false, the result cell normals will not be unit length normals and the point normals will be different.

SetAutoOrientNormals/GetAutoOrientNormals If true, any generated point and/or cell normals will be oriented to point outwards from the surface. This requires a closed manifold surface or else the behavior is undefined. This is off by default.

SetFlipNormals/GetFlipNormals When AutoOrientNormals is true, this option will reverse the point and cell normals to point inward. This is off by default.

SetConsistency/GetConsistency When GenerateCellNormals is true, this option will ensure that the triangle vertices in the output dataset are wound counter-clockwise around the generated cell normal. This only affects triangles. This is on by default.

SetCellNormalsName/GetCellNormalsName Specifies the output cell normals field name. If no cell or point normal name is specified, "Normals" is used.

SetPointNormalsName/GetPointNormalsName Specifies the output point normals field name. If no cell or point normal name is specified, "Normals" is used.

SetActiveField/GetActiveFieldName Specifies the name of the field to use as input.

SetUseCoordinateSystemAsField/GetUseCoordinateSystemAsField Specifies a Boolean flag that determines whether to use point coordinates as the input field. Set to false by default. When true, the values for the active field are ignored.

SetActiveCoordinateSystem/GetActiveCoordinateSystemIndex Specifies the index of which coordinate system to use as the input field. The default index is 0 , which is the first coordinate system.

SetOutputFieldName/GetOutputFieldName Specifies the name of the output field generated.

Execute Takes a data set, executes the filter on a device, and returns a data set that contains the result.

SetFieldsToPass/GetFieldsToPass Specifies which fields to pass from input to output. By default all fields are passed. See Section 9.2.2 for more details. 


\subsubsection{Threshold}

A threshold operation removes topology elements from a data set that do not meet a specified criterion. The vtkm: :filter: :Threshold filter removes all cells where the field (provided to Execute) is not between a range of values.

Note that Threshold either passes an entire cell or discards an entire cell. This can consequently lead to jagged surfaces at the interface of the threshold caused by the shape of cells that jut inside or outside the removed region. See Section 9.1.3 for a clipping filter that will clip off a smooth region of the mesh.

Threshold provides the following methods.

SetLowerThreshold/GetLowerThreshold Specifies the lower scalar value. Any cells where the scalar field is less than this value are removed.

SetUpperThreshold/GetUpperThreshold Specifies the upper scalar value. Any cells where the scalar field is more than this value are removed.

SetAllInRange/GetAllInRange When thresholding on a point field, each cell must consider the multiple values associated with all incident points. When this flag is false (the default), the cell is passed if any of the incident points matches the threshold criteria. When this flag is true, the cell is passed only if all the incident points match the threshold criteria.

SetActiveField/GetActiveFieldName Specifies the name of the field to use as input.

SetUseCoordinateSystemAsField/GetUseCoordinateSystemAsField Specifies a Boolean flag that determines whether to use point coordinates as the input field. Set to false by default. When true, the values for the active field are ignored.

SetActiveCoordinateSystem/GetActiveCoordinateSystemIndex Specifies the index of which coordinate system to use as when computing spatial locations in the mesh. The default index is 0 , which is the first coordinate system.

Execute Takes a data set, executes the filter on a device, and returns a data set that contains the result.

SetFieldsToPass/GetFieldsToPass Specifies which fields to pass from input to output. By default all fields are passed. See Section 9.2.2 for more details.

\subsubsection{Tube}

vtkm: :filter: :Tube generates a tube around each line and polyline in the input data set. The radius, number of sides, and end capping can be specified for each tube. The orientation of the geometry of the tube are computed automatically using a heuristic to minimize the twisting along the input data set.

Tube provides the following methods.

SetRadius Specifies the radius of the tube.

SetNumberOfSides Specifies the number of sides for the tube geometry.

SetCapping Specifies if the ends of the tube should be capped.

SetActiveCoordinateSystem/GetActiveCoordinateSystemIndex Specifies the index of which coordinate system to use as when computing spatial locations in the mesh. The default index is 0 , which is the first coordinate system. 
Execute Takes a data set, executes the filter on a device, and returns a data set that contains the result.

SetFieldsToPass/GetFieldsToPass Specifies which fields to pass from input to output. By default all fields are passed. See Section 9.2.2 for more details.

Example 9.8: Using Tube, which is a data set with field filter.

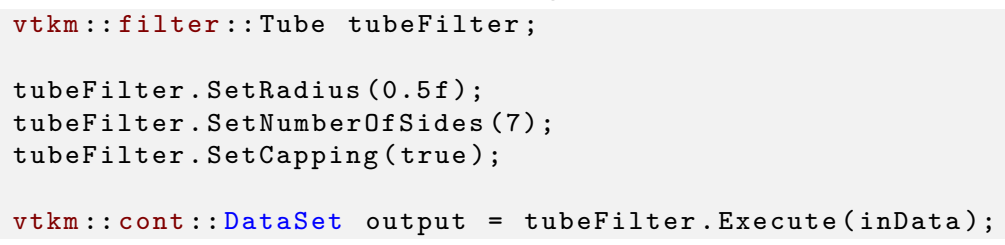

\subsubsection{Vector Magnitude}

vtkm: :filter: :VectorMagnitude takes a field comprising vectors and computes the magnitude for each vector. The vector field is selected as usual with the SetActiveField method. The default name for the output field is "magnitude", but that can be overridden as always using the SetOutputFieldName method.

VectorMagnitude provides the following methods.

SetActiveField/GetActiveFieldName Specifies the name of the field to use as input.

SetUseCoordinateSystemAsField/GetUseCoordinateSystemAsField Specifies a Boolean flag that determines whether to use point coordinates as the input field. Set to false by default. When true, the values for the active field are ignored.

SetActiveCoordinateSystem/GetActiveCoordinateSystemIndex Specifies the index of which coordinate system to use as the input field. The default index is 0 , which is the first coordinate system.

SetOutputFieldName/GetOutputFieldName Specifies the name of the output field generated.

Execute Takes a data set, executes the filter on a device, and returns a data set that contains the result.

SetFieldsToPass/GetFieldsToPass Specifies which fields to pass from input to output. By default all fields are passed. See Section 9.2.2 for more details.

\subsubsection{Vertex Clustering}

vtkm: :filter: :VertexClustering is a filter that simplifies a polygonal mesh. It does so by dividing space into a uniform grid of bin and then merges together all points located in the same bin. The smaller the dimensions of this binning grid, the fewer polygons will be in the output cells and the coarser the representation. This surface simplification is an important operation to support level of detail (LOD) rendering in visualization applications.

VertexClustering provides the following methods.

SetNumberOfDivisions/GetNumberOfDimensions Specifies the dimensions of the uniform grid that establishes the bins used for clustering. Setting smaller numbers of dimensions produces a smaller output, but with a coarser representation of the surface. The dimensions are provided as a vtkm: :Id3. 
SetActiveCoordinateSystem/GetActiveCoordinateSystemIndex Specifies the index of which coordinate system to use as when computing spatial locations in the mesh. The default index is 0 , which is the first coordinate system.

Execute Takes a data set, executes the filter on a device, and returns a data set that contains the result.

SetFieldsToPass/GetFieldsToPass Specifies which fields to pass from input to output. By default all fields are passed. See Section 9.2.2 for more details.

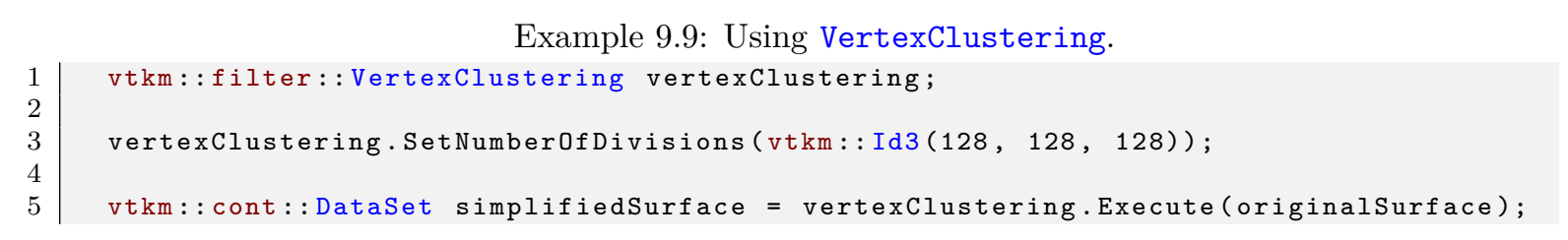

\subsubsection{Warp Scalar}

vtkm: :filter: :WarpScalar is a specialized point transformation filter. The filter transforms points by moving them based on a scalar field and a constant scale factor. This filter is useful for creating carpet plots.

The WarpScalar filter will take a data set, a normal field, a scalar field, and a constant scale factor. The coordinates will be scaled based on the scalar field and the scale factor. If no explicit normal field is provided the filter will search for a field named "normal". If no explicit scalar field is provided the filter will search for a field named "scalarfactor".

The default name for the output field is "warpscalar", but that can be overridden as always using the SetOutputFieldName method.

In addition to the standard SetOutputFieldName and Execute methods, WarpScalar provides the following methods.

SetNormalField This method allows the user to select the name of the normal field. The normal field is the $B$ field in the warp equation of $A+B \times$ scaleAmount $\times$ scalar Factor (where $A$ is the original position of the point).

SetScalarFactorField This method allows the user to select the name of the scale factor field. The scale factor field is the scalarFactor field in the warp equation of $A+B \times$ scaleAmount $\times$ scalarFactor (where $A$ is the original position of the point).

SetActiveField/GetActiveFieldName Specifies the name of the field to use as input.

SetUseCoordinateSystemAsField/GetUseCoordinateSystemAsField Specifies a Boolean flag that determines whether to use point coordinates as the input field. Set to false by default. When true, the values for the active field are ignored.

SetActiveCoordinateSystem/GetActiveCoordinateSystemIndex Specifies the index of which coordinate system to use as the input field. The default index is 0 , which is the first coordinate system.

SetOutputFieldName/GetOutputFieldName Specifies the name of the output field generated.

Execute Takes a data set, executes the filter on a device, and returns a data set that contains the result.

SetFieldsToPass/GetFieldsToPass Specifies which fields to pass from input to output. By default all fields are passed. See Section 9.2.2 for more details. 


\subsubsection{Warp Vector}

vtkm: :filter: :WarpVector is a specialized point transformation filter. The filter transforms points by moving them based on a vector field and a constant scale factor. This filter can be used to highlight interesting features such as flow or deformations.

The WarpScalar filter will take a data set, a vector field, and a constant scale factor. The coordinates will be scaled based on the vector field and the scale factor. If no explicit vector field is provided the filter will search for a field named "normal".

The default name for the output field is "warpvector", but that can be overridden as always using the SetOutputFieldName method.

In addition the standard SetOutputFieldName and Execute methods, WarpVector provides the following methods.

SetVectorField This method allows the user to select the name of the vector field. The vector field is the $B$ field in the warp equation of $A+B$ (where $A$ is the original position of the point).

SetActiveField/GetActiveFieldName Specifies the name of the field to use as input.

SetUseCoordinateSystemAsField/GetUseCoordinateSystemAsField Specifies a Boolean flag that determines whether to use point coordinates as the input field. Set to false by default. When true, the values for the active field are ignored.

SetActiveCoordinateSystem/GetActiveCoordinateSystemIndex Specifies the index of which coordinate system to use as the input field. The default index is 0 , which is the first coordinate system.

SetOutputFieldName/GetOutputFieldName Specifies the name of the output field generated.

Execute Takes a data set, executes the filter on a device, and returns a data set that contains the result.

SetFieldsToPass/GetFieldsToPass Specifies which fields to pass from input to output. By default all fields are passed. See Section 9.2.2 for more details.

\subsubsection{ZFP Compression}

vtkm: :filter::ZFPCompressor takes a 1D, 2D, or 3D field and compresses the values using the compression algorithm ZFP. The field is selected as usual with the SetActiveField method. The rate of compression is set using SetRate. The default name for the output field is "compressed"

ZFPCompressor provides the following methods:

SetRate/GetRate Specifies the rate of compression.

SetActiveField/GetActiveFieldName Specifies the name of the field to use as input.

SetActiveField/GetActiveFieldName Specifies the name of the field to use as input.

SetUseCoordinateSystemAsField/GetUseCoordinateSystemAsField Specifies a Boolean flag that determines whether to use point coordinates as the input field. Set to false by default. When true, the values for the active field are ignored.

SetActiveCoordinateSystem/GetActiveCoordinateSystemIndex Specifies the index of which coordinate system to use as the input field. The default index is 0 , which is the first coordinate system. 
SetOutputFieldName/GetOutputFieldName Specifies the name of the output field generated.

Execute Takes a data set, executes the filter on a device, and returns a data set that contains the result.

SetFieldsToPass/GetFieldsToPass Specifies which fields to pass from input to output. By default all fields are passed. See Section 9.2.2 for more details.

vtkm: :filter: :ZFPDecompressor takes a field of compressed values and decompresses into scalar values using the compression algorithm ZFP. The field is selected as usual with the SetActiveField method. The rate of compression is set using SetRate. The default name for the output field is "decompressed"

ZFPDecompressor provides the following methods:

SetRate Specifies the rate of compression.

SetActiveField/GetActiveFieldName Specifies the name of the field to use as input.

SetActiveField/GetActiveFieldName Specifies the name of the field to use as input.

SetUseCoordinateSystemAsField/GetUseCoordinateSystemAsField Specifies a Boolean flag that determines whether to use point coordinates as the input field. Set to false by default. When true, the values for the active field are ignored.

SetActiveCoordinateSystem/GetActiveCoordinateSystemIndex Specifies the index of which coordinate system to use as the input field. The default index is 0 , which is the first coordinate system.

SetOutputFieldName/GetOutputFieldName Specifies the name of the output field generated.

Execute Takes a data set, executes the filter on a device, and returns a data set that contains the result.

SetFieldsToPass/GetFieldsToPass Specifies which fields to pass from input to output. By default all fields are passed. See Section 9.2.2 for more details.

\subsection{Advanced Field Management}

Most filters work with fields as inputs and outputs to their algorithms. Although in the previous discussions of the filters we have seen examples of specifying fields, these examples have been kept brief in the interest of clarity. In this section we revisit how filters manage fields and provide more detailed documentation of the controls.

Note that not all of the discussion in this section applies to all the aforementioned filters. For example, not all filters have a specified input field. But where possible, the interface to the filter objects is kept consistent.

\subsubsection{Input Fields}

Many of VTK-m's filters have a method named SetActiveField, which selects a field in the input data to use as the data for the filter's algorithm. We have already seen how SetActiveField takes the name of the field as an argument. However, SetActiveField also takes an optional second argument that specifies which topological elements the field is associated with (such as points or cells). If specified, this argument is one of the following.

vtkm: :cont::Field::Association: :ANY Any field regardless of the association. (This is the default if no association is given.) 
vtkm::cont::Field::Association::POINTS A field that applies to points. There is a separate field value attached to each point. Point fields usually represent samples of continuous data that can be reinterpolated through cells. Physical properties such as temperature, pressure, density, velocity, etc. are usually best represented in point fields. Data that deals with the points of the topology, such as displacement vectors, are also appropriate for point data.

vtkm: :cont::Field::Association::CELL_SET A field that applies to cells. There is a separate field value attached to each cell in a cell set. Cell fields usually represent values from an integration over the finite cells of the mesh. Integrated values like mass or volume are best represented in cell fields. Statistics about each cell like strain or cell quality are also appropriate for cell data.

vtkm: :cont: :Field: :Association: :WHOLE_MESH A "global" field that applies to the whole mesh. These often contain summary or annotation information. An example of a whole mesh field could be the volume that the mesh covers.

Example 9.10: Setting a field's active filter with an association.

1 filter.SetActivefield("pointvar", vtkm::cont::Field::Association:: POINTS);

\section{Common Errors}

It is possible to have two fields with the same name that are only differentiable by the association. That is, you could have a point field and a cell field with different data but the same name. Thus, it is best practice to specify the field association when possible. Likewise, it is poor practice to have two fields with the same name, particularly if the data are not equivalent in some way. It is often the case that fields are selected without an association.

It is also possible to set the active scalar field as a coordinate system of the data. A coordinate system essentially provides the spatial location of the points of the data and they have a special place in the vtkm: : cont: : DataSet structure. (See Section 7.4 for details on coordinate systems.) You can use a coordinate system as the active scalars by calling the SetUseCoordinateSystemAsField method with a true flag. Since a DataSet can have multiple coordinate systems, you can select the desired coordinate system with SetActiveCoordinateSystem. (By default, the first coordinate system will be used.)

\subsubsection{Passing Fields from Input to Output}

After a filter successfully executes and returns a new data set, fields are mapped from input to output. Depending on what operation the filter does, this could be a simple shallow copy of an array, or it could be a computed operation. By default, the filter will automatically pass all fields from input to output (performing whatever transformations are necessary). You can control which fields are passed (and equivalently which are not) with the SetFieldsToPass methods of vtkm: :filter: :Filter.

There are multiple ways to to use Filter: :SetFieldsToPass to control what fields are passed. If you want to turn off all fields so that none are passed, call SetFieldsToPass with vtkm::filter::FieldSelection: :MODE_NONE.

Example 9.11: Turning off the passing of all fields when executing a filter.

1 filter.SetFieldsToPass (vtkm::filter::FieldSelection::MODE_NONE);

If you want to pass one specific field, you can pass that field's name to SetFieldsToPass. 
Example 9.12: Setting one field to pass by name.

1 filter.SetFieldsToPass ("pointvar");

Or you can provide a list of fields to pass by giving SetFieldsToPass an initializer list of names.

Example 9.13: Using a list of fields for a filter to pass.

1 filter.SetFieldstopass (\{ "pointvar", "cellvar" $\}$ );

If you want to instead select a list of fields to not pass, you can add vtkm: :filter: :FieldSelection: :MODE_EXCLUDE as an argument to SetFieldsToPass.

Example 9.14: Excluding a list of fields for a filter to pass.

1
2 $\begin{array}{r}\text { filter.SetFieldsToPass ( }\{\text { "pointvar", "cellvar" }\}, \\ \text { vtkm::filter:: FieldSelection::MODE_EXCLUDE); }\end{array}$

Ultimately, Filter::SetFieldsToPass takes a vtkm::filter::FieldSelection object. You can create one directly to select (or exclude) specific fields and their associations.

Example 9.15: Using vtkm: :filter: :FieldSelection.

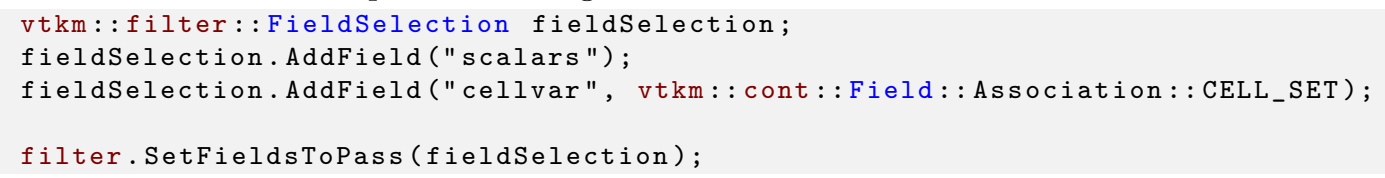

It is also possible to specify field attributions directly to Filter: : SetFieldsToPass. If you only have one field, you can just specify both the name and attribution. If you have multiple fields, you can provide an initializer list of std::pair or vtkm::Pair containing a std::string and a vtkm::cont::Field::AssociationEnum. In either case, you can add an optional last argument of vtkm: :filter::FieldSelection: :MODE_EXCLUDE to exclude the specified filters instead of selecting them.

Example 9.16: Selecting one field and its association for a filter to pass.

1 filter.SetFieldsToPass ("pointvar", vtkm::cont::Field::Association::POINTS);

Example 9.17: Selecting a list of fields and their associations for a filter to pass.

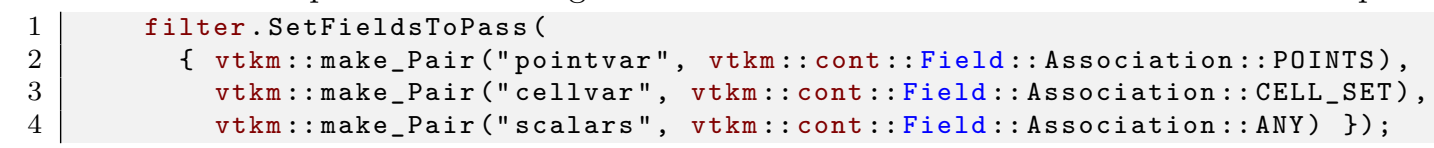





\section{RENDERING}

Rendering, the generation of images from data, is a key component to visualization. To assist with rendering, VTK-m provides a rendering package to produce imagery from data, which is located in the vtkm: :rendering namespace.

The rendering package in VTK-m is not intended to be a fully featured rendering system or library. Rather, it is a lightweight rendering package with two primary use cases:

1. New users getting started with VTK-m need a "quick and dirty" render method to see their visualization results.

2. In situ visualization that integrates VTK-m with a simulation or other data-generation system might need a lightweight rendering method.

Both of these use cases require just a basic rendering platform. Because VTK-m is designed to be integrated into larger systems, it does not aspire to have a fully featured rendering system.

D) Did you know?

\VTK-m's big sister toolkit VTK is already integrated with VTK-m and has its own fully featured rendering system. If you need more rendering capabilities than what VTK-m provides, you can leverage VTK instead.

\subsection{Scenes and Actors}

The primary intent of the rendering package in VTK-m is to visually display the data that is loaded and processed. Data are represented in VTK-m by vtkm: : cont: :DataSet objects, which are described in Chapter 7. They are also the unit created from I/O operations (Chapter 8 and filters (Chapter 9).

To render a DataSet, the data are wrapped in a vtkm: :rendering: :Actor class. The Actor holds the components of the DataSet to render (a cell set, a coordinate system, and a field). A color table can also be optionally be specified, but a default color table will be specified otherwise.

Actors are collected together in an object called vtkm: :rendering: :Scene. An Actor is added to a Scene with the AddActor method. The following example demonstrates creating a Scene with one Actor.

Example 10.1: Creating an Actor and adding it to a Scene. 


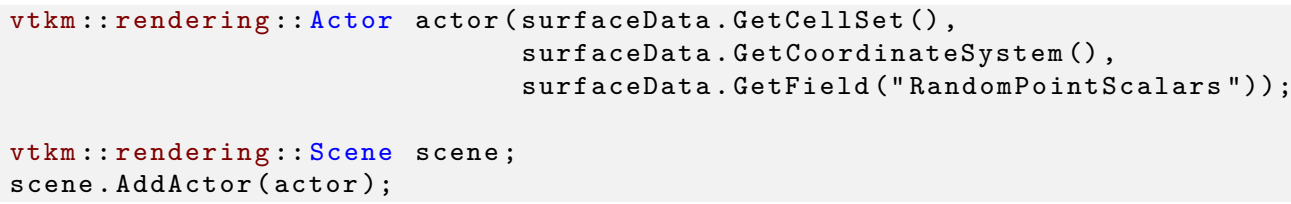

\subsection{Canvas}

A canvas is a unit that represents the image space that is the target of the rendering. The canvas' primary function is to manage the buffers that hold the working image data during the rendering. The canvas also manages the context and state of the rendering subsystem.

vtkm: :rendering: : Canvas is the base class of all canvas objects. Each type of rendering system has its own canvas subclass, but currently the only rendering system provided by VTK-m is the internal ray tracer. The canvas for the ray tracer is vtkm: :rendering: CanvasRayTracer. CanvasRayTracer is typically constructed by giving the width and height of the image to render.

Example 10.2: Creating a canvas for rendering.

1 vtkm: :rendering: : CanvasRayTracer canvas (1920, 1080);

\subsection{Mappers}

A mapper is a unit that converts data (managed by an Actor) and issues commands to the rendering subsystem to generate images. All mappers in VTK-m are a subclass of vtkm: :rendering: : Mapper. Different rendering systems (as established by the Canvas) often require different mappers. Also, different mappers could render different types of data in different ways. For example, one mapper might render polygonal surfaces whereas another might render polyhedra as a translucent volume. Thus, a mapper should be picked to match both the rendering system of the Canvas and the data in the Actor.

The following mappers are provided by VTK-m.

vtkm: :rendering: :MapperRayTracer Uses VTK-m's built in ray tracing system to render the visible surface of a mesh. MapperRayTracer only works in conjunction with CanvasRayTracer.

vtkm: :rendering: :MapperCylinder Uses VTK-m's built in ray tracing system to render cylinders as lines of a mesh. MapperCylinder only works in conjunction with CanvasRayTracer.

vtkm: : rendering: : MapperPoint Uses VTK-m's built in ray tracing system to render the visible points/vertices of a mesh. MapperPoint only works in conjunction with CanvasRayTracer.

vtkm: :rendering: :MapperQuad Uses VTK-m's built in ray tracing system to render the visible quadrilaterals of a mesh. MapperQuad only works in conjunction with CanvasRayTracer.

vtkm: :rendering: : MapperVolume Uses VTK-m's built in ray tracing system to render polyhedra as a translucent volume. MapperVolume only works in conjunction with CanvasRayTracer.

vtkm: :rendering: :MapperWireframer Uses VTK-m's built in ray tracing system to render the cell edges (i.e. the "wireframe") of a mesh. MapperWireframer only works in conjunction with CanvasRayTracer. 


\subsection{Views}

A view is a unit that collects all the structures needed to perform rendering. It contains everything needed to take a Scene (Section 10.1) and use a Mapper (Section 10.3) to render it onto a Canvas (Section 10.2). The view also annotates the image with spatial and scalar properties.

The base class for all views is vtkm: :rendering: :View. View is an abstract class, and you must choose one of the three provided subclasses, vtkm::rendering::View3D, vtkm::rendering::View2D, and vtkm::rendering: :View3D, depending on the type of data being presented. All three view classes take a Scene, a Mapper, and a Canvas as arguments to their constructor.

Example 10.3: Constructing a View.

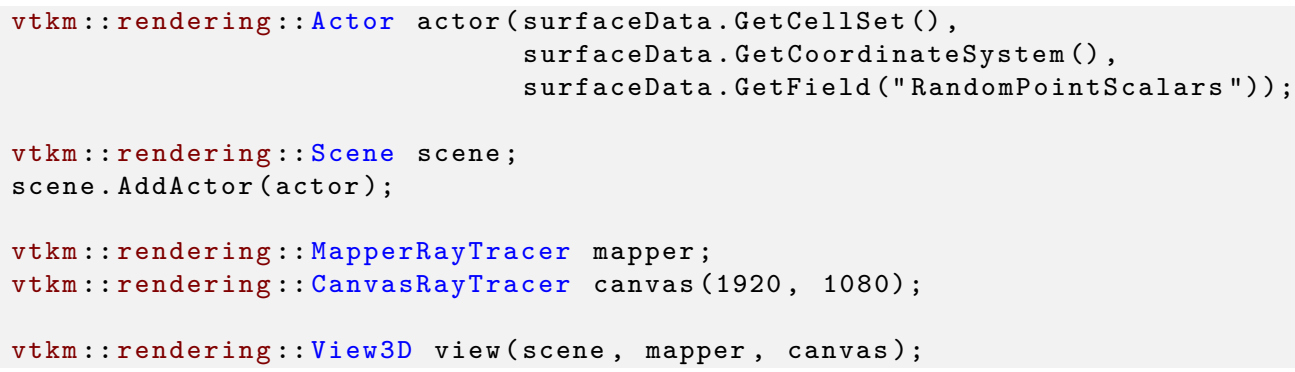

The View also maintains a background color (the color used in areas where nothing is drawn) and a foreground color (the color used for annotation elements). By default, the View has a black background and a white foreground. These can be set in the view's constructor, but it is a bit more readable to set them using the View: :SetBackground and View: :SetForeground methods. In either case, the colors are specified using the vtkm: :rendering: Color helper class, which manages the red, green, and blue color channels as well as an optional alpha channel. These channel values are given as floating point values between 0 and 1 .

Example 10.4: Changing the background and foreground colors of a View.

view.SetBackgroundColor (vtkm: :rendering:: Color (1.0f, $1.0 f, 1.0 f)$ );

view.SetForegroundColor (vtkm: :rendering: : Color (0.0f, $0.0 f, 0.0 f)$ );

\section{Common Errors}

Although the background and foreground colors are set independently, it will be difficult or impossible to see the annotation if there is not enough contrast between the background and foreground colors. Thus, when changing a View's background color, it is always good practice to also change the foreground color.

Once the View is constructed, intialized, and set up, it is ready to render. This is done by calling the View : : Paint method.

$$
1 \text { view.Paint (); }
$$

Example 10.5: Using Canvas: : Paint in a display callback.

Putting together Examples 10.3, 10.4, and 10.5, the final render of a view looks like that in Figure 10.1.

Of course, the vtkm: :rendering::CanvasRayTracer created in 10.3 is an offscreen rendering buffer, so you cannot immediately see the image. When doing batch visualization, an easy way to output the image to a file for later viewing is with the View: :SaveAs method. This method can save the image in either PNG or in the portable pixelmap (PPM) format. 

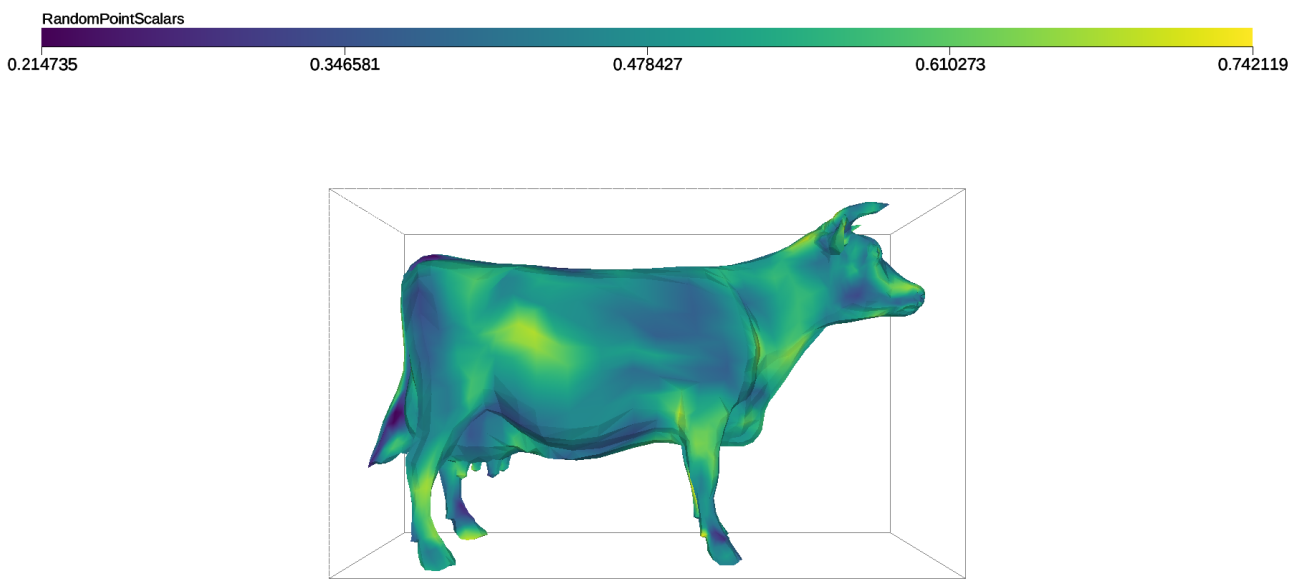

Figure 10.1: Example output of VTK-m's rendering system.

Example 10.6: Saving the result of a render as an image file.

1 view.SaveAs ("BasicRendering.png");

We visit doing interactive rendering in a GUI later in Section 10.7.

\subsection{Changing Rendering Modes}

Example 10.3 constructs the default mapper for ray tracing, which renders the data as an opaque solid. However, you can change the rendering mode by using one of the other mappers listed in Section 10.3. For example, say you just wanted to see a wireframe representation of your data. You can achieve this by using vtkm: :rendering: :MapperWireframer.

Example 10.7: Creating a mapper for a wireframe representation.

1 vtkm: :rendering: : MapperWireframer mapper;

2 vtkm::rendering:: View3D view(scene, mapper, canvas);

Alternatively, perhaps you wish to render just the points of mesh. vtkm: :rendering: : MapperPoint renders the points as spheres and also optionally can scale the spheres based on field values.

Example 10.8: Creating a mapper for point representation.

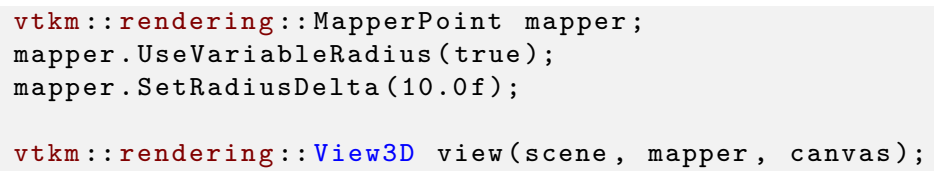

These mappers respectively render the images shown in Figure 10.2. Other mappers, such as those that can render translucent volumes, are also available. 

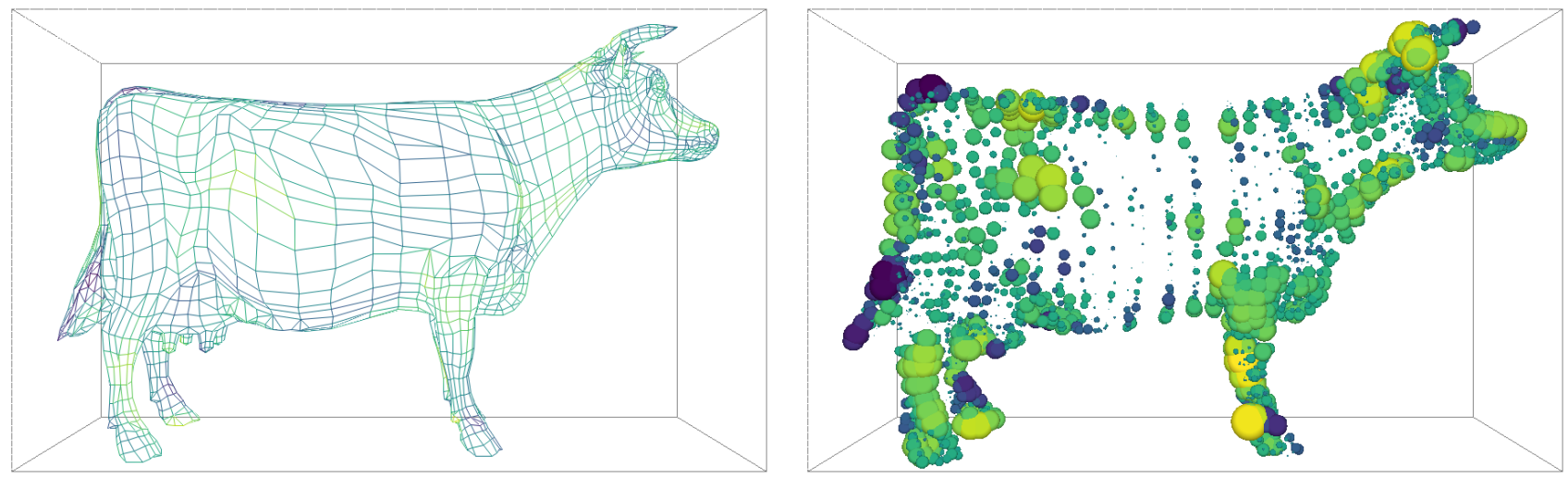

Figure 10.2: Examples of alternate rendering modes using different mappers. The left image is rendered with MapperWireframer. The right image is rendered with MapperPoint.

\subsection{Manipulating the Camera}

The vtkm: :rendering::View uses an object called vtkm::rendering::Camera to describe the vantage point from which to draw the geometry. The camera can be retrieved from the View: GetCamera method. That retrieved camera can be directly manipulated or a new camera can be provided by calling View: : SetCamera. In this section we discuss camera setups typical during view set up. Camera movement during interactive rendering is revisited in Section 10.7.2.

A Camera operates in one of two major modes: 2D mode or 3D mode. 2D mode is designed for looking at flat geometry (or close to flat geometry) that is parallel to the x-y plane. 3D mode provides the freedom to place the camera anywhere in 3D space. The different modes can be set with SetModeTo2D and SetModeTo3D, respectively. The interaction with the camera in these two modes is very different.

\subsubsection{D Camera Mode}

The $2 \mathrm{D}$ camera is restricted to looking at some region of the $\mathrm{x}-\mathrm{y}$ plane.

\section{View Range}

The vantage point of a $2 \mathrm{D}$ camera can be specified by simply giving the region in the $\mathrm{x}-\mathrm{y}$ plane to look at. This region is specified by calling Camera: :SetViewRange2D. This method takes the left, right, bottom, and top of the region to view. Typically these are set to the range of the geometry in world space as shown in Figure 10.3.

There are 3 overloaded versions of the SetViewRange2D method. The first version takes the 4 range values, left, right, bottom, and top, as separate arguments in that order. The second version takes two vtkm: : Range objects specifying the range in the $\mathrm{x}$ and $\mathrm{y}$ directions, respectively. The third version trakes a single vtkm: :Bounds object, which completely specifies the spatial range. (The range in $\mathrm{z}$ is ignored.) The Range and Bounds objects are documented later in Sections 19.3 and 19.4, respectively.

Pan

A camera pan moves the viewpoint left, right, up, or down. A camera pan is performed by calling the Camera: :Pan method. Pan takes two arguments: the amount to pan in $\mathrm{x}$ and the amount to pan in $\mathrm{y}$. 


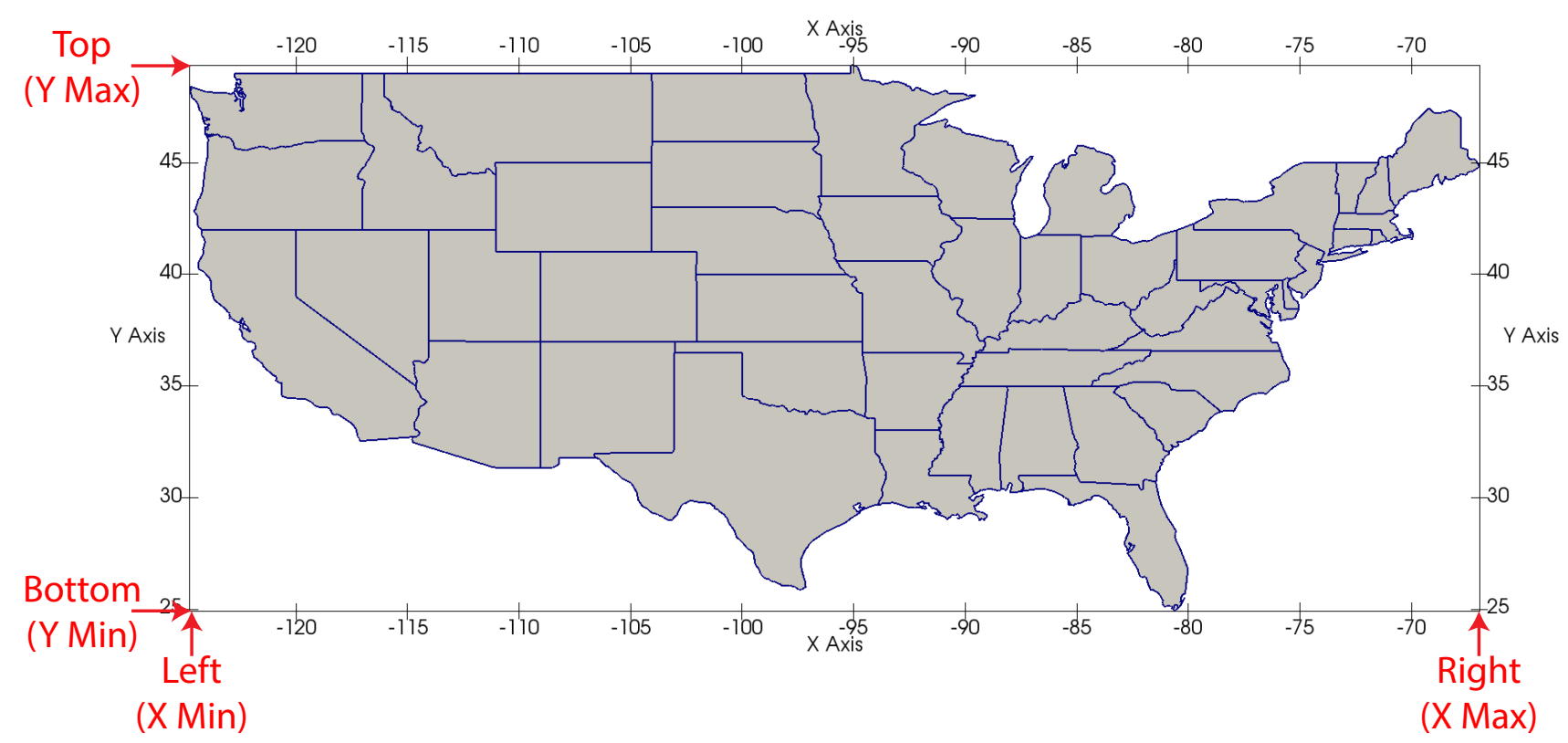

Figure 10.3: The view range bounds to give a Camera.

The pan is given with respect to the projected space. So a pan of 1 in the $\mathrm{x}$ direction moves the camera to focus on the right edge of the image whereas a pan of -1 in the $\mathrm{x}$ direction moves the camera to focus on the left edge of the image.

Example 10.9: Panning the camera.

1 view.GetCamera(). Pan (deltaX, deltaY);

\section{Zoom}

A camera zoom draws the geometry larger or smaller. A camera zoom is performed by calling the Camera: :Zoom method. Zoom takes a single argument specifying the zoom factor. A positive number draws the geometry larger (zoom in), and larger zoom factor results in larger geometry. Likewise, a negative number draws the geometry smaller (zoom out). A zoom factor of 0 has no effect.

Example 10.10: Zooming the camera.

1 view.GetCamera().Zoom(zoomFactor);

\subsubsection{D Camera Mode}

The 3D camera is a free-form camera that can be placed anywhere in 3D space and can look in any direction. The projection of the $3 \mathrm{D}$ camera is based on the pinhole camera model in which all viewing rays intersect a single point. This single point is the camera's position.

\section{Position and Orientation}

The position of the camera, which is the point where the observer is viewing the scene, can be set with the Camera: : SetPosition method. The direction the camera is facing is specified by giving a position to focus on. 
This is called either the "look at" point or the focal point and is specified with the Camera: : SetLookAt method. Figure 10.4 shows the relationship between the position and look at points.

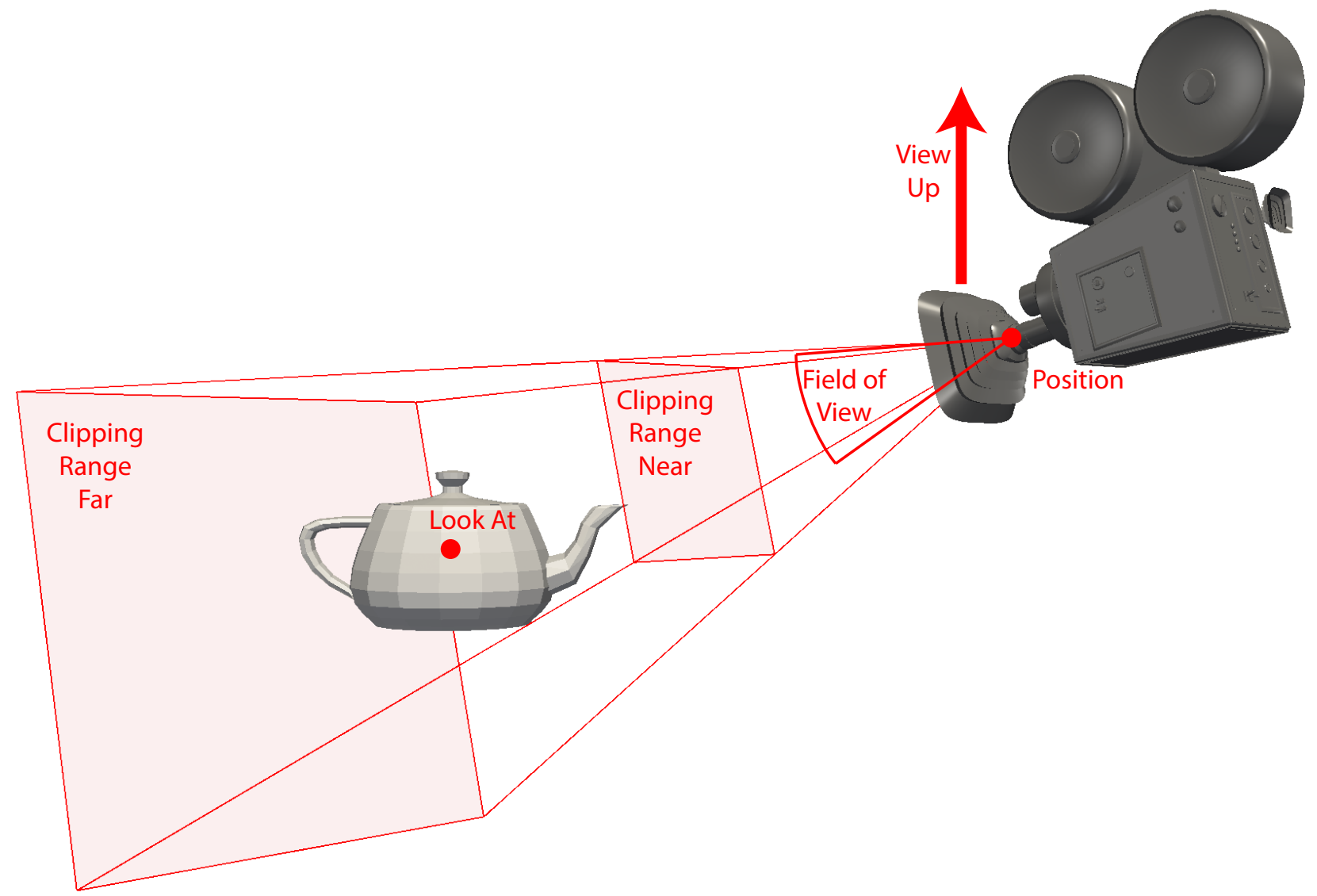

Figure 10.4: The position and orientation parameters for a Camera.

In addition to specifying the direction to point the camera, the camera must also know which direction is considered "up." This is specified with the view up vector using the Camera: : SetViewUp method. The view up vector points from the camera position (in the center of the image) to the top of the image. The view up vector in relation to the camera position and orientation is shown in Figure 10.4.

Another important parameter for the camera is its field of view. The field of view specifies how wide of a region the camera can see. It is specified by giving the angle in degrees of the cone of visible region emanating from the pinhole of the camera to the Camera: : SetFieldOfView method. The field of view angle in relation to the camera orientation is shown in Figure 10.4. A field of view angle of $60^{\circ}$ usually works well.

Finally, the camera must specify a clipping region that defines the valid range of depths for the object. This is a pair of planes parallel to the image that all visible data must lie in. Each of these planes is defined simply by their distance to the camera position. The near clip plane is closer to the camera and must be in front of all geometry. The far clip plane is further from the camera and must be behind all geometry. The distance to both the near and far planes are specified with the Camera: :SetClippingRange method. Figure 10.4 shows the clipping planes in relationship to the camera position and orientation.

Example 10.11: Directly setting vtkm: :rendering: : Camera position and orientation.

1 camera.SetPosition (vtkm: :make_Vec $(10.0,6.0,6.0)$ ); 


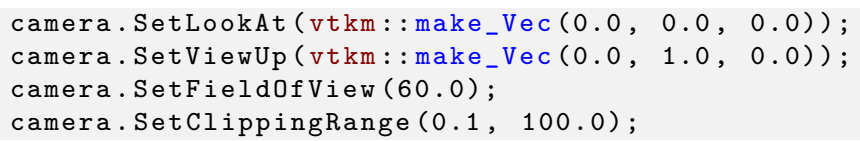

\section{Movement}

In addition to specifically setting the position and orientation of the camera, vtkm: :rendering: : Camera contains several convenience methods that move the camera relative to its position and look at point.

Two such methods are elevation and azimuth, which move the camera around the sphere centered at the look at point. Camera: :Elevation raises or lowers the camera. Positive values raise the camera up (in the direction of the view up vector) whereas negative values lower the camera down. Camera: :Azimuth moves the camera around the look at point to the left or right. Positive values move the camera to the right whereas negative values move the camera to the left. Both Elevation and Azimuth specify the amount of rotation in terms of degrees. Figure 10.5 shows the relative movements of Elevation and Azimuth.

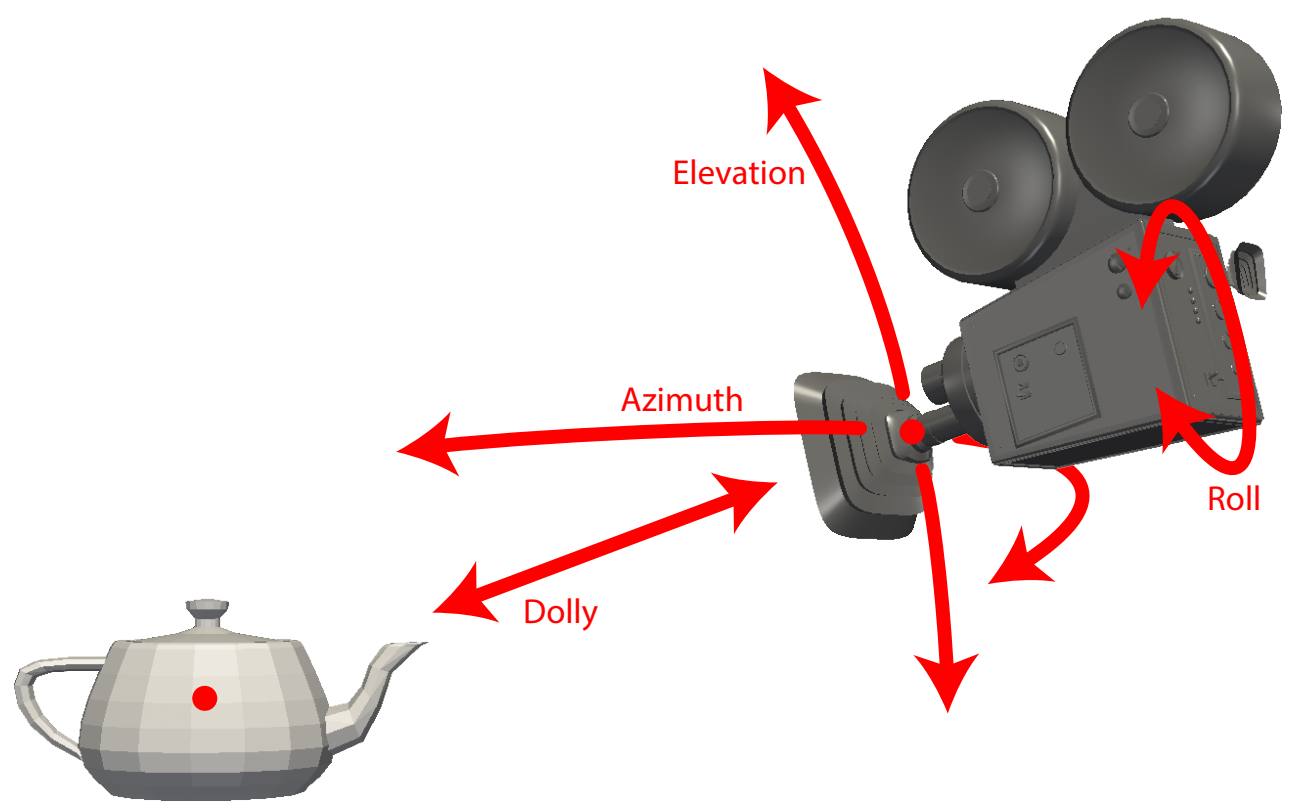

Figure 10.5: Camera movement functions relative to position and orientation.

Example 10.12: Moving the camera around the look at point.

1 view.GetCamera().Azimuth (45.0);

2 view.GetCamera().Elevation (45.0);

The Camera: :Elevation and Camera: :Azimuth methods change the position of the camera, but not the view up vector. This can cause some wild camera orientation changes when the direction of the camera 
view is near parallel to the view up vector, which often happens when the elevation is raised or lowered by about 90 degrees.

In addition to rotating the camera around the look at point, you can move the camera closer or further from the look at point. This is done with the Camera: :Dolly method. The Dolly method takes a single value that is the factor to scale the distance between camera and look at point. Values greater than one move the camera away, values less than one move the camera closer. The direction of dolly movement is shown in Figure 10.5.

Finally, the Camera: :Roll method rotates the camera around the viewing direction. It has the effect of rotating the rendered image. The Roll method takes a single value that is the angle to rotate in degrees. The direction of roll movement is shown in Figure 10.5.

Pan

A camera pan moves the viewpoint left, right, up, or down. A camera pan is performed by calling the Camera: :Pan method. Pan takes two arguments: the amount to pan in $\mathrm{x}$ and the amount to pan in $\mathrm{y}$.

The pan is given with respect to the projected space. So a pan of 1 in the $\mathrm{x}$ direction moves the camera to focus on the right edge of the image whereas a pan of -1 in the $\mathrm{x}$ direction moves the camera to focus on the left edge of the image.

Example 10.13: Panning the camera.

1 view.GetCamera(). Pan(deltaX, deltaY);

\section{Zoom}

A camera zoom draws the geometry larger or smaller. A camera zoom is performed by calling the Camera: :Zoom method. Zoom takes a single argument specifying the zoom factor. A positive number draws the geometry larger (zoom in), and larger zoom factor results in larger geometry. Likewise, a negative number draws the geometry smaller (zoom out). A zoom factor of 0 has no effect.

Example 10.14: Zooming the camera.

1 view.GetCamera( ). Zoom (zoomFactor);

\section{Reset}

Setting a specific camera position and orientation can be frustrating, particularly when the size, shape, and location of the geometry is not known a priori. Typically this involves querying the data and finding a good camera orientation.

To make this process simpler, vtkm: :rendering: :Camera has a convenience method named Camera: :ResetToBounds that automatically positions the camera based on the spatial bounds of the geometry. The most expedient method to find the spatial bounds of the geometry being rendered is to get the vtkm: :rendering: : Scene object and call GetSpatialBounds. The Scene object can be retrieved from the vtkm: :rendering: :View, which, as described in Section 10.4, is the central object for managing rendering.

Example 10.15: Resetting a Camera to view geometry.

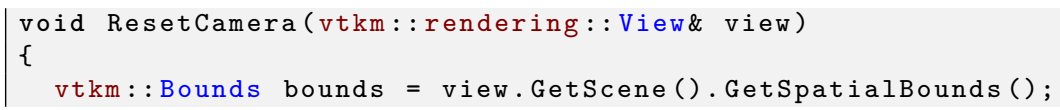


4 view.GetCamera().ResetToBounds (bounds);

$5 \mid\}$

The ResetToBounds method operates by placing the look at point in the center of the bounds and then placing the position of the camera relative to that look at point. The position is such that the view direction is the same as before the call to ResetToBounds and the distance between the camera position and look at point has the bounds roughly fill the rendered image. This behavior is a convenient way to update the camera to make the geometry most visible while still preserving the viewing position. If you want to reset the camera to a new viewing angle, it is best to set the camera to be pointing in the right direction and then calling ResetToBounds to adjust the position.

Example 10.16: Resetting a Camera to be axis aligned.

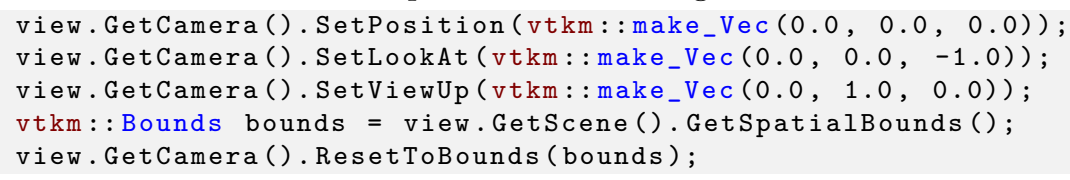

\subsection{Interactive Rendering}

So far in our description of VTK-m's rendering capabilities we have talked about doing rendering of fixed scenes. However, an important use case of scientific visualization is to provide an interactive rendering system to explore data. In this case, you want to render into a GUI application that lets the user interact manipulate the view. The full design of a 3D visualization application is well outside the scope of this book, but we discuss in general terms what you need to plug VTK-m's rendering into such a system.

In this section we discuss two important concepts regarding interactive rendering. First, we need to write images into a GUI while they are being rendered. Second, we want to translate user interaction to camera movement.

\subsubsection{Rendering Into a GUI}

Before being able to show rendering to a user, we need a system rendering context in which to push the images. In this section we demonstrate the display of images using the OpenGL rendering system, which is common for scientific visualization applications. That said, you could also use other rendering systems like DirectX or even paste images into a blank widget.

Creating an OpenGL context varies depending on the OS platform you are using. If you do not already have an application you want to integrate with VTK-m's rendering, you may wish to start with graphics utility API such as GLUT or GLFW. The process of initializing an OpenGL context is not discussed here.

The process of rendering into an OpenGL context is straightforward. First call Paint on the View object to do the actual rendering. Second, get the image color data out of the View's Canvas object. This is done by calling Canvas: :GetColorBuffer. This will return a vtkm: :cont: :ArrayHandle object containing the image's pixel color data. (ArrayHandles are discussed in detail in Chapter 16 and subsequent chapters.) A raw pointer can be pulled out of this ArrayHandle by casting it to the vtkm: : cont: : ArrayHandleBase subclass and calling the GetReadPointer method on that. Third, the pixel color data are pasted into the OpenGL render context. There are multiple ways to do so, but the most straightforward way is to use the glDrawPixels function provided by OpenGL. Fourth, swap the OpenGL buffers. The method to swap OpenGL buffers varies by OS platform. The aforementioned graphics libraries GLUT and GLFW each provide a function for doing so.

Example 10.17: Rendering a View and pasting the result to an active OpenGL context.

1 view.Paint (); 


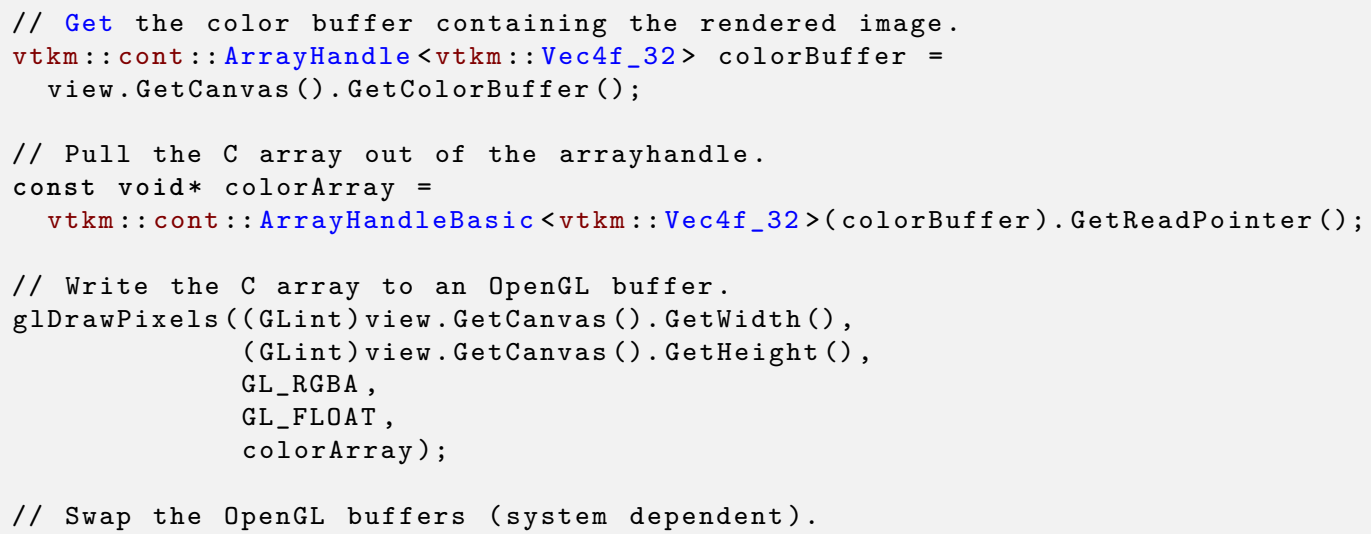

\subsubsection{Camera Movement}

When interactively manipulating the camera in a windowing system, the camera is usually moved in response to mouse movements. Typically, mouse movements are detected through callbacks from the windowing system back to your application. Once again, the details on how this works depend on your windowing system. The assumption made in this section is that through the windowing system you will be able to track the $\mathrm{x}$-y pixel location of the mouse cursor at the beginning of the movement and the end of the movement. Using these two pixel coordinates, as well as the current width and height of the render space, we can make several typical camera movements.

Pixel coordinates in VTK-m's rendering system originate in the lower-left corner of the image. However, windowing systems generally report mouse coordinates with the origin in the upper-left corner. The upshot is that the y coordinates will have to be reversed when translating mouse coordinates to VTK-m image coordinates. This inverting is present in all the following examples.

\section{Rotate}

A common and important mode of interaction with $3 \mathrm{D}$ views is to allow the user to rotate the object under inspection by dragging the mouse. To facilitate this type of interactive rotation, vtkm: :rendering: : Camera provides a convenience method named TrackballRotate. The TrackballRotate method takes a start and end position of the mouse on the image and rotates viewpoint as if the user grabbed a point on a sphere centered in the image at the start position and moved under the end position.

The TrackballRotate method is typically called from within a mouse movement callback. The callback must record the pixel position from the last event and the new pixel position of the mouse. Those pixel positions must be normalized to the range -1 to 1 where the position $(-1,-1)$ refers to the lower left of the image and $(1,1)$ refers to the upper right of the image. The following example demonstrates the typical operations used to establish rotations when dragging the mouse.

Example 10.18: Interactive rotations through mouse dragging with Camera: :TrackballRotate. 


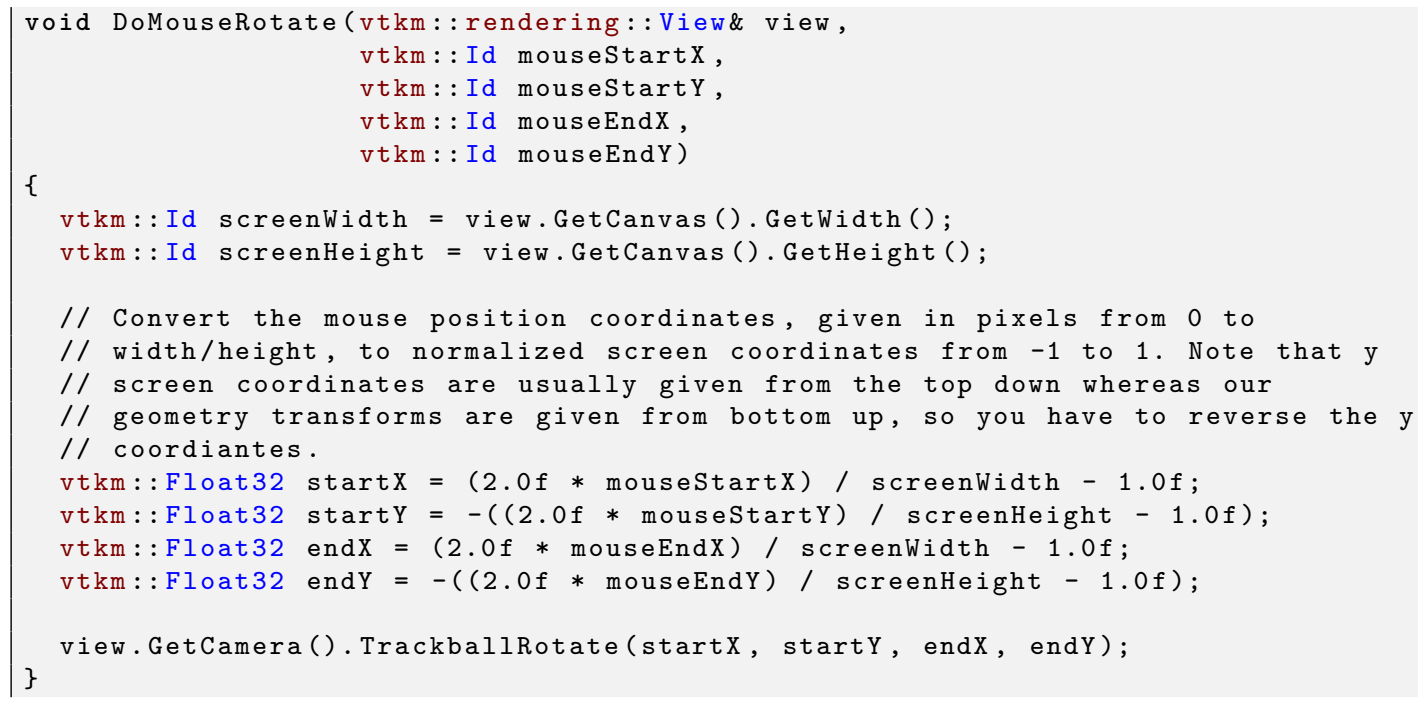

Pan

Panning can be performed by calling Camera: : Pan with the translation relative to the width and height of the canvas. For the translation to track the movement of the mouse cursor, simply scale the pixels the mouse has traveled by the width and height of the image.

Example 10.19: Pan the view based on mouse movements.

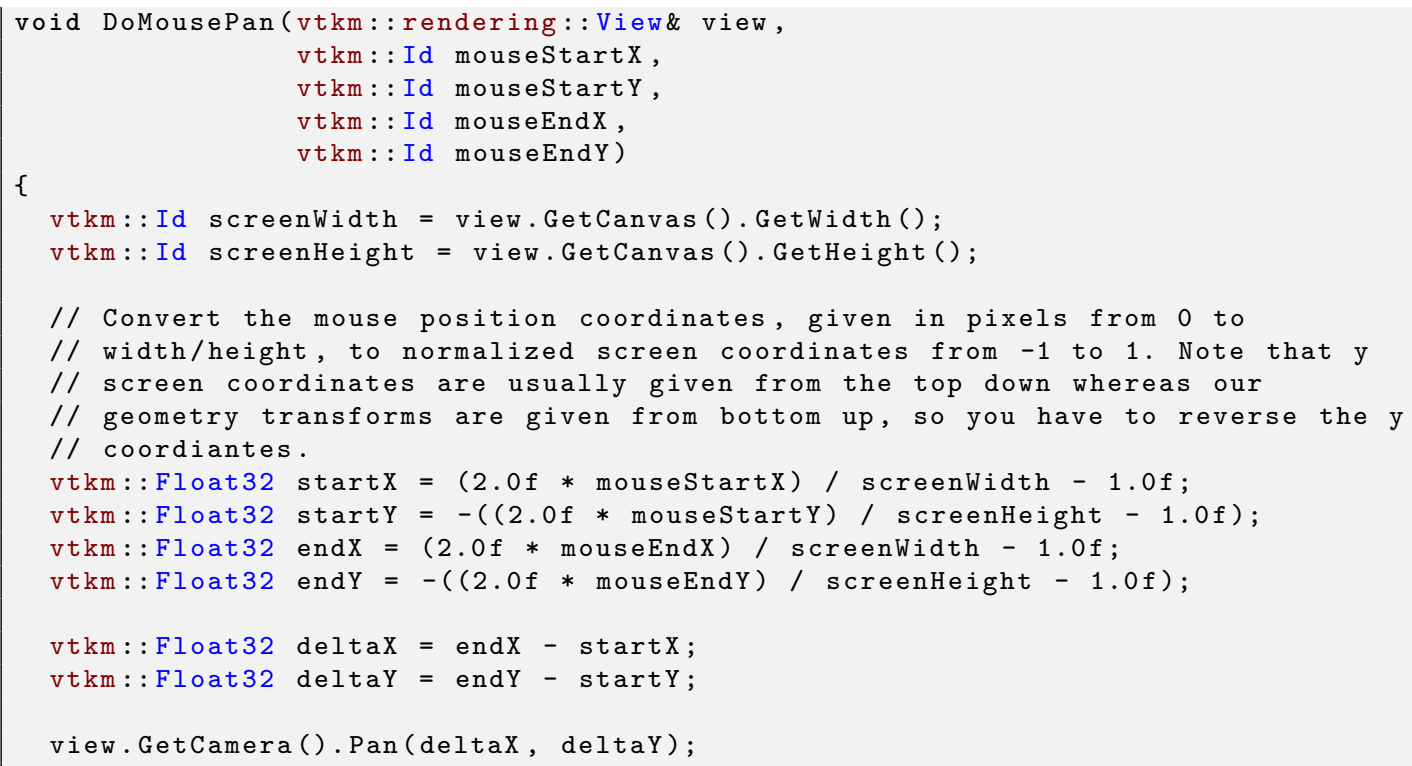

\section{Zoom}

Zooming can be performed by calling Camera: : Zoom with a positive or negative zoom factor. When using Zoom to respond to mouse movements, a natural zoom will divide the distance traveled by the mouse pointer by the width or height of the screen as demonstrated in the following example. 
Example 10.20: Zoom the view based on mouse movements.

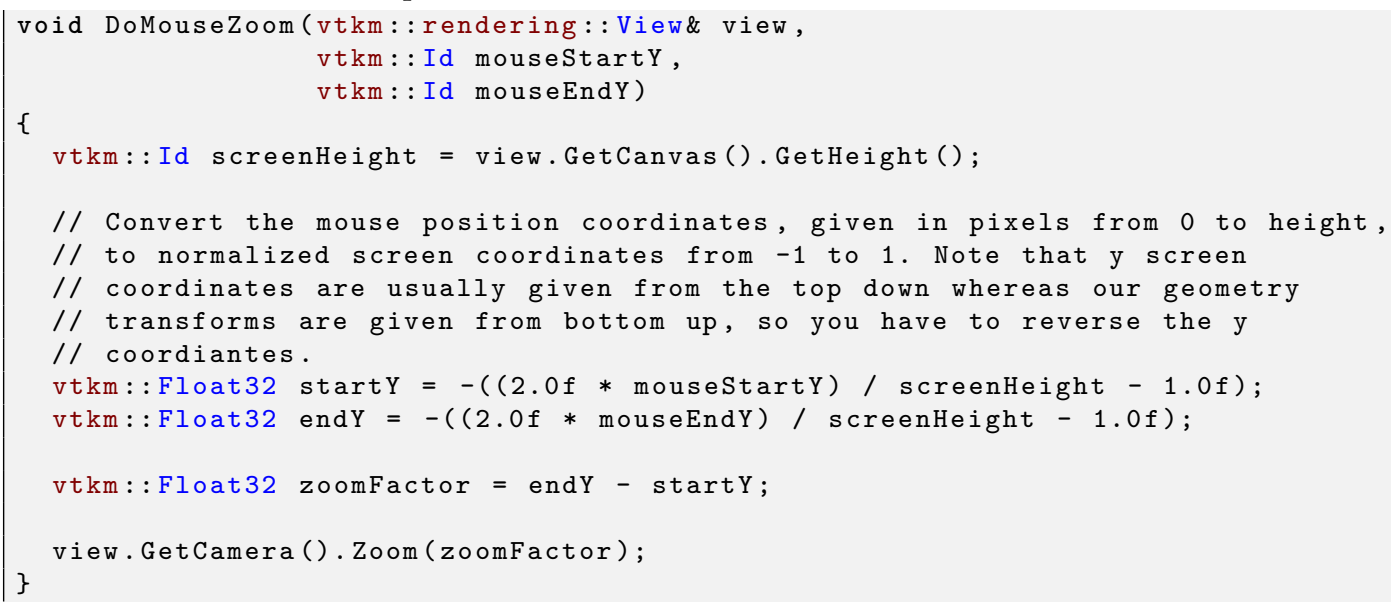

\subsection{Color Tables}

An important feature of VTK-m's rendering units is the ability to pseudocolor objects based on scalar data. This technique maps each scalar to a potentially unique color. This mapping from scalars to colors is defined by a vtkm: : cont::ColorTable object. A ColorTable can be specified as an optional argument when constructing a vtkm: :rendering: :Actor. (Use of Actors is discussed in Section 10.1.)

Example 10.21: Specifying a ColorTable for an Actor.

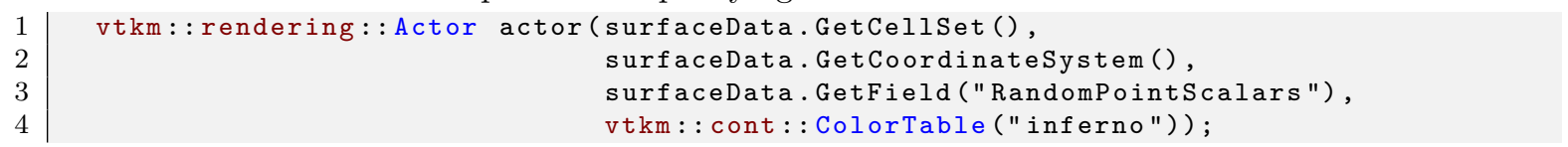

The easiest way to create a ColorTable is to provide the name of one of the many predefined sets of color provided by VTK-m. A list of all available predefined color tables is provided below.

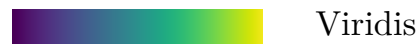

\section{Cool to Warm}

Cool to Warm Extended

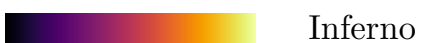

Plasma

Black-Body Radiation
Matplotlib Virdis, which is designed to have perceptual uniformity, accessibility to color blind viewers, and good conversion to black and white. This is the default color map.

A color table designed to be perceptually even, to work well on shaded 3D surfaces, and to generally perform well across many uses.

This colormap is an expansion on cool to warm that moves through a wider range of hue and saturation. Useful if you are looking for a greater level of detail, but the darker colors at the end might interfere with $3 \mathrm{D}$ surfaces.

Matplotlib Inferno, which is designed to have perceptual uniformity, accessibility to color blind viewers, and good conversion to black and white.

Matplotlib Plasma, which is designed to have perceptual uniformity, accessibility to color blind viewers, and good conversion to black and white.

The colors are inspired by the wavelengths of light from black body radiation. The actual colors used are designed to be perceptually uniform. 


\begin{tabular}{|l}
\hline \multicolumn{1}{|l}{ X Ray } \\
Green \\
Black - Blue - White \\
Blue to Orange \\
Gray to Red \\
Cold and Hot \\
Blue - Green - Orange \\
Yellow - Gray - Blue \\
Rainbow Uniform
\end{tabular}

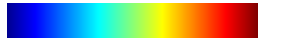

Jet

Rainbow Desaturated
Greyscale colormap useful for making volume renderings similar to what you would expect in an x-ray.

A sequential color map of green varied by saturation.

A sequential color map from black to blue to white.

A double-ended (diverging) color table that goes from dark blues to a neutral white and then a dark orange at the other end.

A double-ended (diverging) color table with black/gray at the low end and orange/red at the high end.

A double-ended color map with a black middle color and diverging values to either side. Colors go from red to yellow on the positive side and through blue on the negative side. A three-part color map with blue at the low end, green in the middle, and orange at the high end.

A three-part color map with yellow at the low end, gray in the middle, and blue at the high end.

A color table that spans the hues of a rainbow. There have been many scientific perceptual studies on the effectiveness of rainbow colors, and they uniformly found them to be ineffective. This color table modifies the hues to make them more perceptually uniform, which should improve the effectiveness of the colors. However, we still recommend the other color tables over this one.

A rainbow color table that adds some darkness for greater perceptual resolution. The ends of the jet color table might be too dark for 3D surfaces.

All the badness of the rainbow color table with periodic dark points added, which can help identify rate of change. 


\section{ERROR HANDLING}

VTK-m contains several mechanisms for checking and reporting error conditions.

\subsection{Runtime Error Exceptions}

VTK-m uses exceptions to report errors. All exceptions thrown by VTK-m will be a subclass of vtkm: : cont: :Error. For simple error reporting, it is possible to simply catch a vtkm::cont::Error and report the error message string reported by the Error: :GetMessage method.

Example 11.1: Simple error reporting.

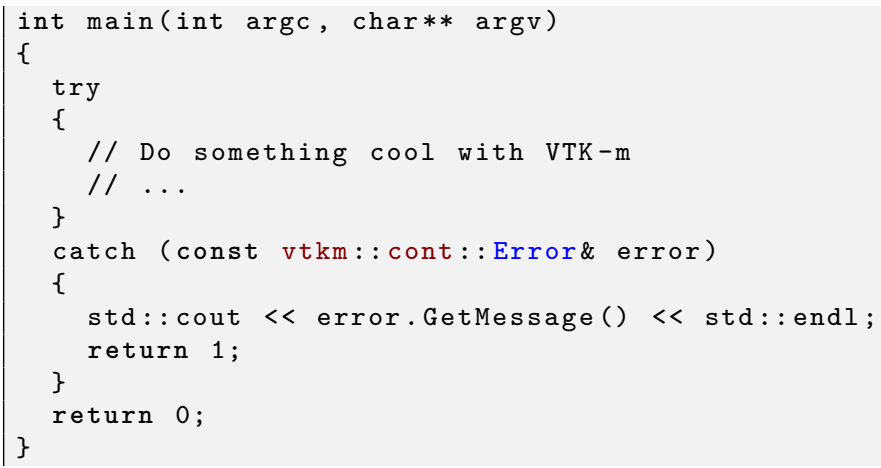

There are several subclasses to vtkm: :cont::Error. The specific subclass gives an indication of the type of error that occurred when the exception was thrown. Catching one of these subclasses may help a program better recover from errors.

vtkm: : cont: :ErrorBadAllocation Thrown when there is a problem accessing or manipulating memory. Often this is thrown when an allocation fails because there is insufficient memory, but other memory access errors can cause this to be thrown as well.

vtkm: : cont: :ErrorBadType Thrown when VTK-m attempts to perform an operation on an object that is of an incompatible type.

vtkm: cont::ErrorBadValue Thrown when a VTK-m function or method encounters an invalid value that inhibits progress.

vtkm: : cont: :ErrorExecution Throw when an error is signaled in the execution environment for example when a worklet is being executed. 
vtkm: :cont: ErrorInternal Thrown when VTK-m detects an internal state that should never be reached. This error usually indicates a bug in VTK-m or, at best, VTK-m failed to detect an invalid input it should have.

vtkm: :io: :ErrorI0 Thrown by a reader or writer when a file error is encountered.

\subsection{Asserting Conditions}

In addition to the aforementioned error signaling, the vtkm/Assert.h header file defines a macro named VTKM_ASSERT. This macro behaves the same as the POSIX assert macro. It takes a single argument that is a condition that is expected to be true. If it is not true, the program is halted and a message is printed. Asserts are useful debugging tools to ensure that software is behaving and being used as expected.

Example 11.2: Using VTKM_ASSERT.

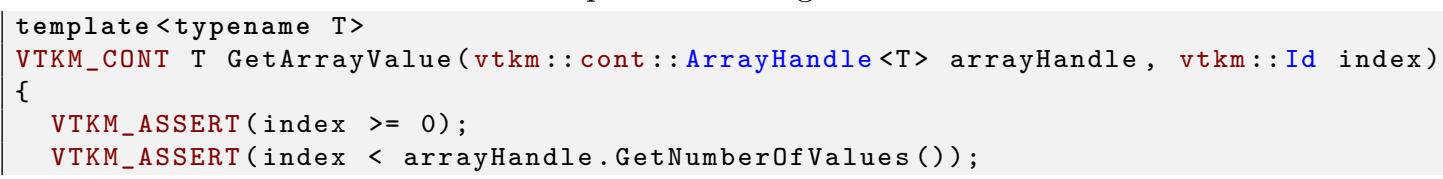

S Like the POSIX assert, if the NDEBUG macro is defined, then VTKM_ASSERT will become an empty expression. Typically NDEBUG is defined with a compiler flag (like-DNDEBUG) for release builds to better optimize the code. CMake will automatically add this flag for release builds.

A helpful warning provided by many compilers alerts you of unused variables. (This warning is commonly enabled on VTK-m regression test nightly builds.) If a function argument is used only in a VTKM_ASSERT, then it will be required for debug builds and be unused in release builds. To get around this problem, add a statement to the function of the form (void) variableName;. This statement will have no effect on the code generated but will suppress the warning for release builds.

\subsection{Compile Time Checks}

Because VTK-m makes heavy use of $\mathrm{C}++$ templates, it is possible that these templates could be used with inappropriate types in the arguments. Using an unexpected type in a template can lead to very confusing errors, so it is better to catch such problems as early as possible. The VTKM_STATIC_ASSERT macro, defined in vtkm/StaticAssert.h makes this possible. This macro takes a constant expression that can be evaluated at compile time and verifies that the result is true.

In the following example, VTKM_STATIC_ASSERT and its sister macro VTKM_STATIC_ASSERT_MSG, which allows you to give a descriptive message for the failure, are used to implement checks on a templated function that is designed to work on any scalar type that is represented by 32 or more bits. 
Example 11.3: Using VTKM_STATIC_ASSERT.

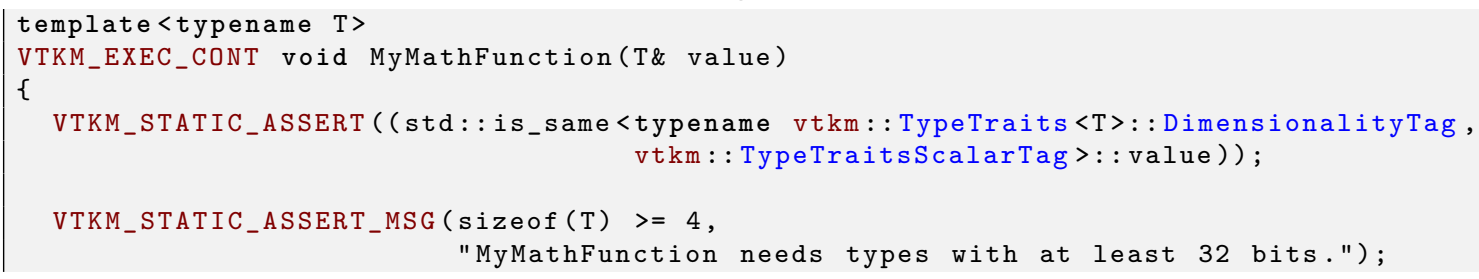

\section{(8) Did you know?}

S In addition to the several trait template classes provided by VTK-m to introspect $C++$ types, the $C++$ standard type_traits header file contains several helpful templates for general queries on types. Example 11.3 demonstrates the use of one such template: std: :is_same.

Many templates used to introspect types resolve to the tags std: :true_type and std: :false_type rather than the constant values true and false that VTKM_STATIC_ASSERT expects. The std::true_type and std: :false_type tags can be converted to the Boolean literal by adding : :value to the end of them. Failing to do so will cause VTKM_STATIC_ASSERT to behave incorrectly. Example 11.3 demonstrates getting the Boolean literal from the result of std: : is_same. 



\section{MANAGING DEVICES}

Multiple vendors vie to provide accelerator-type processors. VTK-m endeavors to support as many such architectures as possible. Each device and device technology requires some level of code specialization, and that specialization is encapsulated in a unit called a device adapter.

So far in Part II we have been writing code that runs on a local serial CPU. In those examples where we run a filter, VTK-m is launching parallel execution in the execution environment. Internally VTK-m uses a device adapter to manage this execution.

A build of VTK-m generally supports multiple device adapters. In this chapter we describe how to represent and manage devices.

\subsection{Device Adapter Tag}

A device adapter is identified by a device adapter tag. This tag, which is simply an empty struct type, is used as the template parameter for several classes in the VTK-m control environment and causes these classes to direct their work to a particular device. The following device adapter tags are available in VTK-m.

vtkm: :cont::DeviceAdapterTagSerial Performs all computation on the same single thread as the control environment. This device is useful for debugging. This device is always available. This tag is defined in vtkm/cont/DeviceAdapterSerial.h.

vtkm: : cont: :DeviceAdapterTagCuda Uses a CUDA capable GPU device. For this device to work, VTK-m must be configured to use CUDA and the code must be compiled by the CUDA nvcc compiler. This tag is defined in vtkm/cont/cuda/DeviceAdapterCuda.h.

vtkm: :cont: :DeviceAdapterTagOpenMP Uses OpenMP compiler extensions to run algorithms on multiple threads. For this device to work, VTK-m must be configured to use OpenMP and the code must be compiled with a compiler that supports OpenMP pragmas. This tag is defined in vtkm/cont/openmp/DeviceAdapterOpenMP.h.

vtkm: :cont: :DeviceAdapterTagTBB Uses the Intel Threading Building Blocks library to run algorithms on multiple threads. For this device to work, VTK-m must be configured to use TBB and the executable must be linked to the TBB library. This tag is defined in vtkm/cont/tbb/DeviceAdapterTBB.h.

vtkm: : cont: :DeviceAdapterTagKokkos Uses the Kokkos library to run algorithms in parallel. For this device to work, VTK-m must be configured to use Kokkos and the executable must be linked to the Kokkos libraries. Vtk-m will use the default execution space of the provided kokkos library build. This tag is defined in vtkm/cont/kokkos/DeviceAdapterKokkos.h. 
The following example uses the tag for the Intel Threading Building blocks device adapter to specify a specific device for VTK-m to use. (Details on specifying devices in VTK-m is provided in Section 12.3.)

Example 12.1: Specifying a device using a device adapter tag.

1 | vtkm: : cont : : ScopedRuntimeDeviceTracker (vtkm: :cont: : DeviceAdapterTagTBB \{\} );

For classes and methods that have a template argument that is expected to be a device adapter tag, the tag type can be checked with the VTKM_IS_DEVICE_ADAPTER_TAG macro to verify the type is a valid device adapter tag. It is good practice to check unknown types with this macro to prevent further unexpected errors.

\subsection{Device Adapter Id}

Using a device adapter tag directly means that the type of device needs to be known at compile time. To store a device adapter type at run time, one can instead use vtkm: :cont: :DeviceAdapterId. DeviceAdapterId is a superclass to all the device adapter tags, and any device adapter tag can be "stored" in a DeviceAdapterId. Thus, it is more common for functions and classes to use DeviceAdapterId then to try to track a specific device with templated code.

In addition to the provided device adapter tags listed previously, a DeviceAdapterId can store some special device adapter tags that do not directly specify a specific device.

vtkm: : cont: : DeviceAdapterTagAny Used to specify that any device may be used for an operation. In practice this is limited to devices that are currently available.

vtkm: : cont: :DeviceAdapterTagUndefined Used to avoid specifying a device. Useful as a placeholder when a device can be specified but none is given.

Any device adapter tag can be used where a device adapter id is expected. Thus, you can use a device adapter tag whenever you want to specify a particular device and pass that to any method expecting a device $i d$. Likewise, it is usually more convenient for classes and methods to manage device adapter ids rather than device adapter tag.

DeviceAdapterId contains several helpful methods to get runtime information about a particular device.

GetName A static method that returns a string description for the device adapter. The string is stored in a type named vtkm::cont::DeviceAdapterNameType, which is currently aliased to std::string. The device adapter name is useful for printing information about a device being used.

GetId A static method taking no arguments that returns a unique integer identifier for the device adapter as a vtkm: : Int8.

IsValueValid A static const bool that is true if the implementation of the integer returned from GetId corresponds to a concrete device. So, for example, the IsValueValid flag for a DeviceAdapterTagSerial is true whereas the IsValueValid flag for a DeviceAdapterTagAny is false. 
Did you know?

As a cheat, all device adapter tags actually inherit from the vtkm: :cont::DeviceAdapterId class. Thus, all of these methods can be called directly on a device adapter tag.

\section{Common Errors}

Just because the DeviceAdapterId: IsValueValid returns true that does not necessarily mean that this device is available to be run on. It simply means that the device is implemented in VTK-m. However, that device might not be compiled, or that device might not be available on the current running system, or that device might not be enabled. Use the device runtime tracker described in Section 12.3 to determine if a particular device can actually be used.

\subsection{Runtime Device Tracker}

It is often the case that you are agnostic about what device VTK-m algorithms run so long as they complete correctly and as fast as possible. Thus, rather than directly specify a device adapter, you would like VTK-m to try using the best available device, and if that does not work try a different device. Because of this, there are many features in VTK-m that behave this way. For example, you may have noticed that running filters, as in the examples of Chapter 9, you do not need to specify a device; they choose a device for you.

However, even though we often would like VTK-m to choose a device for us, we still need a way to manage device preferences. VTK-m also needs a mechanism to record runtime information about what devices are available so that it does not have to continually try (and fail) to use devices that are not available at runtime. These needs are met with the vtkm: :cont: :RuntimeDeviceTracker class. RuntimeDeviceTracker maintains information about which devices can and should be run on. VTK-m maintains a RuntimeDeviceTracker for each thread your code is operating on. To get the runtime device for the current thread, use the vtkm: : cont: :GetRuntimeDeviceTracker method.

RuntimeDeviceTracker has the following methods.

CanRunOn Takes a device adapter tag and returns true if VTK-m was configured for the device and it has not yet been marked as disabled.

DisableDevice Takes a device adapter tag and marks that device to not be used. Future calls to CanRunOn for this device will return false until that device is reset.

ResetDevice Takes a vtkm: :cont: :DeviceAdapterTag and resets the state for that device to its default value. Each device defaults to on as long as VTK-m is configured to use that device and a basic runtime check finds a viable device.

Reset Resets all devices. This equivocally calls ResetDevice for all devices supported by VTK-m.

ForceDevice Takes a device adapter tag and enables that device. All other devices are disabled. This method throws a vtkm: :cont: :ErrorBadValue if the requested device cannot be enabled.

ReportAllocationFailure A device might have less working memory available than the main CPU. If this is the case, memory allocation errors are more likely to happen. This method is used to report a vtkm: :cont: :ErrorBadAllocation and disables the device for future execution. 
ReportBadDeviceFailure It is possible that a device may throw a vtkm: : cont: : ErrorBadDevice failure caused by some erroneous device issue. If this occurs, it is possible to catch the vtkm: :cont: :ErrorBadDevice exception and pass it to ReportBadDeviceFailure along with the vtkm: :cont::DeviceAdapterId to forcefully disable a device.

A RuntimeDeviceTracker can be used to specify which devices to consider for a particular operation. However, a better way to specify devices is to use the vtkm: : cont: : ScopedRuntimeDeviceTracker class. When a ScopedRuntimeDeviceTracker is constructed, it specifies a new set of devices for VTK-m to use. When the ScopedRuntimeDeviceTracker is destroyed as it leaves scope, it restores VTK-m's devices to those that existed when it was created.

The following example demonstrates how the ScopedRuntimeDeviceTracker is used to force the VTK-m operations that happen within a function to operate exclusively with the TBB device.

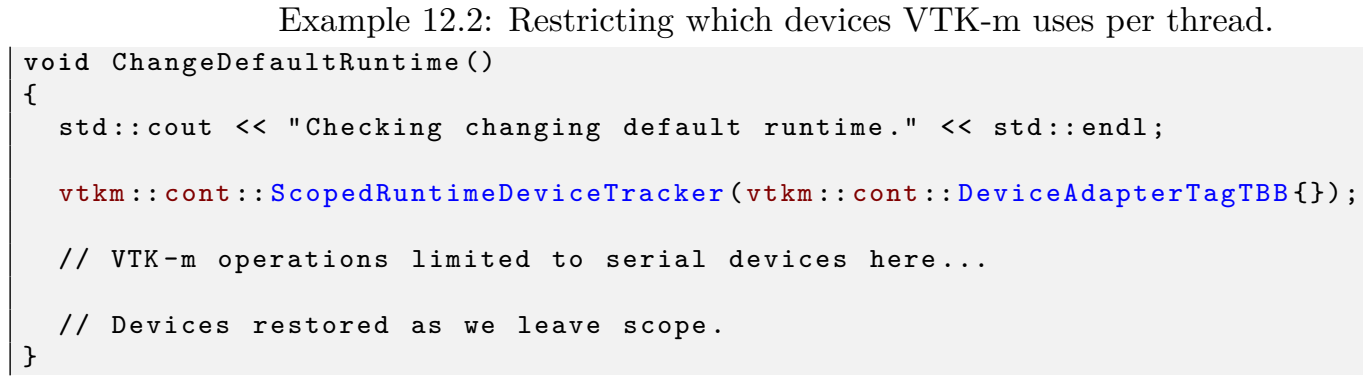

In the previous example we forced VTK-m to use the TBB device. This is the default behavior of ScopedRuntimeDeviceTracker, but the constructor takes an optional second argument that is a value in the vtkm: :cont: :RuntimeDeviceTrackerMode to specify how modify the current device adapter list.

RuntimeDeviceTrackerMode: :Force Replaces the current list of devices to try with the device specified to the ScopedRuntimeDeviceTracker. This has the effect of forcing VTK-m to use the provided device. This is the default behavior for the ScopedRuntimeDeviceTracker.

RuntimeDeviceTrackerMode: :Enable Adds the provided device adapter to the list of devices to try.

RuntimeDeviceTrackerMode: :Disable Removes the provided device adapter from the list of devices to try.

As a motivating example, let us say that we want to perform a deep copy of an array (described in Section 16.2). However, we do not want to do the copy on a CUDA device because we happen to know the data is not on that device and we do not want to spend the time to transfer the data to that device. We can use a vtkm: : cont: :ScopedRuntimeDeviceTracker to temporarily disable the CUDA device for this operation.

Example 12.3: Disabling a device with RuntimeDeviceTracker.

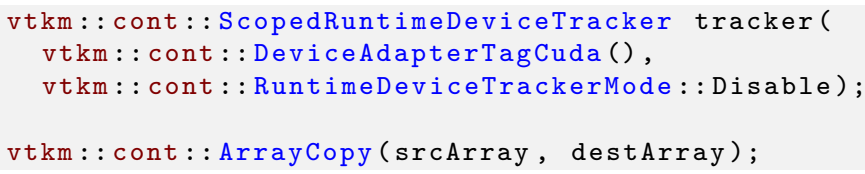


It is often the case that you need to measure the time it takes for an operation to happen. This could be for performing measurements for algorithm study or it could be to dynamically adjust scheduling.

Performing timing in a multi-threaded environment can be tricky because operations happen asynchronously. To ensure that accurate timings can be made, VTK-m provides a vtkm: : cont: : Timer class to provide an accurate measurement of operations that happen on devices that VTK-m can use. By default, Timer will time operations on all possible devices.

The timer is started by calling the Timer: : Start method. The timer can subsequently be stopped by calling Timer: : Stop. The time elapsed between calls to Start and Stop (or the current time if Stop was not called) can be retrieved with a call to the Timer: :GetElapsedTime method. Subsequently calling Start again will restart the timer.

Example 13.1: Using vtkm: : cont: :Timer.

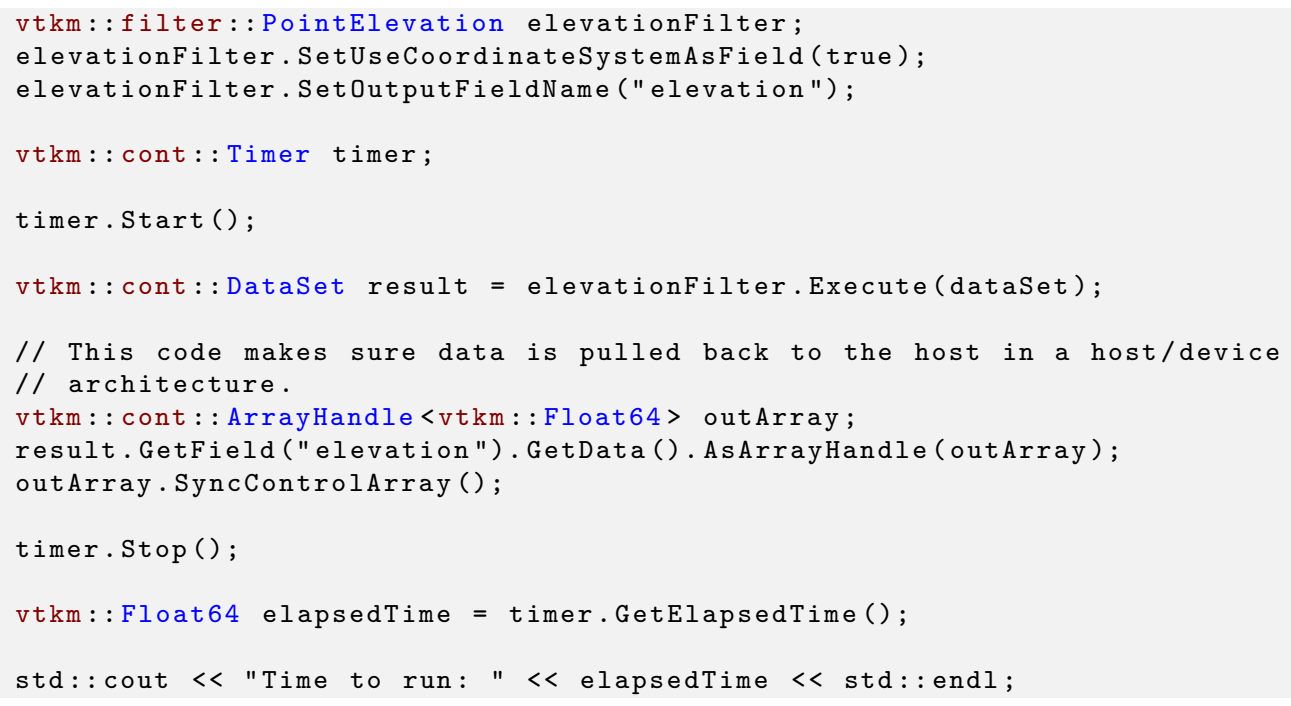

Some device require data to be copied between the host CPU and the device. In this case you might want 
to measure the time to copy data back to the host. This can be done by "touching" the data on the host by getting a control portal.

The VTK-m Timer does its best to capture the time it takes for all parallel operations run between calls to Start and Stop to complete. It does so by synchronizing to concurrent execution on devices that might be in use.

Because Timer synchronizes with devices (essentially waiting for the device to finish executing), that can have an effect on how your program runs. Be aware that using a Timer can itself change the performance of your code. In particular, starting and stopping the timer many times to measure the parts of a sequence of operations can potentially make the whole operation run slower.

By default, Timer will synchronize with all active devices. However, if you want to measure the time for a specific device, then you can pass the device adapter tag or id to vtkm: :cont: :Timer's constructor. You can also change the device being used by passing a device adapter tag or id to the Timer: : Reset method. A device can also be specified through an optional argument to the Timer: :GetElapsedTime method.

The following methods are provided by vtkm: : cont: :Timer.

Start Causes the Timer to begin timing. The elapsed time will record an interval beginning when this method is called.

Started Returns true if Start has been called. It is invalid to try to get the elapsed time if Started is not true.

Stop Causes the Timer to finish timing. The elapsed time will record an interval ending when this method is called. It is invalid to stop the timer if Started is not true.

Stopped Returns true if Stop has been called. If Stopped is true, then the elapsed time will no longer increase. If Stopped is false and Started is true, then the timer is still running.

Ready Returns true if the timer has finished the synchronization required to get the timing result from the device.

GetElapsedTime Returns the amount of time that has elapsed between calling Start and Stop. If Stop was not called, then the amount of time between calling Start and GetElapsedTime is returned. GetElapsedTime can optionally take a device adapter tag or id to specify for which device to return the elapsed time.

Reset Restores the initial state of the Timer. All previous recorded time is erased. Reset optionally takes a device adapter tag or id that specifies on which device to time and synchronize.

GetDevice Returns the id of the device adapter for which this timer is synchronized. If the device adapter has the same id as vtkm: :cont: :DeviceAdapterTagAny (the default), then the timer will synchronize on all devices. 


\section{IMPLICIT FUNCTIONS}

VTK-m's implicit functions are objects that are constructed with values representing $3 \mathrm{D}$ spatial coordinates that often describe a shape. Each implicit function is typically defined by the surface formed where the value of the function is equal to 0. All vtkm: :ImplicitFunction s implement Value and Gradient methods that describe the orientation of a provided point with respect to the vtkm: :ImplicitFunction's shape.

Value The Value method for a vtkm::ImplicitFunction takes a vtkm::Vec3f and returns a vtkm::FloatDefault representing the orientation of the point with respect to the vtkm: :ImplicitFunction's shape. Negative scalar values represent vector points inside of the vtkm: :ImplicitFunction's shape. Positive scalar values represent vector points outside the vtkm: ImplicitFunction's shape. Zero values represent vector points that lie on the surface of the vtkm: : ImplicitFunction

Gradient The Gradient method for a vtkm::ImplicitFunction takes a vtkm: :Vec3f and returns a vtkm: :Vec3f representing the pointing direction from the vtkm: : ImplicitFunction's shape. Gradient calculations are more object shape specific. It is advised to look at the individual shape implementations for specific implicit functions.

Implicit functions are useful when trying to clip regions from a dataset. For example, it is possible to use vtkm: :filter::ClipWithImplicitFunction to remove a region in a provided dataset according to the shape of an implicit function. See Section9.1.4 for more information on clipping with implicit functions.

\subsection{Provided Implicit Functions}

VTK-m has implementations of various implicit functions provided by the following subclasses.

\subsubsection{Plane}

vtkm: :Plane defines an infinite plane. The plane is defined by a pair of vtkm::Vec3f values that represent the origin, which is any point on the plane, and a normal, which is a vector that is tangent to the plane. These are set with the SetOrigin and SetNormal methods, respectively. Planes extend infinitely from the origin point in the direction perpendicular form the Normal. An example vtkm: :Plane is shown in Figure 14.1.

\subsubsection{Sphere}

vtkm: : Sphere defines a sphere. The Sphere is defined by a center location and a radius, which are set with the SetCenter and SetRadius methods, respectively. An example vtkm: :Sphere is shown in Figure 14.2. 


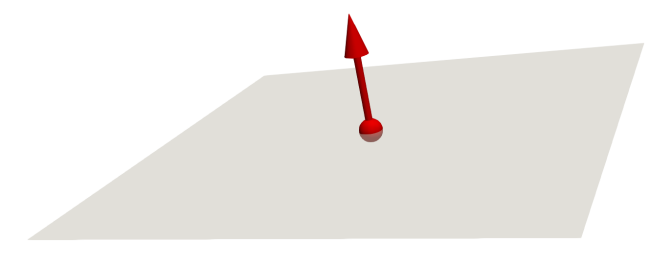

Figure 14.1: Visual Representation of an Implicit Plane. The red dot and arrow represent the origin and normal of the plane, respectively. For demonstrative purposes the plane as shown with limited area, but in actuality the plane extends infinitely.

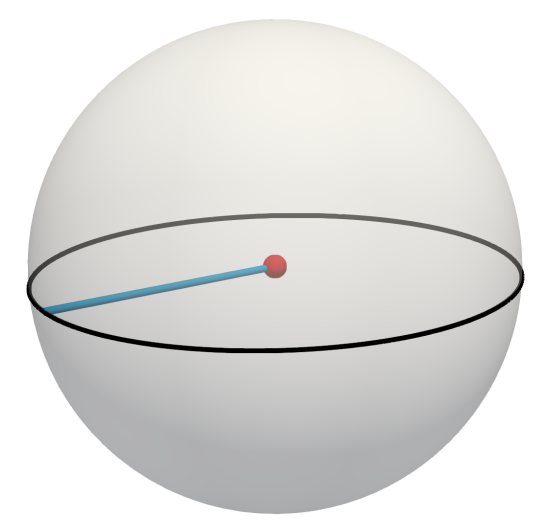

Figure 14.2: Visual Representation of an Implicit Sphere. The red dot represents the center of the sphere. The radius is the length of any line (like the blue one shown here) that extends from the center in any direction to the surface.

\subsubsection{Cylinder}

vtkm: : Cylinder defines a cylinder that extends infinitely along its axis. The cylinder is defined with a center point, a direction of the center axis, and a radius, which are set with SetCenter, SetAxis, and SetRadius, respectively. An example vtkm: : Cylinder is shown in Figure 14.3 with set origin, radius, and axis values.

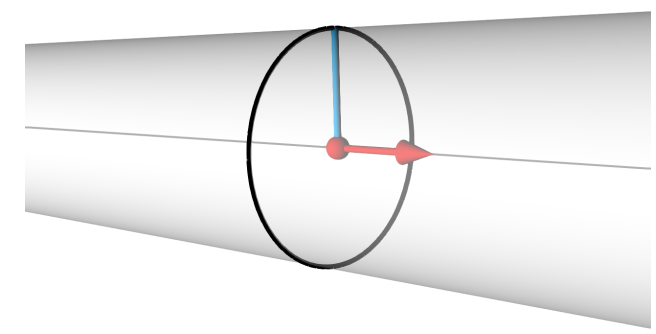

Figure 14.3: Visual Representation of an Implicit Cylinder. The red dot represents the center value, and the red arrow represents the vector that points in the direction of the axis. The radius is the length of any line (like the blue one shown here) that extends perpendicular from the axis to the surface. 


\subsubsection{Box}

vtkm: : Box defines an axis-aligned box. The box is defined with a pair of vtkm: :Vec3f values that represent the minimum point coordinates and maximum point coordinates, which are set with SetMinPoint and SetMaxPoint, respectively. The Box is the shape enclosed by intersecting axis-parallel lines drawn from each point. Alternately, the Box can be specified with a vtkm: :Bounds object using the SetBounds method. An example vtkm::Box is shown in Figure 14.4.

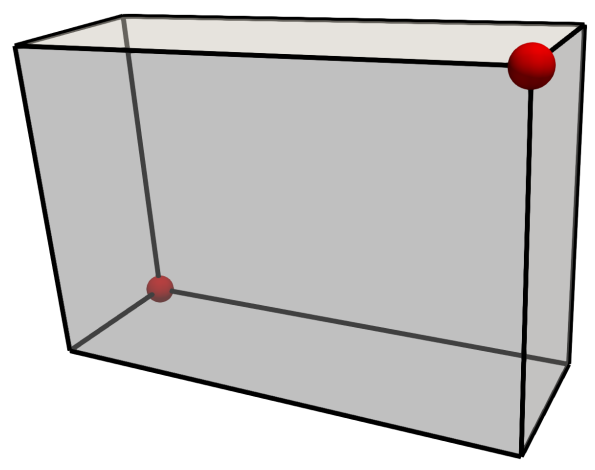

Figure 14.4: Visual Representation of an Implicit Box. The red dots represent the minimum and maximum points.

\subsubsection{Frustum}

vtkm: :Frustum defines a hexahedral region with potentially oblique faces. A Frustum is typically used to define the tapered region of space visible in a perspective camera projection. The frustum is defined by the 6 planes that make up its 6 faces. Each plane is defined by a point and a normal vector, which are set with SetPlane and SetNormal, respectively. Parameters for all 6 planes can be set at once using the SetPlanes and SetNormals methods. Alternately, the Frustum can be defined by the 8 points at the vertices of the enclosing hexahedron using the CreateFromPoints method. The points given to CreateFromPoints must be in hex-cell order where the first four points are assumed to be a plane, and the last four points are assumed to be a plane. An example vtkm: :Frustum is shown in Figure 14.5.

\subsection{General Implicit Functions}

It is often the case when creating code that uses an implicit function that you do not know which implicit function will be desired. For example, the vtkm: filter: ClipWithImplicitFunction filter can be used with any of the implicit functions described here (Plane, Sphere, etc.).

To handle conditions where you want to support multiple implicit functions simultaneously, VTK-m provides vtkm: ImplicitFunctionGeneral. Any of the implicit functions described in this chapter can be copied to a ImplicitFunctionGeneral, which will behave like the specified function. The following example shows shows passing a vtkm: :Sphere to ClipWithImplicitFunction, which internally uses ImplicitFunctionGeneral to manage the implicit function types.

Example 14.1: Passing an implicit function to a filter.

1 // Parameters needed for implicit function 


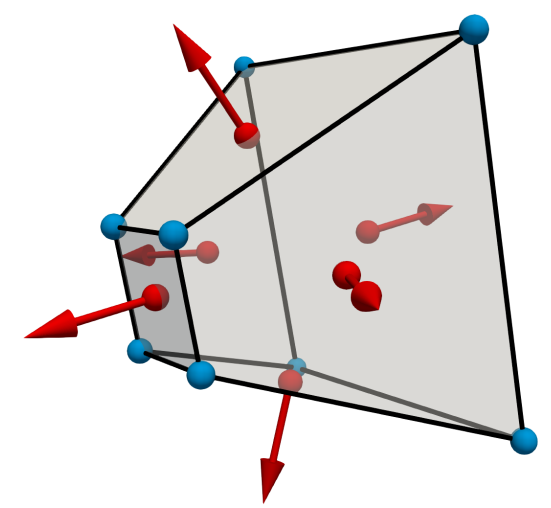

Figure 14.5: Visual Representation of an Implicit Frustum. The red dots and arrows represent the points and normals defining each enclosing plane. The blue dots represent the 8 vertices, which can also be used to define the frustum.

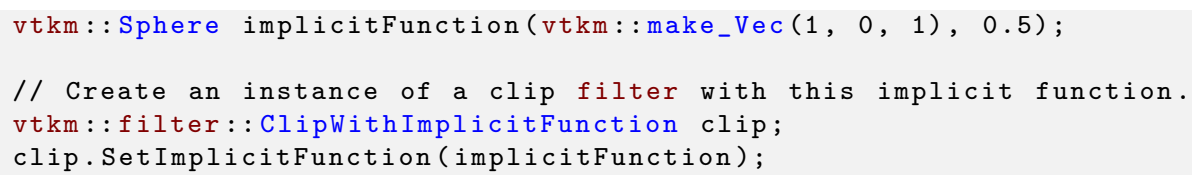




\section{Part III}

\section{Developing Algorithms}





\section{GENERAL APPROACH}

VTK-m is designed to provide a pervasive parallelism throughout all its visualization algorithms, meaning that the algorithm is designed to operate with independent concurrency at the finest possible level throughout. VTK-m provides this pervasive parallelism by providing a programming construct called a worklet, which operates on a very fine granularity of data. The worklets are designed as serial components, and VTK-m handles whatever layers of concurrency are necessary, thereby removing the onus from the visualization algorithm developer. Worklet operation is then wrapped into filters, which provide a simplified interface to end users.

A worklet is essentially a functor or kernel designed to operate on a small element of data. (The name "worklet" means a small amount of the work. We mean small in this sense to be the amount of data, not necessarily the amount of instructions performed.) The worklet is constrained to contain a serial and stateless function. These constraints form three critical purposes. First, the constraints on the worklets allow VTK-m to schedule worklet invocations on a great many independent concurrent threads and thereby making the algorithm pervasively parallel. Second, the constraints allow VTK-m to provide thread safety. By controlling the memory access the toolkit can insure that no worklet will have any memory collisions, false sharing, or other parallel programming pitfalls. Third, the constraints encourage good programming practices. The worklet model provides a natural approach to visualization algorithm design that also has good general performance characteristics.

VTK-m allows developers to design algorithms that are run on massive amounts of threads. However, VTK-m also allows developers to interface to applications, define data, and invoke algorithms that they have written or are provided otherwise. These two modes represent significantly different operations on the data. The operating code of an algorithm in a worklet is constrained to access only a small portion of data that is provided by the framework. Conversely, code that is building the data structures needs to manage the data in its entirety, but has little reason to perform computations on any particular element.

Consequently, VTK-m is divided into two environments that handle each of these use cases. Each environment has its own API, and direct interaction between the environments is disallowed. The environments are as follows.

Execution Environment This is the environment in which the computational portion of algorithms are executed. The API for this environment provides work for one element with convenient access to information such as connectivity and neighborhood as needed by typical visualization algorithms. Code for the execution environment is designed to always execute on a very large number of threads.

Control Environment This is the environment that is used to interface with applications, interface with I/O devices, and schedule parallel execution of the algorithms. The associated API is designed for users that want to use VTK-m to analyze their data using provided or supplied filters. Code for the control environment is designed to run on a single thread (or one single thread per process in an MPI job).

These dual programming environments are partially a convenience to isolate the application from the execution of the worklets and are partially a necessity to support GPU languages with host and device environments. The 
control and execution environments are logically equivalent to the host and device environments, respectively, in CUDA and other associated GPU languages.

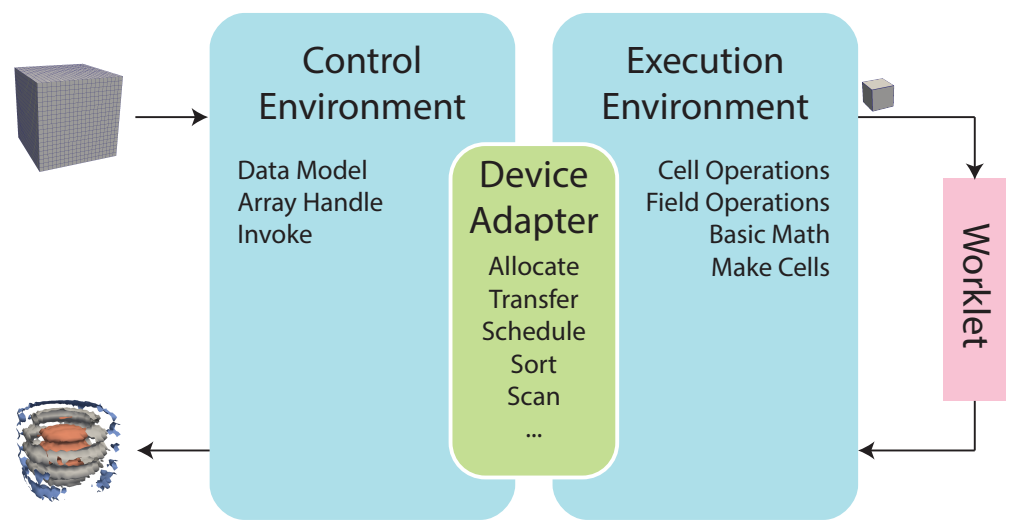

Figure 15.1: Diagram of the VTK-m framework.

Figure 15.1 displays the relationship between the control and execution environment. The typical workflow when using VTK-m is that first the control thread establishes a data set in the control environment and then invokes a parallel operation on the data using a filter. From there the data is logically divided into its constituent elements, which are sent to independent invocations of a worklet. The worklet invocations, being independent, are run on as many concurrent threads as are supported by the device. On completion the results of the worklet invocations are collected to a single data structure and a handle is returned back to the control environment.

0 Did you know?

3 Are you only planning to use filters in VTK-m that already exist? If so, then everything you work with will be in the control environment. The execution environment is only used when implementing algorithms for filters.

\subsection{Package Structure}

VTK-m is organized in a hierarchy of nested packages. VTK-m places definitions in namespaces that correspond to the package (with the exception that one package may specialize a template defined in a different namespace).

The base package is named vtkm. All classes within VTK-m are placed either directly in the vtkm package or in a package beneath it. This helps prevent name collisions between VTK-m and any other library.

As described at the beginning of this chapter, the VTK-m API is divided into two distinct environments: the control environment and the execution environment. The API for these two environments are located in the vtkm: : cont and vtkm: :exec packages, respectively. Items located in the base vtkm namespace are available in both environments.

Although it is conventional to spell out names in identifiers, ${ }^{1}$ there is an exception to abbreviate control and execution to cont and exec, respectively. This is because it is also part of the coding convention to declare the entire namespace when using an identifier that is part of the corresponding package. The shorter names

\footnotetext{
${ }^{1}$ VTK-m coding conventions are outlined in the doc/CodingConventions.md file in the VTK-m source code and at https://gitlab. kitware.com/vtk/vtk-m/blob/master/docs/CodingConventions.md
} 
make the identifiers easier to read, faster to type, and more feasible to pack lines in 80 column displays. These abbreviations are also used instead of more common abbreviations (e.g. ctrl for control) because, as part of actual English words, they are easier to type.

Further functionality in VTK-m is built on top of the base vtkm, vtkm: cont, and vtkm::exec packages. Support classes for building worklets, introduced in Chapter 17, are contained in the vtkm: : worklet package. Other facilities in VTK-m are provided in their own packages such as vtkm::io, vtkm::filter, and vtkm: :rendering. These packages are described in Part II.

VTK-m contains code that uses specialized compiler features, such as those with CUDA, or libraries, such as Intel Threading Building Blocks, that will not be available on all machines. Code for these features are encapsulated in their own packages under the vtkm::cont namespace: vtkm::cont::cuda and vtkm::cont::tbb .

VTK-m contains OpenGL interoperability that allows data generated with VTK-m to be efficiently transferred to OpenGL objects. This feature is encapsulated in the vtkm: :opengl package.

Figure 15.2 provides a diagram of the VTK-m package hierarchy.

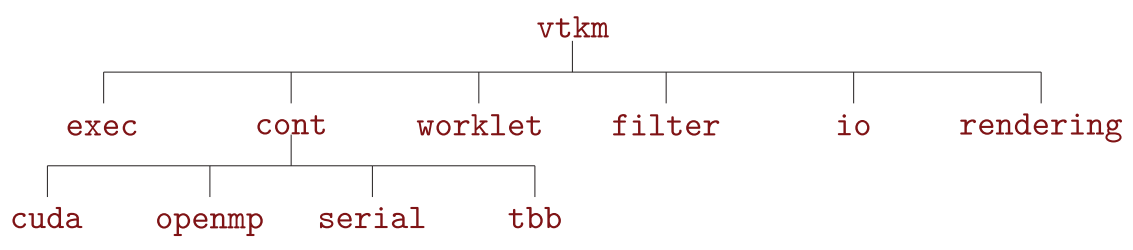

Figure 15.2: VTK-m package hierarchy.

By convention all classes will be defined in a file with the same name as the class name (with a. $\mathrm{h}$ extension) located in a directory corresponding to the package name. For example, the vtkm: : cont: :DataSet class is found in the vtkm/cont/DataSet.h header. There are, however, exceptions to this rule. Some smaller classes and types are grouped together for convenience. These exceptions will be noted as necessary.

Within each namespace there may also be internal and detail sub-namespaces. The internal namespaces contain features that are used internally and may change without notice. The detail namespaces contain features that are used by a particular class but must be declared outside of that class. Users should generally ignore classes in these namespaces.

\subsection{Function and Method Environment Modifiers}

Any function or method defined by VTK-m must come with a modifier that determines in which environments the function may be run. These modifiers are C macros that VTK-m uses to instruct the compiler for which architectures to compile each method. Most user code outside of VTK-m need not use these macros with the important exception of any classes passed to VTK-m. This occurs when defining new worklets, array storage, and device adapters.

VTK-m provides three modifier macros, VTKM_CONT, VTKM_EXEC, and VTKM_EXEC_CONT, which are used to declare functions and methods that can run in the control environment, execution environment, and both environments, respectively. These macros get defined by including just about any VTK-m header file, but including vtkm/Types.h will ensure they are defined.

The modifier macro is placed after the template declaration, if there is one, and before the return type for the function. Here is a simple example of a function that will square a value. Since most types you would use this function on have operators in both the control and execution environments, the function is declared for both places. 
Example 15.1: Usage of an environment modifier macro on a function.

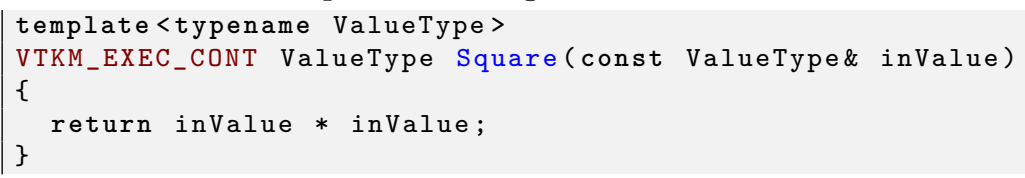

The primary function of the modifier macros is to inject compiler-specific keywords that specify what architecture to compile code for. For example, when compiling with CUDA, the control modifiers have __ host__- in them and execution modifiers have __device__ in them.

It is sometimes the case that a function declared as VTKM_EXEC_CONT has to call a method declared as VTKM_EXEC or VTKM_CONT. Generally functions should not call other functions with incompatible control/execution modifiers, but sometimes a generic VTKM_EXEC_CONT function calls another function determined by the template parameters, and the valid environments of this subfunction may be inconsistent. For cases like this, you can use the VTKM_SUPPRESS_EXEC_WARNINGS to tell the compiler to ignore the inconsistency when resolving the template. When applied to a templated function or method, VTKM_SUPPRESS_EXEC_WARNINGS is placed before the template keyword. When applied to a non-templated method in a templated class, VTKM_SUPPRESS_EXEC_WARNINGS is placed before the environment modifier macro.

Example 15.2: Suppressing warnings about functions from mixed environments.

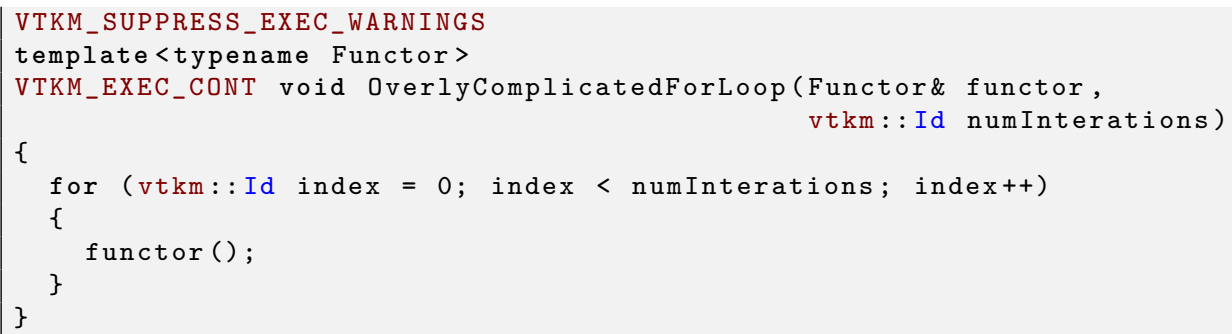




\section{BASIC ARRAY HANDLES}

Chapter 7 describes the basic data sets used by VTK-m. This chapter dives deeper into how VTK-m represents data. Ultimately, data structures like vtkm: : cont: : DataSet can be broken down into arrays of numbers. Arrays in VTK-m are managed by a unit called an array handle.

An array handle, which is implemented with the vtkm: :cont: :ArrayHandle class, manages an array of data that can be accessed or manipulated by VTK-m algorithms. It is typical to construct an array handle in the control environment to pass data to an algorithm running in the execution environment. It is also typical for an algorithm running in the execution environment to populate an array handle, which can then be read back in the control environment. It is also possible for an array handle to manage data created by one VTK-m algorithm and passed to another, remaining in the execution environment the whole time and never copied to the control environment.

\section{(0) Did you know?}

The array handle may have multiple of the array, one for the control environment and one for each device.

Kowever, depending on the device and how the array is being used, the array handle will only have one copy when possible. Copies between the environments are implicit and lazy. They are copied only when an operation needs data in an environment where the data are not.

vtkm: :cont: :ArrayHandle behaves like a shared smart pointer in that when the $\mathrm{C}++$ object is copied, each copy holds a reference to the same array. These copies are reference counted so that when all copies of the vtkm: : cont: : ArrayHandle are destroyed, any allocated memory is released.

An ArrayHandle defines the following methods. Note, however, that the brief overview of this chapter will not cover the use of most of these methods. Further descriptions are given in later chapters that explore even further the features of ArrayHandles.

GetNumberOfValues Returns the number of entries in the array.

Allocate Resizes the array to include the number of entries given. Any previously stored data might be discarded.

ReleaseResourcesExecution If the ArrayHandle is holding any data on a device (such as a GPU), that memory is released to be used elsewhere. No data is lost from this call. Any data on the released resources is copied to the control environment (the local CPU) before the memory is released.

ReleaseResources Releases all memory managed by this ArrayHandle. Any data in this memory is lost. 
SyncControlArray Makes sure any data in the execution environment is also available in the control environment. This method is useful when timing parallel algorithms and you want to include the time to transfer data between parallel devices and their hosts.

ReadPortal Returns an array portal that can be used to access the data in the array handle in the control environment. The data in the array portal can only be read. Array portals are described in Section 27.1.

WritePortal Returns an array portal that can be used to access the data in the array handle in the control environment. The data in the array portal can be both read and written. Array portals are described in Section 27.1.

PrepareForInput Readies the data as input to a parallel algorithm. See Section 27.4 for more details.

PrepareForOutput Readies the data as output to a parallel algorithm. See Section 27.4 for more details.

PrepareForInPlace Readies the data as input and output to a parallel algorithm. See Section 27.4 for more details.

DeepCopyFrom Given an ArrayHandle of the same type, deep copies the data from the provided array to this array. The array will be resized as necessary.

IsOnHost Returns true if the data are available on the host memory (that is, available in the control environment).

IsOnDevice Returns true if the data are available on the specific device.

\subsection{Creating Array Handles}

vtkm: :cont: :ArrayHandle is templated on the type of values being stored in the array. There are multiple ways to create and populate an array handle. The default vtkm: :cont: : ArrayHandle constructor will create an empty array with nothing allocated in either the control or execution environment. This is convenient for creating arrays used as the output for algorithms.

Example 16.1: Creating an ArrayHandle for output data.

1 | vtkm::cont: :ArrayHandle<vtkm::Float32> outputArray;

Chapter 27 describes in detail how to allocate memory and access data in an ArrayHandle. However, you can use the vtkm: : cont: :make_ArrayHandle function for a simplified way to create an ArrayHandle with data.

make_ArrayHandle has many forms. An easy form to use takes an initializer list and creates a basic ArrayHandle with it. This allows you to create short ArrayHandles from literals.

Example 16.2: Creating an ArrayHandle from initially specified values.

1 auto fibonacciArray = vtkm: : cont::make_ArrayHandle $(\{0,1,1,2,3,5,8,13\})$;

One problem with creating an array from an initializer list like this is that it can be tricky to specify the exact value type of the ArrayHandle. The value type of the ArrayHandle will be the same types as the literals in the initializer list, but that might not match the type you actually need. This is particularly true for types like vtkm: :Id and vtkm: :FloatDefault, which can change depending on compile options. To specify the exact value type to use, give that type as a template argument to the vtkm: : cont: :make_ArrayHandle function.

Example 16.3: Creating a typed ArrayHandle from initially specified values.

vtkm: : cont : : ArrayHandle<vtkm: :FloatDefault> inputArray =

vtkm: : cont : :make_ArrayHandle<vtkm::FloatDefault>(\{ 1.4142f, 2.7183f, 3.1416f $\})$; 
Constructing an ArrayHandle that points to a provided $\mathrm{C}$ array is also straightforward. To do this, call make_ArrayHandle with the array pointer, the number of values in the $\mathrm{C}$ array, and a vtkm: :CopyFlag. This last argument can be either CopyFlag: : On to copy the array or CopyFlag: :Off to share the provided buffer.

Example 16.4: Creating an ArrayHandle that points to a provided $\mathrm{C}$ array.

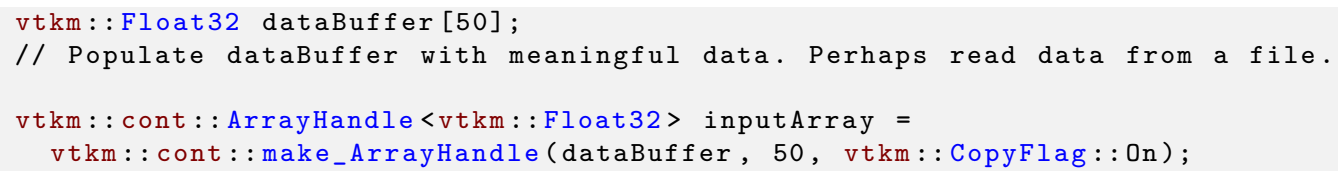

Likewise, you can use make_ArrayHandle to transfer data from a std: : vector to an ArrayHandle. This form of make_ArrayHandle takes the std: : vector as the first argument and a vtkm: :CopyFlag as the second argument.

Example 16.5: Creating an ArrayHandle that points to a provided std: : vector.

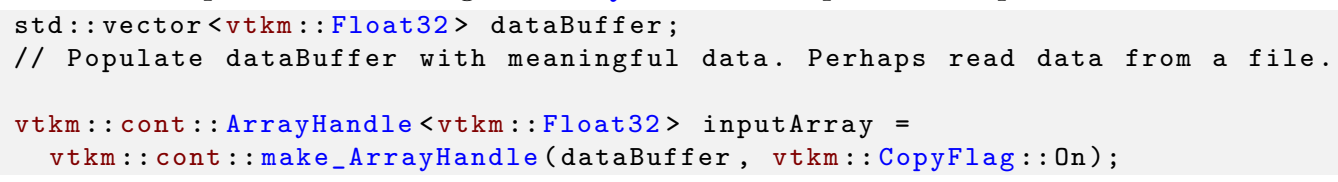

As hinted at earlier, it is possible to send CopyFlag: :Off to make_ArrayHandle to wrap an ArrayHandle around an existing C array or std: : vector. Doing so allows you to send the data to the ArrayHandle without copying it. It also provides a mechanism for VTK-m to write directly into your array. However, be aware that if you change or delete the data provided, the internal state of ArrayHandle becomes invalid and undefined behavior can ensue. A common manifestation of this error happens when a std: : vector goes out of scope. This subtle interaction will cause the vtkm: cont: :ArrayHandle to point to an unallocated portion of the memory heap. The following example provides an erroneous use of ArrayHandle and some ways to fix it.

Example 16.6: Invalidating an ArrayHandle by letting the source std: : vector leave scope.

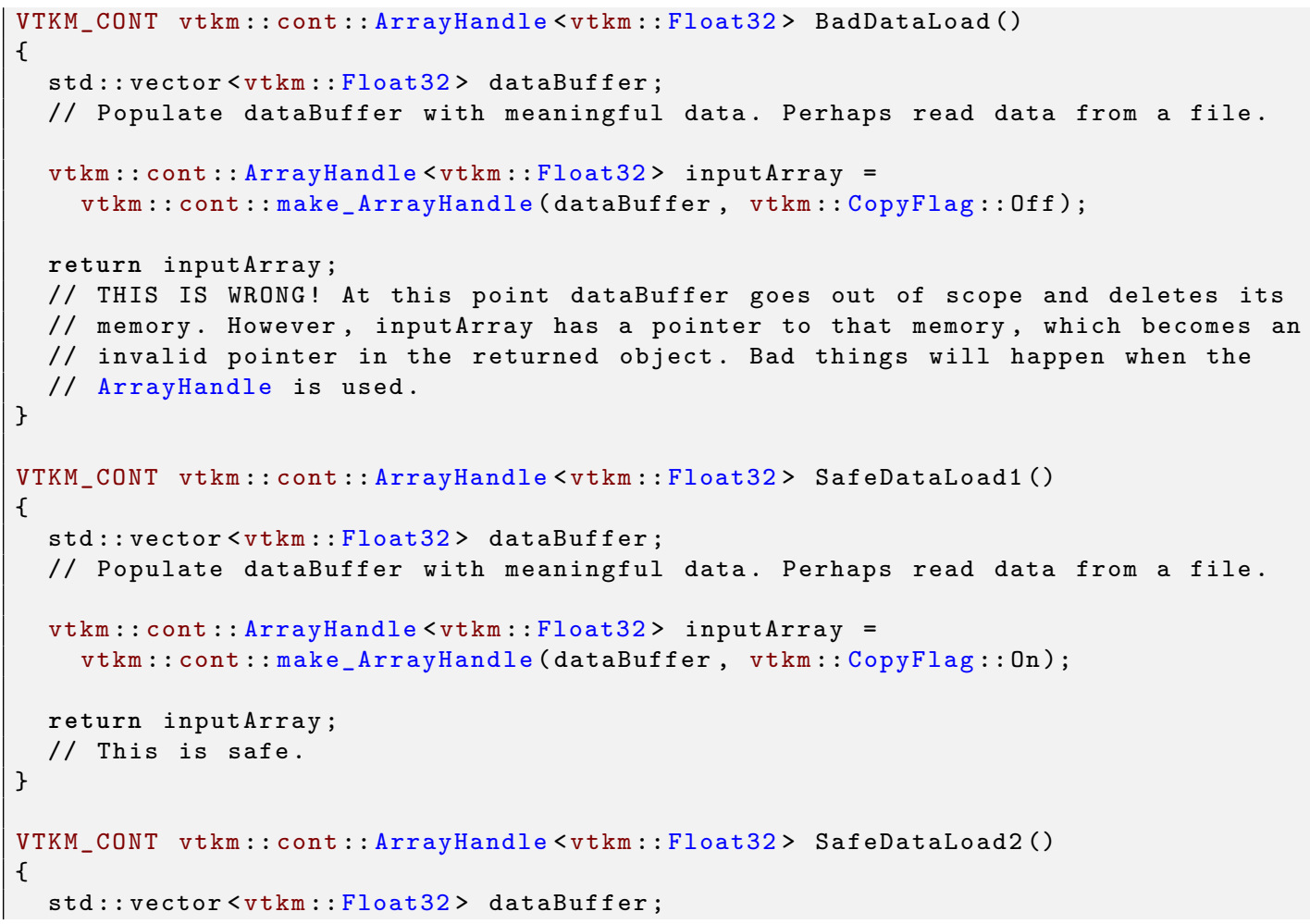




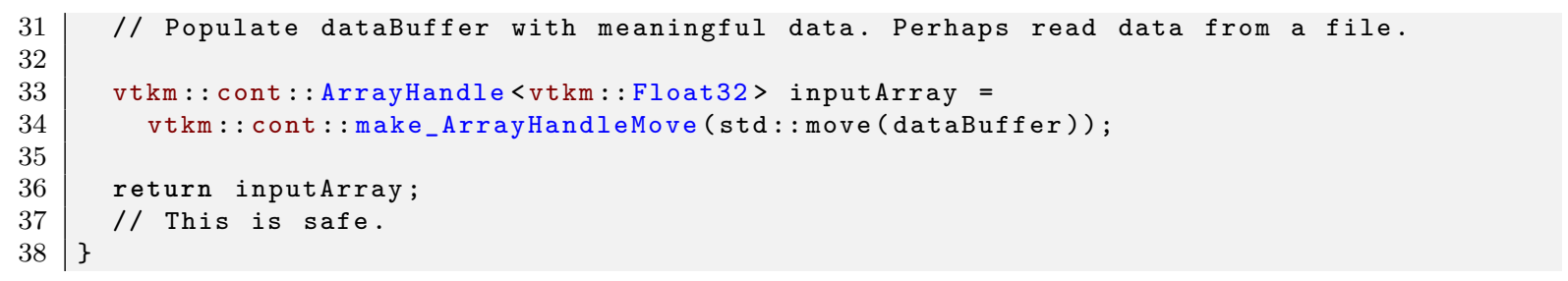

An easy way around the problem of having an ArrayHandle's data going out of scope is to copy the data into the ArrayHandle. Simply make the vtkm: : CopyFlag argument be On to copy the data. This solution is shown on line 22 of Example 16.6.

What if you have a std: : vector that you want to pass to an ArrayHandle and then want to only use in the ArrayHandle? In this case, it is wasteful to have to copy the data, but you also do not want to be responsible for keeping the std: :vector in scope. To handle this, there is a special vtkm: : cont: :make_ArrayHandleMove that will move the memory out of the std: : vector and into the ArrayHandle. make_ArrayHandleMove takes an "rvalue" version of a std: :vector. To create an "rvalue", use the std: :move function provided by C++. Once make_ArrayHandleMove is called, the provided std: : vector becomes invalid and any further access to it is undefined. This solution is shown on line 34 of Example 16.6.

\subsection{Deep Array Copies}

As stated previously, an ArrayHandle object behaves as a smart pointer that copies references to the data without copying the data itself. This is clearly faster and more memory efficient than making copies of the data itself and usually the behavior desired. However, it is sometimes the case that you need to make a separate copy of the data.

The easiest way to copy ArrayHandles is to use the ArrayHandle: :DeepCopyFrom method.

Example 16.7: Deep copy ArrayHandles of the same type.

1 destarray. DeepCopyFrom (srcArray);

However, the DeepCopyFrom method only works if the two ArrayHandles are the exact same type. To simplify copying the data between ArrayHandles of different types, VTK-m comes with the vtkm::cont: :ArrayCopy convenience function defined in vtkm/cont/ArrayCopy.h. ArrayCopy takes the array to copy from (the source) as its first argument and the array to copy to (the destination) as its second argument. The destination array will be properly reallocated to the correct size.

Example 16.8: Using ArrayCopy.

1 | vtkm::cont::ArrayCopy(srcArray, destArray);

\subsection{The Hidden Second Template Parameter}

We have already seen that vtkm: : cont: : ArrayHandle is a templated class with the template parameter indicating the type of values stored in the array. However, ArrayHandle has a second hidden parameter that indicates the storage of the array. We have so far been able to ignore this second template parameter because VTK-m will assign a default storage for us that will store the data in a basic array.

Example 16.9: Declaration of the vtkm: : cont : : ArrayHandle templated class.

1 template<typename $T$, typename StorageTag = VTKM_DEFAULT_STORAGE_TAG>

2 class ArrayHandle; 
Changing the storage of an ArrayHandle lets us do many weird and wonderful things. We will explore these options in later chapters, but for now we can ignore this second storage template parameter. However, there are a couple of things to note concerning the storage.

First, if the compiler gives an error concerning your use of ArrayHandle, the compiler will report the ArrayHandle type with not one but two template parameters. A second template parameter of vtkm: :cont: :StorageTagBasic can be ignored.

Second, if you write a function, method, or class that is templated based on an ArrayHandle type, it is good practice to accept ArrayHandles with non-default storage types. There are two ways to do this. The first way is to template both the value type and the storage type.

Example 16.10: Templating a function on an ArrayHandle's parameters

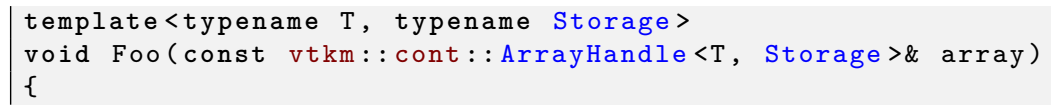

The second way is to template the whole array type rather than the sub types. If you create a template where you expect one of the parameters to be an ArrayHandle, you should use the VTKM_IS_ARRAY_HANDLE macro to verify that the type is indeed an ArrayHandle.

Example 16.11: A template parameter that should be an ArrayHandle.

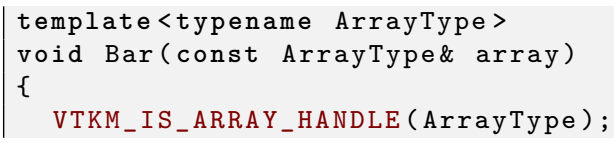

\subsection{Mutability}

One subtle feature of ArrayHandle is that the class is, in principle, a pointer to an array pointer. This means that the data in an ArrayHandle is always mutable even if the class is declared const. You can change the contents of "constant" arrays via methods like WritePortal and PrepareForOutput. It is even possible to change the underlying array allocation with methods like Allocate and ReleaseResources. The upshot is that you can (sometimes) pass output arrays as constant ArrayHandle references.

So if a constant ArrayHandle can have its contents modified, what is the difference between a constant reference and a non-constant reference? The difference is that the constant reference can change the array's content, but not the array itself. Basically, this means that you cannot perform shallow copies into constant ArrayHandles. This can be a pretty big limitation, and many of VTK-m's internal device algorithms still require non-constant references for outputs. 



\section{SIMPLE WORKLETS}

The simplest way to implement an algorithm in VTK-m is to create a worklet. A worklet is fundamentally a functor that operates on an element of data. Thus, it is a class or struct that has an overloaded parenthesis operator (which must be declared const for thread safety). However, worklets are also embedded with a significant amount of metadata on how the data should be managed and how the execution should be structured.

Example 17.1: A simple worklet.

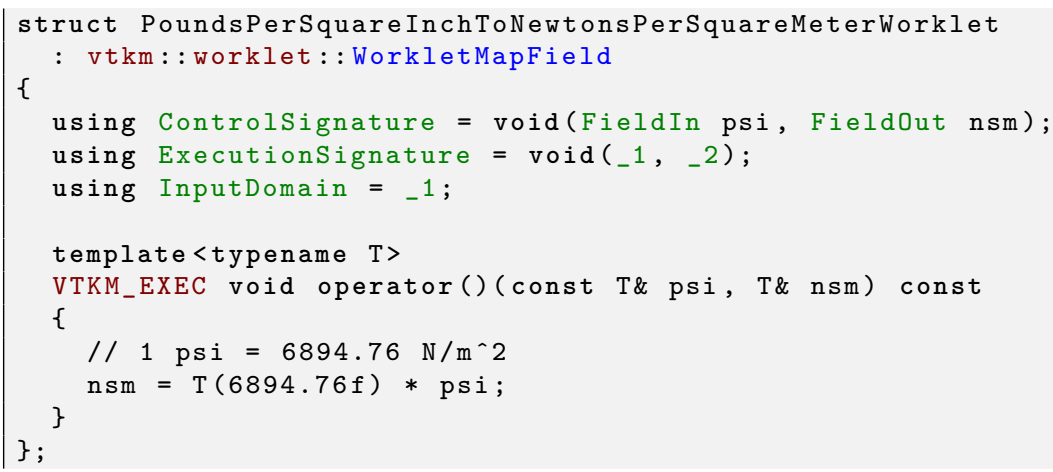

As can be seen in Example 17.1, a worklet is created by implementing a class or struct with the following features.

1. The class must publicly inherit from a base worklet class that specifies the type of operation being performed (line 1).

2. The class must contain a functional type named ControlSignature (line 4), which specifies what arguments are expected when invoking the class in the control environment.

3. The class must contain a functional type named ExecutionSignature (line 5), which specifies how the data gets passed from the arguments in the control environment to the worklet running in the execution environment.

4. The class specifies an InputDomain (line 6), which identifies which input parameter defines the input domain of the data.

5. The class must contain an implementation of the parenthesis operator, which is the method that is executed in the execution environment (lines 8-13). The parenthesis operator must be declared const. 


\subsection{Control Signature}

The control signature of a worklet is a functional type named Controlsignature. The function prototype matches what data are provided when the worklet is invoked (as described in Section 17.5).

Example 17.2: A ControlSignature.

1 using Controlsignature = void(Fieldin psi, Fieldout nsm);

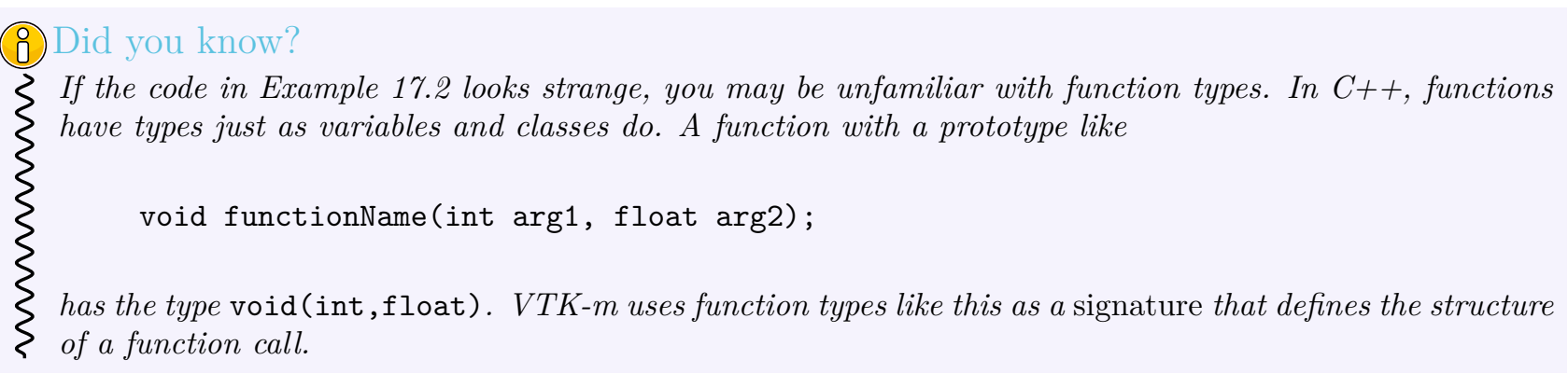

The return type of the function prototype is always void. The parameters of the function prototype are tags that identify the type of data that is expected to be passed to invoke. Controlsignature tags are defined by the worklet type and the various tags are documented more fully in Chapter 21. In the case of Example 17.2, the two tags FieldIn and Fieldout represent input and output data, respectively.

By convention, ControlSignature tag names start with the base concept (e.g. Field or Topology) followed by the domain (e.g. Point or Cell) followed by In or Out. For example, FieldPointIn would specify values for a field on the points of a mesh that are used as input (read only). Although they should be there in most cases, some tag names might leave out the domain or in/out parts if they are obvious or ambiguous.

\subsection{Execution Signature}

Like the control signature, the execution signature of a worklet is a functional type named ExecutionSignature. The function prototype must match the parenthesis operator (described in Section 17.4) in terms of arity and argument semantics.

\section{Example 17.3: An ExecutionSignature. \\ 1 using ExecutionSignature = void (_1, _2);}

The arguments of the ExecutionSignature's function prototype are tags that define where the data come from. The most common tags are an underscore followed by a number, such as $\_1, \ldots 2$, etc. These numbers refer back to the corresponding argument in the ControlSignature. For example, _1 means data from the first control signature argument, _2 means data from the second control signature argument, etc.

Unlike the control signature, the execution signature optionally can declare a return type if the parenthesis operator returns a value. If this is the case, the return value should be one of the numeric tags (i.e. $-1, \ldots 2$, etc.) to refer to one of the data structures of the control signature. If the parenthesis operator does not return a value, then ExecutionSignature should declare the return type as void.

In addition to the numeric tags, there are other execution signature tags to represent other types of data. For example, the WorkIndex tag identifies the instance of the worklet invocation. Each call to the worklet function 
will have a unique WorkIndex. Other such tags exist and are described in the following section on worklet types where appropriate.

\subsection{Input Domain}

All worklets represent data parallel operations that are executed over independent elements in some domain. The type of domain is inherent from the worklet type, but the size of the domain is dependent on the data being operated on.

A worklet identifies the argument specifying the domain with a type alias named InputDomain. The InputDomain must be aliased to one of the execution signature numeric tags (i.e. _1, _2, etc.). By default, the InputDomain points to the first argument, but a worklet can override that to point to any argument.

Example 17.4: An InputDomain declaration.

1 using InputDomain = 1 ;

Different types of worklets can have different types of domain. For example a simple field map worklet has a FieldIn argument as its input domain, and the size of the input domain is taken from the size of the associated field array. Likewise, a worklet that maps topology has a CellSetIn argument as its input domain, and the size of the input domain is taken from the cell set.

Specifying the InputDomain is optional. If it is not specified, the first argument is assumed to be the input domain.

\subsection{Worklet Operator}

A worklet is fundamentally a functor that operates on an element of data. Thus, the algorithm that the worklet represents is contained in or called from the parenthesis operator method.

Example 17.5: An overloaded parenthesis operator of a worklet.

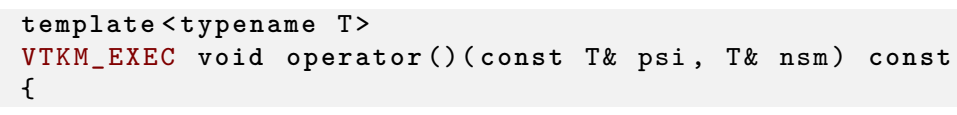

There are some constraints on the parenthesis operator. First, it must have the same arity as the ExecutionSignature, and the types of the parameters and return must be compatible. Second, because it runs in the execution environment, it must be declared with the VTKM_EXEC (or VTKM_EXEC_CONT) modifier. Third, the method must be declared const to help preserve thread safety.

\subsection{Invoking a Worklet}

Previously in this chapter we discussed creating a simple worklet. In this section we describe how to run the worklet in parallel.

A worklet is run using the vtkm: : cont: : Invoker class.

Example 17.6: Invoking a worklet.

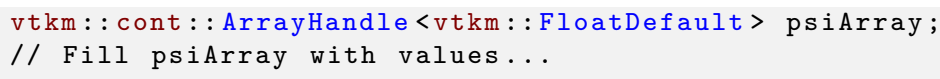




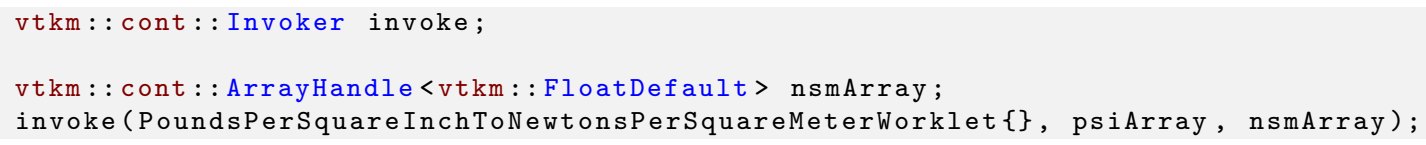

Using an Invoker is simple. First, an Invoker can be simply constructed with no arguments (line 4). Next, the Invoker is called as if it were a function (line 7).

The first argument to the invoke is always an instance of the worklet. The remaining arguments are data that are passed (indirectly) to the worklet. Each of these arguments (after the worklet) match a corresponding argument listed in the ControlSignature. So in the invocation on Example 17.6, line 7, the second and third arguments correspond the the two ControlSignature arguments given in Example 17.2. psiArray corresponds to the FieldIn argument and nmsArray corresponds to the FieldOut argument.

\subsection{Preview of More Complex Worklets}

This chapter demonstrates the creation of a worklet that performs a very simple math operation in parallel. However, we have just scratched the surface of the kinds of algorithms that can be expressed with VTK-m worklets. There are many more execution patterns and data handling constructs. The following example gives a preview of some of the more advanced features of worklets.

Example 17.7: A more complex worklet.

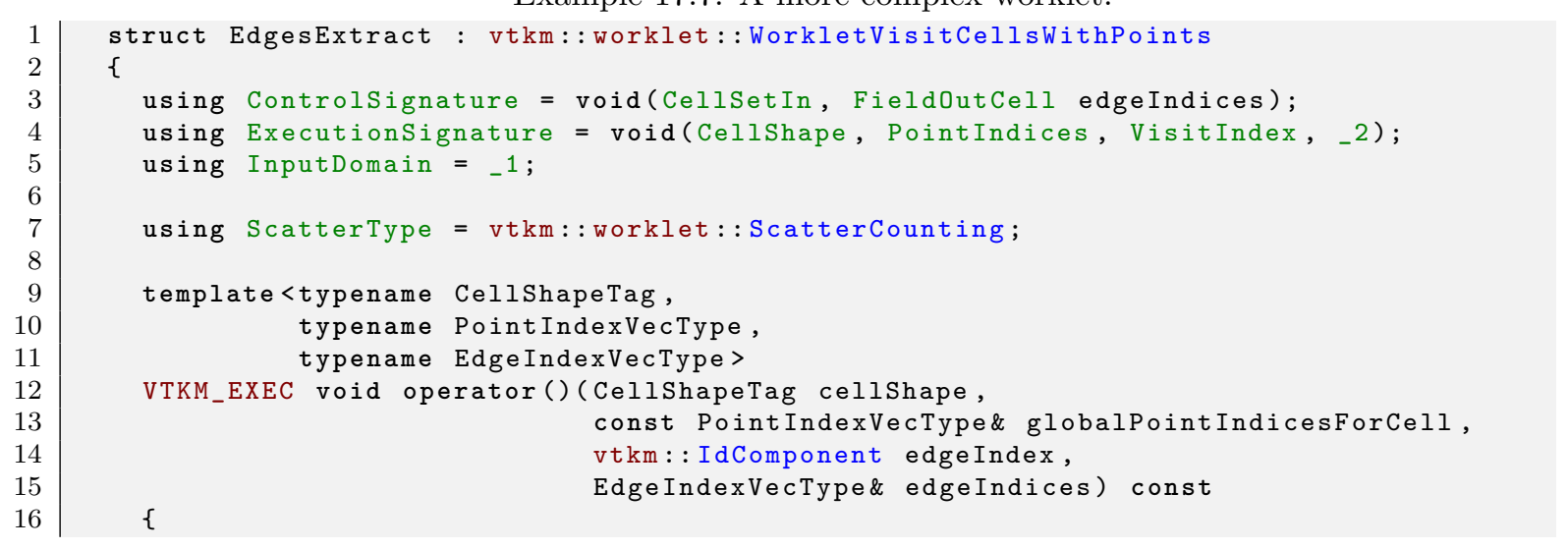

We will discuss the many features available in the worklet framework throughout Part IV. 


\section{BASIC FILTER IMPLEMENTATION}

Chapter 17 introduced the concept of a worklet and demonstrated how to create and run one to execute an algorithm on a device. Although worklets provide a powerful mechanism for designing heavily threaded visualization algorithms, invoking them requires quite a bit of knowledge of the workings of VTK-m. Instead, most users execute algorithms in VTK-m using filters. Thus, to expose algorithms implemented with worklets to general users, we need to implement a filter to encapsulate the worklets. In this chapter we will create a filter that encapsulates the worklet algorithm presented in Chapter 17, which converted the units of a pressure field from pounds per square inch (psi) to Newtons per square meter $\left(\mathrm{N} / \mathrm{m}^{2}\right)$.

Filters in VTK-m are implemented by deriving one of the filter base classes provided in vtkm: :filter . There are multiple base filter classes that we can choose from. These different classes are documented later in Chapter 22. For this example we will use the vtkm: :filter: :FilterField base class, which is used to derive one field from another field.

The following example shows the declaration of our pressure unit conversion filter. By convention, this declaration would be placed in a header file with a. h extension.

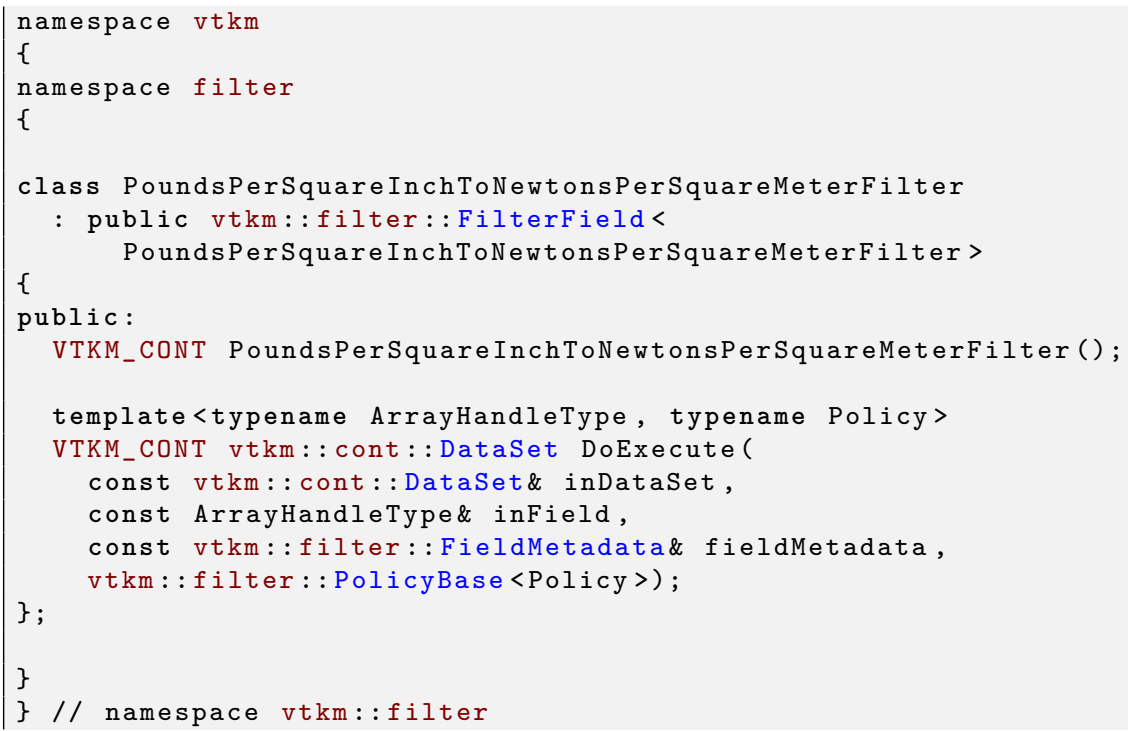

It should be noted that the FilterField superclass is actually a templated class that expects to be templated by the concrete subclass (in this case the PoundsPerSquareInchToNewtonsPerSquareMeterFilter class we are creating). This allows templated methods in the superclass call templated methods in the concrete subclass. 
This technique of templating a superclass based on the subclass is known as the Curiously Recurring Template Pattern (CRTP).

It is typical for a filter to have a constructor to set up its initial state. A filter will also implement a DoExecute method. Different filter types require different arguments for DoExecute, but the FilterField version has 4 arguments: the input DataSet, an ArrayHandle holding the data for the input field, metadata about the input field, and a policy used to pull other information from the input DataSet. (We will not need to use the policy in this example as the superclass will give us all the data we need. Policies are demonstrated in Chapter 22.)

Once the filter class is declared in the $h$ file, the implementation filter is by convention given in a separate .hxx file. Given the definition of our filter in Example 18.1, we will need to provide the implementation for the constructor and the DoExecute method. The constructor is quite simple. It initializes the name of the output field name, which is managed by the superclass.

Example 18.2: Constructor for a simple filter.

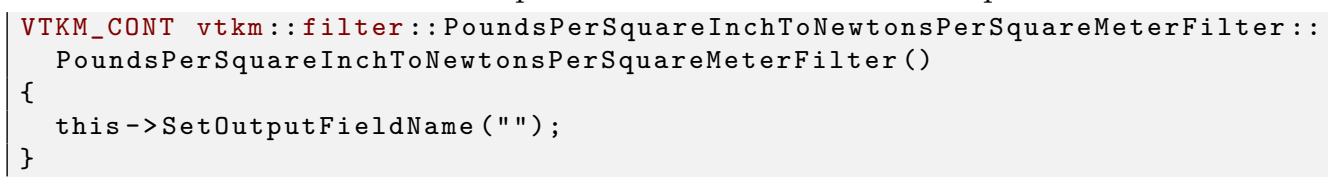

In this case, we are setting the output field name to the empty string. This is not to mean that the default name of the output field should be the empty string, which is not a good idea. Rather, as we will see later, we will use the empty string to flag an output name that should be derived from the input name.

The meat of the filter implementation is located in the DoExecute method.

Example 18.3: Implementation of DoExecute for a simple filter.

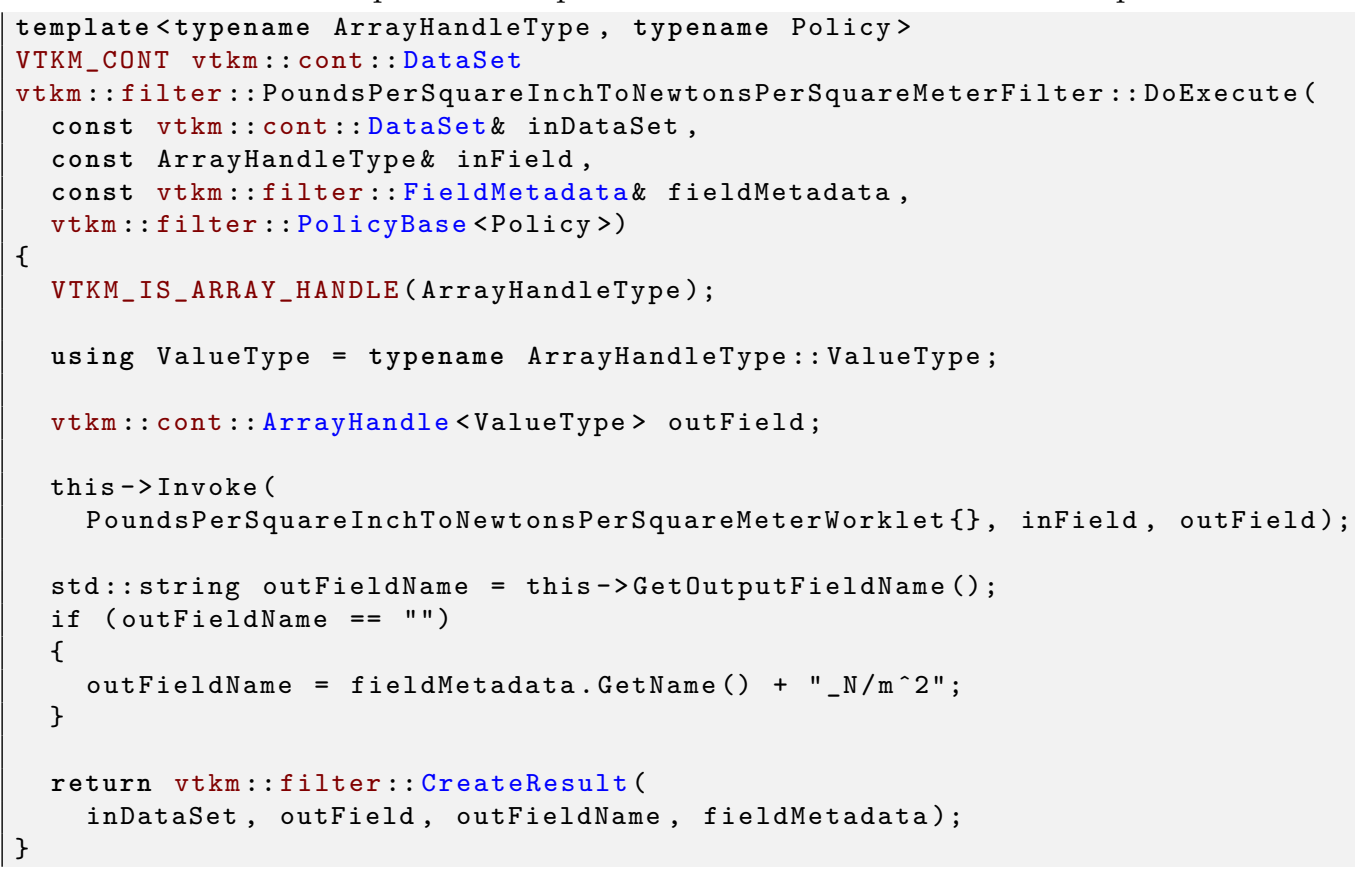

The second argument to DoExecute is a vtkm: :cont: :ArrayHandle that contains the data for the input field. This argument is templated because, as described in Chapter 16, ArrayHandles are templated classes that can change based on the types of values and how they are stored. The superclass has determined what these types are and has passed a concrete version of the ArrayHandle to DoExecute. It is good practice to check that the type 
we get is in fact an ArrayHandle using the VTKM_IS_ARRAY_HANDLE macro (demonstrated in line 9 of Example 18.3).

The first operation our filter does is to create a new ArrayHandle to store the new computed field (line 13). The type of the computed output field is typically derived from the type of the input array.

Once the filter has both the input and output ArrayHandles, it is ready to invoke the worklet to compute the output. For convenience, all filter superclasses provide an Invoke member that is a vtkm: : cont: : Invoker class. Thus, worklets can be invoked within a filter by calling this->Invoke as if it were a method. This is shown starting on line 15. Recall that the worklet created in Chapter 17 takes two parameters: an input array and an output array, which are shown in this invocation.

With the output data created, the filter has to build the output structure to return. All implementations of DoExecute must return a vtkm: : cont: : DataSet, and for a simple field filter like this we want to return the same DataSet as the input with the output field added. The output field needs a name, and we get the appropriate name from the superclass (line 18). However, we would like a special case where if the user does not specify an output field name we construct one based on the input field name. Recall from Example 18.2 that by default we set the output field name to the empty string. Thus, our filter checks for this empty string, and if it is encountered, it builds a field name by appending "_N/M^2" to it.

Finally, our filter constructs the output DataSet using the vtkm: :filter: :CreateResult helper function. There are multiple versions of CreateResult, but the version we are using is adding our new array as a field as the same type as the input.

This chapter has just provided a brief introduction to creating filters. There are several more filter superclasses to help express algorithms of different types. After some more worklet concepts to implement more complex algorithms are introduced in Part IV, we will see a more complete documentation of the types of filters in Chapter 22 . 



\section{Part IV}

Advanced Development 



\section{ADVANCED TYPES}

Chapter 4 introduced some of the base data types defined for use in VTK-m. However, for simplicity Chapter 4 just briefly touched the high-level concepts of these types. In this chapter we dive into much greater depth and introduce several more types.

\subsection{Single Number Types}

As described in Chapter 4, VTK-m provides aliases for all the base $\mathrm{C}$ types to ensure the representation matches the variable use. When a specific type width is not required, then the most common types to use are vtkm: :FloatDefault for floating-point numbers, vtkm: :Id for array and similar indices, and vtkm: : IdComponent for shorter-width vector indices.

If a specific type width is desired, then one of the following is used to clearly declare the type and width.

\begin{tabular}{rlll} 
bytes & floating point & signed integer & unsigned integer \\
\hline 1 & & vtkm::Int8 & vtkm::UInt8 \\
2 & & vtkm::Int16 & vtkm::UInt16 \\
4 & vtkm::Float32 & vtkm::Int32 & vtkm::UInt32 \\
8 & vtkm::Float64 & vtkm::Int64 & vtkm::UInt64
\end{tabular}

These VTK-m-defined types should be preferred over basic C types like int or float.

\subsection{Vector Types}

Visualization algorithms also often require operations on short vectors. Arrays indexed in up to three dimensions are common. Data are often defined in 2-space and 3-space, and transformations are typically done in homogeneous coordinates of length 4 . To simplify these types of operations, VTK-m provides the vtkm: :Vec $\langle\mathrm{T}$, Size $>$ templated type, which is essentially a fixed length array of a given type.

The default constructor of vtkm: :Vec objects leaves the values uninitialized. All vectors have a constructor with one argument that is used to initialize all components. All vtkm: :Vec objects also have a constructor that allows you to set the individual components (one per argument). All vtkm: :Vec objects with a size that is greater than 4 are constructed at run time and support an arbitrary number of initial values. Likewise, there is a vtkm: :make_Vec convenience function that builds initialized vector types with an arbitrary number of components. Once created, you can use the bracket operator to get and set component values with the same syntax as an array.

Example 19.1: Creating vector types. 


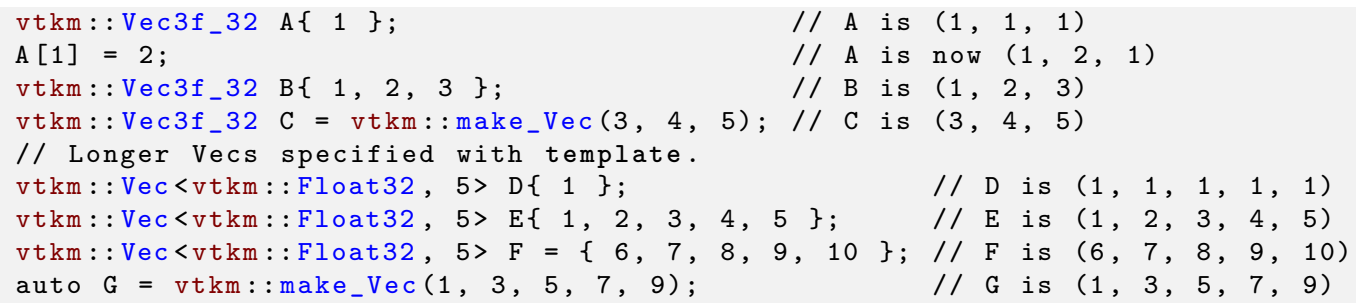

The types vtkm::Id2, vtkm::Id3, and vtkm::Id4 are type aliases of vtkm::Vec <vtkm::Id,2>, vtkm::Vec <vtkm::Id,3>, and vtkm::Vec <vtkm::Id,4>. These are used to index arrays of 2,3 , and 4 dimensions, which is common. Likewise, vtkm::IdComponent2, vtkm::IdComponent4, and vtkm: :IdComponent4 are type aliases of vtkm: :Vec <vtkm: :IdComponent, 2>, vtkm: :Vec <vtkm: :IdComponent, 3>, and vtkm: :Vec <vtkm: :IdComponent, $4>$.

Because declaring vtkm: :Vec $<\mathrm{T}$, Size $>$ with all of its template parameters can be cumbersome, VTK-m provides easy to use aliases for small vectors of base types. As introduced in Section 4.3, the following type aliases are available.

\begin{tabular}{rrlll}
\multicolumn{2}{c}{ bytes size } & floating point & signed integer & unsigned integer \\
\hline default & 2 & vtkm::Vec2f & vtkm::Vec2i & vtkm::Vec2ui \\
& 3 & vtkm::Vec3f & vtkm::Vec3i & vtkm::Vec3ui \\
& 4 & vtkm::Vec4f & vtkm::Vec4i & vtkm::Vec4ui \\
1 & 2 & & vtkm::Vec2i_8 & vtkm::Vec2ui_8 \\
& 3 & & vtkm::Vec3i_8 & vtkm::Vec3ui_8 \\
& 4 & & vtkm::Vec4i_8 & vtkm::Vec4ui_8 \\
2 & 2 & & vtkm::Vec2i_16 & vtkm::Vec2ui_16 \\
& 3 & & vtkm::Vec3i_16 & vtkm::Vec3ui_16 \\
& 4 & & vtkm::Vec4i_16 & vtkm::Vec4ui_16 \\
4 & 2 & vtkm::Vec2f_32 & vtkm::Vec2i_32 & vtkm::Vec2ui_32 \\
& 3 & vtkm::Vec3f_32 & vtkm::Vec3i_32 & vtkm::Vec3ui_32 \\
& 4 & vtkm::Vec4f_32 & vtkm::Vec4i_32 & vtkm::Vec4ui_32 \\
8 & 2 & vtkm::Vec2f_64 & vtkm::Vec2i_64 & vtkm::Vec2ui_64 \\
3 & vtkm::Vec3f_64 & vtkm::Vec3i_64 & vtkm::Vec3ui_64 \\
& 4 & vtkm::Vec4f_64 & vtkm::Vec4i_64 & vtkm::Vec4ui_64
\end{tabular}

vtkm: :Vec supports component-wise arithmetic using the operators for plus (+), minus (-), multiply (*), and divide (/). It also supports scalar to vector multiplication with the multiply operator. The comparison operators equal $(==)$ is true if every pair of corresponding components are true and not equal $(!=)$ is true otherwise. A special vtkm: : Dot function is overloaded to provide a dot product for every type of vector.

Example 19.2: Vector operations.

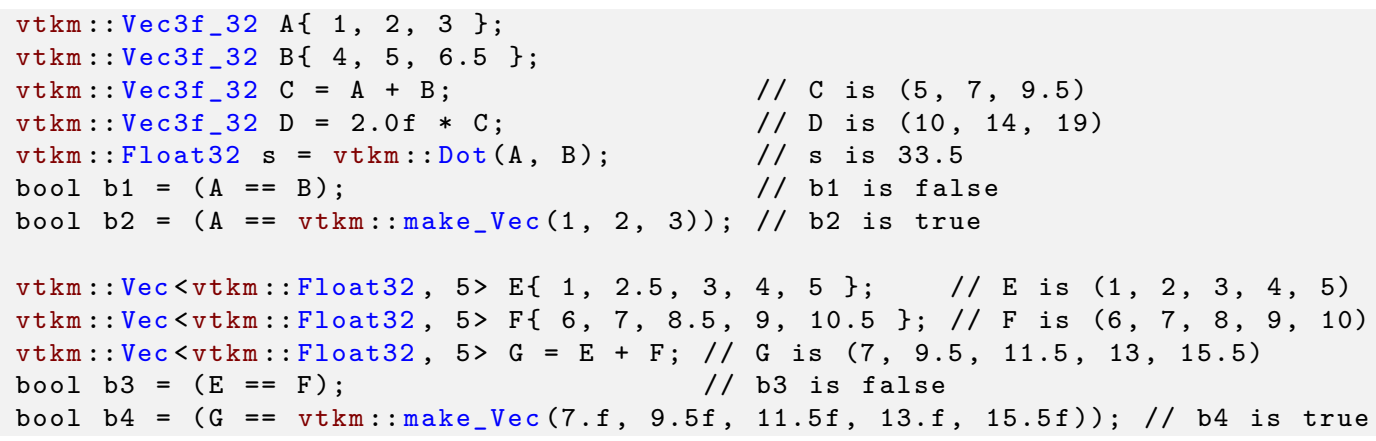


These operators, of course, only work if they are also defined for the component type of the vtkm::Vec. For example, the multiply operator will work fine on objects of type vtkm: :Vec <char, 3>, but the multiply operator will not work on objects of type vtkm: :Vec <std: :string, 3> because you cannot multiply objects of type std: : string.

In addition to generalizing vector operations and making arbitrarily long vectors, vtkm: :Vec can be repurposed for creating any sequence of homogeneous objects. Here is a simple example of using vtkm: : Vec to hold the state of a polygon.

Example 19.3: Repurposing a vtkm: :Vec.

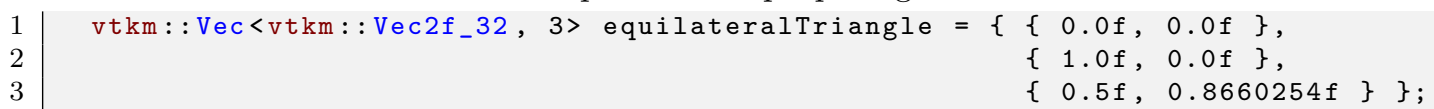

The vtkm: :Vec class provides a convenient structure for holding and passing small vectors of data. However, there are times when using Vec is inconvenient or inappropriate. For example, the size of vtkm: :Vec must be known at compile time, but there may be need for a vector whose size is unknown until compile time. Also, the data populating a vtkm: :Vec might come from a source that makes it inconvenient or less efficient to construct a vtkm::Vec. For this reason, VTK-m also provides several Vec-like objects that behave much like vtkm::Vec but are a different class. These Vec-like objects have the same interface as vtkm: :Vec except that the NuM_COMPONENTS constant is not available on those that are sized at run time. Vec-like objects also come with a CopyInto method that will take their contents and copy them into a standard Vec class. (The standard Vec class also has a CopyInto method for consistency.)

The first Vec-like object is vtkm: :VecC, which exposes a C-type array as a Vec. The constructor for vtkm: :VecC takes a $\mathrm{C}$ array and a size of that array. There is also a constant version of VecC named vtkm: : VecCConst, which takes a constant array and cannot be mutated. The vtkm/Types.h header defines both VecC and VecCConst as well as multiple versions of vtkm: :make_VecC to easily convert a C array to either a VecC or VecCConst.

The following example demonstrates converting values from a constant table into a vtkm: :VecCConst for further consumption. The table and associated methods define how 8 points come together to form a hexahedron.

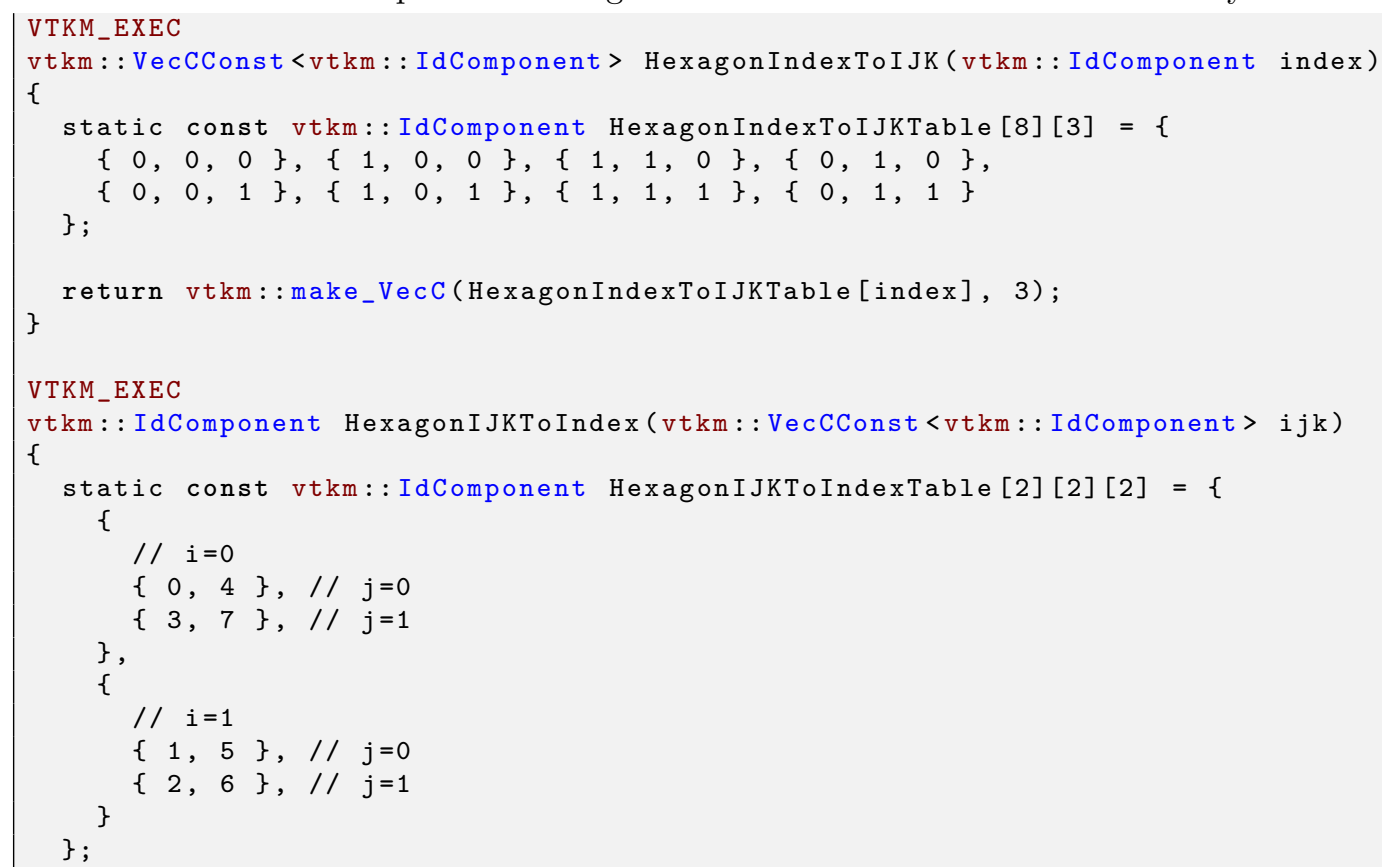




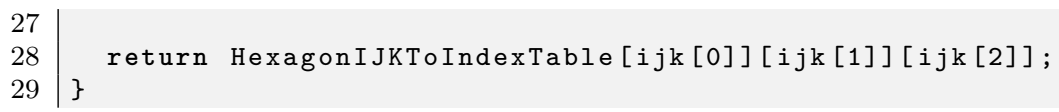

\section{Common Errors}

The vtkm: :VecC and vtkm: :VecCConst classes only hold a pointer to a buffer that contains the data. They do not manage the memory holding the data. Thus, if the pointer given to vtkm: :VecC or vtkm: :VecCConst becomes invalid, then using the object becomes invalid. Make sure that the scope of the vtkm::VecC or vtkm: :VecCConst does not outlive the scope of the data it points to.

The next Vec-like object is vtkm: :VecVariable, which provides a Vec-like object that can be resized at run time to a maximum value. Unlike VecC, VecVariable holds its own memory, which makes it a bit safer to use. But also unlike VecC, you must define the maximum size of VecVariable at compile time. Thus, VecVariable is really only appropriate to use when there is a predetermined limit to the vector size that is fairly small.

The following example uses a vtkm: :VecVariable to store the trace of edges within a hexahedron. This example uses the methods defined in Example 19.4.

Example 19.5: Using vtkm: :VecVariable.

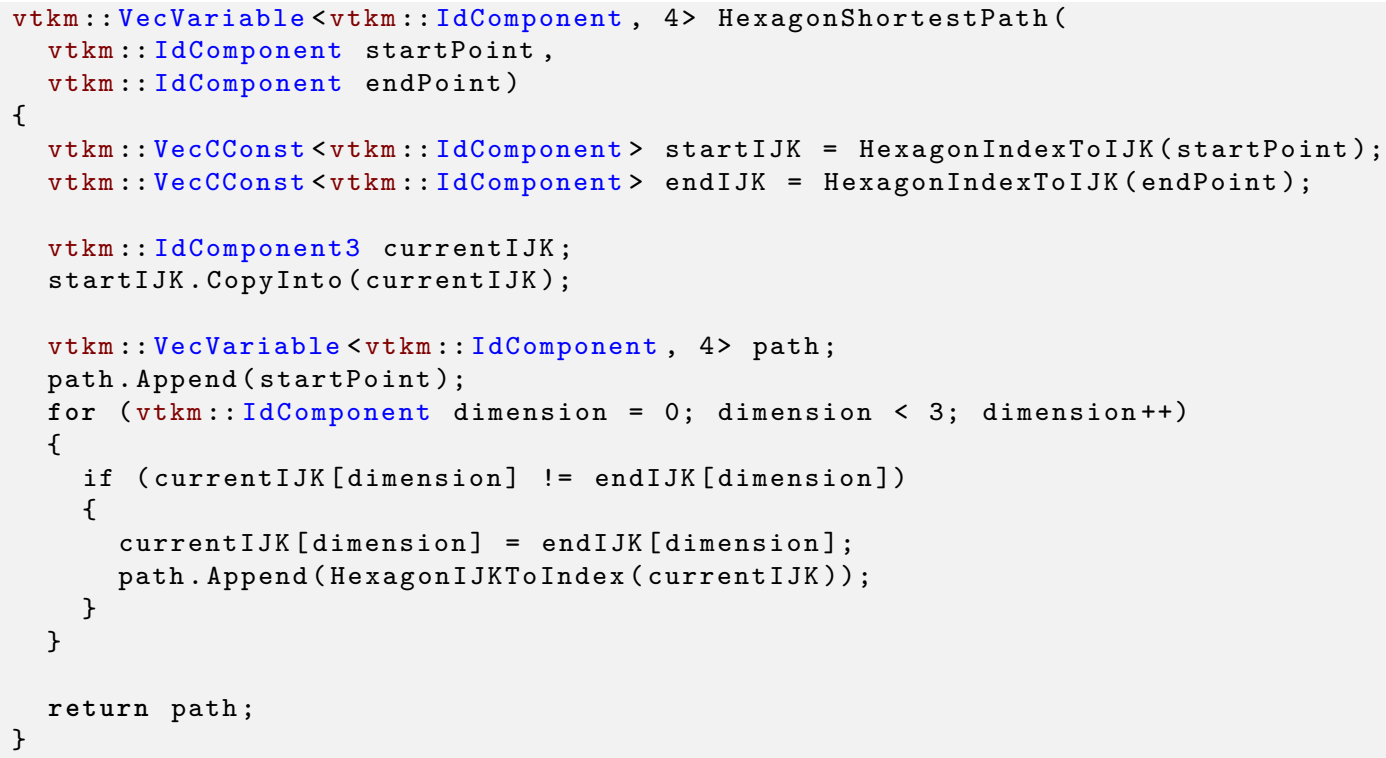

VTK-m provides further examples of Vec-like objects as well. For example, the vtkm::VecFromPortal and vtkm: :VecFromPortalPermute objects allow you to treat a subsection of an arbitrarily large array as a Vec. These objects work by attaching to array portals, which are described in Section 27.1. Another example of a Vec-like object is vtkm::VecRectilinearPointCoordinates, which efficiently represents the point coordinates in an axis-aligned hexahedron. Such shapes are common in structured grids. These and other data sets are described in Chapter 7. 


\subsection{Range}

VTK-m provides a convenience structure named vtkm: :Range to help manage a range of values. The Range struct contains two data members, Min and Max, which represent the ends of the range of numbers. Min and Max are both of type vtkm::Float64. Min and Max can be directly accessed, but Range also comes with the following helper functions to make it easier to build and use ranges. Note that all of these functions treat the minimum and maximum value as inclusive to the range.

IsNonEmpty Returns true if the range covers at least one value.

Contains Takes a single number and returns true if that number is contained within the range.

Length Returns the distance between Min and Max. Empty ranges return a length of 0 . Note that if the range is non-empty and the length is 0, then Min and Max must be equal, and the range contains exactly one number.

Center Returns the number equidistant to Min and Max. If the range is empty, NaN is returned.

Include Takes either a single number or another range and modifies this range to include the given number or range. If necessary, the range is grown just enough to encompass the given argument. If the argument is already in the range, nothing changes.

Union A nondestructive version of Include, which builds a new Range that is the union of this range and the argument. The + operator is also overloaded to compute the union.

The following example demonstrates the operation of vtkm: :Range.

Example 19.6: Using vtkm: :Range.

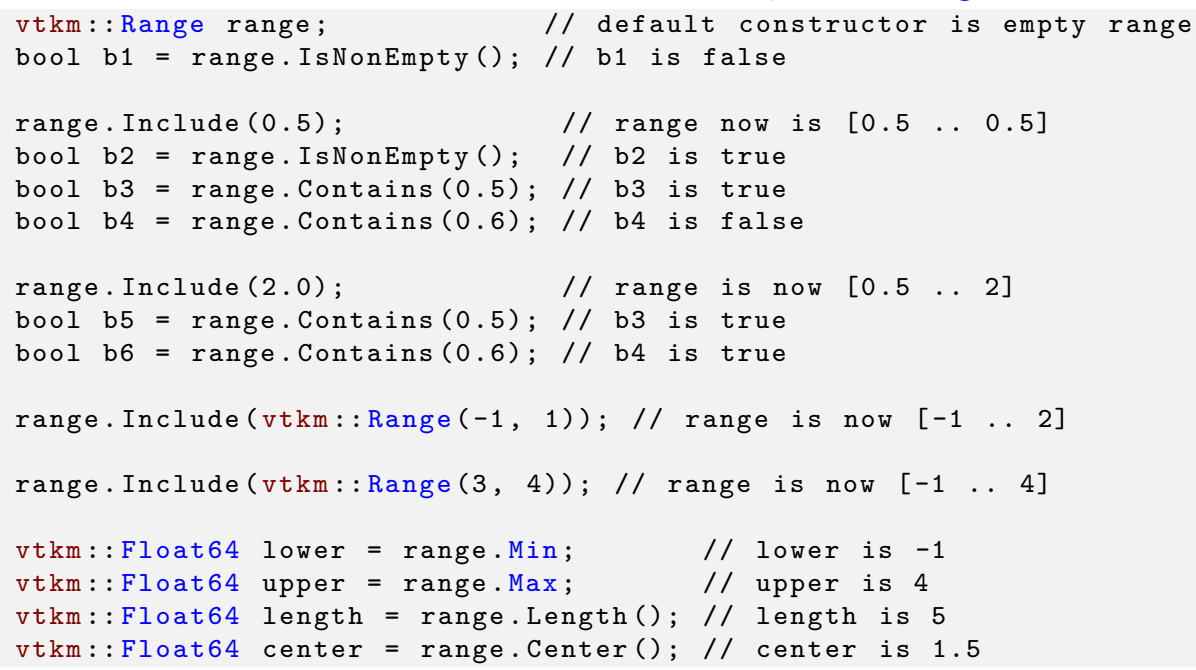

\subsection{Bounds}

VTK-m provides a convenience structure named vtkm: :Bounds to help manage an axis-aligned region in 3D space. Among other things, this structure is often useful for representing a bounding box for geometry. The Bounds struct contains three data members, X, Y, and Z, which represent the range of the bounds along each 
respective axis. All three of these members are of type vtkm: : Range, which is discussed previously in Section 19.3. $\mathrm{X}, \mathrm{Y}$, and $\mathrm{Z}$ can be directly accessed, but Bounds also comes with the following helper functions to make it easier to build and use ranges.

IsNonEmpty Returns true if the bounds cover at least one value.

Contains Takes a vtkm: :Vec of size 3 and returns true if those point coordinates are contained within the range.

Center Returns the point at the center of the range as a vtkm: :Vec <vtkm: :Float64,3>.

Include Takes either a vtkm: :Vec of size 3 or another bounds and modifies this bounds to include the given point or bounds. If necessary, the bounds are grown just enough to encompass the given argument. If the argument is already in the bounds, nothing changes.

Union A nondestructive version of Include, which builds a new Bounds that is the union of this bounds and the argument. The + operator is also overloaded to compute the union.

The following example demonstrates the operation of vtkm: :Bounds.

Example 19.7: Using vtkm: :Bounds.

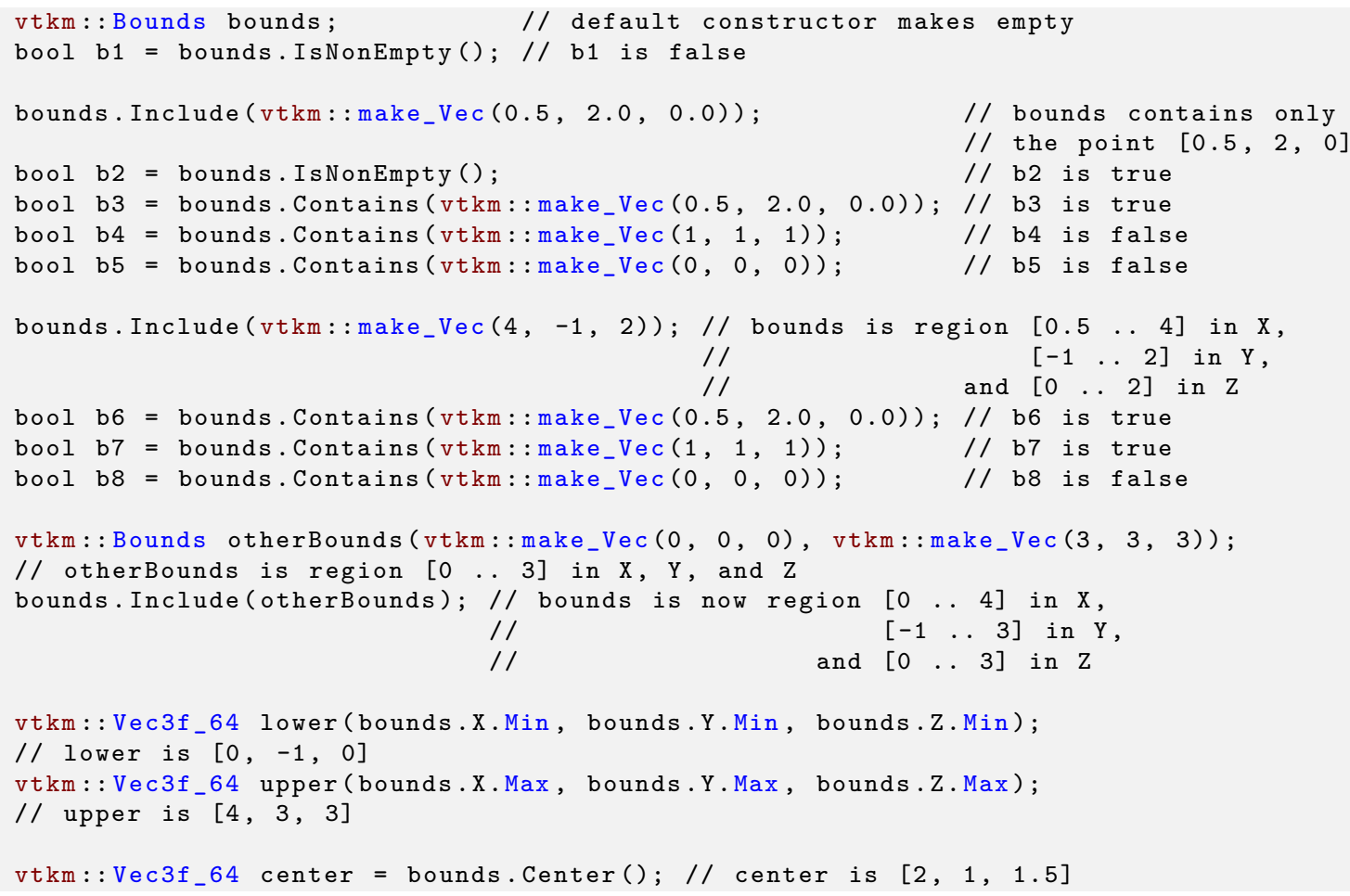

\subsection{Traits}

When using templated types, it is often necessary to get information about the type or specialize code based on general properties of the type. VTK-m uses traits classes to publish and retrieve information about types. A traits class is simply a templated structure that provides type aliases for tag structures, empty types used for identification. The traits classes might also contain constant numbers and helpful static functions. See Effective $C++$ Third Edition by Scott Meyers for a description of traits classes and their uses. 


\subsubsection{Type Traits}

The vtkm: :TypeTraits <T> templated class provides basic information about a core type. These type traits are available for all the basic C++ types as well as the core VTK-m types described in Chapter 4. vtkm: :TypeTraits contains the following elements.

NumericTag This type is set to either vtkm: :TypeTraitsRealTag or vtkm::TypeTraitsIntegerTag to signal that the type represents either floating point numbers or integers.

DimensionalityTag This type is set to either vtkm: :TypeTraitsScalarTag or vtkm: :TypeTraitsVectorTag to signal that the type represents either a single scalar value or a tuple of values.

ZeroInitialization A static member function that takes no arguments and returns 0 (or the closest equivalent to it) cast to the type.

The definition of vtkm: :TypeTraits for vtkm: :Float32 could like something like this.

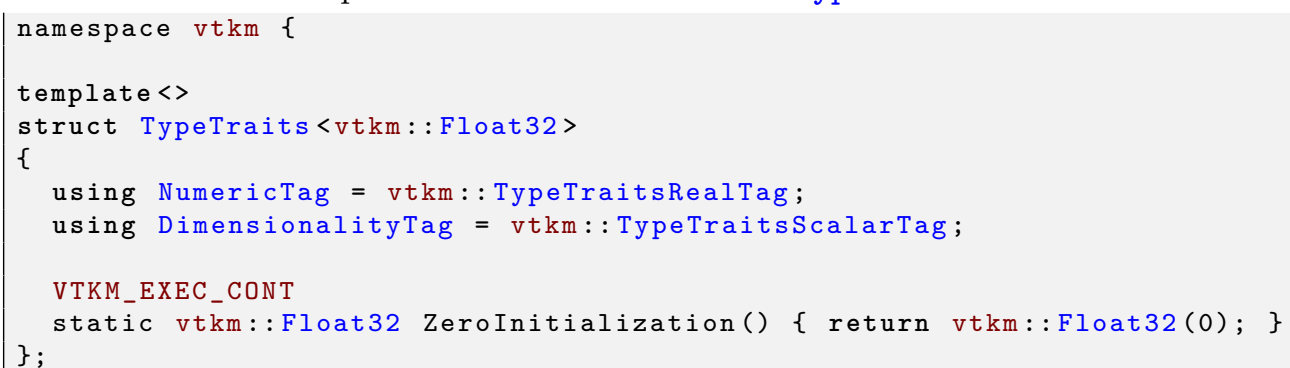

Here is a simple example of using vtkm: :TypeTraits to implement a generic function that behaves like the remainder operator $(\%)$ for all types including floating points and vectors.

Example 19.9: Using TypeTraits for a generic remainder.

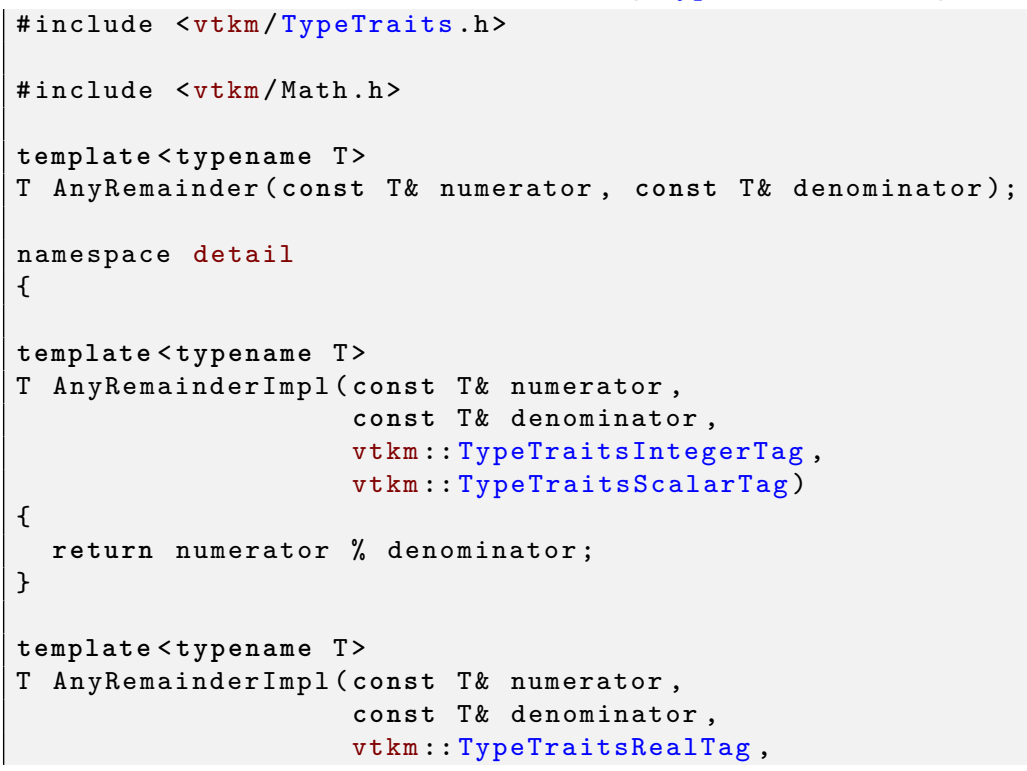




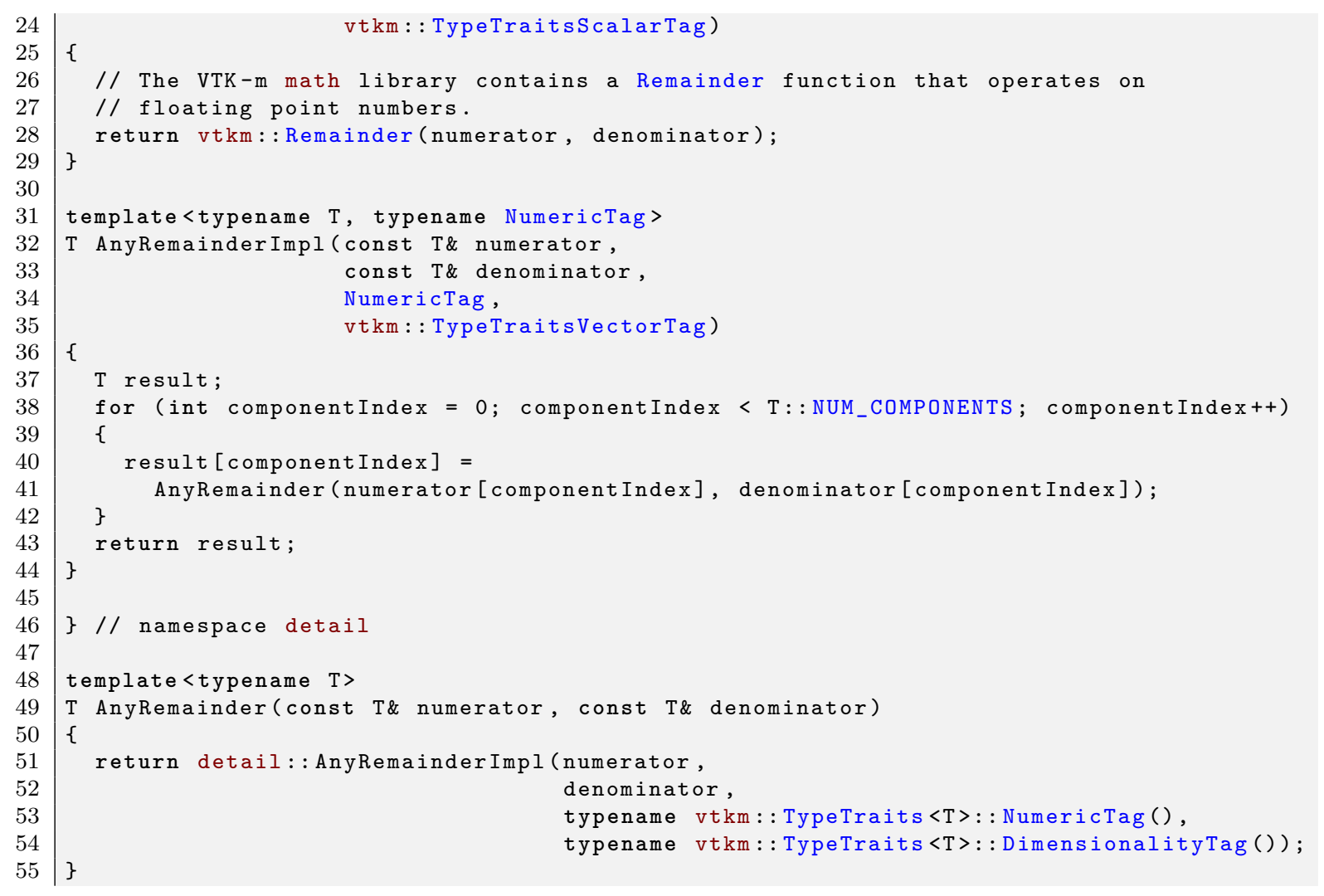

\subsubsection{Vector Traits}

The templated vtkm: :Vec class contains several items for introspection (such as the component type and its size). However, there are other types that behave similarly to Vec objects but have different ways to perform this introspection.

For example, VTK-m contains Vec-like objects that essentially behave the same but might have different features. Also, there may be reason to interchangeably use basic scalar values, like an integer or floating point number, with vectors. To provide a consistent interface to access these multiple types that represents vectors, the vtkm: :VecTraits $<\mathrm{T}>$ templated class provides information and accessors to vector types.It contains the following elements.

ComponentType This type is set to the type for each component in the vector. For example, a vtkm::Id3 has ComponentType defined as vtkm: :Id.

IsSizeStatic This type is set to either vtkm: :VecTraitsTagSizeStatic if the vector has a static number of components that can be determined at compile time or set to vtkm::VecTraitsTagSizeVariable if the size of the vector is determined at run time. If IsSizeStatic is set to VecTraitsTagSizeVariable, then VecTraits will be missing some information that cannot be determined at compile time.

HasMultipleComponents This type is set to either vtkm: :VecTraitsTagSingleComponent if the vector length is size 1 or vtkm: :VecTraitsTagMultipleComponents otherwise. This tag can be useful for creating specialized functions when a vector is really just a scalar. If the vector type is of variable size (that is, IsSizeStatic is VecTraitsTagSizeVariable), then HasMultipleComponents might be VecTraitsTagMultipleComponents even when at run time there is only one component. 
NUM_COMPONENTS An integer specifying how many components are contained in the vector. NUM_COMPONENTS is not available for vector types of variable size (that is, IsSizeStatic is VecTraitsTagSizeVariable).

GetNumberOfComponents A static method that takes an instance of a vector and returns the number of components the vector contains. The result of GetNumberOfComponents is the same value of NUM_COMPONENTS for vector types that have a static size (that is, IsSizeStatic is VecTraitsTagSizeStatic). But unlike NUM_COMPONENTS, GetNumberOfComponents works for vectors of any type.

GetComponent A static method that takes a vector and returns a particular component.

SetComponent A static method that takes a vector and sets a particular component to a given value.

CopyInto A static method that copies the components of a vector to a vtkm: :Vec.

The definition of vtkm: :VecTraits for vtkm: : Id3 could look something like this.

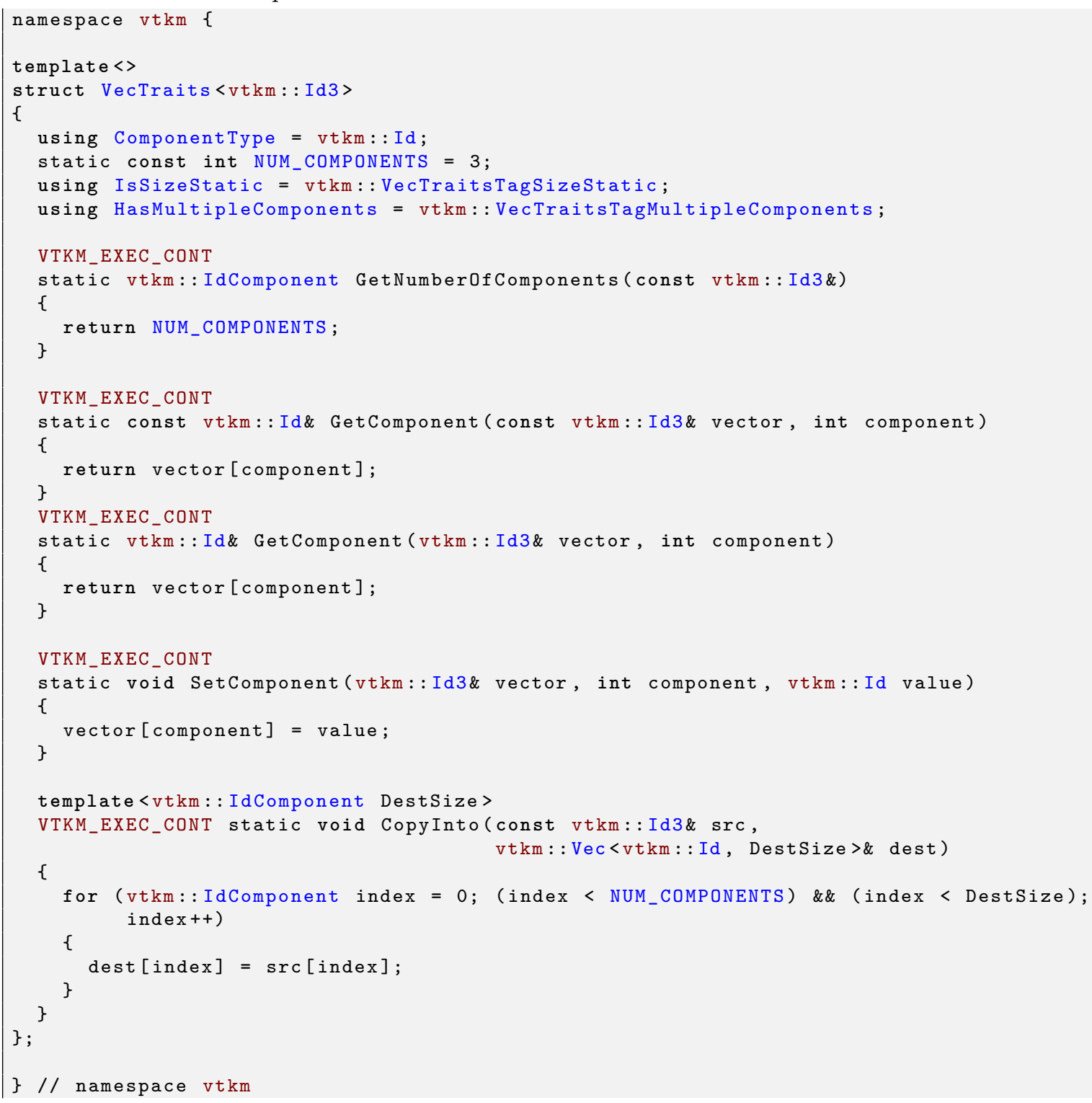


The real power of vector traits is that they simplify creating generic operations on any type that can look like a vector. This includes operations on scalar values as if they were vectors of size one. The following code uses vector traits to simplify the implementation of less functors that define an ordering that can be used for sorting and other operations.

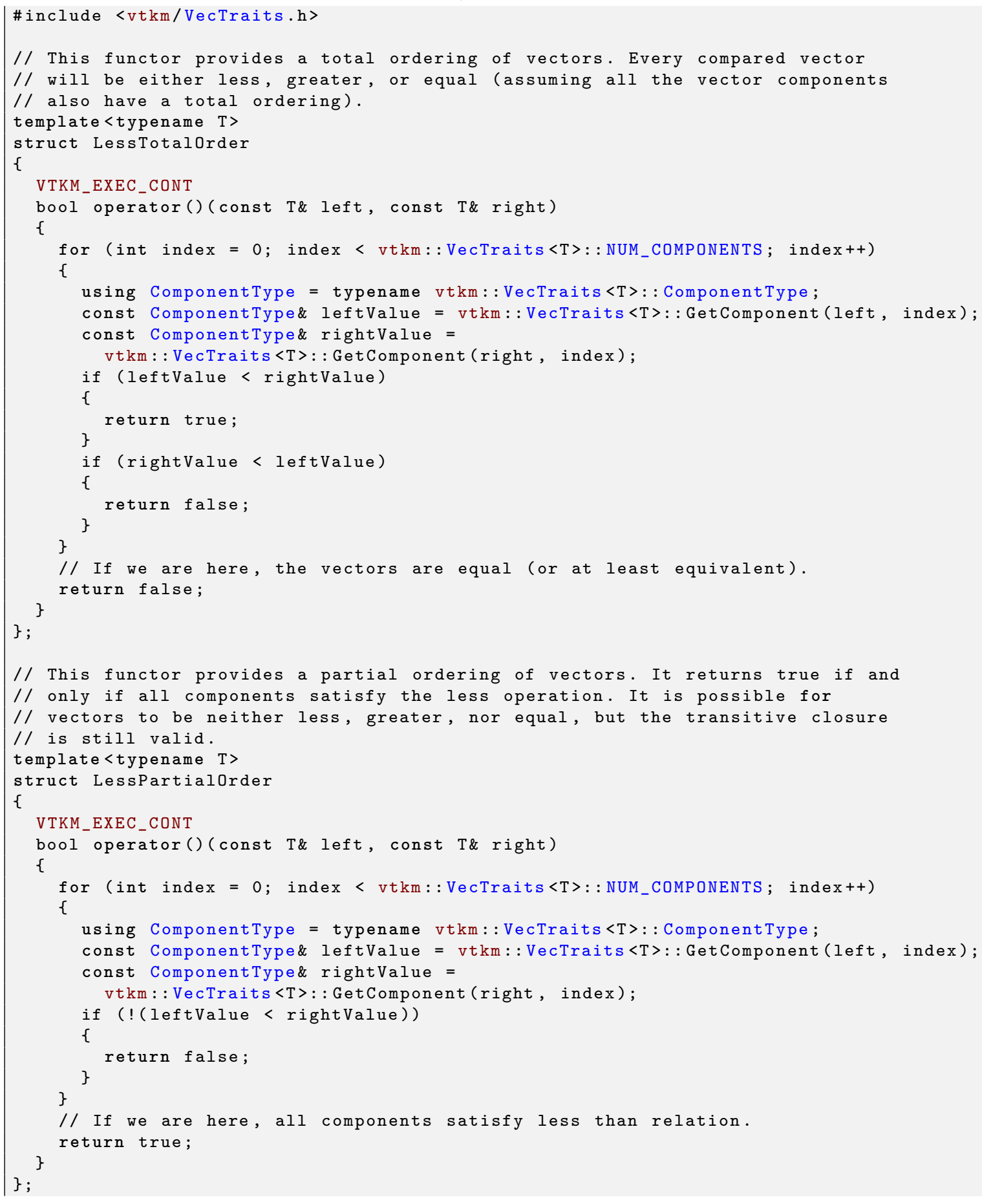




\subsection{List Templates}

VTK-m internally uses template metaprogramming, which utilizes $\mathrm{C}++$ templates to run source-generating programs, to customize code to various data and compute platforms. One basic structure often uses with template metaprogramming is a list of class names (also sometimes called a tuple or vector, although both of those names have different meanings in VTK-m).

Many VTK-m users only need predefined lists, such as the type lists specified in Section 19.6.2. Those users can skip most of the details of this section. However, it is sometimes useful to modify lists, create new lists, or operate on lists, and these usages are documented here.

\subsubsection{Building Lists}

A basic list is defined with the vtkm: :List $<\mathrm{T}, \ldots>$ template, which is defined in the vtkm/List.h header. It is common (but not necessary) to use the using keyword to define an alias for a list with a particular meaning.

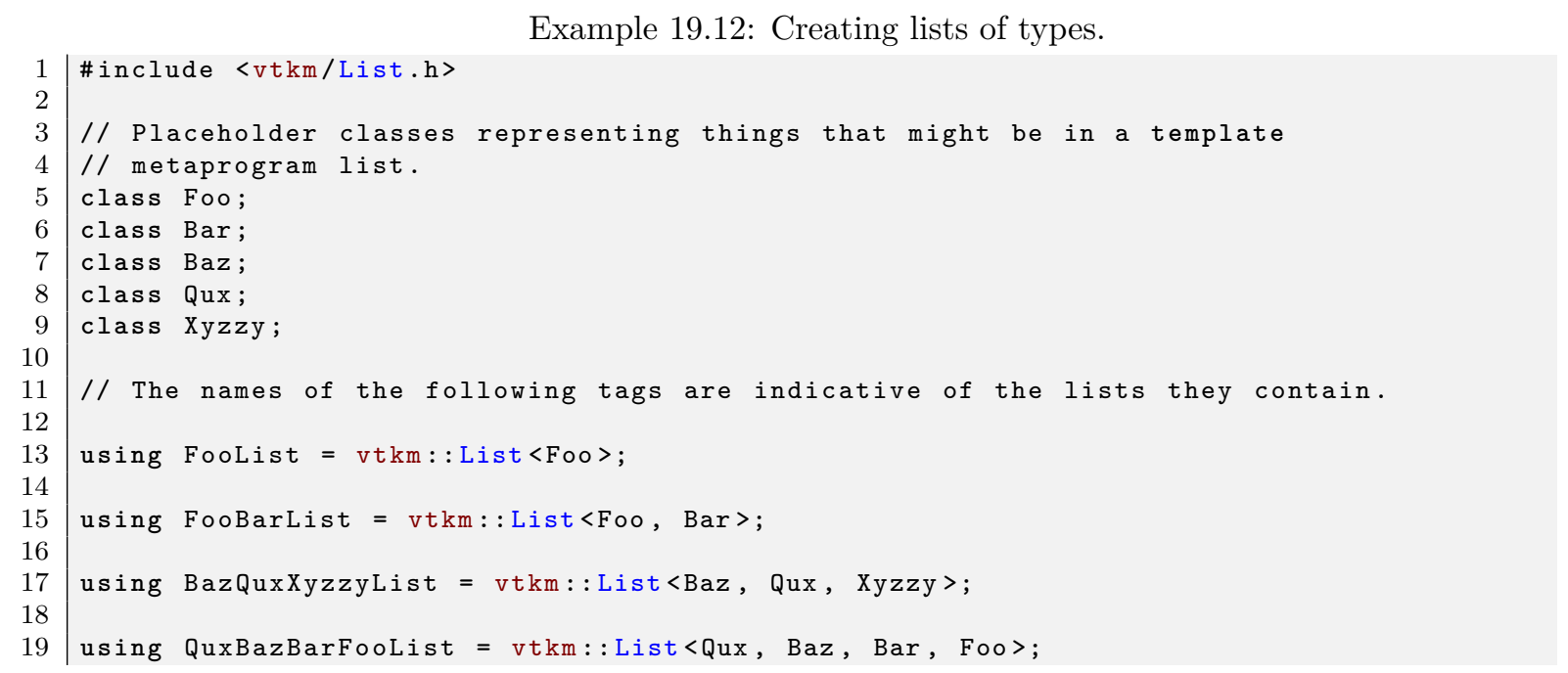

VTK-m defines the convenience class vtkm: :ListEmpty, which is simply an empty list (i.e. vtkm: :List <>).

VTK-m also provides a special identifier named vtkm: ListUniversal. ListUniversal is a conceptual list containing all possible types. Operations on ListUniversal will behave as if it contains all types where possible, but some operations (such as getting the size of the list) are ill-defined and will fail.

\subsubsection{Type Lists}

One of the major use cases for template metaprogramming lists in VTK-m is to identify a set of potential data types for arrays. The vtkm/TypeList.h header contains predefined lists for known VTK-m types. Although technically all these lists are of $\mathrm{C}++$ types, the types we refer to here are those data types stored in data arrays. The following lists are provided.

vtkm: :TypeListId Contains the single item vtkm: :Id.

vtkm: :TypeListId2 Contains the single item vtkm::Id2.

vtkm: :TypeListId3 Contains the single item vtkm: :Id3. 
vtkm: :TypeListIdComponent Contains the single item vtkm: : IdComponent.

vtkm::TypeListIndex A list of all types used to index arrays. Contains vtkm::Id, vtkm: :Id2, and vtkm: :Id3.

vtkm: :TypeListFieldScalar A list containing types used for scalar fields. Specifically, it contains floating point numbers of different widths (i.e. vtkm: :Float32 and vtkm: :Float64).

vtkm: :TypeListFieldVec2 A list containing types for values of fields with 2 dimensional vectors. All these vectors use floating point numbers.

vtkm: :TypeListFieldVec3 A list containing types for values of fields with 3 dimensional vectors. All these vectors use floating point numbers.

vtkm: :TypeListFieldVec4 A list containing types for values of fields with 4 dimensional vectors. All these vectors use floating point numbers.

vtkm: :TypeListField A list containing all the types generally used for fields. It is the combination of vtkm: :TypeListFieldScalar, vtkm::TypeListFieldVec2, vtkm::TypeListFieldVec3, and vtkm: :TypeListFieldVec4.

vtkm: :TypeListScalarAll A list of all scalar types. It contains signed and unsigned integers of widths from 8 to 64 bits. It also contains floats of 32 and 64 bit widths.

vtkm: :TypeListVecCommon A list of the most common vector types. It contains all vtkm: :Vec class of size 2 through 4 containing components of unsigned bytes, signed 32-bit integers, signed 64-bit integers, 32-bit floats, or 64 -bit floats.

vtkm: :TypeListVecAll A list of all vtkm: :Vec classes with standard integers or floating points as components and lengths between 2 and 4 .

vtkm: :TypeListall A list of all types included in vtkm/Types.h with vtkm: :Vec s with up to 4 components.

vtkm::TypeListCommon A list containing only the most used types in visualization. This includes signed integers and floats that are 32 or 64 bit. It also includes 3 dimensional vectors of floats. This is the default list used when resolving the type in arrays of unknown type (described in Chapter 33).

If these lists are not sufficient, it is possible to build new type lists using the existing type lists and the list bases from Section 19.6.1 as demonstrated in the following example.

Example 19.13: Defining new type lists.

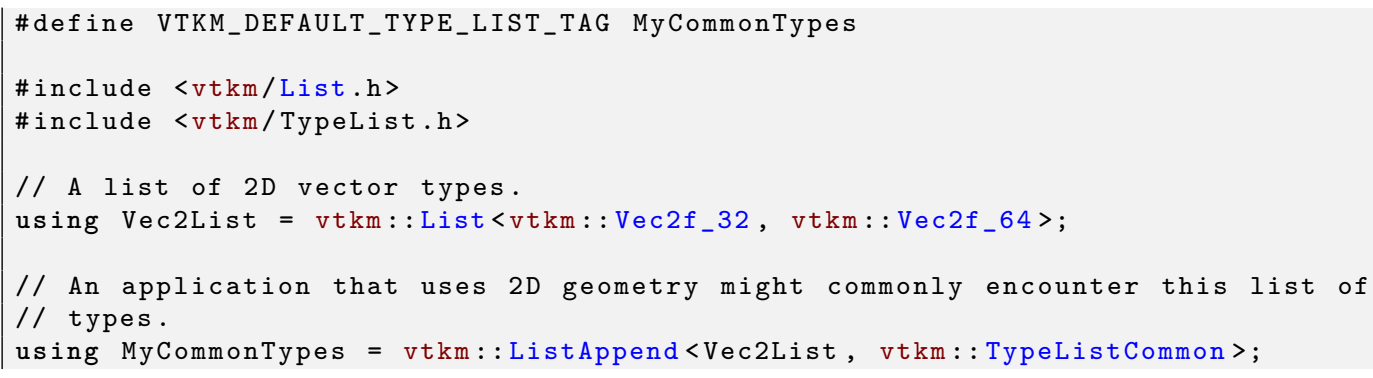

The vtkm/TypeList.h header also defines a macro named VTKM_DEFAULT_TYPE_LIST that defines a default list of types to use when, for example, determining the type of a field array. This list can be overridden by defining the VTKM_DEFAULT_TYPE_LIST macro before any VTK-m headers are included. If included after a VTK-m header, the list is not likely to take effect. Do not ignore compiler warnings about the macro being redefined, which you will not get if defined correctly. Example 19.13 also contains an example of overriding the VTKM_DEFAULT_TYPE_LIST macro. 


\subsubsection{Querying Lists}

vtkm/List.h contains some templated classes to help get information about a list type. This are particularly useful for lists that are provided as templated parameters for which you do not know the exact type.

The VTKM_IS_LIST does a compile-time check to make sure a particular type is actually a vtkm: : List of types. If the compile-time check fails, then a build error will occur. This is a good way to verify that a templated class or method that expects a list actually gets a list.

Example 19.14: Checking that a template parameter is a valid List.

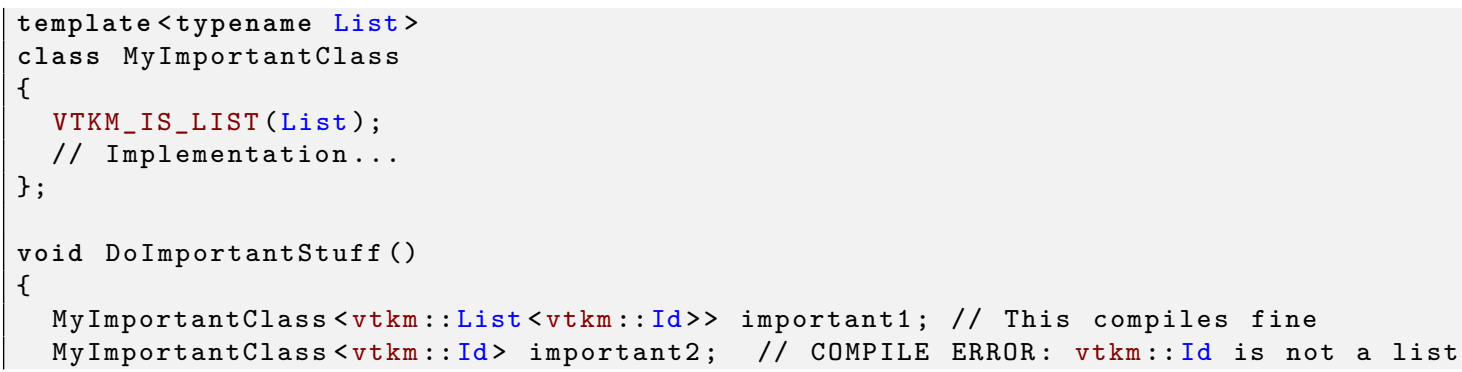

The size of a list can be determined by using the vtkm: :ListSize template. The type of the template will resolve to a std::integral_constant<vtkm: IdComponent, $\mathrm{N}>$ where $\mathrm{N}$ is the number of types in the list. vtkm: :ListSize does not work with vtkm: :ListUniversal.

Example 19.15: Getting the size of a List.

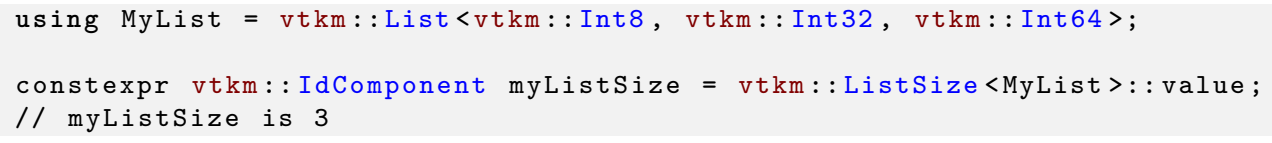

The vtkm: :ListHas template can be used to determine if a vtkm::List contains a particular type. ListHas takes two template parameters. The first parameter is a form of vtkm: :List. The second parameter is any type to check to see if it is in the list. If the type is in the list, then ListHas resolves to std: :true_type. Otherwise it resolves to std: :false_type. vtkm::ListHas always returns true for vtkm::ListUniversal.

Example 19.16: Determining if a List contains a particular type.

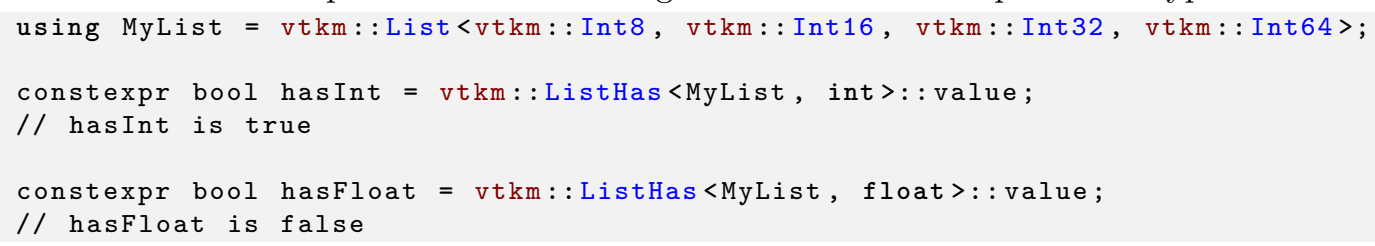

The vtkm: :ListIndexOf template can be used to get the index of a particular type in a vtkm: :List. ListIndexOf takes two template parameters. The first parameter is a form of vtkm::List. The second parameter is any type to check to see if it is in the list. The type of the template will resolve to a std::integral_constant<vtkm: : IdComponent, $\mathrm{N}>$ where $\mathrm{N}$ is the index of the type. If the requested type is not in the list, then ListIndexOf becomes std: : integral_constant<vtkm: : IdComponent,-1>.

Conversely, the vtkm: :Listat template can be used to get the type for a particular index. The two template parameters for ListAt are the List and an index for the list.

Neither vtkm: :ListIndexOf nor vtkm: :ListAt works with vtkm::ListUniversal.

Example 19.17: Using indices with List. 


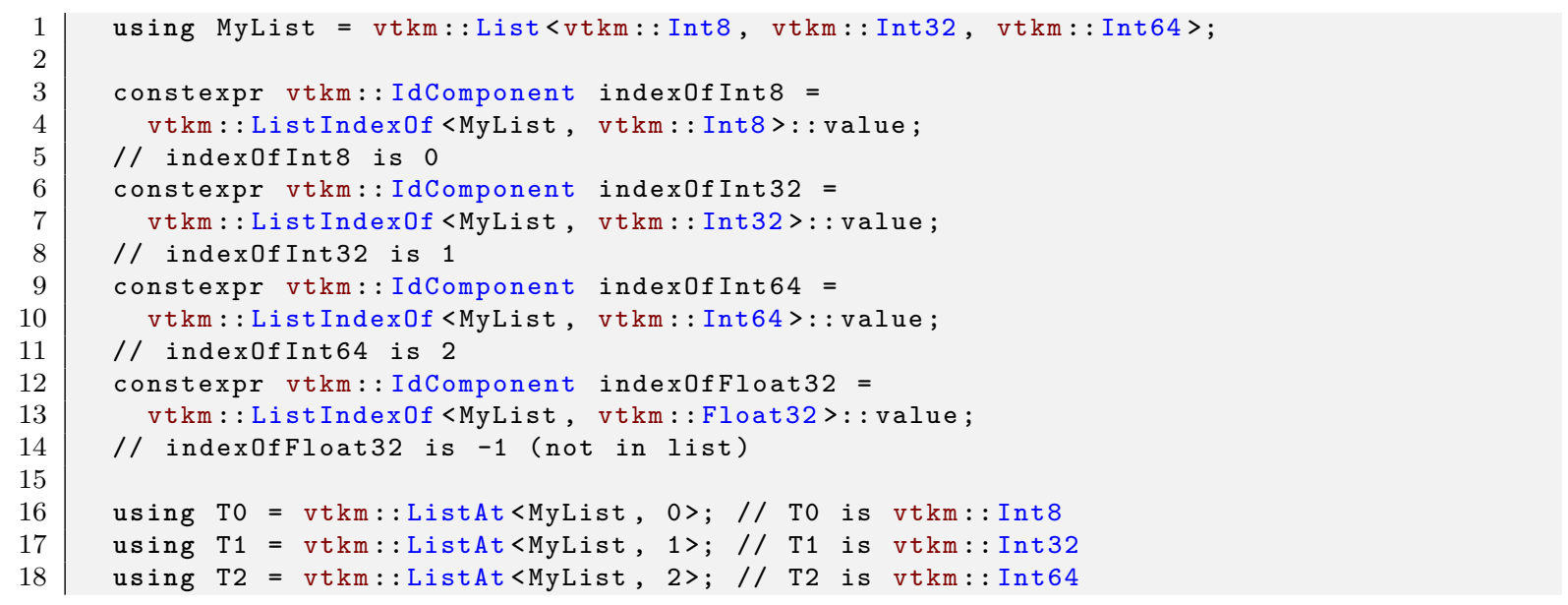

\subsubsection{Operating on Lists}

In addition to providing the base templates for defining and querying lists, vtkm/List.h also contains several features for operating on lists.

The vtkm: ListAppend template joins together 2 or more Lists. The items are concatenated in the order provided to ListAppend. ListAppend does not work with vtkm: :ListUniversal.

Example 19.18: Appending Lists.

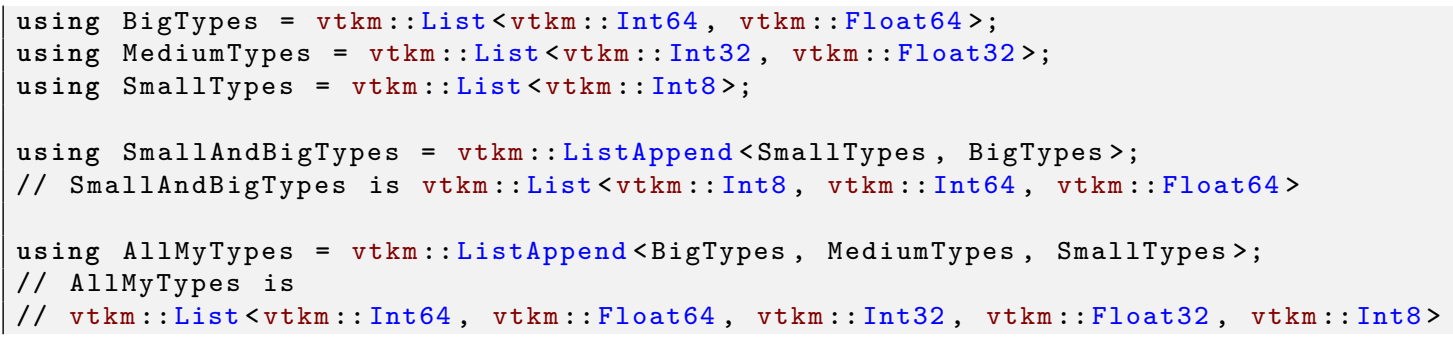

The vtkm::ListIntersect template takes two Lists and becomes a vtkm::List containing all types in both lists. If one of the lists is vtkm: :ListUniversal, the contents of the other list used.

Example 19.19: Intersecting Lists.

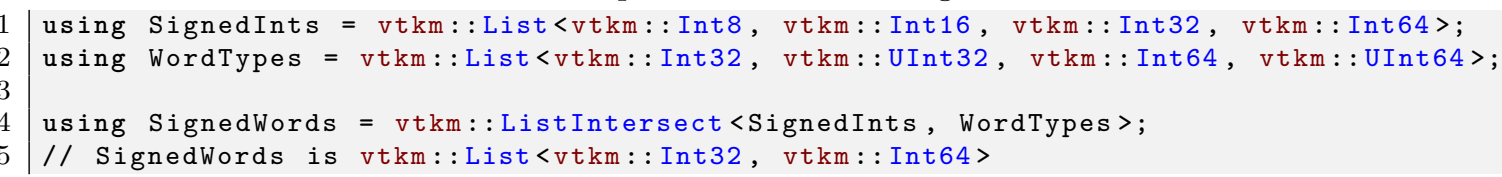

The vtkm::ListApply template transfers all of the types in a vtkm::List to another template. The first template argument of ListApply is the List to apply. The second template argument is another template to apply to. ListApply becomes an instance of the passed template with all the types in the List. ListApply can be used to convert a List to some other template. ListApply cannot be used with vtkm: :ListUniversal.

Example 19.20: Applying a List to another template.

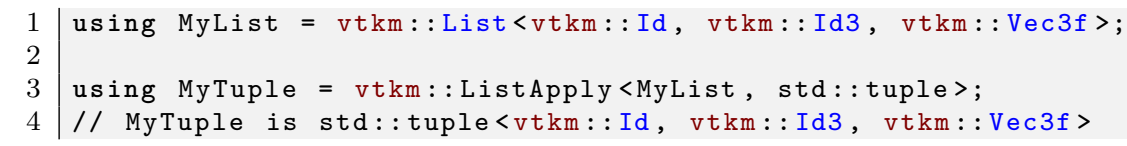


The vtkm: :ListTransform template applies each item in a vtkm: : List to another template and constructs a list from all these applications. The first template argument of ListTransform is the List to apply. The second template argument is another template to apply to. ListTransform becomes an instance of a new vtkm: : List containing the passed template each type. ListTransform cannot be used with vtkm: :ListUniversal.

Example 19.21: Transforming a List using a custom template.

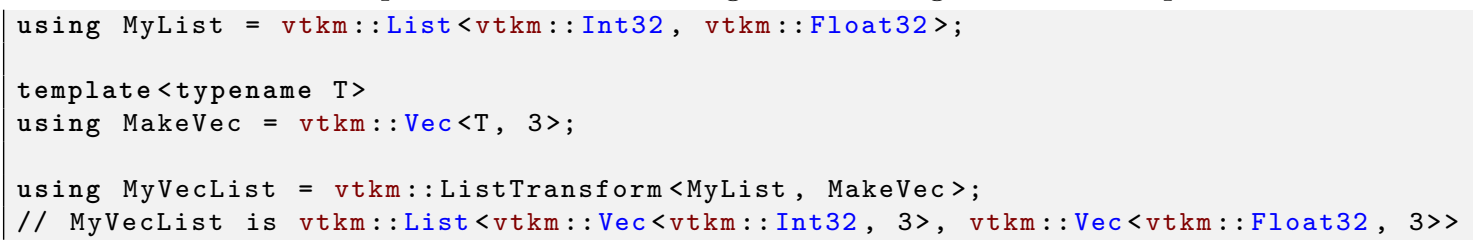

The vtkm: :ListRemoveIf template removes items from a vtkm: :List given a predicate. The first template argument of ListRemoveIf is the List. The second argument is another template that is used as a predicate to determine if the type should be removed or not. The predicate should become a type with a value member that is a static true or false value. Any type in the list that the predicate evaluates to true is removed. ListRemoveIf cannot be used with vtkm: :ListUniversal.

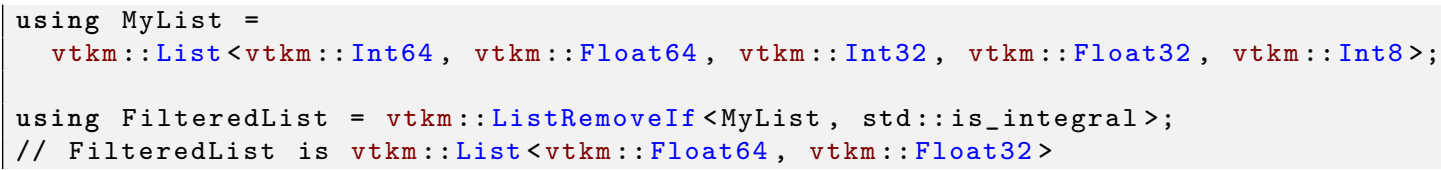

The vtkm: : ListCross takes two lists and performs a cross product of them. It does this by creating a new vtkm: : List that contains nested Lists, each of length 2 and containing all possible pairs of items in the first list with items in the second list. ListCross is often used in conjunction with another list processing command, such as ListTransform to build templated types of many combinations. ListCross cannot be used with vtkm: :ListUniversal.

Example 19.23: Creating the cross product of 2 Lists.

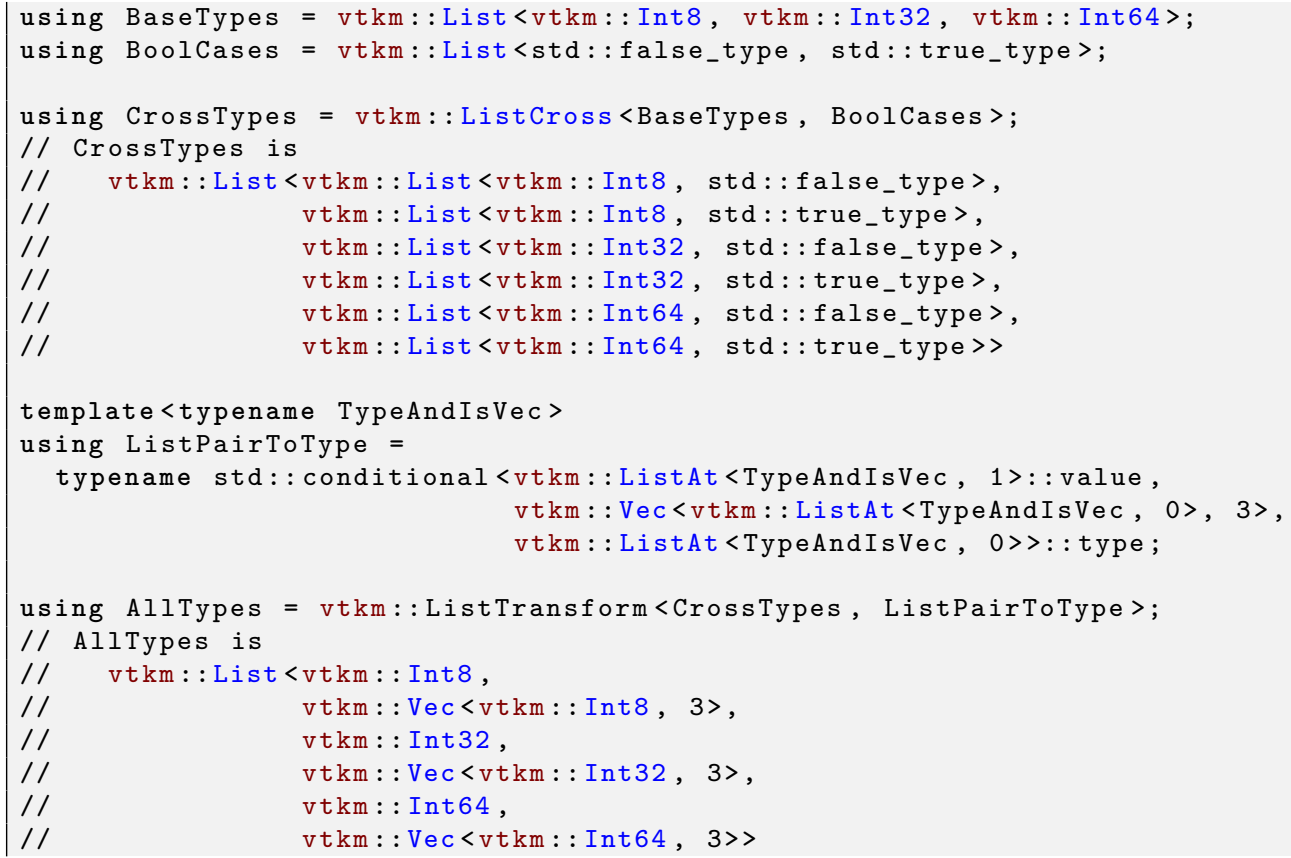


The vtkm: :ListForEach function takes a functor object and a vtkm: :List. It then calls the functor object with the default object of each type in the list. This is most typically used with $\mathrm{C}++$ run-time type information to convert a run-time polymorphic object to a statically typed (and possibly inlined) call.

The following example shows a rudimentary version of converting a dynamically-typed array to a statically-typed array similar to what is done in VTK-m classes like vtkm: : cont: :UnknownArrayHandle (which is documented in Chapter 33).

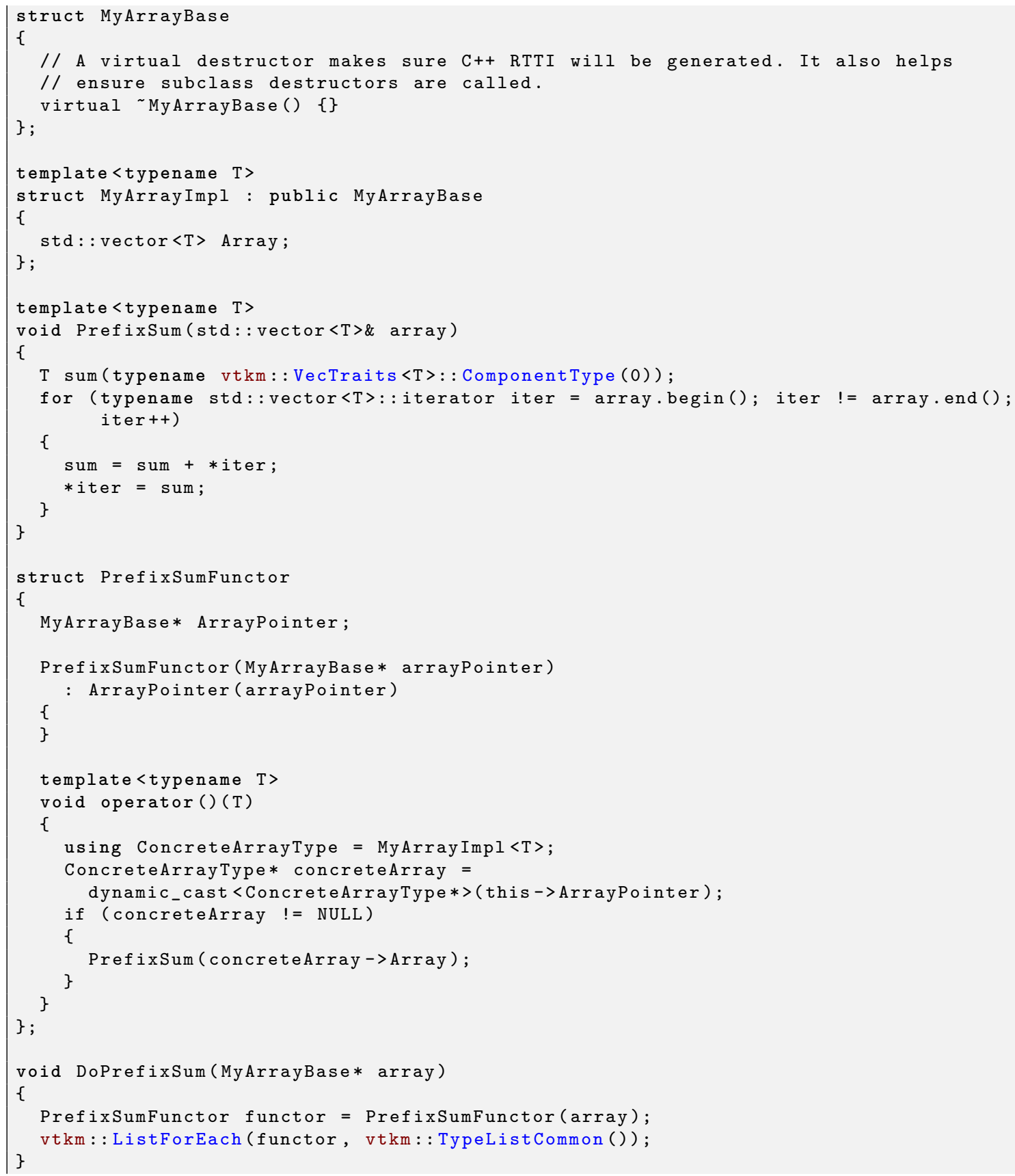




\subsection{Pair}

VTK-m defines a vtkm: :Pair <T1, T2> templated object that behaves just like std: :pair from the standard template library. The difference is that vtkm: :Pair will work in both the execution and control environments, whereas the STL std: :pair does not always work in the execution environment.

The VTK-m version of vtkm: :Pair supports the same types, fields, and operations as the STL version. VTK-m also provides a vtkm: :make_Pair function for convenience.

\subsection{Tuple}

VTK-m defines a vtkm: Tuple templated object that behaves like std: tuple from the standard template library. The main difference is that vtkm: :Tuple will work in both the execution and control environments, whereas the STL std: : tuple does not always work in the execution environment.

\subsubsection{Defining and Constructing}

vtkm: :Tuple takes any number of template parameters that define the objects stored the tuple.

Example 19.25: Defining a Tuple.

1 vtkm::Tuple<vtkm::Id, vtkm::Vec3f, vtkm::cont::ArrayHandle<vtkm:: Int32>> myTuple;

You can construct a vtkm: :Tuple with arguments that will be used to initialize the respective objects. As a convenience, you can use vtkm: :MakeTuple to construct a vtkm: :Tuple of types based on the arguments.

Example 19.26: Initializing values in a Tuple.

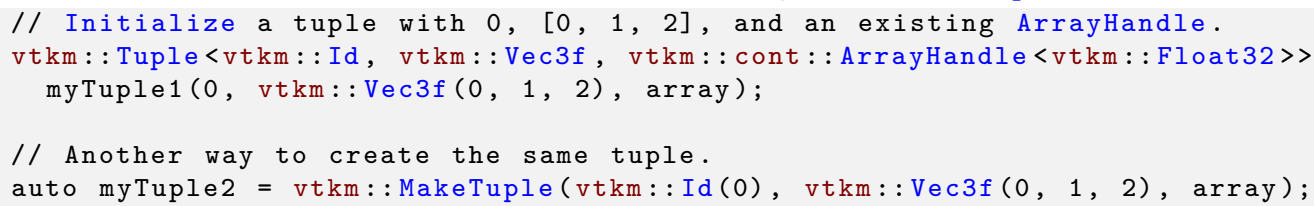

\subsubsection{Querying}

The size of a vtkm: :Tuple can be determined by using the vtkm::TupleSize template, which resolves to an std: : integral_constant. The types at particular indices can be determined with vtkm: :TupleElement.

Example 19.27: Querying Tuple types.

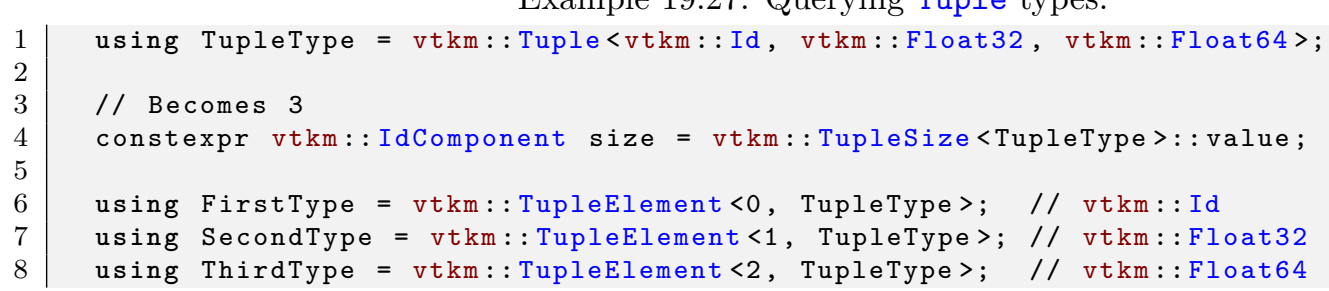

The function vtkm: :Get can be used to retrieve an element from the vtkm: :Tuple. Get returns a reference to the element, so you can set a vtkm: :Tuple element by Geting the value. 
Example 19.28: Retrieving values from a Tuple.

\subsubsection{For Each}

Tuple: :ForEach is a method that takes a function or functor and calls it for each of the items in the tuple. Nothing is returned from ForEach and any return value from the function is ignored. ForEach can be used to check the validity of each item.

Example 19.29: Using Tuple: :ForEach to check the contents.

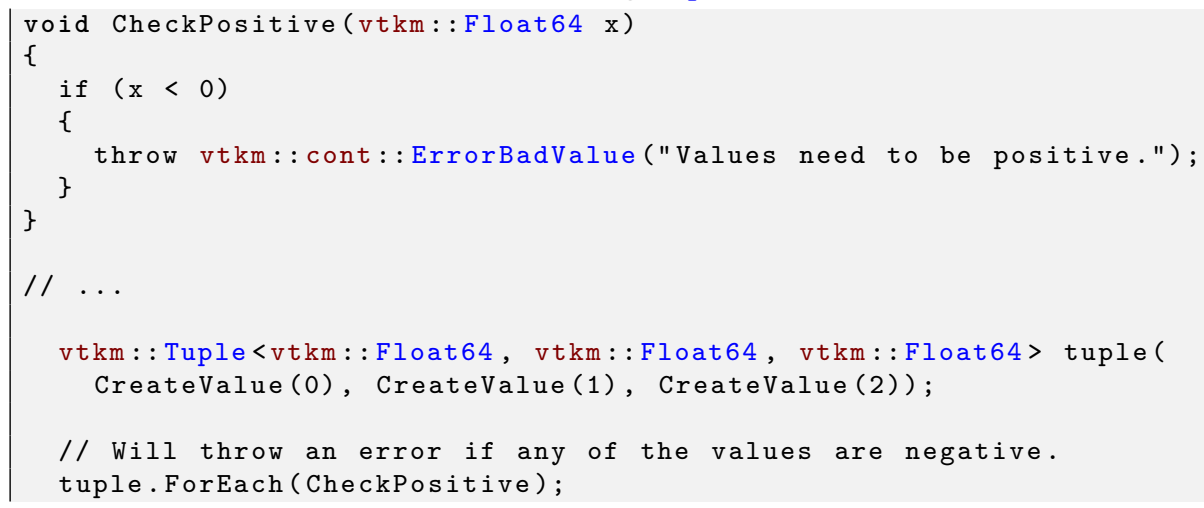

Tuple: : ForEach can also be used to aggregate values.

Example 19.30: Using Tuple: :ForEach to aggregate.

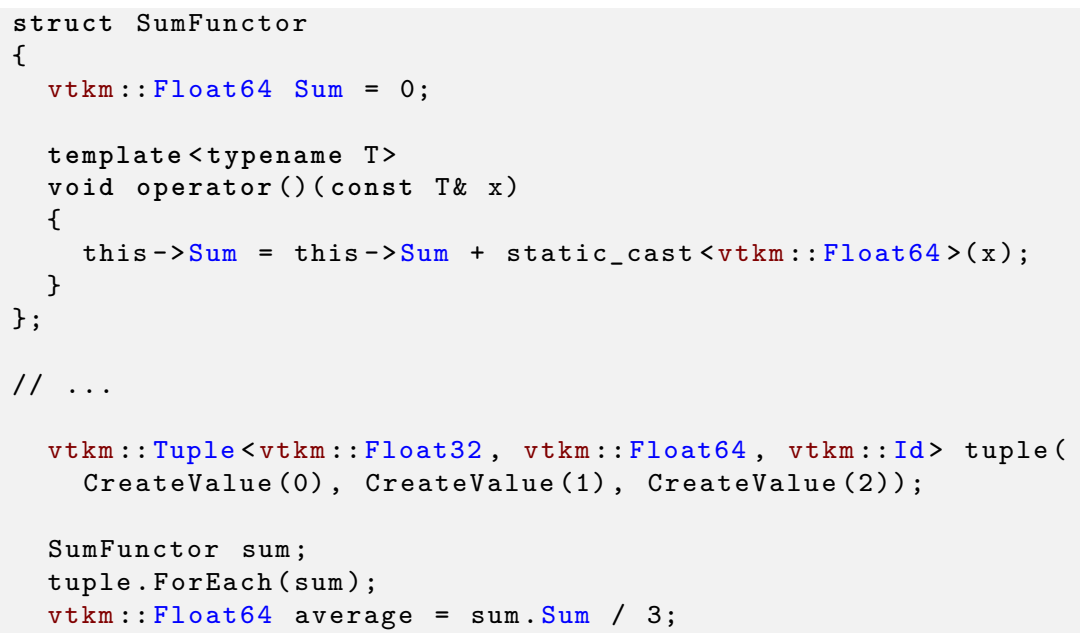




\subsubsection{Transform}

Tuple: :Transform is a method that builds a new Tuple by calling a function or functor on each of the items. The return value is placed in the corresponding part of the resulting Tuple, and the type is automatically created from the return type of the function.

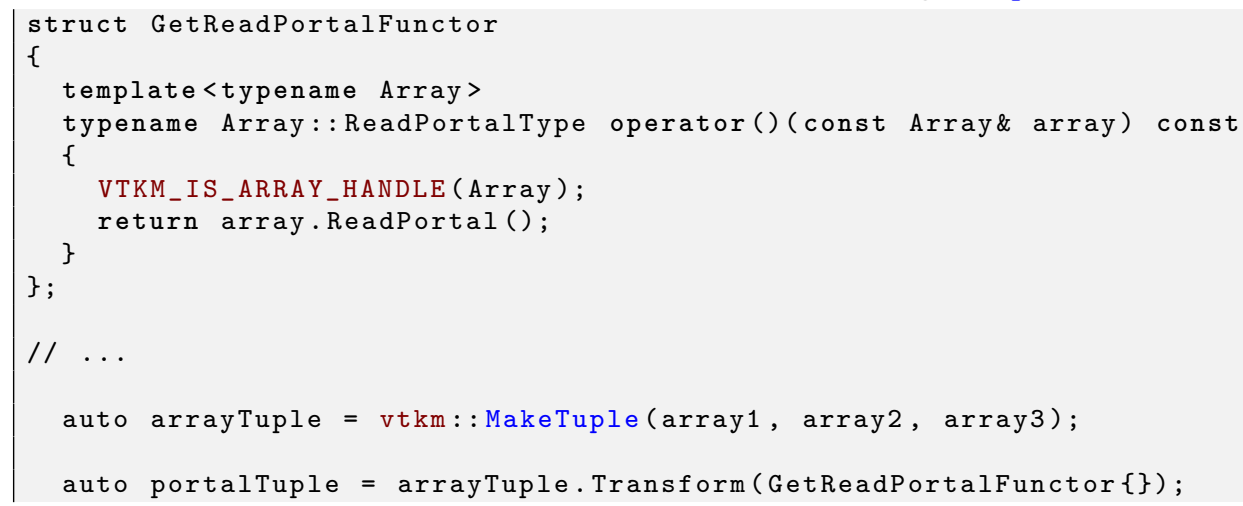

\subsubsection{Apply}

Tuple: : Apply is a method that calls a function or functor using the objects in the Tuple as the arguments. If the function returns a value, that value is returned from Apply.

Example 19.32: Applying a Tuple as arguments to a function.

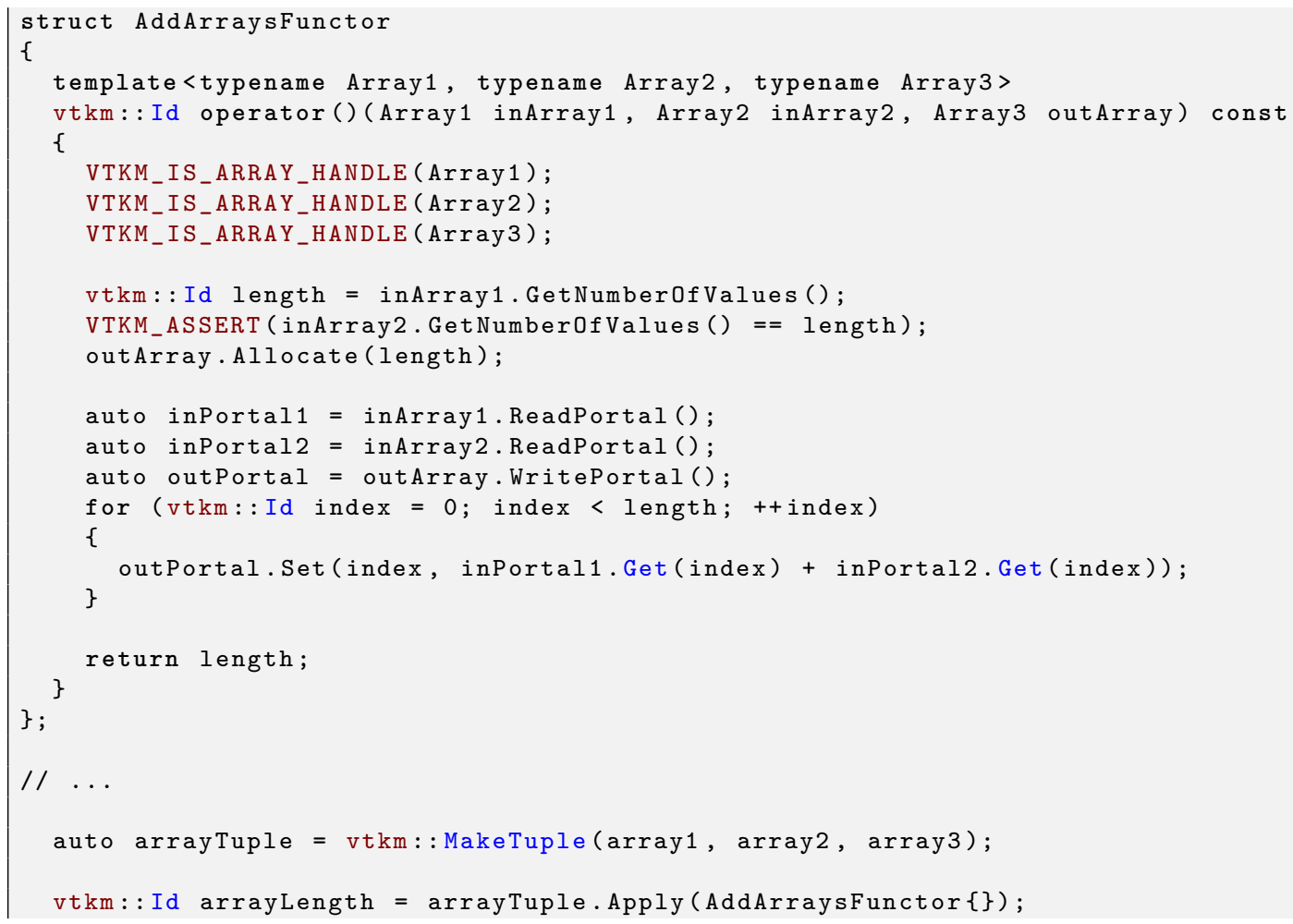


If additional arguments are given to Apply, they are also passed to the function (before the objects in the vtkm: :Tuple). This is helpful for passing state to the function.

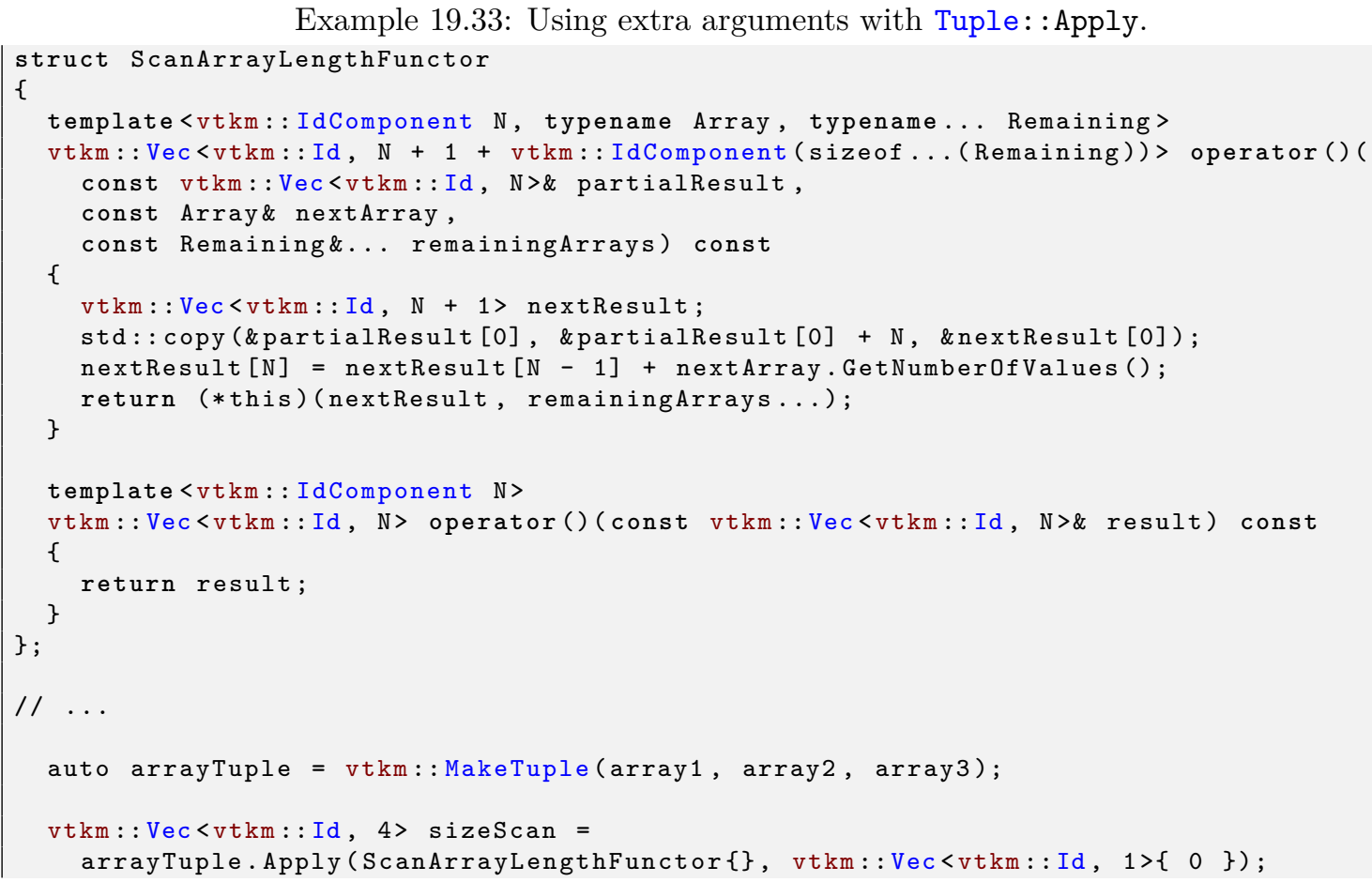

\subsection{Error Codes}

For operations that occur in the control environment, VTK-m uses exceptions to report errors as described in Chapter 11. However, when operating in the execution environment, it is not feasible to throw exceptions. Thus, for operations designed for the execution environment, the status of an operation that can fail is returned as an vtkm: :ErrorCode, which is an enum. An ErrorCode can be one of the following enumerators.

ErrorCode: :Success The operation completed successfully.

ErrorCode: : InvalidShapeId An operation on a cell was given a shape identifier that is not recognized.

ErrorCode: InvalidNumberOfPoints The wrong number of points was provided for a given cell type. For example, if a triangle has 4 points associated with it, you are likely to get this error.

ErrorCode: : InvalidPointId A bad point identifier was detected while operating on a cell.

ErrorCode: : InvalidEdgeId A bad edge identifier was detected while operating on a cell.

ErrorCode: : InvalidFaceId A bad face identifier was detected while operating on a cell.

ErrorCode: : SolutionDidNotConverge An iterative operation did not find an appropriate solution. The result is not likely to be accurate.

ErrorCode: :MatrixFactorizationFailed A solution was not found for a linear system. 
ErrorCode: :DegenerateCellDetected A cell's parameters have degenerated it to another type. For example, if two vertices of a tetrahedron are the same, it degenerates into a triangle.

ErrorCode: :MalformedCellDetected The structure of a cell is incorrect. For example, if the vertices of a cell are listed in the wrong order, you might encounter this error.

ErrorCode: :OperationOnEmptyCell There is an "empty" cell placeholder type to be used when other cell types cannot be applied. Because it is a placeholder, operations on these types of cells are undefined.

ErrorCode: :CellNotFound A locate operation failed to find a cell given the search criteria.

If a function or method returns an ErrorCode, it is a good practice to check to make sure that the returned value is Success. If it is not, you can use the vtkm: :ErrorString function to convert the ErrorCode to a descriptive $\mathrm{C}$ string. The easiest thing to do from within a worklet is to call the worklet's RaiseError method.

Example 19.34: Checking an ErrorCode and reporting errors in a worklet.

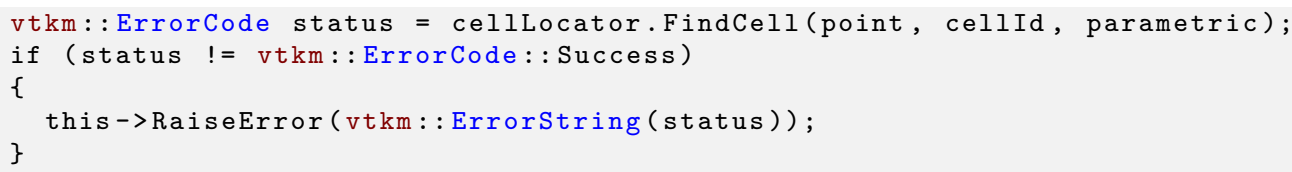



VTK-m features a logging system that allows status updates and timing. VTK-m uses the loguru project to provide runtime logging facilities. ${ }^{1}$ Logging is enabled by setting the CMake variable VTKm_ENABLE_LOGGING. When this flag is enabled, any messages logged to the Info, Warn, Error, and Fatal levels are printed to stderr by default.

\subsection{Initializing Logging}

Additional logging features are enabled by calling vtkm: : cont: : Initialize as described in Chapter 6. Although calling Initialize is not strictly necessary for output messages, initialization adds the following features.

- Set human-readable names for the log levels in the output.

- Allow the stderr logging level to be set at runtime by passing a $-\mathrm{v}$ [level] argument to the executable (if provided).

- Name the main thread.

- Print a preamble with details of the program's startup (arguments, etc).

- Install signal handlers to automatically print stack traces and error contexts (Linux only) on crashes.

Example 20.1 in the following section provides an example of initializing with additional logging setup.

The logging implementation is thread-safe. When working in a multithreaded environment, each thread may be assigned a human-readable name using vtkm::cont::SetThreadName (which can later be retrieved with vtkm: : cont: :GetThreadName. This name will appear in the log output so that per-thread messages can be easily tracked.

\subsection{Logging Levels}

The logging in VTK-m provides several "levels" of logging. Logging levels are ordered by precedence. When selecting which log message to output, a single logging level is provided. Any logging message with that or a higher precedence is output. For example, if warning messages are on, then error messages are also outputted

\footnotetext{
${ }^{1} \mathrm{~A}$ sample of the log output can be found at https://gitlab.kitware.com/snippets/427.
} 
because errors are a higher precedence than warnings. Likewise, if information messages are on, then error and warning messages are also outputted.

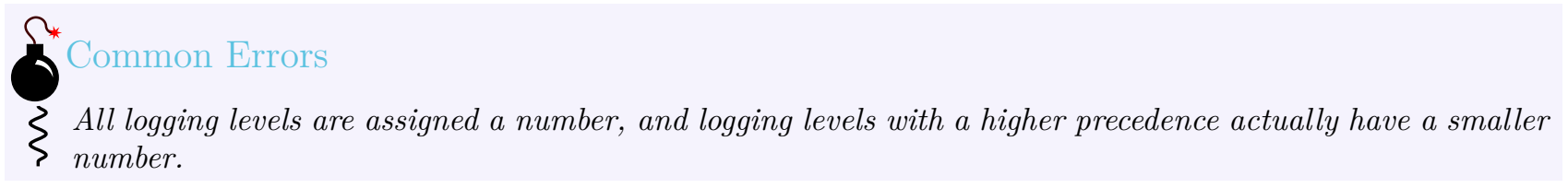

All logging levels are listed in the vtkm: :cont::LogLevel enum. The available logging levels, in order of precedence, are as follows.

LogLevel: :Off A placeholder used to silence all logging.

LogLevel: :Fatal Fatal errors that should abort execution.

LogLevel: :Error Important but non-fatal errors, such as device fail-over.

LogLevel: :Warn Less important user errors, such as out-of-bounds parameters.

LogLevel: : Info Information messages (detected hardware, etc) and temporary debugging output.

LogLevel: :UserFirst The first in a range of logging levels reserved for code that uses VTK-m. Internal VTK-m code will not log on these levels but will report these logs.

LogLevel: :UserLast The last in a range of logging levels reserved for code that uses VTK-m.

LogLevel::Perf General timing data and algorithm flow information, such as filter execution, worklet dispatches, and device algorithm calls.

LogLevel: :MemCont Host-side resource memory allocations and frees such as ArrayHandle control buffers.

LogLevel: :MemExec Device-side resource memory allocations and frees such as ArrayHandle device buffers)

LogLevel: : MemTransfer Transferring of data between a host and device.

LogLevel:: Cast Report when a dynamic object is (or is not) resolved via a CastAndCall or other casting method.

LogLevel: :UserVerboseFirst The first in a range of logging levels reserved for code that uses VTK-m. Internal VTK-m code will not log on these levels but will report these logs. These are used similarly to those in the UserFirst range but are at a lower precedence that also includes more verbose reporting from VTK-m.

LogLevel: :UserVerboseLast The last in a range of logging levels reserved for code that uses VTK-m.

When VTK-m outputs an entry in its log, it annotates the message with the logging level. VTK-m will automatically provide descriptions for all log levels described in vtkm: : cont: :LogLevel. A custom log level can be described by calling the vtkm: : cont: : SetLogLevelName function. (The log name can likewise be retrieved with vtkm: : cont: : GetLogLevelName.) 


\section{Common Errors}

The SetLogLevelName function must be called before vtkm: :cont: : Initialize to have an effect.

\section{Common Errors}

The descriptions for each log level are only set up if vtkm: :cont: : Initialize is called. If it is not, then all log levels will be represented with a numerical value.

If vtkm: :cont: : Initialize is called with argc/argv, then the user can control the logging level with the "vtkm-log-level" command line argument. Alternatively, you can control which logging levels are reported with the vtkm: : cont: : SetStderrLogLevel.

Example 20.1: Initializing logging.

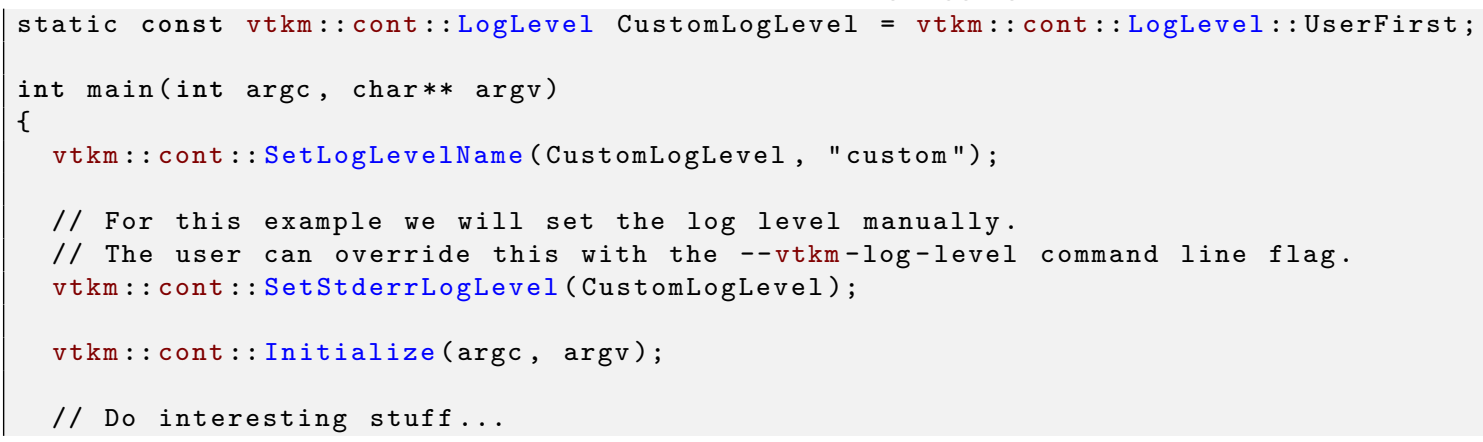

\subsection{Log Entries}

Log entries are created with a collection of macros provided in vtkm/cont/Logging.h. In addition to basic log entries, VTK-m logging can also provide conditional logging, scope levels of logs, and generate special logs on crashes.

\subsubsection{Basic Log Entries}

The main logging entry points are the macros VTKM_LOG_S and VTKM _LOG_F, which use $\mathrm{C}++$ stream and printf syntax, respectively. Both macros take a logging level as the first argument. The remaining arguments specify the message printed to the log. VTKM_LOG_S takes a single argument with a $\mathrm{C}++$ stream expression (so $<<$ operators can exist in the expression). VTKM_LOG_F takes a $\mathrm{C}$ string as its second argument that has printf-style formatting codes. The remaining arguments fulfill those codes.

Example 20.2: Basic logging.

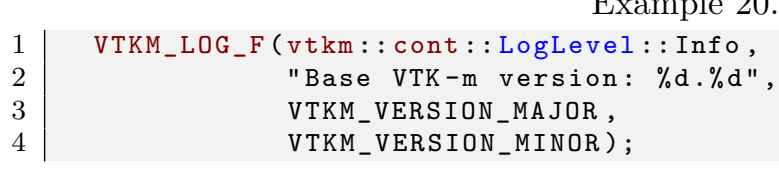


VTKM_LOG_S (vtkm: : cont : : LogLevel : : Info,

6 "Full VTK-m version: " $<$ VTKM_VERSION_FULL);

\subsubsection{Conditional Log Entries}

The macros VTKM_LOG_IF_S VTKM_LOG_IF_F behave similarly to VTKM_LOG_S and VTKM_LOG_F, respectively, except they have an extra argument that contains the condition. If the condition is true, then the log entry is created. If the condition is false, then the statement is ignored and nothing is recorded in the log.

Example 20.3: Conditional logging.

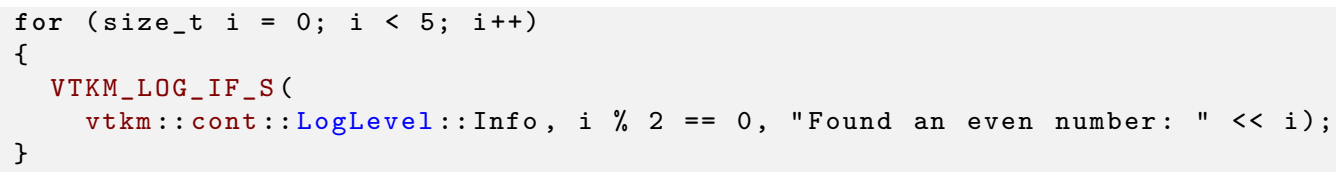

\subsubsection{Scoped Log Entries}

The logging back end supports the concept of scopes. Scopes allow the nesting of log messages, which allows a complex operation to report when it starts, when it ends, and what log messages happen in the middle. Scoped log entries are also timed so you can get an idea of how long operations take. Scoping can happen to arbitrary depths.

\section{Common Errors}

Although the timing reported in scoped log entries can give an idea of the time each operation takes, the reported time should not be considered accurate in regards to timing parallel operations. If a parallel algorithm is invoked inside a log scope, the program may return from that scope before the parallel algorithm is complete. See Chapter 13 for information on more accurate timers.

Scoped log entries follow the same scoping of your C++ code. A scoped log can be created with the VTKM_LOG_SCOPE macro. This macro behaves similarly to VTKM_LOG_F except that it creates a scoped log that starts when VTKM_LOG_SCOPE and ends when the program leaves the given scope.

Example 20.4: Scoped logging.

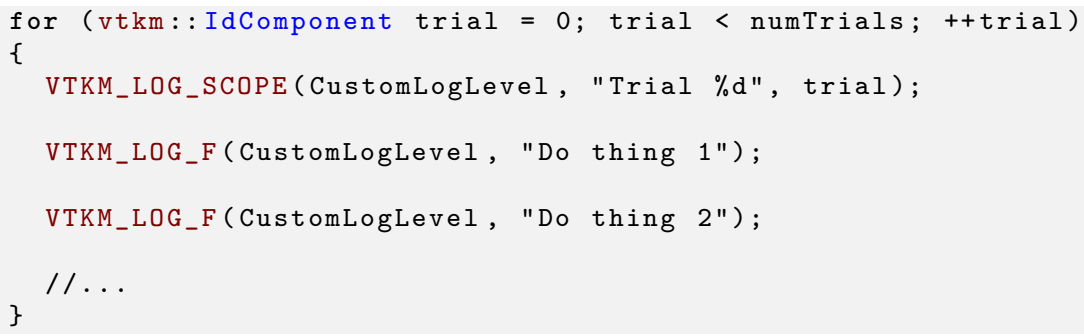

It is also common, and typically good code structure, to structure scoped concepts around functions or methods. Thus, VTK-m provides VTKM_LOG_SCOPE_FUNCTION. When placed at the beginning of a function or macro, VTKM_LOG_SCOPE_FUNCTION will automatically create a scoped log around it. 


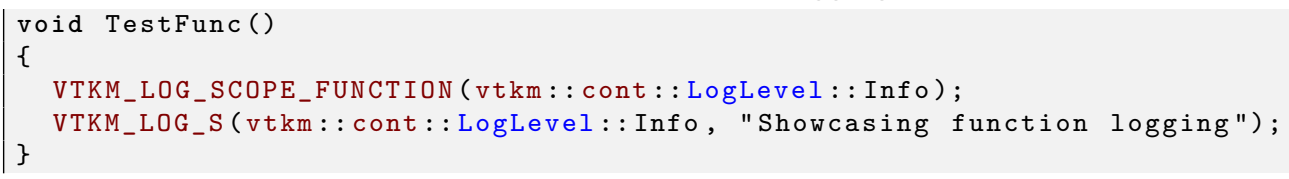

Example 20.5: Scoped logging in a function.

\subsubsection{Error Context}

The VTK-m logging is capable of capturing some crashes and writing information to the log before the program terminates. The VTKM_LOG_ERROR_CONTEXT can be used to record some information that should be reported if an error occurs. If the program terminates successfully, then information is never recorded to the log.

Example 20.6: Providing an error context for logging.

1 // This message is only logged if a crash occurs

2 VTKM_LOG_ERROR_CONTEXT("Some variable value", 42);

\subsection{Helper Functions}

The vtkm/cont/Logging.h header file also contains several helper functions that provide useful functions when reporting information about the system.

\section{(8) Did you know?}

\ Although provided with the logging utilities, these functions can be useful in contexts outside of the logging as well. These functions are available even if VTK-m is compiled with logging off.

The vtkm: : cont: :TypeToString function provides run-time type information (RTTI) based type-name information. TypeToString is a templated function for which you have to explicitly declare the type. TypeToString returns a std: : string containing a representation of the type provided. When logging is enabled, TypeToString uses the logging back end to demangle symbol names on supported platforms.

The vtkm: : cont: :GetHumanReadableSize takes a size of memory in bytes and returns a human readable string (for example "64 bytes", "1.44 MiB", "128 GiB", etc). vtkm: : cont: :GetSizeString is a similar function that returns the same thing as GetHumanReadableSize followed by "(\# bytes)" (with \# replaced with the number passed to the function). Both GetHumanReadableSize and GetSizeString take an optional second argument that is the number of digits of precision to display. By default, they display 2 digits of precision.

The vtkm: : cont: :GetStackTrace function returns a string containing a trace of the stack, which can be helpful for debugging. GetStackTrace takes an optional argument for the number of stack frames to skip. Reporting the stack trace is not available on all platforms. On platforms that are not supported, a simple string reporting that the stack trace is unavailable is returned.

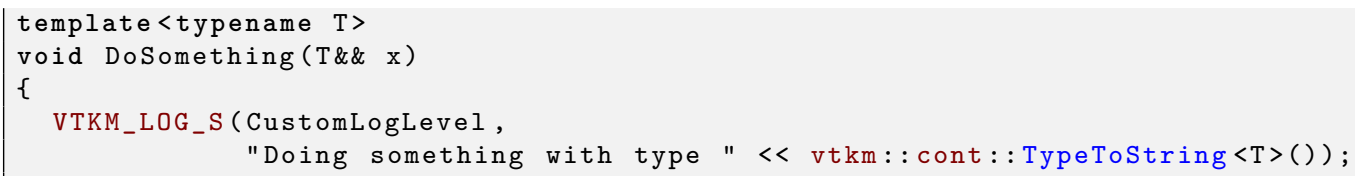




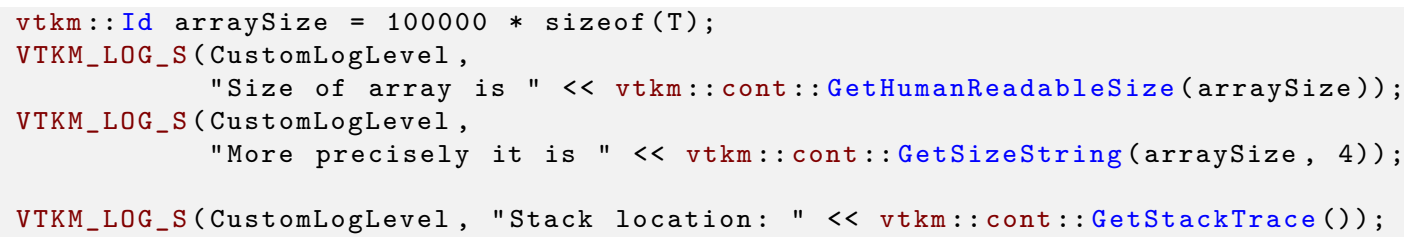




\section{WORKLET TYPE REFERENCE}

Chapter 17 introduces worklets and provides a simple example of creating a worklet to run an algorithm on a many core device. Different operations in visualization can have different data access patterns, perform different execution flow, and require different provisions. VTK-m manages these different accesses, execution, and provisions by grouping visualization algorithms into common classes of operation and supporting each class with its own worklet type.

Each worklet type has a generic superclass that worklets of that particular type must inherit. This makes the type of the worklet easy to identify. The following list describes each worklet type provided by VTK-m and the superclass that supports it.

Field Map A worklet deriving vtkm: :worklet: WorkletMapField performs a basic mapping operation that applies a function (the operator in the worklet) on all the field values at a single point or cell and creates a new field value at that same location. Although the intention is to operate on some variable over a mesh, a WorkletMapField may actually be applied to any array. Thus, a field map can be used as a basic map operation.

Topology Map A worklet deriving vtkm: :worklet: :WorkletMapTopology or one of its child classes performs a mapping operation that applies a function (the operator in the worklet) on all elements of a particular type (such as points or cells) and creates a new field for those elements. The basic operation is similar to a field map except that in addition to access fields being mapped on, the worklet operation also has access to incident fields.

There are multiple convenience classes available for the most common types of topology mapping. vtkm: :worklet: :WorkletVisitCellsWithPoints calls the worklet operation for each cell and makes every incident point available. This type of map also has access to cell structures and can interpolate point fields. Likewise, vtkm: : worklet: :WorkletVisitPointsWithCells calls the worklet operation for each point and makes every incident cell available.

Point Neighborhood A worklet deriving from vtkm::worklet::WorkletPointNeighborhood performs a mapping operation that applies a function (the operator in the worklet) on all points of a structured mesh. The basic operation is similar to a field map except that in addition to having access to the point being operated on, you can get the field values of nearby points within a neighborhood of a given size. Point neighborhood worklets can only applied to structured cell sets.

Reduce by Key A worklet deriving vtkm: :worklet: :WorkletReduceByKey operates on an array of keys and one or more associated arrays of values. When a reduce by key worklet is invoked, all identical keys are collected and the worklet is called once for each unique key. Each worklet invocation is given a Vec-like containing all values associated with the unique key. Reduce by key worklets are very useful for combining like items such as shared topology elements or coincident points. 
The remainder of this chapter provides details on how to create worklets of each type. It is also possible to create new worklet types in VTK-m. This is an advanced topic covered in Chapter 41.

\subsection{Field Map}

A worklet deriving vtkm: : worklet: :WorkletMapField performs a basic mapping operation that applies a function (the operator in the worklet) on all the field values at a single point or cell and creates a new field value at that same location. Although the intention is to operate on some variable over the mesh, a WorkletMapField can actually be applied to any array.

A field map worklet supports the following tags in the parameters of its Controlsignature.

FieldIn This tag represents an input field. A FieldIn argument expects an ArrayHandle or an UnknownArrayHandle in the associated parameter of the Invoker. Each invocation of the worklet gets a single value out of this array.

The worklet's InputDomain can be set to a FieldIn argument. In this case, the input domain will be the size of the array.

FieldOut This tag represents an output field. A FieldOut argument expects an ArrayHandle or an UnknownArrayHandle in the associated parameter of the Invoker. The array is resized before scheduling begins, and each invocation of the worklet sets a single value in the array.

FieldInOut This tag represents field that is both an input and an output. A FieldInOut argument expects an ArrayHandle or an UnknownArrayHandle in the associated parameter of the Invoker. Each invocation of the worklet gets a single value out of this array, which is replaced by the resulting value after the worklet completes.

The worklet's InputDomain can be set to a FieldInOut argument. In this case, the input domain will be the size of the array.

WholeArrayIn This tag represents an array where all entries can be read by every worklet invocation. A WholeArrayIn argument expects an ArrayHandle in the associated parameter of the Invoker. An array portal capable of reading from any place in the array is given to the worklet. Whole arrays are discussed in detail in Section 28.1 starting on page 237.

WholeArrayOut This tag represents an array where any entry can be written by any worklet invocation. A WholeArrayOut argument expects an ArrayHandle in the associated parameter of the Invoker. An array portal capable of writing to any place in the array is given to the worklet. Developers should take care when using writable whole arrays as introducing race conditions is possible. Whole arrays are discussed in detail in Section 28.1 starting on page 237.

WholeArrayInOut This tag represents an array where any entry can be read or written by any worklet invocation. A WholeArrayInOut argument expects an ArrayHandle in the associated parameter of the Invoker. An array portal capable of reading from or writing to any place in the array is given to the worklet. Developers should take care when using writable whole arrays as introducing race conditions is possible. Whole arrays are discussed in detail in Section 28.1 starting on page 237.

AtomicArrayInOut This tag represents an array where any entry can be read or written by any worklet invocation. A AtomicArrayInOut argument expects an ArrayHandle in the associated parameter of the Invoker. A vtkm: :exec: :AtomicArray object capable of performing atomic operations to the entries in the array is given to the worklet. Atomic arrays can help avoid race conditions but can slow down the running of a parallel algorithm. Atomic arrays are discussed in detail in Section 28.2 starting on page 239. 
WholeCellSetIn This tag represents the connectivity of a cell set. A WholeCellSetIn argument expects a vtkm: : cont: :Cellset in the associated parameter of the Invoker. A connectivity object capable of finding elements of one type that are incident on elements of a different type. Accessing whole cell set connectivity is discussed in detail in Section 28.3.

ExecObject This tag represents an execution object that is passed directly from the control environment to the worklet. A ExecObject argument expects a subclass of vtkm: :exec: :ExecutionObjectBase. Subclasses of ExecutionObjectBase behave like a factory for objects that work on particular devices. They do this by implementing a PrepareForExecution method that takes a device adapter tag and returns an object that works on that device. That device-specific object is passed directly to the worklet. Execution objects are discussed in detail in Section 29 starting on page 245.

A field map worklet supports the following tags in the parameters of its ExecutionSignature.

$\_1, \_2, \ldots$ These reference the corresponding parameter in the Controlsignature.

WorkIndex This tag produces a vtkm: : Id that uniquely identifies the invocation of the worklet.

VisitIndex This tag produces a vtkm: : IdComponent that uniquely identifies when multiple worklet invocations operate on the same input item, which can happen when defining a worklet with scatter (as described in Section 31.1).

InputIndex This tag produces a vtkm: : Id that identifies the index of the input element, which can differ from the WorkIndex in a worklet with a scatter (as described in Section 31.1).

OutputIndex This tag produces a vtkm: Id that identifies the index of the output element. (This is generally the same as WorkIndex.)

ThreadIndices This tag produces an internal object that manages indices and other metadata of the current thread. Thread indices objects are described in Section 41.2, but most users can get the information they need through other signature tags.

Field maps most commonly perform basic calculator arithmetic, as demonstrated in the following example.

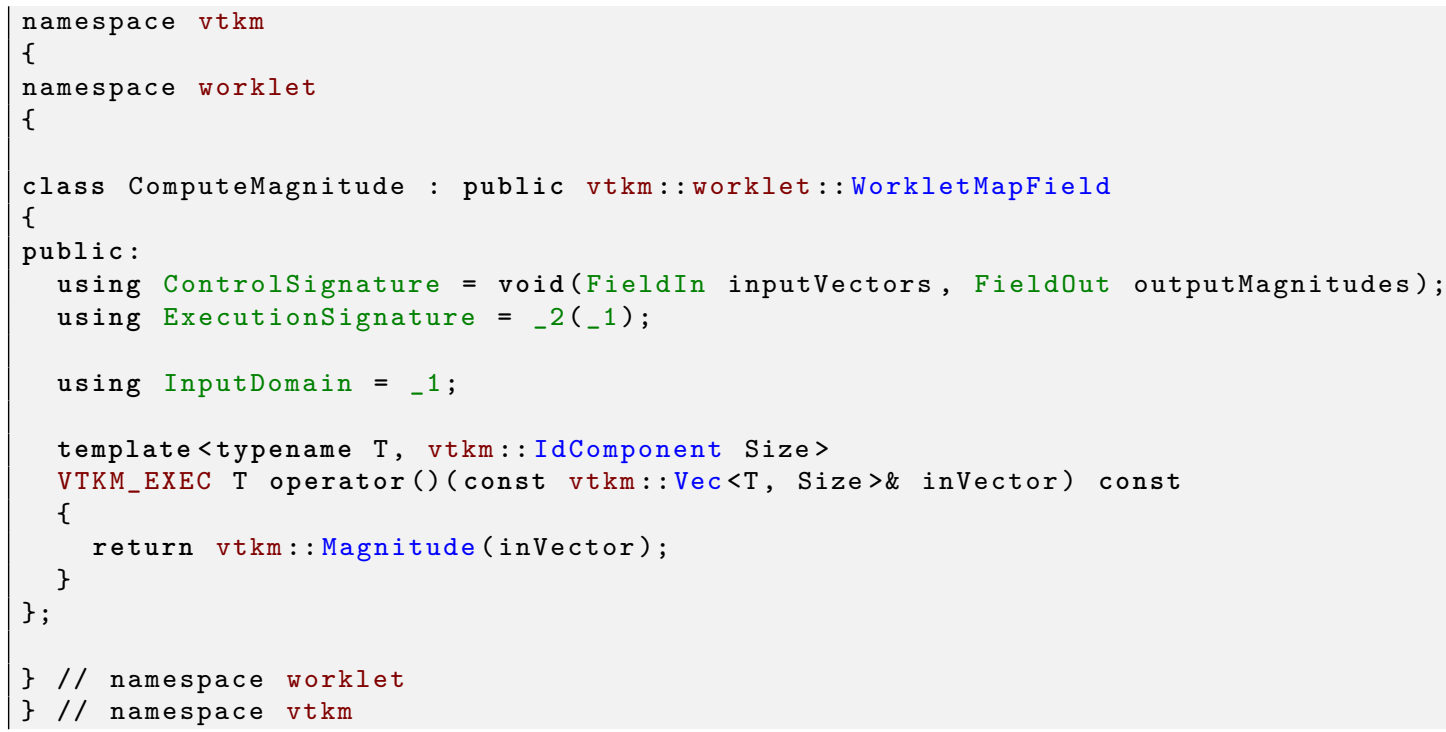


Although simple, the WorkletMapField worklet type can be used (and abused) as a general parallelfor/scheduling mechanism. In particular, the WorkIndex execution signature tag can be used to get a unique index, the WholeArray* tags can be used to get random access to arrays, and the ExecObject control signature tag can be used to pass execution objects directly to the worklet. Whole arrays and execution objects are talked about in more detail in Chapters 28 and 29, respectively, in more detail, but here is a simple example that uses the random access of WholeArrayOut to make a worklet that copies an array in reverse order.

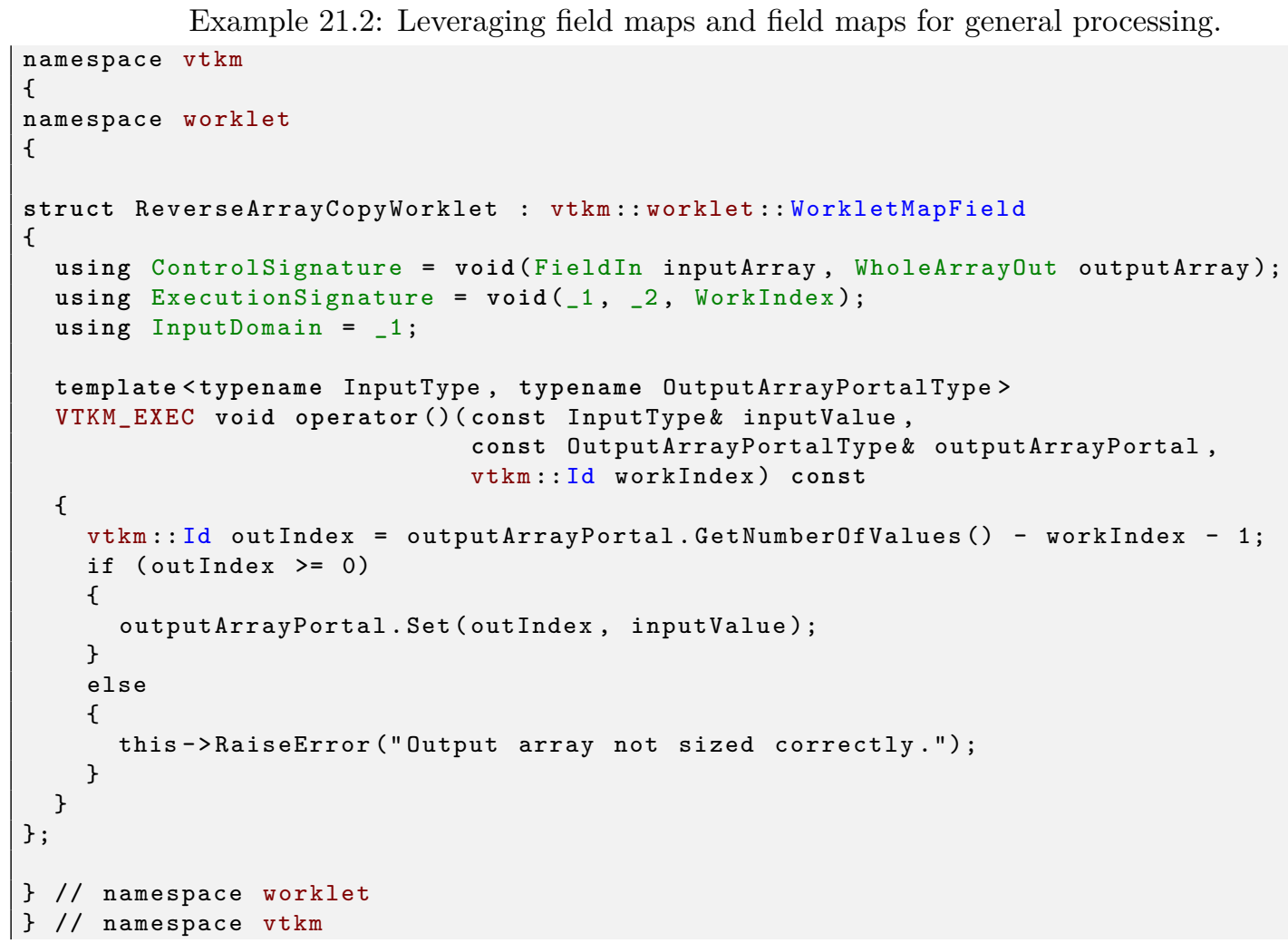

\subsection{Topology Map}

A topology map performs a mapping that it applies a function (the operator in the worklet) on all the elements of a DataSet of a particular type (i.e. point, edge, face, or cell). While operating on the element, the worklet has access to data from all incident elements of another type.

There are several versions of topology maps that differ in what type of element being mapped from and what type of element being mapped to. The subsequent sections describe these different variations of the topology maps.

\subsubsection{Visit Cells with Points}

A worklet deriving vtkm: : worklet: :WorkletVisitCellsWithPoints performs a mapping operation that applies a function (the operator in the worklet) on all the cells of a DataSet. While operating on the cell, the worklet has access to fields associated both with the cell and with all incident points. Additionally, the worklet can get information about the structure of the cell and can perform operations like interpolation on it. 
A visit cells with points worklet supports the following tags in the parameters of its ControlSignature.

CellSetIn This tag represents the cell set that defines the collection of cells the map will operate on. A CellSetIn argument expects a CellSet subclass or a DynamicCellset in the associated parameter of the Invoker. Each invocation of the worklet gets a cell shape tag. (Cell shapes and the operations you can do with cells are discussed in Chapter 25.)

There must be exactly one CellSetIn argument, and the worklet's InputDomain must be set to this argument.

FieldInPoint This tag represents an input field that is associated with the points. A FieldinPoint argument expects an ArrayHandle or an UnknownArrayHandle in the associated parameter of the Invoker. The size of the array must be exactly the number of points.

Each invocation of the worklet gets a Vec-like object containing the field values for all the points incident with the cell being visited. The order of the entries is consistent with the defined order of the vertices for the visited cell's shape. If the field is a vector field, then the provided object is a Vec of Vecs.

FieldInCell This tag represents an input field that is associated with the cells. A FieldInCell argument expects an ArrayHandle or an UnknownArrayHandle in the associated parameter of the Invoker. The size of the array must be exactly the number of cells. Each invocation of the worklet gets a single value out of this array.

FieldOutCell This tag represents an output field, which is necessarily associated with cells. A FieldOutCell argument expects an ArrayHandle or an UnknownArrayHandle in the associated parameter of the Invoker. The array is resized before scheduling begins, and each invocation of the worklet sets a single value in the array.

FieldOut is an alias for FieldOutCell (since output arrays can only be defined on cells).

FieldInOutCell This tag represents field that is both an input and an output, which is necessarily associated with cells. A FieldInOutCell argument expects an ArrayHandle or an UnknownArrayHandle in the associated parameter of the Invoker. Each invocation of the worklet gets a single value out of this array, which is replaced by the resulting value after the worklet completes.

FieldInOut is an alias for FieldInOutCell (since output arrays can only be defined on cells).

WholeArrayIn This tag represents an array where all entries can be read by every worklet invocation. A WholeArrayIn argument expects an ArrayHandle in the associated parameter of the Invoker. An array portal capable of reading from any place in the array is given to the worklet. Whole arrays are discussed in detail in Section 28.1 starting on page 237.

WholeArrayOut This tag represents an array where any entry can be written by any worklet invocation. A WholeArrayOut argument expects an ArrayHandle in the associated parameter of the Invoker. An array portal capable of writing to any place in the array is given to the worklet. Developers should take care when using writable whole arrays as introducing race conditions is possible. Whole arrays are discussed in detail in Section 28.1 starting on page 237.

WholeArrayInOut This tag represents an array where any entry can be read or written by any worklet invocation. A WholeArrayInOut argument expects an ArrayHandle in the associated parameter of the Invoker. An array portal capable of reading from or writing to any place in the array is given to the worklet. Developers should take care when using writable whole arrays as introducing race conditions is possible. Whole arrays are discussed in detail in Section 28.1 starting on page 237.

AtomicArrayInOut This tag represents an array where any entry can be read or written by any worklet invocation. A AtomicArrayInOut argument expects an ArrayHandle in the associated parameter of the Invoker. 
A vtkm: :exec: :AtomicArray object capable of performing atomic operations to the entries in the array is given to the worklet. Atomic arrays can help avoid race conditions but can slow down the running of a parallel algorithm. Atomic arrays are discussed in detail in Section 28.2 starting on page 239.

WholeCellSetIn This tag represents the connectivity of a cell set. A WholeCellSetIn argument expects a vtkm: :cont::Cellset in the associated parameter of the Invoker. A connectivity object capable of finding elements of one type that are incident on elements of a different type. Accessing whole cell set connectivity is discussed in detail in Section 28.3.

ExecObject This tag represents an execution object that is passed directly from the control environment to the worklet. A ExecObject argument expects a subclass of vtkm: :exec: :ExecutionObjectBase. Subclasses of ExecutionObjectBase behave like a factory for objects that work on particular devices. They do this by implementing a PrepareForExecution method that takes a device adapter tag and returns an object that works on that device. That device-specific object is passed directly to the worklet. Execution objects are discussed in detail in Section 29 starting on page 245.

A visit cells with points worklet supports the following tags in the parameters of its ExecutionSignature.

$\_1, \ldots 2, \ldots$ These reference the corresponding parameter in the ControlSignature.

CellShape This tag produces a shape tag corresponding to the shape of the visited cell. (Cell shapes and the operations you can do with cells are discussed in Chapter 25.) This is the same value that gets provided if you reference the CellSetIn parameter.

PointCount This tag produces a vtkm: IdComponent equal to the number of points incident on the cell being visited. The Vecs provided from a FieldInPoint parameter will be the same size as PointCount.

PointIndices This tag produces a Vec-like object of vtkm: :Id s giving the indices for all incident points. Like values from a FieldInPoint parameter, the order of the entries is consistent with the defined order of the vertices for the visited cell's shape.

WorkIndex This tag produces a vtkm: : Id that uniquely identifies the invocation of the worklet.

VisitIndex This tag produces a vtkm: : IdComponent that uniquely identifies when multiple worklet invocations operate on the same input item, which can happen when defining a worklet with scatter (as described in Section 31.1).

InputIndex This tag produces a vtkm: :Id that identifies the index of the input element, which can differ from the WorkIndex in a worklet with a scatter (as described in Section 31.1).

OutputIndex This tag produces a vtkm: : Id that identifies the index of the output element. (This is generally the same as WorkIndex.)

Threadindices This tag produces an internal object that manages indices and other metadata of the current thread. Thread indices objects are described in Section 41.2, but most users can get the information they need through other signature tags.

Point to cell field maps are a powerful construct that allow you to interpolate point fields throughout the space of the data set. See Chapter 25 for a description on how to work with the cell information provided to the worklet. The following example provides a simple demonstration that finds the geometric center of each cell by interpolating the point coordinates to the cell centers. 


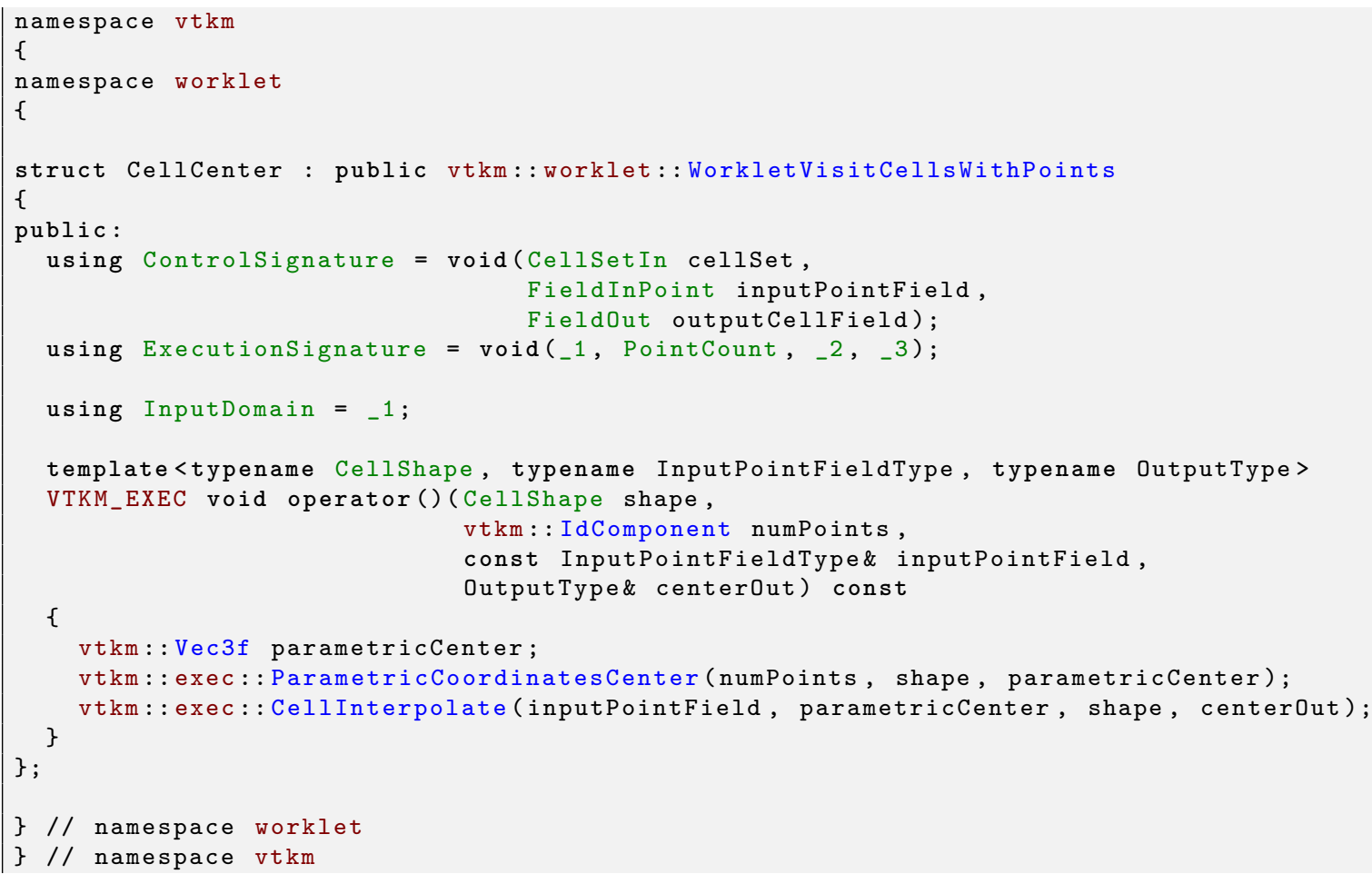

\subsubsection{Visit Points with Cells}

A worklet deriving vtkm: : worklet: :WorkletVisitPointsWithCells performs a mapping operation that applies a function (the operator in the worklet) on all the points of a DataSet. While operating on the point, the worklet has access to fields associated both with the point and with all incident cells.

A visit points with cells worklet supports the following tags in the parameters of its Controlsignature.

CellSetIn This tag represents the cell set that defines the collection of points the map will operate on. A CellSetIn argument expects a Cellset subclass or a DynamicCellset in the associated parameter of the Invoker.

There must be exactly one CellSetIn argument, and the worklet's InputDomain must be set to this argument.

FieldInCell This tag represents an input field that is associated with the cells. A FieldInCell argument expects an ArrayHandle or an UnknownArrayHandle in the associated parameter of the Invoker. The size of the array must be exactly the number of cells.

Each invocation of the worklet gets a Vec-like object containing the field values for all the cells incident with the point being visited. The order of the entries is arbitrary but will be consistent with the values of all other FieldInCell arguments for the same worklet invocation. If the field is a vector field, then the provided object is a Vec of Vecs.

FieldInPoint This tag represents an input field that is associated with the points. A FieldInPoint argument expects an ArrayHandle or an UnknownArrayHandle in the associated parameter of the Invoker. The size of the array must be exactly the number of points. Each invocation of the worklet gets a single value out of this array. 
FieldOutPoint This tag represents an output field, which is necessarily associated with points. A FieldOutPoint argument expects an ArrayHandle or an UnknownArrayHandle in the associated parameter of the Invoker. The array is resized before scheduling begins, and each invocation of the worklet sets a single value in the array.

FieldOut is an alias for FieldOutPoint (since output arrays can only be defined on points).

FieldInOutPoint This tag represents field that is both an input and an output, which is necessarily associated with points. A FieldInOutPoint argument expects an ArrayHandle or an UnknownArrayHandle in the associated parameter of the Invoker. Each invocation of the worklet gets a single value out of this array, which is replaced by the resulting value after the worklet completes.

FieldInOut is an alias for FieldInOutPoint (since output arrays can only be defined on points).

WholeArrayIn This tag represents an array where all entries can be read by every worklet invocation. A WholeArrayIn argument expects an ArrayHandle in the associated parameter of the Invoker. An array portal capable of reading from any place in the array is given to the worklet. Whole arrays are discussed in detail in Section 28.1 starting on page 237.

WholeArrayOut This tag represents an array where any entry can be written by any worklet invocation. A WholeArrayOut argument expects an ArrayHandle in the associated parameter of the Invoker. An array portal capable of writing to any place in the array is given to the worklet. Developers should take care when using writable whole arrays as introducing race conditions is possible. Whole arrays are discussed in detail in Section 28.1 starting on page 237.

WholeArrayInOut This tag represents an array where any entry can be read or written by any worklet invocation. A WholeArrayInOut argument expects an ArrayHandle in the associated parameter of the Invoker. An array portal capable of reading from or writing to any place in the array is given to the worklet. Developers should take care when using writable whole arrays as introducing race conditions is possible. Whole arrays are discussed in detail in Section 28.1 starting on page 237.

AtomicArrayInOut This tag represents an array where any entry can be read or written by any worklet invocation. A AtomicArrayInOut argument expects an ArrayHandle in the associated parameter of the Invoker. A vtkm: :exec: :AtomicArray object capable of performing atomic operations to the entries in the array is given to the worklet. Atomic arrays can help avoid race conditions but can slow down the running of a parallel algorithm. Atomic arrays are discussed in detail in Section 28.2 starting on page 239.

WholeCellSetIn This tag represents the connectivity of a cell set. A WholeCellsetIn argument expects a vtkm: :cont: Cellset in the associated parameter of the Invoker. A connectivity object capable of finding elements of one type that are incident on elements of a different type. Accessing whole cell set connectivity is discussed in detail in Section 28.3.

ExecObject This tag represents an execution object that is passed directly from the control environment to the worklet. A ExecObject argument expects a subclass of vtkm: :exec: :ExecutionObjectBase. Subclasses of ExecutionObjectBase behave like a factory for objects that work on particular devices. They do this by implementing a PrepareForExecution method that takes a device adapter tag and returns an object that works on that device. That device-specific object is passed directly to the worklet. Execution objects are discussed in detail in Section 29 starting on page 245.

A visit points with cells worklet supports the following tags in the parameters of its ExecutionSignature.

$\_1, \_2, \ldots$ These reference the corresponding parameter in the ControlSignature.

CellCount This tag produces a vtkm: IdComponent equal to the number of cells incident on the point being visited. The Vecs provided from a FieldInCell parameter will be the same size as CellCount. 
CellIndices This tag produces a Vec-like object of vtkm: :Id s giving the indices for all incident cells. The order of the entries is arbitrary but will be consistent with the values of all other FieldInCell arguments for the same worklet invocation.

WorkIndex This tag produces a vtkm: : Id that uniquely identifies the invocation of the worklet.

VisitIndex This tag produces a vtkm: : IdComponent that uniquely identifies when multiple worklet invocations operate on the same input item, which can happen when defining a worklet with scatter (as described in Section 31.1).

InputIndex This tag produces a vtkm: : Id that identifies the index of the input element, which can differ from the WorkIndex in a worklet with a scatter (as described in Section 31.1).

OutputIndex This tag produces a vtkm: : Id that identifies the index of the output element. (This is generally the same as WorkIndex.)

ThreadIndices This tag produces an internal object that manages indices and other metadata of the current thread. Thread indices objects are described in Section 41.2, but most users can get the information they need through other signature tags.

Cell to point field maps are typically used for converting fields associated with cells to points so that they can be interpolated. The following example does a simple averaging, but you can also implement other strategies such as a volume weighted average.

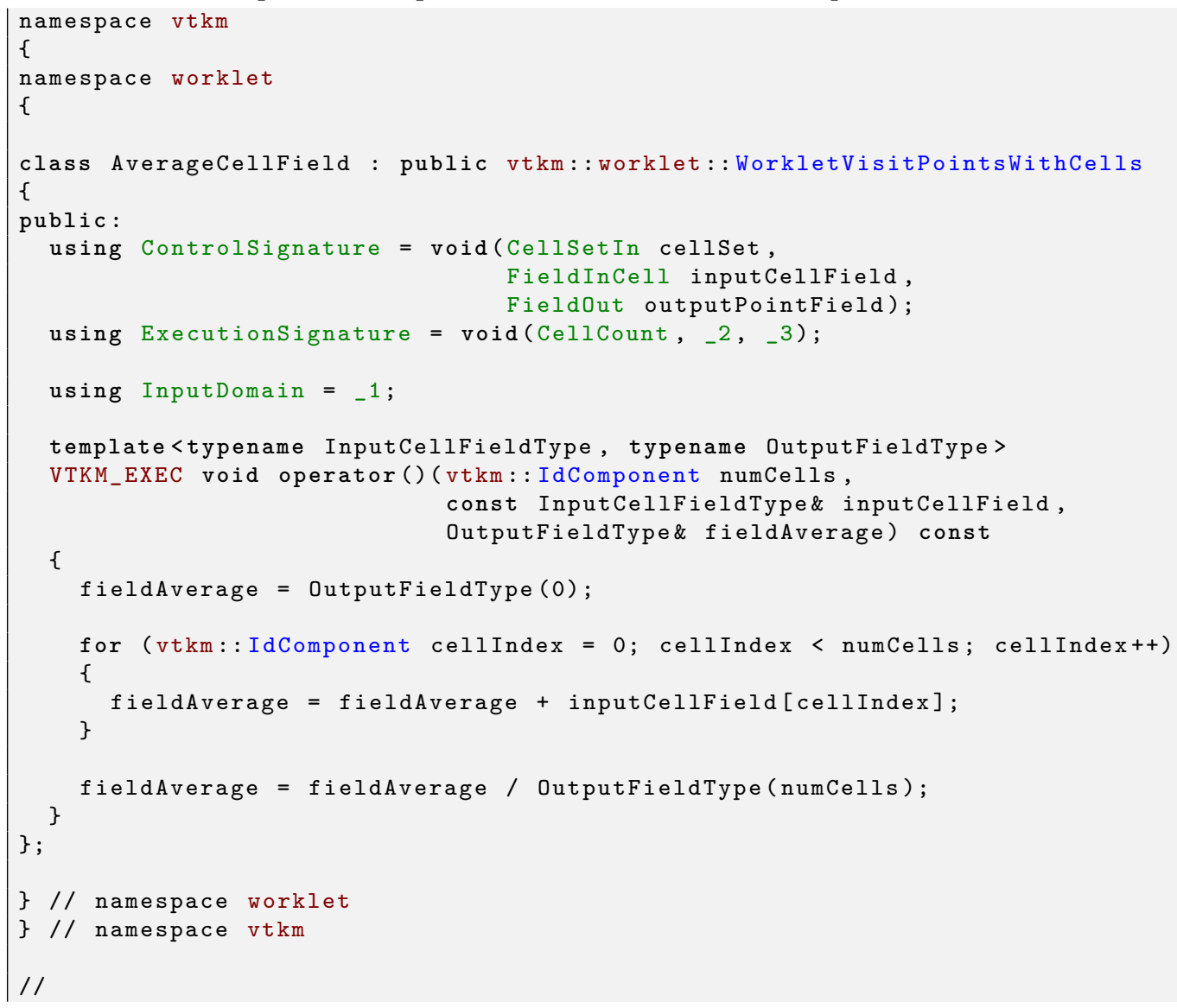




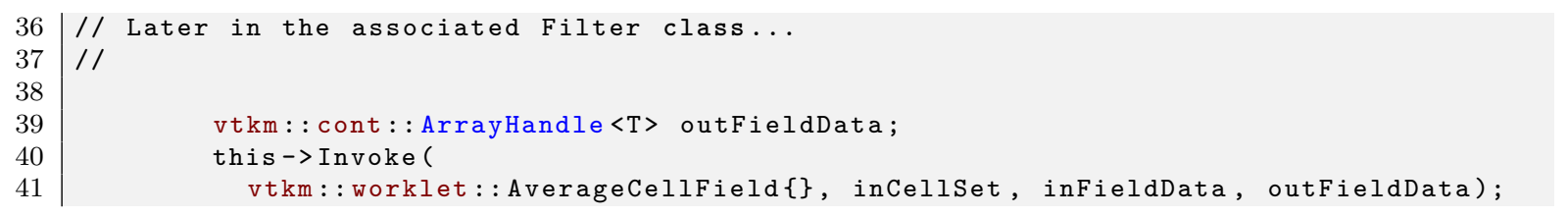

\subsubsection{General Topology Maps}

A worklet deriving vtkm: : worklet: :WorkletMapTopology performs a mapping operation that applies a function (the operator in the worklet) on all the elements of a specified type from a DataSet. While operating on each element, the worklet has access to fields associated both with that element and with all incident elements of a different specified type.

The WorkletMapTopology class is a template with two template parameters. The first template parameter specifies the "visit" topology element, and the second parameter specifies the "incident" topology element. The worklet is scheduled such that each instance is associated with a particular "visit" topology element and has access to "incident" topology elements.

These visit and incident topology elements are specified with topology element tags, which are defined in the vtkm/TopologyElementTag.h header file. The available topology element tags are vtkm: :TopologyElementTagCell, vtkm: :TopologyElementTagPoint, vtkm: :TopologyElementTagEdge, and vtkm: :TopologyElementTagFace, which represent the cell, point, edge, and face elements, respectively.

WorkletMapTopology is a generic form of a topology map, and it can perform identically to the aforementioned forms of topology map with the correct template parameters. For example,

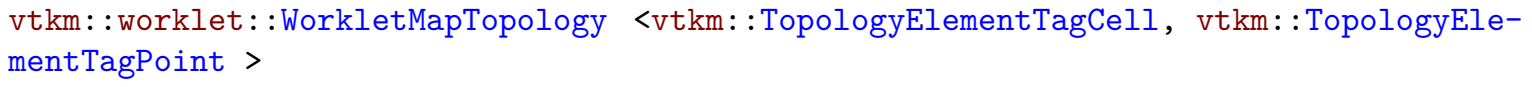

is equivalent to the vtkm: : worklet: :WorkletVisitCellsWithPoints class except the signature tags have different names. The names used in the specific topology map superclasses (such as WorkletVisitCellsWithPoints) tend to be easier to read and are thus preferable. However, the generic WorkletMapTopology is available for topology combinations without a specific superclass or to support more general mappings in a worklet.

The general topology map worklet supports the following tags in the parameters of its Controlsignature, which are equivalent to tags in the other topology maps but with different (more general) names.

CellSetIn This tag represents the cell set that defines the collection of elements the map will operate on. A CellSetIn argument expects a Cellset subclass or a DynamicCellset in the associated parameter of the Invoker. Each invocation of the worklet gets a cell shape tag. (Cell shapes and the operations you can do with cells are discussed in Chapter 25.)

There must be exactly one CellSetIn argument, and the worklet's InputDomain must be set to this argument.

FieldInVisit This tag represents an input field that is associated with the "visit" element. A FieldInVisit argument expects an ArrayHandle or an UnknownArrayHandle in the associated parameter of the Invoker. The size of the array must be exactly the number of cells. Each invocation of the worklet gets a single value out of this array.

FieldInIncident This tag represents an input field that is associated with the "incident" elements. A FieldInIncident argument expects an ArrayHandle or an UnknownArrayHandle in the associated parameter of the Invoker. The size of the array must be exactly the number of "incident" elements. 
Each invocation of the worklet gets a Vec-like object containing the field values for all the "incident" elements incident with the "visit" element being visited. If the field is a vector field, then the provided object is a Vec of Vecs.

FieldOut This tag represents an output field, which is necessarily associated with "visit" elements. A FieldOut argument expects an ArrayHandle or an UnknownArrayHandle in the associated parameter of the Invoker. The array is resized before scheduling begins, and each invocation of the worklet sets a single value in the array.

FieldInOut This tag represents field that is both an input and an output, which is necessarily associated with "visit" elements. A FieldInOut argument expects an ArrayHandle or an UnknownArrayHandle in the associated parameter of the Invoker. Each invocation of the worklet gets a single value out of this array, which is replaced by the resulting value after the worklet completes.

WholeArrayIn This tag represents an array where all entries can be read by every worklet invocation. A WholeArrayIn argument expects an ArrayHandle in the associated parameter of the Invoker. An array portal capable of reading from any place in the array is given to the worklet. Whole arrays are discussed in detail in Section 28.1 starting on page 237.

WholeArrayOut This tag represents an array where any entry can be written by any worklet invocation. A WholeArrayOut argument expects an ArrayHandle in the associated parameter of the Invoker. An array portal capable of writing to any place in the array is given to the worklet. Developers should take care when using writable whole arrays as introducing race conditions is possible. Whole arrays are discussed in detail in Section 28.1 starting on page 237.

WholeArrayInOut This tag represents an array where any entry can be read or written by any worklet invocation. A WholeArrayInOut argument expects an ArrayHandle in the associated parameter of the Invoker. An array portal capable of reading from or writing to any place in the array is given to the worklet. Developers should take care when using writable whole arrays as introducing race conditions is possible. Whole arrays are discussed in detail in Section 28.1 starting on page 237.

AtomicArrayInOut This tag represents an array where any entry can be read or written by any worklet invocation. A AtomicArrayInOut argument expects an ArrayHandle in the associated parameter of the Invoker. A vtkm: :exec: :AtomicArray object capable of performing atomic operations to the entries in the array is given to the worklet. Atomic arrays can help avoid race conditions but can slow down the running of a parallel algorithm. Atomic arrays are discussed in detail in Section 28.2 starting on page 239.

WholeCellSetIn This tag represents the connectivity of a cell set. A WholeCellSetIn argument expects a vtkm: :cont: :CellSet in the associated parameter of the Invoker. A connectivity object capable of finding elements of one type that are incident on elements of a different type. Accessing whole cell set connectivity is discussed in detail in Section 28.3.

ExecObject This tag represents an execution object that is passed directly from the control environment to the worklet. A ExecObject argument expects a subclass of vtkm: :exec: :ExecutionObjectBase. Subclasses of ExecutionObjectBase behave like a factory for objects that work on particular devices. They do this by implementing a PrepareForExecution method that takes a device adapter tag and returns an object that works on that device. That device-specific object is passed directly to the worklet. Execution objects are discussed in detail in Section 29 starting on page 245.

A general topology map worklet supports the following tags in the parameters of its ExecutionSignature.

$\_1, \ldots 2, \ldots$ These reference the corresponding parameter in the Controlsignature. 
CellShape This tag produces a shape tag corresponding to the shape of the visited element. (Cell shapes and the operations you can do with cells are discussed in Chapter 25.) This is the same value that gets provided if you reference the CellSetIn parameter.

If the "visit" element is cells, the CellShape clearly will match the shape of each cell. Other elements will have shapes to match their structures. Points have vertex shapes, edges have line shapes, and faces have some type of polygonal shape.

IncidentElementCount This tag produces a vtkm: IdComponent equal to the number of elements incident on the element being visited. The Vecs provided from a FieldInIncident parameter will be the same size as IncidentElementCount.

IncidentElementIndices This tag produces a Vec-like object of vtkm: : Id s giving the indices for all incident elements. The order of the entries is consistent with the values of all other FieldInIncident arguments for the same worklet invocation.

WorkIndex This tag produces a vtkm: : Id that uniquely identifies the invocation of the worklet.

VisitIndex This tag produces a vtkm: : IdComponent that uniquely identifies when multiple worklet invocations operate on the same input item, which can happen when defining a worklet with scatter (as described in Section 31.1).

InputIndex This tag produces a vtkm: : Id that identifies the index of the input element, which can differ from the WorkIndex in a worklet with a scatter (as described in Section 31.1).

OutputIndex This tag produces a vtkm: Id that identifies the index of the output element. (This is generally the same as WorkIndex.)

ThreadIndices This tag produces an internal object that manages indices and other metadata of the current thread. Thread indices objects are described in Section 41.2, but most users can get the information they need through other signature tags.

\subsection{Point Neighborhood}

A worklet deriving vtkm: : worklet: :WorkletPointNeighborhood performs a mapping operation that applies a function (the operator in the worklet) on all the points of a DataSet. While operating on the point, the worklet has access to field values on nearby points within a neighborhood.

A point neighborhood worklet supports the following tags in the parameters of its Controlsignature.

CellSetIn This tag represents the cell set that defines the collection of points the map will operate on. A CellSetIn argument expects a vtkm: :cont: :CellSetStructured object in the associated parameter of the Invoker. The object could also be stored in a DynamicCellset, but it is an error to use any object other than CellsetStructured.

There must be exactly one CellSetIn argument, and the worklet's InputDomain must be set to this argument.

FieldIn This tag represents an input field that is associated with the points. A FieldIn argument expects an ArrayHandle or an UnknownArrayHandle in the associated parameter of the Invoker. The size of the array must be exactly the number of points. Each invocation of the worklet gets a single value out of this array. 
FieldInNeighborhood This tag represents an input field that is associated with the points. A FieldInNeighborhood argument expects an ArrayHandle or an UnknownArrayHandle in the Invoker. The size of the array must be exactly the number of points.

What differentiates FieldInNeighborhood from FieldIn is that FieldInNeighborhood allows the worklet function to access the field value at the point it is visiting and the field values in the neighborhood around it. Thus, instead of getting a single value out of the array, each invocation of the worklet gets a vtkm: :exec: :FieldNeighborhood object. FieldNeighborhood objects are described in the Neighborhood Information section starting on page 172 .

FieldOut This tag represents an output field, which is necessarily associated with points. A FieldOut argument expects an ArrayHandle or an UnknownArrayHandle in the associated parameter of the Invoker. The array is resized before scheduling begins, and each invocation of the worklet sets a single value in the array.

FieldInOut This tag represents field that is both an input and an output, which is necessarily associated with points. A FieldInOut argument expects an ArrayHandle or an UnknownArrayHandle in the associated parameter of the Invoker. Each invocation of the worklet gets a single value out of this array, which is replaced by the resulting value after the worklet completes.

WholeArrayIn This tag represents an array where all entries can be read by every worklet invocation. A WholeArrayIn argument expects an ArrayHandle in the associated parameter of the Invoker. An array portal capable of reading from any place in the array is given to the worklet. Whole arrays are discussed in detail in Section 28.1 starting on page 237.

WholeArrayOut This tag represents an array where any entry can be written by any worklet invocation. A WholeArrayOut argument expects an ArrayHandle in the associated parameter of the Invoker. An array portal capable of writing to any place in the array is given to the worklet. Developers should take care when using writable whole arrays as introducing race conditions is possible. Whole arrays are discussed in detail in Section 28.1 starting on page 237.

WholeArrayInOut This tag represents an array where any entry can be read or written by any worklet invocation. A WholeArrayInOut argument expects an ArrayHandle in the associated parameter of the Invoker. An array portal capable of reading from or writing to any place in the array is given to the worklet. Developers should take care when using writable whole arrays as introducing race conditions is possible. Whole arrays are discussed in detail in Section 28.1 starting on page 237.

AtomicArrayInOut This tag represents an array where any entry can be read or written by any worklet invocation. A AtomicArrayInOut argument expects an ArrayHandle in the associated parameter of the Invoker. A vtkm: :exec: :AtomicArray object capable of performing atomic operations to the entries in the array is given to the worklet. Atomic arrays can help avoid race conditions but can slow down the running of a parallel algorithm. Atomic arrays are discussed in detail in Section 28.2 starting on page 239.

WholeCellSetIn This tag represents the connectivity of a cell set. A WholeCellsetIn argument expects a vtkm: : cont: :Cellset in the associated parameter of the Invoker. A connectivity object capable of finding elements of one type that are incident on elements of a different type. Accessing whole cell set connectivity is discussed in detail in Section 28.3.

ExecObject This tag represents an execution object that is passed directly from the control environment to the worklet. A ExecObject argument expects a subclass of vtkm: :exec: :ExecutionObjectBase. Subclasses of ExecutionObjectBase behave like a factory for objects that work on particular devices. They do this by implementing a PrepareForExecution method that takes a device adapter tag and returns an object that works on that device. That device-specific object is passed directly to the worklet. Execution objects are discussed in detail in Section 29 starting on page 245.

A point neighborhood worklet supports the following tags in the parameters of its ExecutionSignature. 
$\_1, \ldots 2, \ldots$ These reference the corresponding parameter in the ControlSignature.

Boundary This tag produces a vtkm: :exec: :arg: :BoundaryState object, which provides information about where the local neighborhood is in relationship to the full mesh. BoundaryState objects are described in the Neighborhood Information section starting on page 172.

WorkIndex This tag produces a vtkm: :Id that uniquely identifies the invocation of the worklet.

VisitIndex This tag produces a vtkm: : IdComponent that uniquely identifies when multiple worklet invocations operate on the same input item, which can happen when defining a worklet with scatter (as described in Section 31.1).

InputIndex This tag produces a vtkm: : Id that identifies the index of the input element, which can differ from the WorkIndex in a worklet with a scatter (as described in Section 31.1).

OutputIndex This tag produces a vtkm: : Id that identifies the index of the output element. (This is generally the same as WorkIndex.)

Threadindices This tag produces an internal object that manages indices and other metadata of the current thread. Thread indices objects are described in Section 41.2, but most users can get the information they need through other signature tags.

\subsubsection{Neighborhood Information}

As stated earlier in this section, what makes a WorkletPointNeighborhood worklet special is its ability to get field information in a neighborhood surrounding a point rather than just the point itself. This is done using the special FieldInNeighborhood ControlSignature tag. When you use this tag, rather than getting the single field value for the point, you get a vtkm: :exec: :FieldNeighborhood object.

The FieldNeighborhood class (which has a single template argument of the array portal type values are stored in) contains a Get method that retrieves a field value relative to the local neighborhood. FieldNeighborhood: : Get takes the $i, j, k$ index of the point with respect to the local point. So, calling FieldNeighborhood: :Get $(0,0,0)$ retrieves at the point being visited. Likewise, $\operatorname{Get}(-1,0,0)$ gets the value to the "left" of the point visited and Get $(1,0,0)$ gets the value to the "right." FieldNeighborhood: :Get is overloaded to accept the index as either three separate vtkm: : IdComponent values or a single vtkm: :Vec <vtkm: : IdComponent, 3>.

Example 21.5: Retrieve neighborhood field value.

$1 \mid$

sum $=$ sum + inputField.Get $(i, j, k)$;

When performing operations on a neighborhood within the mesh, it is often important to know whether the expected neighborhood is contained completely within the mesh or whether the neighborhood extends beyond the borders of the mesh. This can be queried using a vtkm: :exec: :BoundaryState object, which is provided when a Boundary tag is listed in the ExecutionSignature.

Generally, BoundaryState allows you to specify the size of the neighborhood at runtime. The neighborhood size is specified by a radius. The radius specifies the number of items in each direction the neighborhood extends. So, for example, a point neighborhood with radius 1 would contain a $3 \times 3 \times 3$ neighborhood centered around the point. Likewise, a point neighborhood with radius 2 would contain a $5 \times 5 \times 5$ neighborhood centered around the point. BoundaryState provides several methods to determine if the neighborhood is contained in the mesh.

MinNeighborIndices Given a radius for the neighborhood, returns a vtkm: :Vec <vtkm: : IdComponent, 3> for the "lower left" (minimum) index. If the visited point is in the middle of the mesh, the returned triplet is the negative radius for all components. But if the visited point is near the mesh boundary, then the minimum index will be clipped. 
For example, if the visited point is at $[5,5,5]$ and MinNeighborIndices (2) is called, then $[-2,-2,-2]$ is returned. However, if the visited point is at $[0,1,2]$ and MinNeighborIndices (2) is called, then $[0,-1,-2]$ is returned.

MaxNeighborIndices Given a radius for the neighborhood, returns a vtkm::Vec <vtkm: :IdComponent, 3> for the "upper right" (maximum) index. If the visited point is in the middle of the mesh, the returned triplet is the negative radius for all components. But if the visited point is near the mesh boundary, then the maximum index will be clipped.

For example, if the visited point is at $[5,5,5]$ in a $10^{3}$ mesh and MaxNeighborIndices (2) is called, then $[2,2,2]$ is returned. However, if the visited point is at $[7,8,9]$ in the same mesh and MaxNeighborIndices (2) is called, then $[2,1,0]$ is returned.

InBoundary Given a radius for the neighborhood, returns true if the neighborhood is contained completely within the boundary of the mesh, false otherwise.

InXBoundary Given a radius for the neighborhood, returns false if the neighborhood extends beyond the edge of the mesh in the positive or negative $x$ (I) direction, true otherwise.

InYBoundary Given a radius for the neighborhood, returns false if the neighborhood extends beyond the edge of the mesh in the positive or negative $y(\mathrm{~J})$ direction, true otherwise.

InZBoundary Given a radius for the neighborhood, returns false if the neighborhood extends beyond the edge of the mesh in the positive or negative $z(\mathrm{~K})$ direction, true otherwise.

The BoundaryState: :MinNeighborIndices and BoundaryState: :MaxNeighborIndices are particularly useful for iterating over the valid portion of the neighborhood.

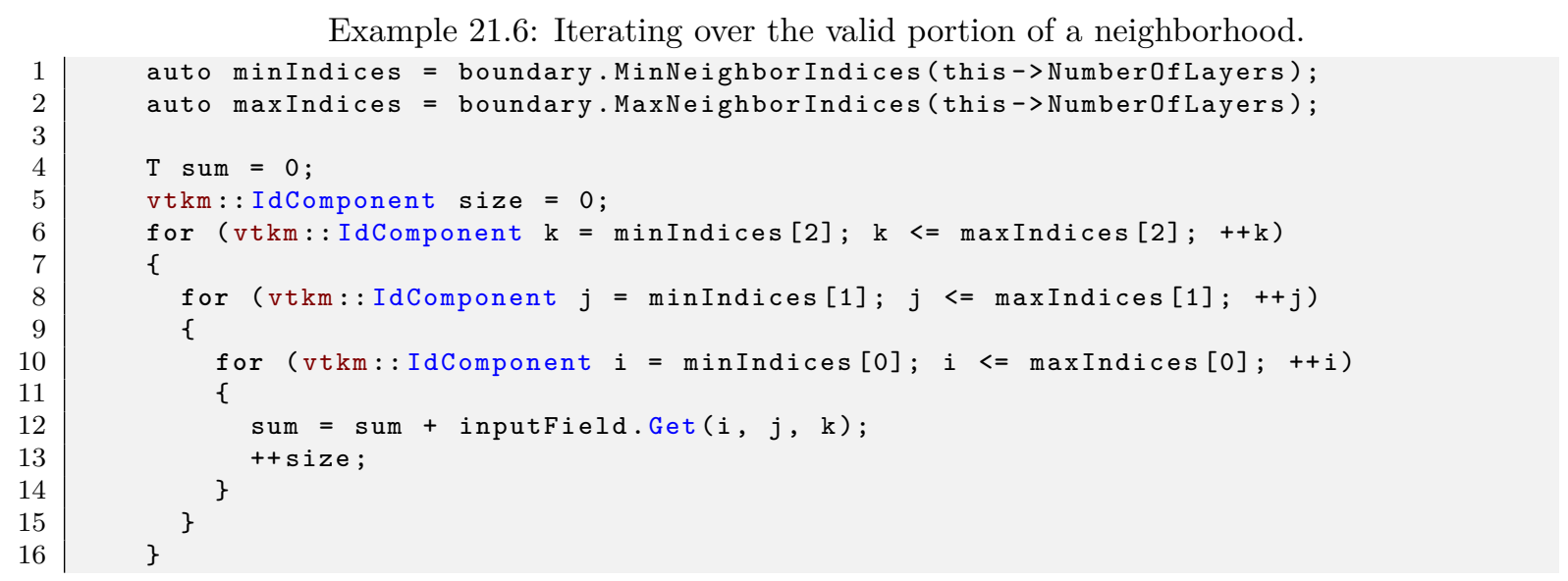

\subsubsection{Convolving Small Kernels}

A common use case for point neighborhood worklets is to convolve a small kernel with a structured mesh. A very simple example of this is averaging out the values the values within some distance to the central point. This has the effect of smoothing out the field (although smoothing filters with better properties exist). The following example shows a worklet that applies this simple "box" averaging.

Example 21.7: Implementation and use of a point neighborhood worklet.

1 class ApplyBoxKernel : public vtkm:: worklet::WorkletPointNeighborhood

2\{ 


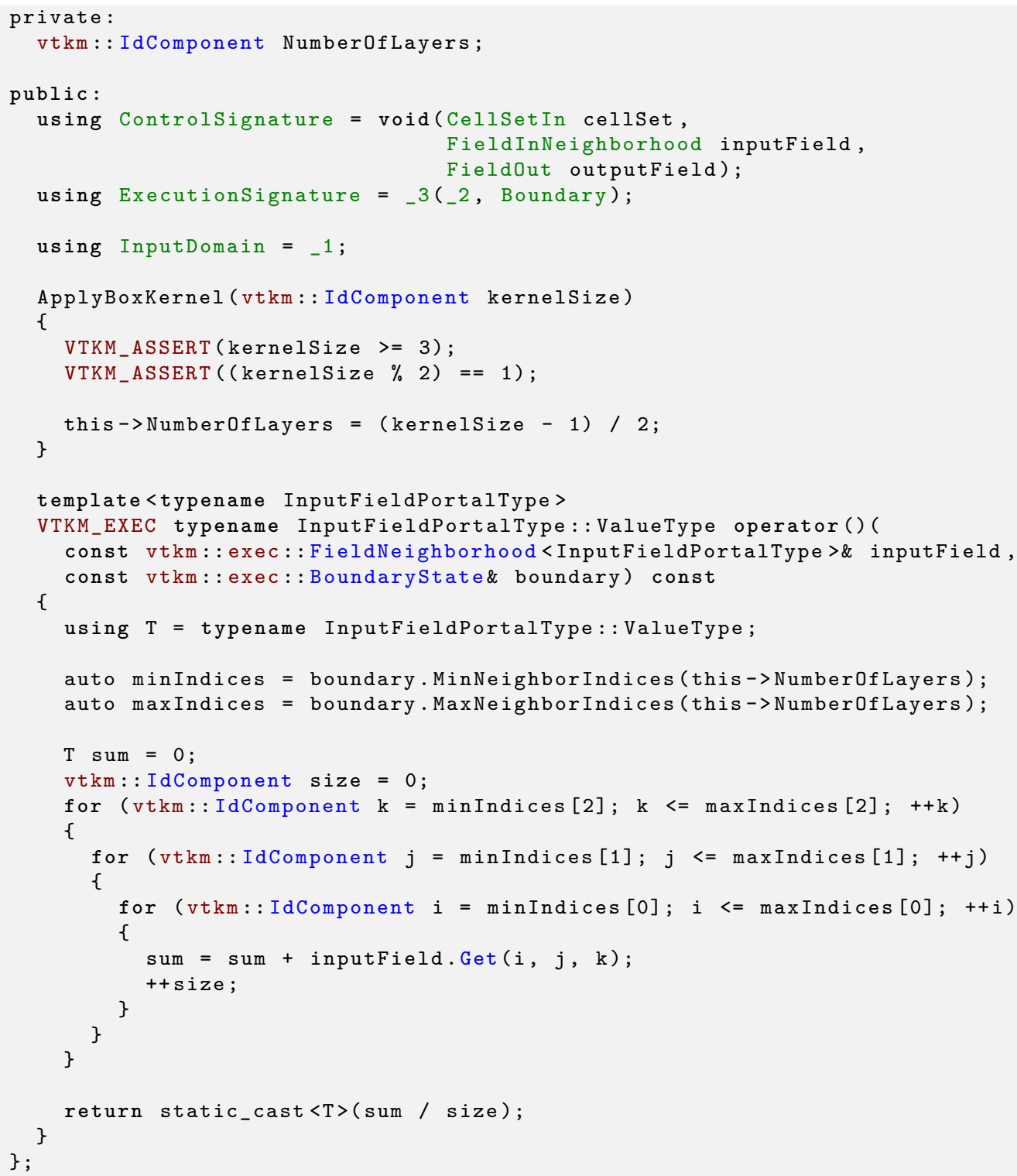

\subsection{Reduce by Key}

A worklet deriving vtkm: : worklet: :WorkletReduceByKey operates on an array of keys and one or more associated arrays of values. When a reduce by key worklet is invoked, all identical keys are collected and the worklet is called once for each unique key. Each worklet invocation is given a Vec-like containing all values associated with the unique key. Reduce by key worklets are very useful for combining like items such as shared topology elements or coincident points.

Figure 21.1 show a pictorial representation of how VTK-m collects data for a reduce by key worklet. All calls to a reduce by key worklet has exactly one array of keys. The key array in this example has 4 unique keys: 0,1 , 2,4 . These 4 unique keys will result in 4 calls to the worklet function. This example also has 2 arrays of values associated with the keys. (A reduce by keys worklet can have any number of values arrays.) 


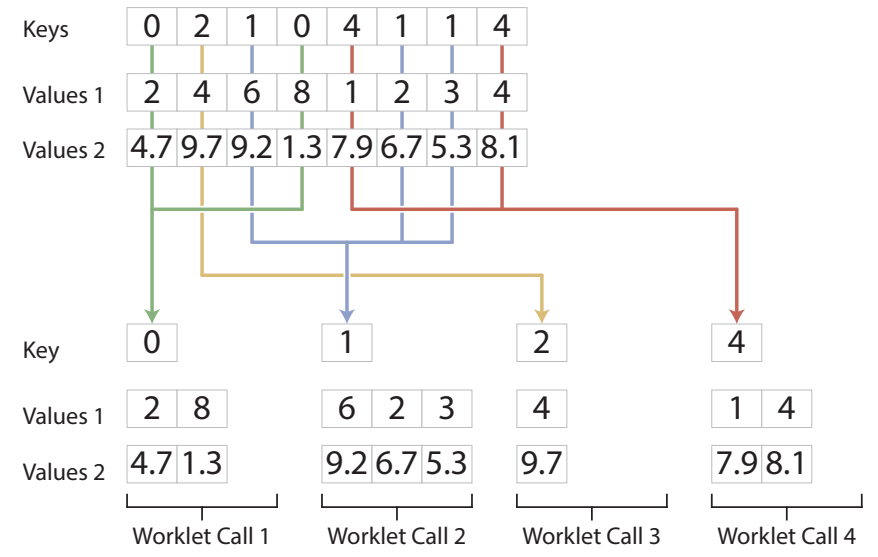

Figure 21.1: The collection of values for a reduce by key worklet.

When the worklet is invoked, all these common keys will be collected with their associated values. The parenthesis operator of the worklet will be called once per each unique key. The worklet call will be given a Vec-like containing all values that have the key.

A reduce by key worklet supports the following tags in the parameters of its Controlsignature.

KeysIn This tag represents the input keys. A KeysIn argument expects a vtkm: : worklet: :Keys object in the associated parameter of the Invoker. The Keys object, which wraps around an ArrayHandle containing the keys and manages the auxiliary structures for collecting like keys, is described later in this section.

Each invocation of the worklet gets a single unique key.

A WorkletReduceByKey object must have exactly one KeysIn parameter in its ControlSignature, and the InputDomain must point to the KeysIn parameter.

ValuesIn This tag represents a set of input values that are associated with the keys. A ValuesIn argument expects an ArrayHandle or an UnknownArrayHandle in the associated parameter of the Invoker. The number of values in this array must be equal to the size of the array used with the Keys In argument. Each invocation of the worklet gets a Vec-like object containing all the values associated with the unique key.

ValuesInOut This tag behaves the same as ValuesIn except that the worklet may write values back into the Veclike object, and these values will be placed back in their original locations in the array. Use of ValuesInOut is rare.

ValuesOut This tag behaves the same as ValuesInOut except that the array is resized appropriately and no input values are passed to the worklet. As with ValuesInOut, values the worklet writes to its Vec-like object get placed in the location of the original arrays. Use of ValuesOut is rare.

ReducedValuesOut This tag represents the resulting reduced values. A ReducedValuesOut argument expects an ArrayHandle or an UnknownArrayHandle in the associated parameter of the Invoker. The array is resized before scheduling begins, and each invocation of the worklet sets a single value in the array.

ReducedValuesIn This tag represents input values that come from (typically) from a previous invocation of a reduce by key. A ReducedValuesOut argument expects an ArrayHandle or an UnknownArrayHandle in the associated parameter of the Invoker. The number of values in the array must equal the number of unique keys. 
A ReducedValuesIn argument is usually used to pass reduced values from one invocation of a reduce by key worklet to another invocation of a reduced by key worklet such as in an algorithm that requires iterative steps.

ReducedValuesInOut This tag behaves the same as ReducedValuesIn except that the worklet may write values back into the array. Make sure that the associated parameter to the worklet operator is a reference so that the changed value gets written back to the array.

WholeArrayIn This tag represents an array where all entries can be read by every worklet invocation. A WholeArrayIn argument expects an ArrayHandle in the associated parameter of the Invoker. An array portal capable of reading from any place in the array is given to the worklet. Whole arrays are discussed in detail in Section 28.1 starting on page 237.

WholeArrayOut This tag represents an array where any entry can be written by any worklet invocation. A WholeArrayOut argument expects an ArrayHandle in the associated parameter of the Invoker. An array portal capable of writing to any place in the array is given to the worklet. Developers should take care when using writable whole arrays as introducing race conditions is possible. Whole arrays are discussed in detail in Section 28.1 starting on page 237.

WholeArrayInOut This tag represents an array where any entry can be read or written by any worklet invocation. A WholeArrayInOut argument expects an ArrayHandle in the associated parameter of the Invoker. An array portal capable of reading from or writing to any place in the array is given to the worklet. Developers should take care when using writable whole arrays as introducing race conditions is possible. Whole arrays are discussed in detail in Section 28.1 starting on page 237.

AtomicArrayInOut This tag represents an array where any entry can be read or written by any worklet invocation. A AtomicArrayInOut argument expects an ArrayHandle in the associated parameter of the Invoker. A vtkm: :exec: :AtomicArray object capable of performing atomic operations to the entries in the array is given to the worklet. Atomic arrays can help avoid race conditions but can slow down the running of a parallel algorithm. Atomic arrays are discussed in detail in Section 28.2 starting on page 239.

WholeCellSetIn This tag represents the connectivity of a cell set. A WholeCellsetIn argument expects a vtkm: : cont: :CellSet in the associated parameter of the Invoker. A connectivity object capable of finding elements of one type that are incident on elements of a different type. Accessing whole cell set connectivity is discussed in detail in Section 28.3.

ExecObject This tag represents an execution object that is passed directly from the control environment to the worklet. A ExecObject argument expects a subclass of vtkm: :exec: :ExecutionObjectBase. Subclasses of ExecutionObjectBase behave like a factory for objects that work on particular devices. They do this by implementing a PrepareForExecution method that takes a device adapter tag and returns an object that works on that device. That device-specific object is passed directly to the worklet. Execution objects are discussed in detail in Section 29 starting on page 245.

A reduce by key worklet supports the following tags in the parameters of its ExecutionSignature.

$\_1, \ldots 2, \ldots$ These reference the corresponding parameter in the Controlsignature.

ValueCount This tag produces a vtkm: IdComponent that is equal to the number of times the key associated with this call to the worklet occurs in the input. This is the same size as the Vec-like objects provided by ValuesIn arguments.

WorkIndex This tag produces a vtkm: : Id that uniquely identifies the invocation of the worklet. 
VisitIndex This tag produces a vtkm: : IdComponent that uniquely identifies when multiple worklet invocations operate on the same input item, which can happen when defining a worklet with scatter (as described in Section 31.1).

InputIndex This tag produces a vtkm: : Id that identifies the index of the input element, which can differ from the WorkIndex in a worklet with a scatter (as described in Section 31.1).

OutputIndex This tag produces a vtkm: : Id that identifies the index of the output element. (This is generally the same as WorkIndex.)

ThreadIndices This tag produces an internal object that manages indices and other metadata of the current thread. Thread indices objects are described in Section 41.2, but most users can get the information they need through other signature tags.

As stated earlier, the reduce by key worklet is useful for collected like values. To demonstrate the reduce by key worklet, we will create a simple mechanism to generate a histogram in parallel. (VTK-m comes with its own histogram implementation, but we create our own version here for a simple example.) The way we can use the reduce by key worklet to compute a histogram is to first identify which bin of the histogram each value is in, and then use the bin identifiers as the keys to collect the information. To help with this example, we will first create a helper class named BinScalars that helps us manage the bins.

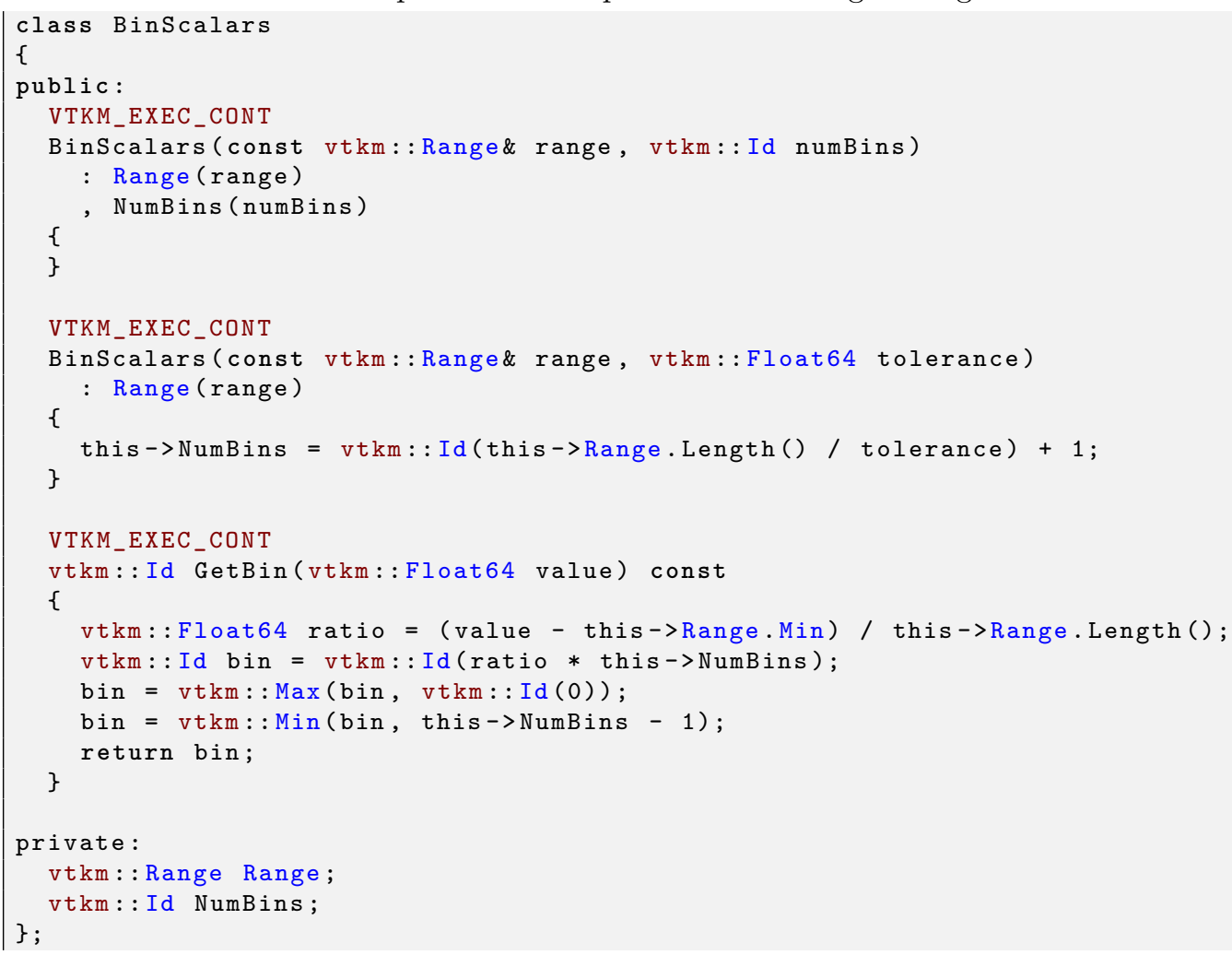

Using this helper class, we can easily create a simple map worklet that takes values, identifies a bin, and writes that result out to an array that can be used as keys.

Example 21.9: A simple map worklet to identify histogram bins, which will be used as keys.

1 struct IdentifyBins : vtkm: : worklet: : WorkletMapField 


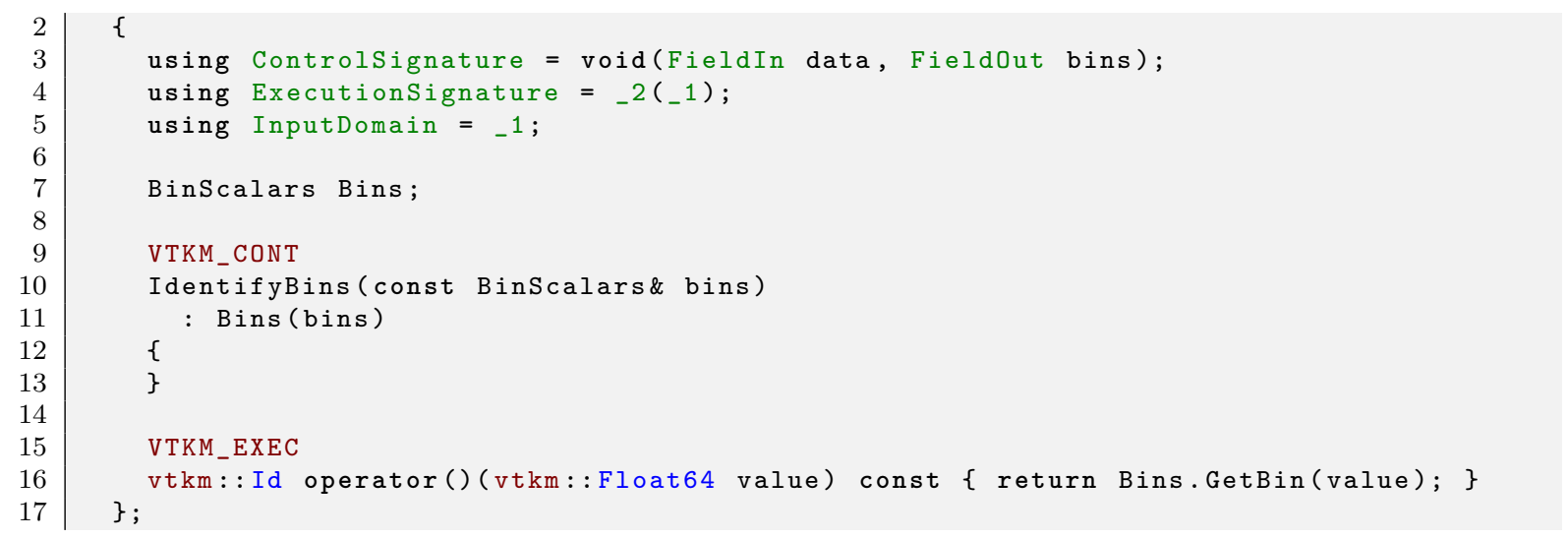

Once you generate an array to be used as keys, you need to make a vtkm: :worklet::Keys object. The Keys object is what will be passed to the Invoker for the argument associated with the KeysIn ControlSignature tag. This of course happens in the control environment after calling the Invoker for our worklet for generating the keys.

Example 21.10: Creating a vtkm: : worklet: :Keys object.

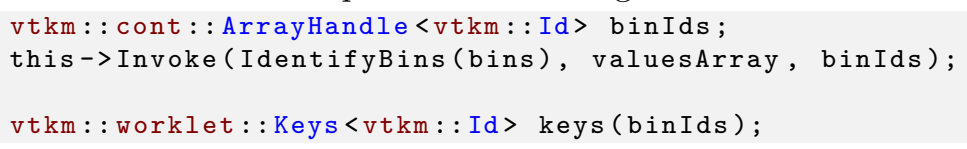

Now that we have our keys, we are finally ready for our reduce by key worklet. A histogram is simply a count of the number of elements in a bin. In this case, we do not really need any values for the keys. We just need the size of the bin, which can be identified with the internally calculated ValueCount.

A complication we run into with this histogram filter is that it is possible for a bin to be empty. If a bin is empty, there will be no key associated with that bin, and the Invoker will not call the worklet for that bin/key. To manage this case, we have to initialize an array with 0's and then fill in the non-zero entities with our reduce by key worklet. We can find the appropriate entry into the array by using the key, which is actually the bin identifier, which doubles as an index into the histogram. The following example gives the implementation for the reduce by key worklet that fills in positive values of the histogram.

Example 21.11: A reduce by key worklet to write histogram bin counts.

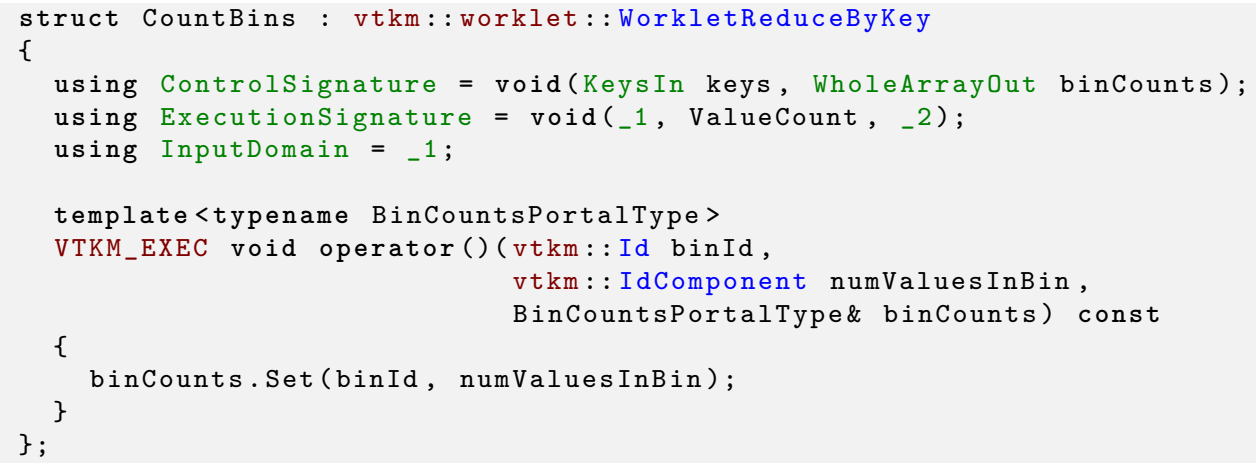

The previous example demonstrates the basic usage of the reduce by key worklet to count common keys. A more common use case is to collect values associated with those keys, do an operation on those values, and provide a "reduced" value for each unique key. The following example demonstrates such an operation by providing a worklet that finds the average of all values in a particular bin rather than counting them. 
Example 21.12: A worklet that averages all values with a common key.

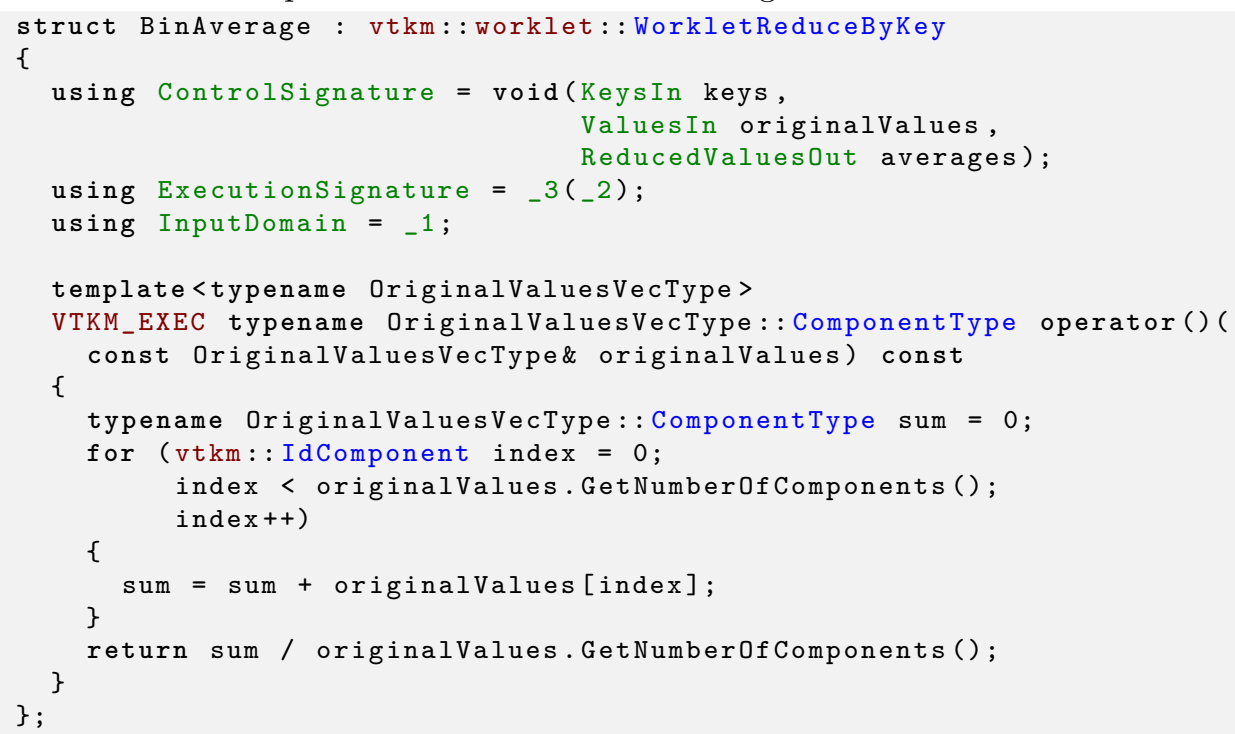

To complete the code required to average all values that fall into the same bin, the following example shows the full code required to invoke such a worklet. Note that this example repeats much of the previous examples, but shows it in a more complete context.

Example 21.13: Using a reduce by key worklet to average values falling into the same bin.

struct IdentifyBins : vtkm: : worklet: : WorkletMapField

\{

using ControlSignature = void(FieldIn data, Fieldout bins);

using Executionsignature = $2\left({ }_{-} 1\right)$;

using InputDomain $=\ldots 1$;

BinScalars Bins;

VTKM_CONT

IdentifyBins (const Binscalars\& bins)

: Bins(bins)

\{

\}

VTKM_EXEC

\};

vtkm::Id operator()(vtkm::Float64 value) const \{return Bins.GetBin(value); \}

struct BinAverage : vtkm: : worklet: : WorkletReduceByKey

\{

using ControlSignature = void(KeysIn keys,

ValuesIn originalValues,

ReducedValuesout averages);

using ExecutionSignature = _3(_2);

using InputDomain $=\ldots 1$;

template<typename OriginalValuesVecType>

VTKM_EXEC typename OriginalValuesVecType: : ComponentType operator()(

\{

const OriginalValuesVecType\& originalValues) const

typename OriginalValuesVecType: : ComponentType sum = 0 ;

for (vtkm: : IdComponent index =0;

index < originalValues. GetNumberOfComponents ();

index++) 


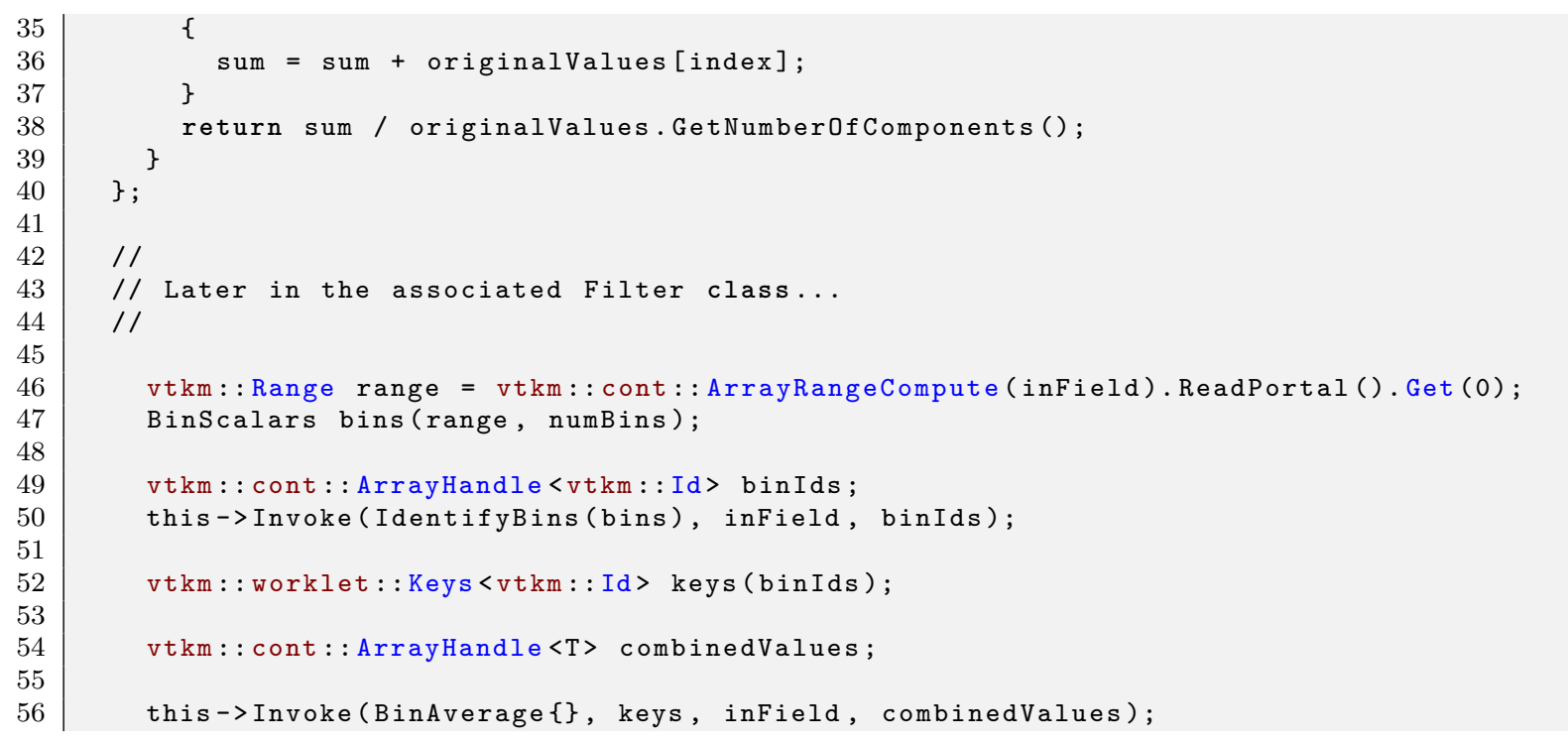




\section{FILTER TYPE REFERENCE}

In Chapters 17 and 21 we discuss how to implement an algorithm in the VTK-m framework by creating a worklet. Worklets might be straightforward to implement and invoke for those well familiar with the appropriate VTK-m API. However, novice users have difficulty using worklets directly. For simplicity, worklet algorithms are generally wrapped in what are called filter objects for general usage. Chapter 9 introduces the concept of filters and documents those that come with the VTK-m library. Chapter 18 gave a brief introduction on implementing filters. This chapter elaborates on building new filter objects by introducing new filter types. These will be used to wrap filters around the extended worklet examples in Chapter 21.

Unsurprisingly, the base filter objects are contained in the vtkm: :filter package. The basic implementation of a filter involves subclassing one of the base filter objects and implementing the DoExecute method. The DoExecute method performs the operation of the filter and returns a new data set containing the result.

As with worklets, there are several flavors of filter types to address different operating behaviors although their is not a one-to-one relationship between worklet and filter types. This chapter is sectioned by the different filter types with an example of implementations for each.

\subsection{Field Filters}

Field filters are a category of filters that generate a new field. These new fields are typically derived from one or more existing fields or point coordinates on the data set. For example, mass, volume, and density are interrelated, and any one can be derived from the other two.

Field filters are implemented in classes that derive the vtkm: filter: :FilterField base class. FilterField is a templated class that has a single template argument, which is the type of the concrete subclass. The propagation of additional fields from the input to the output is handled automatically by vtkm: :filter::FilterField. If the default of automatic propagation isn't desired the caller can select which fields to propagate via SetFieldsToPass.

\section{Did you know? \\ 3 The convention of having a subclass be templated on the derived class' type is known as the Curiously Recurring Template Pattern (CRTP). In the case of FilterField and other filter base classes, VTK-m uses this CRTP behavior to allow the general implementation of these algorithms to run DoExecute in the subclass, which as we see in a moment is itself templated.}

All FilterField subclasses must implement a DoExecute method. The FilterField base class implements an 
Execute method (actually several overloaded versions of Execute), processes the arguments, and then calls the DoExecute method of its subclass. The DoExecute method has the following 4 arguments.

- An input data set contained in a vtkm: :cont: :DataSet object. (See Chapter 7 for details on DataSet objects.)

- The field from the DataSet specified in the Execute method to operate on. The field is always passed as an instance of vtkm: : cont: :ArrayHandle. (See Chapter 16 for details on ArrayHandle objects.) The type of the ArrayHandle is generally not known until the class is used and requires a template type.

- A vtkm: :filter::FieldMetadata object that contains the associated metadata of the field not contained in the ArrayHandle of the second argument. The FieldMetadata contains information like the name of the field and what topological element the field is associated with (such as points or cells).

- A policy class. See Section 22.4 for information on using policies. The type of the policy is generally unknown until the class is used and requires a template type.

In this section we provide an example implementation of a field filter that wraps the "magnitude" worklet provided in Example 21.1 (listed on page 161). By convention, filter implementations are split into two files. The first file is a standard header file with a.$h$ extension that contains the declaration of the filter class without the implementation. So we would expect the following code to be in a file named FieldMagnitude.h.

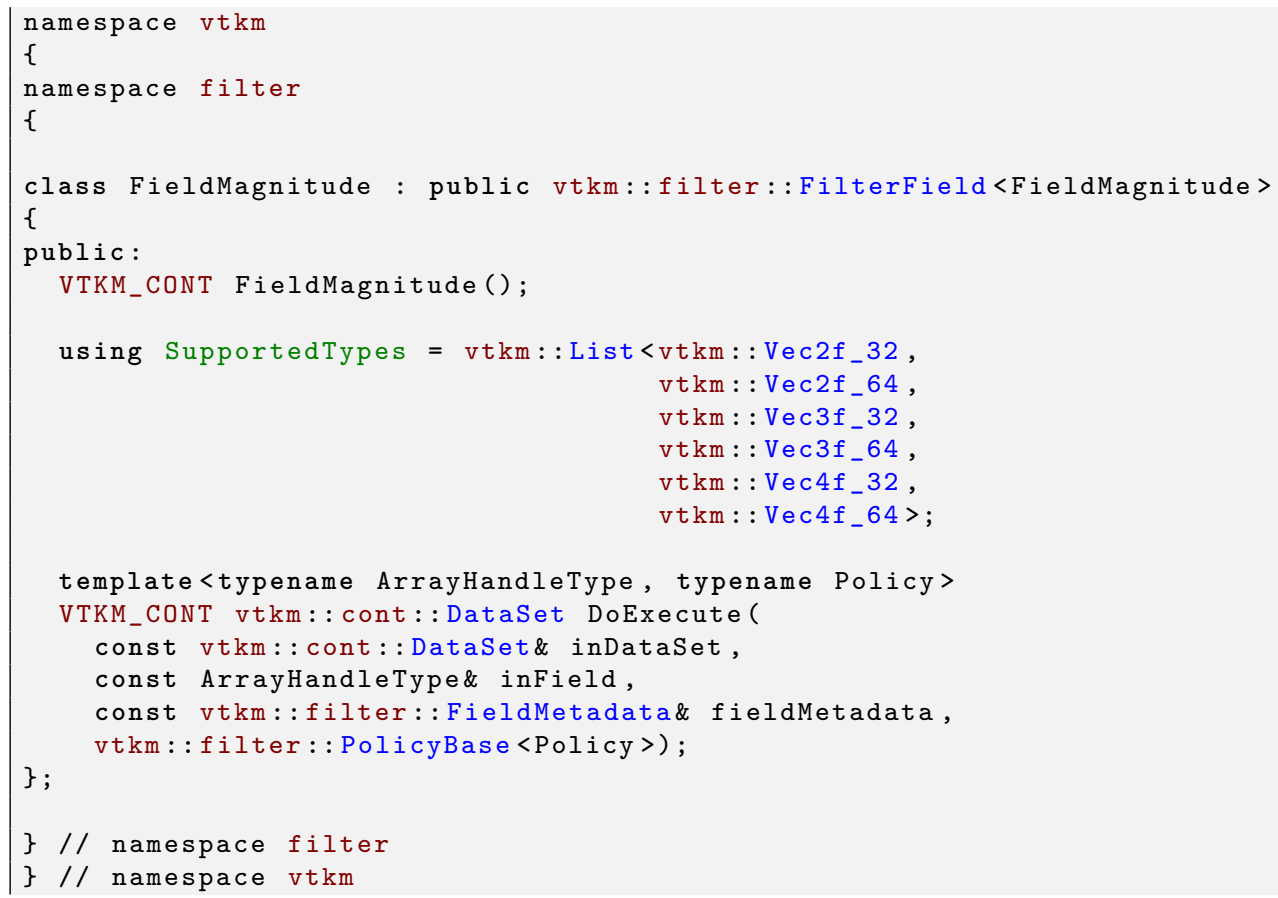

Notice that within the declaration of FieldMagnitude in Example 22.1, there is also the declaration of a type named SupportedTypes (starting on line 11). This type provides a type list containing all the types that are valid for the input field. (Type lists are discussed in detail in Section 19.6.2.) In the particular case of our FieldMagnitude filter, the filter expects to operate on some type of vector field. Thus, SupportedTypes is set to a list of all standard floating point Vecs.

Once the filter class is declared in the. $\mathrm{h}$ file, the implementation filter is by convention given in a separate .hxx file. So the continuation of our example that follows would be expected in a file named FieldMagnitude.hxx. The 
.h file near its bottom needs an include line to the.$h x x$ file. This convention is set up because a near future version of VTK-m will allow the building of filter libraries containing default policies that can be used by only including the header declaration.

The implementation of DoExecute is straightforward. A worklet is invoked to compute a new field array. DoExecute then returns a newly constructed vtkm: cont: :DataSet object containing the result. There are numerous ways to create a DataSet, but to simplify making filters, VTK-m provides the vtkm::filter: :CreateResult function to simplify the process. There are several overloaded versions of vtkm: :filter: :CreateResult (defined in header file vtkm/filter/CreateResult.h), but the simplest one to use for a filter that adds a field takes the following 5 arguments.

- The input data set. This is the same data set passed to the first argument of DoExecute.

- The array containing the data for the new field, which was presumably computed by the filter.

- The name of the new field.

- A vtkm::filter::FieldMetadata object containing information about the association of the array to which topological elements of the input (for example cells or points). Typically, this FieldMetadata object comes from the input parameters of DoExecute.

Note that this form of CreateResult is generally applicable when the field created is of the same type as the input field. For example, when computing the magnitude of a vector field, if the input vectors are on the points, the output scalars will also be on the points and likewise if the input is on the cells. In the case where the field association changes, it is better to use a different form of CreateResult. If the output field is associated with points, such as when averaging cell fields to each point, then it is best to use vtkm: :filter::CreateResultFieldPoint. Likewise, if the output field is associated with cells, such as when computing the volume of each cell, then it is best to use vtkm: :filter: :CreateResultFieldCell. Both functions only need the three arguments of the input data set, the array for the field's data, and the name of the new field.

Note that all fields need a unique name, which is the reason for the third argument to CreateResult. The vtkm: :filter::FilterField base class contains a pair of methods named SetOutputFieldName and GetOutputFieldName to allow users to specify the name of output fields. The DoExecute method should respect the given output field name. However, it is also good practice for the filter to have a default name if none is given. This might be simply specifying a name in the constructor, but it is worthwhile for many filters to derive a name based on the name of the input field.

Example 22.2: Implementation of a field filter.

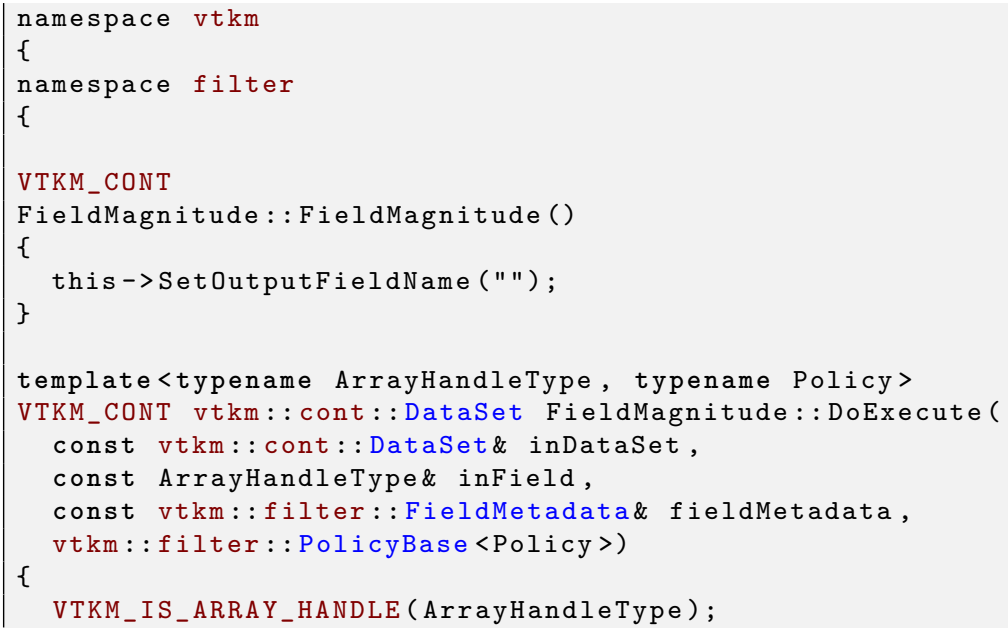




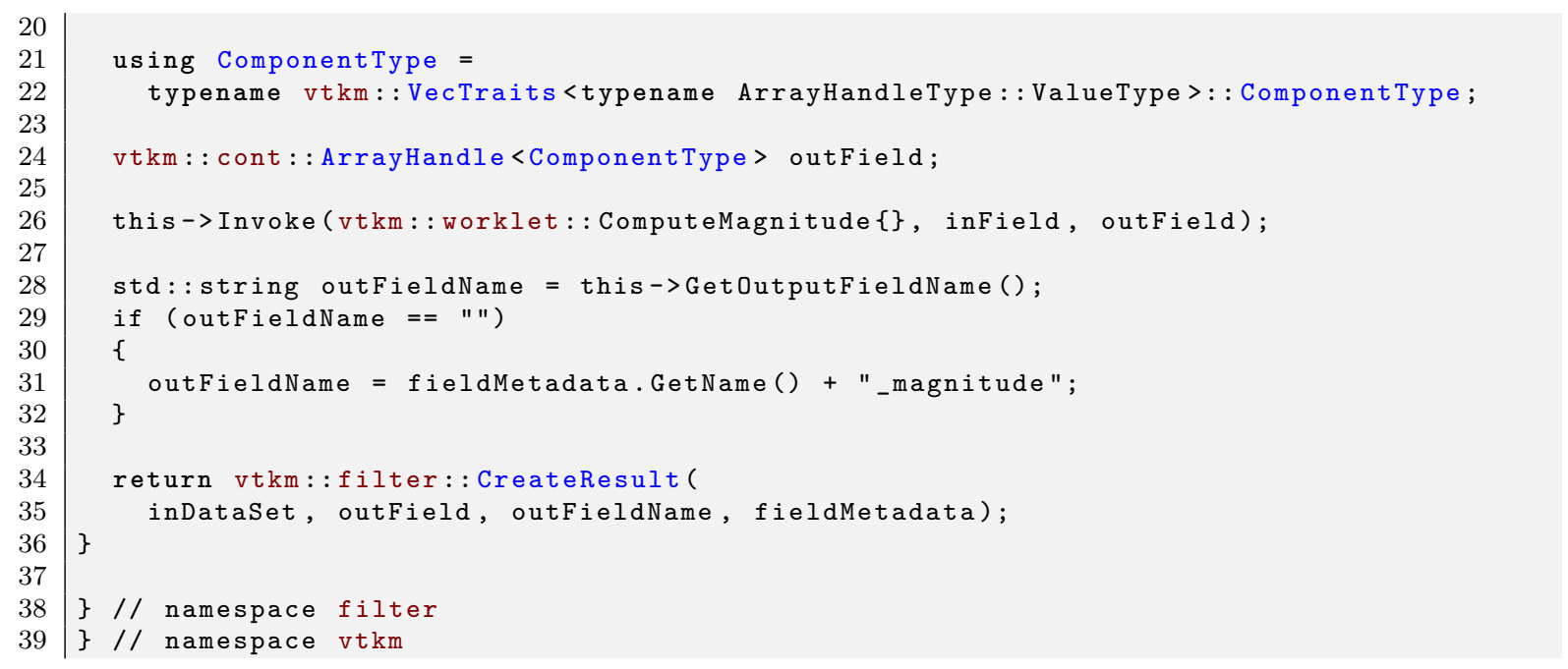

\subsubsection{Using Cell Connectivity}

The previous example performed a simple operation on each element of a field independently. However, it is also common for a "field" filter to take into account the topology of a data set. The interface for the filter is similar, but the implementation involves pulling a vtkm: :cont::CellSet from the input vtkm: :cont: :DataSet and performing operations on fields associated with different topological elements. The steps involve calling DataSet: :GetCellSet to retrieve the CellSet object, applying the policy on that object, and then using topology-based worklets, described in Section 21.2, to operate on them.

In this section we provide an example implementation of a field filter on cells that wraps the "cell center" worklet provided in Example 21.3 (listed on page 165). By convention, filter implementations are split into two files. The first file is a standard header file with a.$h$ extension that contains the declaration of the filter class without the implementation. So we would expect the following code to be in a file named CellCenter.h.

Example 22.3: Header declaration for a field filter using cell topology.

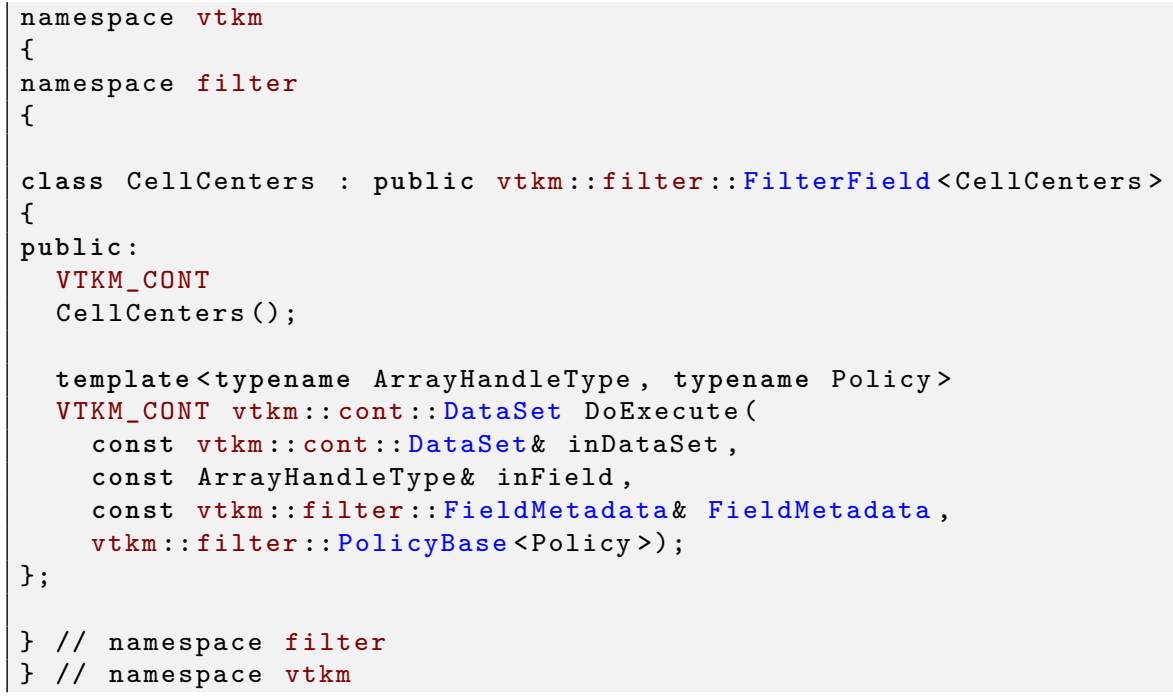


8) Did you know?

You may have noticed that Example 22.1 provided a specification for SupportedTypes but Example 22.3 provides no such specification. This demonstrates that declaring SupportedTypes is optional. If a filter only works on some limited number of types, then it can use SupportedTypes to specify the specific types it supports. But if a filter is generally applicable to many field types, it can simply use the default filter types.

Once the filter class is declared in the.$h$ file, the implementation filter is by convention given in a separate .hxx file. So the continuation of our example that follows would be expected in a file named CellCenter.hxx. The .h file near its bottom needs an include line to the $h \times x$ file. This convention is set up because a near future version of VTK-m will allow the building of filter libraries containing default policies that can be used by only including the header declaration.

As with any subclass of FilterField, the filter implements DoExecute, which in this case invokes a worklet to compute a new field array and then return a newly constructed vtkm: : cont: : DataSet object.

Example 22.4: Implementation of a field filter using cell topology.

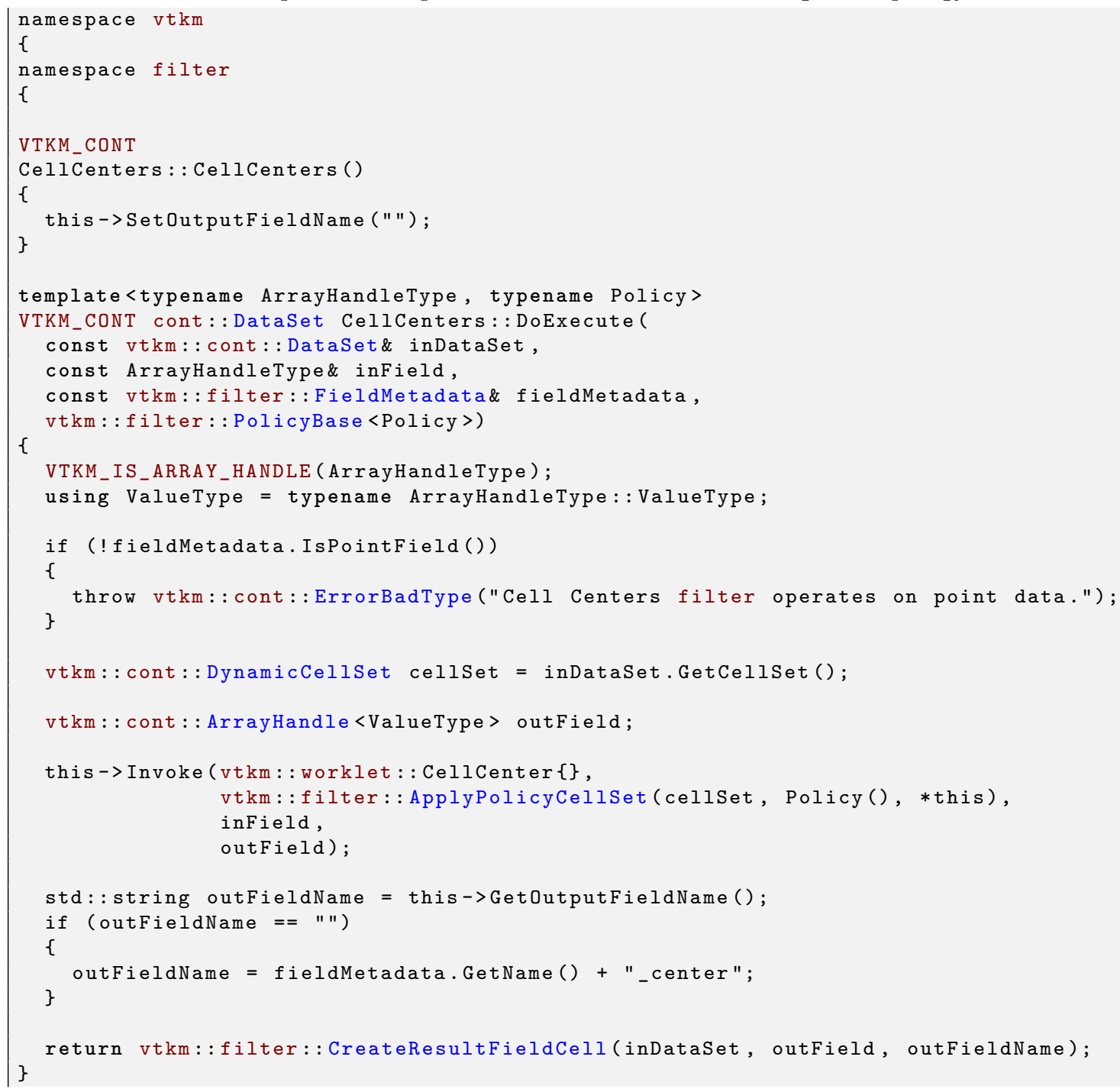




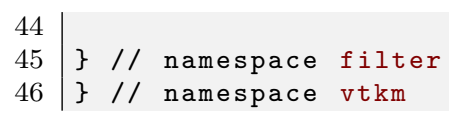

\section{Common Errors}

The policy passed to the DoExecute method contains information on what types of cell sets should be supported by the execution. This list of cell set types could be different than the default types specified ¿ the DynamicCellSet returned from GetCellSet. Thus, it is important to apply the policy to the cell set Before passing it to the Invoke method. The policy is applied by calling the vtkm: :filter: :ApplyPolicy function on the DynamicCellSet. The use of ApplyPolicy is demonstrated in Example 22.4.

\subsection{Data Set Filters}

Data set filters are a category of filters that generate a data set with a new cell set based off the cells of an input data set. For example, a data set can be significantly altered by adding, removing, or replacing cells.

Data set filters are implemented in classes that derive the vtkm: :filter::FilterDataSet base class. FilterDataSet is a templated class that has a single template argument, which is the type of the concrete subclass.

All FilterDataSet subclasses must implement two methods: DoExecute and DoMapField. The FilterDataSet base class implements Execute and MapFieldOntoOutput methods that process the arguments and then call the DoExecute and DoMapField methods, respectively, of its subclass.

Like vtkm: :filter: :FilterField all fields are considered to be eligible for propagation. If this behavior is not desired by the caller they can select which fields to propagate via SetFieldsToPass. The actual transformation of the fields so they are valid is handled by DoMapField.

The DoExecute method has the following 2 arguments.

- An input data set contained in a vtkm: : cont: :DataSet object. (See Chapter 7 for details on DataSet objects.)

- A policy class. See Section 22.4 for information on using policies. The type of the policy is generally unknown until the class is used and requires a template type.

The DoMapField method has the following 4 arguments.

- A vtkm::cont::DataSet object that was returned from the last call to DoExecute. The method both needs information in the DataSet object and writes its results back to it, so the parameter should be declared as a non-const reference. (See Chapter 7 for details on DataSet objects.)

- A field from the DataSet specified in the Execute method to convert so it is applicable to the output DataSet. The field is always passed as an instance of vtkm: :cont: :ArrayHandle. (See Chapter 16 for details on ArrayHandle objects.) The type of the ArrayHandle is generally not known until the class is used and requires a template type.

- A vtkm: :filter::FieldMetadata object that contains the associated metadata of the field not contained in the ArrayHandle of the second argument. The FieldMetadata contains information like the name of the field and what topological element the field is associated with (such as points or cells). 
- A policy class. See Section 22.4 for information on using policies. The type of the policy is generally unknown until the class is used and requires a template type.

\section{Did you know?}

Some FilterDataSet subclasses have no need to process each individual field as they can safely propagate or drop all fields. In these cases it is better to override MapFieldOntoOutput allowing you to perform this logic directly with Field objects and not incur the compile and runtime cost of deducing the underlying ArrayHandle.

In this section we provide an example implementation of a data set filter that wraps the functionality of extracting the edges from a data set as line elements. Many variations of implementing this functionality are given in Chapter 32. For the sake of argument, we are assuming that all the worklets required to implement edge extraction are wrapped up in structure named vtkm: :worklet : :ExtractEdges. Furthermore, we assume that ExtractEdges has a pair of methods, Run and ProcessCellField, that create a cell set of lines from the edges in another cell set and average a cell field from input to output, respectively. The ExtractEdges may hold state. All of these assumptions are consistent with the examples in Chapter 32.

By convention, filter implementations are split into two files. The first file is a standard header file with a.$h$ extension that contains the declaration of the filter class without the implementation. So we would expect the following code to be in a file named ExtractEdges.h.

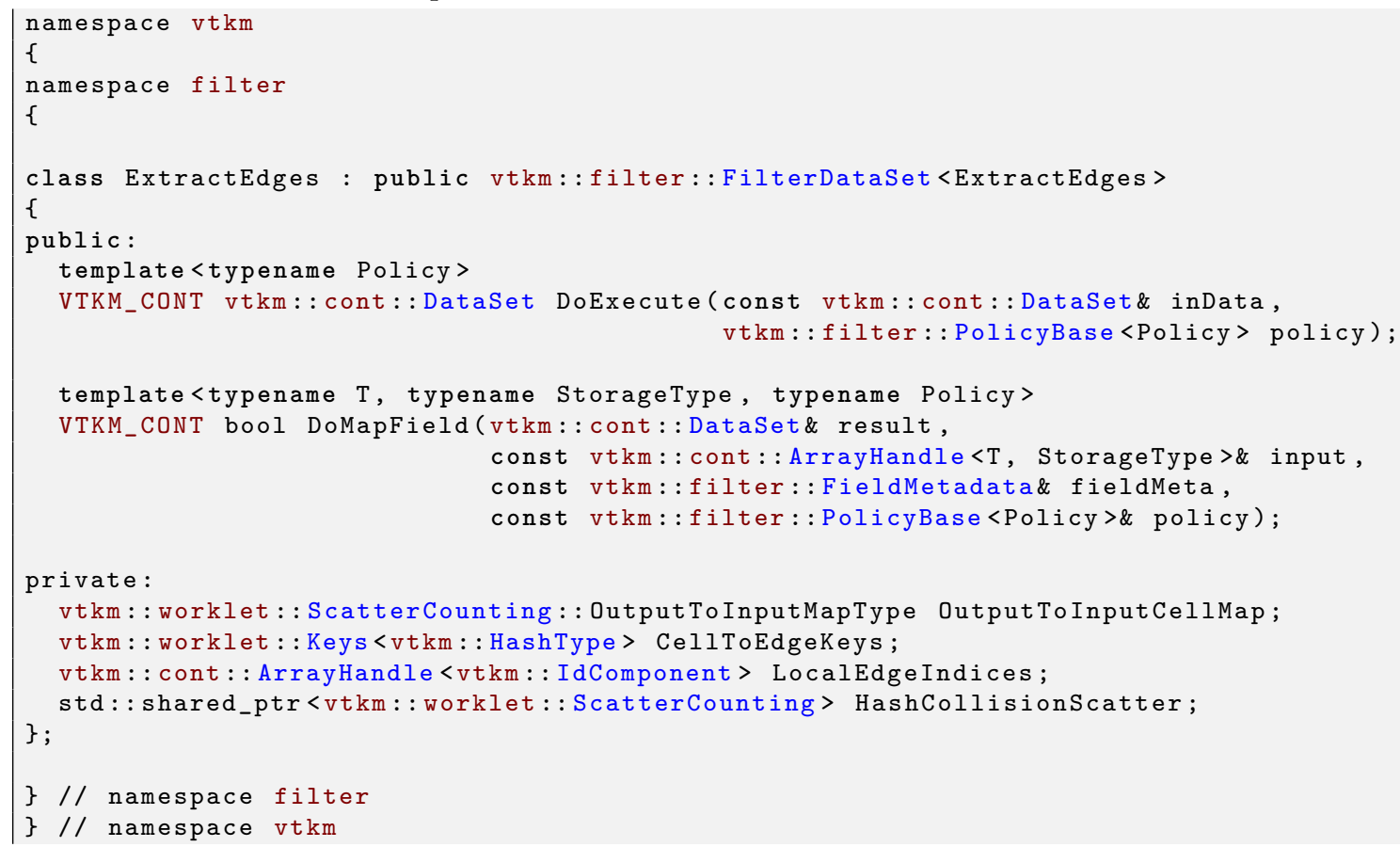

Once the filter class is declared in the $\mathrm{h}$ file, the implementation of the filter is by convention given in a separate .hxx file. So the continuation of our example that follows would be expected in a file named ExtractEdges.hxx. The.$h$ file near its bottom needs an include line to the.$h x x$ file. This convention is set up because a near future version of VTK-m will allow the building of filter libraries containing default policies that can be used by only including the header declaration. 
The implementation of DoExecute first calls the worklet methods to generate a new CellSet class. It then constructs a DataSet containing this CellSet. It also has to pass all the coordinate systems to the new DataSet. Finally, it returns the DataSet.

Example 22.6: Implementation of the DoExecute method of a data set filter.

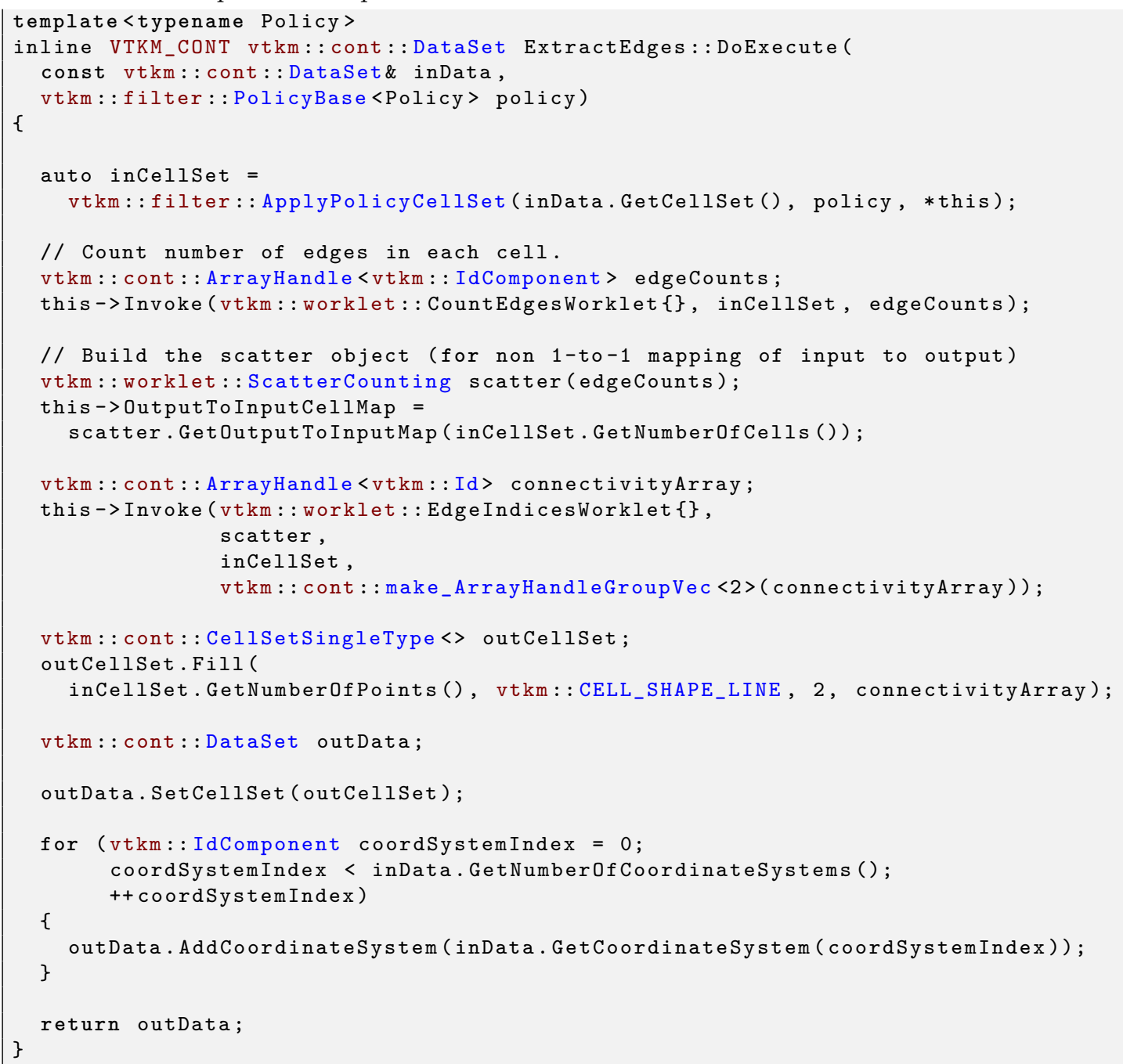

The implementation of DoMapField checks to see what elements the given field is associated with (e.g. points or cells), processes the field data as necessary, and adds the field to the DataSet. The vtkm::filter::FieldMetadata passed to DoMapField provides some features to make this process easier. FieldMetadata contains methods to query what the field is associated with such as IsPointField and FieldMetadata: :IsCellField. FieldMetadata also has a method named FieldMetadata: : AsField that creates a new vtkm: :cont: :Field object with a new data array and metadata that matches the input.

Example 22.7: Implementation of the DoMapField method of a data set filter.

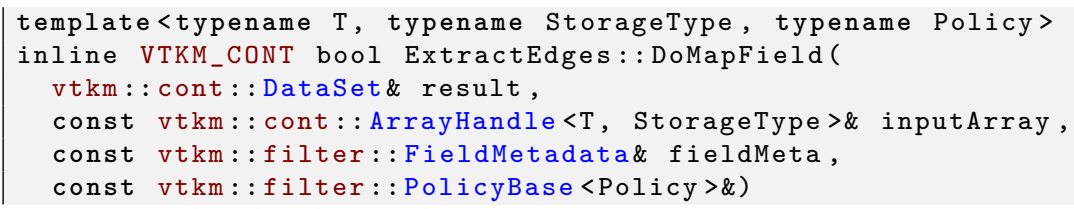




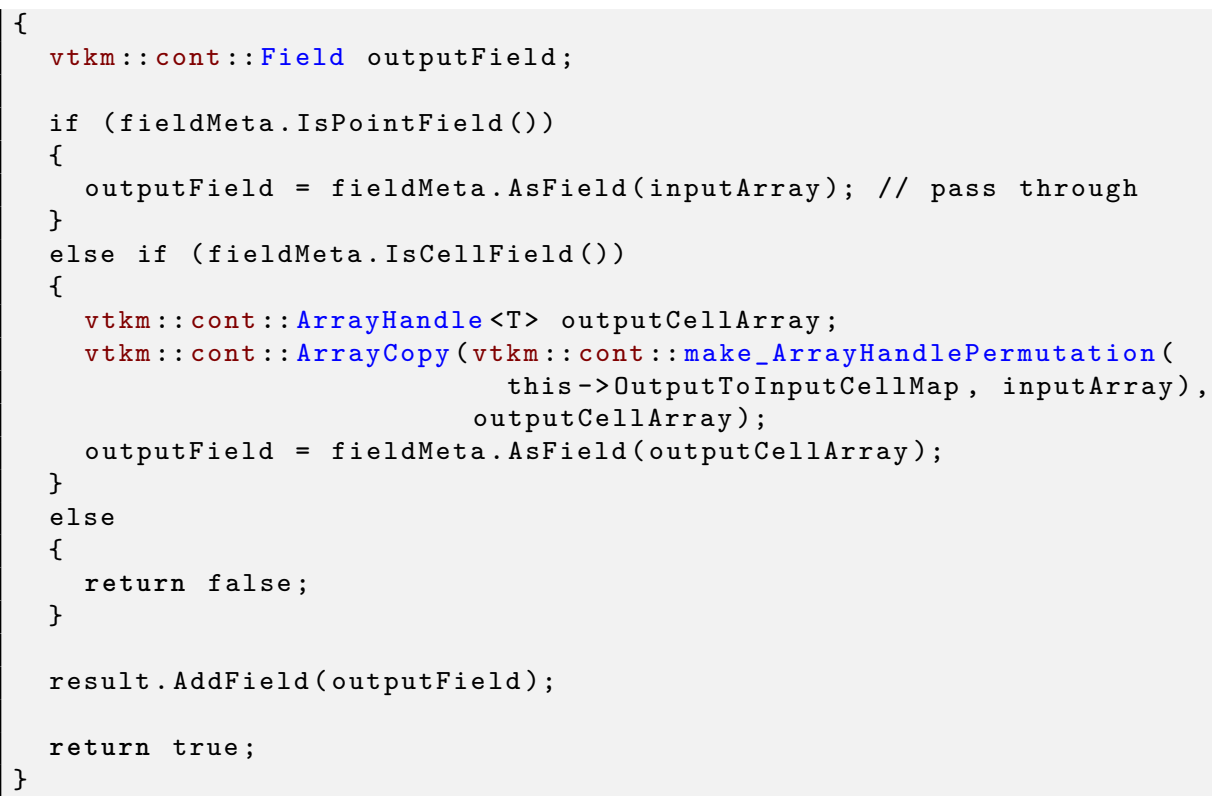

\subsection{Data Set with Field Filters}

Data set with field filters are a category of filters that generate a data set with a new cell set based off the cells of an input data set along with the data in at least one field. For example, a field might determine how each cell is culled, clipped, or sliced.

Data set with field filters are implemented in classes that derive the vtkm: :filter: :FilterDataSetWithField base class. FilterDataSetWithField is a templated class that has a single template argument, which is the type of the concrete subclass.

All FilterDataSetWithField subclasses must implement two methods: DoExecute and DoMapField. The FilterDataSetWithField base class implements Execute and MapFieldOntoOutput methods that process the arguments and then call the DoExecute and DoMapField methods, respectively, of its subclass.

Like vtkm: :filter: :FilterField all fields are considered to be eligible for propagation. If this behavior is not desired by the caller they can select which fields to propagate via SetFieldsToPass. The actual transformation of the fields so they are valid is handled by DoMapField.

The DoExecute method has the following 4 arguments. (They are the same arguments for DoExecute of field filters as described in Section 22.1.)

- An input data set contained in a vtkm: :cont: :DataSet object. (See Chapter 7 for details on DataSet objects.)

- The field from the DataSet specified in the Execute method to operate on. The field is always passed as an instance of vtkm: :cont: :ArrayHandle. (See Chapter 16 for details on ArrayHandle objects.) The type of the ArrayHandle is generally not known until the class is used and requires a template type.

- A vtkm: :filter: :FieldMetadata object that contains the associated metadata of the field not contained in the ArrayHandle of the second argument. The FieldMetadata contains information like the name of the field and what topological element the field is associated with (such as points or cells). 
- A policy class. See Section 22.4 for information on using policies. The type of the policy is generally unknown until the class is used and requires a template type.

The DoMapField method has the following 4 arguments. (They are the same arguments for DoExecute of field filters as described in Section 22.2.)

- A vtkm::cont: :DataSet object that was returned from the last call to DoExecute. The method both needs information in the DataSet object and writes its results back to it, so the parameter should be declared as a non-const reference. (See Chapter 7 for details on DataSet objects.)

- A field from the DataSet specified in the Execute method to convert so it is applicable to the output DataSet. The field is always passed as an instance of vtkm: :cont: :ArrayHandle. (See Chapter 16 for details on ArrayHandle objects.) The type of the ArrayHandle is generally not known until the class is used and requires a template type.

- A vtkm::filter: :FieldMetadata object that contains the associated metadata of the field not contained in the ArrayHandle of the second argument. The FieldMetadata contains information like the name of the field and what topological element the field is associated with (such as points or cells).

- A policy class. See Section 22.4 for information on using policies. The type of the policy is generally unknown until the class is used and requires a template type.

\section{Did you know?}

Some FilterDataSetWithField subclasses have no need to process each individual field as they can safely propagate or drop all fields. In these cases it is better to override MapFieldOntoOutput allowing you to perform this logic directly with Field objects and not incur the compile and runtime cost of deducing the underlying ArrayHandle.

In this section we provide an example implementation of a data set with field filter that blanks the cells in a data set based on a field that acts as a mask (or stencil). Any cell associated with a mask value of zero will be removed.

We leverage the worklets in the VTK-m source code that implement the Threshold functionality. The threshold worklets are templated on a predicate class that, given a field value, returns a flag on whether to pass or cull the given cell. The vtkm: :filter: :Threshold class uses a predicate that is true for field values within a range. Our blank cells filter will instead use the predicate class vtkm: :NotZeroInitialized that will cull all values where the mask is zero.

By convention, filter implementations are split into two files. The first file is a standard header file with a.$h$ extension that contains the declaration of the filter class without the implementation. So we would expect the following code to be in a file named BlankCells.h.

Example 22.8: Header declaration for a data set with field filter.

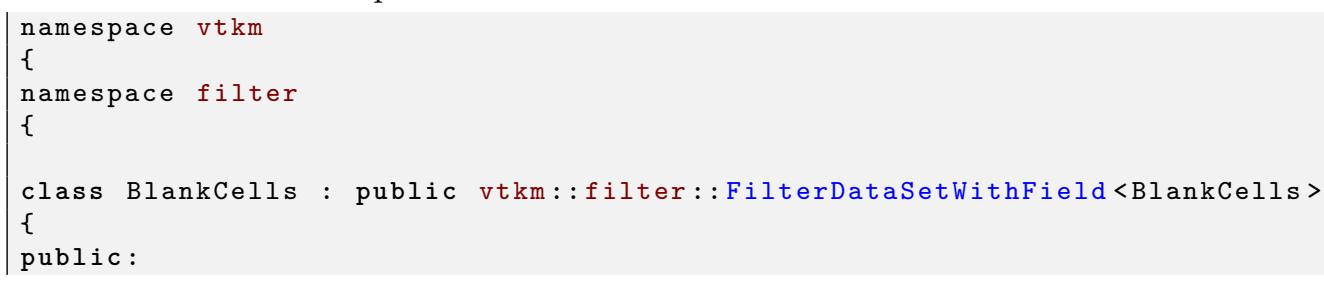




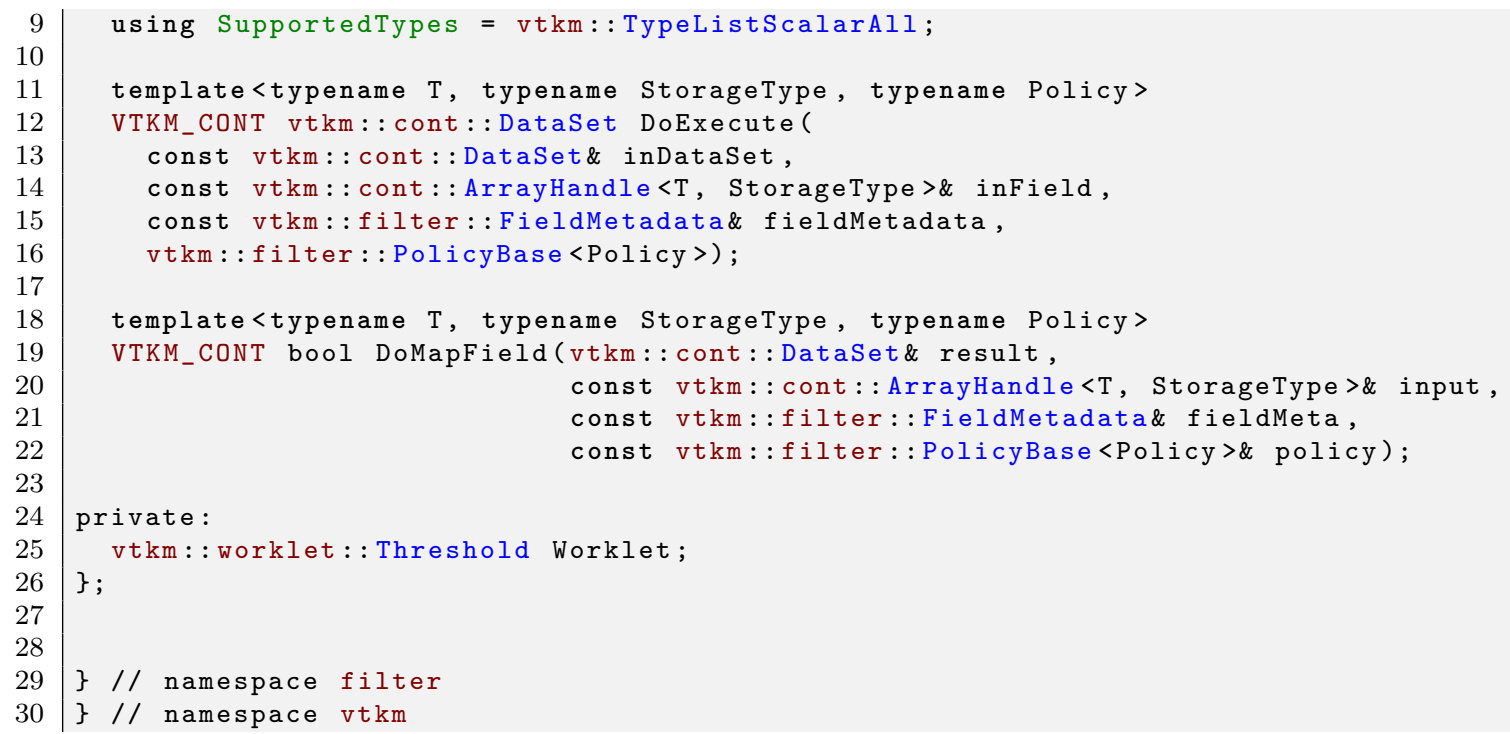

Note that the filter class declaration contains a specification for SupportedTypes to specify that the field is meant to operate specifically on scalar types. Once the filter class is declared in the.$h$ file, the implementation of the filter is by convention given in a separate.$h x x$ file. So the continuation of our example that follows would be expected in a file named BlankCells.hxx. The .h file near its bottom needs an include line to the .hxx file. This convention is set up because a near future version of VTK-m will allow the building of filter libraries containing default policies that can be used by only including the header declaration.

The implementation of DoExecute first calls the worklet methods to generate a new Cellset class. It then constructs a DataSet containing this CellSet. It also has to pass all the coordinate systems to the new DataSet. Finally, it returns the DataSet.

Example 22.9: Implementation of the DoExecute method of a data set with field filter.

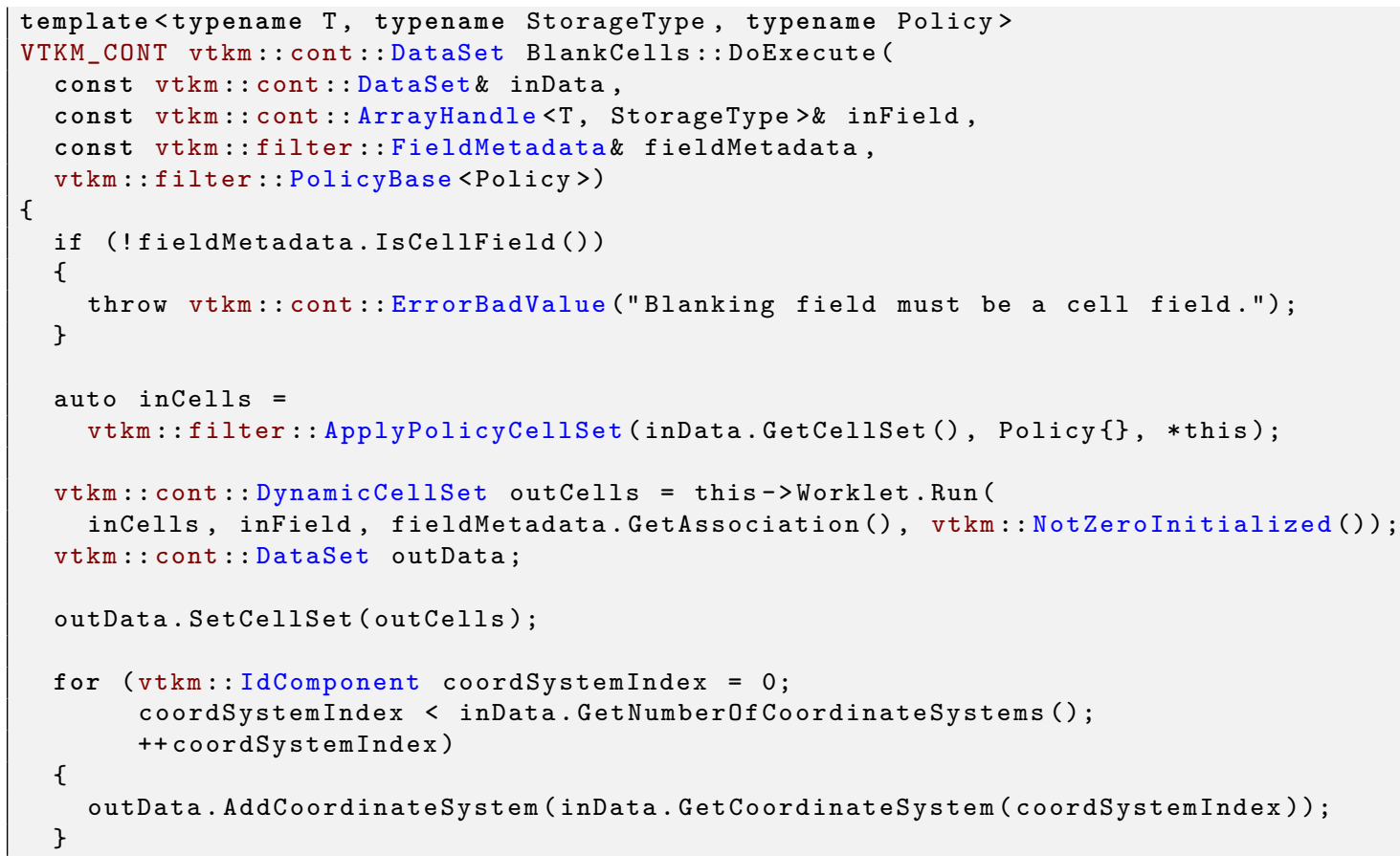




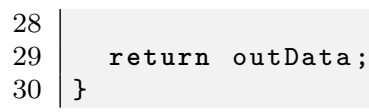

The implementation of DoMapField checks to see what elements the given field is associated with (e.g. points or cells), processes the field data as necessary, and adds the field to the DataSet. The vtkm::filter::FieldMetadata passed to DoMapField provides some features to make this process easier. FieldMetadata contains methods to query what the field is associated with such as IsPointField and IsCellField. FieldMetadata also has a method named AsField that creates a new vtkm: cont: :Field object with a new data array and metadata that matches the input.

Example 22.10: Implementation of the DoMapField method of a data set with field filter.

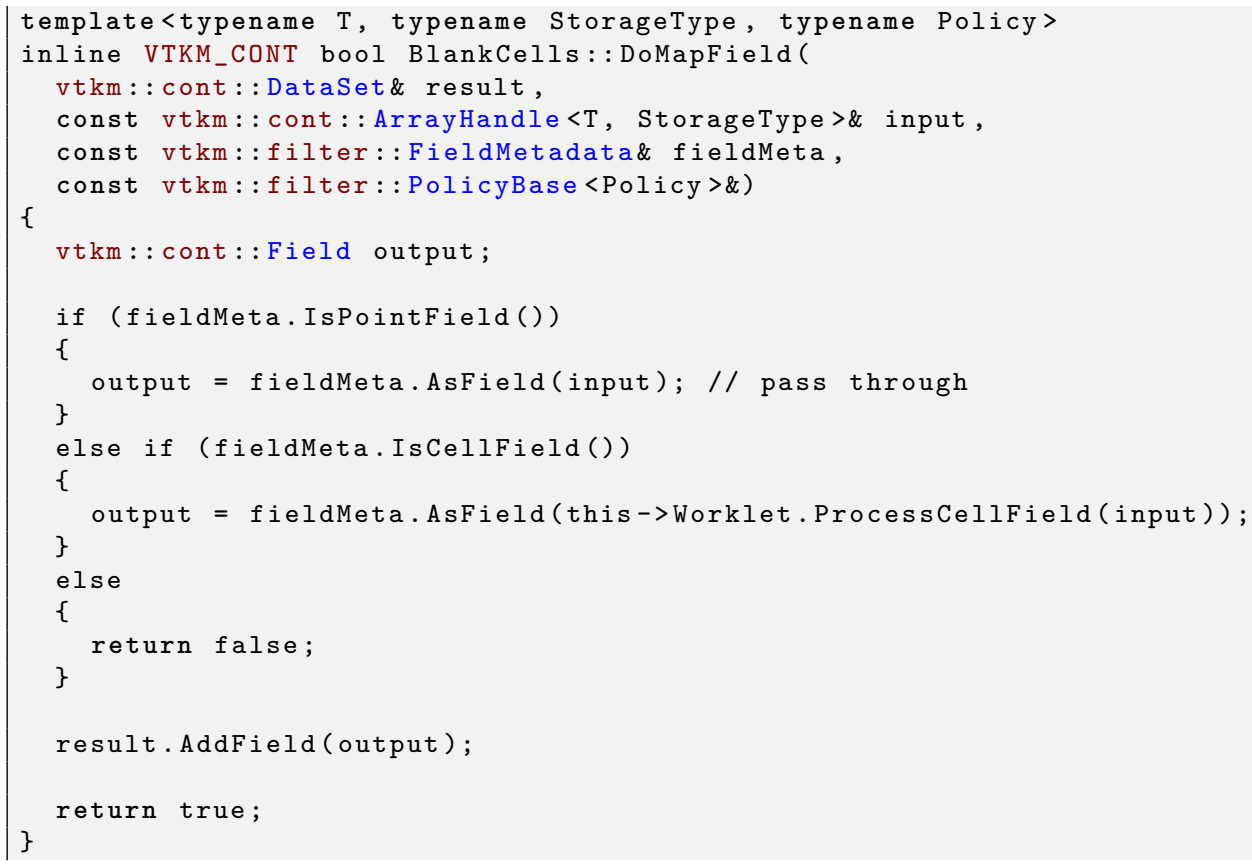

\subsection{Applying Policies}

As seen throughout this chapter, filters define their operation by implementing a DoExecute method. The prototype and information passed to the method varies across the different filter types discussed later in this chapter, but one thing they all in common is that they all receive a vtkm: : cont: : DataSet object that was passed to the Execute method. Although some data extracted from the DataSet may also be passed to DoExecute, it is often necessary to gather further information from the DataSet.

The problem will retrieving data from a DataSet is that the specific types of the data are not known. For example, you can get a vtkm: :cont: Field object from the DataSet, but to do anything of interest with the Field, you have to then pull the array out of the field. The problem is that you do not know what type of values are stored in the array. Nor do you know the storage mechanism for the array.

This is where policies come into play. All DoExecute methods also receive a policy object. The policy object is really just an empty structure, but it contains important compile-time type information that can be used to deduce which types to expect when pulling fields and cell sets from a DataSet. The policy can be applied by using one of the following functions, all of which are defined in vtkm/filter/PolicyBase.h. 


\section{Common Errors}

Policies define a finite list of types to try when retrieving objects from a DataSet. If the DataSet holds an object of an uncommon type not checked, then a runtime error will occur.

\subsubsection{Getting Field Data}

Filter types like FilterField and FilterDataSetWithField will automatically pull an ArrayHandle from a field in the input DataSet and pass it to the subclass' DoExecute. However, sometimes the filter will need more than one field. Any secondary fields that the filter needs will have to be pulled from the DataSet within the implementation of the DoExecute method.

The best way to do this is to use the vtkm: :filter: :ApplyPolicyFieldOfType method. This method takes 3 arguments: a vtkm: :cont::Field object (presumably pulled from the DataSet), the policy, and the filter (i.e. *this). ApplyPolicyFieldOfType also requires an explicit template argument that specifies the value type desired. Typically this type will be the same type as the type of the primary field's ArrayHandle provided to DoExecute.

Example 22.11: Using a policy to get the ArrayHandle out of a Field.

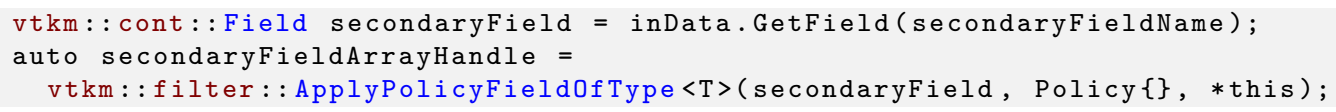

The object returned from ApplyPolicyFieldOfType is an ArrayHandle. The value type of the ArrayHandle will be the same as the type specified in the template to ApplyPolicyField0fType. So the type of secondaryFieldArrayHandle, defined on line 2 of example 22.11 is vtkm: : cont: : ArrayHandle $\langle\mathrm{T}, \ldots\rangle$. The storage type of the returned ArrayHandle is rather complex and not documented here.

Did you know?

3 When using ApplyPolicyFieldOfType, the value type requested does not have to match exactly with the type in the underlying array. For example, if the Field contains an ArrayHandle with a value type of vtkm: : Int32 and you use ApplyPolicyFieldOfType to get an array with a value type of vtkm::Float32, then you will get an ArrayHandle that converts the type from vtkm::Int32 to vtkm::Float32. This is not done with a deep copy. Rather, this is done any time a value is retrieved from the array (usually during worklet invocation).

\subsubsection{Getting Cell Sets}

Any filter that does operations on the topology of a DataSet needs to retrieve a vtkm: : cont: : CellSet it. Of course, Cellsets come in many varieties, and the correct one must be deduced to invoke a worklet on it. This can be done with the vtkm: filter: :ApplyPolicyCellset function.

ApplyPolicyCellSet takes 3 arguments: a vtkm: : cont: : DynamicCellSet (presumably pulled with DataSet: :GetCellSet), the policy, and the filter object itself. 
Example 22.12: Using a policy to get the Cellset from a DataSet.

1 auto inCells $=$

2 vtkm::filter::ApplyPolicyCellSet(inData.GetCellSet(), Policy\{\}, *this);

There are also instances where the algorithm on the Cellset is different depending on whether the Cellset is structured or unstructured (or the algorithm may only work on one or the other). In this case you can use either vtkm: :filter: :ApplyPolicyCellSetStructured or vtkm: :filter: :ApplyPolicyCellSetUnstructured to only get Cellsets that match that type.

Example 22.13: Using a policy to get on structured cell sets.

auto cellset = vtkm:: filter: : ApplyPolicyCellSetStructured (

inDataSet.GetCellSet(), Policy \{\}$, * t h i s)$; 


\section{WORKLET ERROR HANDLING}

It is sometimes the case during the execution of an algorithm that an error condition can occur that causes the computation to become invalid. At such a time, it is important to raise an error to alert the calling code of the problem. Since VTK-m uses an exception mechanism to raise errors, we want an error in the execution environment to throw an exception.

However, throwing exceptions in a parallel algorithm is problematic. Some accelerator architectures, like CUDA, do not even support throwing exceptions. Even on architectures that do support exceptions, throwing them in a thread block can cause problems. An exception raised in one thread may or may not be thrown in another, which increases the potential for deadlocks, and it is unclear how uncaught exceptions progress through thread blocks.

VTK-m handles this problem by using a flag and check mechanism. When a worklet (or other subclass of vtkm: :exec: :FunctorBase) encounters an error, it can call its RaiseError method to flag the problem and record a message for the error. Once all the threads terminate, the scheduler checks for the error, and if one exists it throws a vtkm: : cont: :ErrorExecution exception in the control environment. Thus, calling RaiseError looks like an exception was thrown from the perspective of the control environment code that invoked it.

Example 23.1: Raising an error in the execution environment.

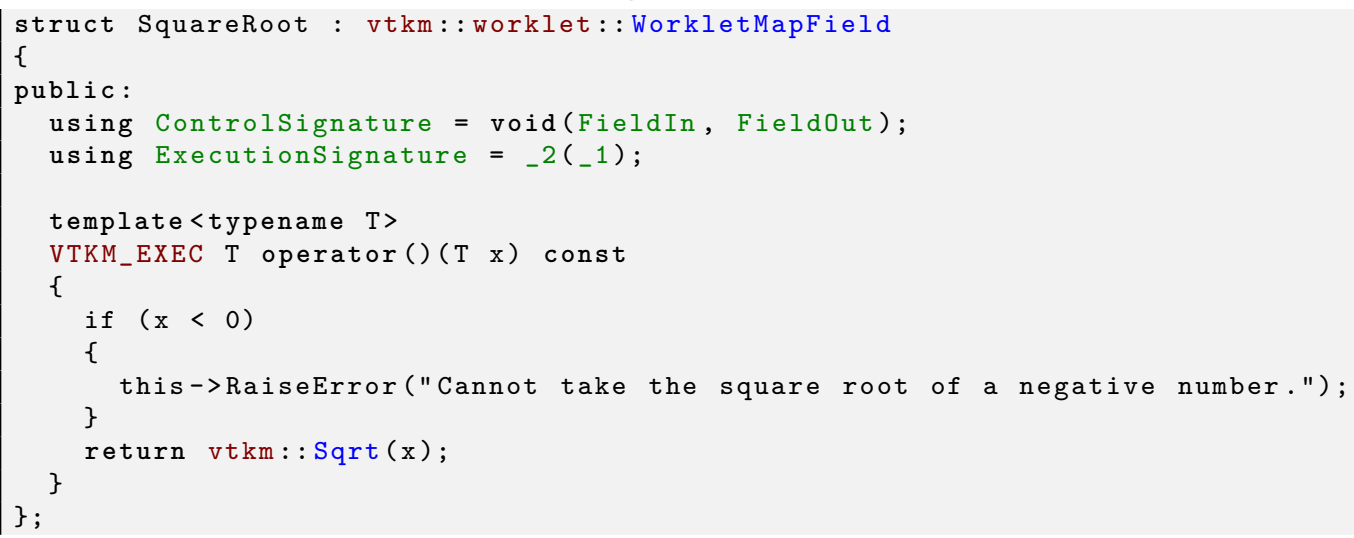

It is also worth noting that the VTKM_ASSERT macro described in Section 11.2 also works within worklets and other code running in the execution environment. Of course, a failed assert will terminate execution rather than just raise an error so is best for checking invalid conditions for debugging purposes. 

VTK-m comes with several math functions that tend to be useful for visualization algorithms. The implementation of basic math operations can vary subtly on different accelerators, and these functions provide cross platform support.

All math functions are located in the vtkm package. The functions are most useful in the execution environment, but they can also be used in the control environment when needed.

\subsection{Basic Math}

The vtkm/Math.h header file contains several math functions that replicate the behavior of the basic POSIX math functions as well as related functionality.

Did you know?

When writing worklets, you should favor using these math functions provided by VTK-m over the standard math functions in math.h. VTK-m's implementation manages several compiling and efficiency issues when porting.

vtkm: :Abs Returns the absolute value of the single argument. If given a vector, performs a component-wise operation.

vtkm: :ACos Returns the arccosine of a ratio in radians. If given a vector, performs a component-wise operation.

vtkm: : ACosH Returns the hyperbolic arccossine. If given a vector, performs a component-wise operation.

vtkm: :ASin Returns the arcsine of a ratio in radians. If given a vector, performs a component-wise operation.

vtkm: :ASinH Returns the hyperbolic arcsine. If given a vector, performs a component-wise operation.

vtkm: :ATan Returns the arctangent of a ratio in radians. If given a vector, performs a component-wise operation.

vtkm: :ATan2 Computes the arctangent of $y / x$ where $y$ is the first argument and $x$ is the second argument. ATan2 uses the signs of both arguments to determine the quadrant of the return value. ATan2 is only defined for floating point types (no vectors). 
vtkm: :ATanH Returns the hyperbolic arctangent. If given a vector, performs a component-wise operation.

vtkm: :Cbrt Takes one argument and returns the cube root of that argument. If called with a vector type, returns a component-wise cube root.

vtkm: Ceil Rounds and returns the smallest integer not less than the single argument. If given a vector, performs a component-wise operation.

vtkm: : CopySign Copies the sign of the second argument onto the first argument and returns that. If the second argument is positive, returns the absolute value of the first argument. If the second argument is negative, returns the negative absolute value of the first argument.

vtkm: : Cos Returns the cosine of an angle given in radians. If given a vector, performs a component-wise operation.

vtkm: : CosH Returns the hyperbolic cosine. If given a vector, performs a component-wise operation.

vtkm: :Epsilon Returns the difference between 1 and the least value greater than 1 that is representable by a floating point number. Epsilon is useful for specifying the tolerance one should have when considering numerical error. The Epsilon method is templated to specify either a 32 or 64 bit floating point number. The convenience methods Epsilon32 and Epsilon64 are non-templated versions that return the precision for a particular precision.

vtkm: : Exp Computes $e^{x}$ where $x$ is the argument to the function and $e$ is Euler's number (approximately 2.71828). If called with a vector type, returns a component-wise exponent.

vtkm: :Exp10 Computes $10^{x}$ where $x$ is the argument. If called with a vector type, returns a component-wise exponent.

vtkm: :Exp2 Computes $2^{x}$ where $x$ is the argument. If called with a vector type, returns a component-wise exponent.

vtkm: : ExpM1 Computes $e^{x}-1$ where $x$ is the argument to the function and $e$ is Euler's number (approximately $2.71828)$. The accuracy of this function is good even for very small values of $x$. If called with a vector type, returns a component-wise exponent.

vtkm::FloatDistance Computes the number of representables between two floating point numbers. This function is non-negative and symmetric in its arguments. If either argument is non-finite, the value returned is the maximum value allowed by 64 -bit unsigned integers: $2^{64}-1$.

vtkm: :Floor Rounds and returns the largest integer not greater than the single argument. If given a vector, performs a component-wise operation.

vtkm::FMod Computes the remainder on the division of 2 floating point numbers. The return value is numerator $-n \cdot$ denominator, where numerator is the first argument, denominator is the second argument, and $n$ is the quotient of numerator divided by denominator rounded towards zero to an integer. For example, FMod $(6.5,2.3)$ returns 1.9 , which is $6.5-2 \cdot 4.6$. If given vectors, FMod performs a componentwise operation. FMod is similar to Remainder except that the quotient is rounded toward 0 instead of the nearest integer.

vtkm: Infinity Returns the representation for infinity. The result is greater than any other number except another infinity or NaN. When comparing two infinities or infinity to NaN, neither is greater than, less than, nor equal to the other. The Infinity method is templated to specify either a 32 or 64 bit floating point number. The convenience methods Infinity32 and Infinity64 are non-templated versions that return the precision for a particular precision. 
vtkm: IsFinite Returns true if the argument is a normal number (neither a NaN nor an infinite).

vtkm: : IsInf Returns true if the argument is either positive infinity or negative infinity.

vtkm: : IsNan Returns true if the argument is not a number $(\mathrm{NaN})$.

vtkm: : IsNegative Returns true if the single argument is less than zero, false otherwise.

vtkm: : Log Computes the natural logarithm (i.e. logarithm to the base $e$ ) of the single argument. If called with a vector type, returns a component-wise logarithm.

vtkm: : Log10 Computes the logarithm to the base 10 of the single argument. If called with a vector type, returns a component-wise logarithm.

vtkm: : Log1P Computes $\ln (1+x)$ where $x$ is the single argument and $\ln$ is the natural logarithm (i.e. logarithm to the base $e$ ). The accuracy of this function is good for very small values. If called with a vector type, returns a component-wise logarithm.

vtkm: :Log2 Computes the logarithm to the base 2 of the single argument. If called with a vector type, returns a component-wise logarithm.

vtkm: : Max Takes two arguments and returns the argument that is greater. If called with a vector type, returns a component-wise maximum.

vtkm: : Min Takes two arguments and returns the argument that is lesser. If called with a vector type, returns a component-wise minimum.

vtkm: :ModF Returns the integral and fractional parts of the first argument. The second argument is a reference in which the integral part is stored. The return value is the fractional part. If given vectors, ModF performs a component-wise operation.

vtkm: : Nan Returns the representation for not-a-number (NaN). A NaN represents an invalid value or the result of an invalid operation such as $0 / 0$. A NaN is neither greater than nor less than nor equal to any other number including other NaNs. The NaN method is templated to specify either a 32 or 64 bit floating point number. The convenience methods Nan32 and NaN64 are non-templated versions that return the precision for a particular precision.

vtkm: : NegativeInfinity Returns the representation for negative infinity. The result is less than any other number except another negative infinity or NaN. When comparing two negative infinities or negative infinity to NaN, neither is greater than, less than, nor equal to the other. The NegativeInfinity method is templated to specify either a 32 or 64 bit floating point number. The convenience methods NegativeInfinity32 and NegativeInfinity64 are non-templated versions that return the precision for a particular precision.

vtkm: :Pi Returns the constant $\pi$ (about 3.14159).

vtkm::Pi_2 Returns the constant $\pi / 2$ (about 1.570796).

vtkm::Pi_3 Returns the constant $\pi / 3$ (about 1.047197).

vtkm: :Pi_4 Returns the constant $\pi / 4$ (about 0.785398).

vtkm: : Pow Takes two arguments and returns the first argument raised to the power of the second argument.

This function is only defined for vtkm: :Float32 and vtkm: :Float64. 
vtkm: : QuadraticRoots Takes the coefficients $a, b, c$ of the quadratic equation $a x^{2}+b x+c=0$ and returns the real roots in a vtkm: $:$ Vec of size 2 . If there are no roots, then both returned values are NaNs. If there are two real roots, the first element is less than or equal to the second. If compiled with FMA support, each root is accurate to 3 ulps; otherwise the discriminant is prone to catastrophic subtractive cancellation and no accuracy guarantees can be provided.

vtkm: :RCbrt Takes one argument and returns the cube root of that argument. The result of this function is equivalent to $1 / \operatorname{Cbrt}(\mathrm{x})$. However, on some devices it is faster to compute the reciprocal cube root than the regular cube root. Thus, you should use this function whenever dividing by the cube root.

vtkm: :Remainder Computes the remainder on the division of 2 floating point numbers. The return value is numerator $-n$-denominator, where numerator is the first argument, denominator is the second argument, and $n$ is the quotient of numerator divided by denominator rounded towards the nearest integer. For example, FMod $(6.5,2.3)$ returns -0.4 , which is $6.5-3 \cdot 2.3$. If given vectors, Remainder performs a component-wise operation. Remainder is similar to FMod except that the quotient is rounded toward the nearest integer instead of toward 0 .

vtkm: : RemainderQuotient Performs an operation identical to Reminder. In addition, this function takes a third argument that is a reference in which the quotient is given.

vtkm: : Round Rounds and returns the integer nearest the single argument. If given a vector, performs a component-wise operation.

vtkm: :RSqrt Takes one argument and returns the square root of that argument. The result of this function is equivalent to $1 / \operatorname{Sqrt}(\mathrm{x})$. However, on some devices it is faster to compute the reciprocal square root than the regular square root. Thus, you should use this function whenever dividing by the square root.

vtkm: :SignBit Returns a nonzero value if the single argument is negative.

vtkm: : Sin Returns the sine of an angle given in radians. If given a vector, performs a component-wise operation.

vtkm: : SinH Returns the hyperbolic sine. If given a vector, performs a component-wise operation.

vtkm: :Sqrt Takes one argument and returns the square root of that argument. If called with a vector type, returns a component-wise square root. On some hardware it is faster to find the reciprocal square root, so RSqrt should be used if you actually plan to divide by the square root.

vtkm: :Tan Returns the tangent of an angle given in radians. If given a vector, performs a component-wise operation.

vtkm: : TanH Returns the hyperbolic tangent. If given a vector, performs a component-wise operation.

vtkm: :TwoPi Returns the constant $2 \pi$ (about 6.283185).

\subsection{Vector Analysis}

Visualization and computational geometry algorithms often perform vector analysis operations. The vtkm/VectorAnalysis.h header file provides functions that perform the basic common vector analysis operations.

vtkm: : Cross Returns the cross product of two vtkm: :Vec of size 3. If compiled with FMA support, it uses Kahan's difference of products algorithm to achieve a maximum error of 1.5 ulps in each component. 
vtkm: Lerp Given two values $x$ and $y$ in the first two parameters and a weight $w$ as the third parameter, interpolates between $x$ and $y$. Specifically, the linear interpolation is $(y-x) w+x$ although Lerp might compute the interpolation faster than using the independent arithmetic operations. The two values may be scalars or equal sized vectors. If the two values are vectors and the weight is a scalar, all components of the vector are interpolated with the same weight. If the weight is also a vector, then each component of the value vectors are interpolated with the respective weight component.

vtkm: :Magnitude Returns the magnitude of a vector. This function works on scalars as well as vectors, in which case it just returns the scalar. It is usually much faster to compute MagnitudeSquared, so that should be substituted when possible (unless you are just going to take the square root, which would be besides the point). On some hardware it is also faster to find the reciprocal magnitude, so RMagnitude should be used if you actually plan to divide by the magnitude.

vtkm: : MagnitudeSquared Returns the square of the magnitude of a vector. It is usually much faster to compute the square of the magnitude than the length, so you should use this function in place of Magnitude or RMagnitude when needing the square of the magnitude or any monotonically increasing function of a magnitude or distance. This function works on scalars as well as vectors, in which case it just returns the square of the scalar.

vtkm: Normal Returns a normalized version of the given vector. The resulting vector points in the same direction as the argument but has unit length.

vtkm: : Normalize Takes a reference to a vector and modifies it to be of unit length. Normalize(v) is functionally equivalent to $\mathrm{v} *=$ RMagnitude $(\mathrm{v})$.

vtkm: :RMagnitude Returns the reciprocal magnitude of a vector. On some hardware RMagnitude is faster than Magnitude, but neither is as fast as MagnitudeSquared. This function works on scalars as well as vectors, in which case it just returns the reciprocal of the scalar.

vtkm: :TriangleNormal Given three points in space (contained in vtkm: :Vec s of size 3) that compose a triangle return a vector that is perpendicular to the triangle. The magnitude of the result is equal to twice the area of the triangle. The result points away from the "front" of the triangle as defined by the standard counter-clockwise ordering of the points.

\subsection{Matrices}

Linear algebra operations on small matrices that are done on a single thread are located in vtkm/Matrix.h.

This header defines the vtkm: :Matrix templated class. The template parameters are first the type of component, then the number of rows, then the number of columns. The overloaded parentheses operator can be used to retrieve values based on row and column indices. Likewise, the bracket operators can be used to reference the Matrix as a 2D array (indexed by row first). The following example builds a Matrix that contains the values

$$
\begin{array}{ccc}
0 & 1 & 2 \\
10 & 11 & 12
\end{array}
$$

Example 24.1: Creating a Matrix.

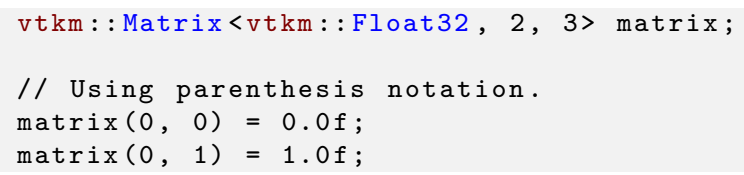




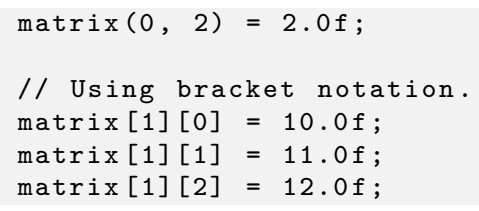

The vtkm/Matrix.h header also defines the following functions that operate on matrices.

vtkm: : MatrixDeterminant Takes a square Matrix as its single argument and returns the determinant of that matrix.

vtkm: : MatrixGetColumn Given a Matrix and a column index, returns a vtkm::Vec of that column. This function might not be as efficient as vtkm: :MatrixRow. (It performs a copy of the column).

vtkm: :MatrixGetRow Given a Matrix and a row index, returns a vtkm: :Vec of that row.

vtkm: : MatrixIdentity Returns the identity matrix. If given no arguments, it creates an identity matrix and returns it. (In this form, the component type and size must be explicitly set.) If given a single square matrix argument, fills that matrix with the identity.

vtkm: :MatrixInverse Finds and returns the inverse of a given matrix. The function takes two arguments. The first argument is the matrix to invert. The second argument is a reference to a Boolean that is set to true if the inverse is found or false if the matrix is singular and the returned matrix is incorrect.

vtkm: :MatrixMultiply Performs a matrix-multiply on its two arguments. Overloaded to work for matrixmatrix, vector-matrix, or matrix-vector multiply.

vtkm: : MatrixSetColumn Given a Matrix, a column index, and a vtkm::Vec, sets the column of that index to the values of the Tuple.

vtkm: :MatrixSetRow Given a Matrix, a row index, and a vtkm: :Vec, sets the row of that index to the values of the Tuple.

vtkm: :MatrixTranspose Takes a Matrix and returns its transpose.

vtkm: : SolveLinearSystem Solves the linear system $A x=b$ and returns $x$. The function takes three arguments. The first two arguments are the matrix $A$ and the vector $b$, respectively. The third argument is a reference to a Boolean that is set to true if a single solution is found, false otherwise.

\subsection{Newton's Method}

VTK-m's matrix methods (documented in Section 24.3) provide a method to solve a small linear system of equations. However, sometimes it is necessary to solve a small nonlinear system of equations. This can be done with the vtkm: : NewtonsMethod function defined in the vtkm/NewtonsMethod.h header.

The NewtonsMethod function assumes that the number of variables equals the number of equations. Newton's method operates on an iterative evaluate and search. Evaluations are performed using the functors passed into the NewtonsMethod. The function takes the following 6 parameters (three of which are optional).

1. A functor whose operation takes a vtkm: :Vec and returns a vtkm: :Matrix containing the math function's Jacobian vector at that point. 
2. A functor whose operation takes a vtkm: Vec and returns the evaluation of the math function at that point as another vtkm: :Vec.

3. The vtkm: :Vec that represents the desired output of the function.

4. A vtkm: :Vec to use as the initial guess. If not specified, the origin is used.

5. The convergence distance. If the iterative method changes all values less than this amount, then it considers the solution found. If not specified, set to $10^{-3}$.

6. The maximum amount of iterations to run before giving up and returning the best solution. If not specified, set to 10 .

The NewtonsMethod function returns a vtkm: : NewtonsMethodResult object. NewtonsMethodResult is a struct templated on the type and number of input values of the nonlinear system. NewtonsMethodResult contains the following items.

Valid A bool that is set to false if the solution runs into a singularity so that no possible solution is found.

Converged A bool that is set to true if a solution is found that is within the convergence distance specified. It is set to false if the method did not convert in the specified number of iterations.

Solution A vtkm: Vec containing the solution to the nonlinear system. If Converged is false, then this value is likely inaccurate. If Valid is false, then this value is undefined.

Example 24.2: Using NewtonsMethod to solve a small system of nonlinear equations.

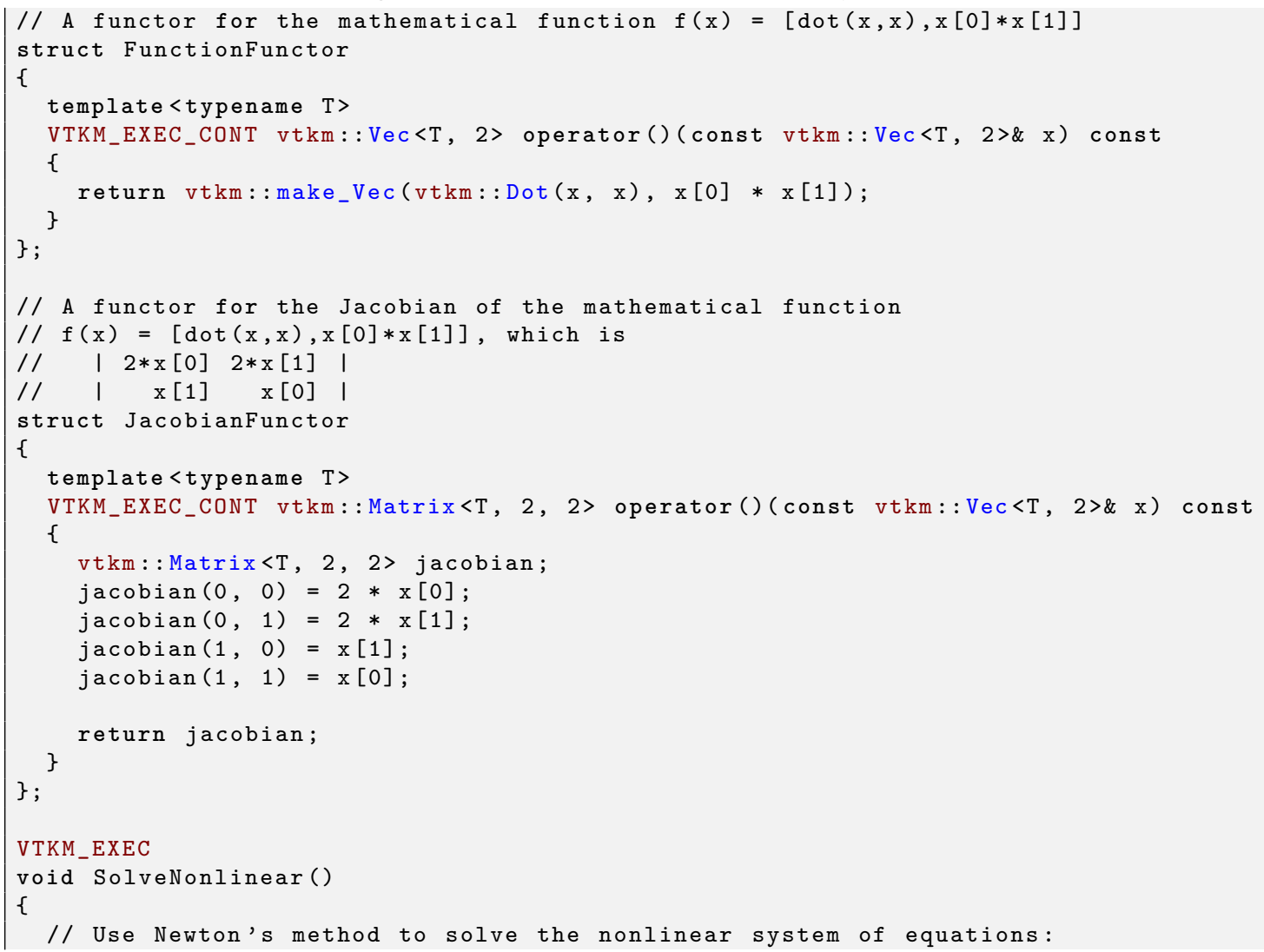




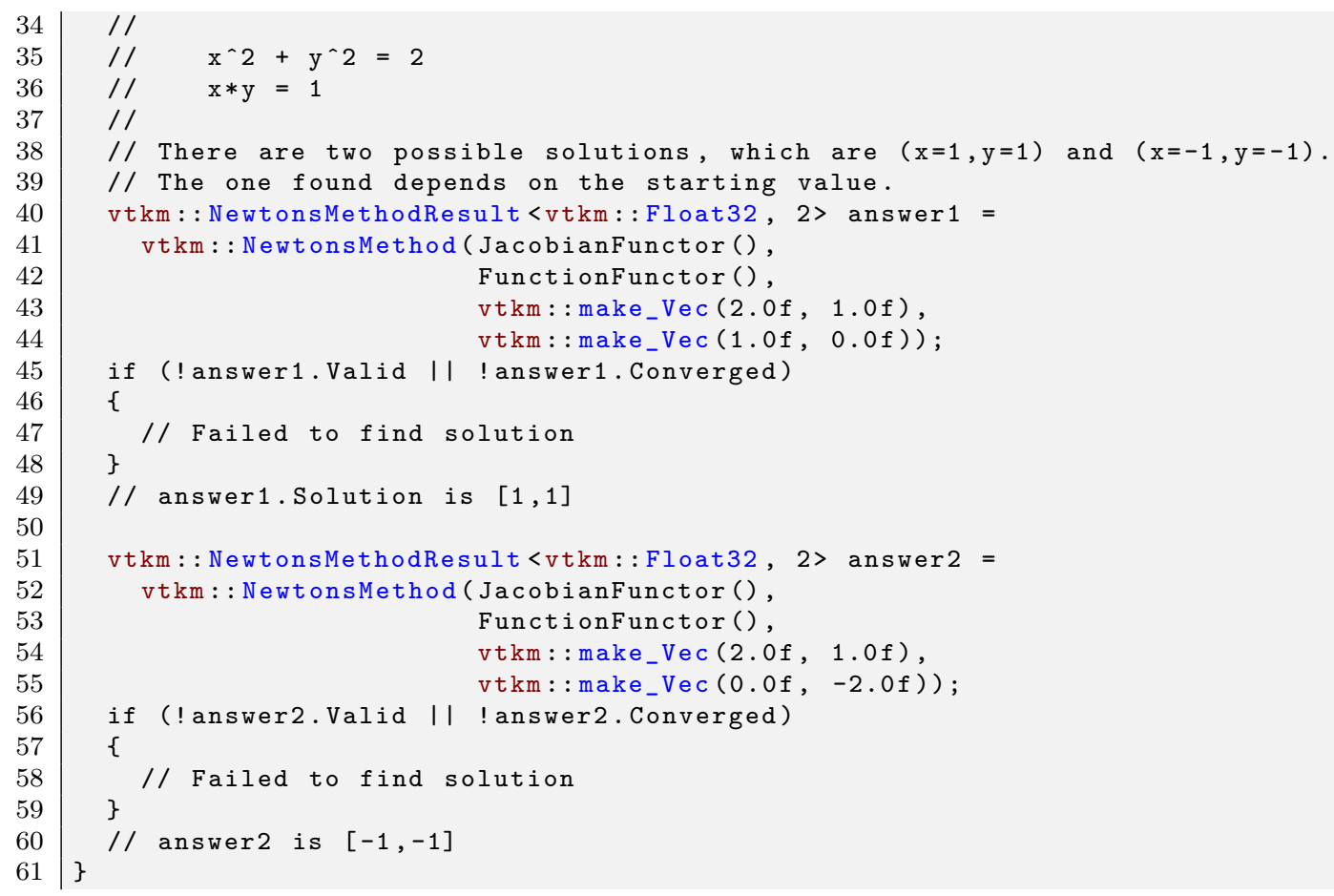




\section{WORKING WITH CELLS}

In the control environment, data is defined in mesh structures that comprise a set of finite cells. (See Section 7.2 starting on page 36 for information on defining cell sets in the control environment.) When worklets that operate on cells are scheduled, these grid structures are broken into their independent cells, and that data is handed to the worklet. Thus, cell-based operations in the execution environment exclusively operate on independent cells.

Unlike some other libraries such as VTK, VTK-m does not have a cell class that holds all the information pertaining to a cell of a particular type. Instead, VTK-m provides tags or identifiers defining the cell shape, and companion data like coordinate and field information are held in separate structures. This organization is designed so a worklet may specify exactly what information it needs, and only that information will be loaded.

\subsection{Cell Shape Tags and Ids}

Cell shapes can be specified with either a tag (defined with a struct with a name like CellShapeTag*) or an enumerated identifier (defined with a constant number with a name like CELL_SHAPE_*). These shape tags and identifiers are defined in vtkm/CellShape.h and declared in the vtkm namespace (because they can be used in either the control or the execution environment). Figure 25.1 gives both the identifier and the tag names.

In addition to the basic cell shapes, there is a special "empty" cell with the identifier vtkm: :CELL_SHAPE_EMPTY and tag vtkm: :CellShapeTagEmpty. This type of cell has no points, edges, or faces and can be thought of as a placeholder for a null or void cell.

There is also a special cell shape "tag" named vtkm: :CellShapeTagGeneric that is used when the actual cell shape is not known at compile time. CellShapeTagGeneric actually has a member variable named Id that stores the identifier for the cell shape. There is no equivalent identifier for a generic cell; cell shape identifiers can be placed in a vtkm: : IdComponent at runtime.

When using cell shapes in templated classes and functions, you can use the VTKM_IS_CELL_SHAPE_TAG to ensure a type is a valid cell shape tag. This macro takes one argument and will produce a compile error if the argument is not a cell shape tag type.

\subsubsection{Converting Between Tags and Identifiers}

Every cell shape tag has a member variable named Id that contains the identifier for the cell shape. This provides a convenient mechanism for converting a cell shape tag to an identifier. Most cell shape tags have their Id member as a compile-time constant, but CellShapeTagGeneric is set at run time.

vtkm/CellShape.h also declares a templated class named vtkm: CellshapeIdToTag that converts a cell shape 
vtkm: :CELL_SHAPE_VERTEX vtkm: : CellShapeTagVertex

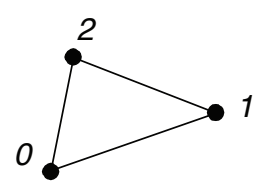

vtkm: :CELL_SHAPE_TRIANGLE vtkm: :CellShapeTagTriangle

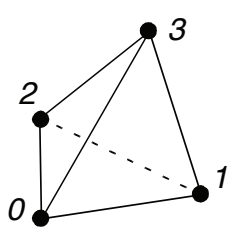

vtkm: :CELL_SHAPE_TETRA vtkm: :CellShapeTagTetra

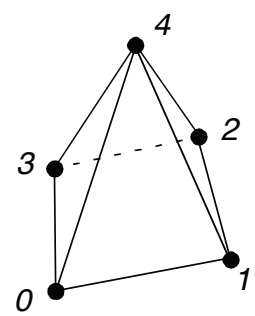

vtkm: :CELL_SHAPE_PYRAMID vtkm: :CellShapeTagPyramid

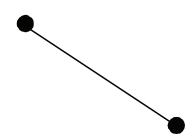

vtkm: :CELL_SHAPE_LINE

vtkm: CellShapeTagLine

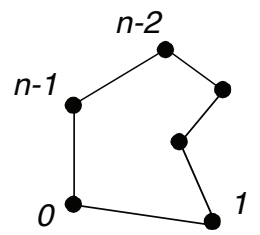

vtkm: :CELL_SHAPE_POLYGON vtkm: : CellShapeTagPolygon

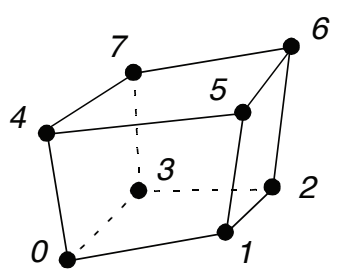

vtkm: :CELL_SHAPE_HEXAHEDRON vtkm: :CellShapeTagHexahedron

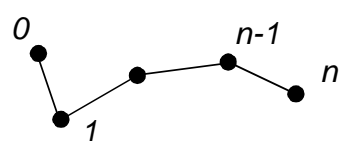

vtkm: :CELL_SHAPE_POLY_LINE vtkm: : CellShapeTagPolyLine

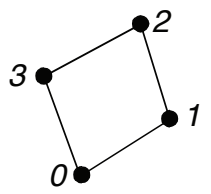

vtkm: :CELL_SHAPE_QUAD vtkm: :CellShapeTagQuad

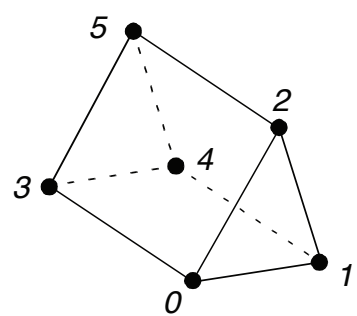

vtkm: :CELL_SHAPE_WEDGE vtkm: : CellShapeTagWedge

Figure 25.1: Basic Cell Shapes

identifier to a cell shape tag. CellShapeIdToTag has a single template argument that is the identifier. Inside the class is a type named Tag that is the type of the correct tag.

Example 25.1: Using CellShapeIdToTag.

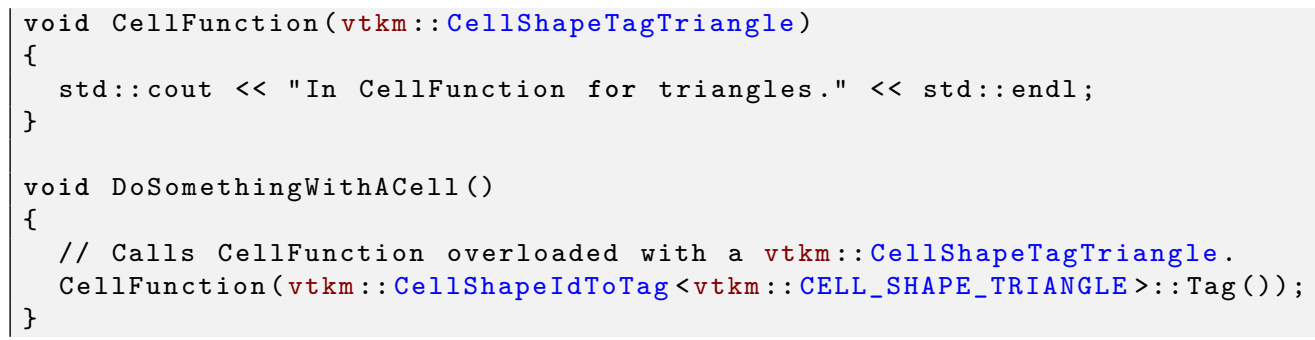

However, CellShapeIdToTag is only viable if the identifier can be resolved at compile time. In the case where a cell identifier is stored in a variable or an array or the code is using a CellShapeTagGeneric, the correct cell 
shape is not known at run time. In this case, vtkmGenericCellShapeMacro can be used to check all possible conditions. This macro is embedded in a switch statement where the condition is the cell shape identifier. vtkmGenericCellShapeMacro has a single argument, which is an expression to be executed. Before the expression is executed, a type named CellShapeTag is defined as the type of the appropriate cell shape tag. Often this method is used to implement the condition for a CellShapeTagGeneric in a function overloaded for cell types. A demonstration of vtkmGenericCellShapeMacro is given in Example 25.2.

\subsubsection{Cell Traits}

The vtkm/CellTraits.h header file contains a traits class named vtkm: :CellTraits that provides information about a cell. Each specialization of CellTraits contains the following members.

TOPOLOGICAL_DIMENSIONS Defines the topological dimensions of the cell type. This is 3 for polyhedra, 2 for polygons, 1 for lines, and 0 for points.

TopologicalDimensionsTag A type set to either vtkm::CellTopologicalDimensionsTag <3>, CellTopologicalDimensionsTag<2>, CellTopologicalDimensionsTag<1>, or CellTopologicalDimensionsTag<0>. The number is consistent with TOPOLOGICAL_DIMENSIONS. This tag is provided for convenience when specializing functions.

IsSizeFixed Set to either vtkm: :CellTraitsTagSizeFixed for cell types with a fixed number of points (for example, triangle) or vtkm: :CellTraitsTagSizeVariable for cell types with a variable number of points (for example, polygon).

NUM_POINTS A vtkm: :IdComponent set to the number of points in the cell. This member is only defined when there is a constant number of points (i.e. IsSizeFixed is set to vtkm: :CellTraitsTagSizeFixed).

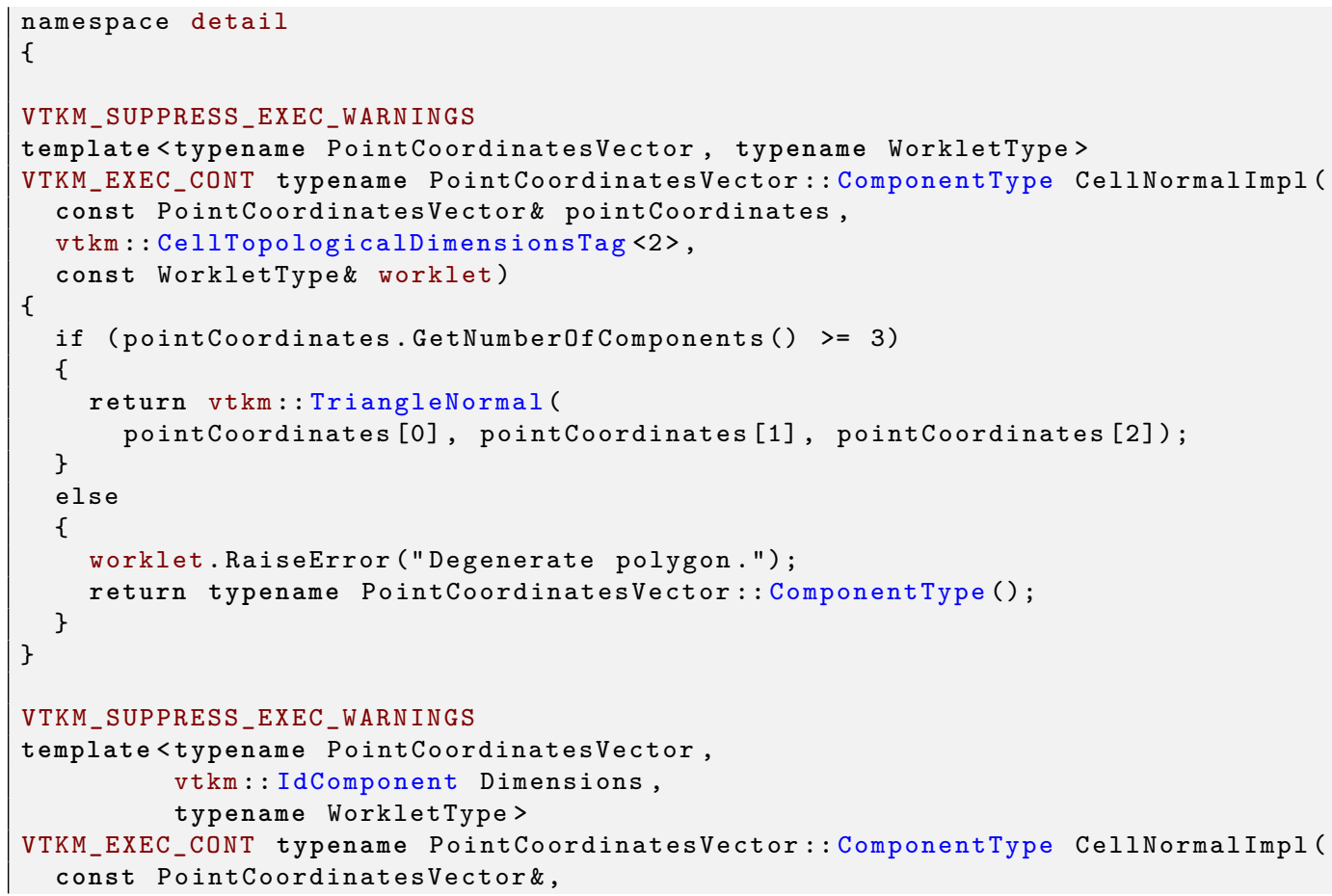




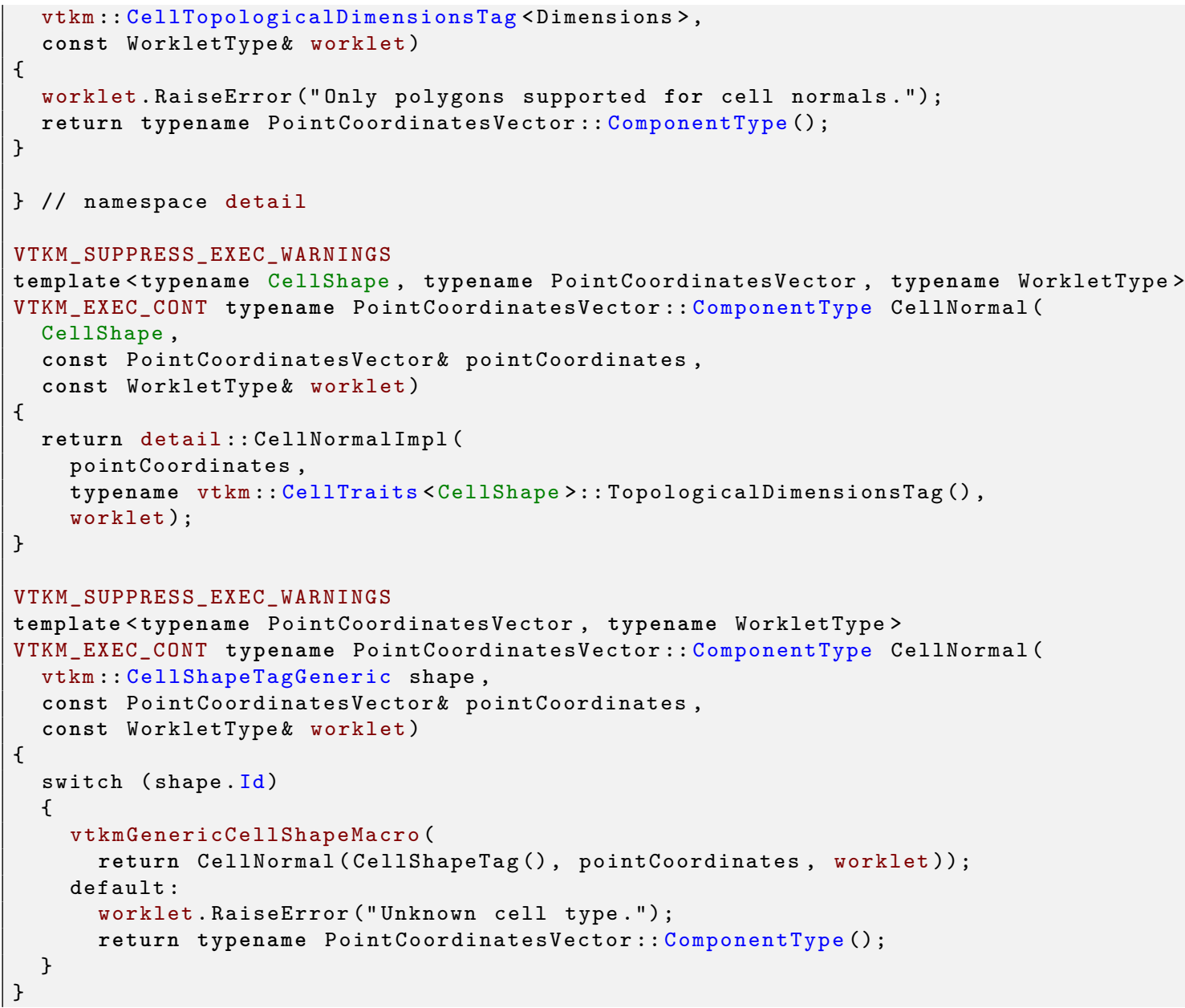

\subsection{Parametric and World Coordinates}

Each cell type supports a one-to-one mapping between a set of parametric coordinates in the unit cube (or some subset of it) and the points in 3D space that are the locus contained in the cell. Parametric coordinates are useful because certain features of the cell, such as vertex location and center, are at a consistent location in parametric space irrespective of the location and distortion of the cell in world space. Also, many field operations are much easier with parametric coordinates.

The vtkm/exec/ParametricCoordinates.h header file contains the following functions for working with parametric coordinates.

vtkm::exec::ParametricCoordinatesCenter Returns the parametric coordinates for the center of a given shape. It takes 3 arguments: the number of points in the cell, a shape tag, and a vtkm: :Vec of size 3 to store the results. An vtkm: :ErrorCode is returned (see Section 19.9).

vtkm: :exec: :ParametricCoordinatesPoint Returns the parametric coordinates for a given point of a given shape. It takes 4 arguments: the number of points in the cell, the index of the point to query, a shape tag, and a vtkm: :Vec of size 3 to store the results. An vtkm: :ErrorCode is returned (see Section 19.9). 
vtkm: :exec::ParametricCoordinatesToWorldCoordinates Converts parametric coordinates (coordinates relative to the cell) to world coordinates (coordinates in the global system). It takes a vector of point coordinates (usually given by a FieldPointIn worklet argument), a vtkm: : Vec of size 3 containing parametric coordinates, a shape tag, and a vtkm: :Vec of size 3 to store the resulting world coordinates. An vtkm: : ErrorCode is returned (see Section 19.9).

vtkm: :exec: :WorldCoordinatesToParametricCoordinates Converts world coordinates (coordinates in the global system) to parametric coordinates (coordinates relative to the cell). It takes a vector of point coordinates (usually given by a FieldPointIn worklet argument), a vtkm: : Vec of size 3 containing world coordinates, a shape tag, and a vtkm: :Vec of size 3 to store the resulting parametric coordinates. An vtkm: :ErrorCode is returned (see Section 19.9). This function can be slow for cell types with nonlinear interpolation (which is anything that is not a simplex).

\subsection{Interpolation}

The shape of every cell is defined by the connections of some finite set of points. Field values defined on those points can be interpolated to any point within the cell to estimate a continuous field.

The vtkm/exec/Celllnterpolate.h header contains the function vtkm: :exec: :CellInterpolate that takes a vector of point field values (usually given by a FieldPointIn worklet argument), a vtkm::Vec of size 3 containing parametric coordinates, a shape tag. The result of the interpolation is placed in the reference passed as the fourth argument. CellInterpolate returns an vtkm: :ErrorCode for the status of the operation.

Example 25.3: Interpolating field values to a cell's center.

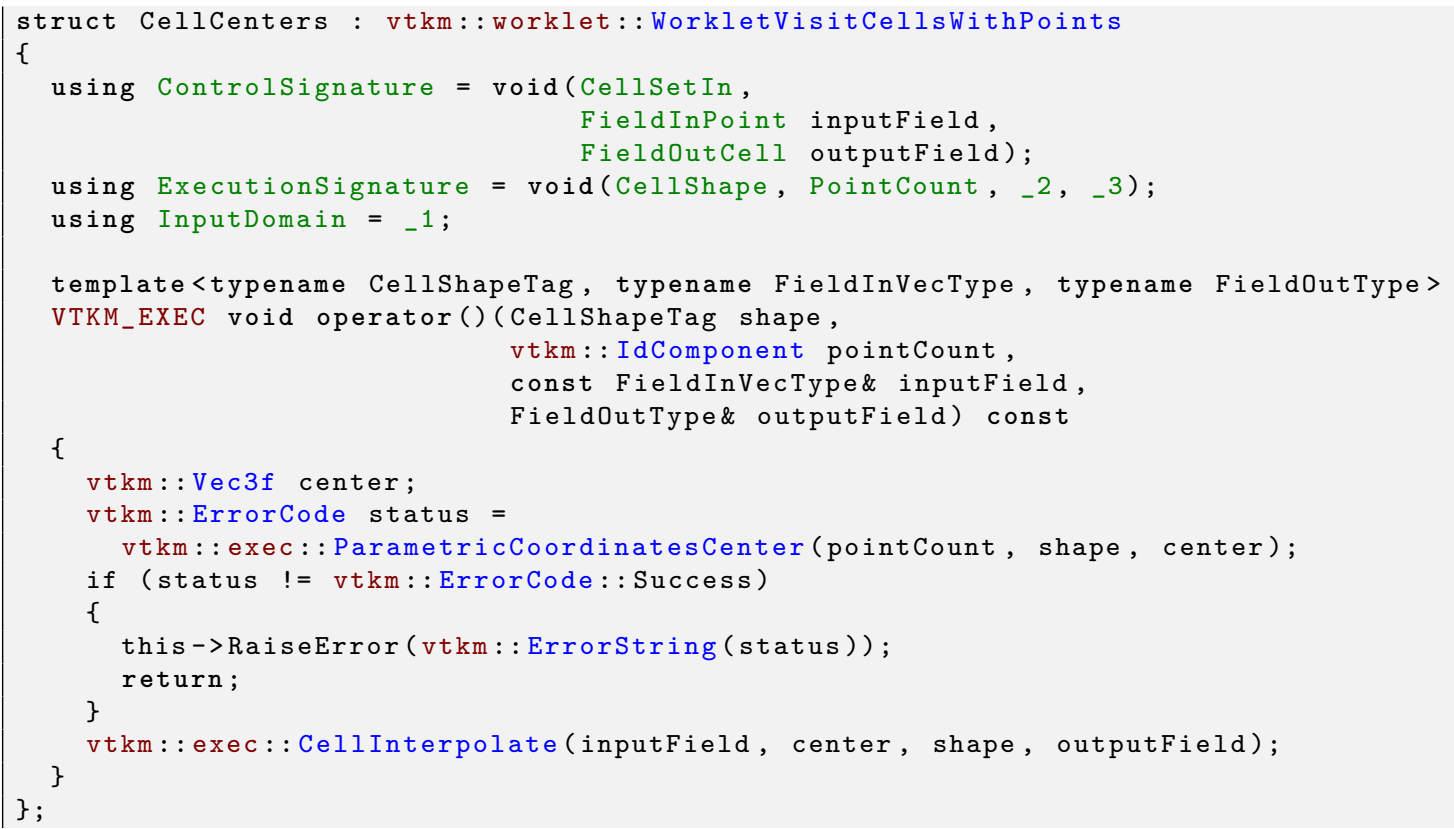

\subsection{Derivatives}

Since interpolations provide a continuous field function over a cell, it is reasonable to consider the derivative of this function. The vtkm/exec/CellDerivative.h header contains the function vtkm: :exec: :CellDerivative that 
takes a vector of scalar point field values (usually given by a FieldPointIn worklet argument), a vtkm: :Vec of size 3 containing parametric coordinates, a shape tag, and a worklet object (for raising errors). It returns the field derivative at the location represented by the given parametric coordinates. The derivative is return in a vtkm: : Vec of size 3 corresponding to the partial derivatives in the $x, y$, and $z$ directions. This derivative is equivalent to the gradient of the field.

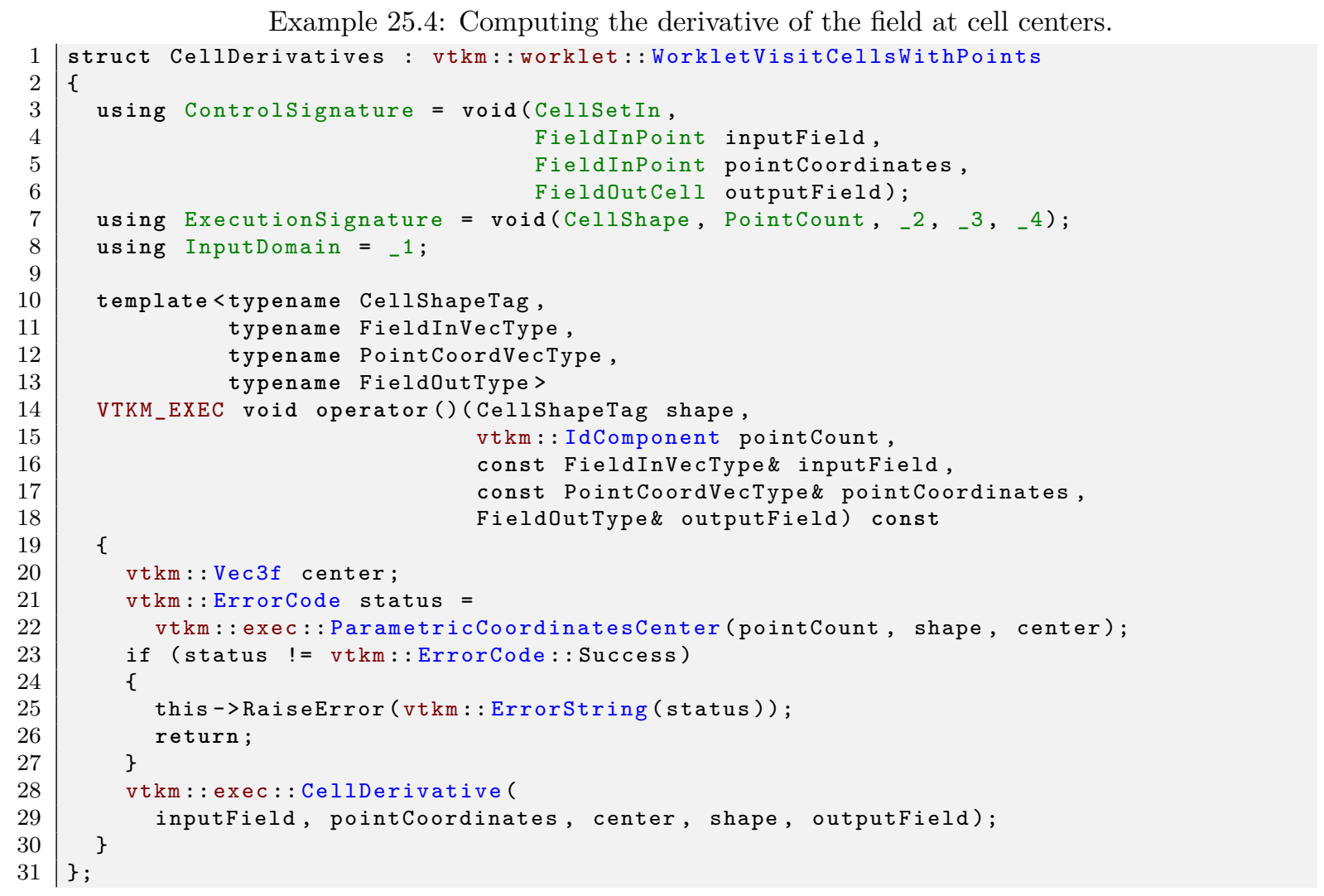

\subsection{Edges and Faces}

As explained earlier in this chapter, a cell is defined by a collection of points and a shape identifier that describes how the points come together to form the structure of the cell. The cell shapes supported by VTK-m are documented in Section 25.1. It contains Figure 25.1 on page 206, which shows how the points for each shape form the structure of the cell.

Most cell shapes can be broken into subelements. 2D and 3D cells have pairs of points that form edges at the boundaries of the cell. Likewise, 3D cells have loops of edges that form faces that encase the cell. Figure 25.2 demonstrates the relationship of these constituent elements for some example cell shapes.

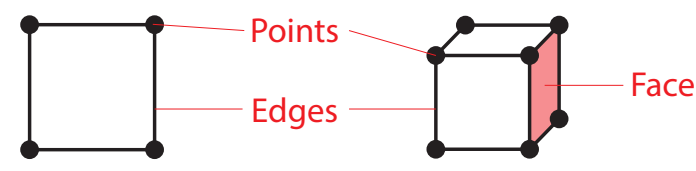

Figure 25.2: The constituent elements (points, edges, and faces) of cells. 
The header file vtkm/exec/CellEdge.h contains a collection of functions to help identify the edges of a cell. The first such function is vtkm: :exec: :CellEdgeNumberOfEdges. This function takes the number of points in the cell, the shape of the cell, and places the number of edges the cell as in its third argument (which is a reference to a vtkm: :IdComponent). The function returns a vtkm: :ErrorCode (section 19.9) for the status of the operation.

The second function is vtkm: :exec: :CellEdgeLocalIndex. This function takes, respectively, the number of points, the local index of the point in the edge $(0$ or 1$)$, the local index of the edge ( 0 to the number of edges in the cell), the shape of the cell, and a reference to a vtkm: : IdComponent to put the result. The result is the local index (between 0 and the number of points in the cell) of the requested point in the edge. This local point index is consistent with the point labels in Figure 25.2. To get the point indices relative to the data set, the edge indices should be used to reference a PointIndices list. The function returns a vtkm: :ErrorCode (section 19.9) for the status of the operation.

The third function is vtkm: :exec: :CellEdgeCanonicalId. This function takes the number of points, the local index of the edge, the shape of the cell, a Vec-like containing the global id of each cell point, and a reference to a vtkm: :Id2 to put the result. The result is a pair of numbers that is globally unique to that edge. If CellEdgeCanonicalld is called on an edge for a different cell, the two will be the same if and only if the two cells share that edge. CellEdgeCanonicalld is useful for finding coincident components of topology.

The following example demonstrates a pair of worklets that use the cell edge functions. As is typical for operations of this nature, one worklet counts the number of edges in each cell and another uses this count to generate the data.

Example 25.5 demonstrates one of many techniques for creating cell sets in a worklet. Chapter 32 describes this and many more such techniques.

Example 25.5: Using cell edge functions.

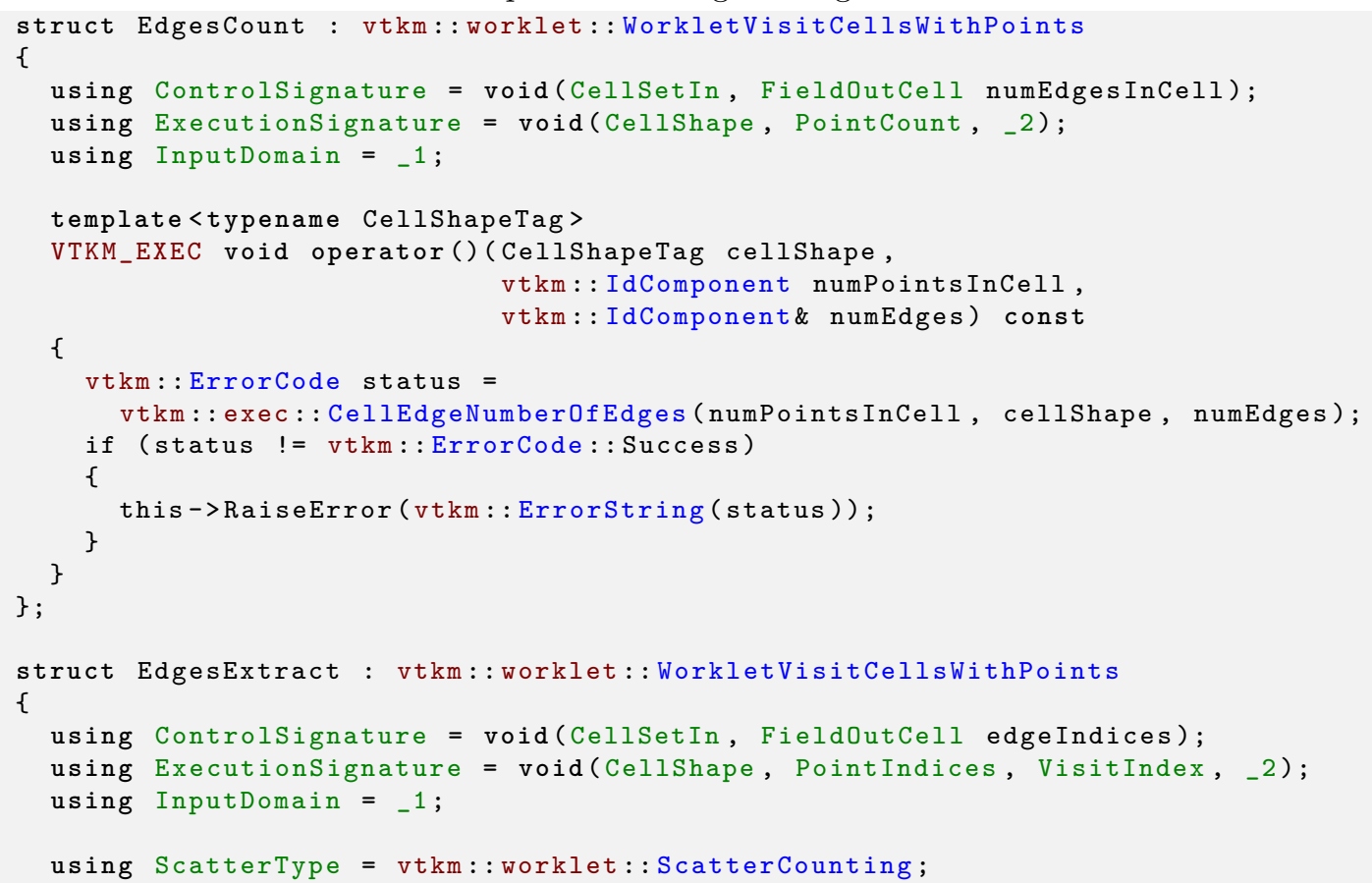




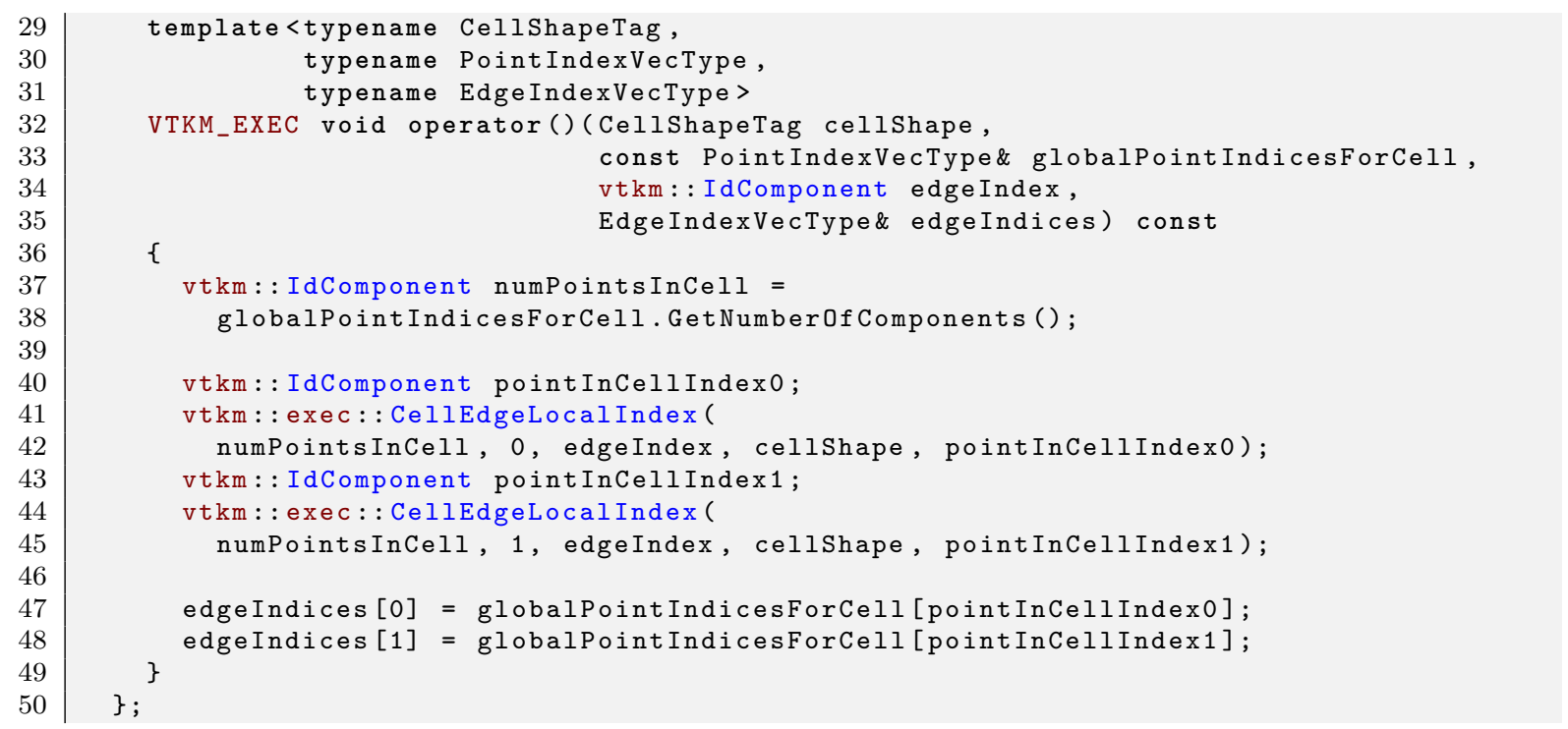

The header file vtkm/exec/CellFace.h contains a collection of functions to help identify the faces of a cell. The first such function is vtkm: :exec: :CellFaceNumberOfFaces. This function takes the shape of the cell and places the number of faces in the cell as its second argument (which is a reference to a vtkm: : IdComponent. The function returns a vtkm: ErrorCode (section 19.9) for the status of the operation.

The second function is vtkm: :exec: :CellFaceNumberOfPoints. This function takes the local index of the face ( 0 to the number of faces in the cell), the shape of the cell, and a reference to a vtkm::IdComponent to put the result. The result is the number of points the specified face has. The function returns a vtkm: :ErrorCode (section 19.9) for the status of the operation.

The third function is vtkm: :exec: :CellFaceLocalIndex. This function takes, respectively, the local index of the point in the face ( 0 to the number of points in the face), the local index of the face ( 0 to the number of faces in the cell), the shape of the cell, and a reference to a vtkm: IdComponent to put the result. The result is the local index (between 0 and the number of points in the cell) of the requested point in the face. The points are indexed in counterclockwise order when viewing the face from the outside of the cell. This local point index is consistent with the point labels in Figure 25.2. To get the point indices relative to the data set, the face indices should be used to reference a PointIndices list. The function returns a vtkm: :ErrorCode (section 19.9) for the status of the operation.

The fourth function is vtkm::exec::CellFaceCanonicalld. This function takes the local index of the face, the shape of the cell, a Vec-like containing the global id of each cell point, and a reference to a vtkm::Id3 to put the result. The result is a triplet of numbers that is globally unique to that face. If CellFaceCanonicalId is called on a face for a different cell, the two will be the same if and only if the two cells share that face. CellFaceCanonicalld is useful for finding coincident components of topology.

The following example demonstrates a triple of worklets that use the cell face functions. As is typical for operations of this nature, the worklets are used in steps to first count entities and then generate new entities. In this case, the first worklet counts the number of faces and the second worklet counts the points in each face. The third worklet generates cells for each face.

Example 25.6: Using cell face functions.

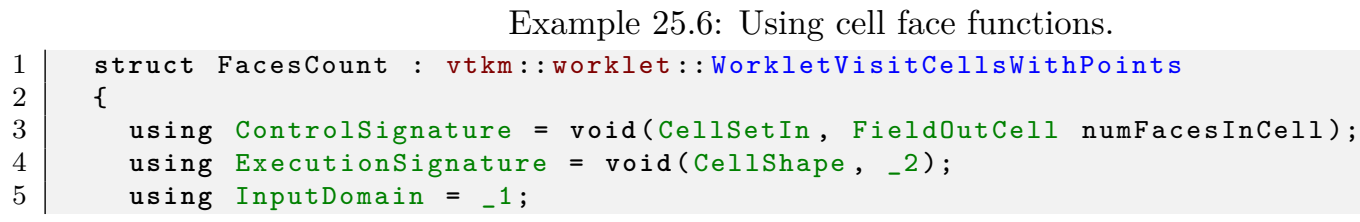




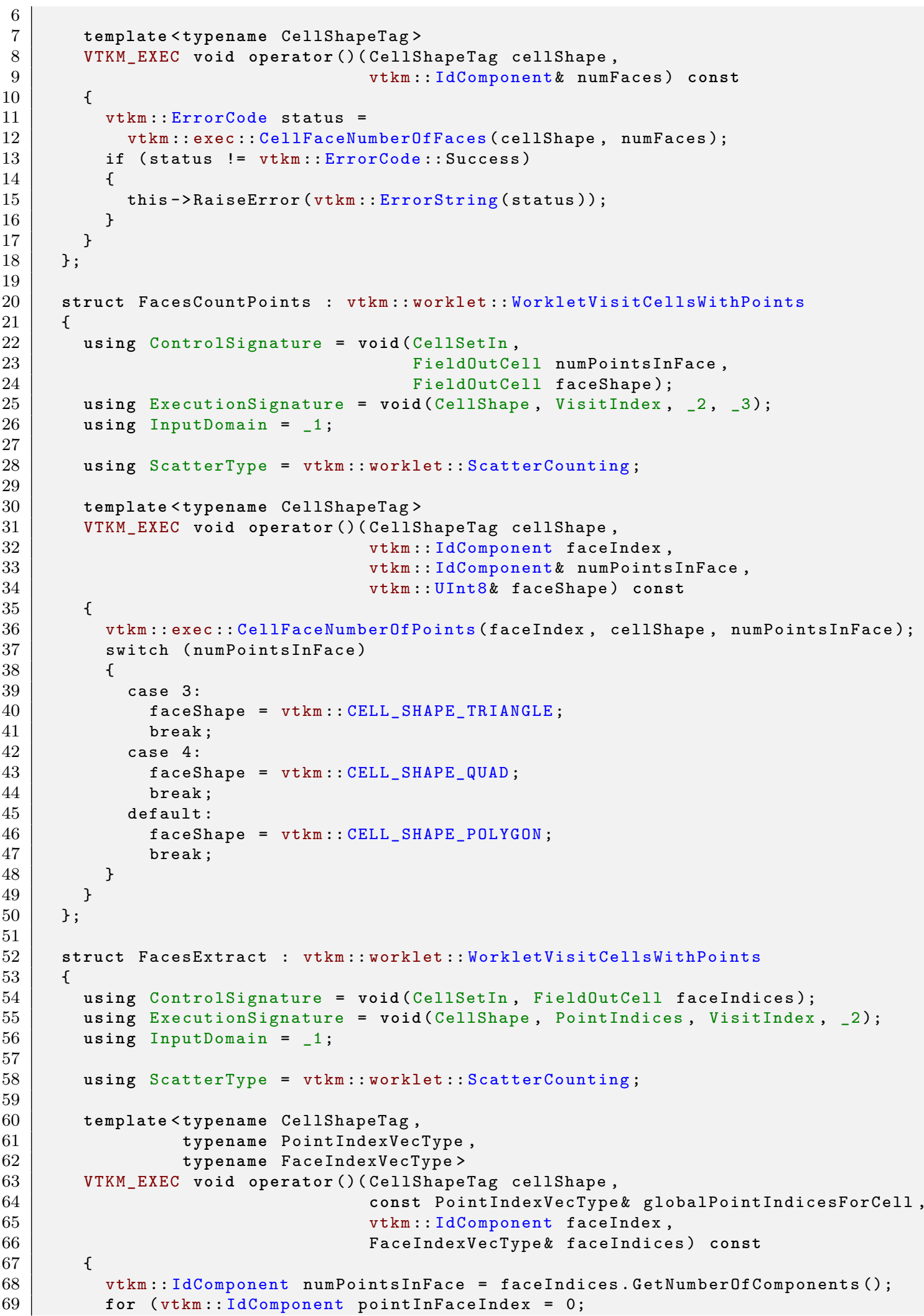




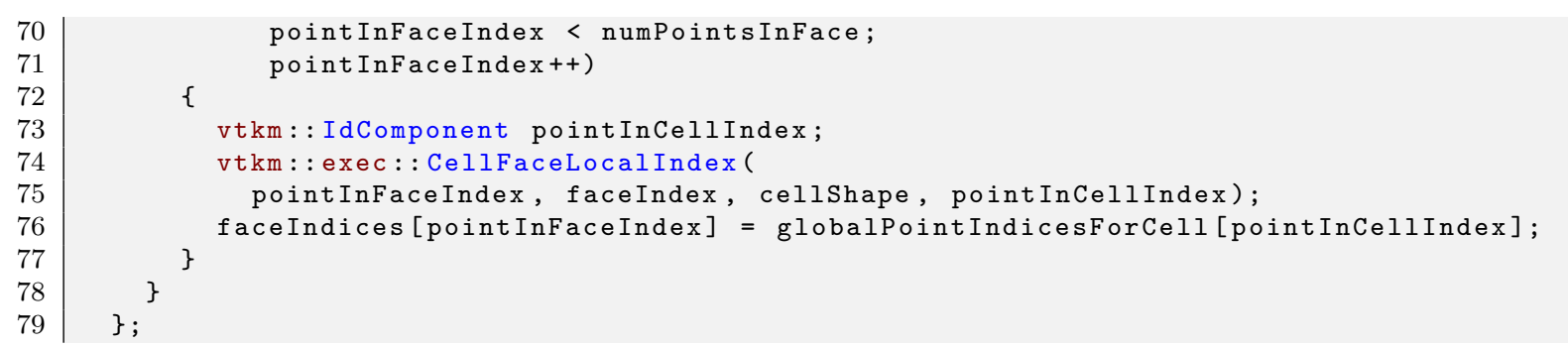




\section{FANCY ARRAY HANDLES}

One of the features of using ArrayHandles is that they hide the implementation and layout of the array behind a generic interface. This gives us the opportunity to replace a simple $\mathrm{C}$ array with some custom definition of the data and the code using the ArrayHandle is none the wiser.

This gives us the opportunity to implement fancy arrays that do more than simply look up a value in an array. For example, arrays can be augmented on the fly by mutating their indices or values. Or values could be computed directly from the index so that no storage is required for the array at all.

VTK-m provides many of the fancy arrays, which we explore in this section. Later in Chapter 35 we explore how to create custom arrays that adapt new memory layouts or augment other types of arrays.

\section{Did you know?}

S One of the advantages of VTK-m's implementation of fancy arrays is that they can define whole arrays without actually storing and values. For example, ArrayHandleConstant, ArrayHandleCounting, and ArrayHandleIndex do not store data in any array in memory. Rather, they construct the value for an index at runtime. Likewise, arrays like ArrayHandlePermutation construct new arrays from the values of other arrays without having to create a copy of the data.

\subsection{Constant Arrays}

A constant array is a fancy array handle that has the same value in all of its entries. The constant array provides this array without actually using any memory.

Specifying a constant array in VTK-m is straightforward. VTK-m has a class named vtkm: :cont: : ArrayHandleConstant. ArrayHandleConstant is a templated class with a single template argument that is the type of value for each element in the array. The constructor for ArrayHandleConstant takes the value to provide by the array and the number of values the array should present. The following example is a simple demonstration of the constant array handle.

Example 26.1: Using ArrayHandleConstant.

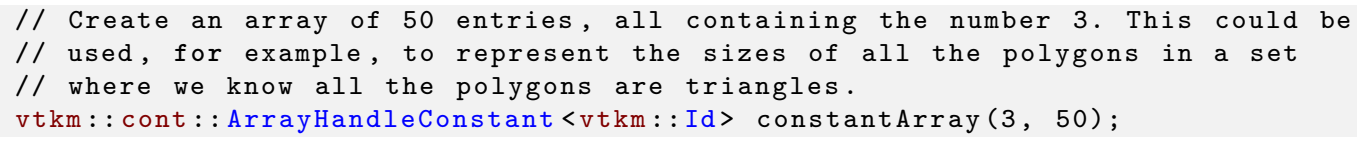


The vtkm/cont/ArrayHandleConstant.h header also contains the templated convenience function vtkm: : cont : :make_ArrayHandleConstant that takes a value and a size for the array. This function can sometimes be used to avoid having to declare the full array type.

Example 26.2: Using make_ArrayHandleConstant.

1 // Create an array of 50 entries, all containing the number 3.

2 vtkm: : cont : : make_ArrayHandleConstant (3, 50)

\subsection{ArrayHandleView}

An array handle view is a fancy array handle that returns a subset of an already existing array handle. The array handle view uses the same memory as the existing array handle the view was created from. This means that changes to the data in the array handle view will also change the data in the original array handle.

To use the ArrayHandleView you must supply an ArrayHandle to the vtkm::cont: :ArrayHandleView class constructor. ArrayHandleView is a templated class with a single template argument that is the ArrayHandle type of the array that the view is being created from. The constructor for ArrayHandleView takes a target array, starting index, and length. The following example shows a simple usage of the array handle view.

Example 26.3: Using ArrayHandleView.

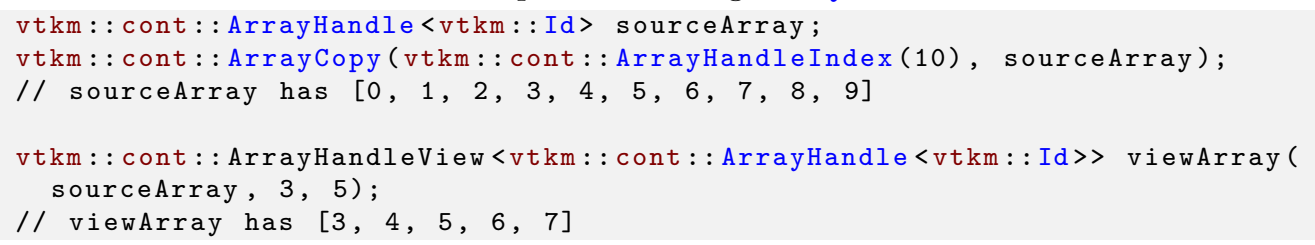

The vtkm/cont/ArrayHandleView.h header contains a templated convenience function vtkm: :cont: :make_ArrayHandleView that takes a target array, index, and length.

Example 26.4: Using make_ArrayHandleView.

1 vtkm: : cont : : make_ArrayHandleView (sourceArray, 3, 5)

\subsection{Uniform Random Bits Array}

An uniform random bits array is a fancy array handle that generates pseudo random bits as vtkm: :Unit64 in its entries. The uniform random bits array provides this array without actually using any memory.

The constructor for ArrayHandleRandomUniformBits takes two arguments: the first argument is the length of the array handle, the second is a seed of type vtkm: :Vec<Uint32, 1>. If the seed is not specified, the $\mathrm{C}++11$ std: :random_device is used as default.

Example 26.5: Using ArrayHandleRandomUniformBits.

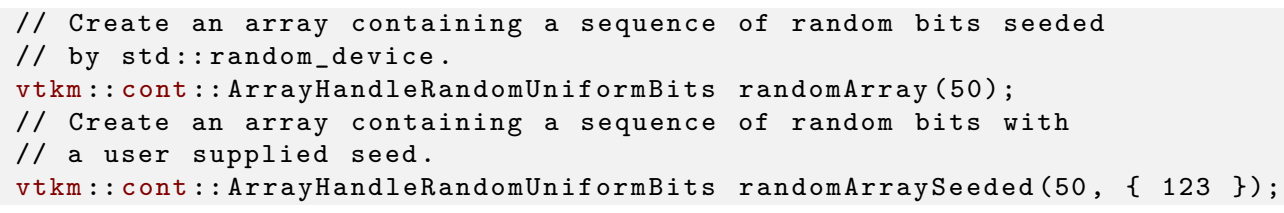


ArrayHandleRandomUniformBits is functional, in the sense that once an instance of ArrayHandleRandomUniformBits is created, its content does not change and always returns the same vtkm: :UInt64 value given the same index.

Example 26.6: ArrayHandleRandomUniformBits is functional

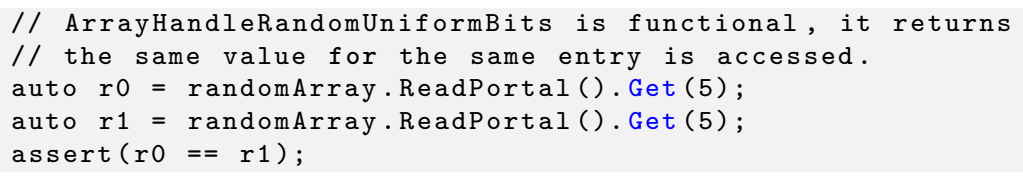

To generate a new set of random bits, we need to create another instance of ArrayHandleRandomUniformBits with a different seed, we can either let std: :random_device provide a unique seed or use some unique identifier such as iteration number as the seed.

Example 26.7: Independent ArrayHandleRandomUniformBits.

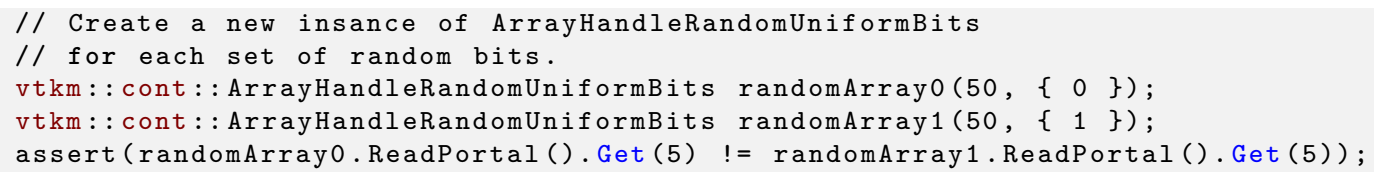

\subsection{Counting Arrays}

A counting array is a fancy array handle that provides a sequence of numbers. These fancy arrays can represent the data without actually using any memory.

VTK-m provides two versions of a counting array. The first version is an index array that provides a specialized but common form of a counting array called an index array. An index array has values of type vtkm: : Id that start at 0 and count up by 1 (i.e. $0,1,2,3, \ldots$ ). The index array mirrors the array's index.

Specifying an index array in VTK-m is done with a class named vtkm: : cont : : ArrayHandleIndex. The constructor for ArrayHandleIndex takes the size of the array to create. The following example is a simple demonstration of the index array handle.

Example 26.8: Using ArrayHandleIndex.

1 // Create an array containing $[0,1,2,3, \ldots, 49]$.

2 vtkm: : cont: : ArrayHandleIndex indexArray (50);

The vtkm: : cont: : ArrayHandleCounting class provides a more general form of counting. ArrayHandleCounting is a templated class with a single template argument that is the type of value for each element in the array. The constructor for ArrayHandleCounting takes three arguments: the start value (used at index 0), the step from one value to the next, and the length of the array. The following example is a simple demonstration of the counting array handle.

Example 26.9: Using ArrayHandleCounting.

// Create an array containing $[-1.0,-0.9,-0.8, \ldots, 0.9,1.0]$

vtkm: : cont: : ArrayHandleCounting<vtkm::Float32> sampleArray (-1.0f, 0.1f, 21); 
D Did you know?

In addition to being simpler to declare, ArrayHandleIndex is slightly faster than ArrayHandleCounting. Thus, when applicable, you should prefer using ArrayHandleIndex.

The vtkm/cont/ArrayHandleCounting.h header also contains the templated convenience function vtkm: : cont: :make_ArrayHandleCounting that also takes the start value, step, and length as arguments. This function can sometimes be used to avoid having to declare the full array type.

Example 26.10: Using make_ArrayHandleCounting.

1 // Create an array containing $[-1.0,-0.9,-0.8, \ldots, 0.9,1.0]$

2 vtkm: : cont : : make_ArrayHandleCounting ( $-1.0 \mathrm{f}, 0.1 \mathrm{f}, 21)$

There are no fundamental limits on how ArrayHandleCounting counts. For example, it is possible to count backwards.

Example 26.11: Counting backwards with ArrayHandleCounting.

1 // Create an array containing [49, 48, 47, 46, .., 0].

2 vtkm::cont: :ArrayHandleCounting <vtkm::Id> backwardIndexArray (49, $-1,50)$;

It is also possible to use ArrayHandleCounting to make sequences of vtkm: :Vec values with piece-wise counting in each of the components.

Example 26.12: Using ArrayHandleCounting with vtkm: : Vec objects.

// Create an array containg $[(0,-3,75),(1,2,25),(3,7,-25)]$

vtkm: : cont : : make_ArrayHandleCounting (

vtkm: : make_Vec $(0,-3,75)$, vtkm::make_Vec $(1,5,-50), 3)$

\subsection{Cast Arrays}

A cast array is a fancy array that changes the type of the elements in an array. The cast array provides this re-typed array without actually copying or generating any data. Instead, casts are performed as the array is accessed.

VTK-m has a class named vtkm: : cont: : ArrayHandleCast to perform this implicit casting. ArrayHandleCast is a templated class with two template arguments. The first argument is the type to cast values to. The second argument is the type of the original ArrayHandle. The constructor to ArrayHandleCast takes the ArrayHandle to modify by casting.

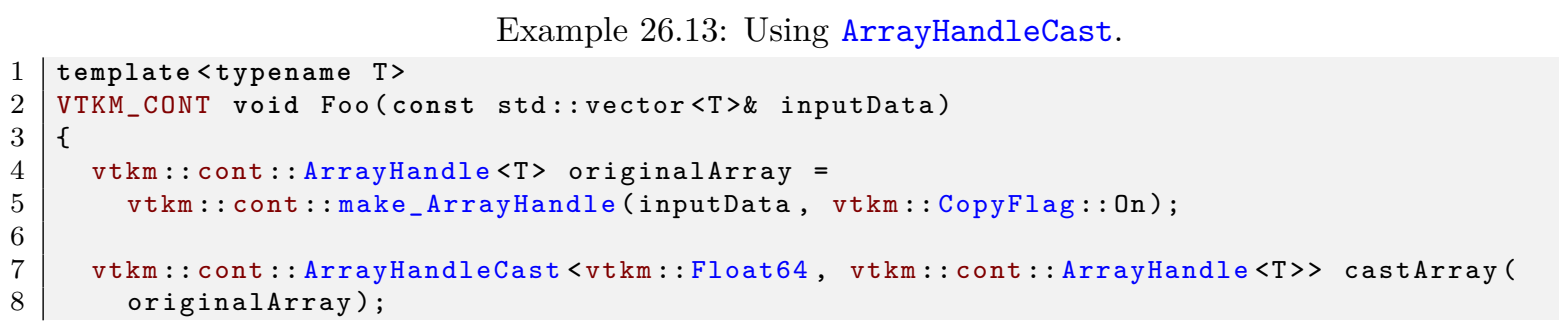

The vtkm/cont/ArrayHandleCast.h header also contains the templated convenience function vtkm: : cont: : make_ArrayHandleCast that constructs the cast array. The first argument is the original ArrayHandle original array to cast. The optional second argument is of the type to cast to (or you can optionally specify the cast-to type as a template argument. 
Example 26.14: Using make_ArrayHandleCast.

1 vtkm: : cont : :make_ArrayHandleCast<vtkm: :Float64>(originalArray)

\subsection{Discard Arrays}

It is sometimes the case where you will want to run an operation in VTK-m that fills values in two (or more) arrays, but you only want the values that are stored in one of the arrays. It is possible to allocate space for both arrays and then throw away the values that you do not want, but that is a waste of memory. It is also possible to rewrite the functionality to output only what you want, but that is a poor use of developer time.

To solve this problem easily, VTK-m provides vtkm: :cont: :ArrayHandleDiscard. This array behaves similar to a regular ArrayHandle in that it can be "allocated" and has size, but any values that are written to it are immediately discarded. ArrayHandleDiscard takes up no memory.

Example 26.15: Using ArrayHandleDiscard.

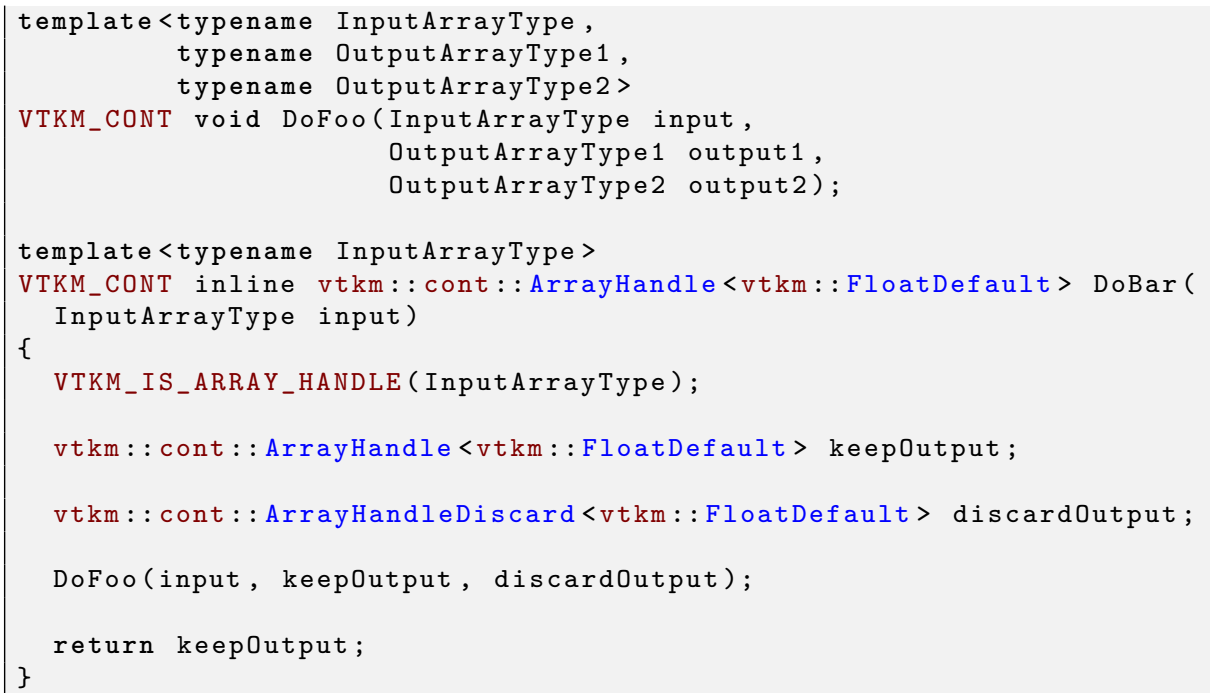

\subsection{Permuted Arrays}

A permutation array is a fancy array handle that reorders the elements in an array. Elements in the array can be skipped over or replicated. The permutation array provides this reordered array without actually coping any data. Instead, indices are adjusted as the array is accessed.

Specifying a permutation array in VTK-m is straightforward. VTK-m has a class named vtkm: :cont: :ArrayHandlePermutation that takes two arrays: an array of values and an array of indices that maps an index in the permutation to an index of the original values. The index array is specified first. The following example is a simple demonstration of the permutation array handle.

Example 26.16: Using ArrayHandlePermutation.

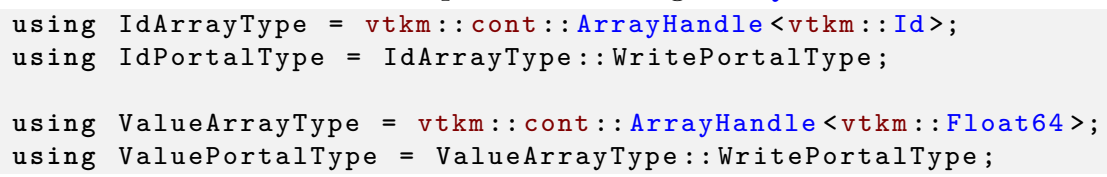




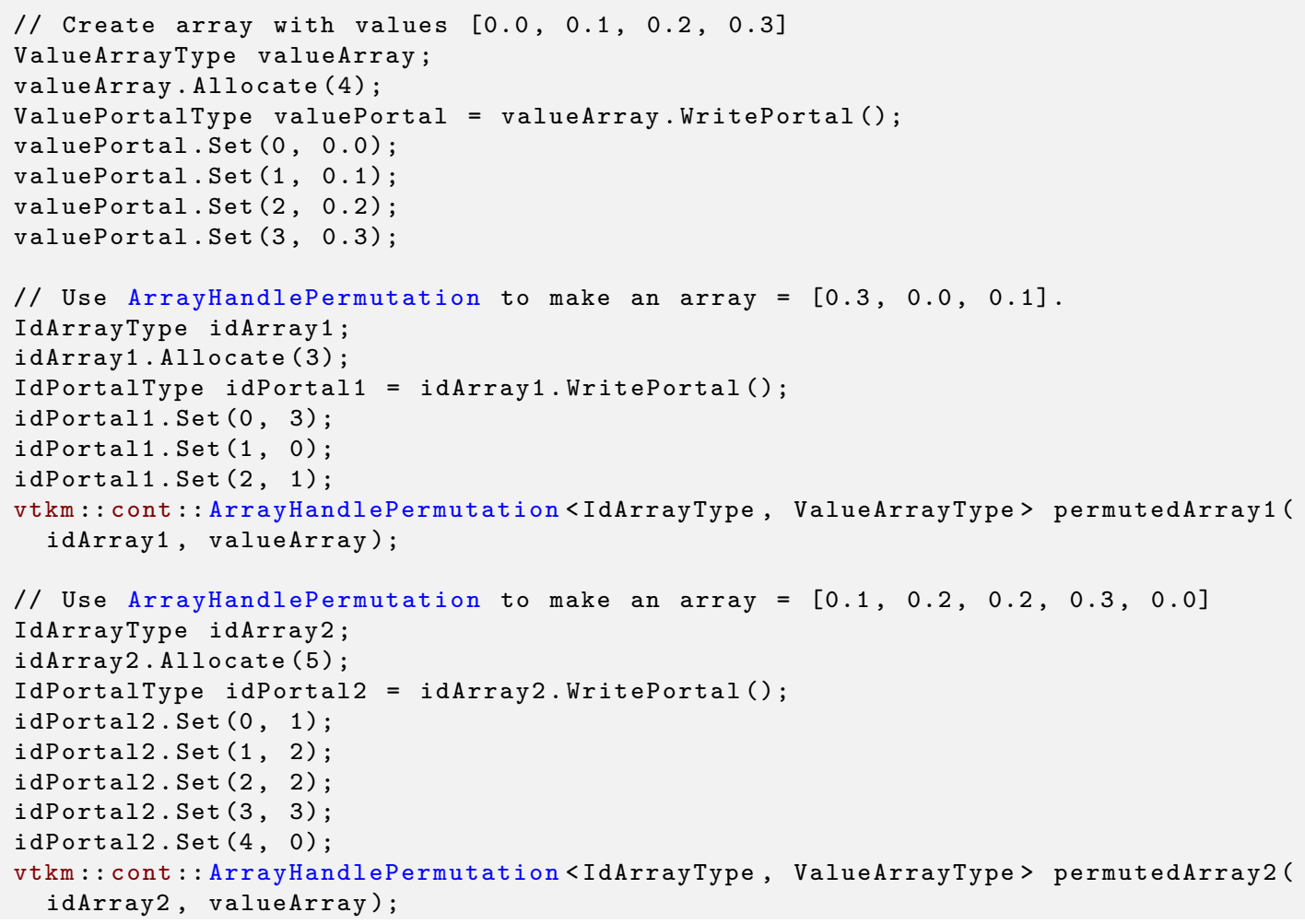

The vtkm/cont/ArrayHandlePermutation.h header also contains the templated convenience function vtkm: :cont: :make_ArrayHandlePermutation that takes instances of the index and value array handles and returns a permutation array. This function can sometimes be used to avoid having to declare the full array type.

Example 26.17: Using make_ArrayHandlePermutation.

1 vtkm: : cont : :make_ArrayHandlePermutation(idArray, valueArray)

\section{Common Errors}

When using an ArrayHandlePermutation, take care that all the provided indices in the index array point to valid locations in the values array. Bad indices can cause reading from or writing to invalid memory locations, which can be difficult to debug. Also, be wary about having duplicate indices, which means that multiple array entries point to the same memory location. This will work fine when using the array as input, but will cause a dangerous race condition if used as an output.

\section{(0) Did you know?}

You can write to a ArrayHandlePermutation by, for example, using it as an output array. Writes to \} the ArrayHandlePermutation will go to the respective location in the source array. However, ArrayHandlePermutation cannot be resized. 


\subsection{Zipped Arrays}

A zip array is a fancy array handle that combines two arrays of the same size to pair up the corresponding values. Each element in the zipped array is a vtkm: : Pair containing the values of the two respective arrays. These pairs are not stored in their own memory space. Rather, the pairs are generated as the array is used. Writing a pair to the zipped array writes the values in the two source arrays.

Specifying a zipped array in VTK-m is straightforward. VTK-m has a class named vtkm: :cont: :ArrayHandleZip that takes the two arrays providing values for the first and second entries in the pairs. The following example is a simple demonstration of creating a zip array handle.

Example 26.18: Using ArrayHandleZip.

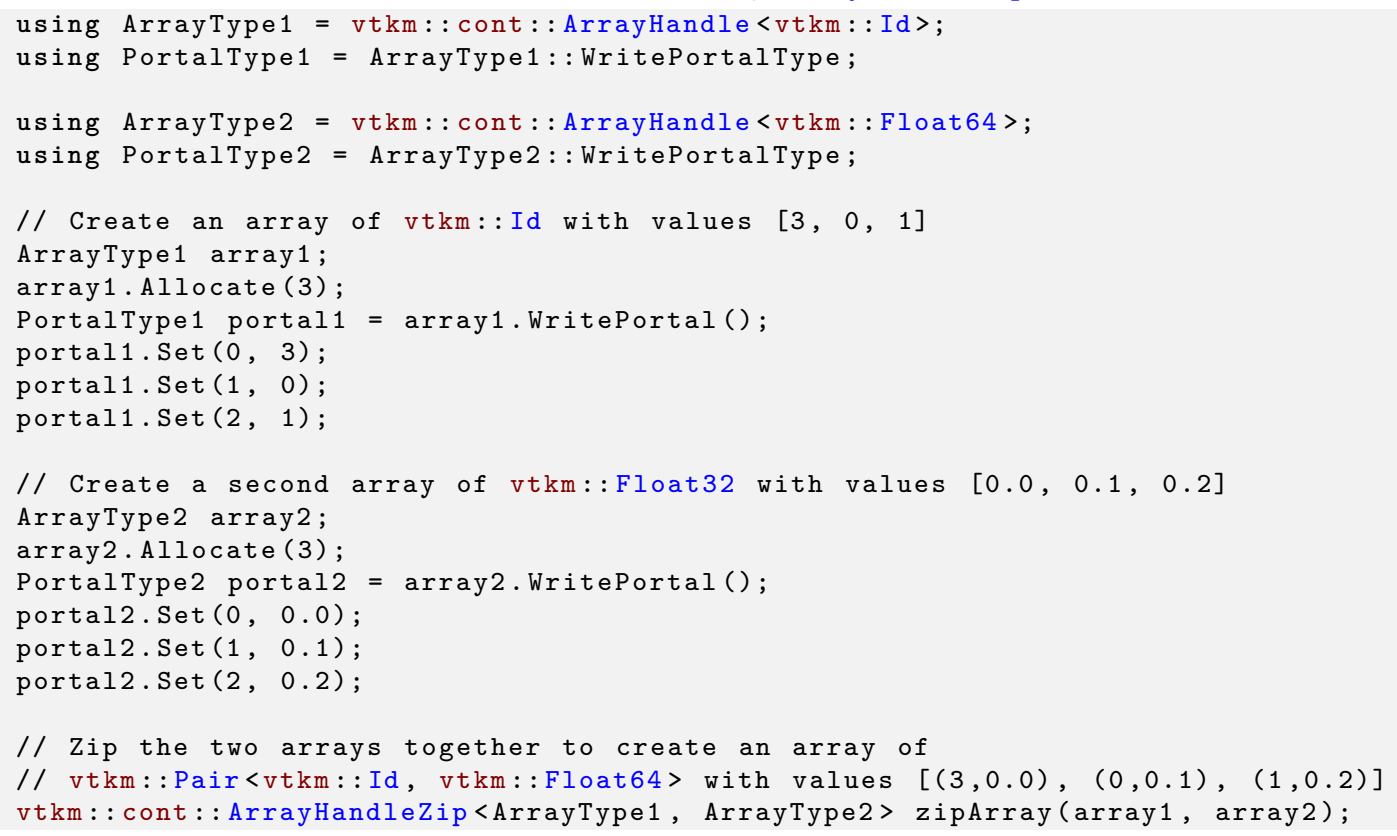

The vtkm/cont/ArrayHandleZip.h header also contains the templated convenience function vtkm: : cont: : make_ArrayHandleZip that takes instances of the two array handles and returns a zip array. This function can sometimes be used to avoid having to declare the full array type.

Example 26.19: Using make_ArrayHandleZip.

1 vtkm: : cont: : make_ArrayHandleZip (array1, array2)

\subsection{Coordinate System Arrays}

Many of the data structures we use in VTK-m are described in a 3D coordinate system. Although, as we will see in Chapter 7, we can use any ArrayHandle to store point coordinates, including a raw array of 3D vectors, there are some common patterns for point coordinates that we can use specialized arrays to better represent the data.

There are two fancy array handles that each handle a special form of coordinate system. The first such array handle is vtkm: : cont: : ArrayHandleUniformPointCoordinates, which represents a uniform sampling of space. The constructor for ArrayHandleUniformPointCoordinates takes three arguments. The first argument is a vtkm: : Id3 that specifies the number of samples in the $x, y$, and $z$ directions. The second argument, which is 
optional, specifies the origin (the location of the first point at the lower left corner). If not specified, the origin is set to $[0,0,0]$. The third argument, which is also optional, specifies the distance between samples in the $x, y$, and $z$ directions. If not specified, the spacing is set to 1 in each direction.

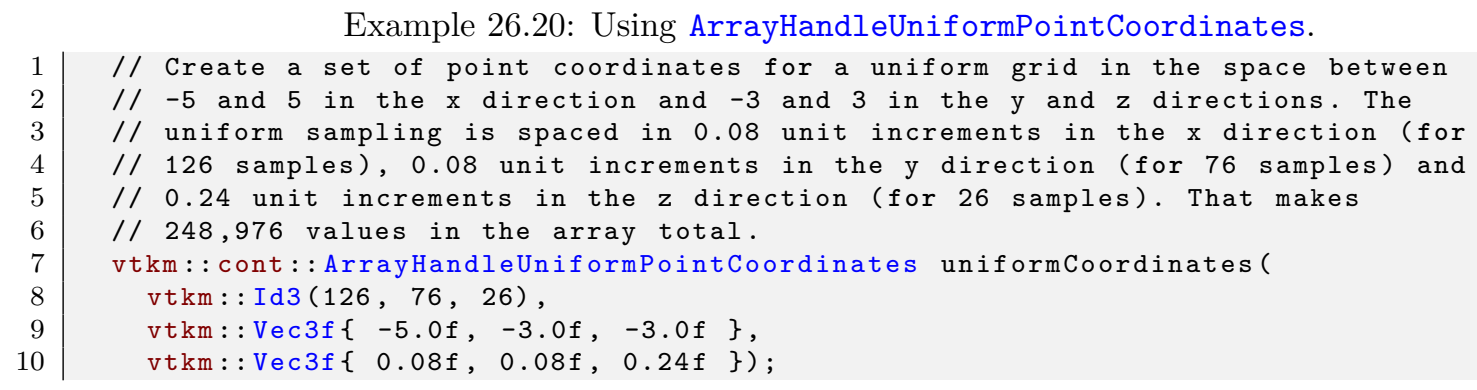

The second fancy array handle for special coordinate systems is vtkm: : cont : : ArrayHandleCartesianProduct, which represents a rectilinear sampling of space where the samples are axis aligned but have variable spacing. Sets of coordinates of this type are most efficiently represented by having a separate array for each component of the axis, and then for each $[i, j, k]$ index of the array take the value for each component from each array using the respective index. This is equivalent to performing a Cartesian product on the arrays.

ArrayHandleCartesianProduct is a templated class. It has three template parameters, which are the types of the arrays used for the $x, y$, and $z$ axes. The constructor for ArrayHandleCartesianProduct takes the three arrays.

Example 26.21: Using a ArrayHandleCartesianProduct.

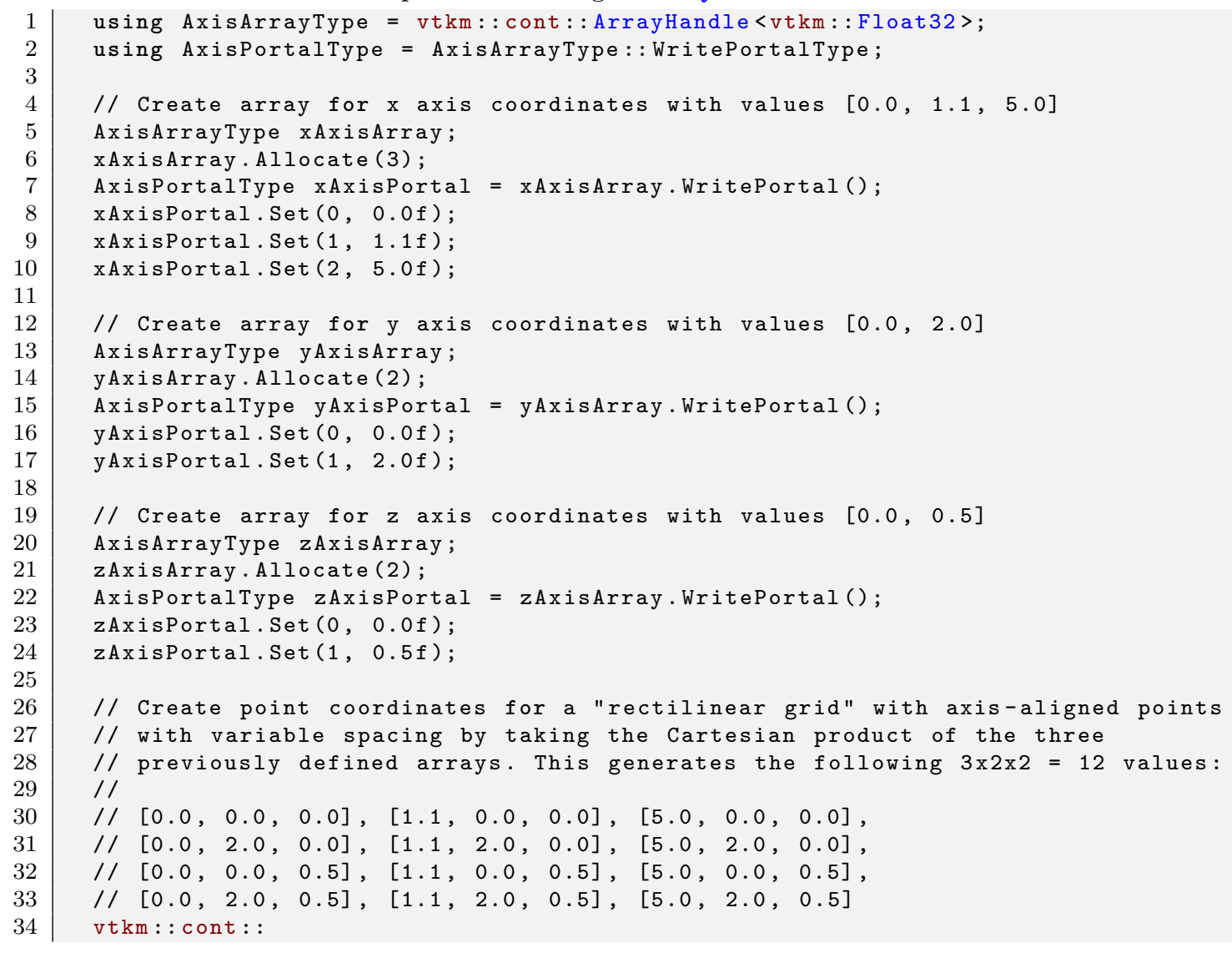


The vtkm/cont/ArrayHandleCartesianProduct.h/header also contains the templated convenience function vtkm: :cont : : make_ArrayHandleCartesianProduct that takes the three axis arrays and returns an array of the Cartesian product. This function can sometimes be used to avoid having to declare the full array type.

Example 26.22: Using make_ArrayHandleCartesianProduct.

1 vtkm: : cont : : make_ArrayHandleCartesianProduct (xAxisArray, yAxisArray, zAxisArray)

\section{(D) Did you know?}

3 These specialized arrays for coordinate systems greatly reduce the code duplication in VTK-m. Most scientific visualization systems need separate implementations of algorithms for uniform, rectilinear, and unstructured grids. But in VTK-m an algorithm can be written once and then applied to all these different grid structures by using these specialized array handles and letting the compiler's templates optimize the code.

\subsection{Composite Vector Arrays}

A composite vector array is a fancy array handle that combines two to four arrays of the same size and value type and combines their corresponding values to form a vtkm::Vec. A composite vector array is similar in nature to a zipped array (described in Section 26.8) except that values are combined into vtkm: :Vec s instead of vtkm::Pair s. The created vtkm::Vec s are not stored in their own memory space. Rather, the Vecs are generated as the array is used. Writing Vecs to the composite vector array writes values into the components of the source arrays.

A composite vector array can be created using the vtkm::cont::ArrayHandleCompositeVector class. This class has a variadic template argument that is a "signature" for the arrays to be combined. The constructor for ArrayHandleCompositeVector takes instances of the array handles to combine.

Example 26.23: Using ArrayHandleCompositeVector.

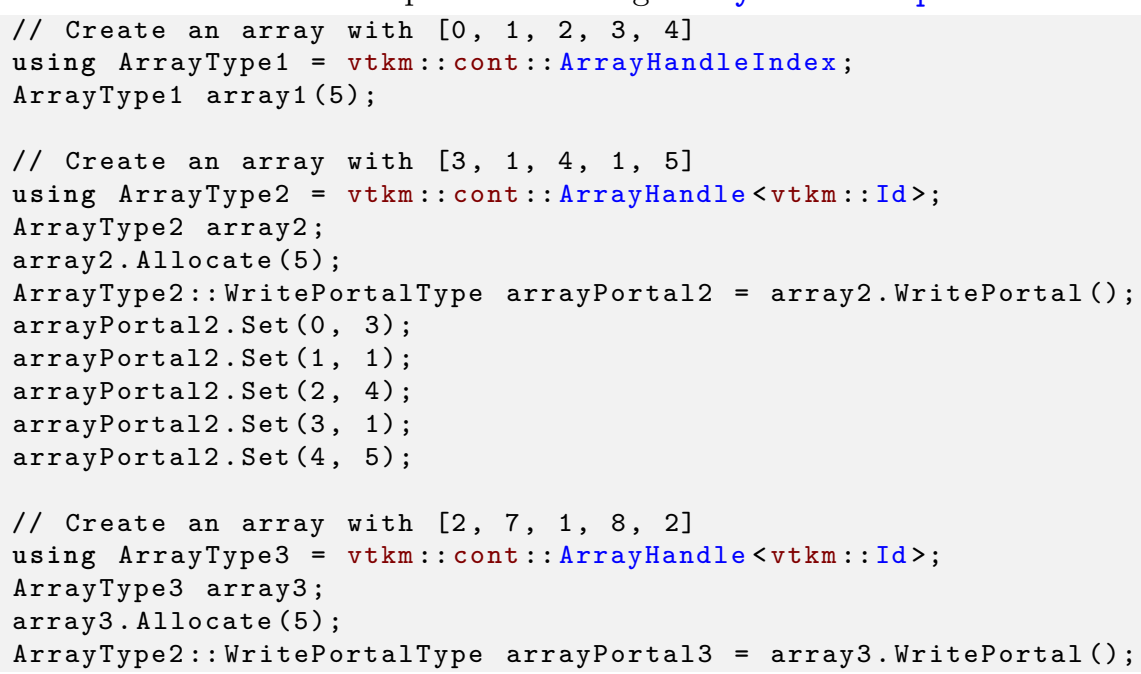




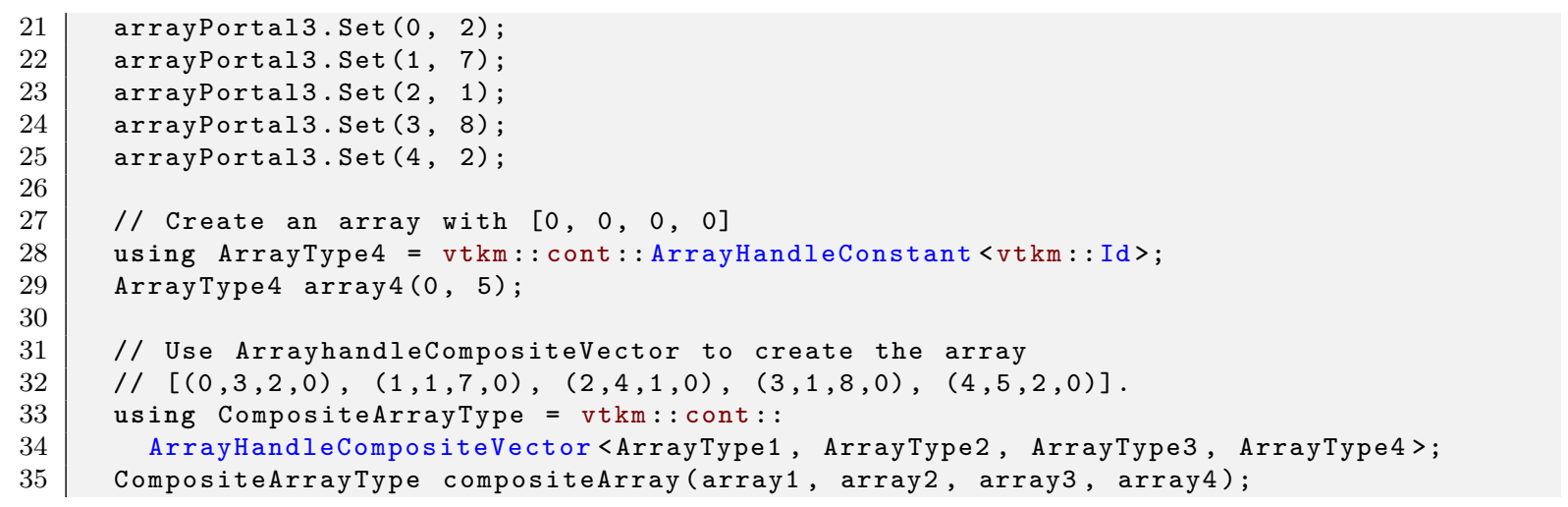

The vtkm/cont/ArrayHandleCompositeVector.h header also contains the templated convenience function vtkm: :cont::make_ArrayHandleCompositeVector which takes a variable number of array handles and returns an ArrayHandleCompositeVector. This function can sometimes be used to avoid having to declare the full array type. ArrayHandleCompositeVector is also often used to combine scalar arrays into vector arrays.

Example 26.24: Using make_ArrayHandleCompositeVector.

1 vtkm::cont::make_ArrayHandleCompositeVector(array1, array2, array3, array4)

\subsection{Extract Component Arrays}

Component extraction allows access to a single component of an ArrayHandle with a vtkm: :Vec ValueType. vtkm: : cont : : ArrayHandleExtractComponent allows one component of a vector array to be extracted without creating a copy of the data. ArrayHandleExtractComponent can also be combined with ArrayHandleCompositeVector (described in Section 26.10) to arbitrarily stitch several components from multiple arrays together.

As a simple example, consider an ArrayHandle containing 3D coordinates for a collection of points and a filter that only operates on the points' elevations ( $\mathrm{Z}$, in this example). We can easily create the elevation array on-the-fly without allocating a new array as in the following example.

Example 26.25: Extracting components of Vecs in an array with ArrayHandleExtractComponent.

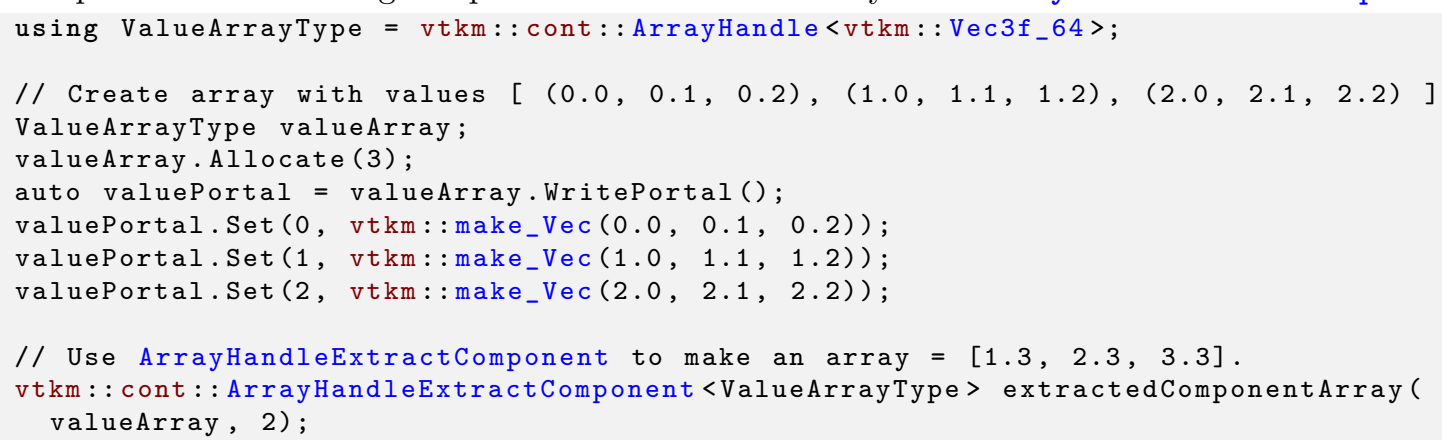

The vtkm/cont/ArrayHandleExtractComponent.h header also contains the templated convenience function vtkm: : cont: :make_ArrayHandleExtractComponent that takes an ArrayHandle of Vecs and vtkm: :IdComponent which returns an appropriately typed ArrayHandleExtractComponent containing the values for a specified component. The index of the component to extract is provided as an argument to make_ArrayHandleExtractComponent, which is required. The use of make_ArrayHandleExtractComponent can be used to avoid having to declare the full array type. 
Example 26.26: Using make_ArrayHandleExtractComponent.

1 vtkm: : cont: :make_ArrayHandleExtractComponent (valueArray , 2)

\subsection{Swizzle Arrays}

It is often useful to reorder or remove specific components from an ArrayHandle with a vtkm: :Vec ValueType. vtkm: : cont: : ArrayHandleSwizzle provides an easy way to accomplish this.

The template parameters of ArrayHandleSwizzle specify a "component map," which defines the swizzle operation. This map consists of the components from the input ArrayHandle, which will be exposed in the ArrayHandleSwizzle. For instance, vtkm: : cont: :ArrayHandleSwizzle <Some3DArrayType, 3> with Some3DArray and vtkm: :Vec <vtkm: : IdComponent, 3>(0,2,1) as constructor arguments will allow access to a 3D array, but with the $\mathrm{Y}$ and $\mathrm{Z}$ components exchanged. This rearrangement does not create a copy, and occurs on-the-fly as data are accessed through the ArrayHandleSwizzle's portal. This fancy array handle can also be used to eliminate unnecessary components from an ArrayHandle's data, as shown below.

Example 26.27: Swizzling components of Vecs in an array with ArrayHandleSwizzle.

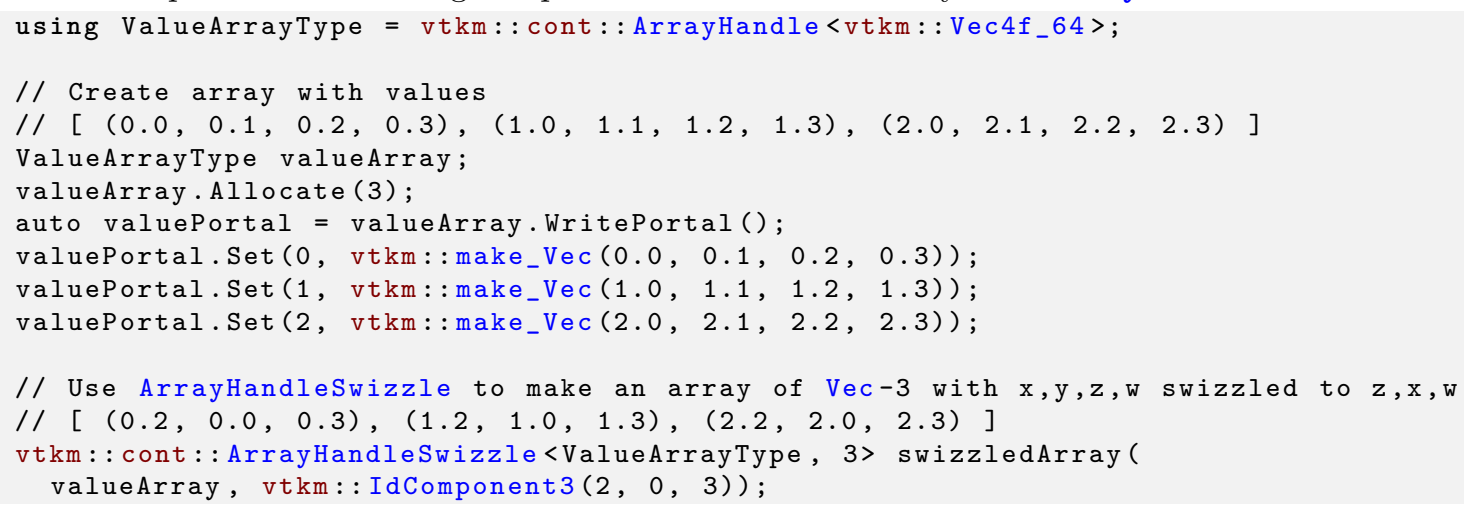

The vtkm/cont/ArrayHandleSwizzle.h header also contains the templated convenience function vtkm: : cont: :make_ArrayHandleSwizzle that takes an ArrayHandle of Vecs and returns an appropriately typed ArrayHandleSwizzle containing swizzled vectors. The indices of the swizzled components are provided as arguments to make_ArrayHandleSwizzle after the ArrayHandle. The use of make_ArrayHandleSwizzle can be used to avoid having to declare the full array type.

Example 26.28: Using make_ArrayHandleSwizzle.

1 vtkm: : cont : :make_ArrayHandleSwizzle (valueArray, 2, 0, 3)

\subsection{Grouped Vector Arrays}

A grouped vector array is a fancy array handle that groups consecutive values of an array together to form a vtkm::Vec. The source array must be of a length that is divisible by the requested Vec size. The created vtkm: :Vec s are not stored in their own memory space. Rather, the Vecs are generated as the array is used. Writing Vecs to the grouped vector array writes values into the the source array.

A grouped vector array is created using the vtkm: : cont: :ArrayHandleGroupVec class. This templated class has two template arguments. The first argument is the type of array being grouped and the second argument is an integer specifying the size of the Vecs to create (the number of values to group together). 
Example 26.29: Using ArrayHandleGroupVec.

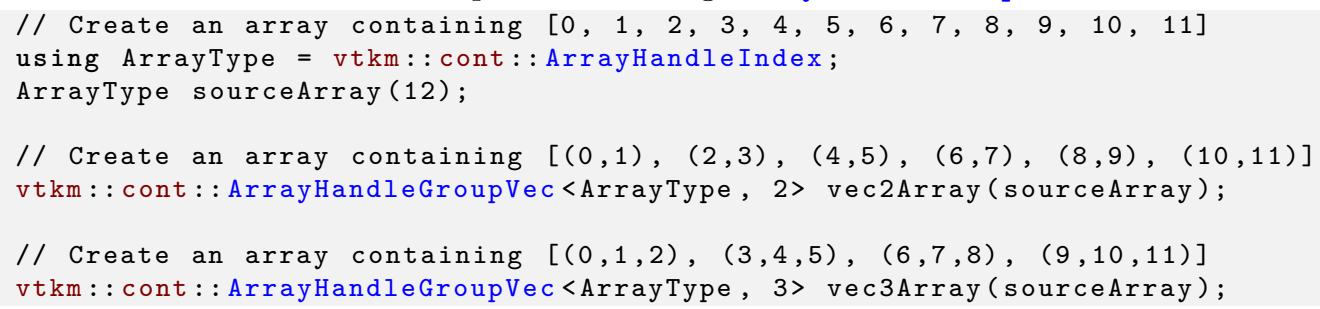

The vtkm/cont/ArrayHandleGroupVec.h header also contains the templated convenience function vtkm: : cont: :make_ArrayHandleGroupVec that takes an instance of the array to group into Vecs. You must specify the size of the Vecs as a template parameter when using vtkm: : cont : :make_ArrayHandleGroupVec.

Example 26.30: Using make_ArrayHandleGroupVec.

\begin{tabular}{l|l}
1 & // Create an array containing $[(0,1,2,3),(4,5,6,7),(8,9,10,11)]$ \\
2 & vtkm: : cont : : make_ArrayHandleGroupVec $<4>(\operatorname{sourceArray})$
\end{tabular}

ArrayHandleGroupVec is handy when you need to build an array of vectors that are all of the same length, but what about when you need an array of vectors of different lengths? One common use case for this is if you are defining a collection of polygons of different sizes (triangles, quadrilaterals, pentagons, and so on). We would like to define an array such that the data for each polygon were stored in its own Vec (or, rather, Vec-like) object. vtkm: : cont : :ArrayHandleGroupVecVariable does just that.

ArrayHandleGroupVecVariable takes two arrays. The first array, identified as the "source" array, is a flat representation of the values (much like the array used with ArrayHandleGroupVec). The second array, identified as the "offsets" array, provides for each vector the index into the source array where the start of the vector is. The offsets array must be monotonically increasing. The size of the offsets array is one greater than the number of vectors in the resulting array. The first offset is always 0 and the last offset is always the size of the input source array. The first and second template parameters to ArrayHandleGroupVecVariable are the types for the source and offset arrays, respectively.

It is often the case that you will start with a group of vector lengths rather than offsets into the source array. If this is the case, then the vtkm: :cont::ConvertNumComponentsToOffsets helper function can convert an array of vector lengths to an array of offsets. The first argument to this function is always the array of vector lengths. The second argument, which is optional, is a reference to a ArrayHandle into which the offsets should be stored. If this offset array is not specified, an ArrayHandle will be returned from the function instead. The third argument, which is also optional, is a reference to a vtkm: :Id into which the expected size of the source array is put. Having the size of the source array is often helpful, as it can be used to allocate data for the source array or check the source array's size. It is also OK to give the expected size reference but not the offset array reference.

Example 26.31: Using ArrayHandleGroupVecVariable.

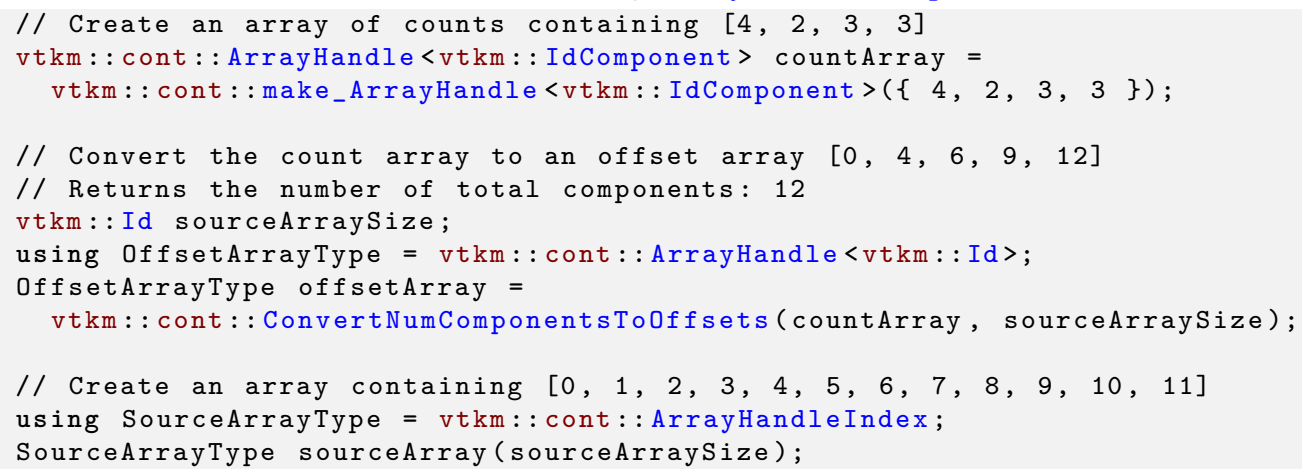




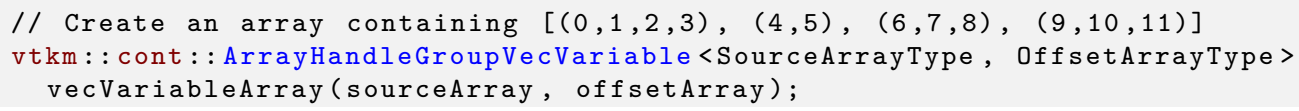

The vtkm/cont/ArrayHandleGroupVecVariable.h header also contains the templated convenience function vtkm: :cont: :make_ArrayHandleGroupVecVariable that takes an instance of the source array to group into Vec-like objects and the offset array.

Example 26.32: Using MakeArrayHandleGroupVecVariable.

// Create an array containing $[(0,1,2,3),(4,5),(6,7,8),(9,10,11)]$

vtkm : : cont : : make_ArrayHandleGroupVecVariable (sourceArray, offsetArray)

\section{Did you know?}

You can write to ArrayHandleGroupVec and ArrayHandleGroupVecVariable by, for example, using it as an output array. Writes to these arrays will go to the respective location in the source array. ArrayHandleGroupVec can also be allocated and resized (which in turn causes the source array to be allocated). However, ArrayHandleGroupVecVariable cannot be resized and the source array must be pre-allocated. You can use the source array size value returned from ConvertNumComponentsToOffsets to allocate source arrays.

\section{Common Errors}

Keep in mind that the values stored in a ArrayHandleGroupVecVariable are not actually vtkm::Vec objects. Rather, they are "Vec-like" objects, which has some subtle but important ramifications. First, the type will not match the $\mathrm{vtkm::Vec}$ template, and there is no automatic conversion to $\mathrm{vtkm::Vec}$ objects. Thus, many functions that accept vtkm: :Vec objects as parameters will not accept the Vec-like object. Second, the size of Vec-like objects are not known until runtime. See Sections 4.3 and 19.5.2 for more information on the difference between $\mathrm{vtkm:} \mathrm{Vec} \mathrm{and} \mathrm{Vec-like} \mathrm{objects.}$ 



\section{ACCESSING AND ALLOCATING ARRAY HANDLES}

So far we have seen examples of creating vtkm: : cont: :ArrayHandle s from normal C++ arrays (Chapter 16) and creating some special arrays (Chapter 26). However, we have so far avoided discussing how to access the actual data in ArrayHandles. So far we have only accessed ArrayHandle data indirectly through other VTK-m features such as worklets.

In this chapter we describe how to more directly access the data in an ArrayHandle, how to allocate space for data in an ArrayHandle, and how data is transferred to the execution environment to be used in a worklet.

\subsection{Array Portals}

An array handle defines auxiliary structures called array portals that provide direct access into its data. An array portal is a simple object that is somewhat functionally equivalent to an STL-type iterator, but with a much simpler interface. Array portals can be read-only or read-write and they can be accessible from either the control environment or the execution environment. All these variants have similar interfaces although some features that are not applicable can be left out.

An array portal object contains each of the following:

ValueType The type for each item in the array.

GetNumberOfValues A method that returns the number of entries in the array.

Get A method that returns the value at a given index.

Set A method that changes the value at a given index. This method does not need to exist for read-only array portals.

The following code example defines an array portal for a simple $\mathrm{C}$ array of scalar values. This definition has no practical value (it is covered by the more general vtkm: :cont: : internal: : ArrayPortalFromIterators), but demonstrates the function of each component.

Example 27.1: A simple array portal implementation.

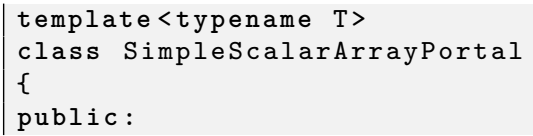




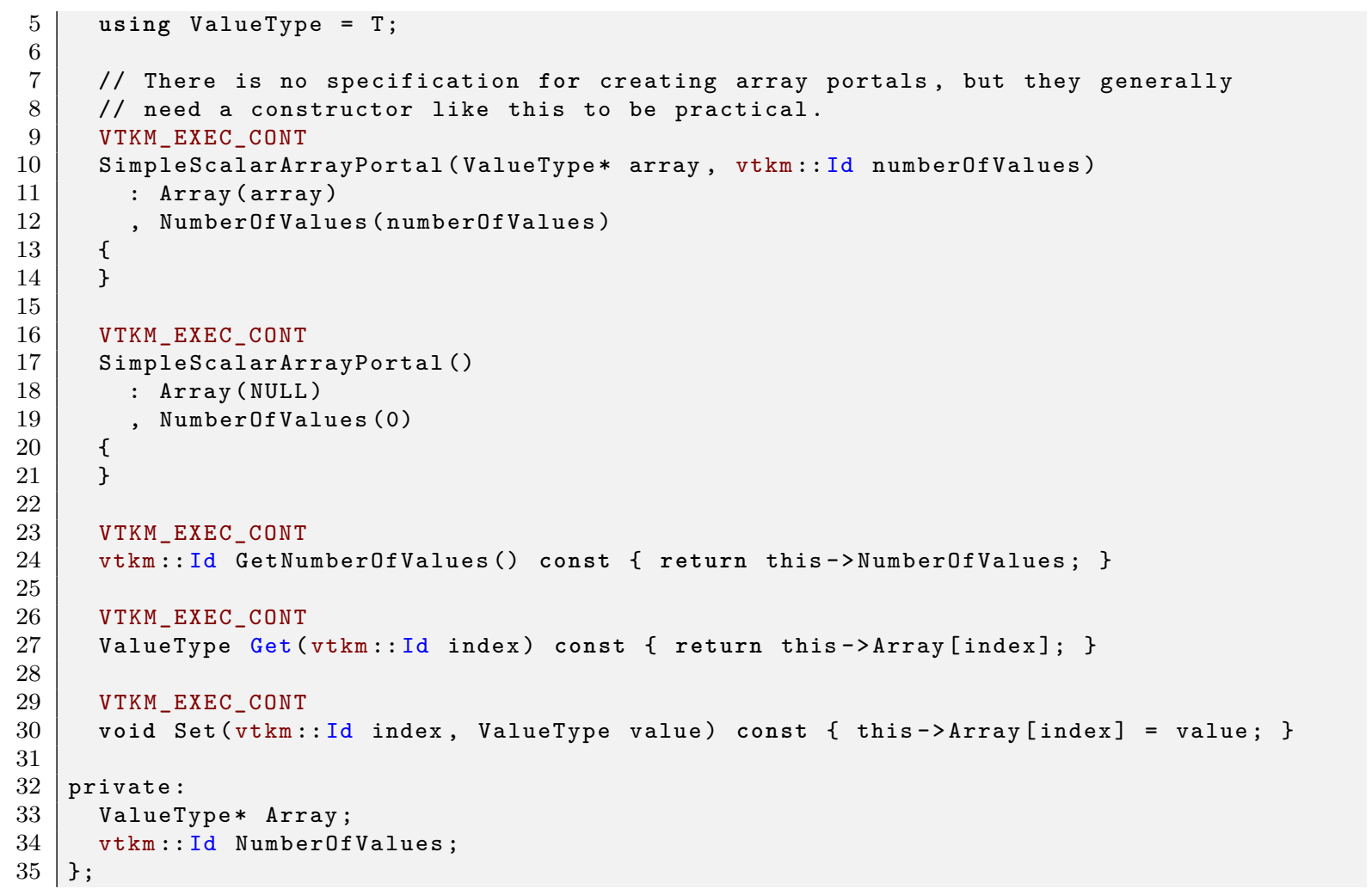

Although array portals are simple to implement and use, and array portals' functionality is similar to iterators, there exists a great deal of code already based on STL iterators and it is often convenient to interface with an array through an iterator rather than an array portal. The vtkm: :cont: :ArrayPortalToIterators class can be used to convert an array portal to an STL-compatible iterator. The class is templated on the array portal type and has a constructor that accepts an instance of the array portal. It contains the following features.

IteratorType The type of an STL-compatible random-access iterator that can provide the same access as the array portal.

GetBegin A method that returns an STL-compatible iterator of type IteratorType that points to the beginning of the array.

GetEnd A method that returns an STL-compatible iterator of type IteratorType that points to the end of the array.

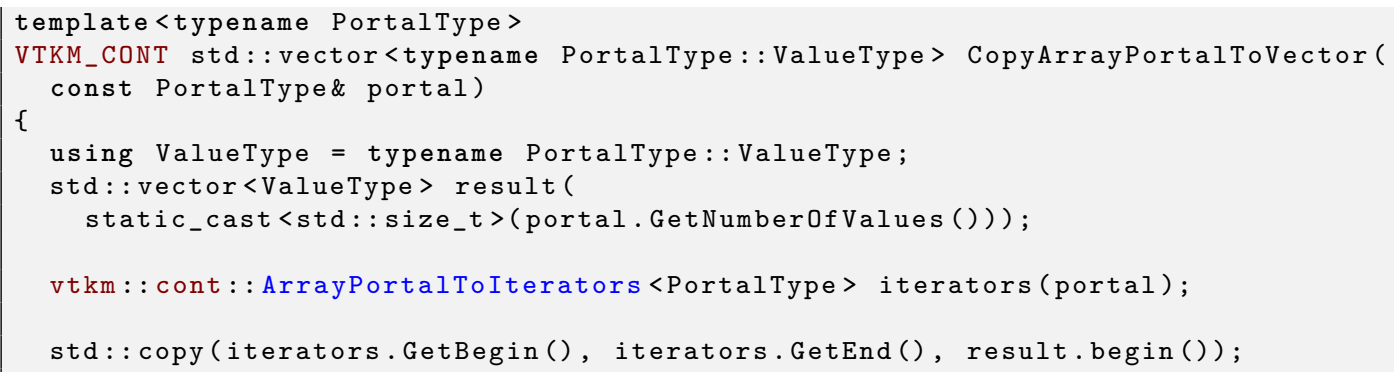


\begin{tabular}{l|l}
13 & return result;
\end{tabular}

As a convenience, vtkm/cont/ArrayPortalTolterators.h also defines a pair of functions named vtkm: : cont: : ArrayPortalToIteratorBegin() and vtkm: : cont: :ArrayPortalToIteratorEnd() that each take an array portal as an argument and return a begin and end iterator, respectively.

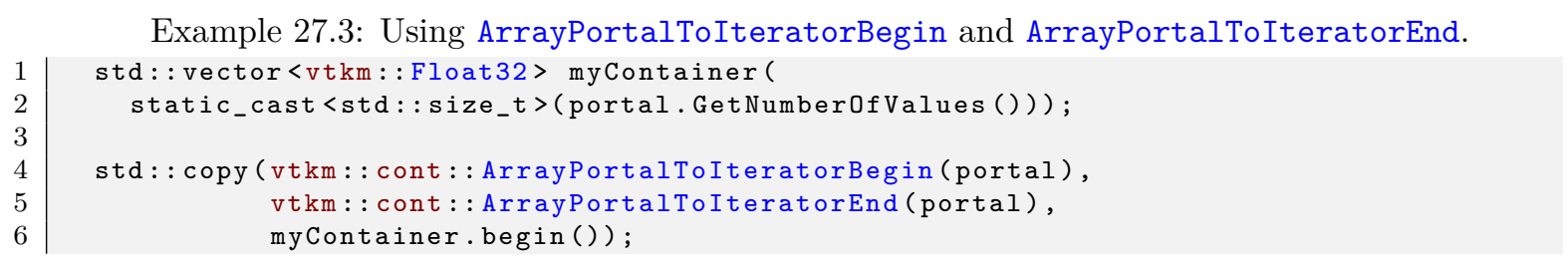

ArrayHandle contains two internal type definitions for array portal types that are capable of interfacing with the underlying data in the control environment. These are WritePortalType and ReadPortalType, which define read-write and read-only array portals, respectively.

ArrayHandle provides the methods ReadPortal and WritePortal to get the associated array portal objects to access the data in the control environment. These methods also have the side effect of refreshing the control environment copy of the data as if you called SyncControlArray. Be aware that calling WritePortal will invalidate any copy in the execution environment, meaning that any subsequent use will cause the data to be copied back again.

Example 27.4: Using portals from an ArrayHandle.

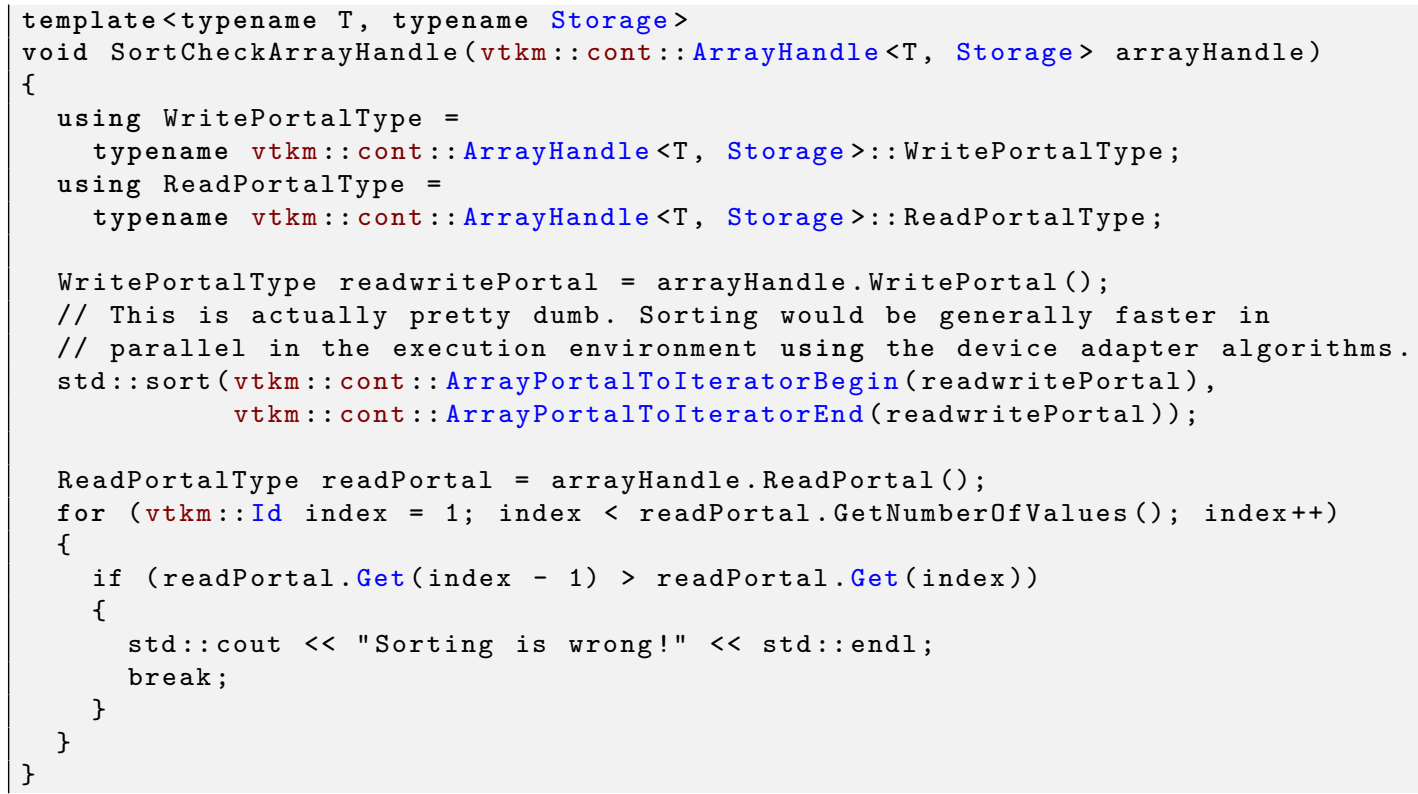

\section{(0)Did you know?}

3 Most operations on arrays in VTK-m should really be done in the execution environment. Keep in mind that whenever doing an operation using a control array portal, that operation will likely be slow for large arrays. However, some operations, like performing file I/O, make sense in the control environment. 


\section{Common Errors}

The portal returned from ReadPortal or WritePortal is only good as long as the data in the ArrayHandle are not moved or reallocated. For example, if you call ArrayHandle: :Allocate, any previously created array portals are likely to become invalid, and using them will result in undefined behavior. Thus, you should keep portals only as long as is necessary to complete an operation.

\subsection{Allocating and Populating Array Handles}

vtkm: : cont: :ArrayHandle is capable of allocating its own memory. The most straightforward way to allocate memory is to call the ArrayHandle: :Allocate method. The Allocate method takes a single argument, which is the number of elements to make the array.

Example 27.5: Allocating an ArrayHandle.

\begin{tabular}{l|l}
1 \\
2 \\
3 \\
4
\end{tabular}

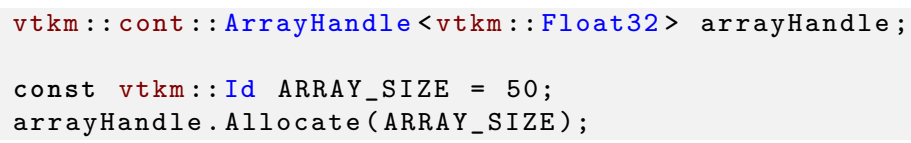

By default when you Allocate an array, it potentially destroys any existing data in it. However, there are cases where you wish to grow or shrink an array while preserving the existing data. To preserve the existing data when allocating an array, pass vtkm: :CopyFlag: : On as an optional second argument.

Example 27.6: Resizing an ArrayHandle.

1 // Add space for 10 more values at the end of the array.

2 arrayHandle.Allocate (arrayHandle.GetNumberOfValues () + 10, vtkm::CopyFlag::0n);

\section{Did you know?}

The ability to allocate memory is a key difference between ArrayHandle and many other common forms of smart pointers. When one ArrayHandle allocates new memory, all other ArrayHandles pointing to the same managed memory get the newly allocated memory. This feature makes it possible to pass an ArrayHandle to a method to be reallocated and filled without worrying about $C++$ details on how to reference the ArrayHandle object itself.

Once an ArrayHandle is allocated, it can be populated by using the portal returned from ArrayHandle: :WritePortal, as described in Section 27.1. This is roughly the method used by the readers in the I/O package (Chapter 8).

Example 27.7: Populating a newly allocated ArrayHandle.

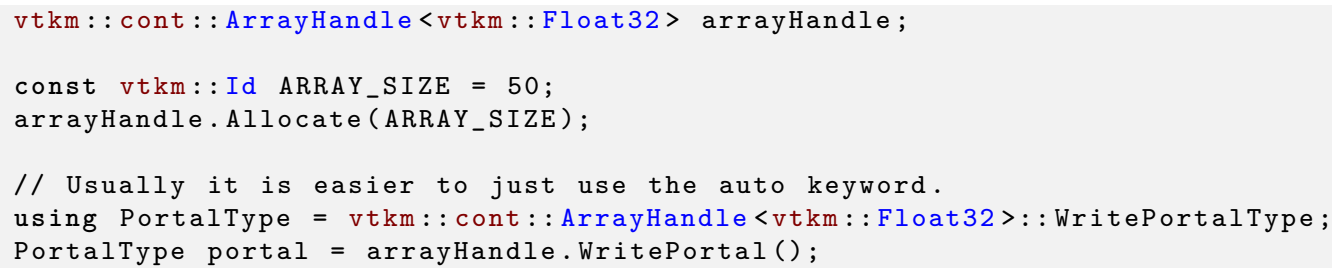




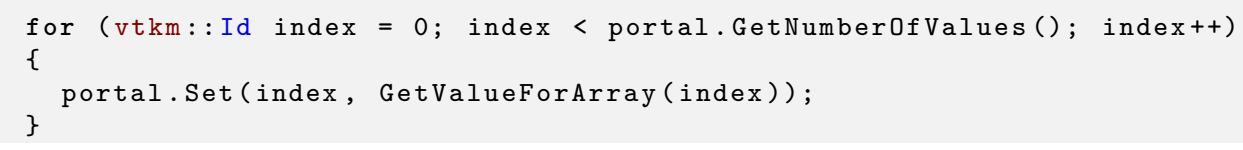

\subsection{Compute Array Range}

It is common to need to know the minimum and/or maximum values in an array. To help find these values, VTK-m provides the vtkm: : cont: : ArrayRangeCompute convenience function defined in vtkm/cont/ArrayRangeCompute.h. ArrayRangeCompute simply takes an ArrayHandle on which to find the range of values.

If given an array with vtkm: :Vec values, ArrayRangeCompute computes the range separately for each component of the Vec. The return value for ArrayRangeCompute is vtkm::cont::ArrayHandle <vtkm: :Range >. This returned array will have one value for each component of the input array's type. So for example if you call ArrayRangeCompute on a vtkm: : cont: :ArrayHandle <vtkm: Id3 >, the returned array of Ranges will have 3 values in it. Of course, when ArrayRangeCompute is run on an array of scalar types, you get an array with a single value in it.

Each value of vtkm: :Range holds the minimum and maximum value for that component. The Range object is documented in Section 19.3.

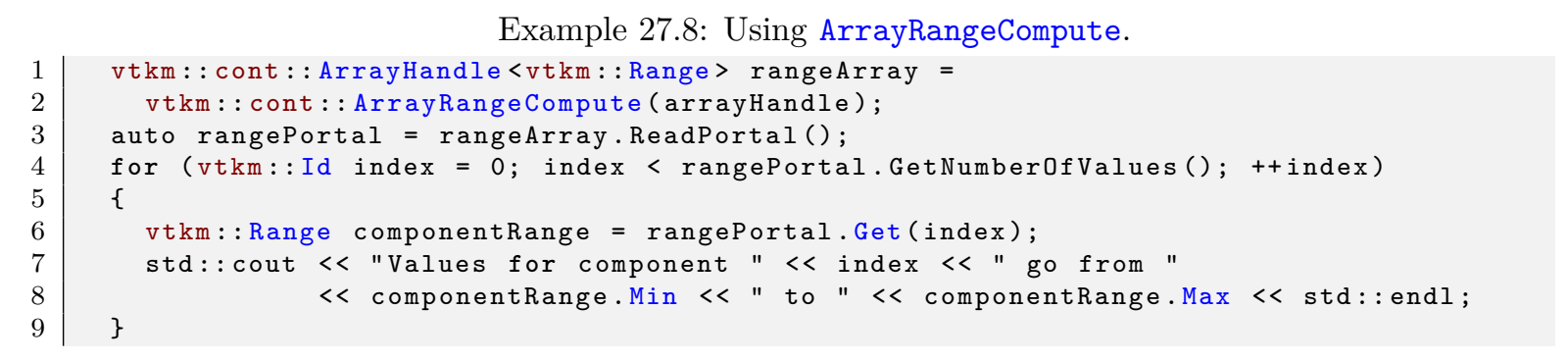

\section{Q Did you know?}

$\zeta$ ArrayRangeCompute will compute the minimum and maximum values in parallel. If desired, you can specify the parallel hardware device used for the computation as an optional second argument to ArrayRangeCompute. You can specify the device using a runtime device tracker, which is documented in Section 12.3.

\subsection{Interface to Execution Environment}

One of the main functions of the array handle is to allow an array to be defined in the control environment and then be used in the execution environment. When using an ArrayHandle with filters, worklets, or algorithms, this transition is handled automatically. However, it is also possible to invoke the transfer for a known device. This is most useful when creating execution objects, as discussed in Chapter 29.

The ArrayHandle class manages the transition from control to execution with a set of three methods that allocate, transfer, and ready the data in one operation. These methods all start with the prefix Prepare and are meant to be called before some operation happens in the execution environment. The methods are as follows. 
ArrayHandle: :PrepareForInput Copies data from the control to the execution environment, if necessary, and readies the data for read-only access.

ArrayHandle: :PrepareForInPlace Copies the data from the control to the execution environment, if necessary, and readies the data for both reading and writing.

ArrayHandle: :PrepareForOutput Allocates space (the size of which is given as a parameter) in the execution environment, if necessary, and readies the space for writing.

The PrepareForInput and PrepareForInPlace methods each take two arguments. The first argument is the device adapter tag where execution will take place (see Section 12.1 for more information on device adapter tags). The second argument is a reference to a vtkm: :cont::Token, which scopes the returned array portal. While the given Token exists, the returned portal is guaranteed to be valid and any conflicting operations on the ArrayHandle will block. Once the Token is destroyed, the associated array portal becomes invalid.

PrepareForOutput takes three arguments: the size of the space to allocate, the device adapter tag, and a reference to a Token object.

Each of these Prepare methods returns an array portal that can be used in the execution environment. PrepareForInput returns an object of type ArrayHandle: : ReadPortalType whereas PrepareForInPlace and PrepareForOutput each return an object of type ArrayHandle: :WritePortalType.

Although these Prepare methods are called in the control environment, the returned array portal can only be used in the execution environment. Thus, the portal must be passed to an invocation of the execution environment.

Most of the time, the passing of ArrayHandle data to the execution environment is handled automatically by VTK-m. The most common need to call one of these Prepare methods is to build execution objects (Chapter 29) or to construct derived array types (Section 35.4).

The following example is a contrived example for preparing arrays for the execution environment. It is contrived because it would be easier to create a worklet or transform array handle to have the same effect, and in those cases VTK-m would take of the transfers internally. More realistic examples can be found in Chapter 29 and Section 35.4.

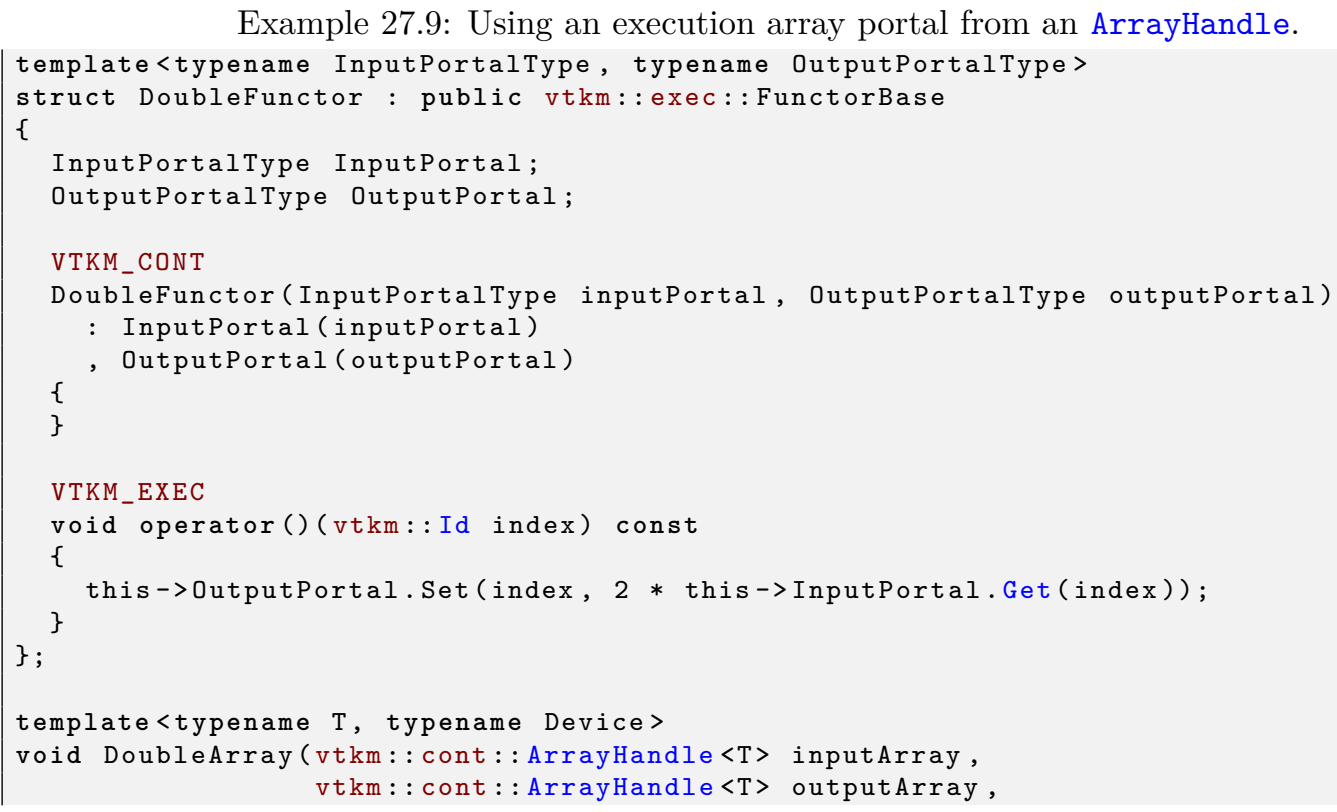




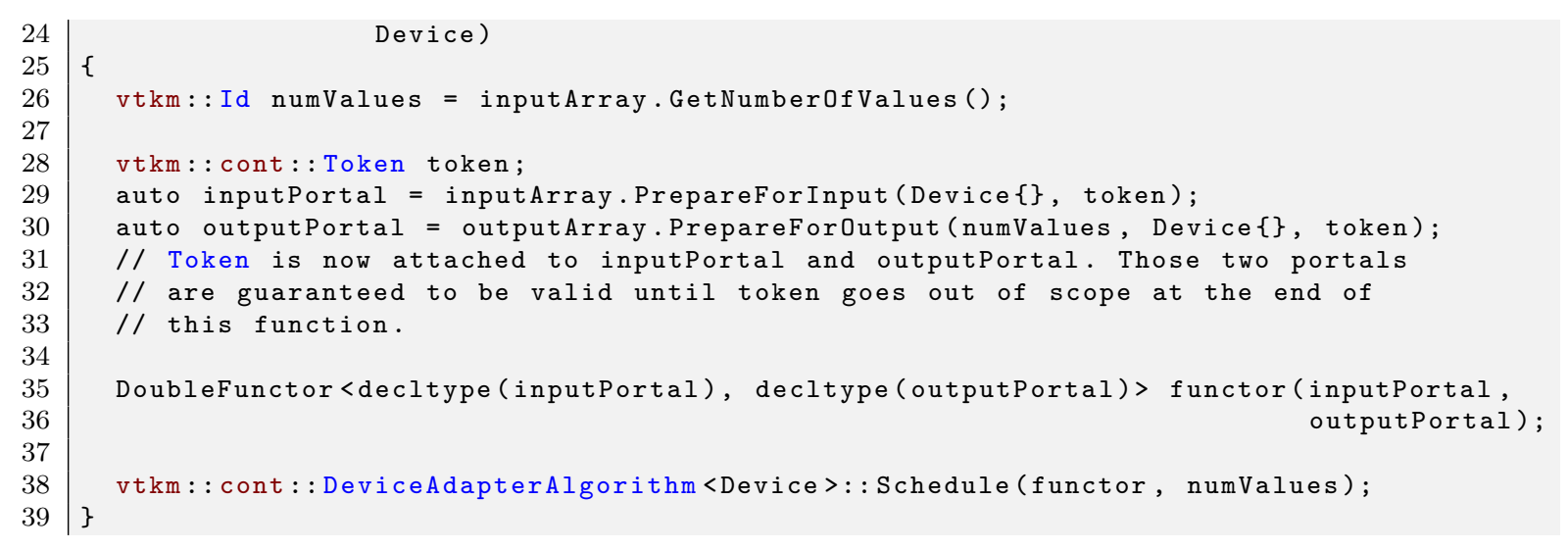

\section{$\delta$}

S Once one of the Prepare methods have been called, further operations on the ArrayHandle that might cause access hazards will block. This opens the possibility of deadlock. To help prevent deadlock, the attached Token object should be scoped to last only as long as necessary. 



\section{GLOBAL ARRAYS AND TOPOLOGY}

When writing an algorithm in VTK-m by creating a worklet, the data each instance of the worklet has access to is intentionally limited. This allows VTK-m to provide safety from race conditions and other parallel programming difficulties. However, there are times when the complexity of an algorithm requires all threads to have shared global access to a global structure. This chapter describes worklet tags that can be used to pass data globally to all instances of a worklet.

\subsection{Whole Arrays}

A whole array argument to a worklet allows you to pass in an ArrayHandle. All instances of the worklet will have access to all the data in the ArrayHandle.

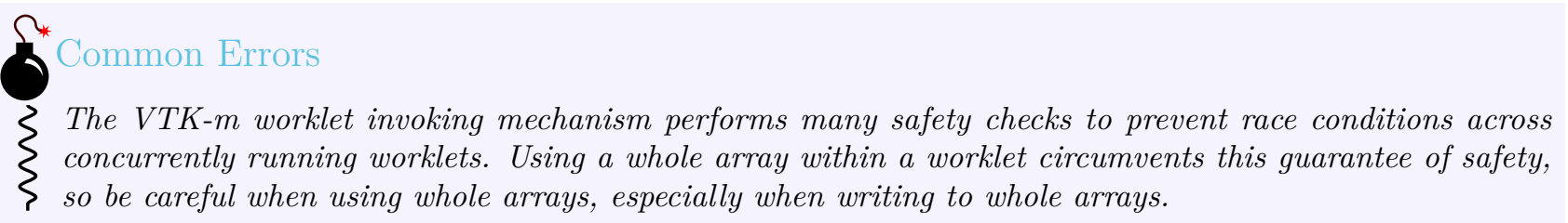

A whole array is declared by adding a WholeArrayIn, a WholeArrayInOut, or a WholeArrayOut to the ControlSignature of a worklet. The corresponding argument to the Invoker should be an ArrayHandle. The ArrayHandle must already be allocated in all cases, including when using WholeArrayOut. When the data are passed to the operator of the worklet, it is passed as an array portal object. (Array portals are discussed in Section 27.1.) This means that the worklet can access any entry in the array with Get and/or Set methods.

We have already seen a demonstration of using a whole array in Example 21.2 to perform a simple array copy. Here we will construct a more thorough example of building functionality that requires random array access.

Let's say we want to measure the quality of triangles in a mesh. A common method for doing this is using the equation

$$
q=\frac{4 a \sqrt{3}}{h_{1}^{2}+h_{2}^{2}+h_{3}^{2}}
$$

where $a$ is the area of the triangle and $h_{1}, h_{2}$, and $h_{3}$ are the lengths of the sides. We can easily compute this in a cell to point map, but what if we want to speed up the computations by reducing precision? After all, we probably only care if the triangle is good, reasonable, or bad. So instead, let's build a lookup table and then retrieve the triangle quality from that lookup table based on its sides. 
The following example demonstrates creating such a table lookup in an array and using a worklet argument tagged with WholeArrayIn to make it accessible.

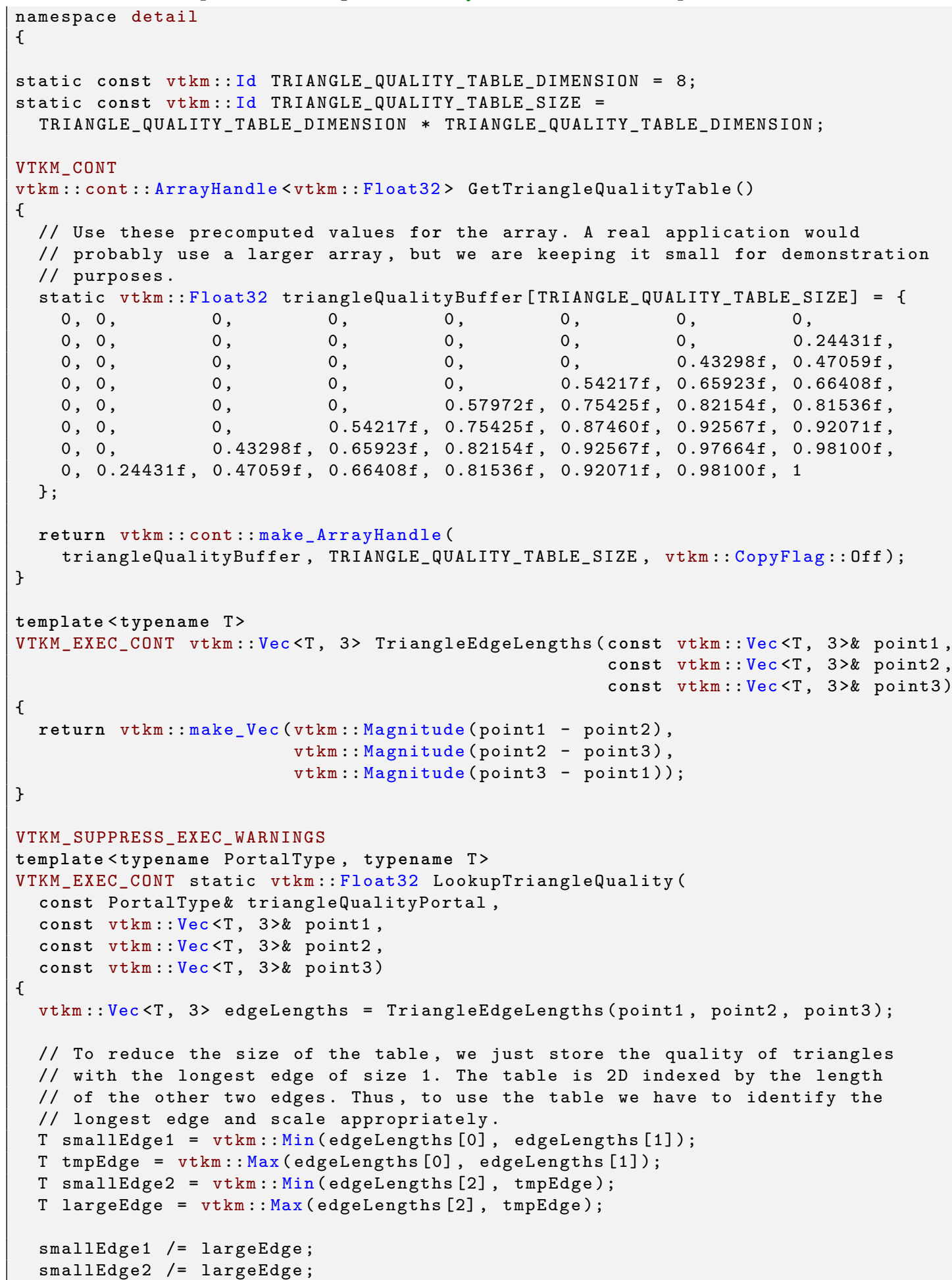




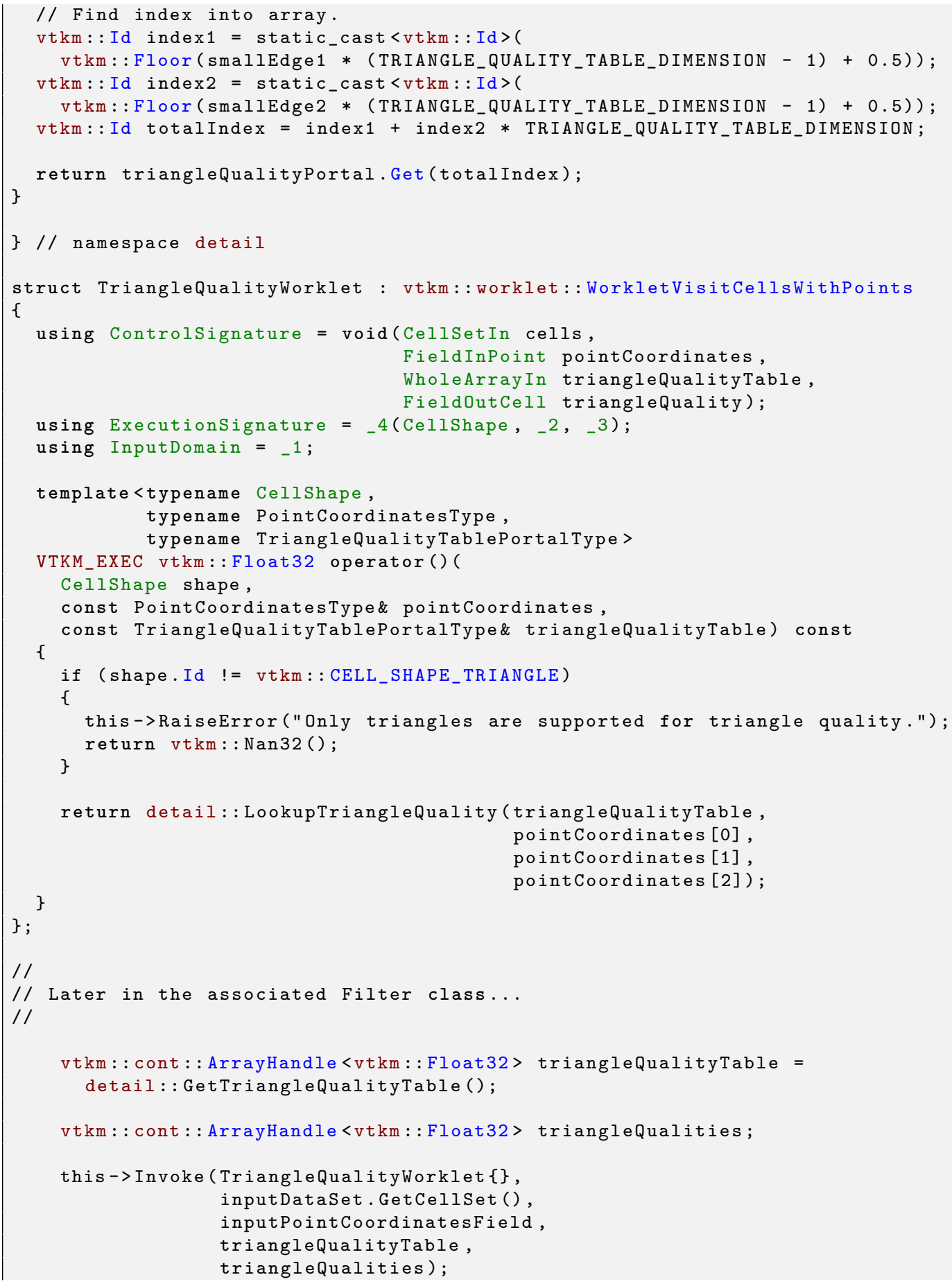

\subsection{Atomic Arrays}

One of the problems with writing to whole arrays is that it is difficult to coordinate the access to an array from multiple threads. If multiple threads are going to write to a common index of an array, then you will probably 
need to use an atomic array.

An atomic array allows random access into an array of data, similar to a whole array. However, the operations on the values in the atomic array allow you to perform an operation that modifies its value that is guaranteed complete without being interrupted and potentially corrupted.

\section{Common Errors
Due to limitations in available atomic operations, atomic arrays can currently only contain vtkm: : Int32
or vtkm: : Int64 values.}

To use an array as an atomic array, first add the AtomicArrayInOut tag to the worklet's ControlSignature. The corresponding argument to the Invoker should be an ArrayHandle, which must already be allocated and initialized with values.

When the data are passed to the operator of the worklet, it is passed in a vtkm::exec: :AtomicArrayExecutionObject structure. AtomicArrayExecutionObject has two important methods:

Add Takes as arguments an index and a value. The entry in the array corresponding to the index will have the value added to it. If multiple threads attempt to add to the same index in the array, the requests will be serialized so that the final result is the sum of all the additions. AtomicArrayExecutionObject: : Add returns the value that was replaced. That is, it returns the value right before the addition.

CompareAndSwap Takes as arguments an index, a new value, and an old value. If the entry in the array corresponding to the index has the same value as the "old value," then it is changed to the "new value" and the original value is return from the method. If the entry in the array is not the same as the "old value," then nothing happens to the array and the value that is actually stored in the array is returned. If multiple threads attempt to compare and swap to the same index in the array, the requests are serialized.

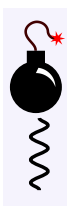

\section{Common Errors}

Atomic arrays help resolve hazards in parallel algorithms, but they come at a cost. Atomic operations are more costly than non-thread-safe ones, and they can slow a parallel program immensely if used incorrectly.

The following example uses an atomic array to count the bins in a histogram. It does this by making the array of histogram bins an atomic array and then using an atomic add. Note that this is not the fastest way to create a histogram. We gave an implementation in Section 21.4 that is generally faster (unless your histogram happens to be very sparse). VTK-m also comes with a histogram worklet that uses a similar approach.

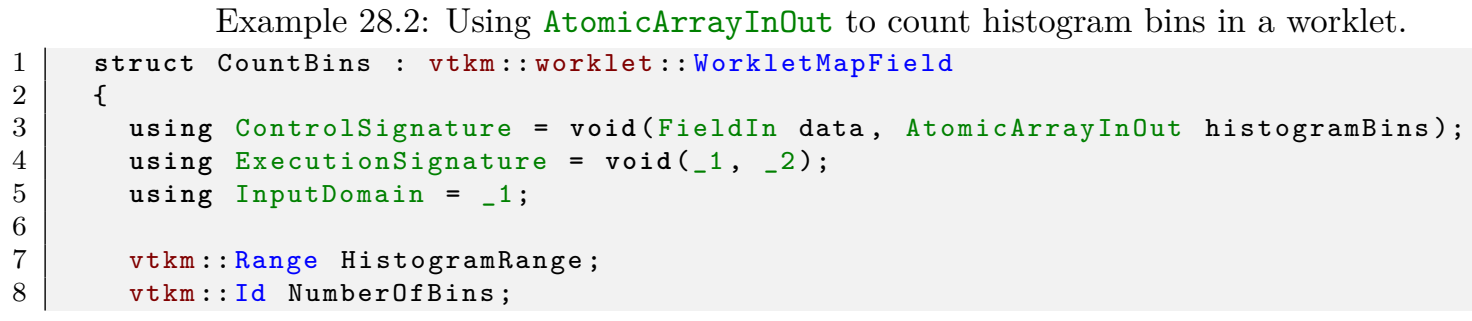

Example 28.2: Using AtomicArrayInOut to count histogram bins in a worklet. 


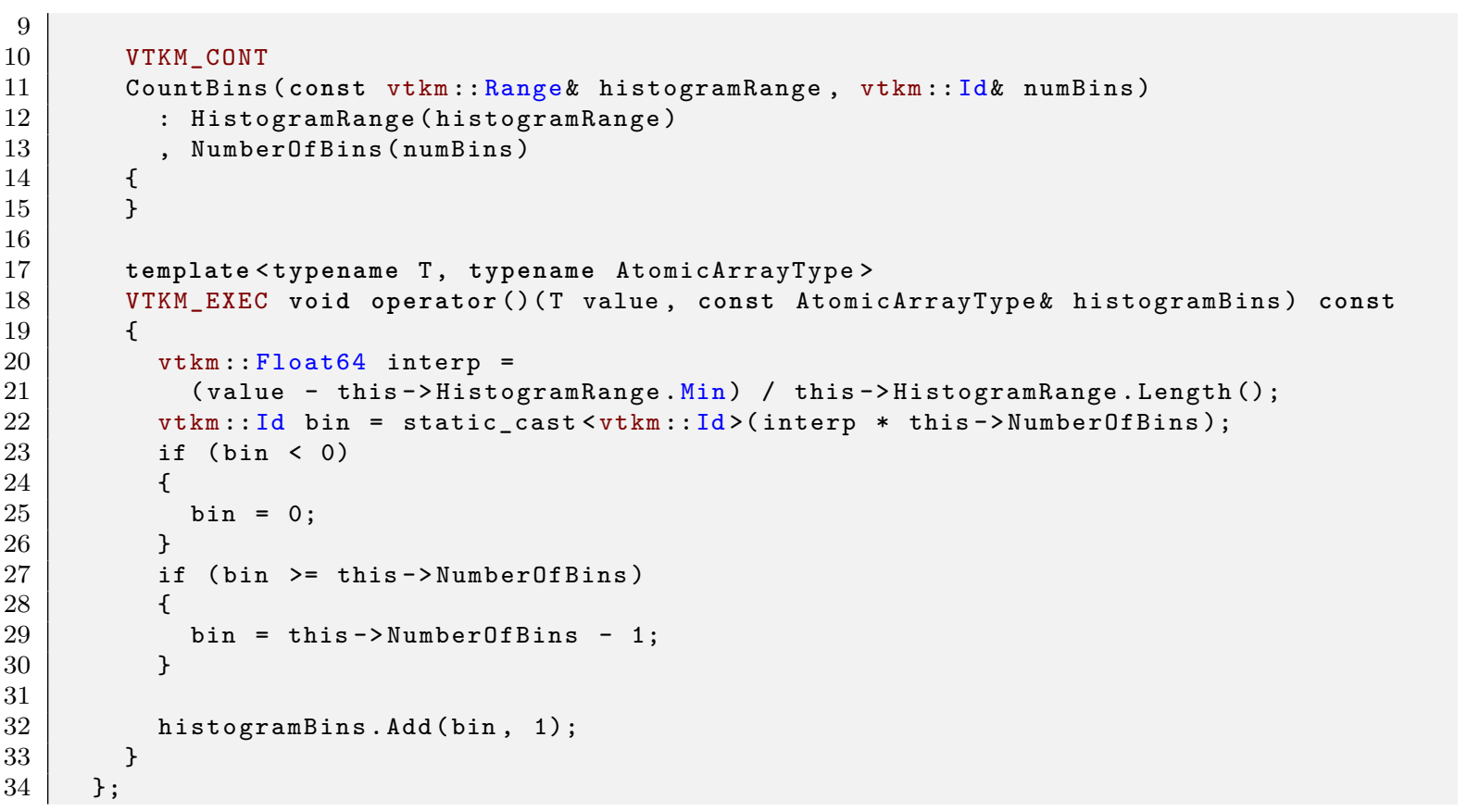

\subsection{Whole Cell Sets}

Section 21.2 describes how to make a topology map filter that performs an operation on cell sets. The worklet has access to a single cell element (such as point or cell) and its immediate connections. But there are cases when you need more general queries on a topology. For example, you might need more detailed information than the topology map gives or you might need to trace connections from one cell to the next. To do this VTK-m allows you to provide a whole cell set argument to a worklet that provides random access to the entire topology.

A whole cell set is declared by adding a WholeCellSetIn to the worklet's ControlSignature. The corresponding argument to the Invoker should be a Cellset subclass or a DynamicCellset (both of which are described in Section 7.2).

The WholeCellSetIn is templated and takes two arguments: the "visit" topology type and the "incident" topology type, respectively. These template arguments must be one of the topology element tags, but for convenience you can use Point and Cell in lieu of vtkm::TopologyElementTagPoint and vtkm::TopologyElementTagCell, respectively. The "visit" and "incident" topology types define which topological elements can be queried (visited) and which incident elements are returned. The semantics of the "visit" and "incident" topology is the same as that for the general topology maps described in Section 21.2.3. You can look up an element of the "visit" topology by index and then get all of the "incident" elements from it.

For example, a WholeCellSetIn<Cell, Point> allows you to find all the points that are incident on each cell (as well as querying the cell shape). Likewise, a WholeCellSetIn<Point, Cell> allows you to find all the cells that are incident on each point. The default parameters of WholeCellsetIn are visiting cells with incident points. That is, WholeCellSetIn<> is equivalent to WholeCellSetIn<Cell, Point>.

When the cell set is passed to the operator of the worklet, it is passed in a special connectivity object. The actual object type depends on the cell set, but vtkm: : exec: :CellSetStructured and are two common examples vtkm: : exec: :CellSetExplicit. All these connectivity objects share a common interface. First, they all declare the following public types. 
CellShapeTag The tag for the cell shapes of the cell set. (Cell shape tags are described in Section 25.1.) If the connectivity potentially contains more than one type of cell shape, then this type will be vtkm: :CellShapeTagGeneric.

IndicesType A Vec-like type that stores all the incident indices.

Second they all provide the following methods.

GetNumberOfElements Get the number of "to" topology elements in the cell set. All the other methods require an element index, and this represents the range of valid indices. The return type is vtkm: :Id.

GetCellShape Takes an index for an element and returns a CellShapeTag object of the corresponding cell shape. If the "to" topology elements are not strictly cell, then a reasonably close shape is returned. For example, if the "to" topology elements are points, then the shape is returned as a vertex.

GetNumberOfIndices Takes an index for an element and returns the number of incident "from" elements are connected to it. The returned type is vtkm: : IdComponent.

GetIndices Takes an index for an element and returns a Vec-like object of type IndicesType containing the indices of all incident "from" elements. The size of the Vec-like object is the same as that returned from GetNumberOfIndicices.

VTK-m comes with several functions to work with the shape and index information returned from these connectivity objects. Most of these methods are documented in Chapter 25.

Let us use the whole cell set feature to help us determine the "flatness" of a polygonal mesh. We will do this by summing up all the angles incident on each on each point. That is, for each point, we will find each incident polygon, then find the part of that polygon using the given point, then computing the angle at that point, and then summing for all such angles. So, for example, in the mesh fragment shown in Figure 28.1 one of the angles attached to the middle point is labeled $\theta_{j}$.

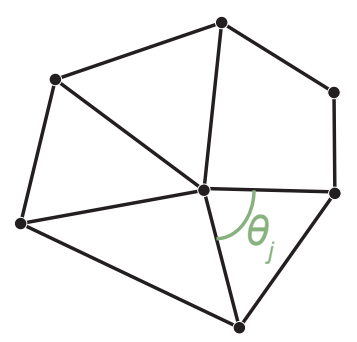

Figure 28.1: The angles incident around a point in a mesh.

We want a worklet to compute $\sum_{j} \theta$ for all such attached angles. This measure is related (but not the same as) the curvature of the surface. A flat surface will have a sum of $2 \pi$. Convex and concave surfaces have a value less than $2 \pi$, and saddle surfaces have a value greater than $2 \pi$.

To do this, we create a visit points with cells worklet (Section 21.2.2) that visits every point and gives the index of every incident cell. The worklet then uses a whole cell set to inspect each incident cell to measure the attached angle and sum them together.

Example 28.3: Using WholeCellSetIn to sum the angles around each point.

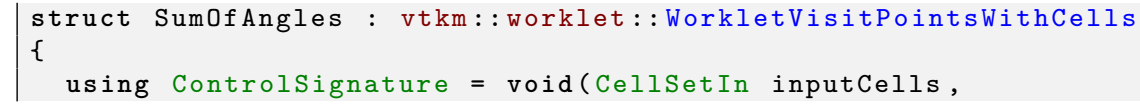




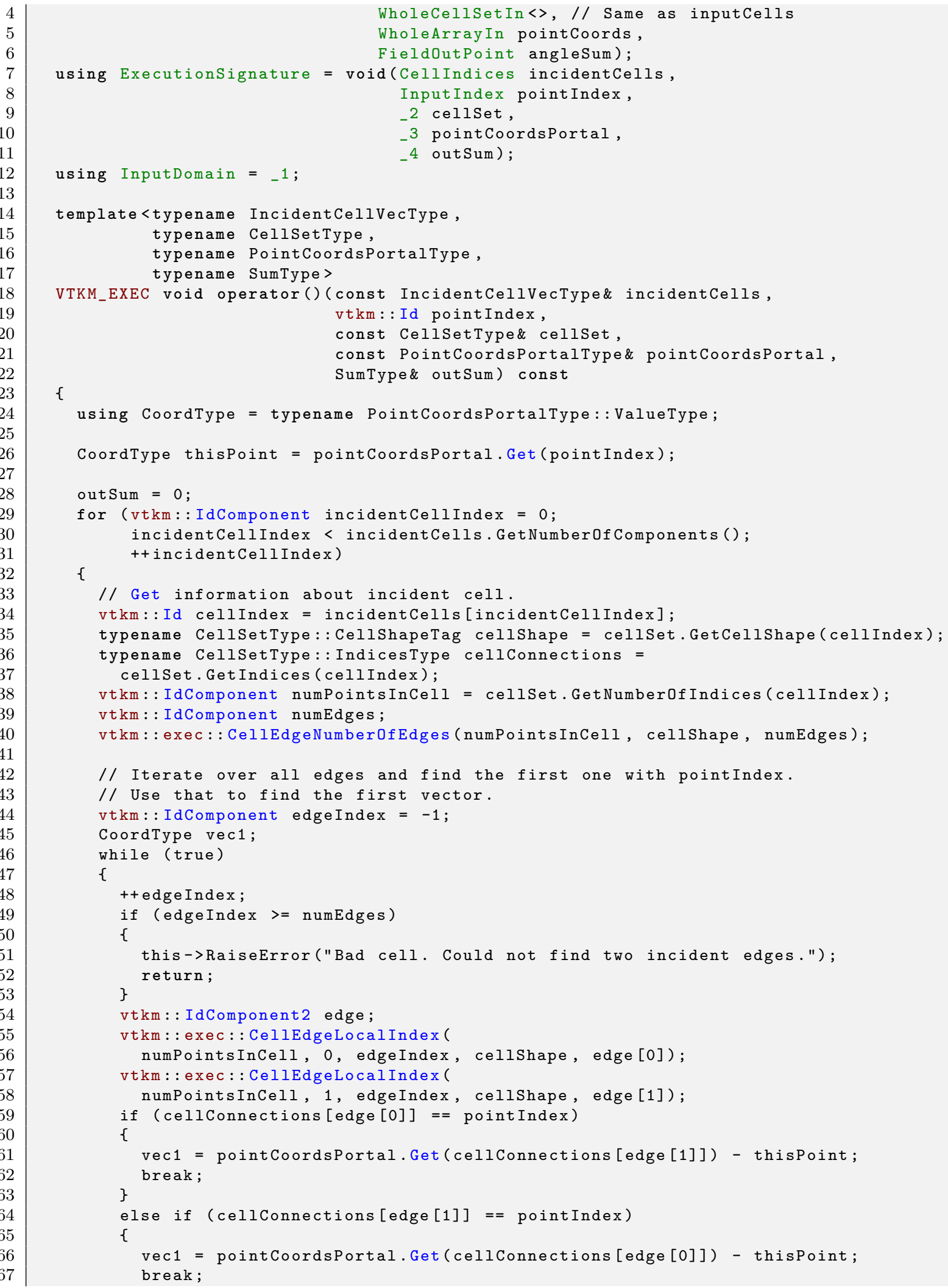




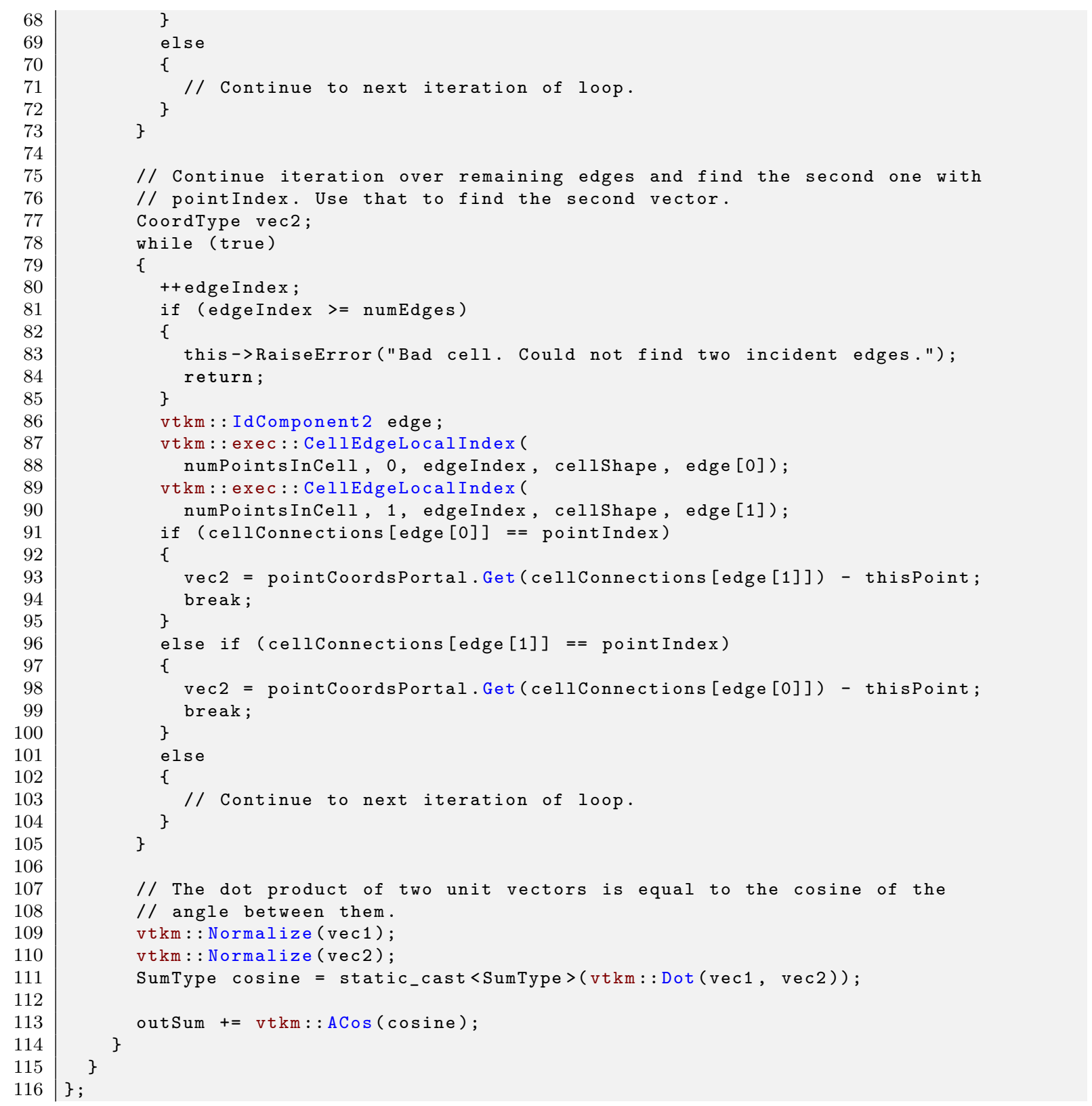




\section{EXECUTION OBJECTS}

Although passing whole arrays and cell sets into a worklet is a convenient way to provide data to a worklet that is not divided by the input or output domain, they are sometimes not the best structures to represent data. Thus, all worklets support a another type of argument called an execution object, or exec object for short, that provides a user-defined object directly to each invocation of the worklet. This is defined by an ExecObject tag in the ControlSignature.

The execution object must be a subclass of vtkm: :cont: :ExecutionObjectBase. Also, it must implement a PrepareForExecution method declared with VTKM_CONT. PrepareForExecution should take two arguments. The first argument is the device adapter tag. The second argument is a vtkm: : cont: : Token object that should be used to scope any execution objects created internally.

The PrepareForExecution function creates an execution object that can be passed from the control environment to the execution environment and be usable in the execution environment, and any method of the produced object used within the worklet must be declared with VTKM_EXEC or VTKM_EXEC_CONT.

An execution object can refer to an array, but the array reference must be through an array portal for the execution environment. This can be retrieved from the ArrayHandle: :PrepareForInput method as described in Section 27.4. Other VTK-m data objects, such as the subclasses of vtkm: :cont: Cellset, have similar methods.

Returning to the example we have in Section 28.1, we are computing triangle quality quickly by looking up the value in a table. In Example 28.1 (page 238) the table is passed directly to the worklet as a whole array. However, there is some additional code involved to get the appropriate index into the table for a given triangle. Let us say that we want to have the ability to compute triangle quality in many different worklets. Rather than pass in a raw array, it would be better to encapsulate the functionality in an object.

We can do that by creating an execution object with a PrepareForExecution method that creates an object that has the table stored inside and methods to compute the triangle quality. The following example uses the table built in Example 28.1 to create such an object.

Example 29.1: Using ExecObject to access a lookup table in a worklet.

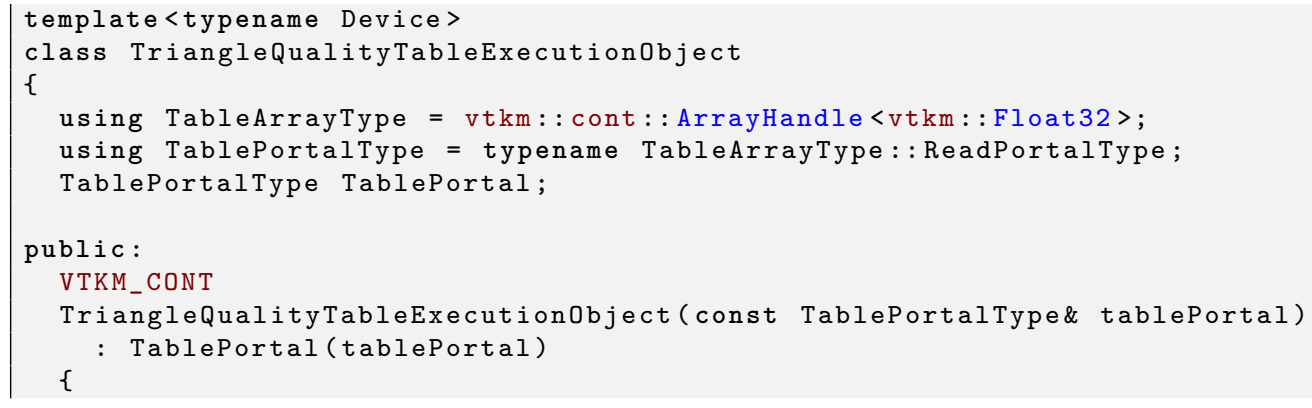




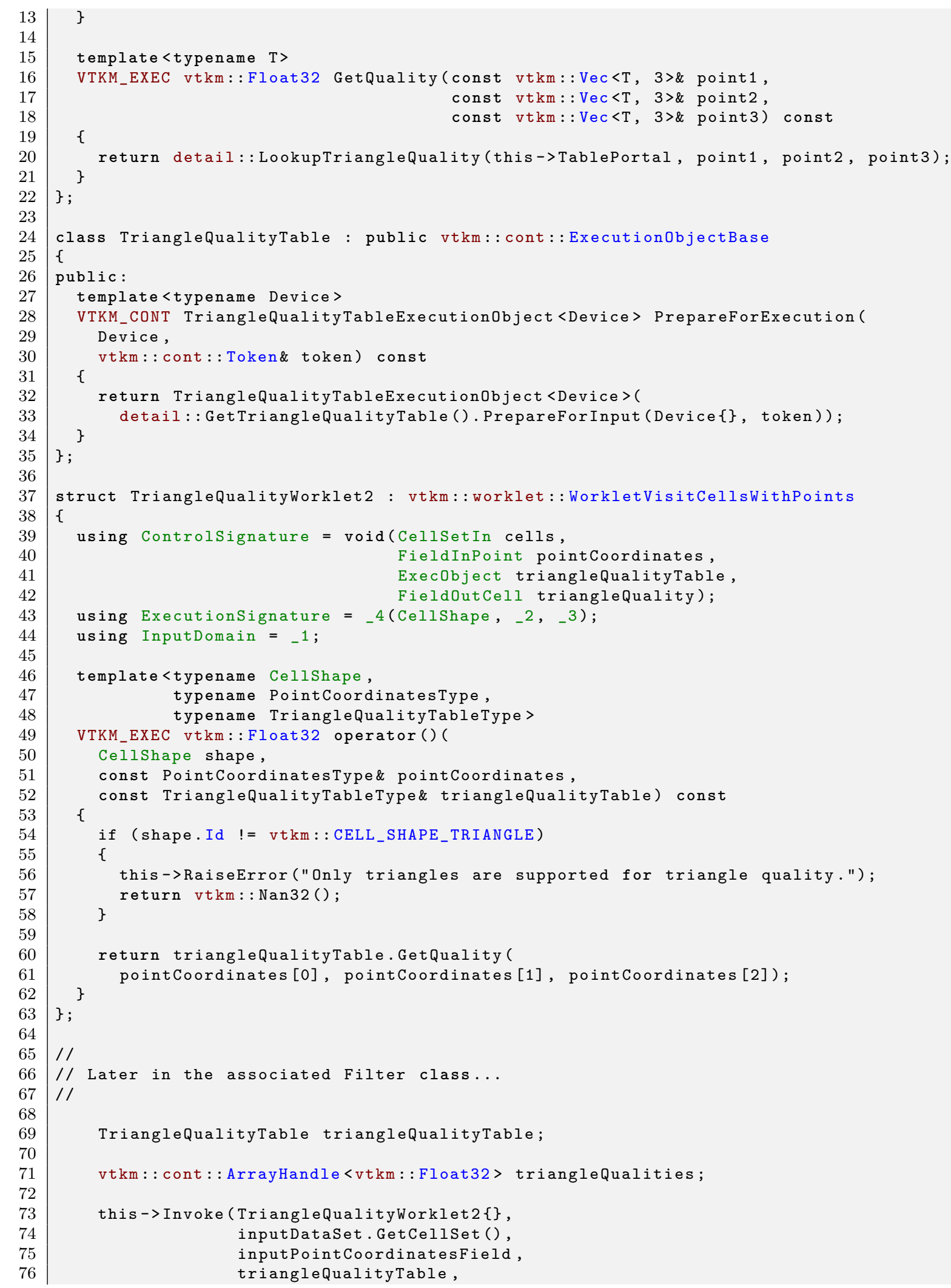





\section{LOCATORS}

Locators are a special type of structure that allows you to take a point coordinate in space and then find a topological element that contains or is near that coordinate. VTK-m comes with multiple types of locators, which are categorized by the type of topological element that they find. For example, a cell locator takes a coordinate in world space and finds the cell in a vtkm: : cont: :DataSet that contains that cell. Likewise, a point locator takes a coordinate in world space and finds a point from a vtkm: : cont : : CoordinateSystem nearby.

Different locators differ in their interface slightly, but they all follow the same basic operation. First, they are constructed and provided with one or more elements of a vtkm: :cont: :DataSet. Then they are built with a call to an Update method. The locator can then be passed to a worklet as an ExecObject, which will cause the worklet to get a special execution version of the locator that can do the queries.

Q Did you know?

¿ther visualization libraries, like VTK-m's big sister toolkit VTK, provide similar locator structures that allow iterative building by adding one element at a time. VTK-m explicitly disallows this use case. Although iteratively adding elements to a locator is undoubtedly useful, such an operation will inevitably bottleneck a highly threaded algorithm in critical sections. This makes iterative additions to locators too costly to support in VTK-m.

\subsection{Cell Locators}

Cell Locators in VTK-m provide a means of building spatial search structures that can later be used to find a cell containing a certain point. This could be useful in scenarios where the application demands the cell to which a point belongs to to achieve a certain functionality. For example, while tracing a particle's path through a vector field, after every step we lookup which cell the particle has entered to interpolate the velocity at the new location to take the next step.

Using cell locators is a two step process. The first step is to build the search structure. This is done by instantiating one of the CellLocator classes, providing a cell set and coordinate system (usually from a vtkm: :cont: : DataSet), and then updating the structure. Once the cell locator is built, it can be used in the execution environment within a filter or worklet. 


\subsubsection{Building a Cell Locator}

All cell locators in VTK-m share the same basic interface for the required features of cell locators. This generic interface provides methods to set the cell set (with SetCellset and GetCellSet) and to set the coordinate system (with SetCoordinates and GetCoordinates). Once the cell set and coordinates are provided, you may call Update to construct the search structures. Although Update is called from the control environment, the search structure will be built on parallel devices.

Example 30.1: Constructing a CellLocator.

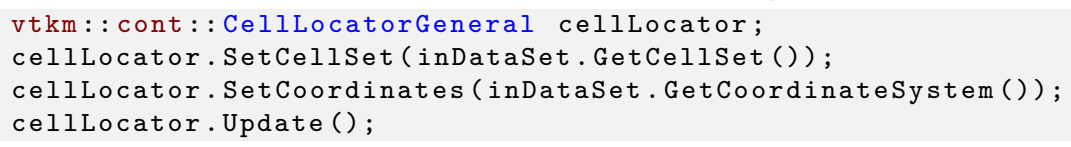

VTK-m currently exposes the implementations of the following Cell Locators.

vtkm: :cont::CellLocatorGeneral This locator will automatically select another locator to use as its implementation. CellLocatorGeneral allows you to automatically select cell locators optimized for certain cell structures without knowing the cell set type. You can change how CellLocatorGeneral selects a CellLocator by providing a function to the SetConfigurator method. If no configurator is set, then a default one is used.

vtkm: : cont: :CellLocatorUniformGrid This locator is optimized for structured data that has uniform axisaligned spacing. For this cell locator to work, it has to be given a cell set of type CellsetStructured and a coordinate system using an ArrayHandleUniformPointCoordinates for its data.

vtkm: : cont: :CellLocatorRectilinearGrid This locator is optimized for structured data that has nonuniform axis-aligned spacing. For this cell locator to work, it has to be given a cell set of type CellSetStructured and a coordinate system using an ArrayHandleCartesianProduct for its data.

vtkm: :cont: :CellLocatorTwoLevel This locator builds a 2-level hierarchy of uniform bins. The first level is a coarse partitioning of the space. Each bin in the first level has a second grid who's size depends on the number of cells in the first level. The density (number of cells expected in each bin) for each level can be set with SetDensityL1 and SetDensityL2. Their default values are 32 and 2, respectively.

vtkm: :cont: : CellLocatorBoundingIntervalHierarchy This locator is based on the bounding interval hierarchy spatial search structure. CellLocatorBoundingIntervalHierarchy takes two parameters: the number of splitting planes used to split the cells uniformly along an axis at each level and the maximum leaf size, which determines if a node needs to be split further. These parameters can set through the SetNumberOfPlanes and SetMaxLeafSize methods.

vtkm: :cont::CellLocatorChooser This locator is similar to CellLocatorGeneral in that it automatically selects an appropriate locator based on the type of cell structure being used. However, unlike the other locator, CellLocatorChooser is a templated class that chooses the correct locator based on the cell set type and the coordinates array type provided as template arguments. This means that you have to know the data types at compile time. CellLocatorChooser is a good choice in applications where you know your DataSet is of a particular type.

\subsubsection{Using Cell Locators in a Worklet}

The CellLocator interface implements vtkm: :cont: :ExecutionObjectBase. This means that any CellLocator can be used in worklets as an ExecObject argument (as defined in the ControlSignature). See Chapter 29 for information on ExecObject arguments to worklets. 
When a vtkm: : cont: : CellLocator class is passed as an ExecObject argument to a worklet Invoke, the worklet receives a different object defined in the vtkm::exec namespace. This vtkm::exec::CellLocator object provides a FindCell method that identifies a containing cell given a point location in space.

\section{$\int^{*}$ Common Errors \\ Note that the vtkm: : cont: :CellLocator and vtkm::exec::CellLocator classes are different objects with different interfaces despite the similar names.}

The CellLocator: FindCell method takes 3 arguments. The first argument is an input query point. The second argument is used to return the id of the cell containing this point (or - 1 if the point is not found in any cell). The third argument is used to return the parametric coordinates for the point within the cell (assuming it is found in any cell). FindCell returns an ErrorCode to indicate the status of the query. If the cell and the location within the cell are found, ErrorCode: :Success is returned. If the point is not inside any cell, ErrorCode: :CellNotFound is likely to be returned.

The following example defines a simple worklet to get the value of a point field interpolated to a group of query point coordinates provided.

Example 30.2: Using a CellLocator in a worklet.

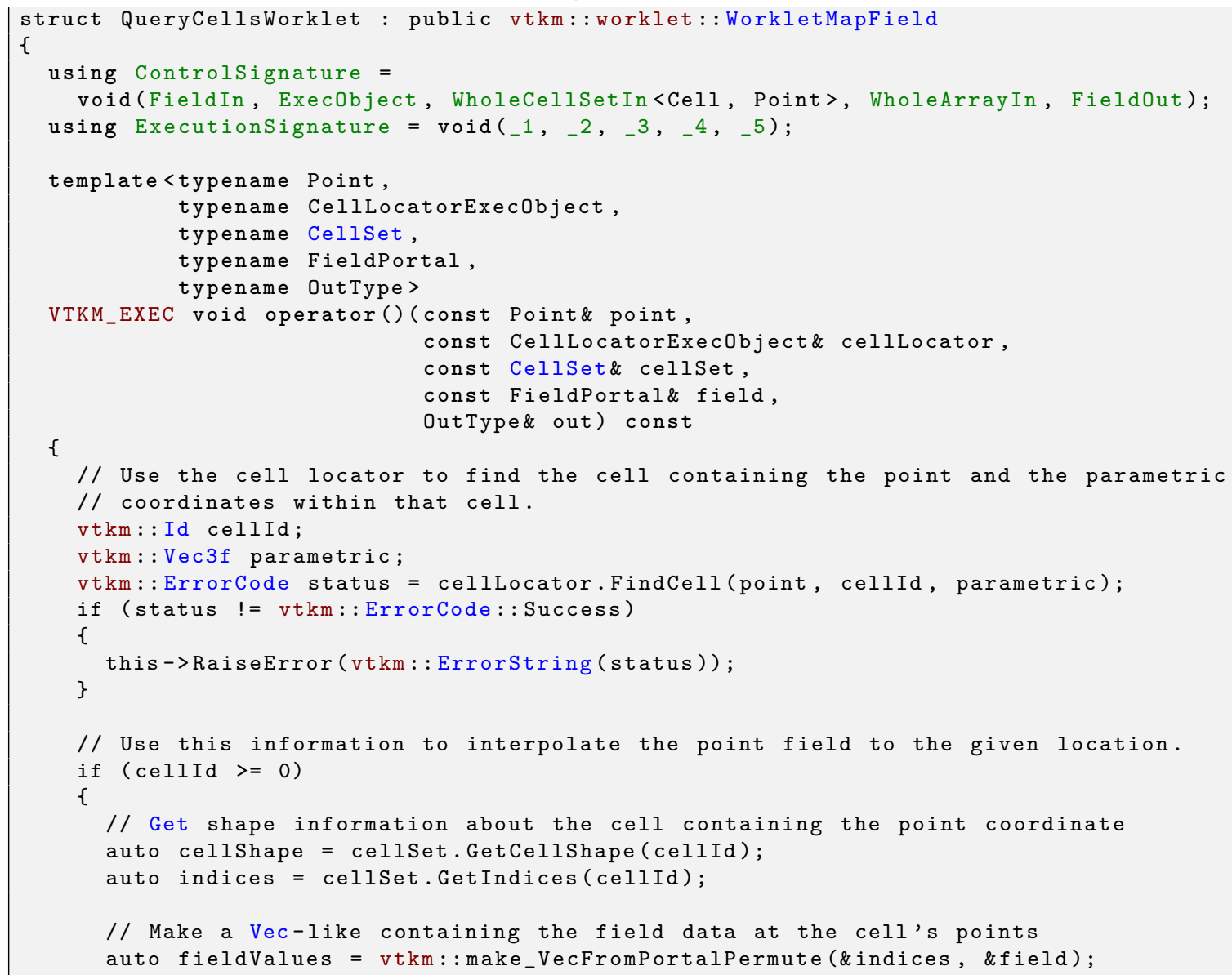




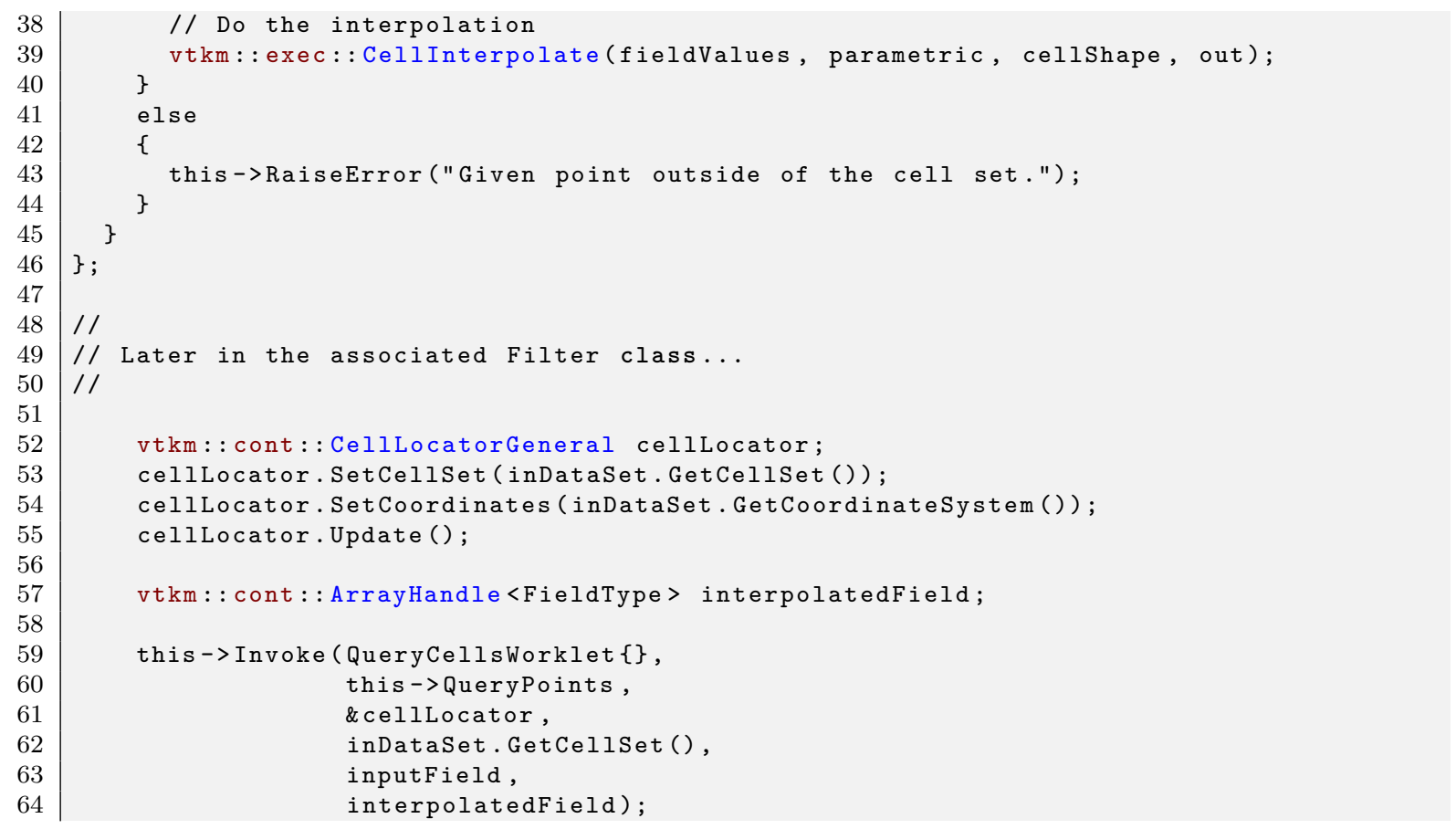

\subsection{Point Locators}

Point Locators in VTK-m provide a means of building spatial search structures that can later be used to find the nearest neighbor a certain point. This could be useful in scenarios where the closest pairs of points are needed. For example, during halo finding of particles in cosmology simulations, pairs of nearest neighbors within certain linking length are used to form clusters of particles.

Using point locators is a two step process. The first step is to build the search structure. This is done by instantiating one of the vtkm: cont: :PointLocator classes, providing a coordinate system (usually from a vtkm: : cont: :DataSet) representing the location of points that can later be found through queries, and then updating the structure. Once the point locator is built, it can be used in the execution environment within a filter or worklet.

\subsubsection{Building Point Locators}

All point locators in VTK-m share the same basic interface for the required features of point locators. This generic interface provides methods to set the coordinate system (with SetCoordinates and GetCoordinates) of training points. Once the coordinates are provided, you may call Update to construct the search structures. Although Update is called from the control environment, the search structure will be built on parallel devices

Example 30.3: Constructing a PointLocator.

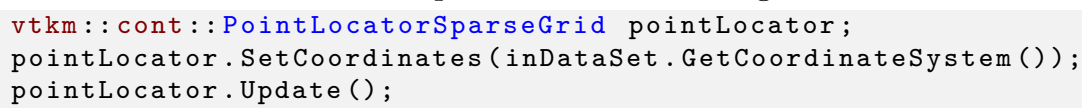

VTK-m currently exposes the implementations of the following Point Locators. 
vtkm: : cont: :PointLocatorSparseGrid This point locator is based on the uniform grid search structure. It divides the search space into a uniform grid of bins. A search for a point near a given coordinate starts in the bin containing the search coordinates. If a candidate point is not found in that bin, points are searched in an expanding neighborhood of grid bins. The size of the grid used by the locator to partition the space can be set with SetNumberOfBins. By default, PointLocatorSparseGrid uses a $32^{3}$ grid. It is also possible to set the physical space over which the search space is constructed with the SetRange method. If the range is not set, it will automatically be set to the space of the coordinates.

\subsubsection{Using Point Locators in a Worklet}

The PointLocator interface implements vtkm: :cont: :ExecutionObjectBase. This means that any PointLocator can be used in worklets as an ExecObject argument (as defined in the ControlSignature). See Chapter 29 for information on ExecObject arguments to worklets.

When a vtkm::cont::PointLocator class is passed as an ExecObject argument to a worklet Invoke, the worklet receives a different object defined in the vtkm::exec namespace. This vtkm::exec::PointLocator object provides a FindNearestNeighbor method that identifies the nearest neighbor point given a coordinate in space.

\section{S* Common Errors}

Note that vtkm: : cont: :PointLocator and vtkm: :exec: :PointLocator are different objects with different interfaces despite the similar names.

The FindNearestNeighbor method takes 3 arguments. The first argument is an input query point. The second argument is used to return the id of the nearest neighbor point (or -1 if no nearby point is found, for example, in the case of an empty set of data set points). The third argument is used to return the squared distance for the query point to its nearest neighbor.

Example 30.4: Using a PointLocator in a worklet.

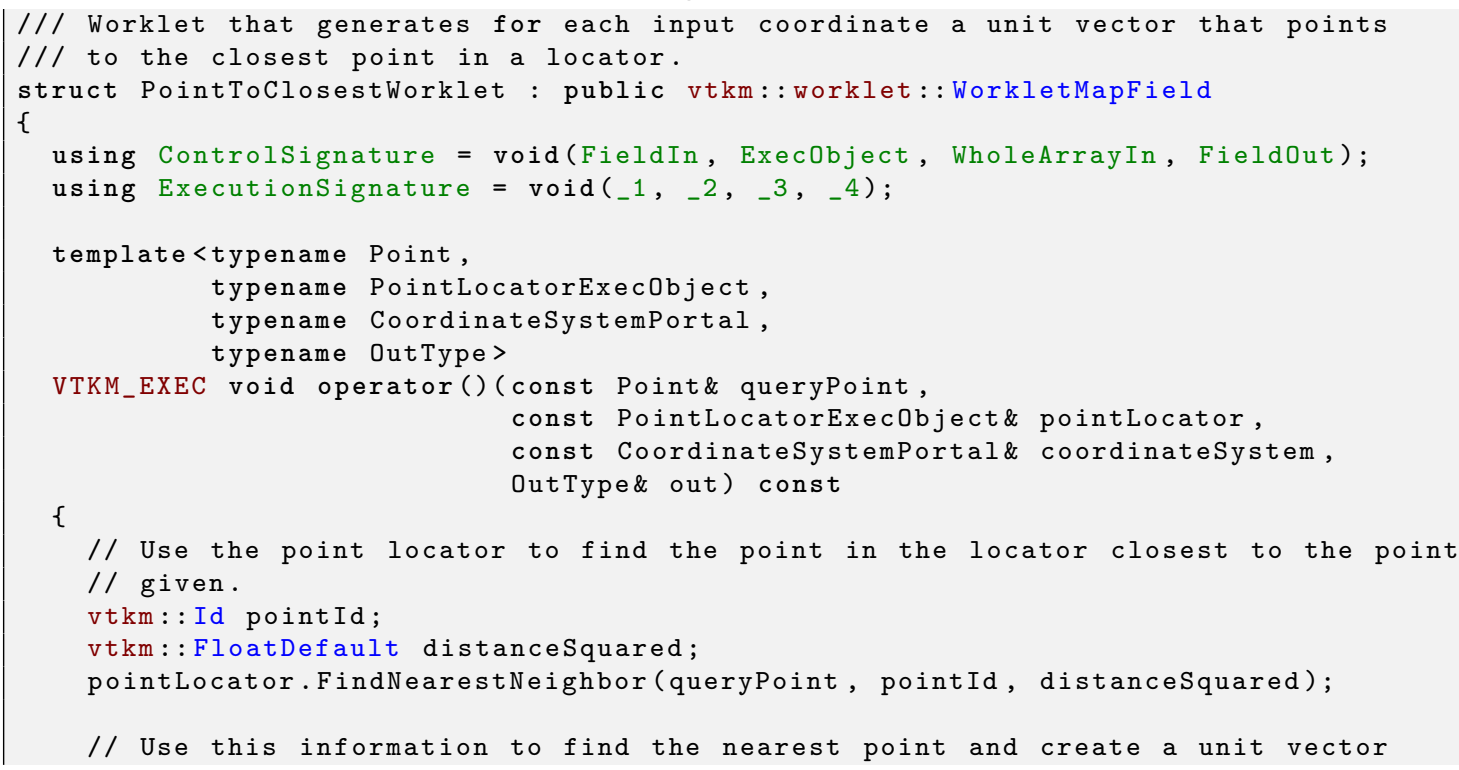




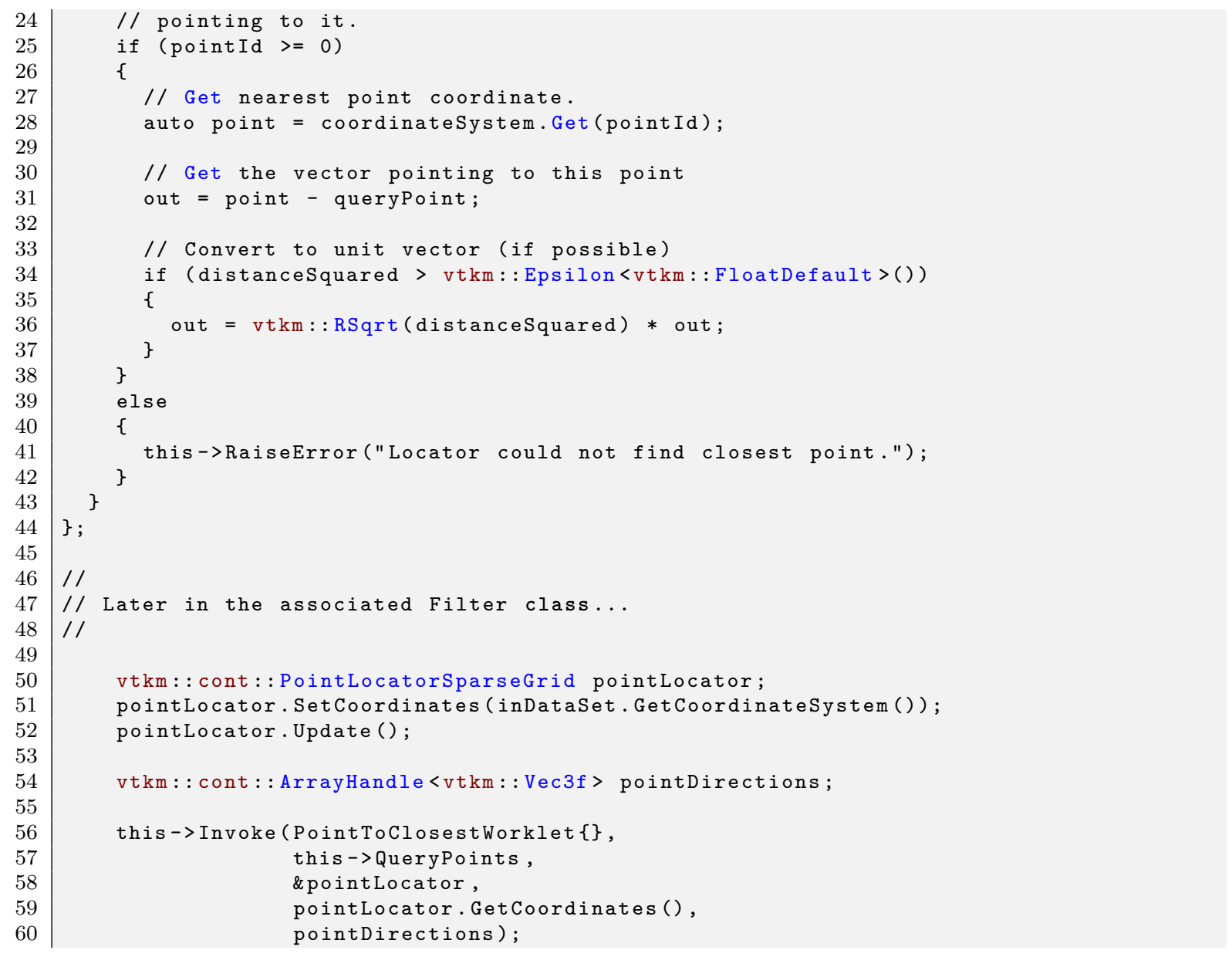




\section{WORKLET INPUT OUTPUT SEMANTICS}

The default scheduling of a worklet provides a 1 to 1 mapping from the input domain to the output domain. For example, a vtkm: :worklet: :WorkletMapField gets run once for every item of the input array and produces one item for the output array. Likewise, vtkm: :worklet::WorkletVisitCellsWithPoints gets run once for every cell in the input topology and produces one associated item for the output field.

However, there are many operations that do not fall well into this 1 to 1 mapping procedure. The operation might need to pass over elements that produce no value or the operation might need to produce multiple values for a single input element. Such non 1 to 1 mappings can be achieved by defining a scatter or a mask (or both) on a worklet.

\subsection{Scatter}

A scatter allows you to specify for each input element how many output elements should be created. For example, a scatter allows you to create two output elements for every input element. A scatter could also allow you to drop every other input element from the output. The following types of scatter are provided by VTK-m.

vtkm: : worklet: :ScatterIdentity Provides a basic 1 to 1 mapping from input to output. This is the default scatter used if none is specified.

vtkm: : worklet: :ScatterUniform Provides a 1 to many mapping from input to output with the same number of outputs for each input. A template parameter provides the number of output values to produce per input.

vtkm: : worklet: :ScatterCounting Provides a 1 to any mapping from input to output with different numbers of outputs for each input. The constructor takes an ArrayHandle that is the same size as the input containing the count of output values to produce for each input. Values can be zero, in which case that input will be skipped.

vtkm: : worklet: :ScatterPermutation Reorders the indices. The constructor takes a permutation ArrayHandle that is sized to the number of output values and maps output indices to input indices. For example, if index $i$ of the permutation array contains $j$, then the worklet invocation for output $i$ will get the $j^{\text {th }}$ input values. The reordering does not have to be 1 to 1 . Any input not referenced by the permutation array will be dropped, and any input referenced by the permutation array multiple times will be duplicated. However, unlike ScatterCounting VisitIndex is always 0 even if an input value happens to be duplicated. 
Did you know?

3 Scatters are often used to create multiple outputs for a single input, but they can also be used to remove inputs from the output. In particular, if you provide a count of 0 in a ScatterCounting count array, no outputs will be created for the associated input. To simply mask out some elements from the input, provide ScatterCounting with a stencil array of 0 's and 1's with a 0 for every element you want to remove and a 1 for every element you want to pass. You can also mix 0's with counts larger than 1 to drop some elements and add multiple results for other elements. ScatterPermutation can similarly be used to remove input values by leaving them out of the permutation.

To define a scatter procedure, the worklet must provide a type definition named ScatterType. The ScatterType must be set to one of the aforementioned Scatter* classes. It is common, but optional, to also provide a static method named MakeScatter that generates an appropriate scatter object for the worklet if you cannot use the default constructor for the scatter. This static method can be used by users of the worklet to set up the scatter for the Invoker.

Example 31.1: Declaration of a scatter type in a worklet.

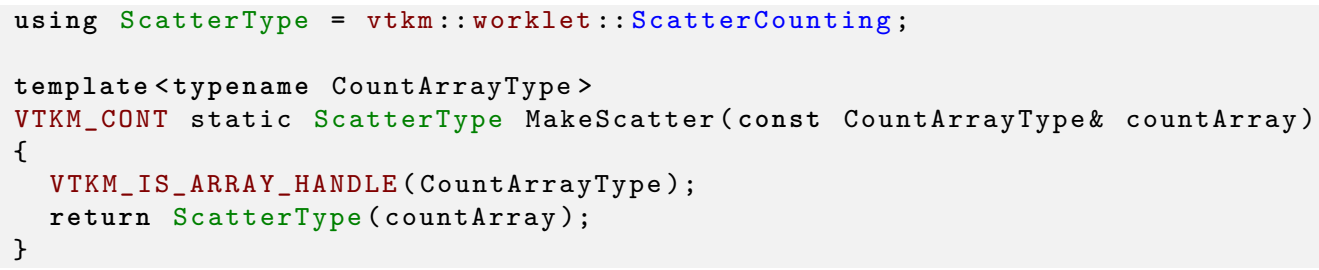

When using a scatter that produces multiple outputs for a single input, the worklet is invoked multiple times with the same input values. In such an event the worklet operator needs to distinguish these calls to produce the correct associated output. This is done by declaring one of the ExecutionSignature arguments as VisitIndex. This tag will pass a vtkm: : IdComponent to the worklet that identifies which invocation is being called.

It is also the case that the when a scatter can produce multiple outputs for some input that the index of the input element is not the same as the WorkIndex. If the index to the input element is needed, you can use the InputIndex tag in the ExecutionSignature. It is also good practice to use the OutputIndex tag if the index to the output element is needed.

Most Scatter objects have a state, and this state must be passed to the vtkm: : cont: : Invoker when invoking the worklet. In this case, the Scatter object should be passed as the second object to the call to the Invoker (after the worklet object).

Example 31.2: Invoking with a custom scatter.

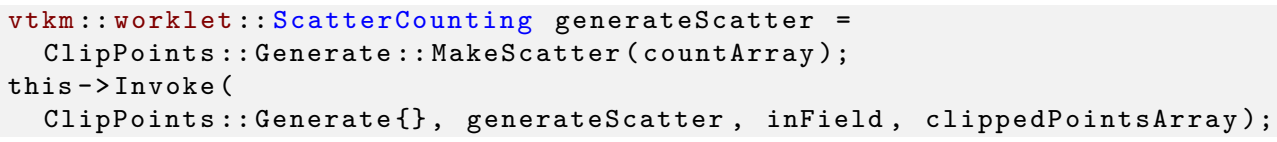

A scatter object does not have to be tied to a single worklet/invoker instance. In some cases it makes sense to use the same scatter object multiple times for worklets that have the same input to output mapping. Although this is not common, it can save time by reusing the set up computations of ScatterCounting. 
To demonstrate using scatters with worklets, we provide some contrived but illustrative examples. The first example is a worklet that takes a pair of input arrays and interleaves them so that the first, third, fifth, and so on entries come from the first array and the second, fourth, sixth, and so on entries come from the second array. We achieve this by using a vtkm: : worklet: : ScatterUniform of size 2 and using the VisitIndex to determine from which array to pull a value.

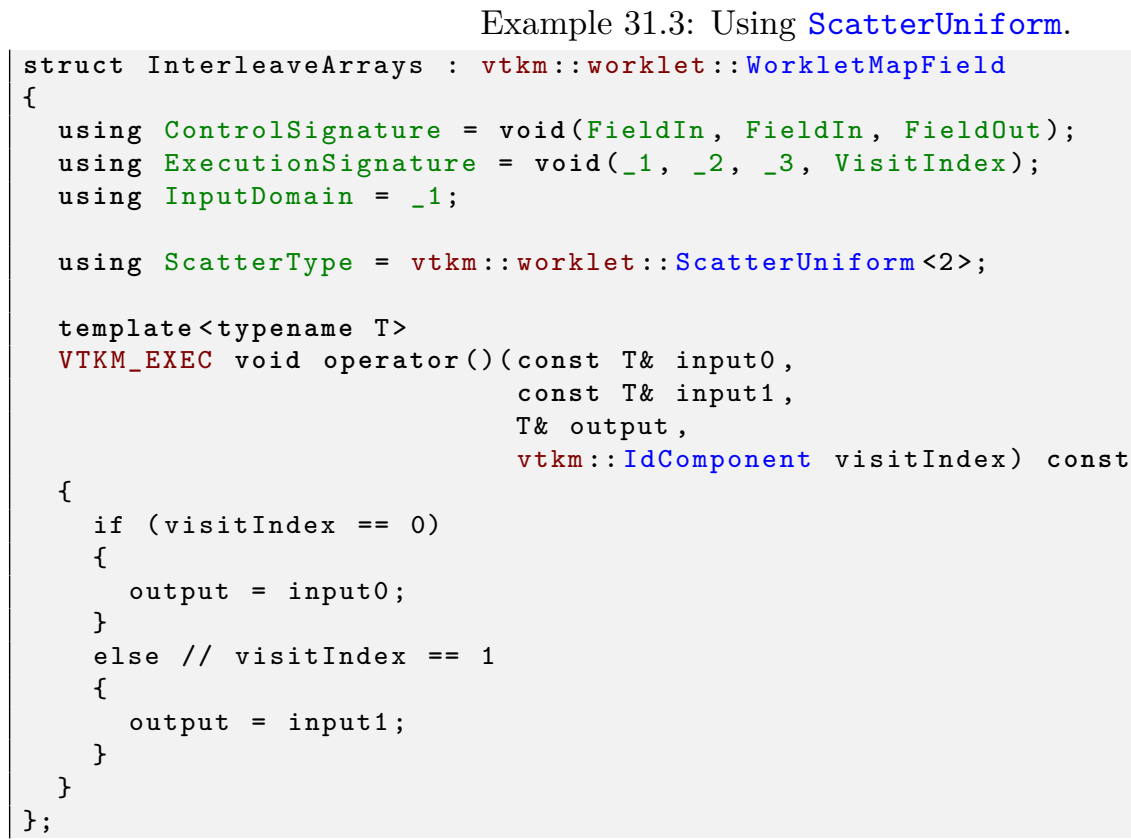

Example 31.3: Using ScatterUniform.

The second example takes a collection of point coordinates and clips them by an axis-aligned bounding box. It does this using a vtkm: :worklet: :ScatterCounting with an array containing 0 for all points outside the bounds and 1 for all points inside the bounds. As is typical with this type of operation, we use another worklet with a default identity scatter to build the count array.

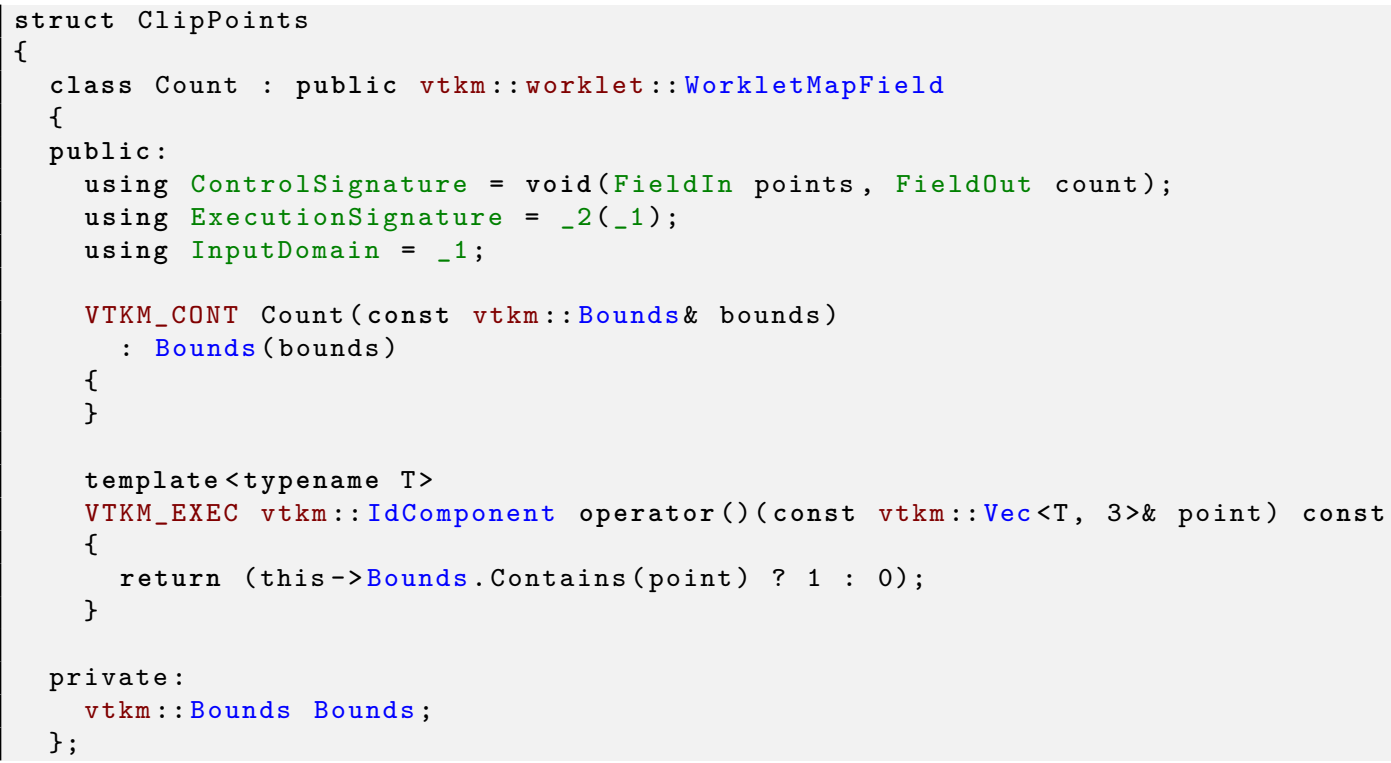




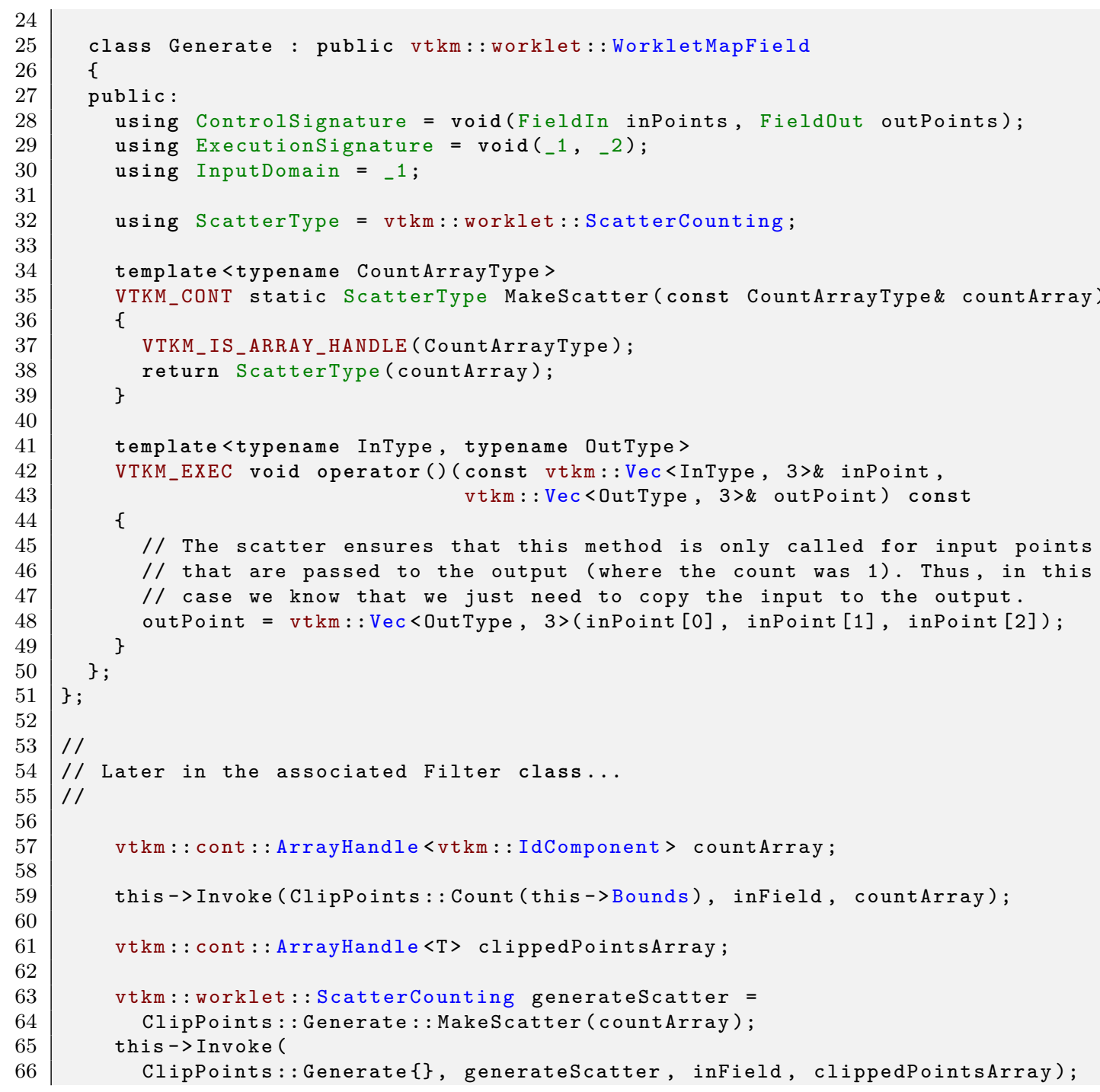

The third example takes an input array and reverses the ordering. It does this using a vtkm: :worklet: :ScatterPermutation with a permutation array generated from a vtkm: : cont: : ArrayHandleCounting counting down from the input array size to 0 .

Example 31.5: Using ScatterPermutation.

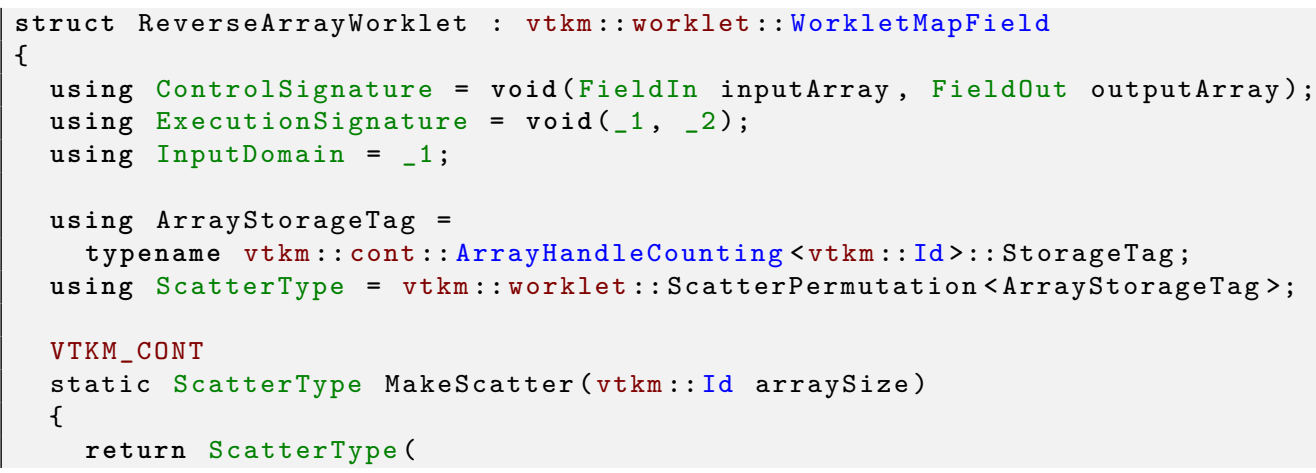




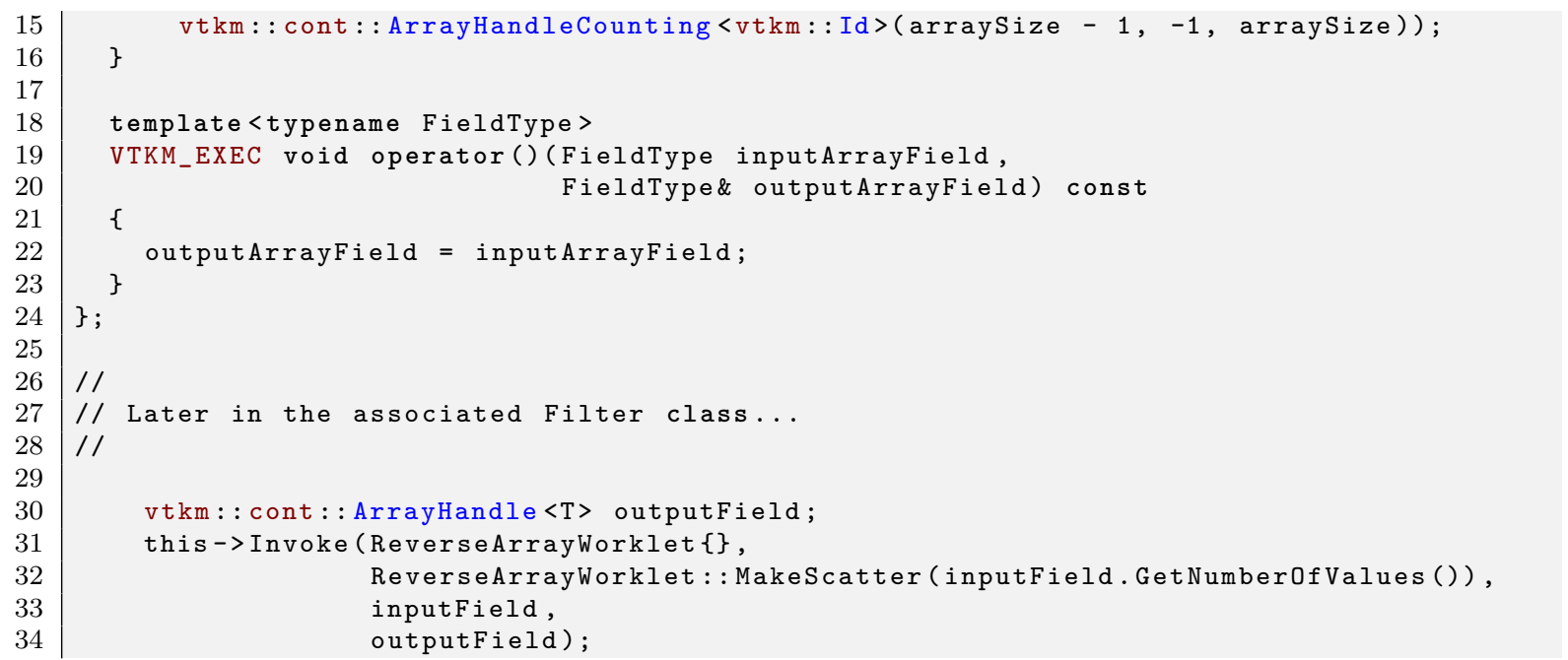

\section{Did you know?}

A vtkm: : worklet: :ScatterPermutation can have less memory usage than a vtkm: :worklet: : ScatterCounting when zeroing indices. By default, a vtkm: :worklet::ScatterPermutation will omit all fields that are not specified in the input permutation, whereas vtkm: :worklet: :ScatterCounting requires 0 values. If mapping an input to an output that omits fields, consider using a vtkm: :worklet::ScatterPermutation to save memory.

\section{Common Errors}

A permutation array provided to vtkm: :worklet: :ScatterPermutation can be filled with arbitrary id values. If an input permutation id exceeds the bounds of an input provided to a worklet, an out of bounds error will occur in the worklet functor. To prevent this kind of error, you should ensure that ids in the vtkm: : worklet: :ScatterPermutation do not exceed the bounds of provided inputs. 



\section{GENERATING CELL SETS}

This chapter describes techniques for designing algorithms in VTK-m that generate cell sets to be inserted in a vtkm: : cont: :DataSet. Although Chapter 7 on data sets describes how to create a data set, including defining its set of cells, these are serial functions run in the control environment that are not designed for computing geometric structures. Rather, they are designed for specifying data sets built from existing data arrays, from inherently slow processes (such as file I/O), or for small test data. In this chapter we discuss how to write worklets that create new mesh topologies by writing data that can be incorporated into a vtkm: :cont: : CellSet.

This chapter is constructed as a set of patterns that are commonly employed to build cell sets. These techniques apply the worklet structures documented in Chapter 21. Although it is possible for these worklets to generate data of its own, the algorithms described here follow the more common use case of deriving one topology from another input data set. This chapter is not (and cannot be) completely comprehensive by covering every possible mechanism for building cell sets. Instead, we provide the basic and common patterns used in scientific visualization.

\subsection{Single Cell Type}

For our first example of algorithms that generate cell sets is one that creates a set of cells in which all the cells are of the same shape and have the same number of points. Our motivating example is an algorithm that will extract all the edges from a cell set. The resulting cell set will comprise a collection of line cells that represent the edges from the original cell set. Since all cell edges can be represented as lines with two endpoints, we know all the output cells will be of the same type. As we will see later in the example, we can use a vtkm: :cont: :CellsetSingleType to represent the data.

It is rare that an algorithm generating a cell set will generate exactly one output cell for each input cell. Thus, the first step in an algorithm generating a cell set is to count the number of cells each input item will create. In our motivating example, this is the the number of edges for each input cell.

Example 32.1: A simple worklet to count the number of edges on each cell.

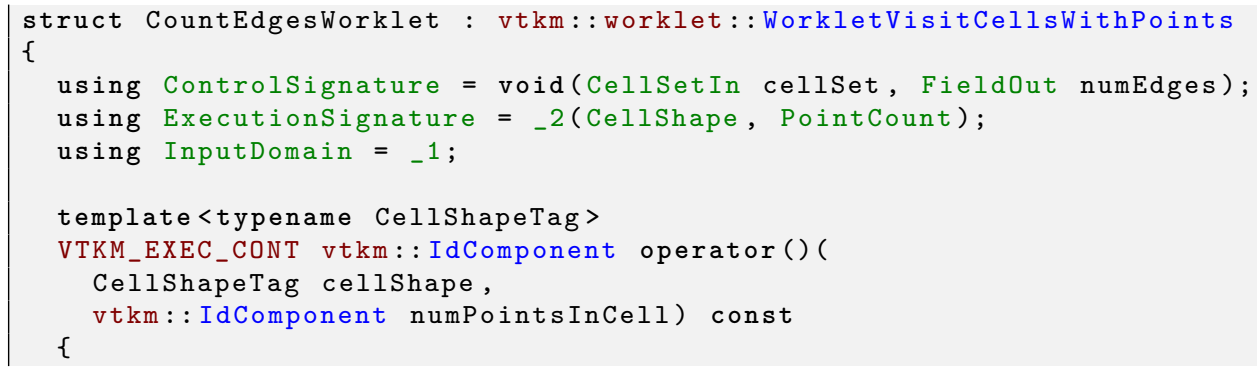




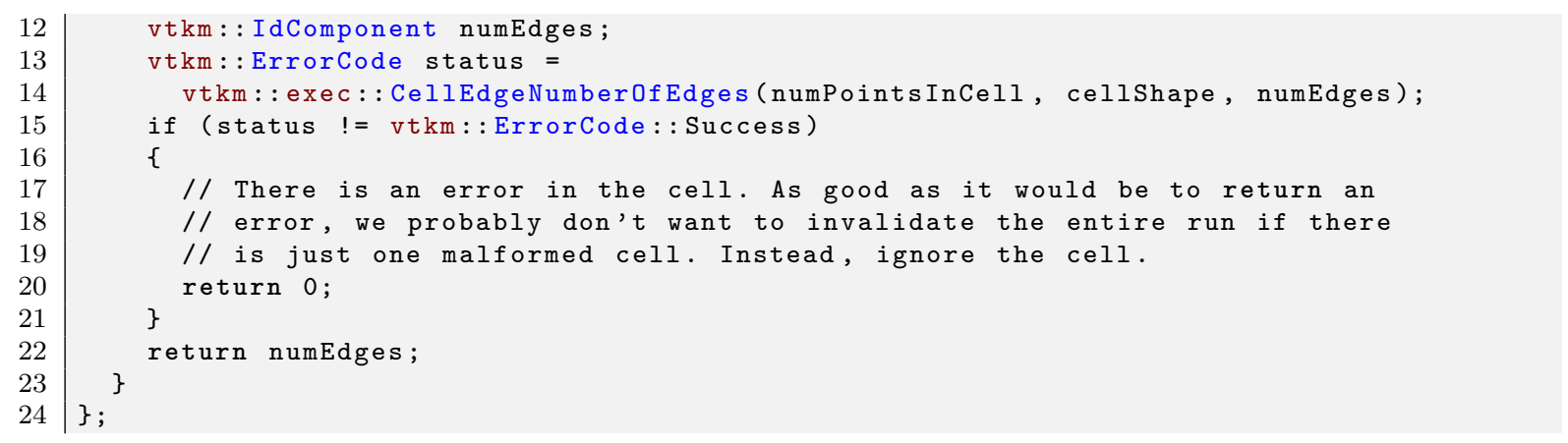

This count array generated in Example 32.1 can be used in a vtkm: : worklet: : ScatterCounting of a subsequent worklet that generates the output cells. (See Section 31.1 for information on using a scatter with a worklet.) We will see this momentarily.

\section{Did you know?}

If you happen to have an operation that you know will have the same count for every input cell, then you can skip the count step and use a vtkm: : worklet: : ScatterUniform instead of ScatterCount. Doing so will simplify the code and skip some computation. We cannot use ScatterUniform in this example because different cell shapes have different numbers of edges and therefore different counts. However, if we were theoretically to make an optimization for $3 D$ structured grids, we know that each cell is a hexahedron with 12 edges and could use a ScatterUniform<12> for that.

The second and final worklet we need to generate our wireframe cells is one that outputs the indices of an edge. The worklet parenthesis' operator takes information about the input cell (shape and point indices) and an index of which edge to output. The aforementioned ScatterCounting provides a VisitIndex that signals which edge to output. The worklet parenthesis operator returns the two indices for the line in, naturally enough, a vtkm: : Id2.

Example 32.2: A worklet to generate indices for line cells.

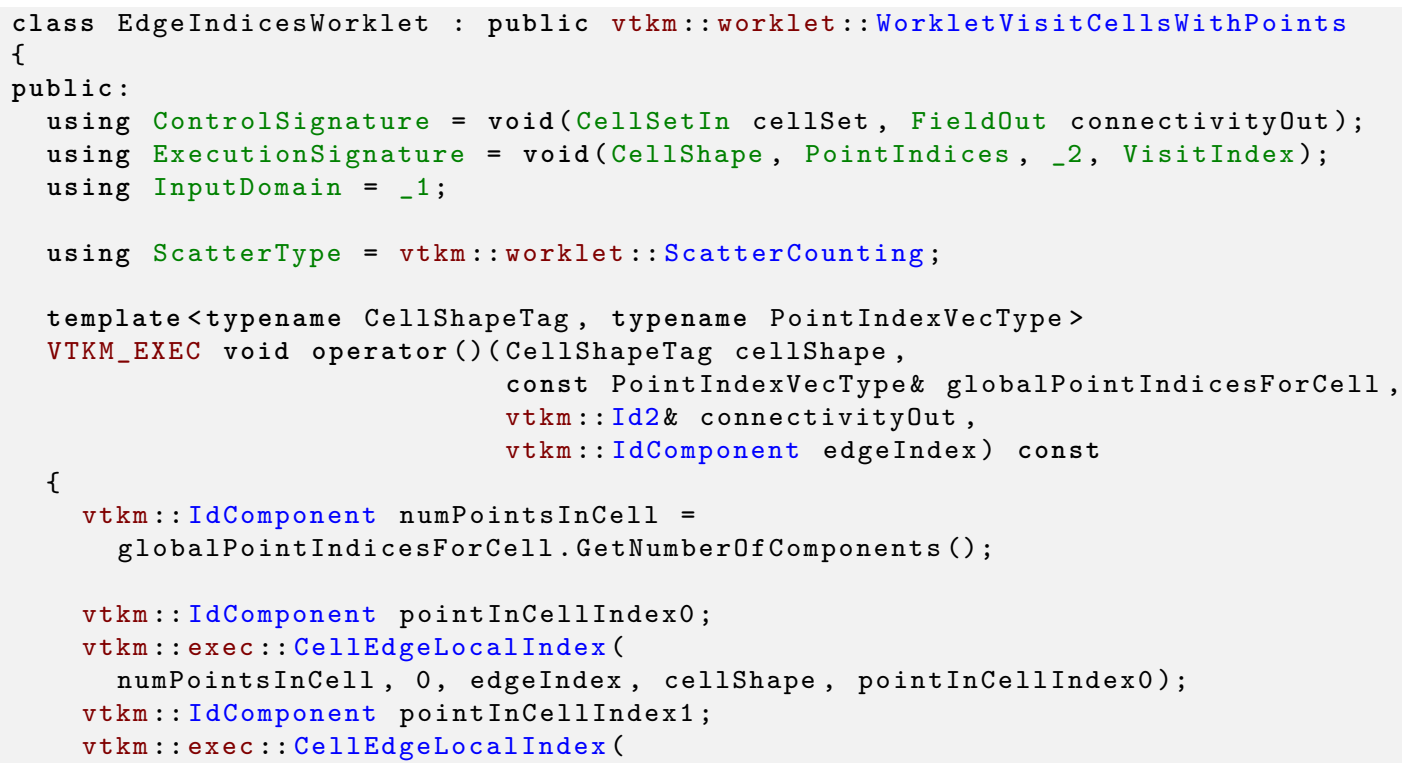




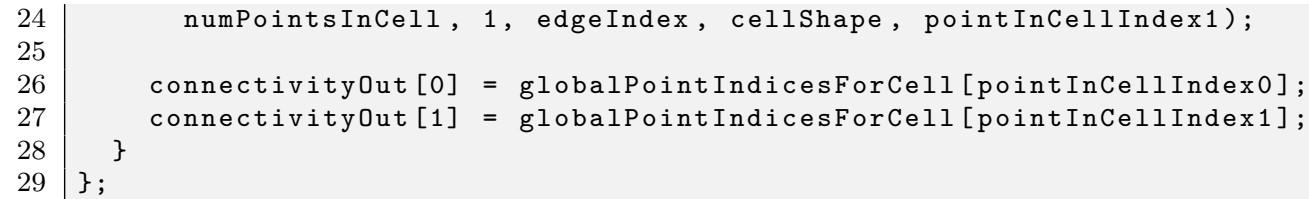

Our ultimate goal is to fill a vtkm: : cont: :CellSetSingleType object with the generated line cells. A CellSetSingleType requires 4 items: the number of points, the constant cell shape, the constant number of points in each cell, and an array of connection indices. The first 3 items are trivial. The number of points can be taken from the input cell set as they are the same. The cell shape and number of points are predetermined to be line and 2, respectively. The last item, the array of connection indices, is what we are creating with the worklet in Example 32.2.

However, there is a complication. The connectivity array for CellSetSingleType is expected to be a flat array of vtkm: :Id indices, not an array of Vec objects. We could jump through some hoops adjusting the ScatterCounting to allow the worklet to output only one index of one cell rather than all indices of one cell. But that would be overly complicated and inefficient.

A simpler approach is to use the vtkm: :cont: :ArrayHandleGroupVec fancy array handle (described in Section 26.13) to make a flat array of indices look like an array of Vec objects. The following example shows what the DoExecute method in the associated filter would look like. Note the use make_ArrayHandleGroupVec when calling Invoke on line 20 to make this conversion.

Example 32.3: Invoking worklets to extract edges from a cell set.

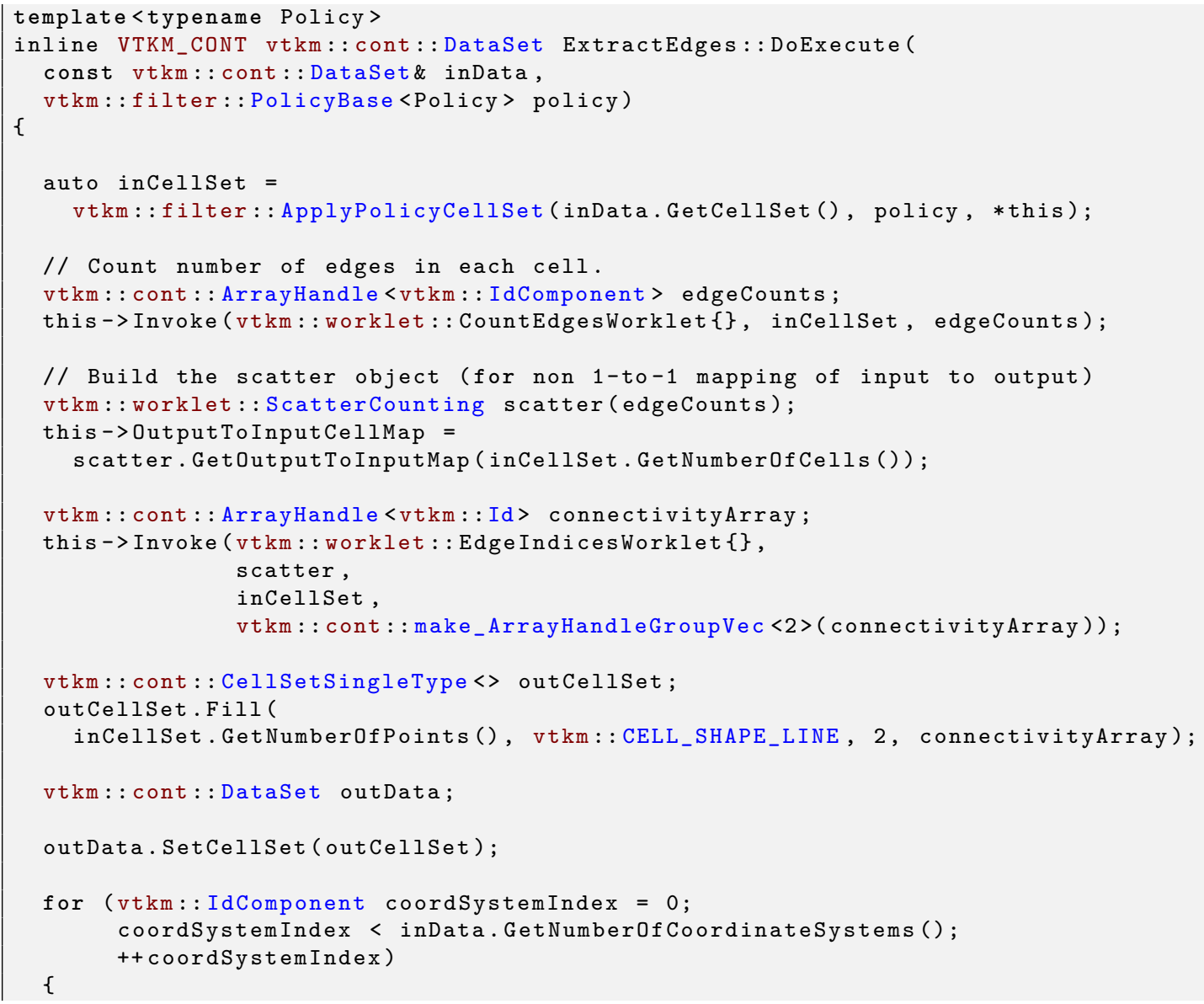




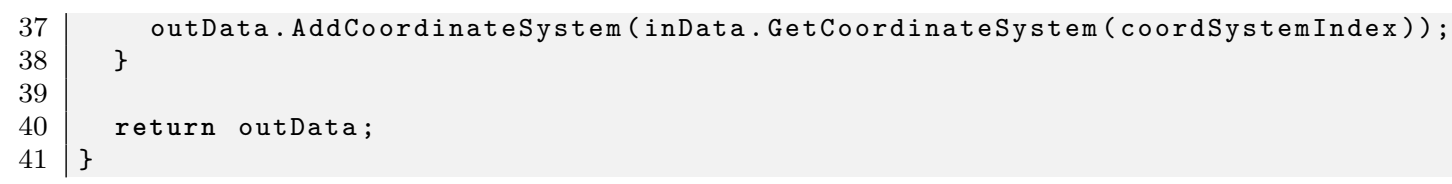

Another feature to note in Example 32.3 is that the method calls GetOutputToInputMap on the Scatter object it creates and squirrels the map array away for later use. The reason for this behavior is to implement mapping fields that are attached on the input cells to the indices of the output. In practice, DoExecute is called on DataSet objects to create new DataSet objects. The method in Example 32.3 creates a new CellSet, but we also need a method to transform the Fields on the data set. The saved OutputToInputCellMap array allows us to transform input fields to output fields.

The following example shows the implementation of DoMapField in the associated filter that takes this saved OutputToInputCellMap array and converts an array from an input cell field to an output cell field array. Note that we can do this by using the OutputToInputCellMap as an index array in a vtkm: : cont : : ArrayHandlePermutation.

Example 32.4: Converting cell fields using a simple permutation.

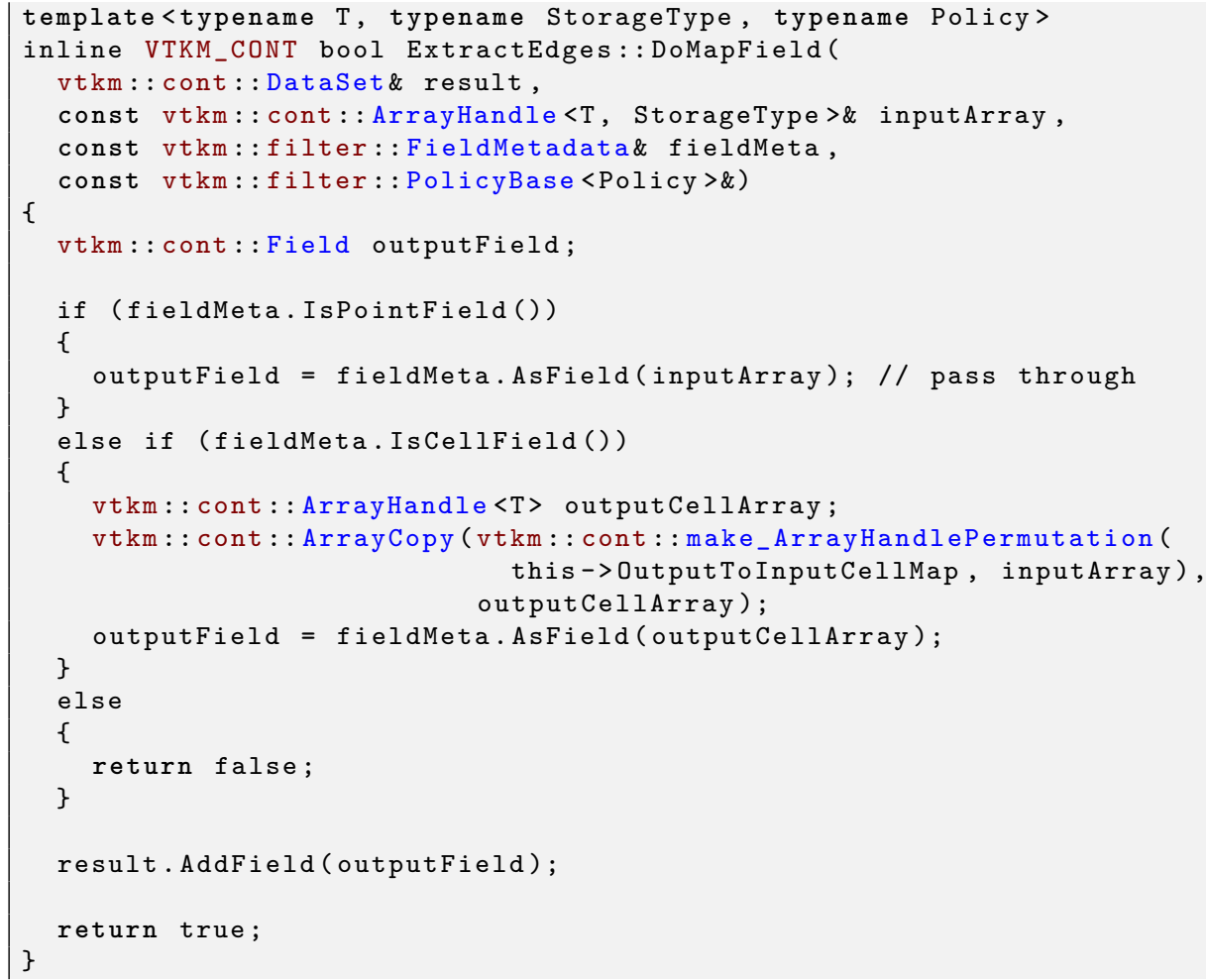

\subsection{Combining Like Elements}

Our motivating example in Section 32.1 created a cell set with a line element representing each edge in some input data set. However, on close inspection there is a problem with our algorithm: it is generating a lot of duplicate elements. The cells in a typical mesh are connected to each other. As such, they share edges with each other. That is, the edge of one cell is likely to also be part of one or more other cells. When multiple cells contain the same edge, the algorithm we created in Section 32.1 will create multiple overlapping lines, one for 
each cell using the edge, as demonstrated in Figure 32.1. What we really want is to have one line for every edge in the mesh rather than many overlapping lines.
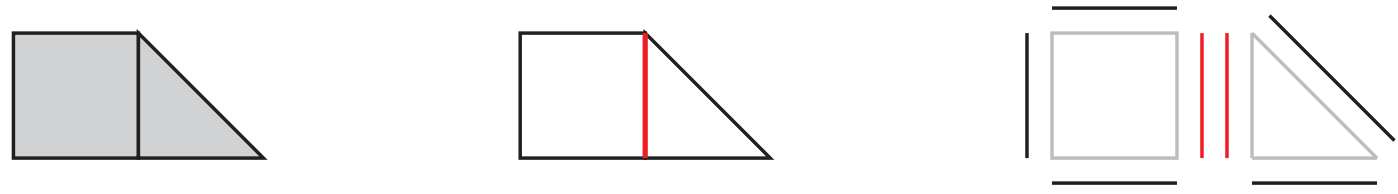

Figure 32.1: Duplicate lines from extracted edges. Consider the small mesh at the left comprising a square and a triangle. If we count the edges in this mesh, we would expect to get 6 . However, our naïve implementation in Section 32.1 generates 7 because the shared edge (highlighted in red in the wireframe in the middle) is duplicated. As seen in the exploded view at right, one line is created for the square and one for the triangle.

In this section we will re-implement the algorithm to generate a wireframe by creating a line for each edge, but this time we will merge duplicate edges together. Our first step is the same as before. We need to count the number of edges in each input cell and use those counts to create a vtkm: : worklet: : ScatterCounting for subsequent worklets. Counting the edges is a simple worklet.

Example 32.5: A simple worklet to count the number of edges on each cell.

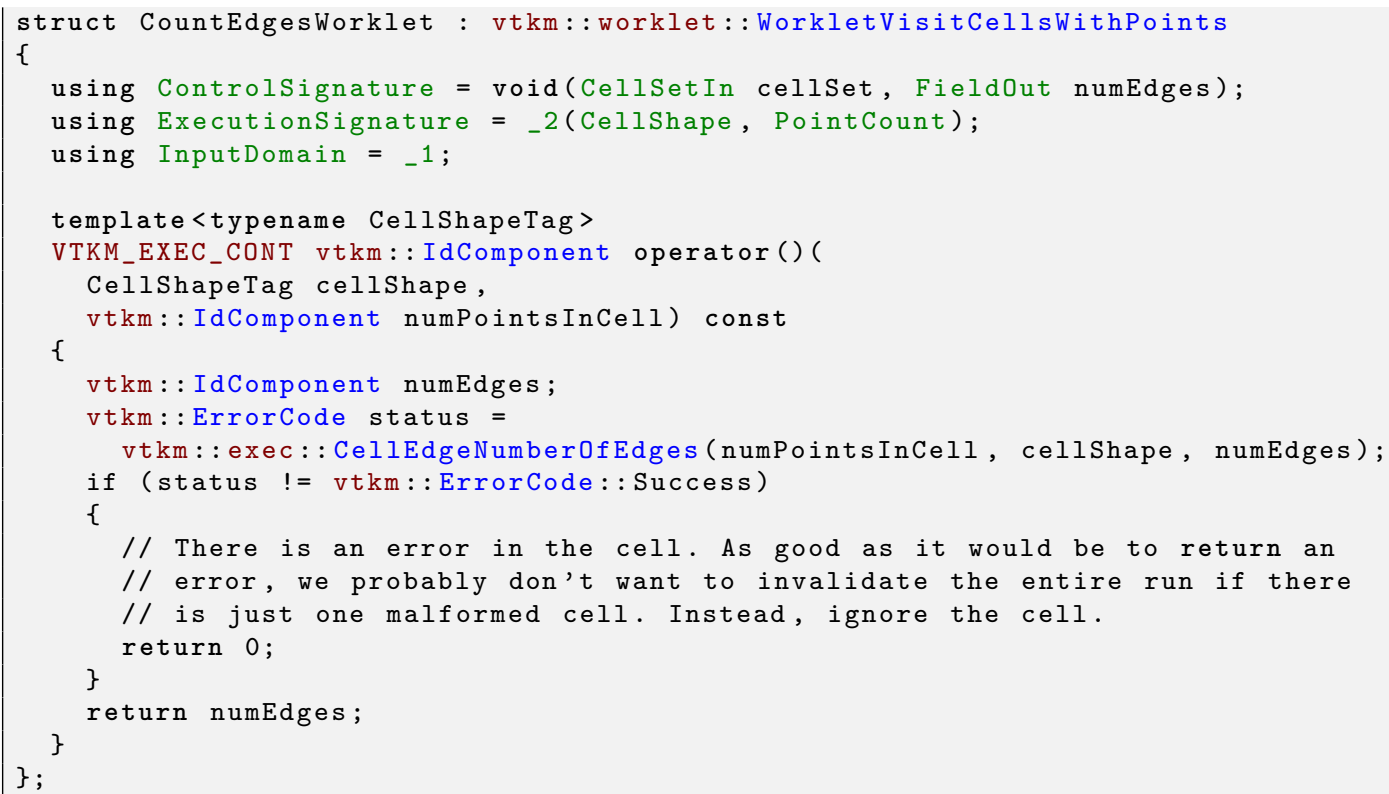

In our previous version, we used the count to directly write out the lines. However, before we do that, we want to identify all the unique edges and identify which cells share this edge. This grouping is exactly the function that the reduce by key worklet type (described in Section 21.4) is designed to accomplish. The principal idea is to write a "key" that uniquely identifies the edge. The reduce by key worklet can then group the edges by the key and allow you to combine the data for the edge.

Thus, our goal of finding duplicate edges hinges on producing a key where two keys are identical if and only if the edges are the same. One straightforward key is to use the coordinates in 3D space by, say, computing the midpoint of the edge. The main problem with using this point coordinates approach is that a computer can hold a point coordinate only with floating point numbers of limited precision. Computer floating point computations are notorious for providing slightly different answers when the results should be the same. For example, if an edge has endpoints at $p_{1}$ and $p_{2}$ and two different cells compute the midpoint as $\left(p_{1}+p_{2}\right) / 2$ and $\left(p_{2}+p_{1}\right) / 2$, 
respectively, the answer is likely to be slightly different. When this happens, the keys will not be the same and we will still produce 2 edges in the output.

Fortunately, there is a better choice for keys based on the observation that in the original cell set each edge is specified by endpoints that each have unique indices. We can combine these 2 point indices to form a "canonical" descriptor of an edge (correcting for order). ${ }^{1}$ VTK-m comes with a helper function, vtkm: : exec: :CellEdgeCanonicalId, defined in vtkm/exec/CellEdge.h, to produce these unique edge keys as vtkm: : Id2 s. Our second worklet produces these canonical edge identifiers.

Example 32.6: Worklet generating canonical edge identifiers.

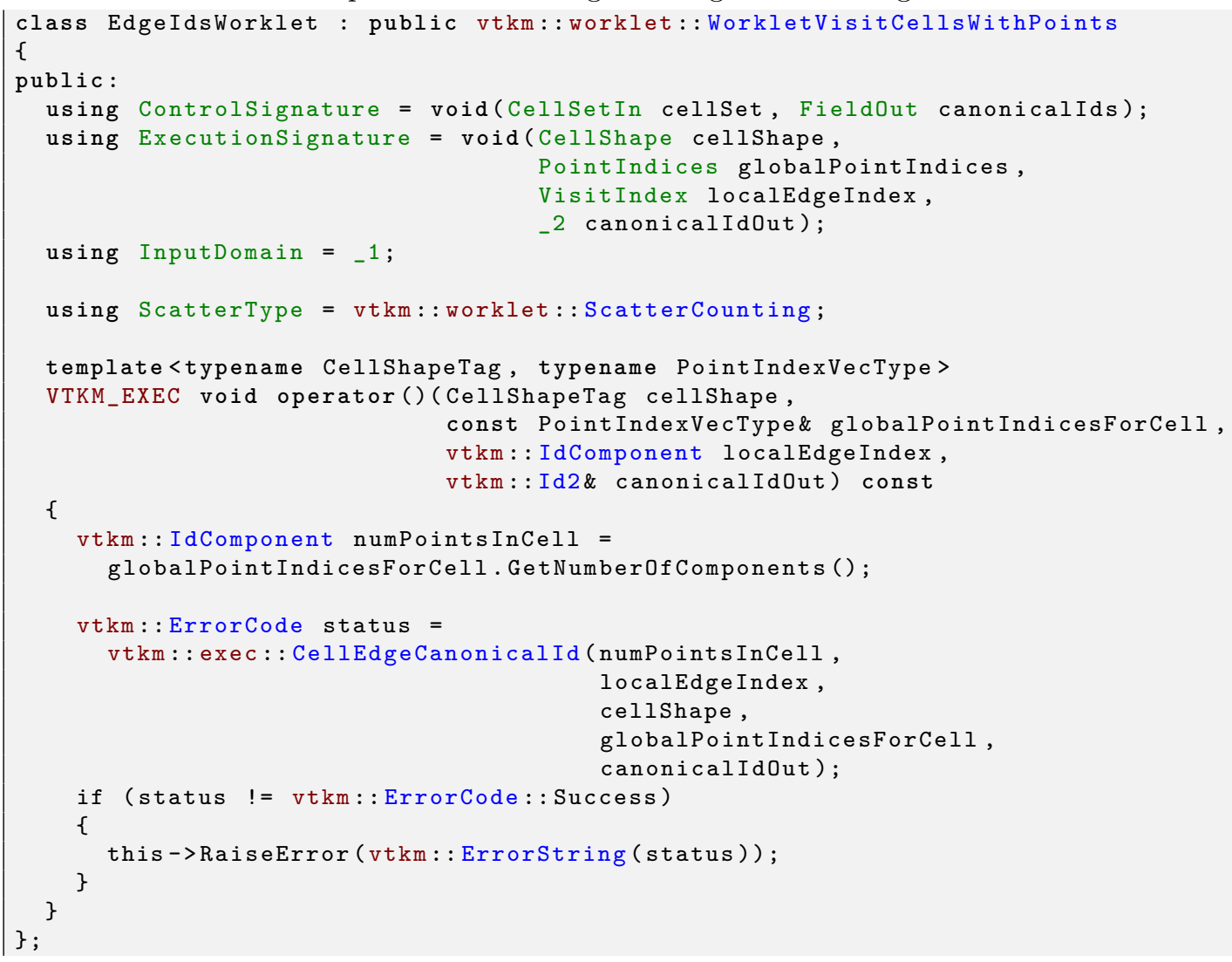

Our third and final worklet generates the line cells by outputting the indices of each edge. As hinted at earlier, this worklet is a reduce by key worklet (inheriting from vtkm: : worklet::WorkletReduceByKey). When the worklet is invoked, VTK-m will collect the unique keys and call the worklet once for each unique edge. Because there is no longer a consistent mapping from the generated lines to the elements of the input cell set, we need pairs of indices identifying the cells/edges from which the edge information comes. We use these indices along with a connectivity structure produced by a WholeCellSetIn to find the information about the edge. As shown later, these indices of cells and edges can be extracted from the ScatterCounting used to execute the worklet back in Example 32.6.

As we did in Section 32.1, this worklet writes out the edge information in a vtkm: : Id2 (which in some following code will be created with an ArrayHandleGroupVec).

Example 32.7: A worklet to generate indices for line cells from combined edges.

\footnotetext{
${ }^{1}$ Using indices to find common mesh elements is described by Miller et al. in "Finely-Threaded History-Based Topology Computation" (in Eurographics Symposium on Parallel Graphics and Visualization, June 2014).
} 


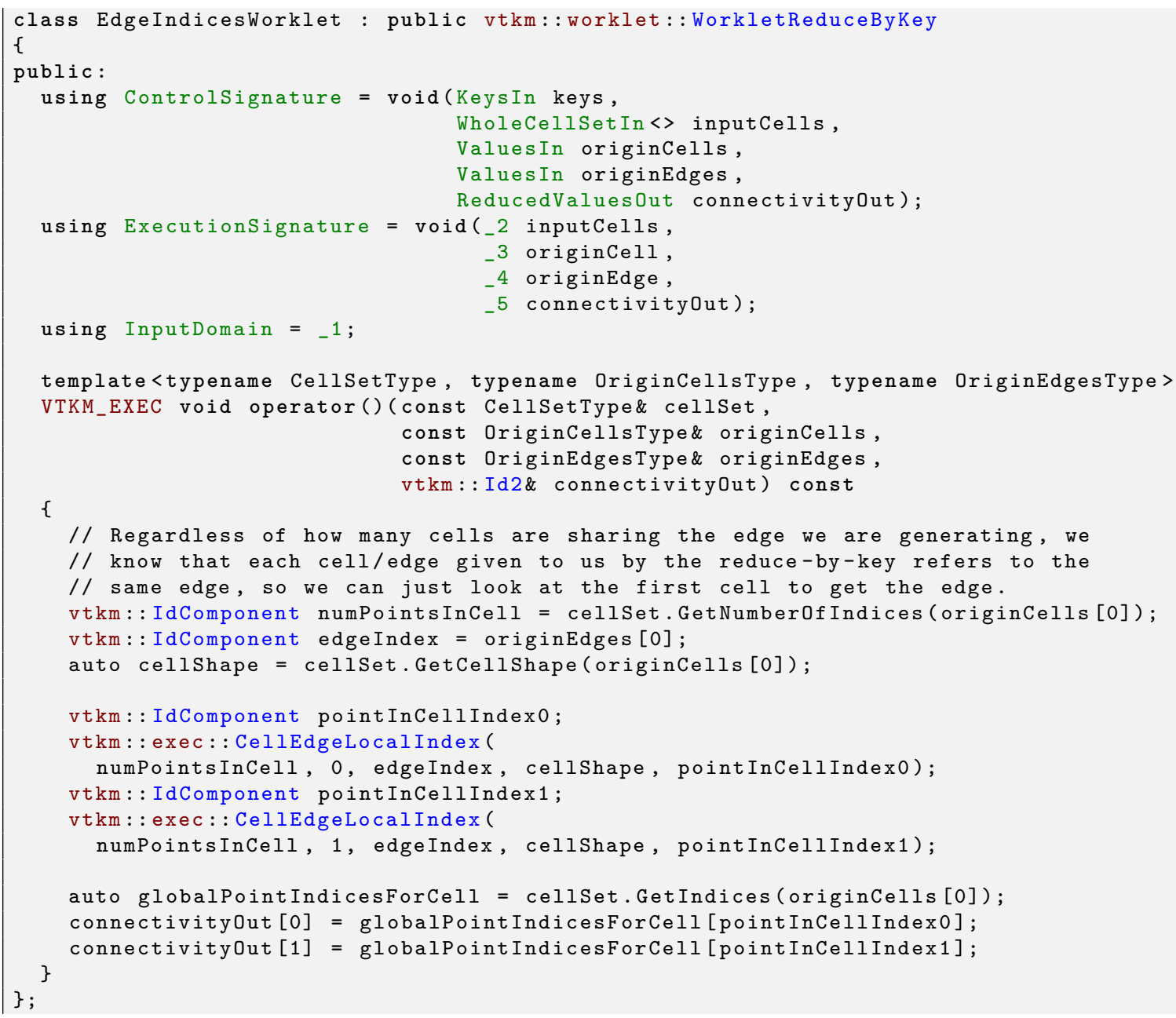

3 It so happens that the vtkm::Id2 s generated by CellEdgeCanonicalId contain the point indices of the two endpoints, which is enough information to create the edge. Thus, in this example it would be possible to forgo the steps of looking up indices through the cell set. That said, this is more often not the case, so for the purposes of this example we show how to construct cells without depending on the structure of the keys.

With these 3 worklets, it is now possible to generate all the information we need to fill a vtkm: :cont: :CellSetSingleType object. A CellSetSingleType requires 4 items: the number of points, the constant cell shape, the constant number of points in each cell, and an array of connection indices. The first 3 items are trivial. The number of points can be taken from the input cell set as they are the same. The cell shape and number of points are predetermined to be line and 2, respectively.

The last item, the array of connection indices, is what we are creating with the worklet in Example 32.7. The connectivity array for CellSetSingleType is expected to be a flat array of vtkm: : Id indices, but the worklet needs to provide groups of indices for each cell (in this case as a Vec object). To reconcile what the worklet 
provides and what the connectivity array must look like, we use the vtkm: : cont: : ArrayHandleGroupVec fancy array handle (described in Section 26.13) to make a flat array of indices look like an array of Vec objects. The following example shows what the DoExecute method in the associated filter would look like. Note the use of make_ArrayHandleGroupVec when calling Invoke on line 30 to make this conversion.

Example 32.8: Invoking worklets to extract unique edges from a cell set.

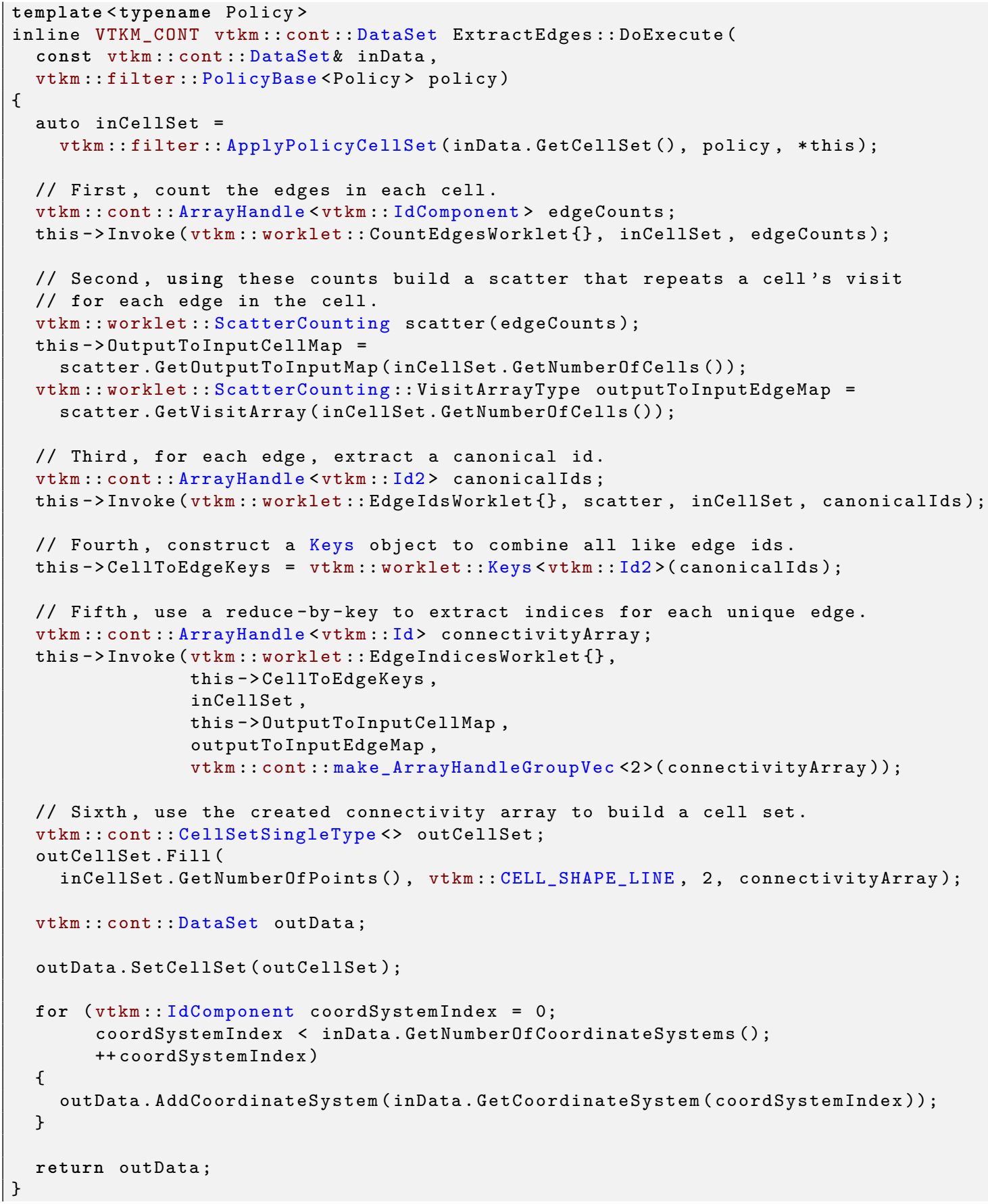

Another feature to note in Example 32.8 is that the method calls GetOutputToInputMap on the Scatter object it creates and squirrels it away for later use. It also saves the vtkm: : worklet: :Keys object created for later use. 
The reason for this behavior is to implement mapping fields that are attached on the input cells to the indices of the output. In practice, DoExecute is called on DataSet objects to create new DataSet objects. The method in Example 32.8 creates a new Cellset, but we also need a method to transform the Fields on the data set. The saved OutputToInputCellMap array and Keys object allow us to transform input fields to output fields.

The following example shows the implementation of DoMapField in the associated filter that takes these saved objects and converts an array from an input cell field to an output cell field array. Because in general there are several cells that contribute to each edge/line in the output, we need a method to combine all these cell values to one. The most appropriate combination is likely an average of all the values. Because this is a common operation, VTK-m provides the vtkm: :worklet: :AverageByKey class to help perform exactly this operation. AverageByKey provides a Run method that takes a Keys object, an array of in values, and a device adapter tag and produces and array of values averaged by key.

Example 32.9: Converting cell fields that average collected values.

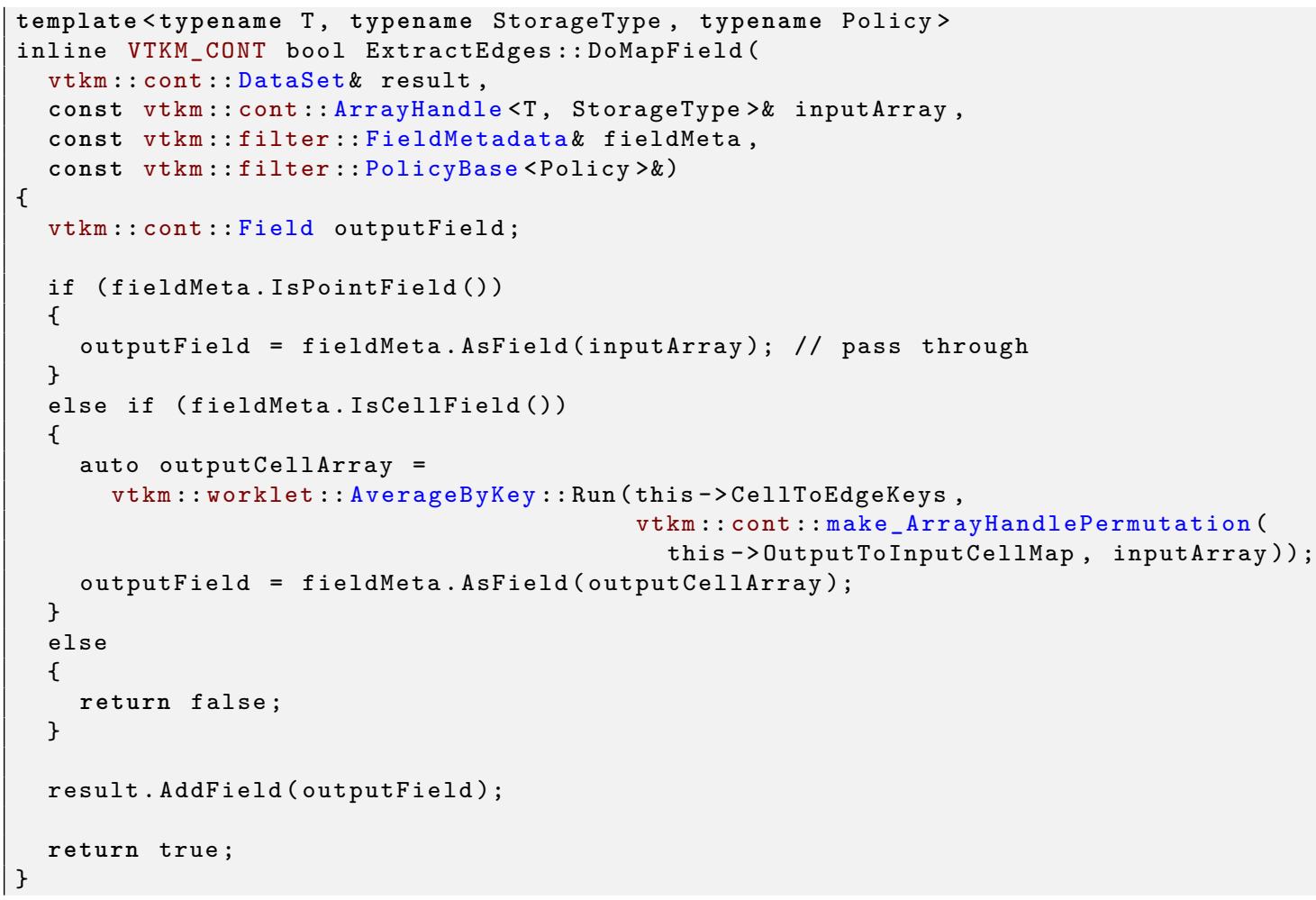

\subsection{Faster Combining Like Elements with Hashes}

In the previous two sections we constructed worklets that took a cell set and created a new set of cells that represented the edges of the original cell set, which can provide a wireframe of the mesh. In Section 32.1 we provided a pair of worklets that generate one line per edge per cell. In Section 32.2 we improved on this behavior by using a reduce by key worklet to find and merge shared edges.

If we were to time all the operations run in the later implementation to generate the wireframe (i.e. the operations in Example 32.8), we would find that the vast majority of the time is not spent in the actual worklets. Rather, the majority of the time is spent in collecting the like keys, which happens in the constructor of the vtkm: :worklet: :Keys object. Internally, keys are collected by sorting them. The most fruitful way to improve the performance of this algorithm is to improve the sorting behavior. 
The details of how the sort works is dependent on the inner workings of the device adapter. It turns out that the performance of the sort of the keys is highly dependent on the data type of the keys. For example, sorting numbers stored in a 32-bit integer is often much faster than sorting groups of 2 or 364 -bit integer. This is particularly true when the sort is capable of performing a radix-based sort.

An easy way to convert collections of indices like those returned from vtkm: :exec: :CellEdgeCanonicalId to a 32-bit integer is to use a hash function. To facilitate the creation of hash values, VTK-m comes with a simple vtkm: : Hash function (in the vtkm/Hash.h header file). Hash takes a Vec or Vec-like object of integers and returns a value of type vtkm: :HashType (an alias for a 32-bit integer). This hash function uses the FNV-1a algorithm that is designed to create hash values that are quasi-random but deterministic. This means that hash values of two different identifiers are unlikely to be the same.

That said, hash collisions can happen and become increasingly likely on larger data sets. Therefore, if we wish to use hash values, we also have to add conditions that manage collisions when they happen. Resolving hash value collisions adds overhead, but the time saved in faster sorting of hash values generally outweighs the overhead added by resolving collisions. ${ }^{2}$ In this section we will improve on the implementation given in Section 32.2 by using hash values for keys and resolving for collisions.

As always, our first step is to count the number of edges in each input cell. These counts are used to create a vtkm: : worklet: : ScatterCounting for subsequent worklets.

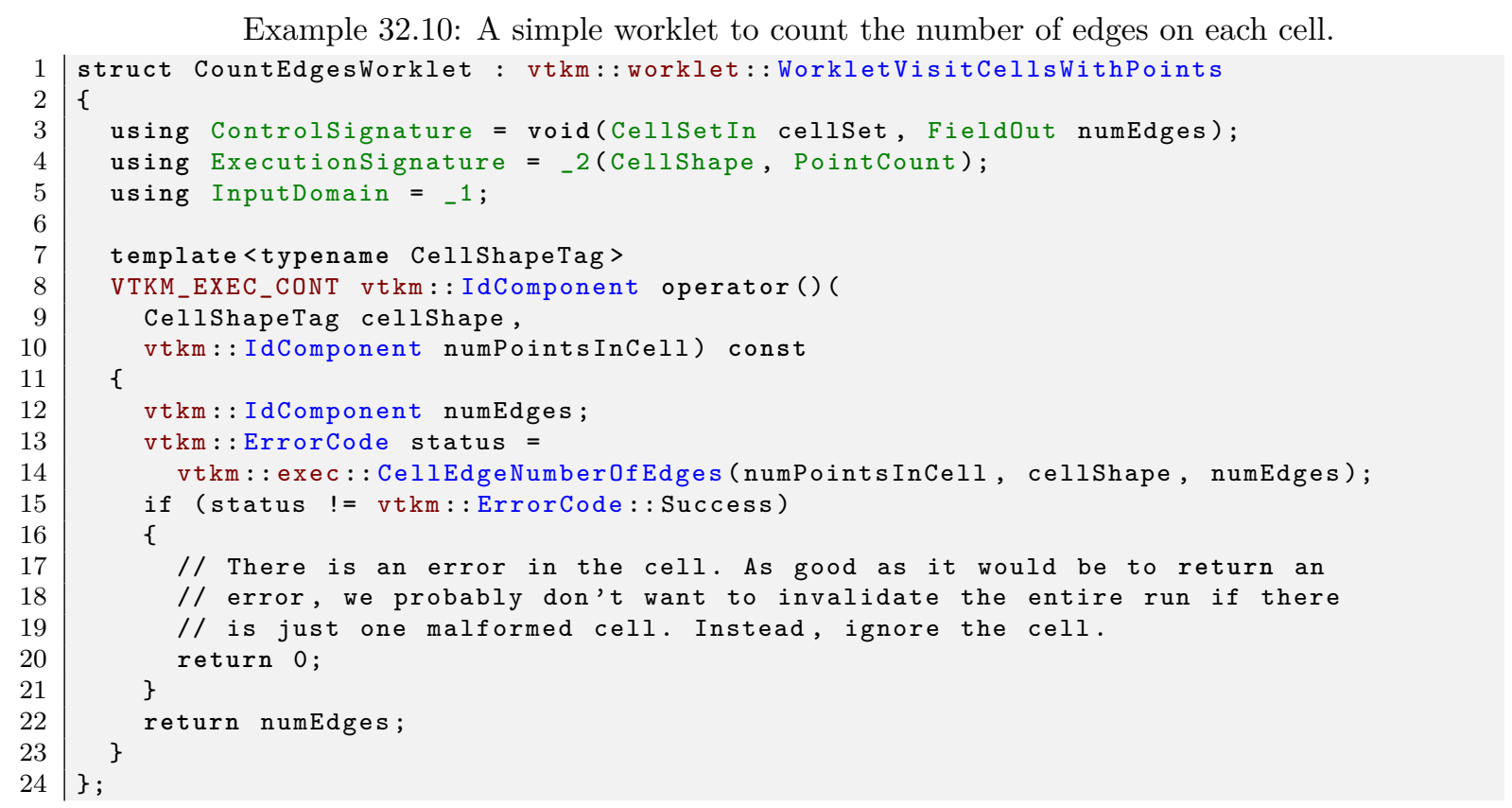

Our next step is to generate keys that can be used to find like elements. As before, we will use the vtkm: :exec: :CellEdgeCanonicalId function to create a unique representation for each edge. However, rather than directly use the value from CellEdgeCanonicalId, which is a vtkm: :Id2, we will instead use that to generate a hash value.

Example 32.11: Worklet generating hash values.

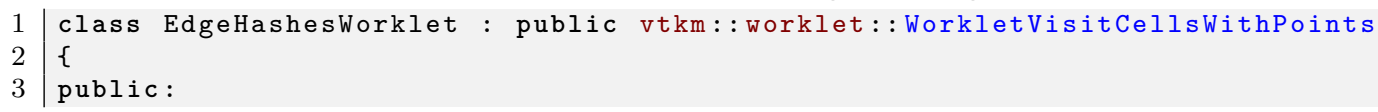

\footnotetext{
${ }^{2} \mathrm{~A}$ comparison of the time required for completely unique keys and hash keys with collisions is studied by Lessley, et al. in "Techniques for Data-Parallel Searching for Duplicate Elements" (in IEEE Symposium on Large Data Analysis and Visualization, October 2017).
} 


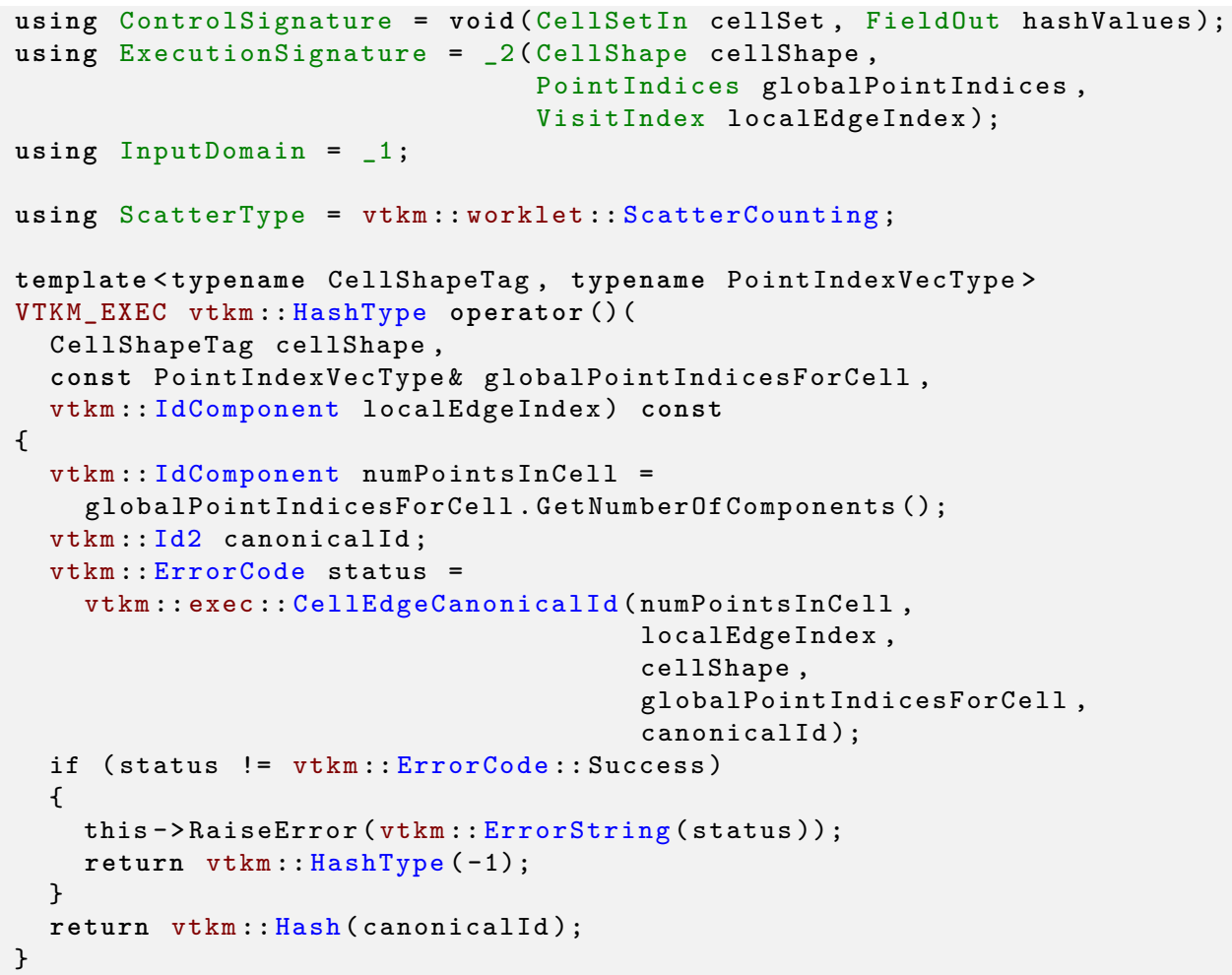

The hash values generated by the worklet in Example 32.11 will be the same for two identical edges. However, it is no longer guaranteed that two distinct edges will have different keys, and collisions of this nature become increasingly common for larger cell sets. Thus, our next step is to resolve any such collisions.

The following example provides a worklet that goes through each group of edges associated with the same hash value (using a reduce by key worklet). It identifies which edges are actually the same as which other edges, marks a local identifier for each unique edge group, and returns the number of unique edges associated with the hash value.

Example 32.12: Worklet to resolve hash collisions occurring on edge identifiers.

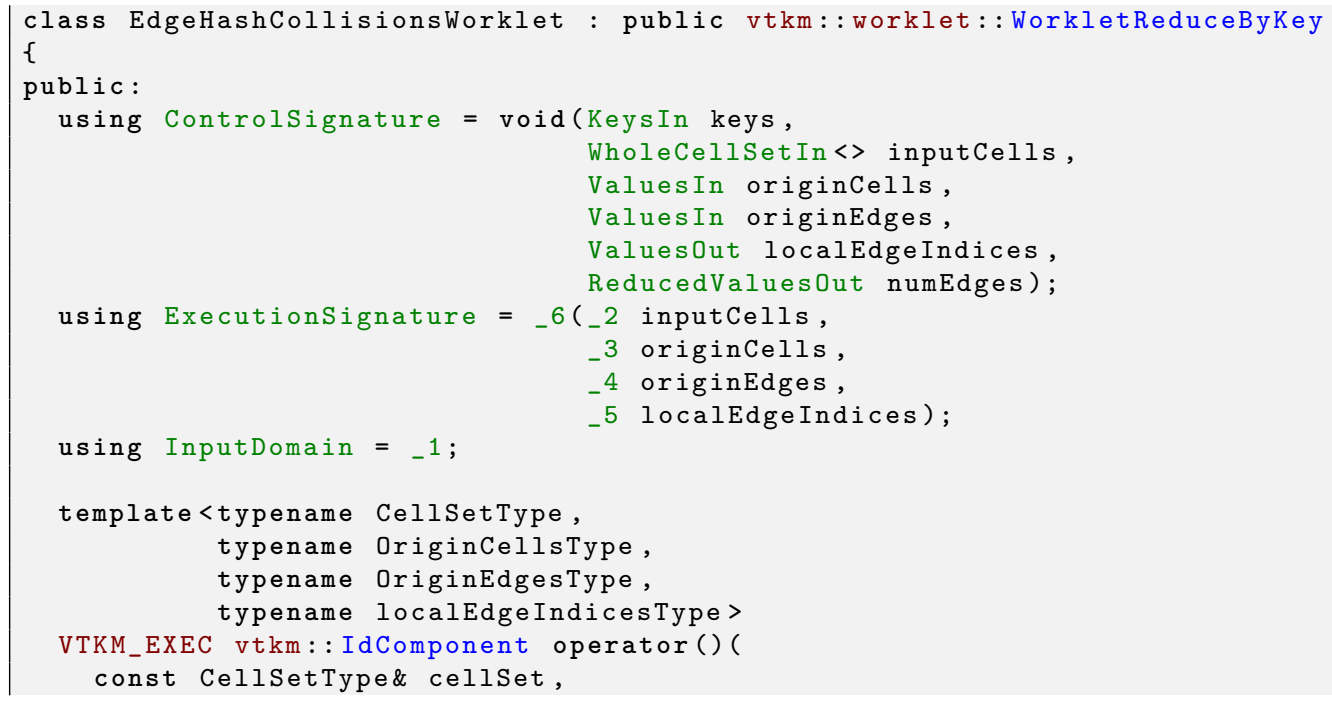




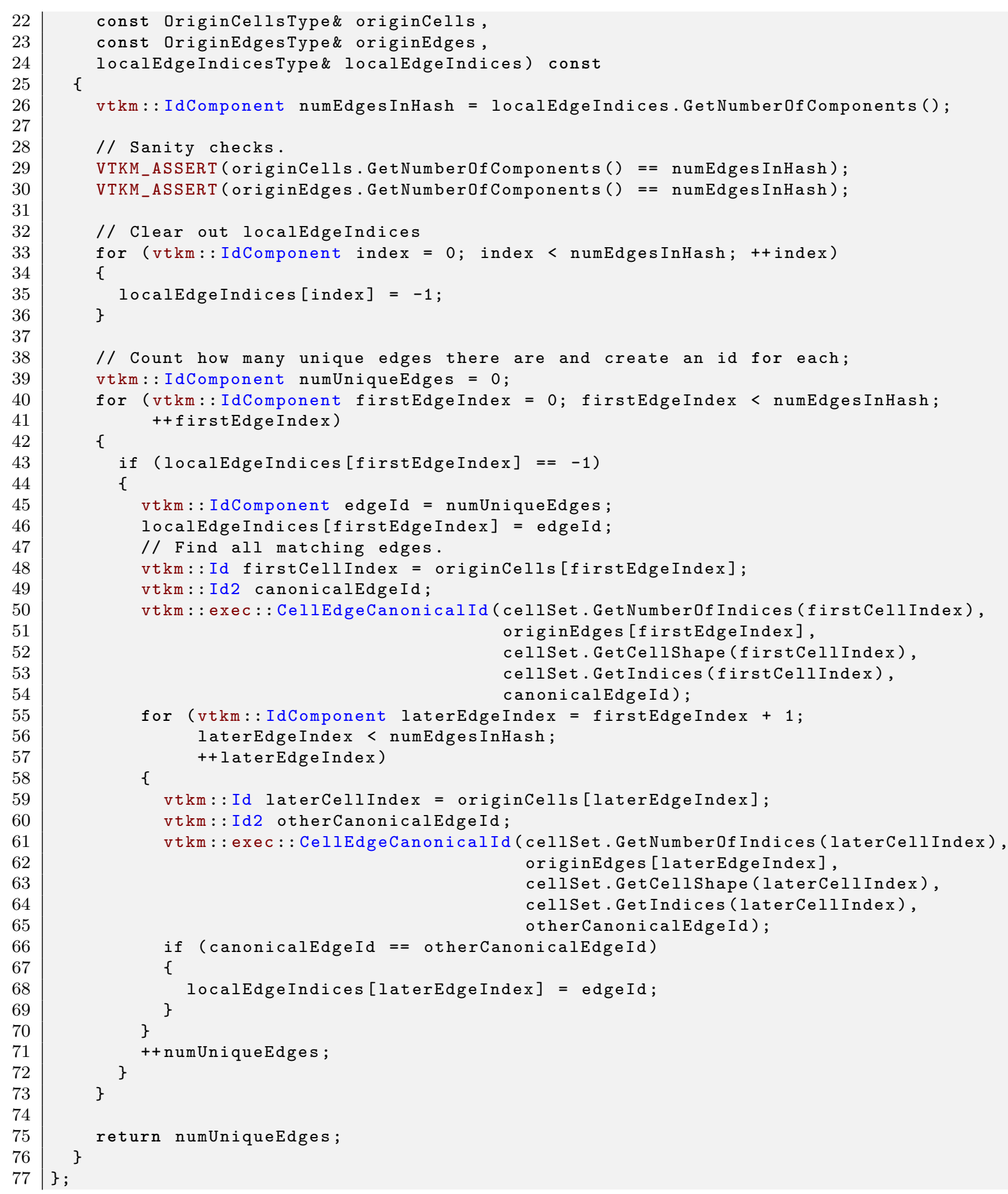

With all hash collisions correctly identified, we are ready to generate the connectivity array for the line elements. This worklet uses a reduce by key worklet like the previous example, but this time we use a ScatterCounting to run the worklet multiple times for hash values that contain multiple unique edges. The worklet takes all the information it needs to reference back to the edges in the original mesh including a WholeCellsetIn, look back indices for the cells and respective edges, and the unique edge group indicators produced by Example 32.11. 
As in the previous sections, this worklet writes out the edge information in a vtkm: : Id2 (which in some following code will be created with an ArrayHandleGroupVec).

Example 32.13: A worklet to generate indices for line cells from combined edges and potential collisions.

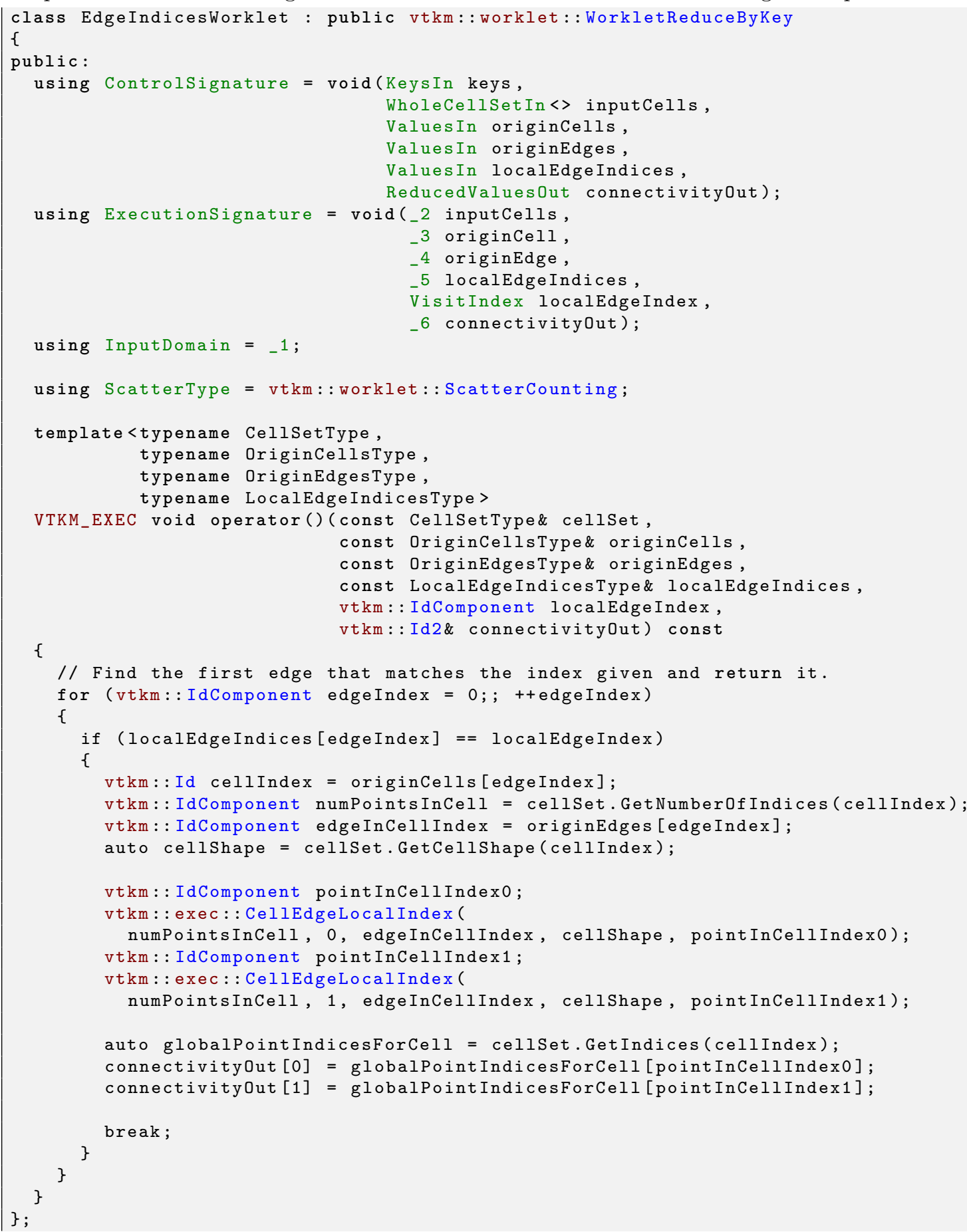

With these 3 worklets, it is now possible to generate all the information we need to fill a vtkm: :cont: :CellSetSingleType object. A CellSetSingleType requires 4 items: the number of points, the constant cell 
shape, the constant number of points in each cell, and an array of connection indices. The first 3 items are trivial. The number of points can be taken from the input cell set as they are the same. The cell shape and number of points are predetermined to be line and 2, respectively.

The last item, the array of connection indices, is what we are creating with the worklet in Example 32.13. The connectivity array for CellSetSingleType is expected to be a flat array of vtkm: : Id indices, but the worklet needs to provide groups of indices for each cell (in this case as a Vec object). To reconcile what the worklet provides and what the connectivity array must look like, we use the vtkm: : cont: : ArrayHandleGroupVec fancy array handle (described in Section 26.13) to make a flat array of indices look like an array of Vec objects.

The following example shows what the DoExecute method in the associated filter would look like.

Example 32.14: Invoking worklets to extract unique edges from a cell set using hash values.

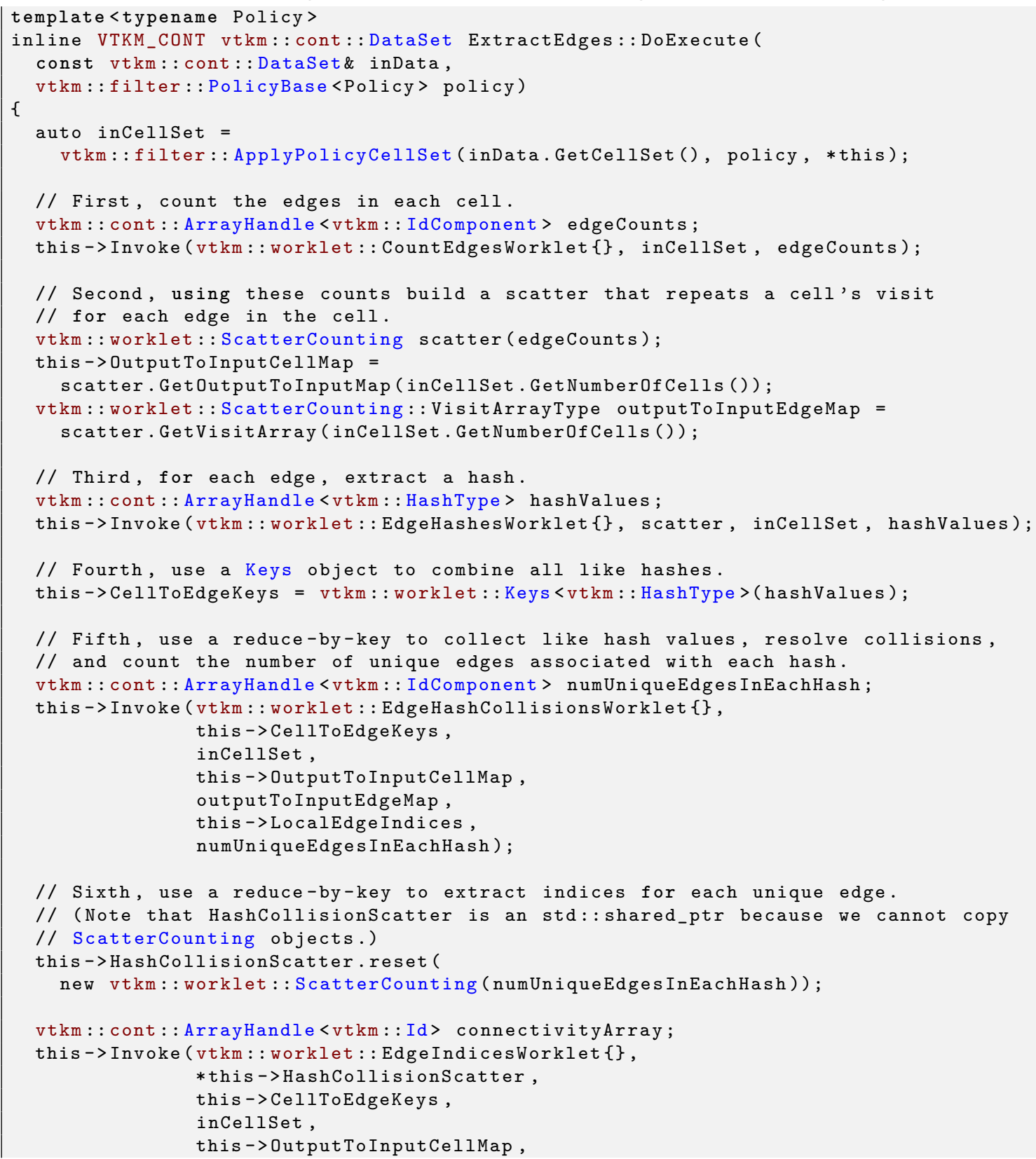




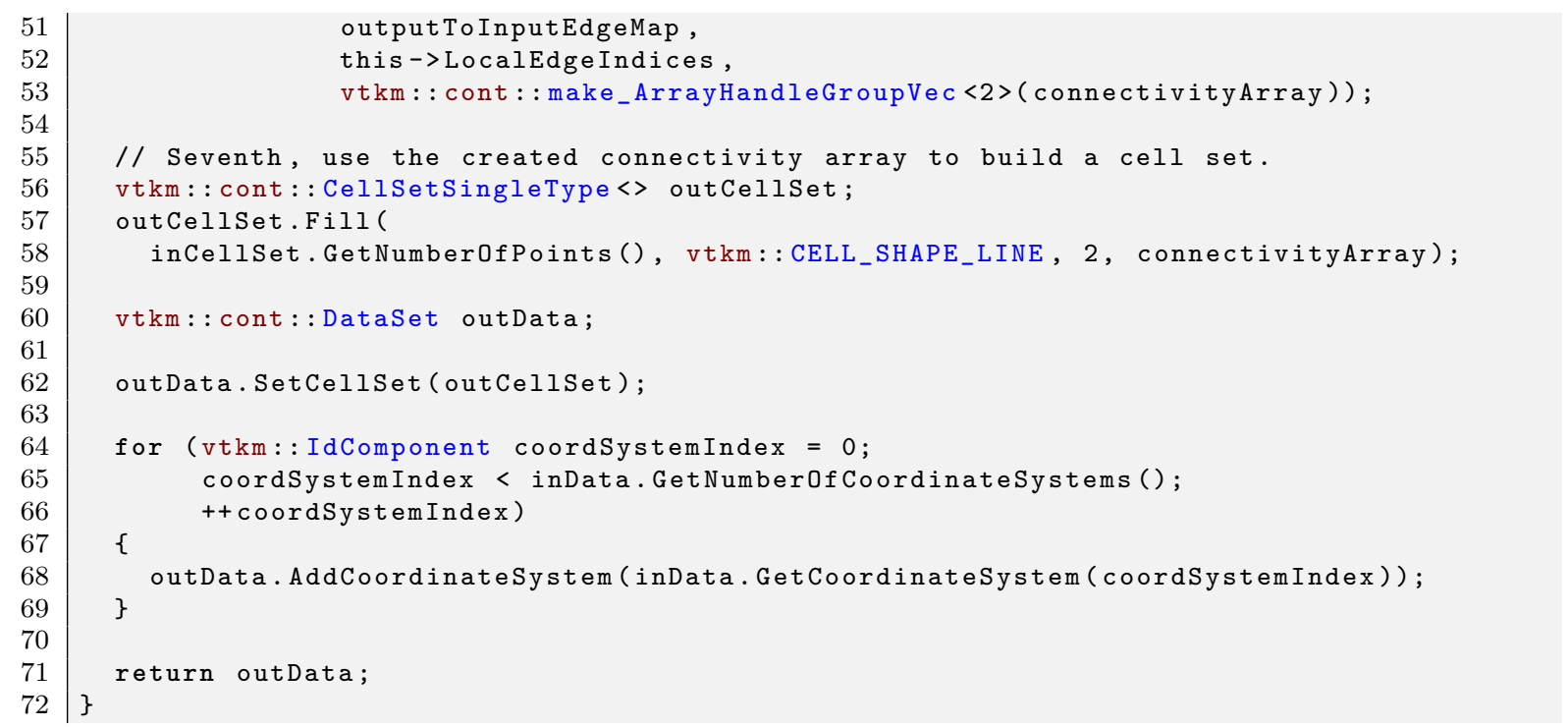

As noted in Section 32.2, in practice DoExecute is called on DataSet objects to create new DataSet objects. Although Example 32.14 creates a new CellSet, we also need a method to transform the Fields on the data set. To do this, we need to save some information. This includes the map from the edge of each cell to its origin cell (OutputToInputCellMap), a Keys object on the hash values (CellToEdgeKeys), an array of indices resolving collisions (LocalEdgeIndices), and a ScatterCounting to repeat edge outputs when unique edges collide on a hash (HashCollisionScatter).

\section{Common Errors \\ 3 Note that HashCollisionScatter is an object of type vtkm: : worklet: : ScatterCounting, which does not \} have a default constructor. That can be a problem since you do not have the data to construct HashCol- lisionScatter until DoExecute is called. To get around this chicken-and-egg issue, it is best to store the ScatterCounter as a pointer. It is best practice to use a smart pointer, like std: :shared_ptr to manage it.}

In Section 32.2 we used a convenience method to average a field attached to cells on the input to each unique edge in the output. Unfortunately, that function does not take into account the collisions that can occur on the keys. Instead we need a custom worklet to average those values that match the same unique edge.

Example 32.15: A worklet to average values with the same key, resolving for collisions.

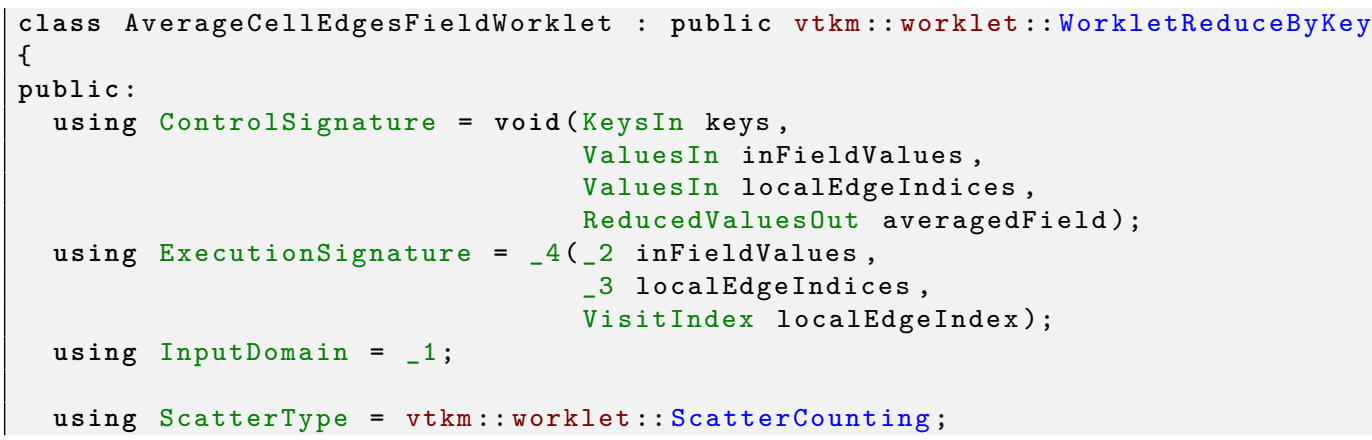




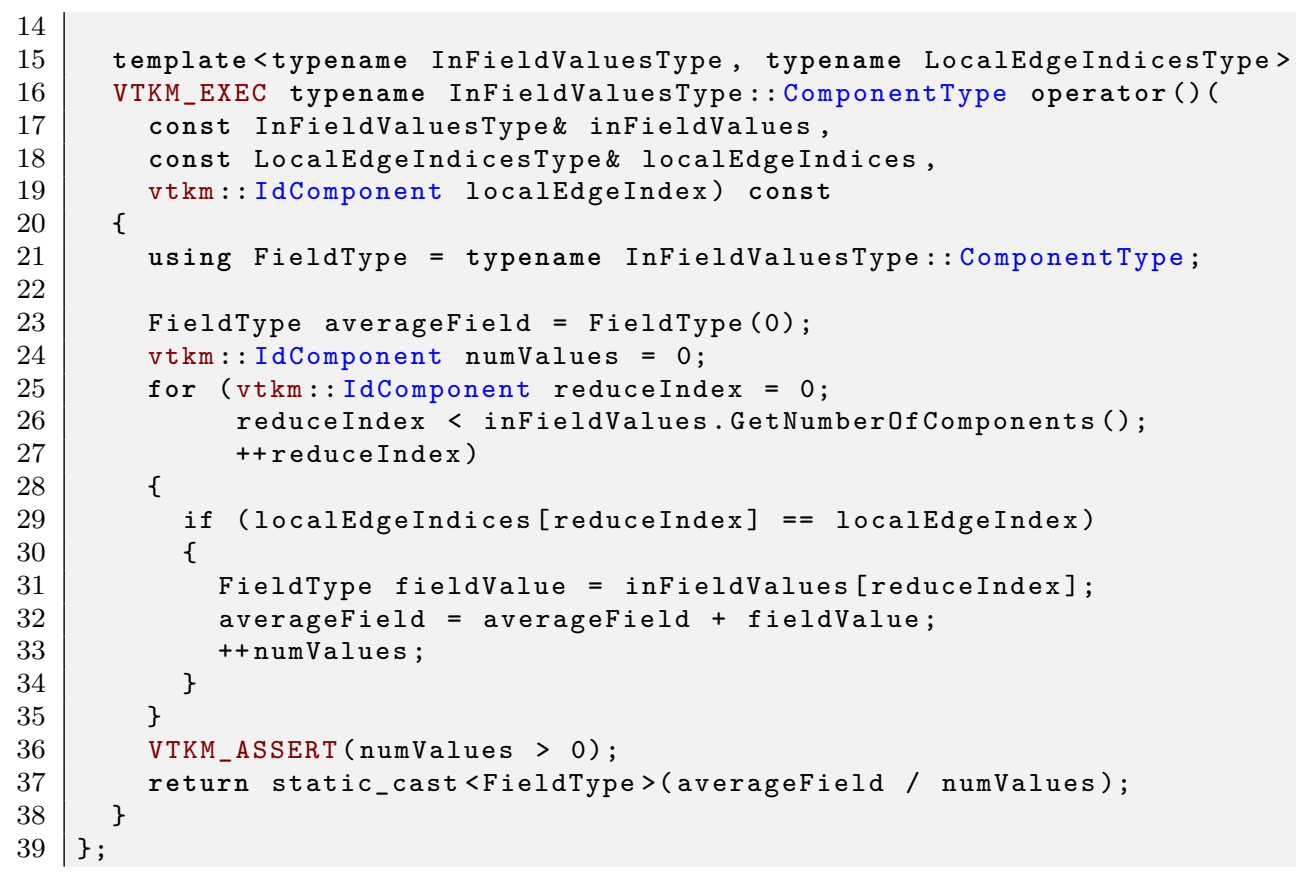

With this worklet, it is straightforward to process cell fields.

Example 32.16: Invoking the worklet to process cell fields, resolving for collisions.

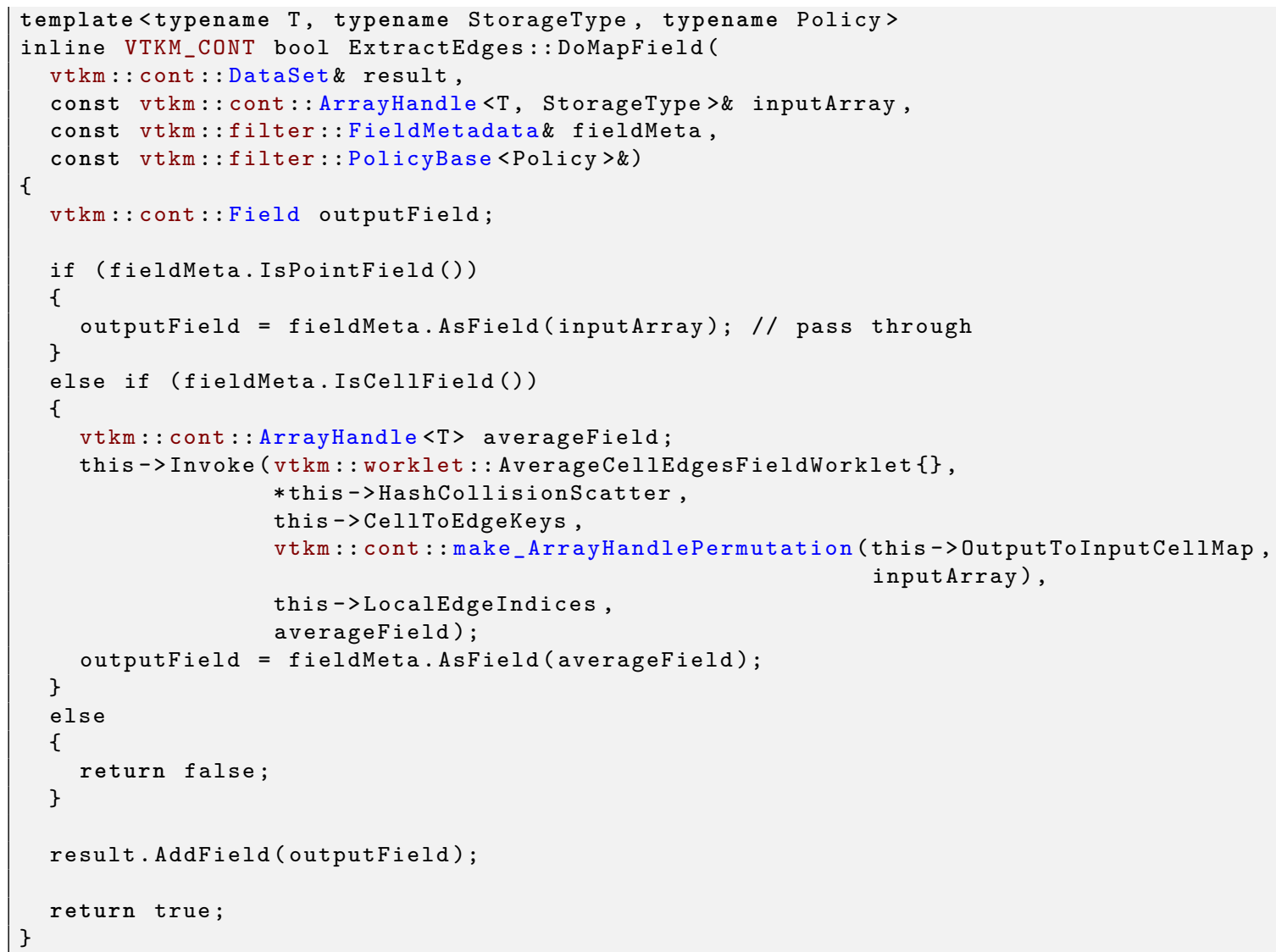




\subsection{Variable Cell Types}

So far in our previous examples we have demonstrated creating a cell set where every cell is the same shape and number of points (i.e. a CellSetSingleType). However, it can also be the case where an algorithm must create cells of a different type (into a vtkm: : cont: :CellSetExplicit). The procedure for generating cells of different shapes is similar to that of creating a single shape. There is, however, an added step of counting the size (in number of points) of each shape to build the appropriate structure for storing the cell connectivity.

Our motivating example is a filter that extracts all the unique faces in a cell set and stores them in a cell set of polygons. This problem is similar to the one addressed in Sections 32.1, 32.2, and 32.3. In both cases it is necessary to find all subelements of each cell (in this case the faces instead of the edges). It is also the case that we expect many faces to be shared among cells in the same way edges are shared among cells. We will use the hash-based approach demonstrated in Section 32.3 except this time applied to faces instead of edges.

The main difference between the two extraction tasks is that whereas all edges are lines with two points, faces can come in different sizes. A tetrahedron has triangular faces whereas a hexahedron has quadrilateral faces. Pyramid and wedge cells have both triangular and quadrilateral faces. Thus, in general the algorithm must be capable of outputting multiple cell types.

Our algorithm for extracting unique cell faces follows the same algorithm as that in Section 32.3. We first need three worklets (used in succession) to count the number of faces in each cell, to generate a hash value for each face, and to resolve hash collisions. These are essentially the same as Examples 32.10, 32.11, and 32.12, respectively, with superficial changes made (like changing Edge to Face). To make it simpler to follow the discussion, the code is not repeated here.

When extracting edges, these worklets provide everything necessary to write out line elements. However, before we can write out polygons of different sizes, we first need to count the number of points in each polygon. The following example does just that. This worklet also writes out the identifier for the shape of the face, which we will eventually require to build a CellSetExplicit. Also recall that we have to work with the information returned from the collision resolution to report on the appropriate unique cell face.

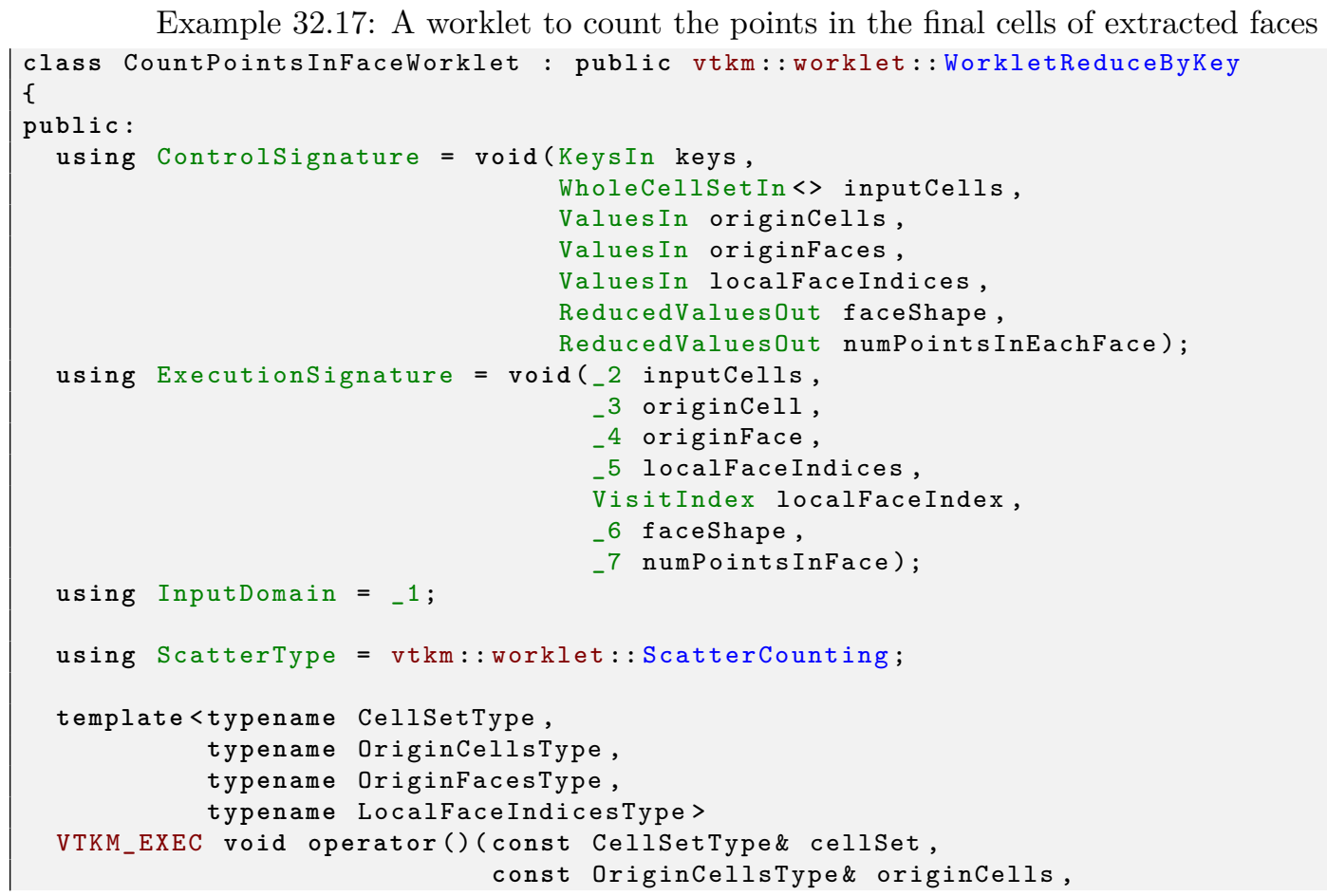

Example 32.17: A worklet to count the points in the final cells of extracted faces 


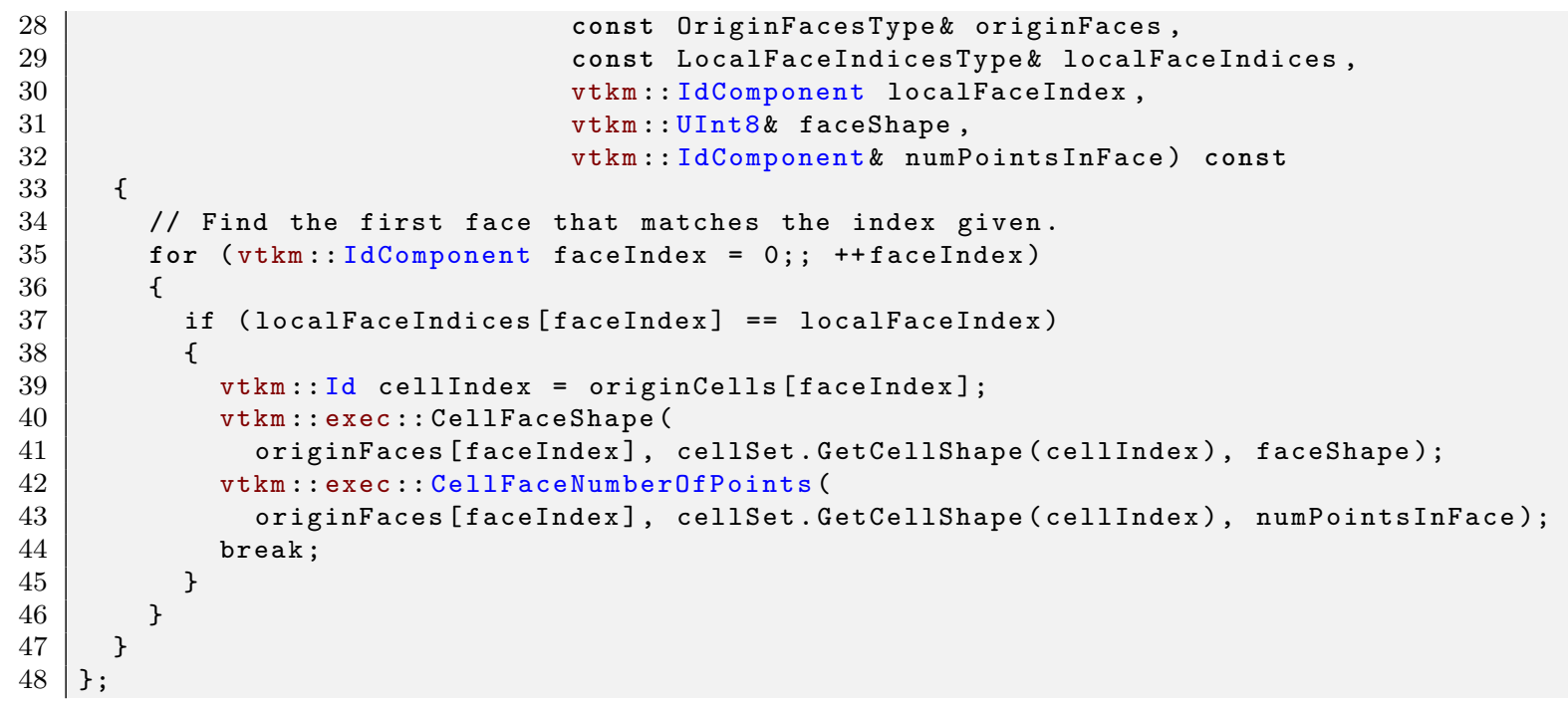

When extracting edges, we converted a flat array of connectivity information to an array of Vecs using an ArrayHandleGroupVec. However, ArrayHandleGroupVec can only create Vecs of a constant size. Instead, for this use case we need to use vtkm::cont: :ArrayHandleGroupVecVariable. As described in Section 26.13, ArrayHandleGroupVecVariable takes a flat array of values and an index array of offsets that points to the beginning of each group to represent as a Vec-like. The worklet in Example 32.17 does not actually give us the array of offsets we need. Rather, it gives us the count of each group. We can get the offsets from the counts by using the vtkm: : cont: : ConvertNumComponentsToOffsets convenience function.

Example 32.18: Converting counts of connectivity groups to offsets for ArrayHandleGroupVecVariable.

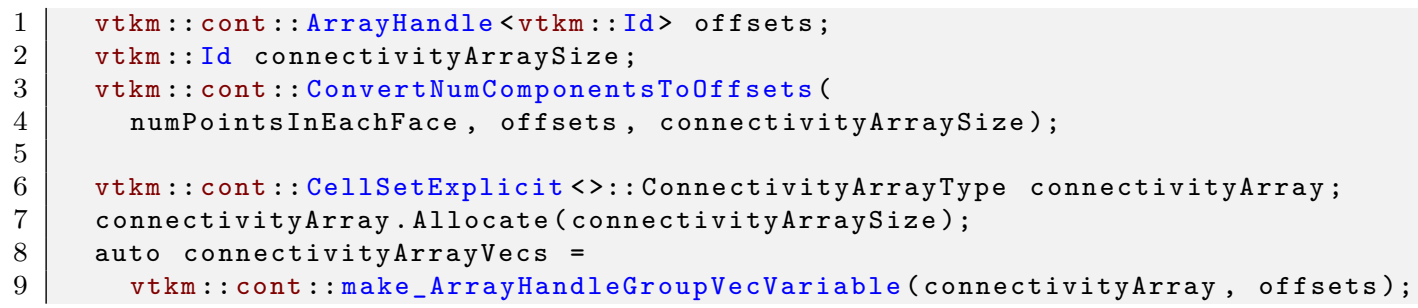

Once we have created an ArrayHandleGroupVecVariable, we can pass that to a worklet that produces the point connections for each output polygon. The worklet is very similar to the one for creating edge lines (shown in Example 32.13), but we have to correctly handle the Vec-like of unknown type and size.

Example 32.19: A worklet to generate indices for polygon cells of different sizes from combined edges and potential collisions.

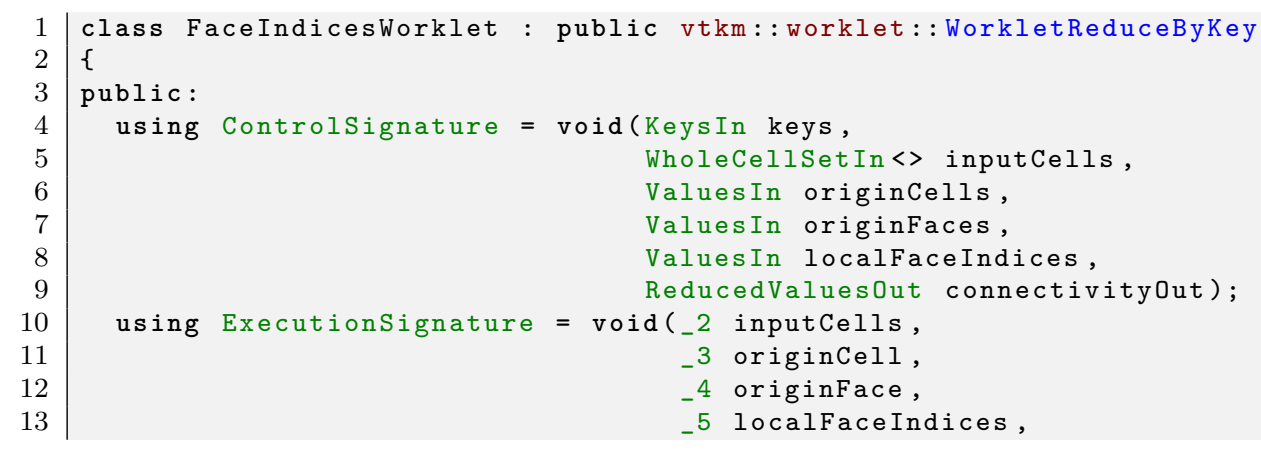




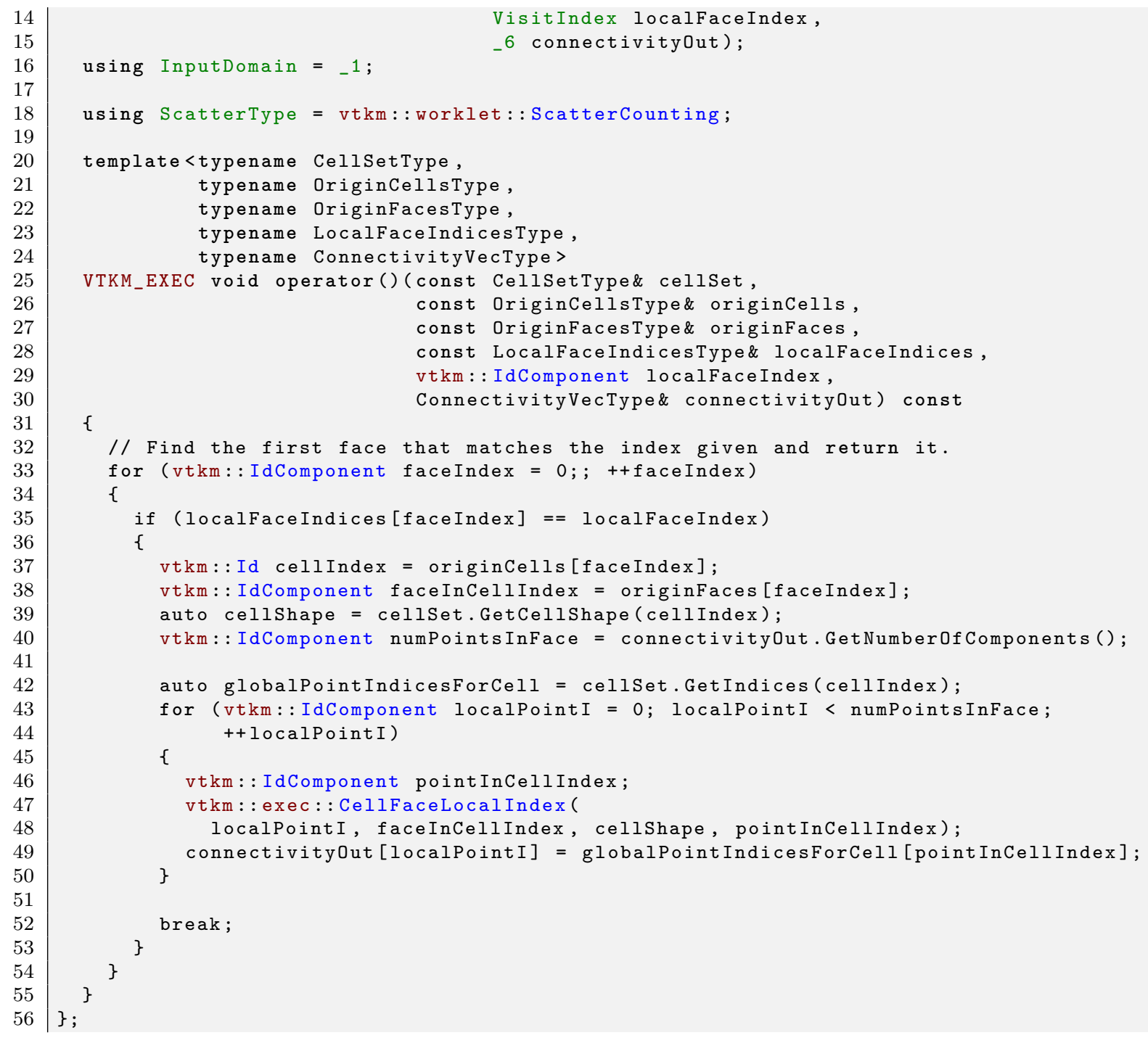

With these worklets in place, we can implement a filter DoExecute as follows.

Example 32.20: Invoking worklets to extract unique faces from a cell set.

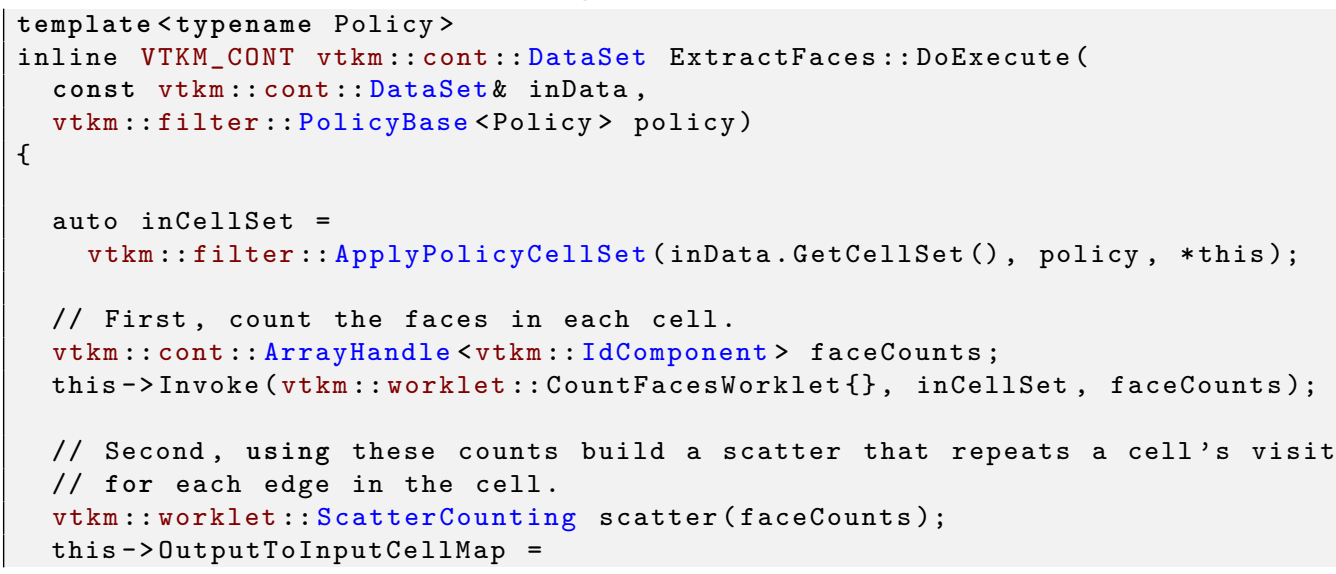




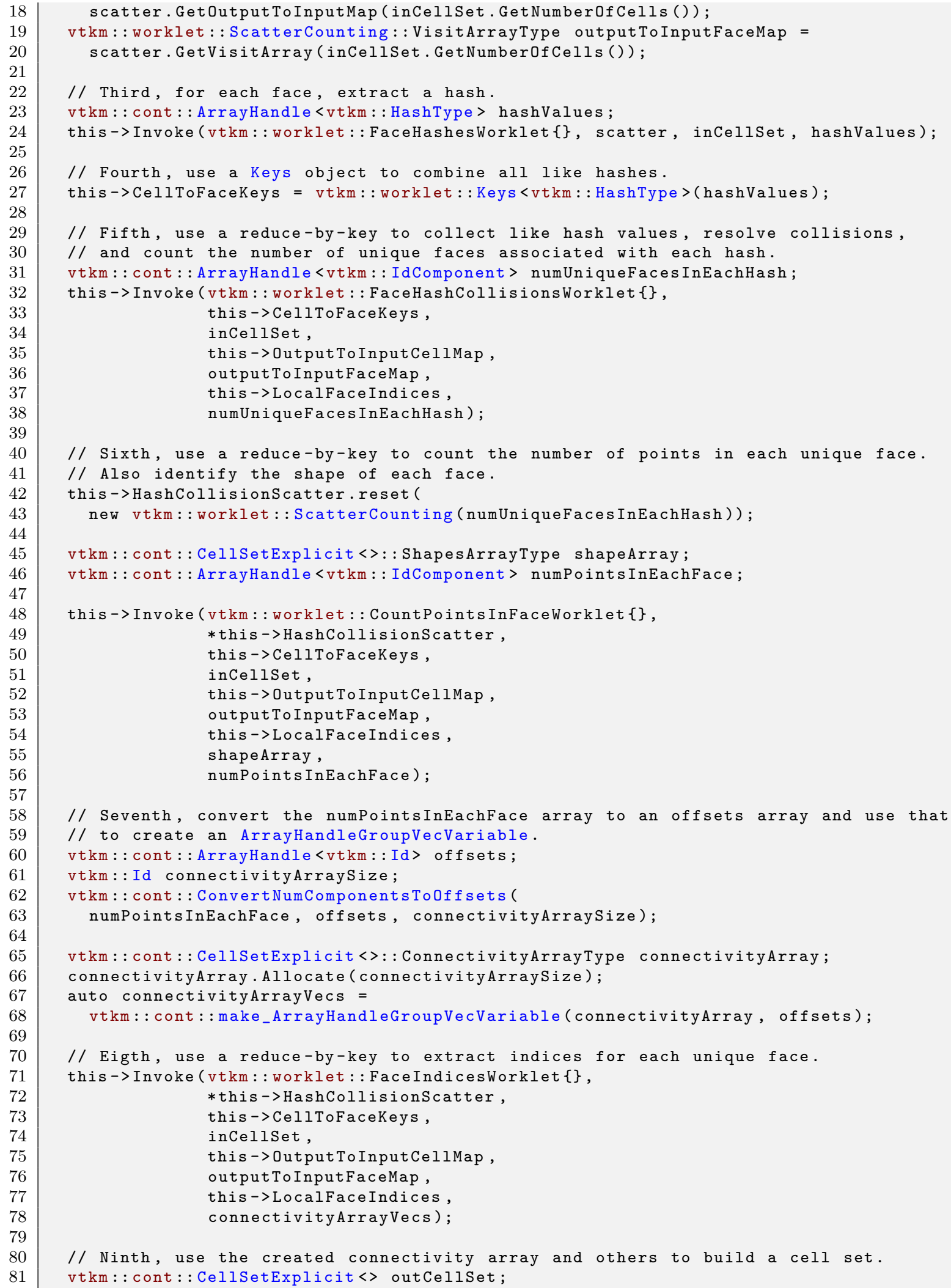




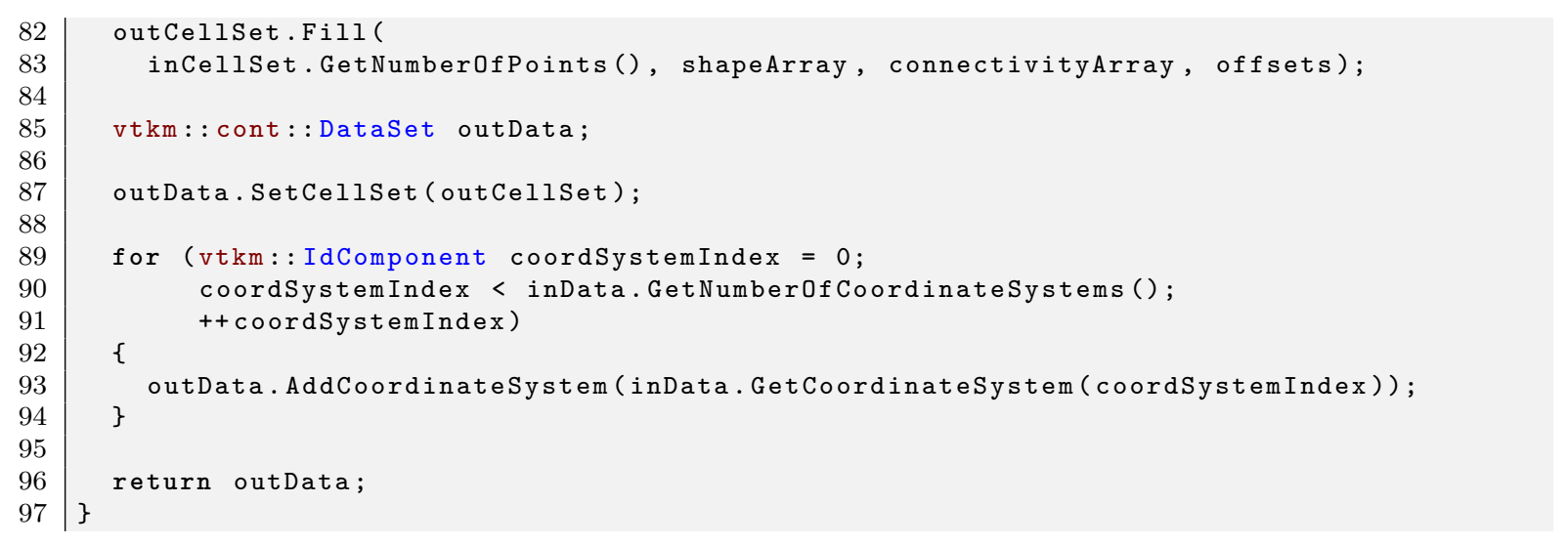

As noted previously, in practice DoExecute is called on DataSet objects to create new DataSet objects. The process for doing so is no different from our previous algorithm as described at the end of Section 32.3 (Examples 32.15 and 32.16$)$. 



\section{UNKNOWN ARRAY HANDLES}

The ArrayHandle class uses templating to make very efficient and type-safe access to data. However, it is sometimes inconvenient or impossible to specify the element type and storage at run-time. The UnknownArrayHandle class provides a mechanism to manage arrays of data with unspecified types.

vtkm: : cont: :UnknownArrayHandle holds a reference to an array. Unlike ArrayHandle, UnknownArrayHandle is not templated. Instead, it uses $\mathrm{C}++$ run-type type information to store the array without type and cast it when appropriate.

An UnknownArrayHandle can be established by constructing it with or assigning it to an ArrayHandle. The following example demonstrates how an UnknownArrayHandle might be used to load an array whose type is not known until run-time.

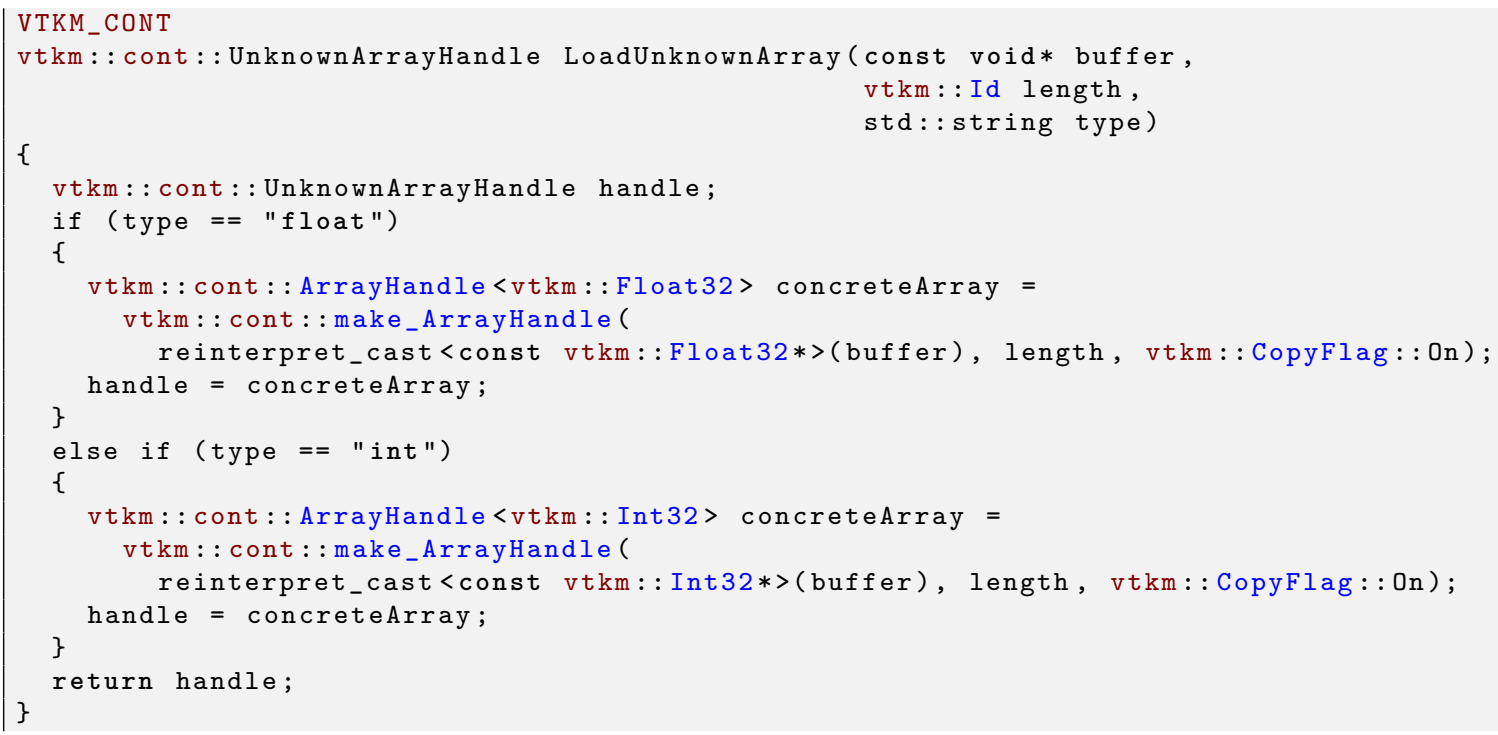

\subsection{Allocation}

Data pointed to by an UnknownArrayHandle is not directly accessible. However, it is still possible to do some type-agnostic manipulation of the array allocations.

First, it is always possible to call UnknownArrayHandle: :GetNumberOfValues to retrieve the current size of 
the array. It is also possible to call UnknownArrayHandle: :Allocate to change the size of an unknown array. UnknownArrayHandle's Allocate works exactly the same as the Allocate in the basic ArrayHandle.

Example 33.2: Checking the size of an ArrayHandle and resizing it.

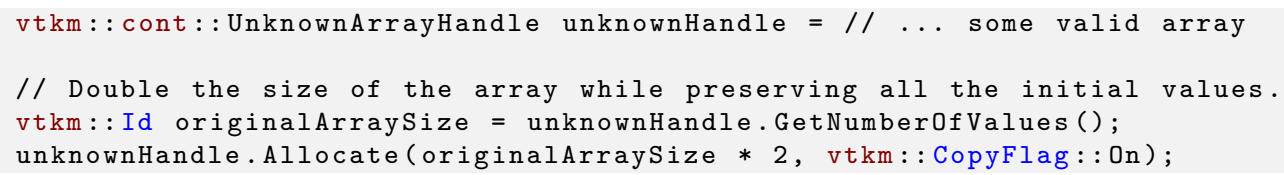

It is often the case where you have an UnknownArrayHandle as the input to an operation and you want to generate an output of the same type. To handle this case, use the NewInstance method to create a new array of the same type (without having to determine the type).

Example 33.3: Creating a new instance of an unknown array handle.

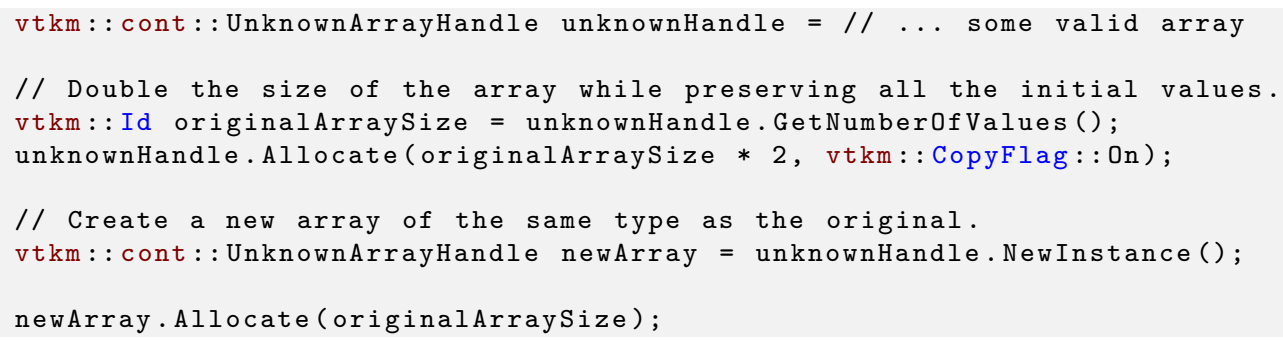

That said, there are many fancy array types (described in Chapter 26) that cannot be used as outputs. Thus, if you do not know the storage of the array, the similar array returned by NewInstance could be infeasible for use as an output. Thus, UnknownArrayHandle also contains the NewInstanceBasic method to create a new array with the same value type but using the basic array storage, which can always be resized and written to.

Example 33.4: Creating a new basic instance of an unknown array handle.

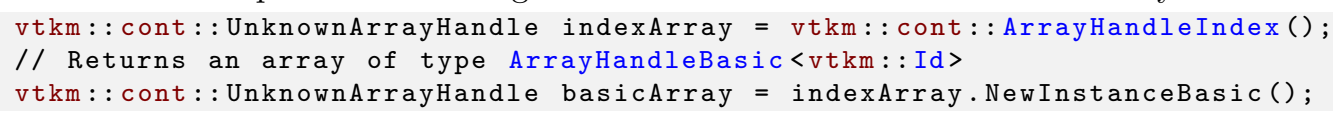

It is occasionally the case that you need a new array of a similar type, but that type has to hold floating point values. For example, if you had an operation that computed a discrete cosine transform on an array, the result would be very inaccurate if stored as integers. In this case, you would actually want to store the result in an array of floating point values. For this case, you can use the NewInstanceFloatBasic to create a new basic ArrayHandle with the component type changed to vtkm: :FloatDefault. For example, if the UnknownArrayHandle stores an ArrayHandle of type vtkm: :Id, NewInstanceFloatBasic will create an ArrayHandle of type vtkm: :FloatDefault. If the UnknownArrayHandle stores an ArrayHandle of type vtkm: :Id3, NewInstanceFloatBasic will create an ArrayHandle of type vtkm: :Vec3f.

Example 33.5: Creating a new array instance with floating point values.

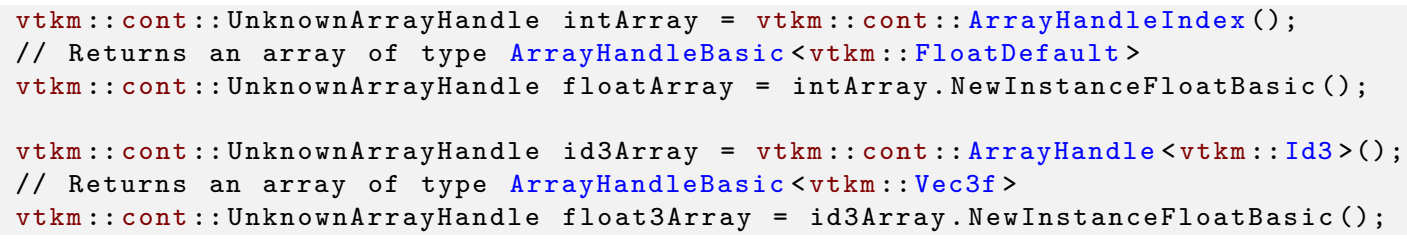




\subsection{Casting to Known Types}

Data pointed to by an UnknownArrayHandle is not directly accessible. To access the data, you need to retrieve the data as an ArrayHandle. If you happen to know (or can guess) the type, you can use the AsArrayHandle method to retrieve the array as a specific type.

Example 33.6: Retrieving an array of a known type from UnknownArrayHandle.

1 vtkm: : cont : : ArrayHandle<vtkm: :Float32> knownArray =

2 unknownArray. AsArrayHandle <vtkm: : cont: : ArrayHandle <vtkm: : Float32>>();

AsArrayHandle actually has two forms. The first form, shown in the previous example, has no arguments and returns the ArrayHandle. This form requires you to specify the type of array as a template parameter. The alternate form has you pass a reference to a concrete ArrayHandle as an argument as shown in the following example. This form can imply the template parameter from the argument.

Example 33.7: Alternate form for retrieving an array of a known type from UnknownArrayHandle.

1 unknownArray. AsArrayHandle (knownArray);

AsArrayHandle treats ArrayHandleCast and ArrayHandleMultiplexer special. If the special ArrayHandle can hold the actual array stored, then AsArrayHandle will return successfully. In the following example, AsArrayHandle returns an array of type vtkm: :Float32 as an ArrayHandleCast that converts the values to vtkm: :Float64.

Example 33.8: Getting a cast array handle from an ArrayHandleCast.

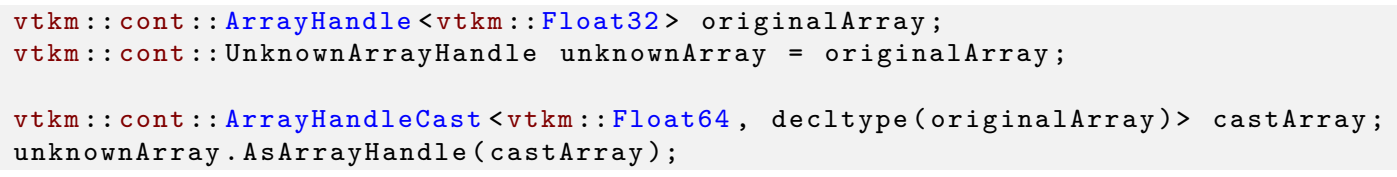

(9) Did you know?

The inverse retrieval works as well. If you create an UnknownArrayHandle with an ArrayHandleCast or ArrayHandleMultiplexer, you can get the underlying array with AsArrayHandle. These relationships also work recursively (e.g. an array placed in a cast array which is placed in a multiplexer).

If the UnknownArrayHandle cannot store its array in the type given to AsArrayHandle, it will throw an exception. Thus, you should not use AsArrayHandle with types that you are not sure about. Use the CanConvert method to determine if a given ArrayHandle type will work with AsArrayHandle.

Example 33.9: Querying whether a given ArrayHandle can be retrieved from an UnknownArrayHandle.

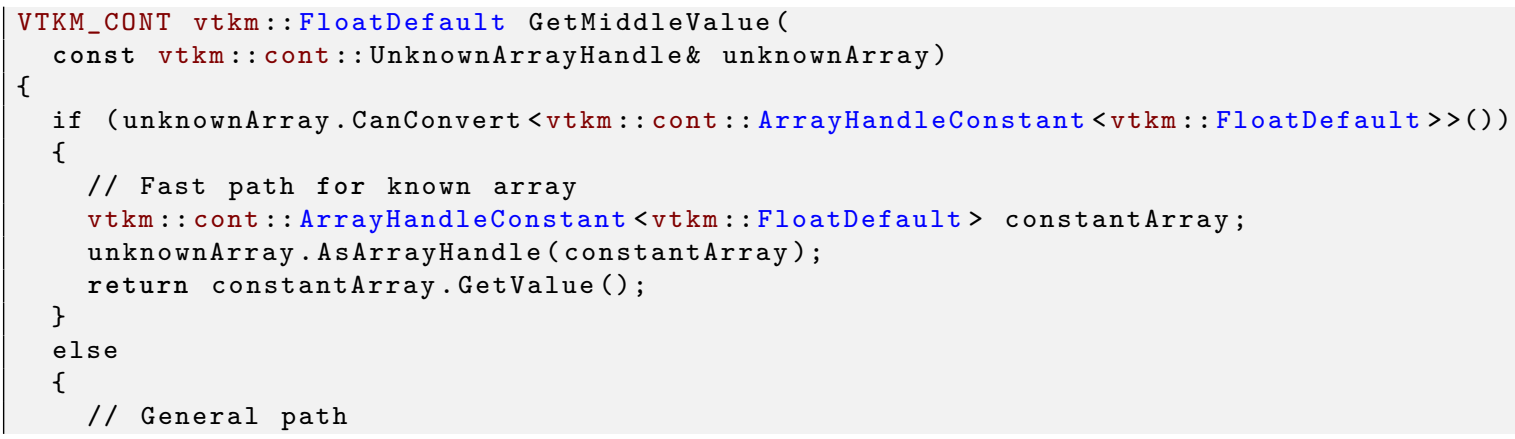




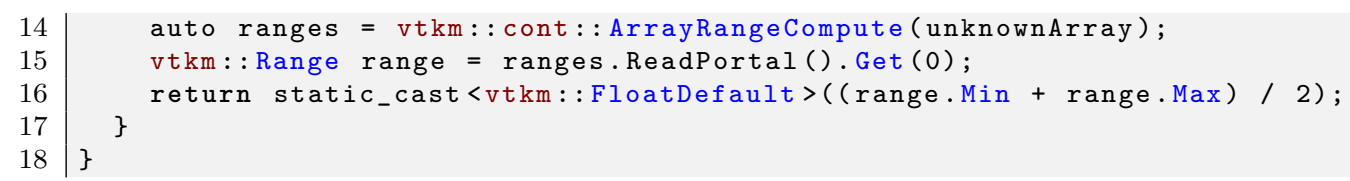

By design, CanConvert will return true for types that are not actually stored in the UnknownArrayHandle but can be retrieved. If you need to know specifically what type is stored in the UnknownArrayHandle, you can use the IsType method instead. If you need to query either the value type or the storage, you can use IsValueType and IsStorageType, respectively. UnknownArrayHandle also provides GetValueTypeName and GetStorageTypeName for debugging purposes.

If you do not know the exact type of the array contained in an UnknownArrayHandle, a brute force method to get the data out is to copy it to an array of a known type. This can be done with the UnknownArrayHandle: :DeepCopyFrom method, which will copy the contents of a target array into an existing array of a (potentially) different type.

CanConvert is almost always safer to use than IsType or its similar methods. Even though IsType reflects the actual array type, CanConvert better describes how UnknownArrayHandle will behave.

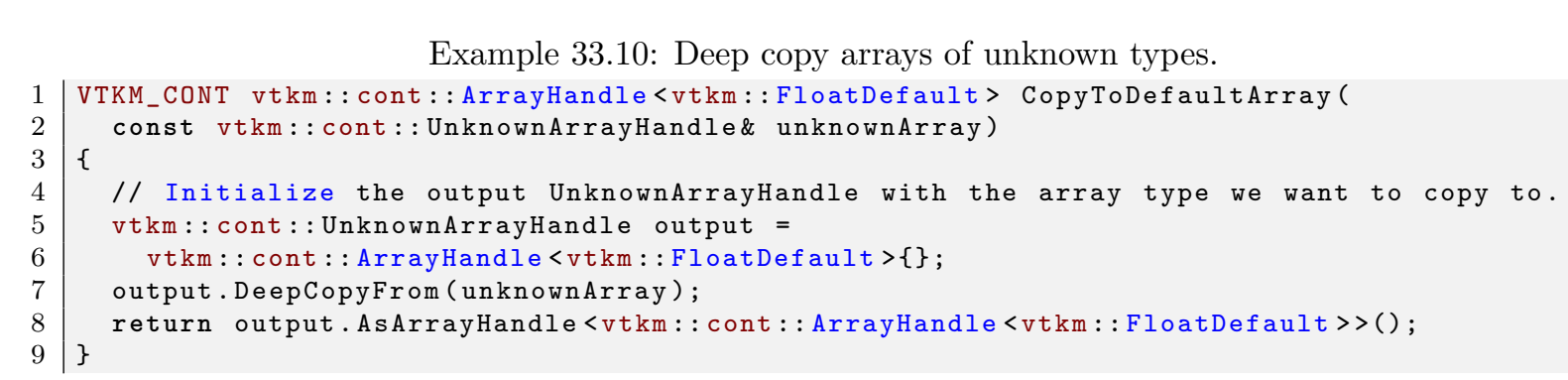

It is often the case that you have good reason to believe that an array is of an expected type, but you have no way to be sure. To simplify code, the most rational thing to do is to get the array as the expected type if that is indeed what it is, or to copy it to an array of that type otherwise. The UnknownArrayHandle: : CopyShallowIfPossible does just that.

Example 33.11: Using ArrayCopyShallowIfPossible to get an unknown array as a particular type.

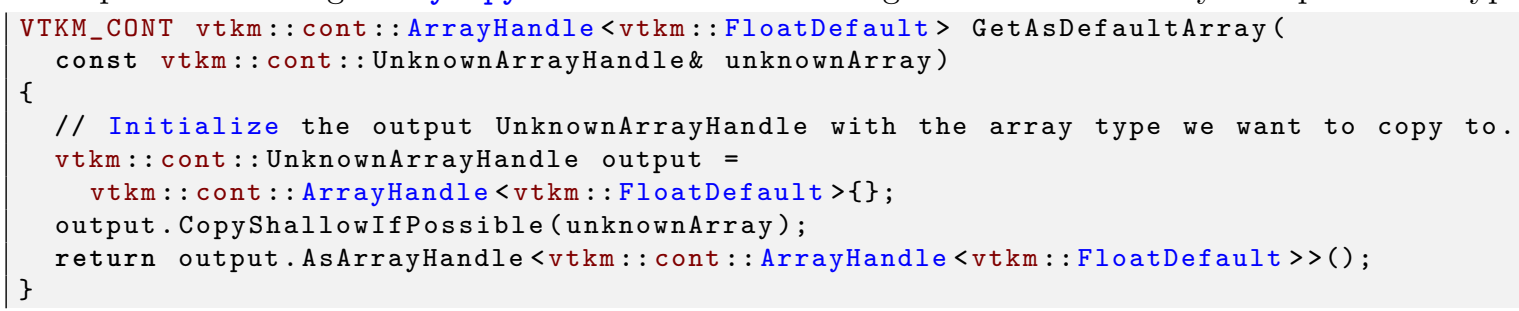


(8) Did you know?

The UnknownArrayHandle copy methods behave similarly to the vtkm: :cont: :ArrayCopy functions. One advantage of using the UnknownArrayHandle methods is that they do not require using a device compiler (such as nvcc). Both versions will (potentially) perform the copy on a device, but the methods for UnknownArrayHandle are sufficiently hidden in a library to avoid calling code needing to compile device instructions.

\subsection{Casting to a List of Potential Types}

Using AsArrayHandle is fine as long as the correct types are known, but often times they are not. For this use case UnknownArrayHandle has a method named CastAndCallForTypes that attempts to cast the array to some set of types.

The CastAndCallForTypes method accepts a functor to run on the appropriately cast array. The functor must have an overloaded const parentheses operator that accepts an ArrayHandle of the appropriate type. You also have to specify two template parameters that specify a vtkm: :List of value types to try and a vtkm: :List of storage types to try, respectively. The macros VTKM_DEFAULT_TYPE_LIST and VTKM_DEFAULT_STORAGE_LIST are often used when nothing more specific is known.

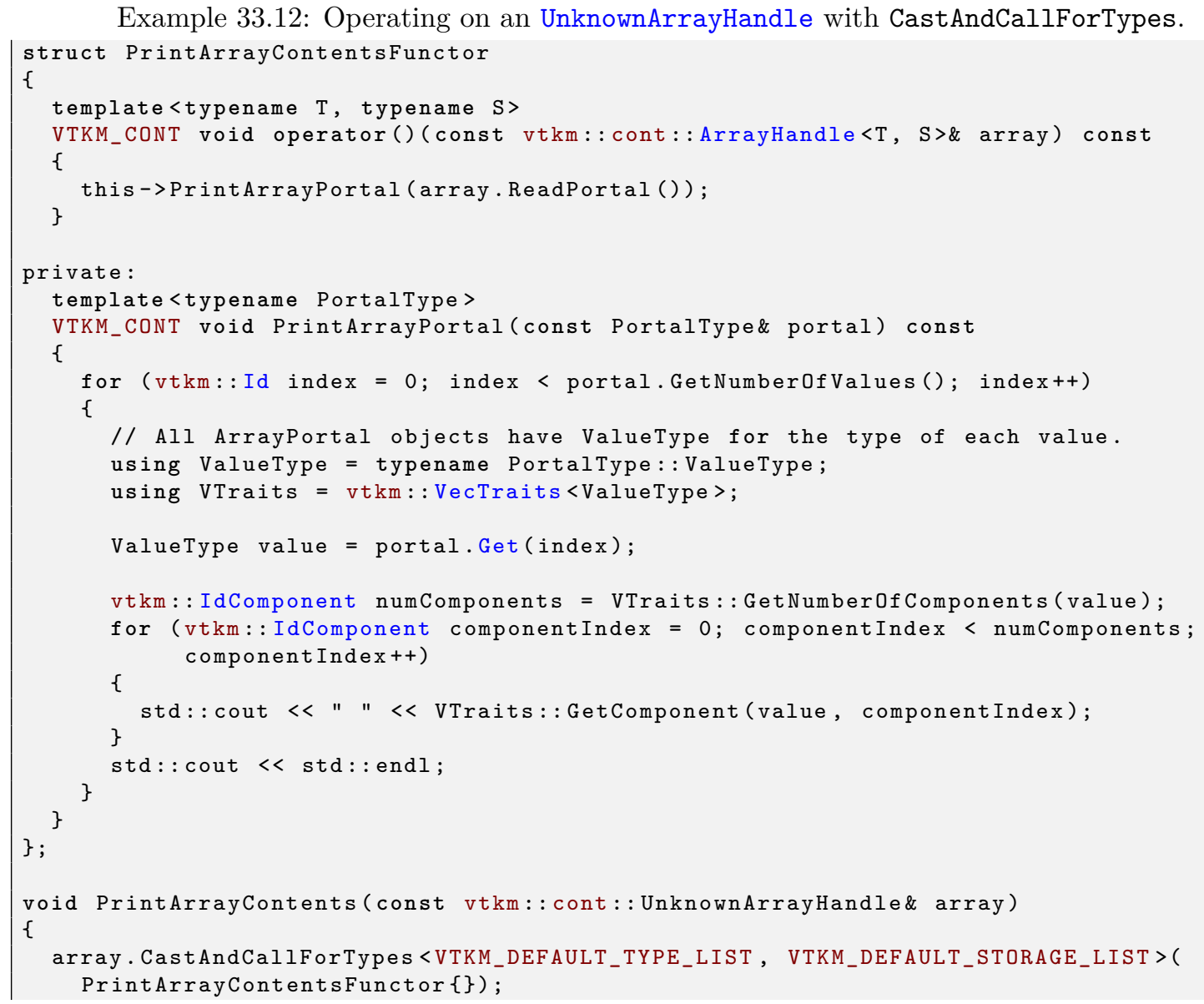


$36 \mid\}$

\section{(0) Did you know?}

3 The first (required) argument to CastAndCallForTypes is the functor to call with the array. You can supply any number of optional arguments after that. Those arguments will be passed directly to the functor. This makes it easy to pass state to the functor.

\section{Did you know?}

3 When an UnknownArrayHandle is used in place of an ArrayHandle as an argument to a worklet invocation, it will internally use CastAndCallForTypes to attempt to call the worklet with an ArrayHandle of the correct type.

UnknownArrayHandle has a simple subclass named vtkm: : cont: :UncertainArrayHandle for use when you can narrow the array to a finite set of types. UncertainArrayHandle has two template parameters that must be specified: a vtkm: :List of value types and a vtkm: :List of storage types. UncertainArrayHandle has a method named CastAndCall that behaves the same as CastAndCallForTypes except that you do not have to specify the types to try. Instead, the types are taken from the template parameters of the UncertainArrayHandle itself.

Example 33.13: Using UncertainArrayHandle to cast and call a functor.

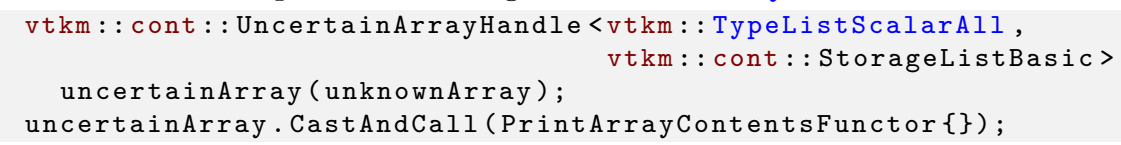

\section{D) Did you know?}

3 Like with UnknownArrayHandle, if an UncertainArrayHandle is used in a worklet invocation, it will internally use CastAndCall. This provides a convenient way to specify what array types the invoker should try.

Both UnknownArrayHandle and UncertainArrayHandle provide a method named ResetTypes to redefine the types to try. ResetTypes has two template parameters that are the vtkm::Lists of value and storage types. ResetTypes returns a new UncertainArrayHandle with the given types. This is a convenient way to pass these types to functions.

Example 33.14: Resetting the types of an UnknownArrayHandle.

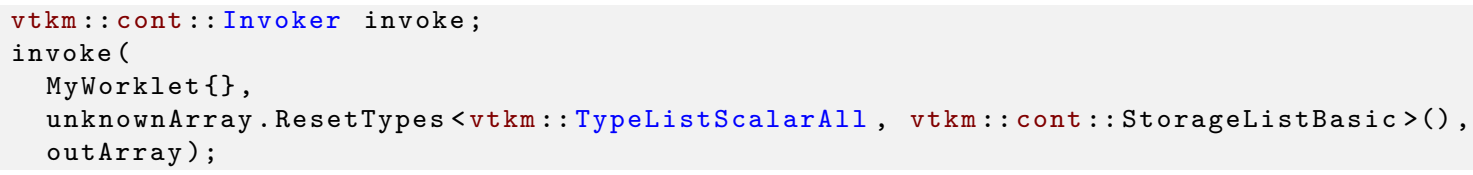




\section{Common Errors}

Because it returns an UncertainArrayHandle, you need to include vtkm/cont/UncertainArrayHandle.h if you use UnknownArrayHandle: :ResetTypes. This is true even if you do not directly use the returned object.

\subsection{Accessing Truly Unknown Arrays}

So far in Sections 33.2 and 33.3 we explored how to access the data in an UnknownArrayHandle when you actually know the array type or can narrow down the array type to some finite number of candidates. But what happens if you cannot practically narrow down the types in the UnknownArrayHandle? For this case, UnknownArrayHandle provides a mechanism for extracting data knowing little or nothing about the types by looking at a single vtkm: :Vec component at a time.

You can use the UnknownArrayHandle: :ExtractComponent method to return an ArrayHandle with the values for a given component for each value in the array. ExtractComponent must be given a template argument for the base component type. The following example extracts the first component of all vtkm::Vec values in an UnknownArrayHandle assuming that the component is of type vtkm: :FloatDefault (line 11).

Example 33.15: Extracting the first component of every value in an UnknownArrayHandle.

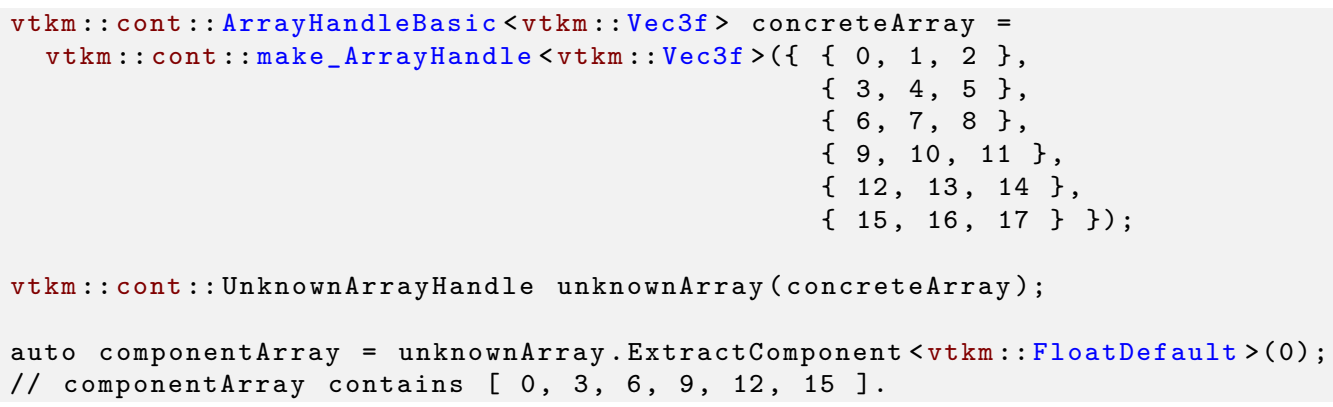

The code in Example 33.15 works with any array with values based on the default floating point type. If the UnknownArrayHandle has an array containing vtkm: :FloatDefault, then the returned array has all the same values. If the UnknownArrayHandle contains values of type vtkm: :Vec3f, then each value in the returned array will be the first component of this array.

If the UnknownArrayHandle really contains an array with incompatible value types (such as ArrayHandle<vtkm: :Id>), then an vtkm: :cont: ErrorBadType will be thrown. To check if the UnknownArrayHandle contains an array of a compatible type, use the IsBaseComponentType method.

Example 33.16: Checking the base component type in an UnknownArrayHandle.

1 unknownArray. IsBaseComponentType<vtkm: : FloatDefault >()

This section started with the motivation of getting data from an UnknownArrayHandle without knowing anything about the type, yet ExtractComponent still requires a type parameter. However, by limiting the type needed to the base component type, you only need to check the base $\mathrm{C}$ types (standard integers and floating points) available in $\mathrm{C}++$. You do not need to know whether these components are arranged in Vecs or the size of the vtkm: :Vec. A general implementation of an algorithm might have to deal with scalars as well as Vecs of size 2,3 , and 4 . If we consider operations on tensors, Vecs of size 6 and 9 can be common as well. But when using ExtractComponent, a single condition can handle any potential Vec size. 
Another advantage of ExtractComponent is that the type of storage does not need to be specified. ExtractComponent works with any type of ArrayHandle storage (with some caveats). So, Example 33.15 works equally as well with ArrayHandleBasic, ArrayHandleSOA, ArrayHandleUniformPointCoordinates, ArrayHandleCartesianProduct, and many others. Trying to capture all reasonable types of arrays could easily require hundreds of conditions, all of which and more can be captured with ExtractComponent and the roughly 12 basic $\mathrm{C}$ data types. In practice, you often only really have to worry about floating point components, which further reduces the cases down to (usually) 2.

UnknownArrayHandle: : ExtractComponent works by returning an ArrayHandleStride. This is a special ArrayHandle that can access data buffers by skipping values at regular intervals. This allows it to access data packed in different ways such as ArrayHandleBasic, ArrayHandleSOA, and many others. That said, ArrayHandleStride is not magic, so if cannot directly access memory, some or all of it may be copied. If you are attempting to use the array from ExtractComponent as an output array, pass vtkm::CopyFlag::Off as a second argument. This will ensure that data are not copied so that any data written will go to the original array (or throw an exception if this cannot be done).

\section{Common Errors}

Although UnknownArrayHandle: : ExtractComponent will technically work with any ArrayHandle (of simple Vec types), it may require a very inefficient memory copy. Pay attention if ExtractComponent issues a warning about an inefficient memory copy. This is likely a serious performance issue, and the data should be retrieved in a different way (or better yet stored in a different way).

Example 33.15 access the first component of each Vec in an array. But in practice you usually want to operate on all components stored in the array. A simple solution is to iterate over each component.

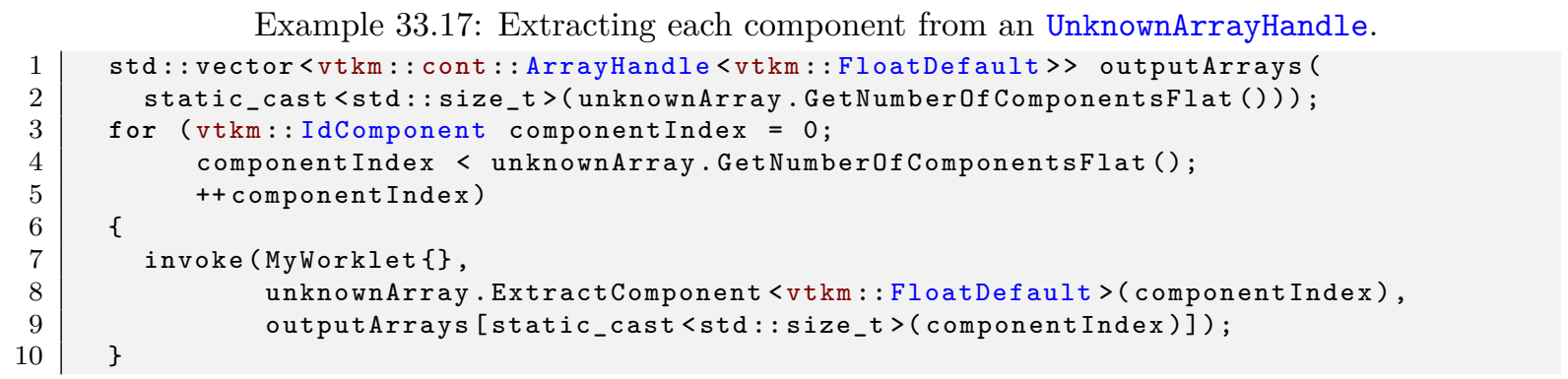

To ensure that the type of the extracted component is a basic C type, the vtkm::Vec values are "flattened." That is, they are treated as if they are a single level vtkm::Vec. For example, if you have a value type of vtkm: :Vec<vtkm: :Id3, 2>, ExtractComponent treats this type as vtkm: Vec<vtkm::Id, 6>. This allows you to extract the components as type vtkm: :Id rather than having a special case for vtkm: :Id3.

Although iterating over components works fine, it can be inconvenient. An alternate mechanism is to use UnknownArrayHandle: :ExtractArrayFromComponents to get all the components at once. ExtractArrayFromComponents works like ExtractComponent except that instead of returning an ArrayHandleStride, it returns a special vtkm: : cont: :ArrayHandleRecombineVec that behaves like an ArrayHandle to reference all component arrays at once.

Example 33.18: Extracting all components from an UnknownArrayHandle at once.

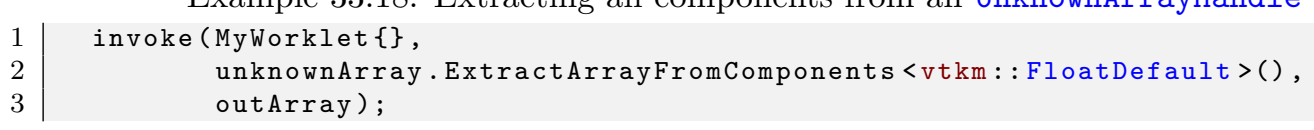


Although it has the same interface as other ArrayHandles, ArrayHandleRecombineVec has a special value type that breaks some conventions. For example, when used in a worklet, the value type passed from this array to the worklet cannot be replicated. That is, you cannot create a temporary stack value of the same type.

Because you still need to specify a base component type, you will likely still need to check several types to safely extract data from an UnknownArrayHandle by component. To do this automatically, you can use the CastAndCallWithExtractedArray. This method behaves similarly to CastAndCall except that it internally uses ExtractArrayFromComponents.

Example 33.19: Calling a functor for nearly any type of array stored in an UnknownArrayHandle.

1 unknownArray.CastAndCallWithExtractedArray (PrintArrayContentsFunctor \{\} );

\subsection{Mutability}

One subtle feature of UnknownArrayHandle is that the class is, in principle, a pointer to an array pointer. This means that the data in an UnknownArrayHandle is always mutable even if the class is declared const. The upshot is that you can pass output arrays as constant UnknownArrayHandle references.

Example 33.20: Using a const UnknownArrayHandle for a function output.

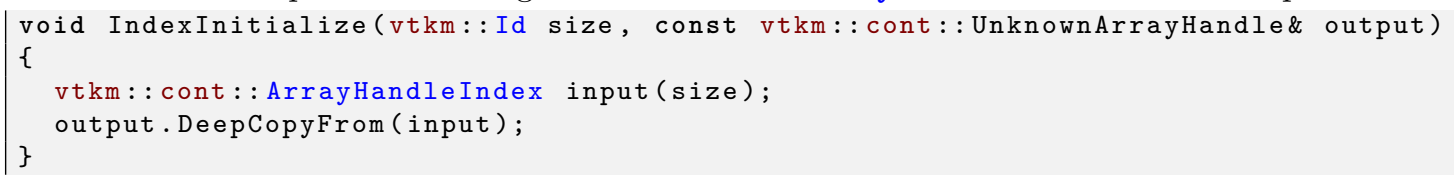

Although it seems strange, there is a good reason to allow output UnknownArrayHandles to be const. It allows a typed ArrayHandle to be used as the argument to the function. In this case, the compiler will automatically convert the ArrayHandle to a UnknownArrayHandle. When $\mathrm{C}++$ creates objects like this, they can only be passed as constant references or by value. So, declaring the output parameter as const UnknownArrayHandle allows it to be used for code like this.

Example 33.21: Passing an ArrayHandle as an output UnknownArrayHandle.

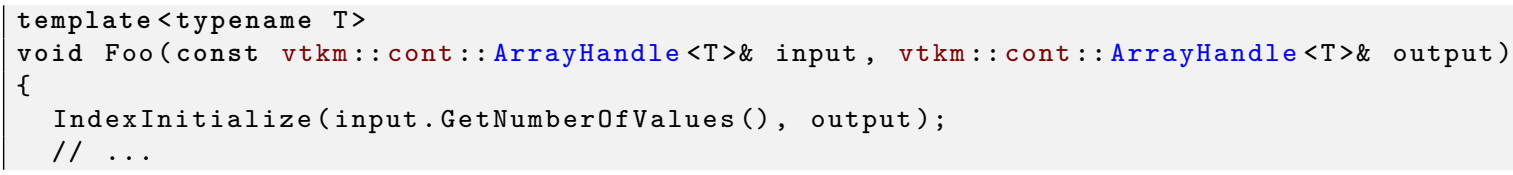

Of course, you could also declare the output by value instead of by reference, but this has the same semantics with extra internal pointer management.

\section{Did you know?}

When possible, it is better to pass UnknownArrayHandles as constant references (or by value) rather than a mutable reference, even if the array contents are going to be modified. This allows the function to support automatic conversion of output ArrayHandles. 
So if a constant UnknownArrayHandle can have its contents modified, what is the difference between a constant reference and a non-constant reference? The difference is that the constant reference can change the array's content, but not the array itself. If you want to do operations like doing a shallow copy or changing the underlying type of the array, a non-constant reference is needed. 


\section{DEVICE ALGORITHMS}

As described in Chapter 15, VTK-m is built around the concept of a device adapter that encapsulates the necessary features of each device on which VTK-m can run. At the core of the device adapter is a collection of basic algorithms optimized for the specific device. Many features of VTK-m, such as worklets, are built on top of these device algorithms. Using these higher level structures simplifies programming.

However, it is sometimes desirable to run directly run these algorithms provided by the device adapter. VTK-m comes with the templated class vtkm: : cont: : Algorithm that provides a set of algorithms that can be invoked in the control environment and are run on the execution environment. All algorithms also accept an optional device adapter argument.

Algorithm contains no state. It only has a set of static methods that implement its algorithms. The following methods are available.

\section{(0) Did you know?}

$\sum$ Many of the following device adapter algorithms take input and output ArrayHandles, and these functions \ill handle their own memory management. This means that it is unnecessary to allocate output arrays. For \{ example, it is unnecessary to call ArrayHandle: :Allocate for the output array passed to the Algorithm: :Copy method.

\subsection{BitFieldToUnorderedSet}

The Algorithm: :BitFieldToUnorderedSet method creates a unique, unsorted list of indices denoting which bits are set in a bitfield. For example, running BitFieldToUnorderedSet on an input of $[0,0,1,0,1,0,1,1,0,1,1,1]$ would return an array containing $[2,4,6,7,9,10,11]$ or those numbers in some other order.

Example 34.1: Using the BitFieldToUnorderedSet algorithm.

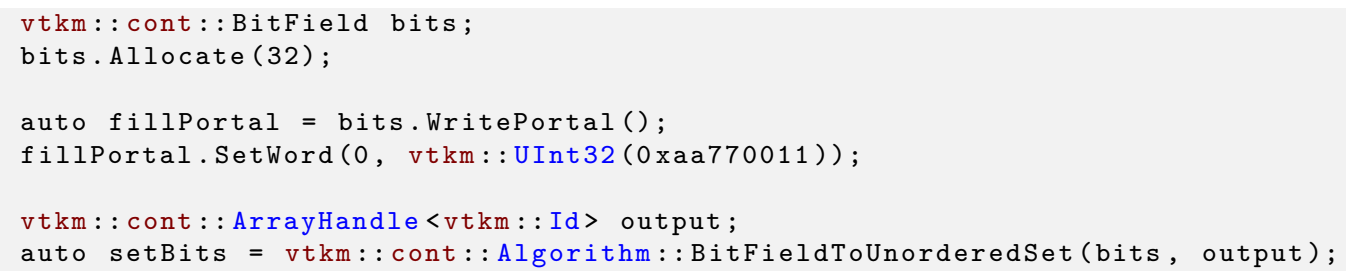




\subsection{Copy}

The Algorithm: : Copy method copies data from an input array to an output array. The copy takes place in the execution environment.

Example 34.2: Using the Copy algorithm.

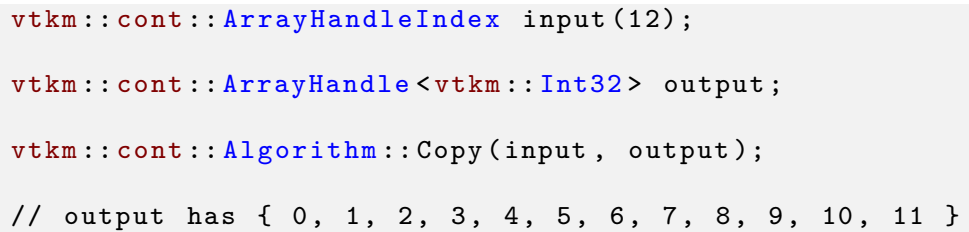

\subsection{Copylf}

The Algorithm: : CopyIf method selectively removes values from an array. The copy if algorithm is also sometimes referred to as stream compact. The first argument, the input, is an ArrayHandle to be compacted (by removing elements). The second argument, the stencil, is an ArrayHandle of equal size with flags indicating whether the corresponding input value is to be copied to the output. The third argument is an output ArrayHandle whose length is set to the number of true flags in the stencil and the passed values are put in order to the output array.

Algorithm: : CopyIf also accepts an optional fourth argument that is a unary predicate to determine what values in the stencil (second argument) should be considered true. See Section 34.23 for more information on unary predicates. The unary predicate determines the true/false value of the stencil that determines whether a given entry is copied. If no unary predicate is given, then CopyIf will copy all values whose stencil value is not equal to 0 (or the closest equivalent to it). More specifically, it copies values not equal to vtkm: :TypeTraits: :ZeroInitialization.

Example 34.3: Using the CopyIf algorithm.

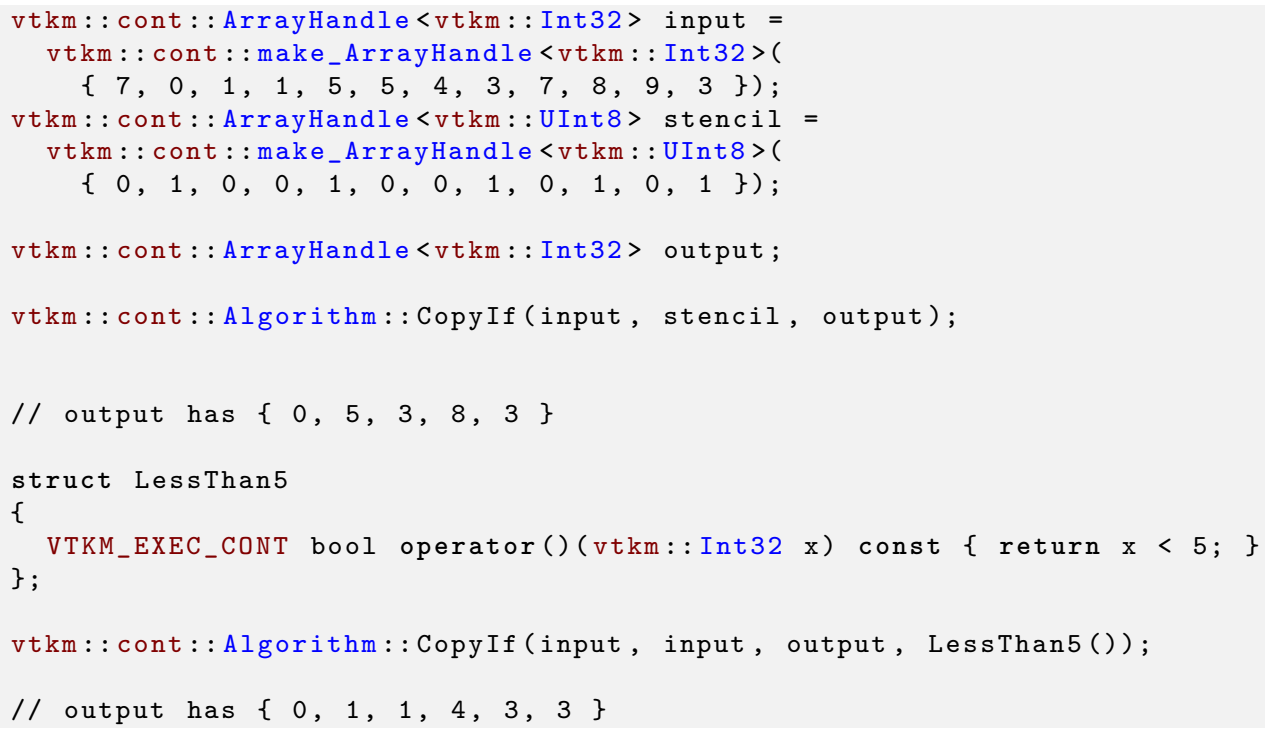




\subsection{CopySubRange}

The Algorithm: :CopySubRange method copies the contents of a section of one ArrayHandle to another. The first argument is the input ArrayHandle. The second argument is the index from which to start copying data. The third argument is the number of values to copy from the input to the output. The fourth argument is the output ArrayHandle, which will be grown if it is not large enough. The fifth argument, which is optional, is the index in the output array to start copying data to. If the output index is not specified, data are copied to the beginning of the output array.

Example 34.4: Using the CopySubRange algorithm.

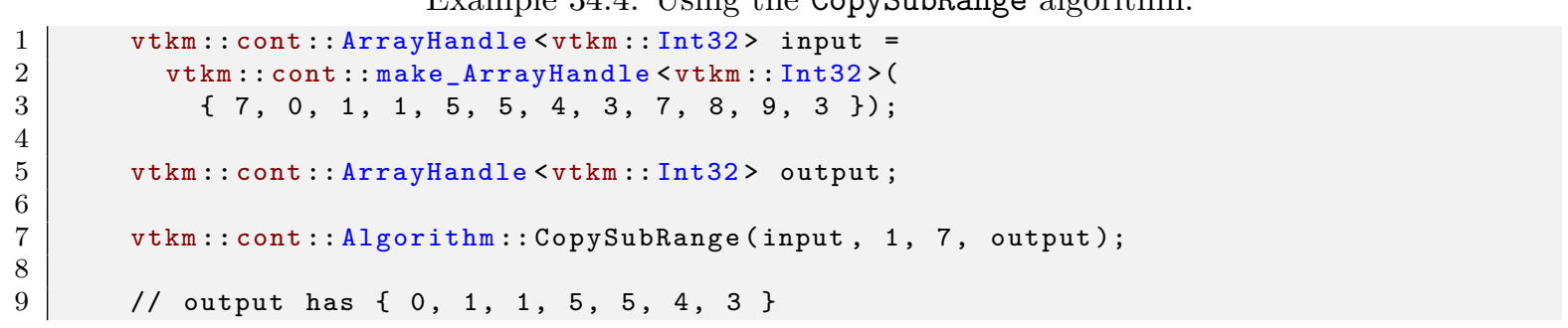

\subsection{CountSetBits}

The Algorithm: : CountSetBits method returns the total number of set bits in a BitField. For example, running BitFieldToUnorderedSet on an input of $[0,0,1,0,1,0,1,1,0,1,1,1]$ would return 7 .

Example 34.5: Using the CountSetBits algorithm.

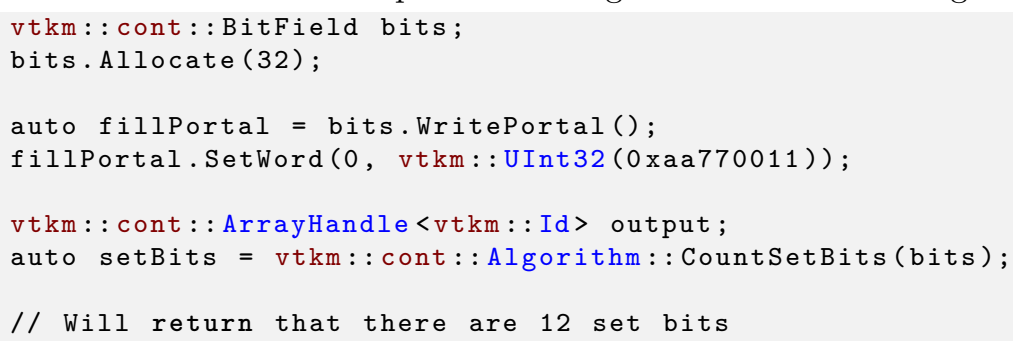

\subsection{Fill}

The Algorithm: :Fill methods fill a BitField or ArrayHandle with a specific pattern of bits/values. For a BitField, it is possible to supply a boolean value or a WordType. For boolean values, all bits are set to 1 if the value is true, 0 if the value is false. For word masks, the WordType must be an unsigned integral type; this value is stamped across the BitField. For a ArrayHandle, the entire array is filled with the provided value. For both types, if a numValues argument is provided the array is resized appropriately and filled with the given value.

Example 34.6: Using the Fill algorithm.

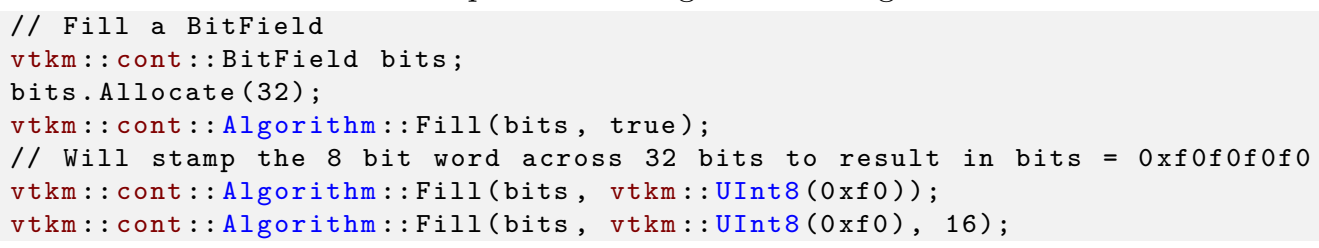




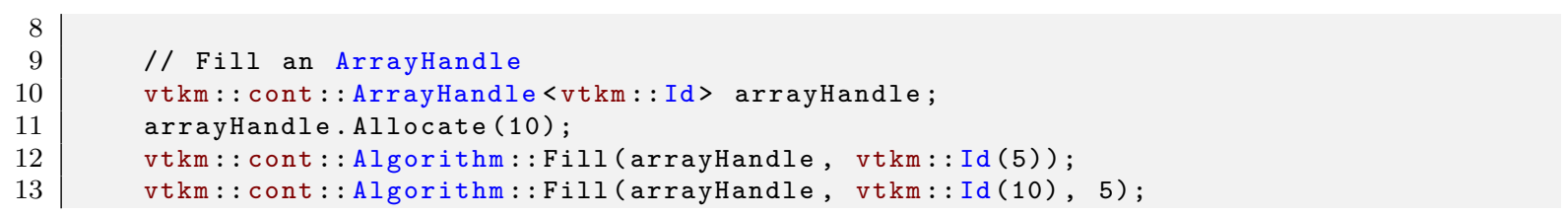

\subsection{LowerBounds}

The Algorithm: :LowerBounds method takes three arguments. The first argument is an ArrayHandle of sorted values. The second argument is another ArrayHandle of items to find in the first array. LowerBounds find the index of the first item that is greater than or equal to the target value, much like the std: :lower_bound STL algorithm. The results are returned in an ArrayHandle given in the third argument.

There are two specializations of Algorithm: :LowerBounds. The first takes an additional comparison function that defines the less-than operation. The second specialization takes only two parameters. The first is an ArrayHandle of sorted vtkm: :Id s and the second is an ArrayHandle of vtkm::Id to find in the first list. The results are written back out to the second array. This second specialization is useful for inverting index maps.

Example 34.7: Using the LowerBounds algorithm.

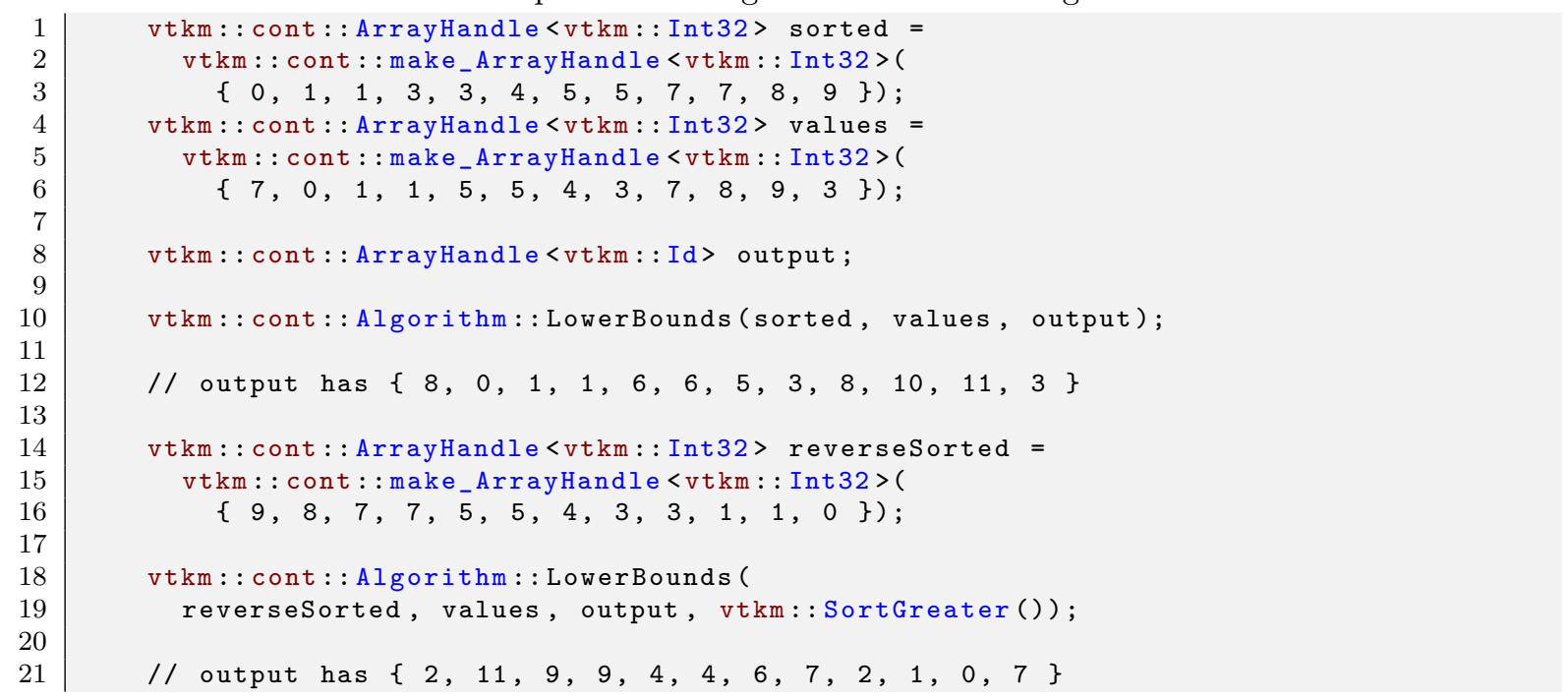

\subsection{Reduce}

The Algorithm: : Reduce method takes an input array, initial value, and a binary function and computes a "total" of applying the binary function to all entries in the array. The provided binary function must be associative (but it need not be commutative). There is a specialization of Reduce that does not take a binary function and computes the sum.

Example 34.8: Using the Reduce algorithm.

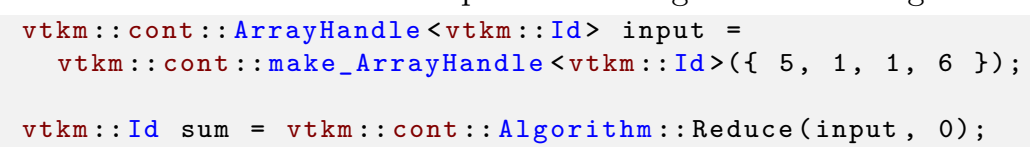




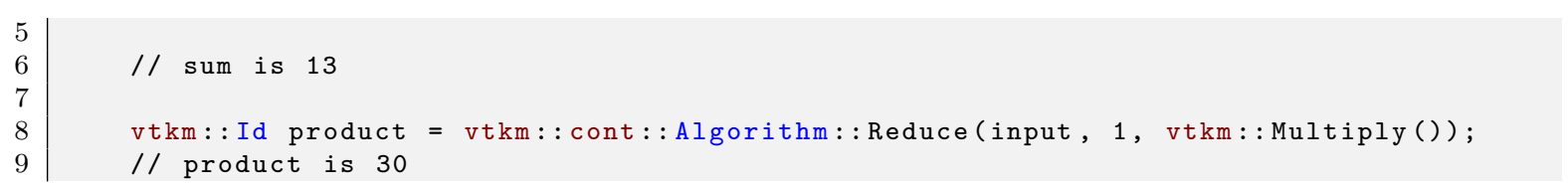

\subsection{ReduceByKey}

The Algorithm: : ReduceByKey method works similarly to the Reduce method except that it takes an additional array of keys, which must be the same length as the values being reduced. The arrays are partitioned into segments that have identical adjacent keys, and a separate reduction is performed on each partition. The unique keys and reduced values are returned in separate arrays.

Example 34.9: Using the ReduceByKey algorithm.

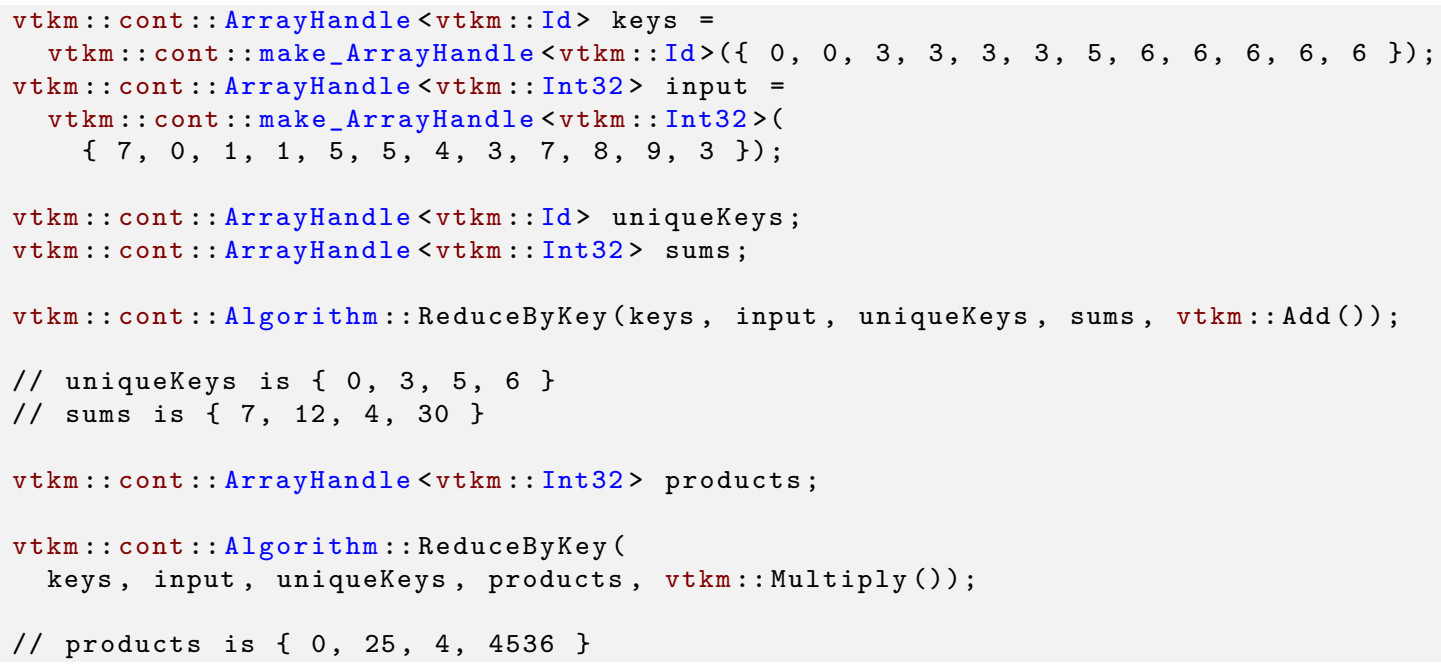

\subsection{Scanlnclusive}

The Algorithm: :ScanInclusive method takes an input and an output ArrayHandle and performs a running sum on the input array. For inclusive scans, the running sum value for position $i$ in the input array includes the element at position $i$. The first value in the output is the same as the first value in the input. The second value in the output is the sum of the first two values in the input. The third value in the output is the sum of the first three values of the input, and so on. If the input and output array are the same, then the operation is done in place. ScanInclusive returns the sum of all values in the input. There are two forms of ScanInclusive: one performs the sum using addition whereas the other accepts a custom binary function to use as the sum operator.

Example 34.10: Using the ScanInclusive algorithm.

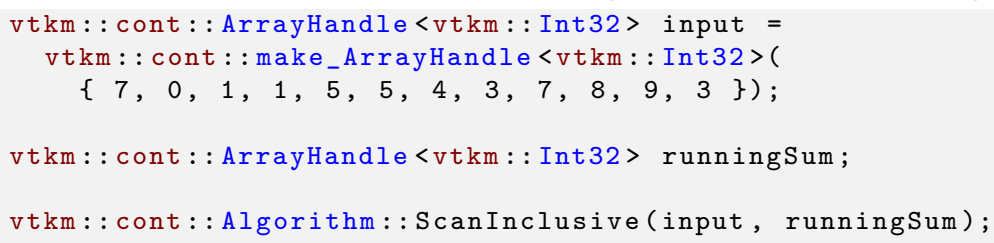




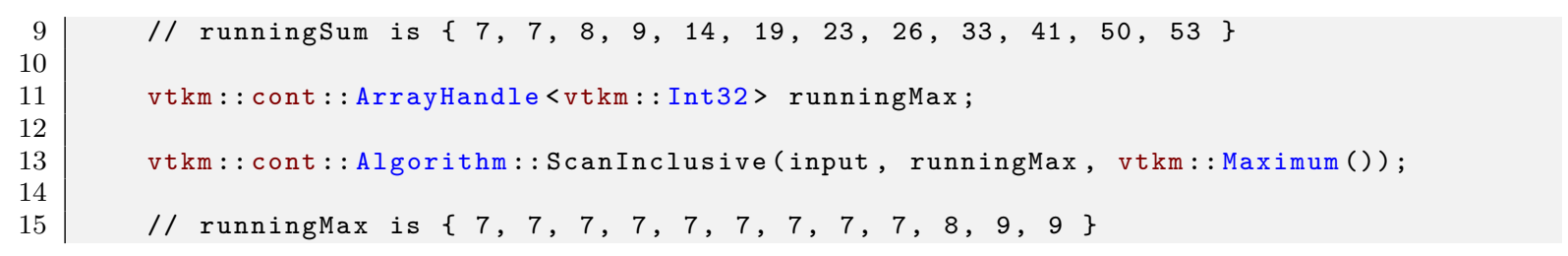

\subsection{ScanInclusiveByKey}

The Algorithm: :ScanInclusiveByKey method works similarly to the ScanInclusive method except that it takes an additional array of keys, which must be the same length as the values being scanned. The arrays are partitioned into segments that have identical adjacent keys, and a separate scan is performed on each partition. Only the scanned values are returned.

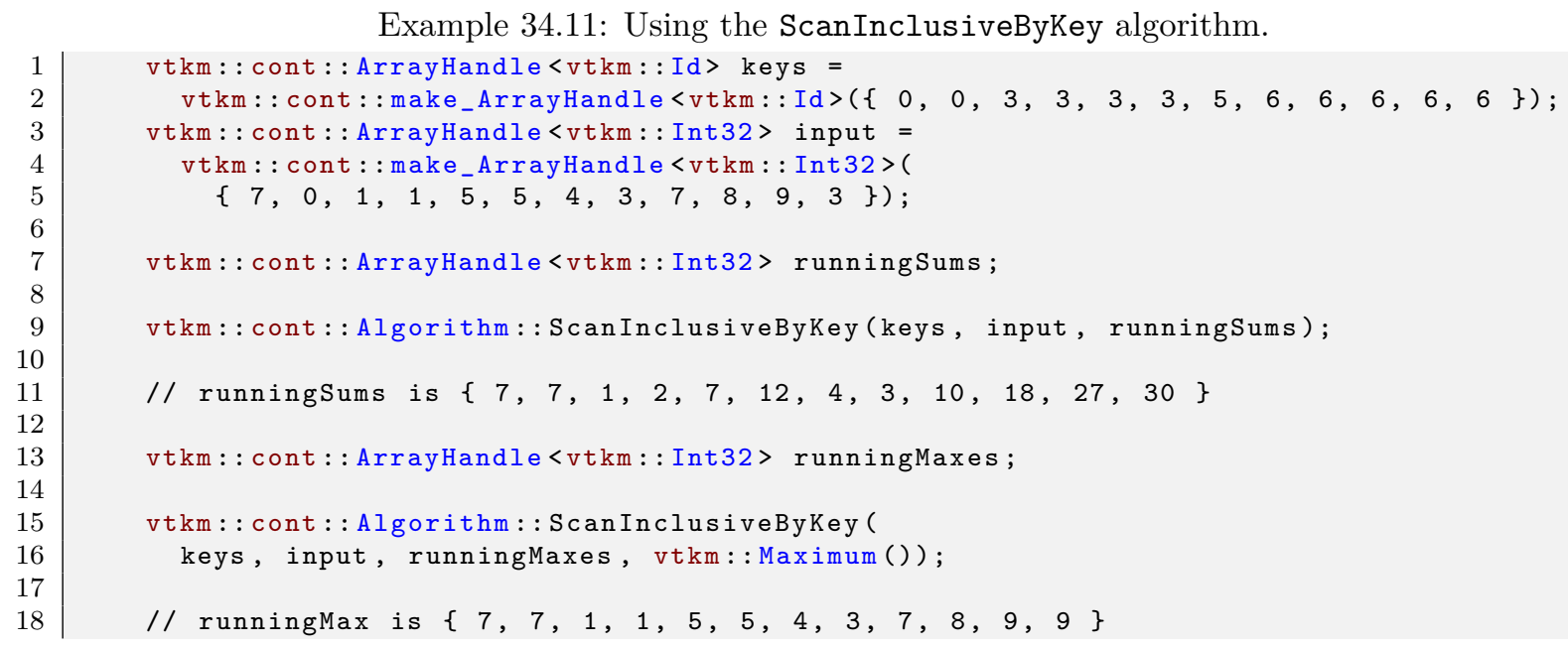

\subsection{ScanExclusive}

The Algorithm: :ScanExclusive method takes an input and an output ArrayHandle and performs a running sum on the input array. For exclusive scans, the running sum value for position $i$ in the input array excludes the element at position $i$. The first value in the output is always 0 . The second value in the output is the same as the first value in the input. The third value in the output is the sum of the first two values in the input. The fourth value in the output is the sum of the first three values of the input, and so on. ScanExclusive returns the sum of all values in the input. If the input and output array are the same, then the operation is done in place. There are two forms of ScanExclusive. The first performs the sum using addition. The second form accepts a custom binary functor to use as the "sum" operator and a custom initial value (instead of 0 ).

Example 34.12: Using the ScanExclusive algorithm.

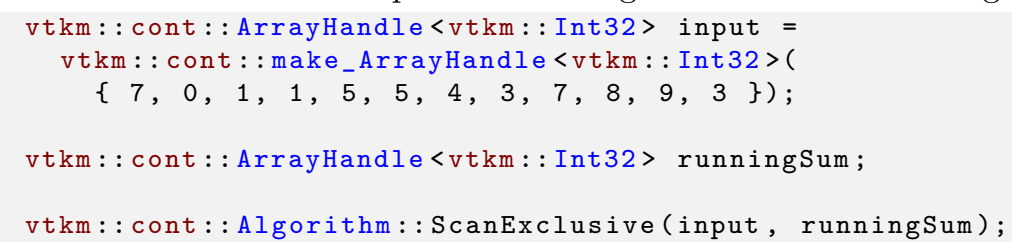




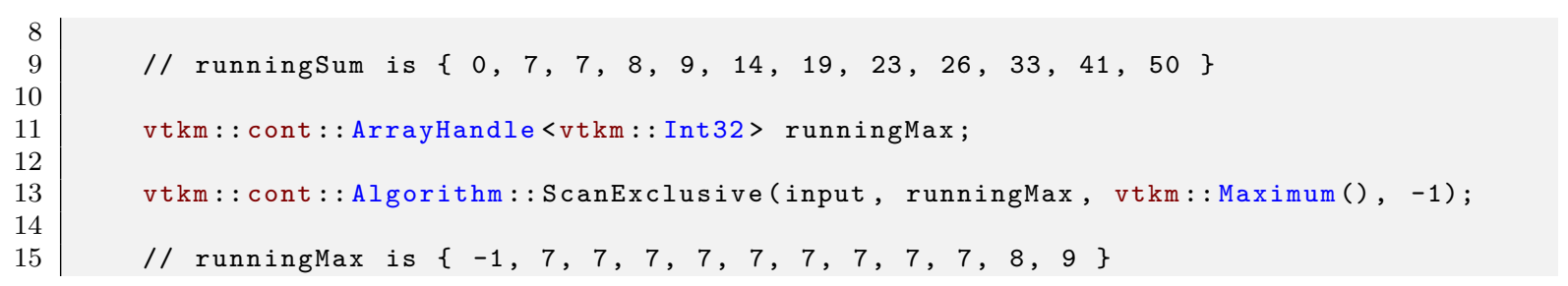

\subsection{ScanExclusiveByKey}

The Algorithm: :ScanExclusiveByKey method works similarly to the ScanExclusive method except that it takes an additional array of keys, which must be the same length as the values being scanned. The arrays are partitioned into segments that have identical adjacent keys, and a separate scan is performed on each partition. Only the scanned values are returned.

Example 34.13: Using ScanExclusiveByKey algorithm.

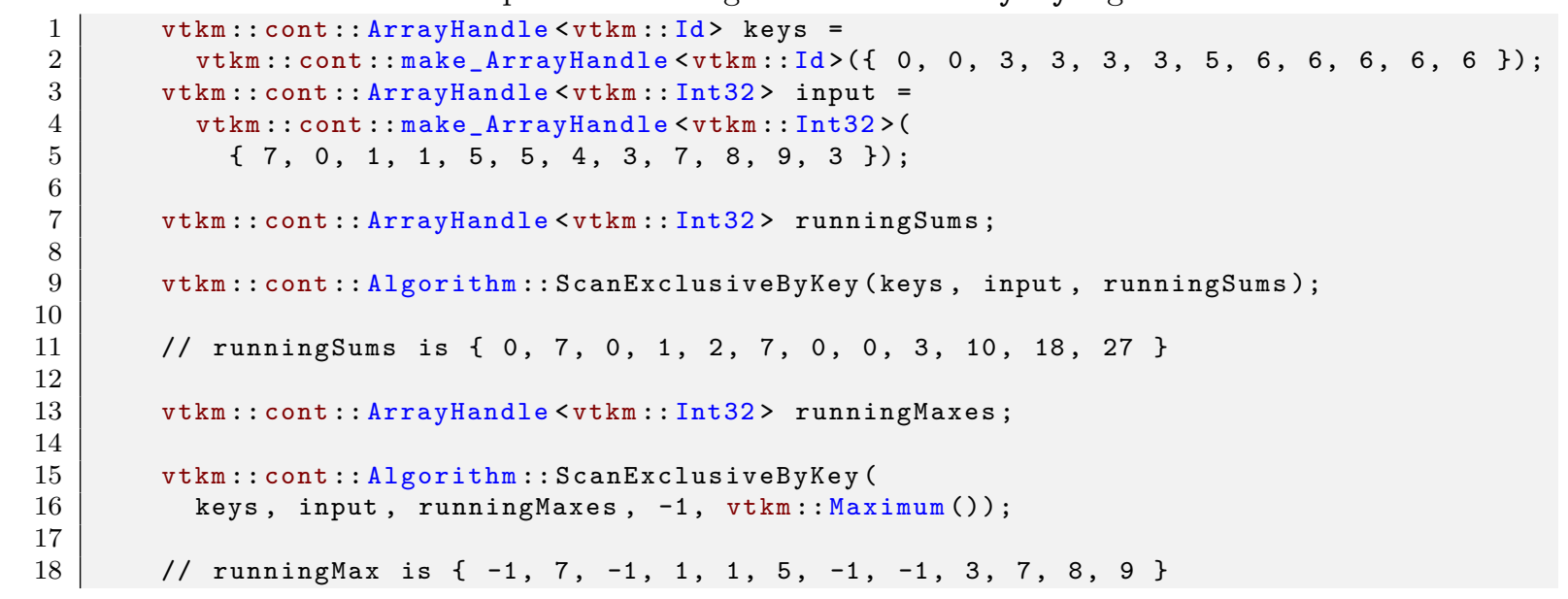

\subsection{ScanExtended}

The Algorithm: ScanExtended computes an extended prefix sum operation on the input ArrayHandle and stores it in a provided output ArrayHandle. The output array has length 1 greater than the input array. Algorithm::ScanExtended is a combination of the Algorithm: ScanInclusive and Algorithm::ScanExclusive methods. The exclusive scan values are stored in indices 0 through size -1 . The inclusive scan values are stored in indices 1 through size. The first entry in the resulting array is 0 (or the specified initial value) like with the exclusive scan. The last entry in the resulting array is the sum total like with the inclusive scan. Unlike the two referenced methods, Algorithm: :ScanExtended does not return the total sum. By using an ArrayHandleView, arrays containing both inclusive and exclusive scans can be generated from an extended scan with minimal memory usage by referencing the correct indices in the extended scan output.

This algorithm may be more efficient than Algorithm::ScanInclusive and Algorithm::ScanExclusive on some devices; this algorithm may be able to avoid copying the total sum to the control environment to return. Algorithm: :ScanExtended is similar to the STL partial sum function, with the exception that it does not perform a serial summation. This means that if you have defined a custom plus operator for $\mathrm{T}$ it must be associative, or you will get inconsistent results. 
The first form performs the sum using addition. The second form accepts a custom binary functor to use as the operator and a custom initial value (instead of 0 ).

Example 34.14: Using ScanExtended algorithm.

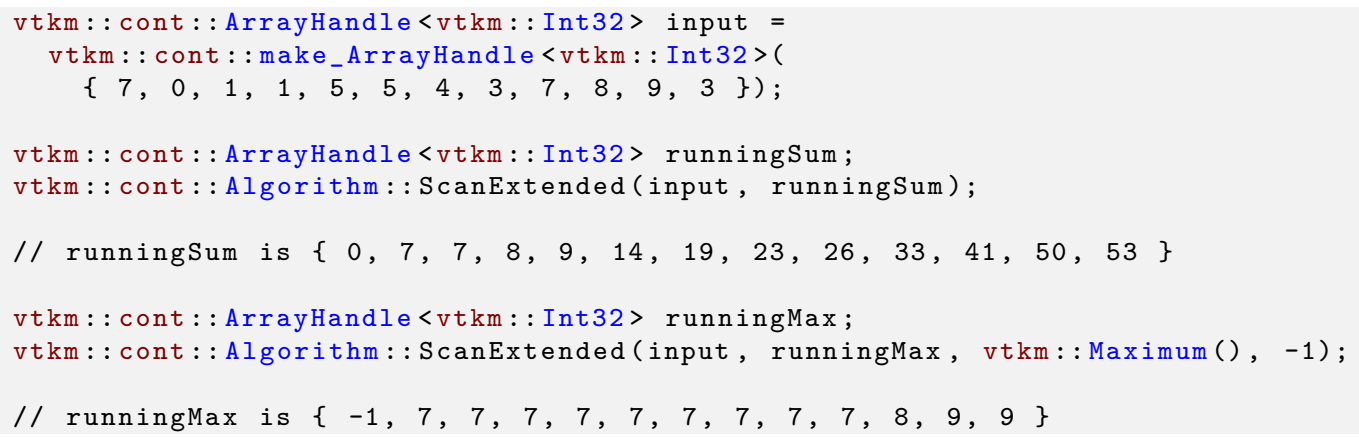

ScanExtended can be used to create a running sum that is quickly reversible. If you subtract to consecutive values of a scan you get back the original value. This is convenient if you need both the input and output of a scan; you can throw away the input and use differences of the output. However, ScanInclusive does not write out the initial value, so you cannot get back the original value at the beginning without a special condition. Likewise, ScanExclusive does not write out the total sum, so you cannot get back the original value at the end without a special condition. ScanExtended solves this problem by extending the array by 1 . The original value for index $i$ can be retrieved by subtracting scan value at index $i$ from the value at index $i+1$ anywhere in the array, including the at the begin and end. This is particularly useful for storing packed arrays in structures like vtkm: : cont: :CellSetExplicit and vtkm: : cont: :ArrayHandleGroupVecVariable.

\subsection{Schedule}

The Algorithm: : Schedule method takes a functor as its first argument and invokes it a number of times specified by the second argument. It should be assumed that each invocation of the functor occurs on a separate thread although in practice there could be some thread sharing.

There are two versions of the Schedule method. The first version takes a vtkm: :Id and invokes the functor that number of times. The second version takes a vtkm: :Id3 and invokes the functor once for every entry in a 3D array of the given dimensions.

The functor is expected to be an object with a const overloaded parentheses operator. The operator takes as a parameter the index of the invocation, which is either a vtkm: :Id or a vtkm: : Id3 depending on what version of Schedule is being used. The functor must also subclass vtkm: :exec: :FunctorBase, which provides the error handling facilities for the execution environment. FunctorBase contains a public method named RaiseError that takes a message and will cause a vtkm: :cont::ErrorExecution exception to be thrown in the control environment.

\subsection{Sort}

The Algorithm: : Sort method provides an unstable sort of an array. There are two forms of the Sort method. The first takes an ArrayHandle and sorts the values in place. The second takes an additional argument that is a functor that provides the comparison operation for the sort.

Example 34.15: Using the Sort algorithm. 


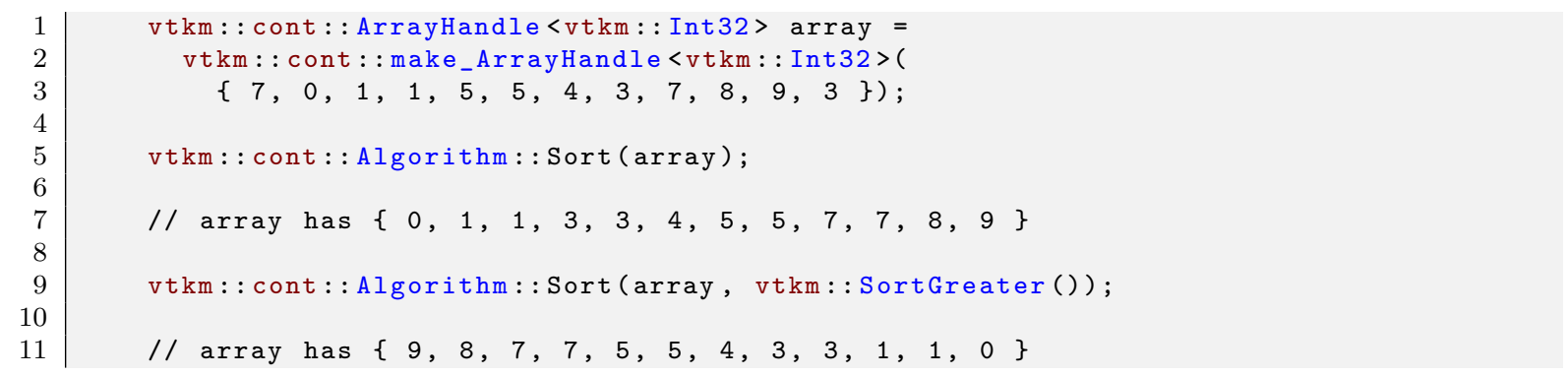

\subsection{SortByKey}

The Algorithm: : SortByKey method works similarly to the Sort method except that it takes two ArrayHandles: an array of keys and a corresponding array of values. The sort orders the array of keys in ascending values and also reorders the values so they remain paired with the same key. Like Sort, SortByKey has a version that sorts by the default less-than operator and a version that accepts a custom comparison functor.

Example 34.16: Using the SortByKey algorithm.

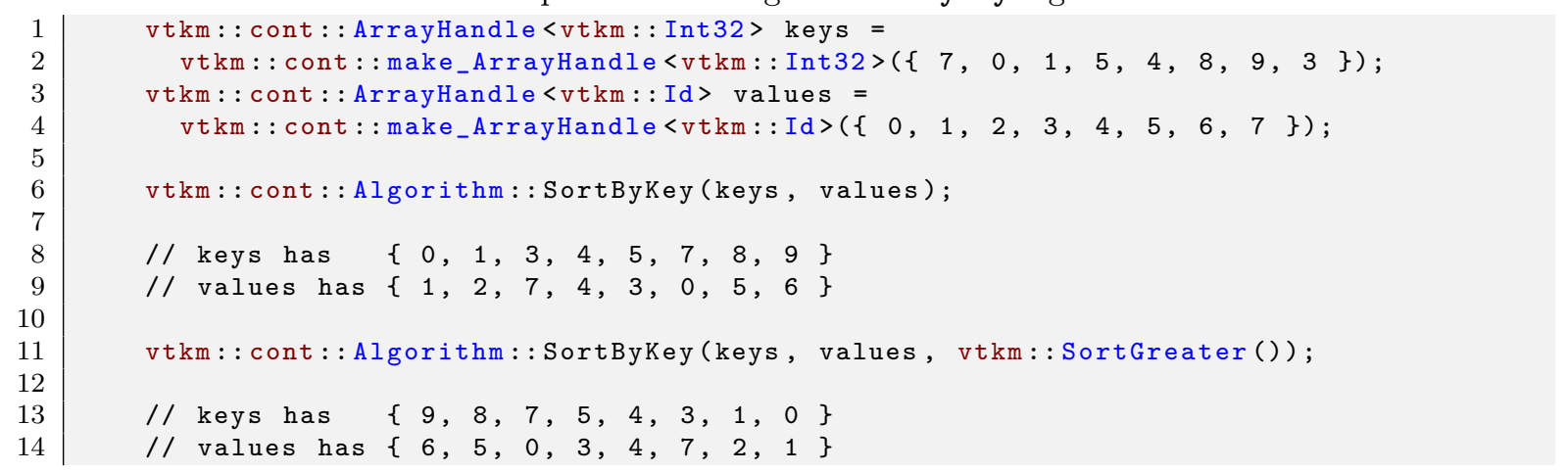

\subsection{Synchronize}

The Synchronize method waits for any asynchronous operations running on the device to complete and then returns.

\subsection{Transform}

The Algorithm: :Transform method applies a given binary operation function element-wise on two input arrays, storing the result in a provided output array. The number of elements in the input arrays do not have to be the same; the output array will have the same number of elements as the smaller of the two input arrays.

Example 34.17: Using the Transform algorithm.

1
2
3
4
5

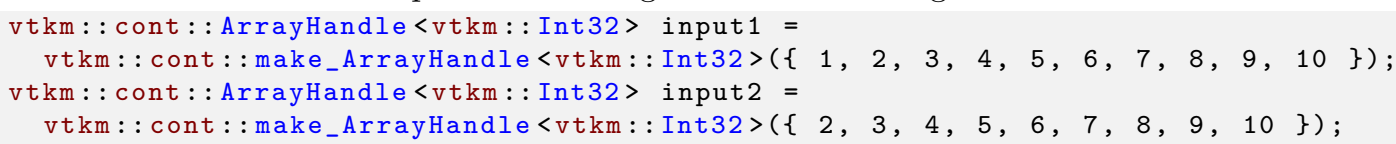




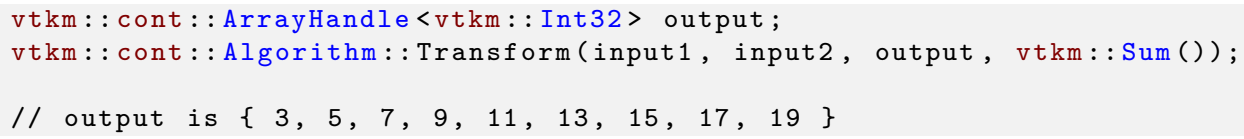

\subsection{Unique}

The Algorithm: :Unique method removes all duplicate values in an ArrayHandle. The method will only find duplicates if they are adjacent to each other in the array. The easiest way to ensure that duplicate values are adjacent is to sort the array first.

There are two versions of Unique. The first uses the equals operator to compare entries. The second accepts a binary functor to perform the comparisons.

Example 34.18: Using the Unique algorithm.

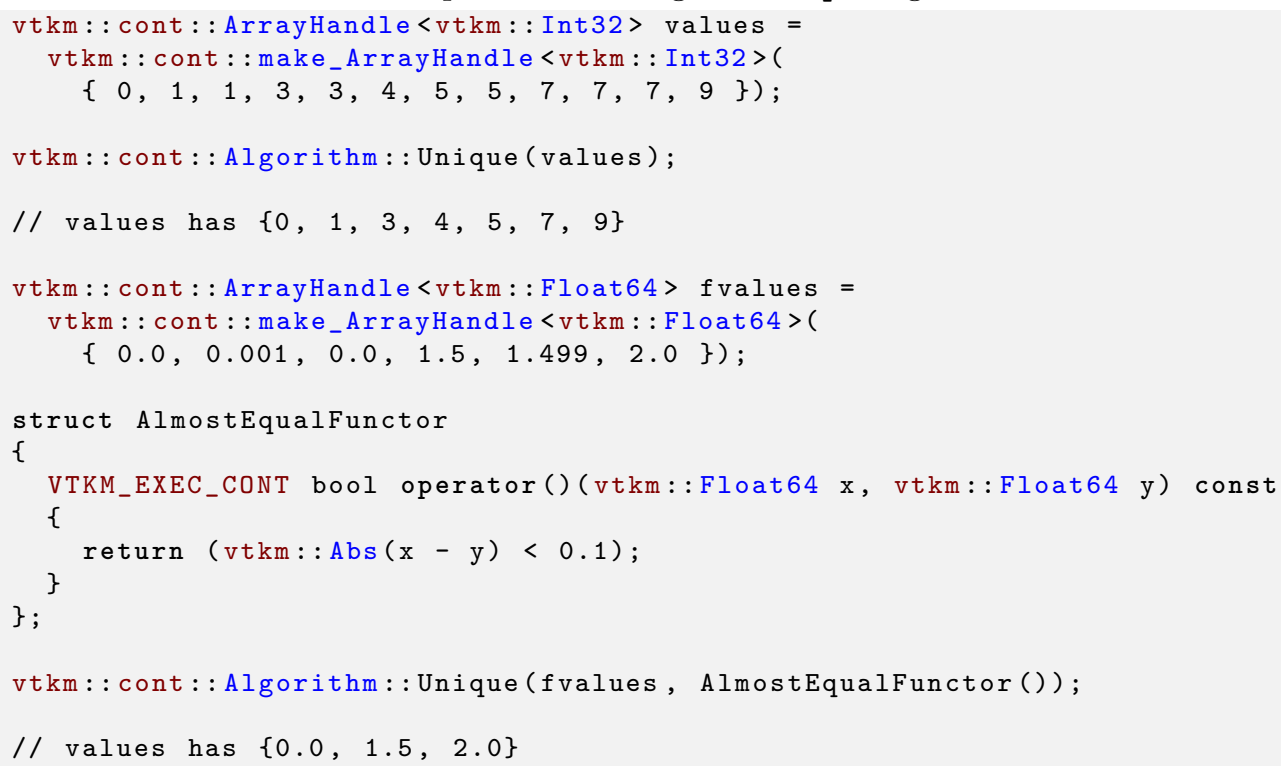

\subsection{UpperBounds}

The Algorithm: :UpperBounds method takes three arguments. The first argument is an ArrayHandle of sorted values. The second argument is another ArrayHandle of items to find in the first array. UpperBounds find the index of the first item that is greater than to the target value, much like the std: : upper_bound STL algorithm. The results are returned in an ArrayHandle given in the third argument.

There are two specializations of UpperBounds. The first takes an additional comparison function that defines the less-than operation. The second takes only two parameters. The first is an ArrayHandle of sorted vtkm: :Id $\mathrm{s}$ and the second is an ArrayHandle of vtkm: :Id s to find in the first list. The results are written back out to the second array. This second specialization is useful for inverting index maps.

Example 34.19: Using the UpperBounds algorithm.

\footnotetext{
1 vtkm: : cont: : ArrayHandle <vtkm:: Int32> sorted =

2 vtkm: : cont: : make_ArrayHandle<vtkm: :Int $32>$ (
} 


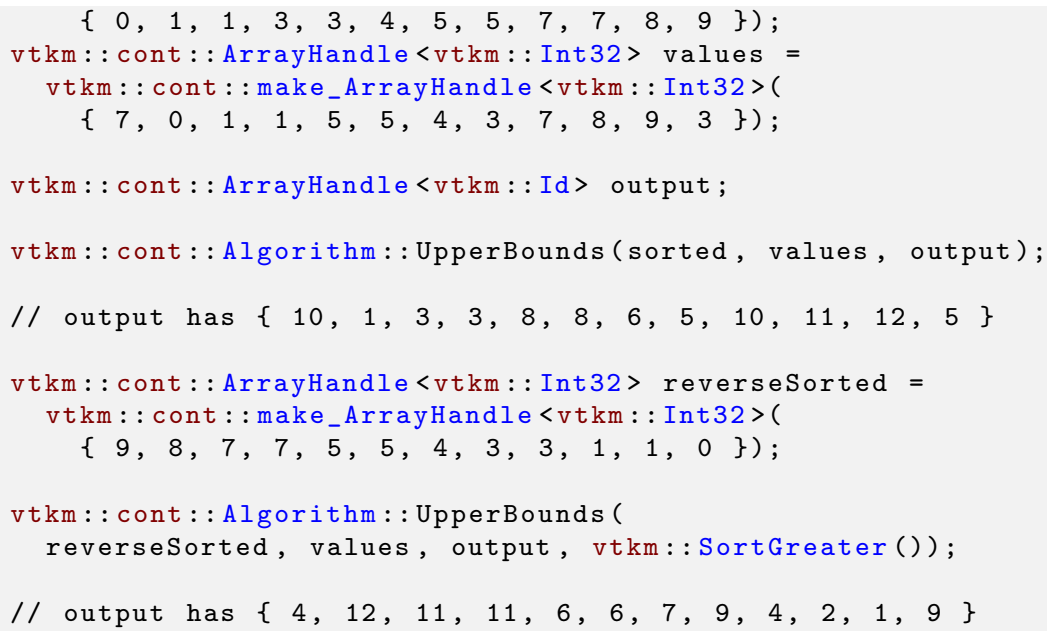

\subsection{Specifying the Device Adapter}

When you call a method in vtkm: :cont: :Algorithm, a device is automatically specified based on available hardware and the VTK-m state. However, if you want to use a specific device, you can specify that device by passing either a vtkm: :cont: :DeviceAdapterId or a device adapter tag as the first argument to any of these methods.

Example 34.20: Using the DeviceAdapter with vtkm: : cont: :Algorithm.

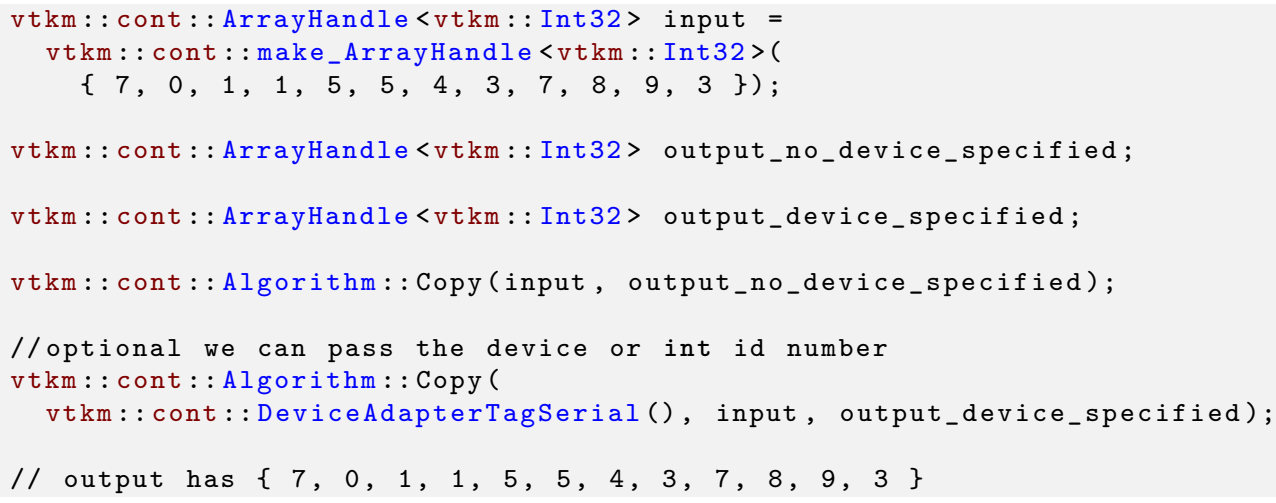

\subsection{Predicates and Operators}

VTK-m follows certain design philosophies consistent with the functional programming paradigm. This assists in making implementations device agnostic and ensuring that various functions operate correctly and efficiently in multiple environments. Many basic operations, such as binary and unary comparisons and predicates, are implemented as templated functors. These are mostly re-implementations of basic C++ STL functors that can be used in the VTK-m execution environment.

Strictly using a functor by itself adds little in the way of functionality to the code. Their use is demonstrated more when used as parameters to one of the vtkm: : cont: : Algorithm methods discussed earlier in this chapter. 
Currently, VTK-m provides 3 categories of functors: Unary Predicates, Binary Predicates, and Binary Operators.

\subsubsection{Unary Predicates}

Unary Predicates are functors that take a single parameter and return a Boolean value. These types of functors are useful in determining if values have been initialized or zeroed out correctly.

vtkm: : IsZeroInitialized Returns True if argument is the identity of its type.

vtkm: : NotZeroInitialized Returns True if the argument is not the identify of its type.

vtkm: : LogicalNot Returns True iff the argument is False. Requires that the argument type is convertible to a Boolean or implements the ! operator.

Example 34.21: Basic Unary Predicate.

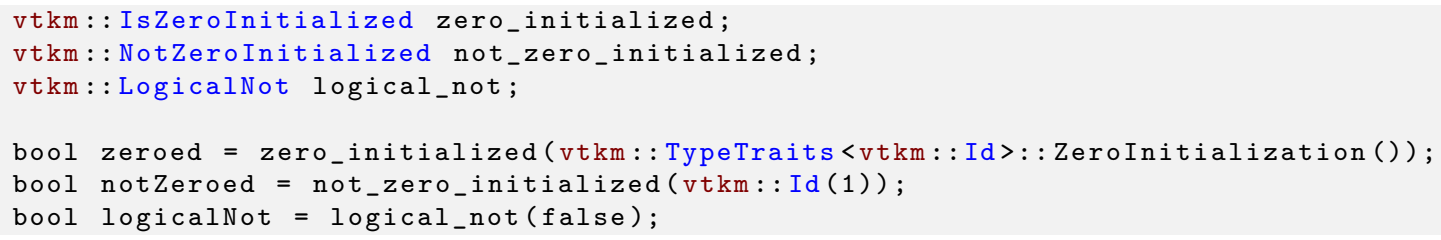

\subsubsection{Binary Predicates}

Binary Predicates take two parameters and return a single Boolean value. These types of functors are used when comparing two different parameters for some sort of condition.

vtkm: :Equal Returns True iff the first argument is equal to the second argument. Requires that the argument type implements the $==$ operator.

vtkm: : NotEqual Returns True iff the first argument is not equal to the second argument. Requires that the argument type implements the $!=$ operator.

vtkm: :SortLess Returns True iff the first argument is less than the second argument. Requires that the argument type implements the < operator.

vtkm: : SortGreater Returns True iff the first argument is greater than the second argument. Requires that the argument type implements the < operator (the comparison is inverted internally).

vtkm: :LogicalAnd Returns True iff the first argument and the second argument are True. Requires that the argument type is convertible to a Boolean or implements the \&\& operator.

vtkm::LogicalOr Returns True iff the first argument or the second argument is True. Requires that the argument type is convertible to a Boolean or implements the II operator.

Example 34.22: Basic Binary Predicate.

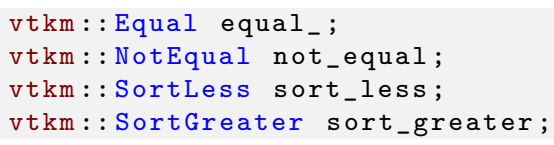




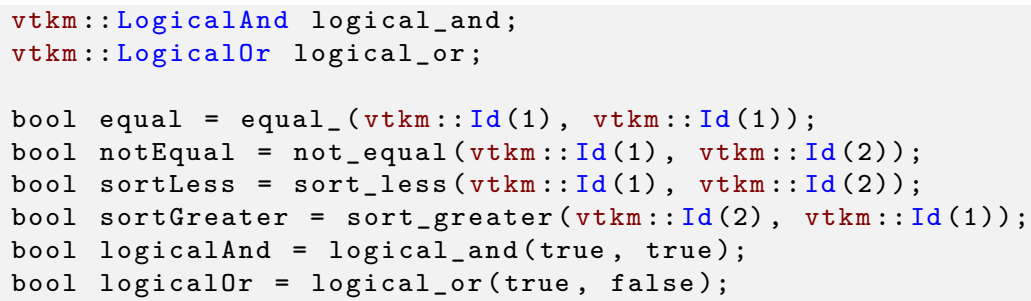

\subsubsection{Binary Operators}

Binary Operators take two parameters and return a single value (usually of the same type as the input arguments). These types of functors are useful when performing reductions or transformations of a dataset.

vtkm: : Sum Returns the sum of two arguments. Requires that the argument type implements the + operator.

vtkm: :Product Returns the product (multiplication) of two arguments. Requires that the argument type implements the $*$ operator.

vtkm: :Maximum Returns the larger of two arguments. Requires that the argument type implements the < operator.

vtkm: :Minimum Returns the smaller of two arguments. Requires that the argument type implements the < operator.

vtkm: :MinAndMax Returns a vtkm::Vec $<\mathrm{T}, 2>$ that represents the minimum and maximum values. Requires that the argument type implements the vtkm: :Min and vtkm: :Max functions.

vtkm: :BitwiseAnd Returns the bitwise and of two arguments. Requires that the argument type implements the \& operator.

vtkm::Bitwise0r Returns the bitwise or of two arguments. Requires that the argument type implements the I operator.

vtkm: :BitwiseXor Returns the bitwise xor of two arguments. Requires that the argument type implements the ${ }^{\wedge}$ operator.

Example 34.23: Basic Binary Operator.

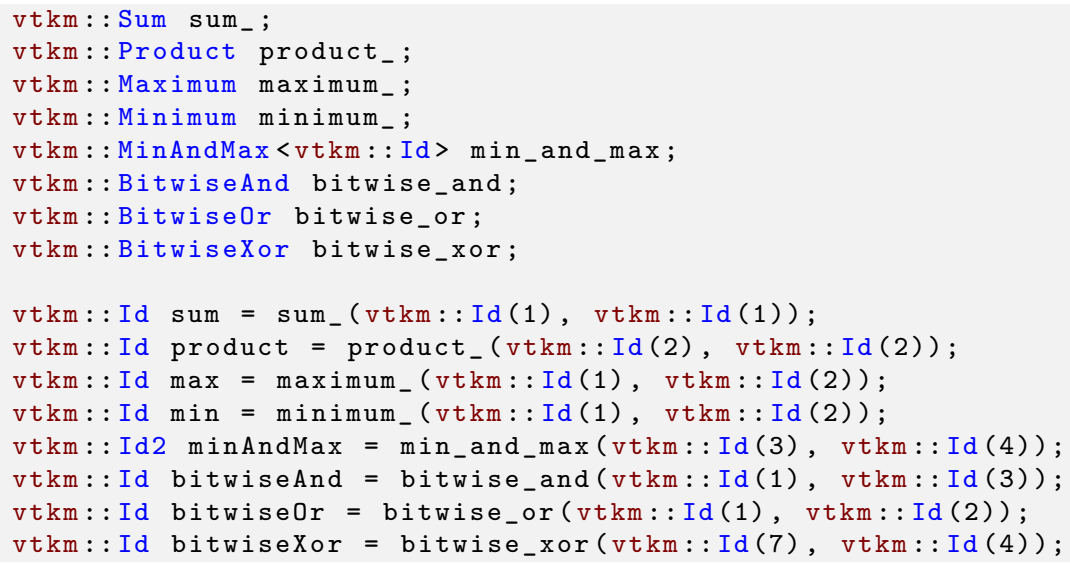




\subsubsection{Creating Custom Comparators}

In addition to using the built in operators and predicates, it is possible to create your own custom functors to be used in one of the vtkm: :cont: :Algorithm. Custom operator and predicate functors can be used to apply specific logic used to manipulate your data. The following example creates a unary predicate that checks if the input is a power of 2 .

Example 34.24: Custom Unary Predicate Implementation.

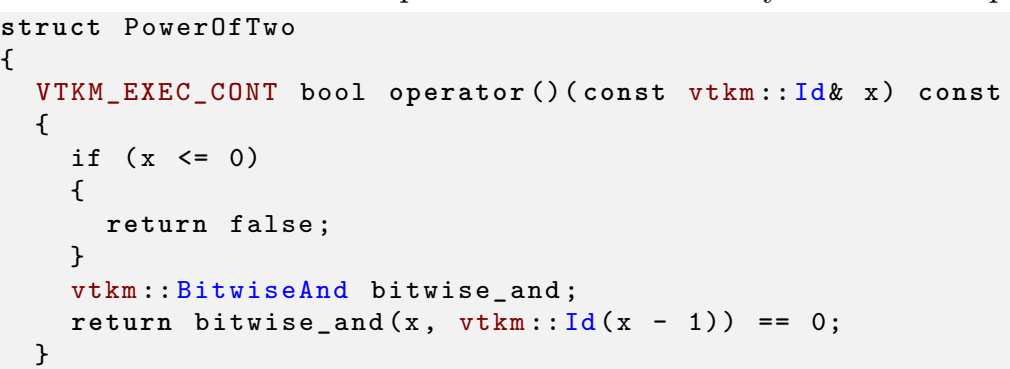

Example 34.25: Custom Unary Predicate Usage.

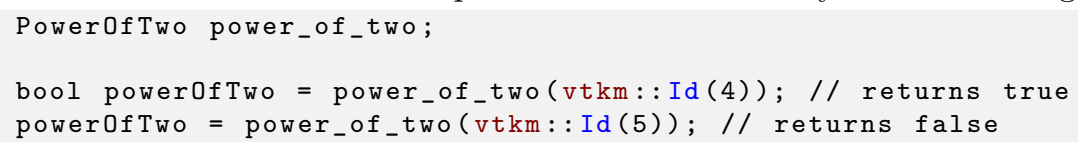




\section{CUSTOM ARRAY STORAGE}

Chapters 16, 26, and 27 introduce the vtkm: :cont: :ArrayHandle class. In them, we learned how an ArrayHandle manages the memory allocation of an array, provides access to the data via array portals, and supervises the movement of data between the control and execution environments.

In addition to these data management features, ArrayHandle also provides a configurable storage mechanism that allows you, through efficient template configuration, to redefine how data are stored and retrieved. The storage object provides an encapsulated interface around the data so that any necessary strides, offsets, or other access patterns may be handled internally. The relationship between array handles and their storage object is shown in Figure 35.1.
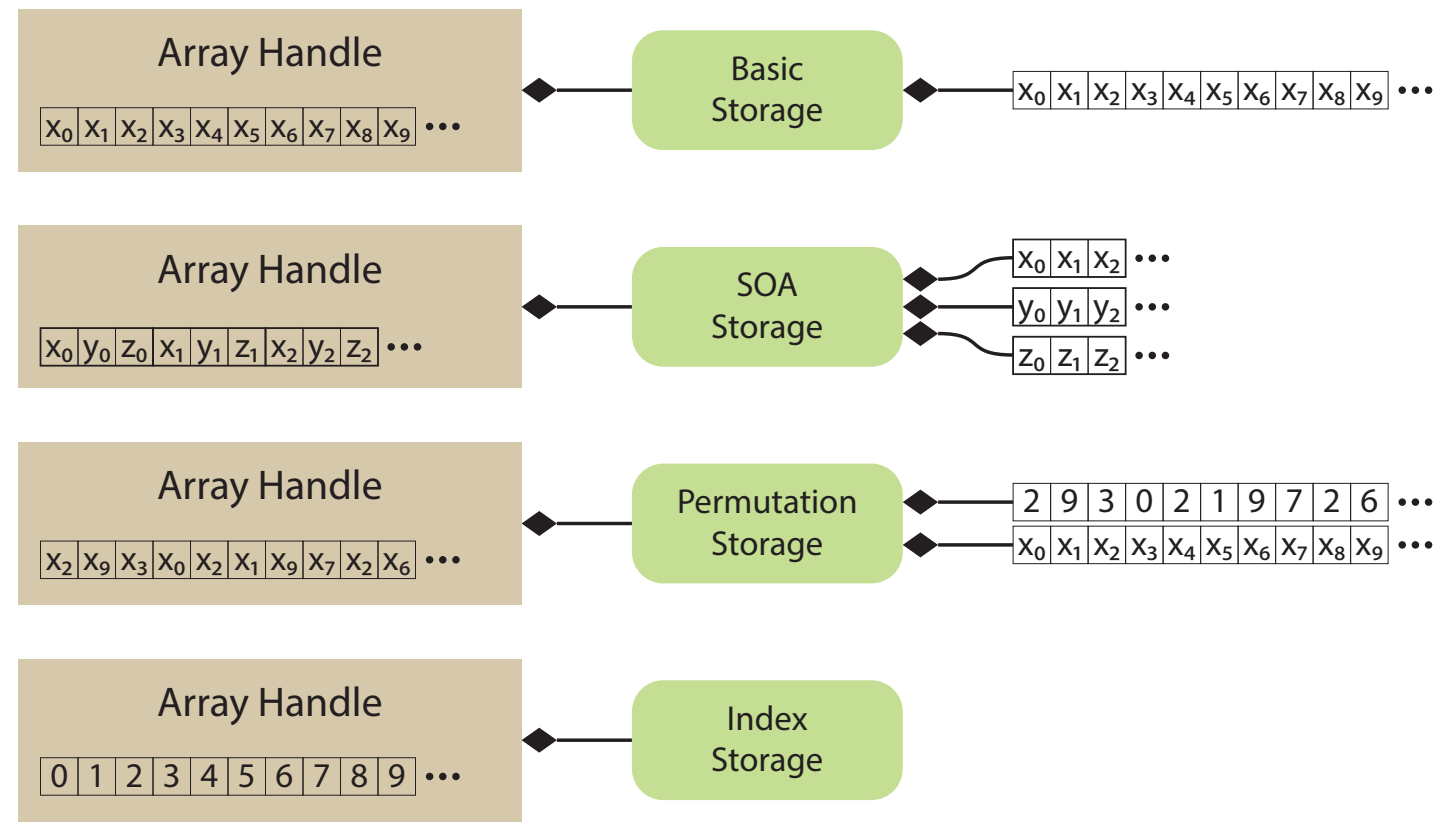

Figure 35.1: Array handles, storage objects, and the underlying data source.

As previously discussed in Chapter 16, vtkm: : cont: : ArrayHandle takes two template arguments.

Example 35.1: Declaration of the vtkm: : cont: : ArrayHandle templated class (again).

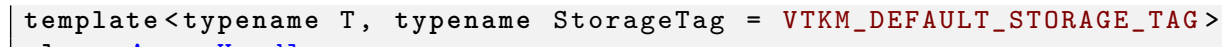


The first argument is the only one required and has been demonstrated multiple times before. The second (optional) argument specifies something called a storage, which provides the interface between the generic vtkm: :cont: : ArrayHandle class and a specific storage mechanism in the control environment. If the storage parameter is not explicitly defined, it is set to VTKM_DEFAULT_STORAGE_TAG, which is a macro that resolves to vtkm: :cont: : StorageTagBasic.

The default storage can always be overridden by specifying an array storage tag. Here is an example of specifying the storage type when declaring an array handle.

Example 35.2: Specifying the storage type for an ArrayHandle.

1 vtkm::cont: :ArrayHandle<vtkm::Float32, vtkm:: cont: :StorageTagBasic> arrayHandle;

Although setting an ArrayHandle's storage explicitly to StorageTagBasic as in Example 35.2 is seldom useful (since this is the default value), setting the storage is a good way to propagate the storage mechanism through template parameters. The remainder of this chapter uses the storage mechanism to customize the representation of arrays.

By replacing the storage template parameter for ArrayHandle, we can change how data are stored in memory. For example, when storing vtkm: :Vec objects, the basic storage writes all vtkm: :Vec s sequentially in a single array. This is known as an array of structures. An alternate representation would be to store each component of the vtkm: Vec $\mathrm{s}$ in a separate array. This alternate layout is known as a structure of arrays. There are reasons one might want to represent vector data in a structure of arrays, and VTK-m provides a separate storage to implement that: vtkm: : cont: :StorageTagSOA. From an interface perspective, the two ArrayHandles behave the same, but they have very different implementations.

As is typical of different types of storage, the basic and SOA storage types have convenience ArrayHandle subclasses: vtkm: : cont: :ArrayHandleBasic and vtkm: : cont: : ArrayHandleSOA, respectively. These are trivial subclasses of vtkm::cont: ArrayHandle with the appropriate storage, but provide convenience methods for construction and data access.

One interesting consequence of using a generic storage object to manage data within an array handle is that the storage can be defined functionally rather than point to data stored in physical memory. Thus, implicit array handles are easily created by adapting to functional "storage." For example, the point coordinates of a uniform rectilinear grid are implicit based on the topological position of the point. Thus, the point coordinates for uniform rectilinear grids can be implemented as an implicit array with the same interface as explicit arrays (where unstructured grid points would be stored). Many examples of this are listed in Chapter 26.

In this chapter we explore the many ways you can manipulate the ArrayHandle storage. There are many ways to create custom storage for ArrayHandle. As we explore these different ways, we will start with the easiest but most restrictive ways and move to the most expressive ways.

\subsection{Implicit Array Handles}

The generic array handle and storage templating in VTK-m allows for any type of operations to retrieve a particular value. Typically this is used to convert an index to some location or locations in memory. However, it is also possible to compute a value directly from an index rather than look up some value in memory. Such an array is completely functional and requires no storage in memory at all. Such a functional array is called an implicit array handle. Implicit arrays are an example of fancy array handles, which are array handles that behave like regular arrays but do special processing under the covers to provide values.

Specifying a functional or implicit array in VTK-m is straightforward. VTK-m has a special class named vtkm: : cont: : ArrayHandleImplicit that makes an implicit array containing values generated by a user-specified functor. A functor is simply a $\mathrm{C}++$ class or struct that contains an overloaded parenthesis operator so that it 
can be used syntactically like a function.

To demonstrate the use of ArrayHandleImplicit, let us say we want an array of even numbers. The array has the values $[0,2,4,6, \ldots]$ (double the index) up to some given size. Although we could easily create this array in memory, we can save space and possibly time by computing these values on demand.

\section{Did you know?}

VTK-m already comes with an implicit array handle named vtkm: : cont: : ArrayHandleCounting that can make implicit even numbers as well as other more general counts. (See Section 26.4 for details.) So in practice you would not have to create a special implicit array, but we are doing so here for demonstrative purposes.

The first step to using ArrayHandleImplicit is to declare a functor. The functor's parenthesis operator should accept a single argument of type vtkm: Id and return a value appropriate for that index. The parenthesis operator should also be declared const because it is not allowed to change the class' state.

Example 35.3: Functor that doubles an index.

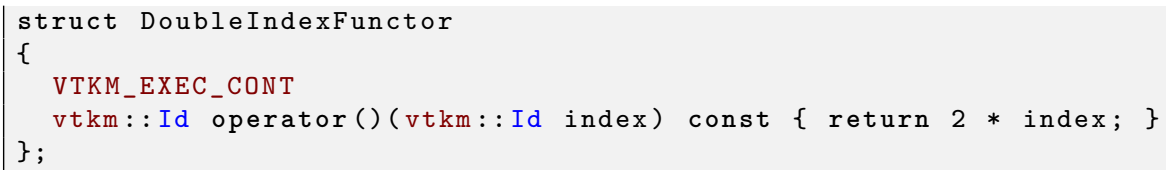

\section{Common Errors}

The functor used with ArrayHandleImplicit may contain state, but it must be trivially copiable across memory spaces. That means it cannot contain any virtual methods nor hold any pointers or references. It also means it cannot hold objects like an ArrayHandle or ArrayPortal. Such behavior may seem to work at first, but will quickly break down on different devices. The point of an implicit array is that it completely computes each value without referencing any external data. Later in this chapter we will explore many ways to create storage that access data stored in memory or other ArrayHandles.

Once the functor is defined, an implicit array can be declared using the templated vtkm: :cont: :ArrayHandleImplicit class. The single template argument is the functor's type.

Example 35.4: Declaring a ArrayHandleImplicit.

1 vtkm: : cont : : ArrayHandleImplicit<DoubleIndexFunctor > implicitArray (
2 DoubleIndexFunctor (), 50);

For convenience, vtkm/cont/ArrayHandlelmplicit.h also declares the vtkm: :cont: :make_ArrayHandleImplicit function. This function takes a functor and the size of the array and returns the implicit array.

Example 35.5: Using make_ArrayHandleImplicit.

1 vtkm: :cont : :make_ArrayHandleImplicit (DoubleIndexFunctor (), 50);

If the implicit array you are creating tends to be generally useful and is something you use multiple times, it might be worthwhile to make a convenience subclass of vtkm: :cont: :ArrayHandleImplicit for your array. 
Example 35.6: Custom implicit array handle for even numbers.

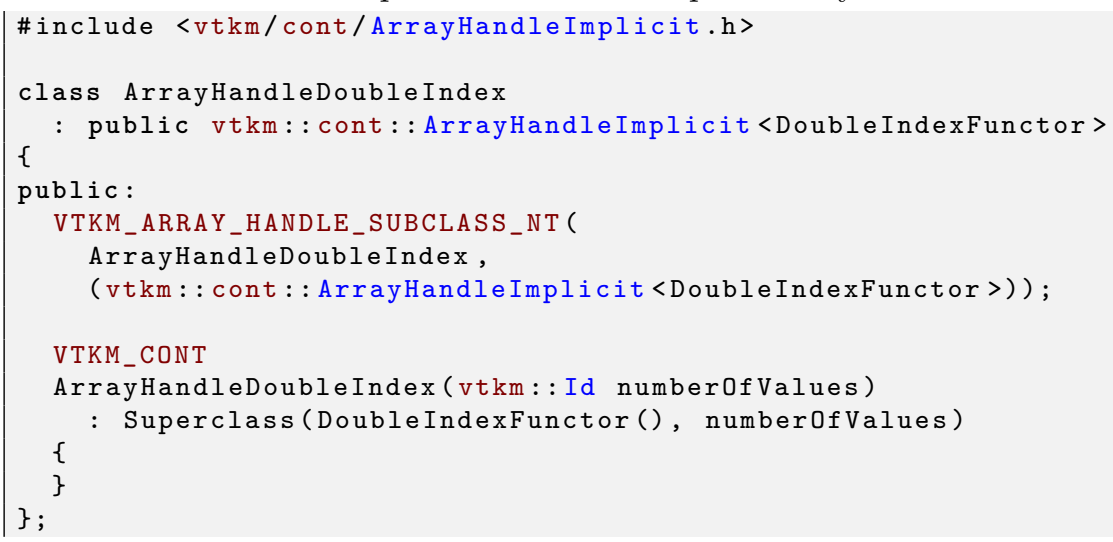

Subclasses of ArrayHandle provide constructors that establish the state of the array handle. All array handle subclasses must also use either the VTKM_ARRAY_HANDLE_SUBCLASS macro or the VTKM_ARRAY_HANDLE_SUBCLASS_NT macro. Both of these macros define the types Superclass, ValueType, and StorageTag as well as a set of constructors and operators expected of all ArrayHandle classes. The difference between these two macros is that VTKM_ARRAY_HANDLE_SUBCLASS is used in templated classes whereas VTKM_ARRAY_HANDLE_SUBCLASS_NT is used in non-templated classes.

The ArrayHandle subclass in Example 35.6 is not templated, so it uses the VTKM_ARRAY_HANDLE_SUBCLASS_NT macro. This macro takes two parameters. The first parameter is the name of the subclass where the macro is defined and the second parameter is the immediate superclass including the full template specification. The second parameter of the macro must be enclosed in parentheses so that the $\mathrm{C}$ pre-processor correctly handles commas in the template specification. (The other macro is described in Section 35.2 on page 312).

\subsection{Transformed Arrays}

Another type of fancy array handle is the transformed array. A transformed array takes another array and applies a function to all of the elements to produce a new array. A transformed array behaves much like a map operation except that a map operation writes its values to a new memory location whereas the transformed array handle produces its values on demand so that no additional storage is required.

Specifying a transformed array in VTK-m is straightforward. VTK-m has a special class named vtkm: : cont: :ArrayHandleTransform that takes an array handle and a functor and provides an interface to a new array comprising values of the functor applied the first array.

To demonstrate the use of ArrayHandleTransform, let us say that we want to scale and bias all of the values in a target array. That is, each value in the target array is going to be multiplied by a given scale and then offset by adding a bias value. (The scale and bias are uniform across all entries.) We could, of course, easily create a worklet to apply this scale and bias to each entry in the target array and save the result in a new array, but we can save space and possibly time by computing these values on demand.

The first step to using ArrayHandleTransform is to declare a functor. The functor's parenthesis operator should accept a single argument of the type of the target array and return the transformed value. For more generally applicable transform functors, it is often useful to make the parenthesis operator a template. The parenthesis operator should also be declared const because it is not allowed to change the class' state.

Example 35.7: Functor to scale and bias a value.

1 template<typename $T>$ 


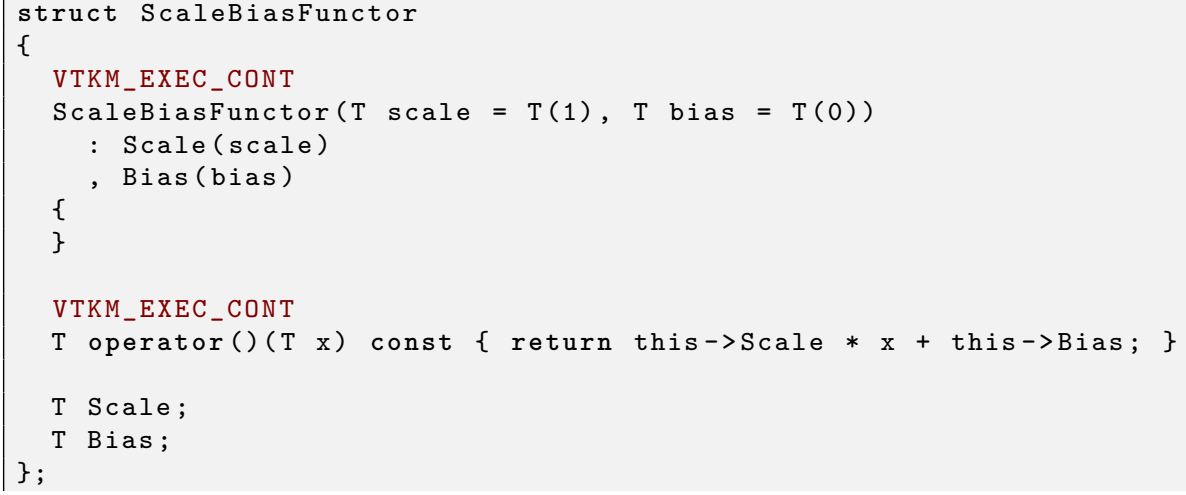

\section{Common Errors}

As with functors for implicit arrays, a functor for ArrayHandleTransform must be trivially copiable. It may not hold in its state reference to any other arrays. The functor may only access the one value from the input array that is passed as an argument to the parenthesis operator. Mechanisms to build arrays with more expressive access to one or more other arrays are described later in this chapter.

Once the functor is defined, a transformed array can be declared using the templated vtkm: :cont: :ArrayHandleTransform class. The first template argument is the type of array being transformed. The second template argument is the type of functor used for the transformation. The third template argument, which is optional, is the type for an inverse functor that provides the inverse operation of the functor in the second argument. This inverse functor is used for writing values into the array. For arrays that will only be read from, there is no need to supply this inverse functor.

That said, it is generally easier to use the vtkm: :cont::make_ArrayHandleTransform convenience function. This function takes an array and a functor (and optionally an inverse functor) and returns a transformed array.

Example 35.8: Using make_ArrayHandleTransform.

\begin{tabular}{l|}
1 \\
2
\end{tabular} vtkm: : cont : : make_ArrayHandleTransform (array, ScaleBiasFunctor <vtkm: : Float $32>(2,3))$

If the transformed array you are creating tends to be generally useful and is something you use multiple times, it might be worthwhile to make a convenience subclass of vtkm: : cont: : ArrayHandleTransform or convenience make_ArrayHandle* function for your array.

Example 35.9: Custom transform array handle for scale and bias.

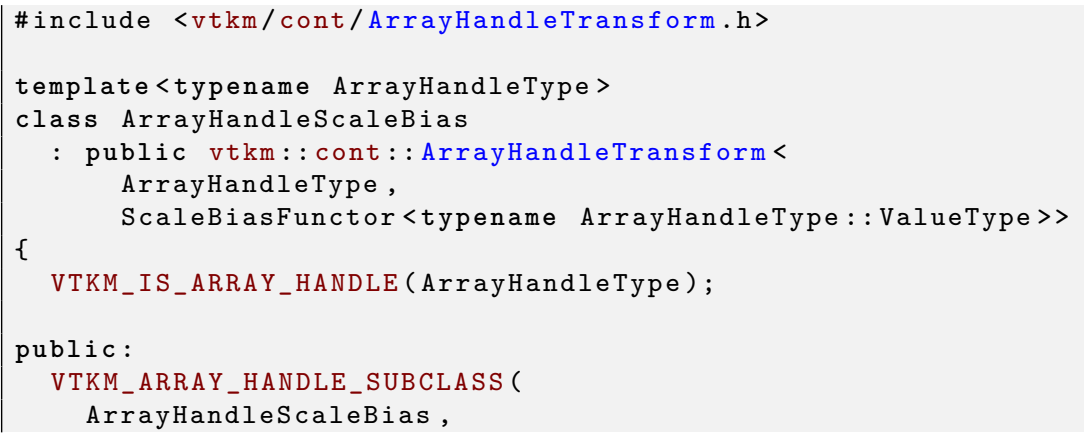




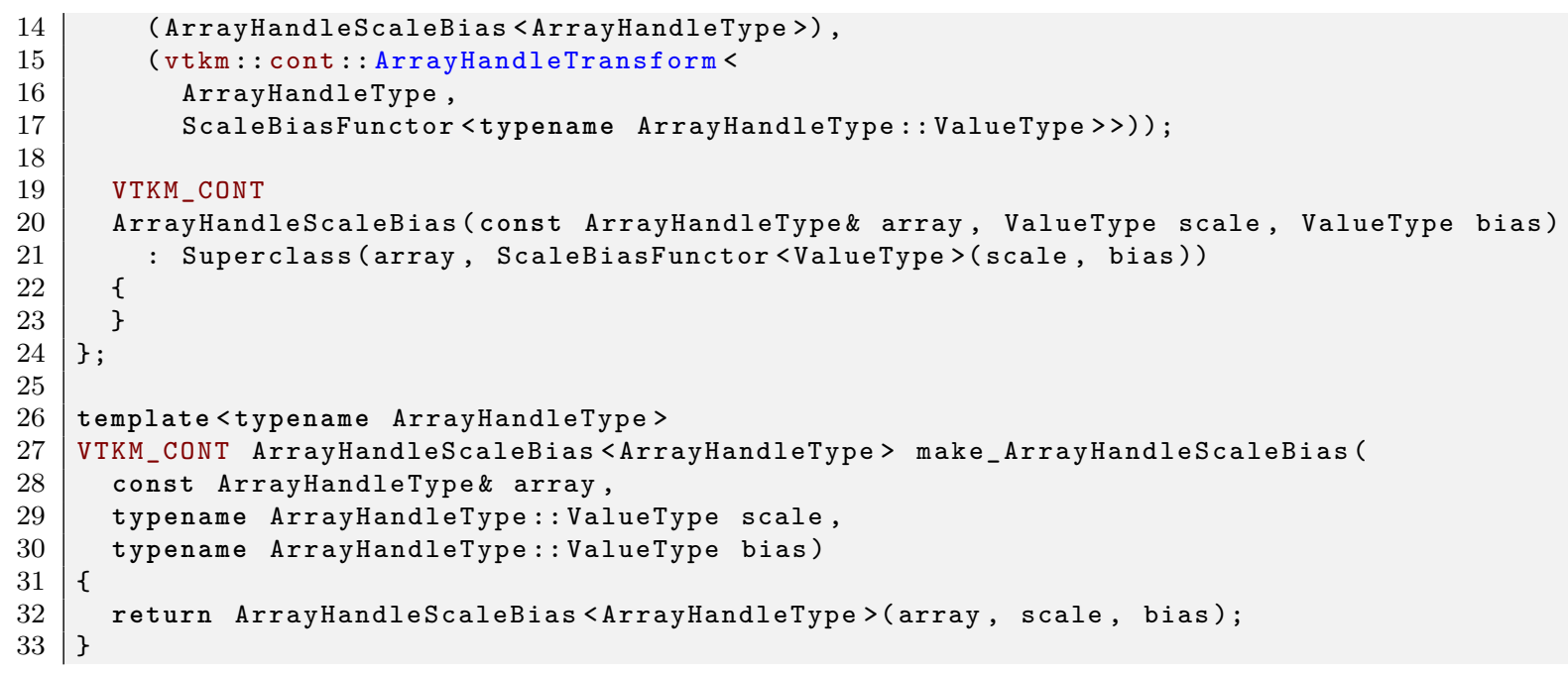

Subclasses of ArrayHandle provide constructors that establish the state of the array handle. All array handle subclasses must also use either the VTKM_ARRAY_HANDLE_SUBCLASS macro or the VTKM_ARRAY_HANDLE_SUBCLASS_NT macro. Both of these macros define the types Superclass, ValueType, and StorageTag as well as a set of constructors and operators expected of all ArrayHandle classes. The difference between these two macros is that VTKM_ARRAY_HANDLE_SUBCLASS is used in templated classes whereas VTKM_ARRAY_HANDLE_SUBCLASS_NT is used in non-templated classes.

The ArrayHandle subclass in Example 35.9 is templated, so it uses the VTKM_ARRAY_HANDLE_SUBCLASS macro. This macro takes three parameters. The first parameter is the name of the subclass where the macro is defined, the second parameter is the type of the subclass including the full template specification, and the third parameter is the immediate superclass including the full template specification. The second and third parameters of the macro must be enclosed in parentheses so that the $\mathrm{C}$ pre-processor correctly handles commas in the template specification. (The other macro is described in Section 35.1 on page 310 ).

\subsection{Decorated Arrays}

In the previous section, we saw how to augment a single array to modify its values in place. However, ArrayHandleTransform is limited in that it only allows you to augment one array at a time, and it does not allow you to adjust the index lookups into the array.

If ArrayHandleTransform is not powerful enough, VTK-m provides vtkm: : cont: : ArrayHandleDecorator for more general augmentation of arrays. ArrayHandleDecorator provides a much more expressive (albeit complicated) interface.

In this section we will demonstrate the steps required to create a more general derived storage. For the purposes of the example in this section, let us say we want 2 array handles to behave as one array with the contents interlaced together. That is, the first $w$ items are the same as the first $w$ of the first array, the second $w$ items are the same as the first $w$ of the second array, the third $w$ items are the same as the second $w$ of the first array, the fourth $w$ items are the same as the second $w$ of the second array, and so on. We could of course actually copy the data, but we can also do it in place.

Using ArrayHandleDecorator requires creating functors for getting and setting data in the arrays plus creating another "interface" structure to manage the functors and data. As always, these can be used to define new ArrayHandle classes. 


\subsubsection{Functors}

Like ArrayHandleImplicit and ArrayHandleTransform, you define the behavior of ArrayHandleDecorator by defining functors. However, unlike the functors for the other ArrayHandles, we relax the restrictions on ArrayHandleDecorator's functors and allow them to hold array portals as part of its state. Note that there are still restrictions on where these portals come from. They are generated internally by ArrayHandleDecorator and are passed to the functors as described in Section 35.3.2.

The decorator needs a functor with a parentheses operator that is given an index and returns a value for that index. The interface is the same as that for ArrayHandleImplicit, but the returned result can come from data in array portals.

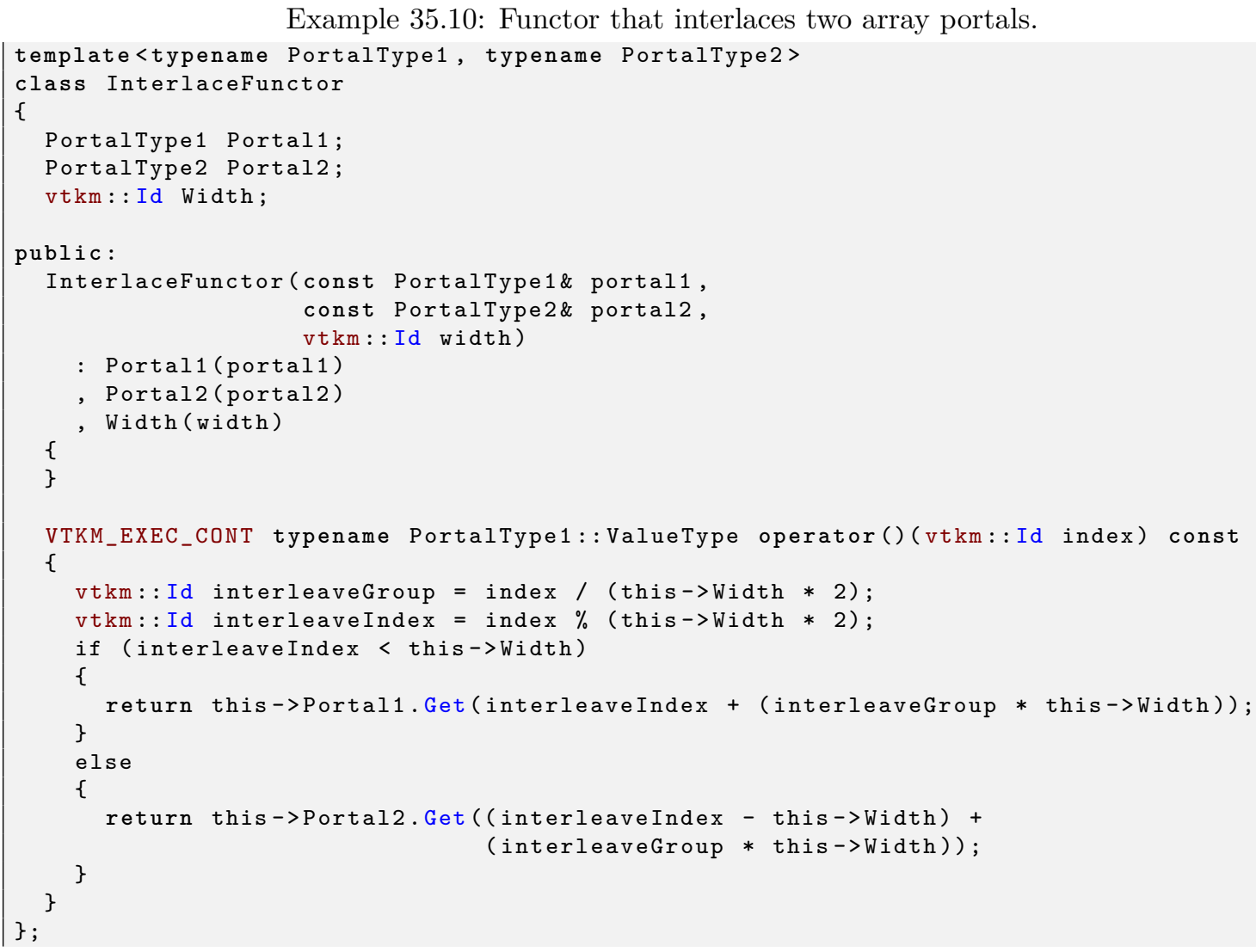

This functor will be used for retrieving data. If the array being generated is intended to be read-only, then this is all you need. However, if you wish to create a writable array, then you need a second "inverse" functor to set data to the array. Fundamentally, there is no reason why we should not support writing data to the interlaced arrays, so we define a secondary inverse functor.

Example 35.11: Inverse functor for writing data to interlaced array portals.

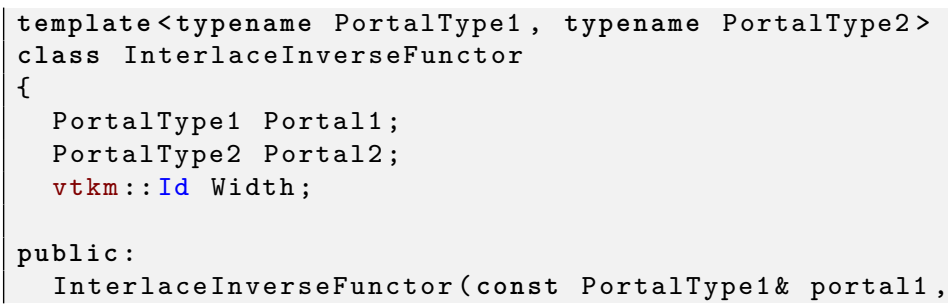




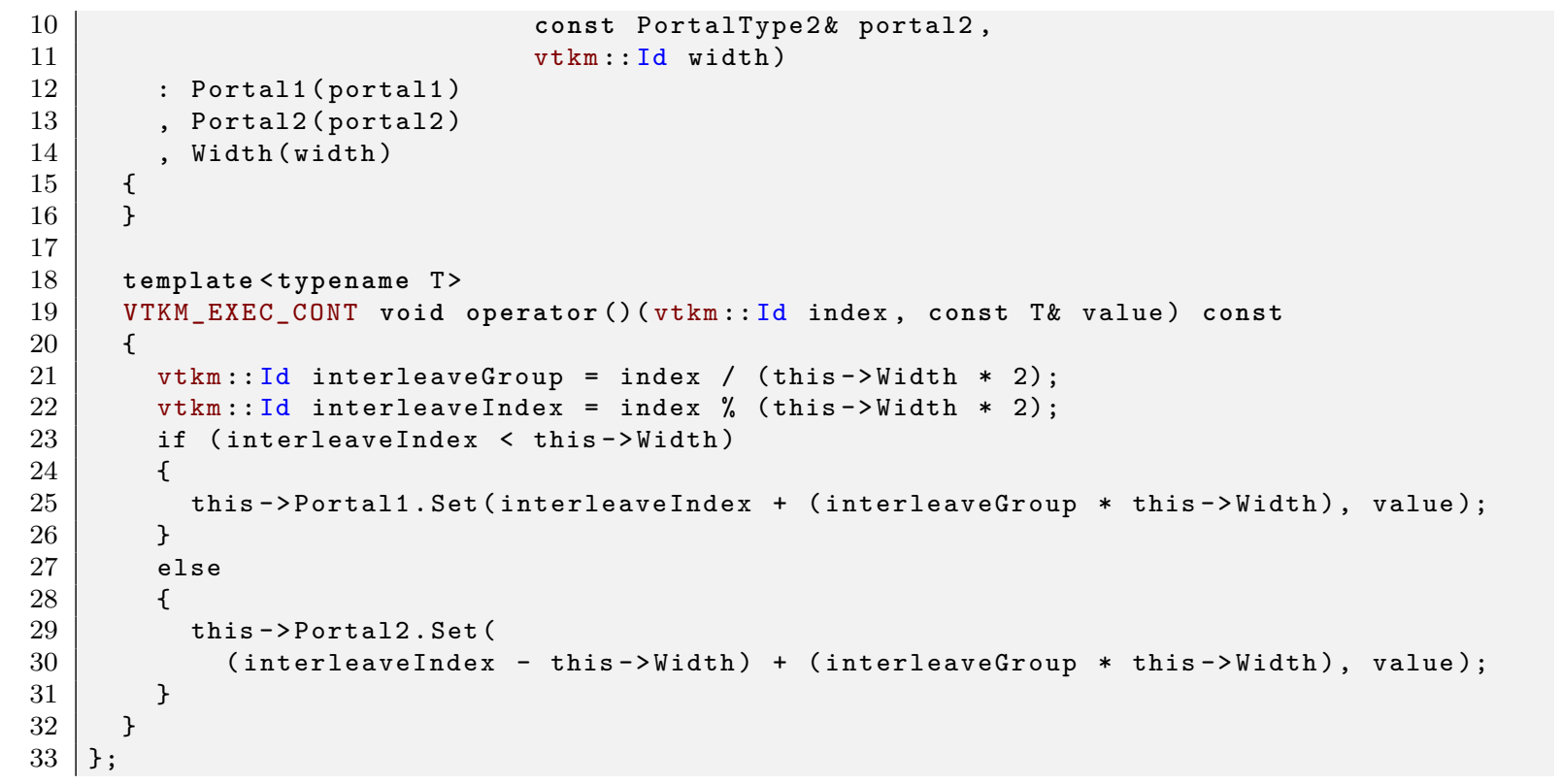

\subsubsection{Interface}

The next step in creating a decorator array is to define an "interface" class that tells ArrayHandleDecorator how to read, write, and resize the array. This is just a normal class or struct containing the following members.

CreateFunctor A method that takes one or more array portals and returns a functor used to get information from these portals. These portals will respectively come from the arrays being decorated. This method is required.

CreateInverseFunctor A method that takes one or more array portals and returns a functor used to set information in these portals. These portals will respectively come from the arrays being decorated. This method is optional. If it is omitted, then the decorated array will be read-only.

AllocateSourceArrays A method that resizes the arrays being decorated. The first three arguments are the new size of the array, a vtkm::CopyFlag indicating whether data should be preserved, and a vtkm: :cont::Token, respectively. The remaining arguments are the arrays being decorated. This method is optional. If it is omitted, then the decorated array cannot be resized after creation.

Our implementation of interlaced array handles produces the functors presented in Exercises 35.10 and 35.11 . It also provides a mechanism to resize the arrays.

Example 35.12: Decorator implementation class for interleaving ArrayHandles.

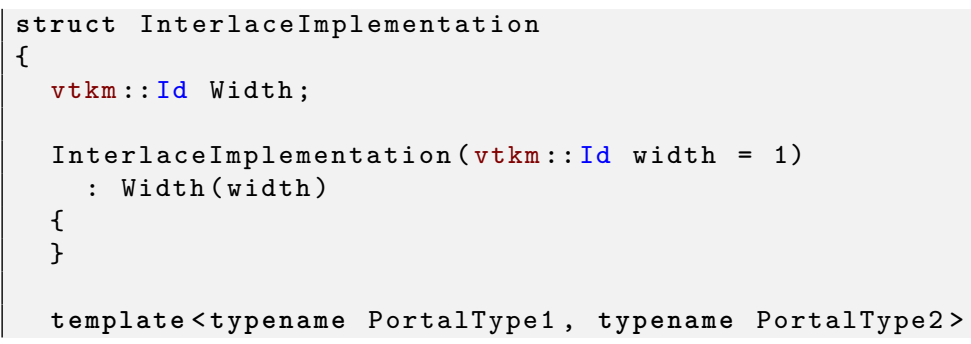




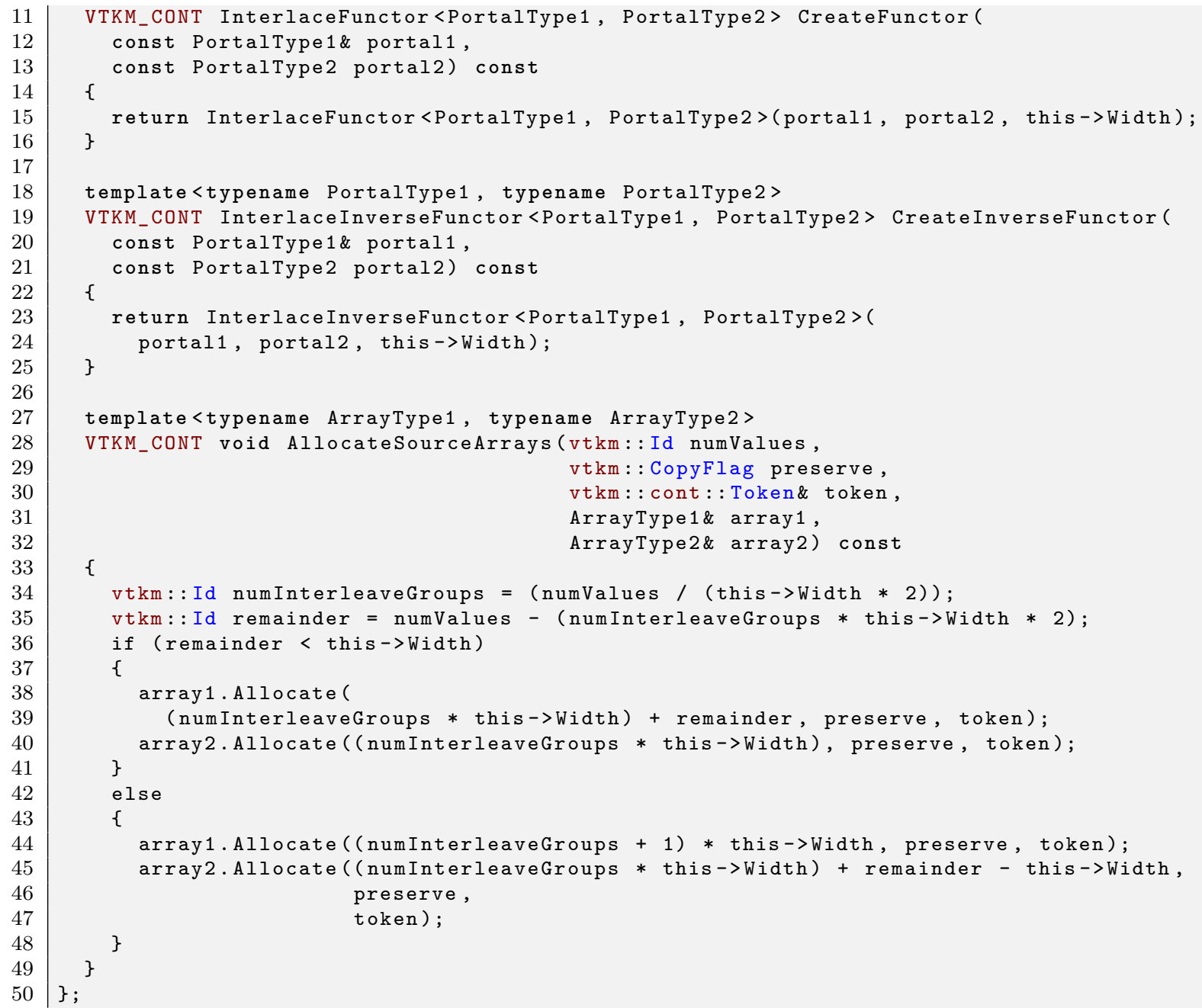

\subsubsection{Subclass}

If the array decorator you are creating tends to be generally useful and is something you use multiple times, it might be worthwhile to make a convenience subclass of vtkm: :cont: :ArrayHandleDecorator or convenience make_ArrayHandle* function for your array.

Example 35.13: Custom decorator array handle for interleaving arrays.

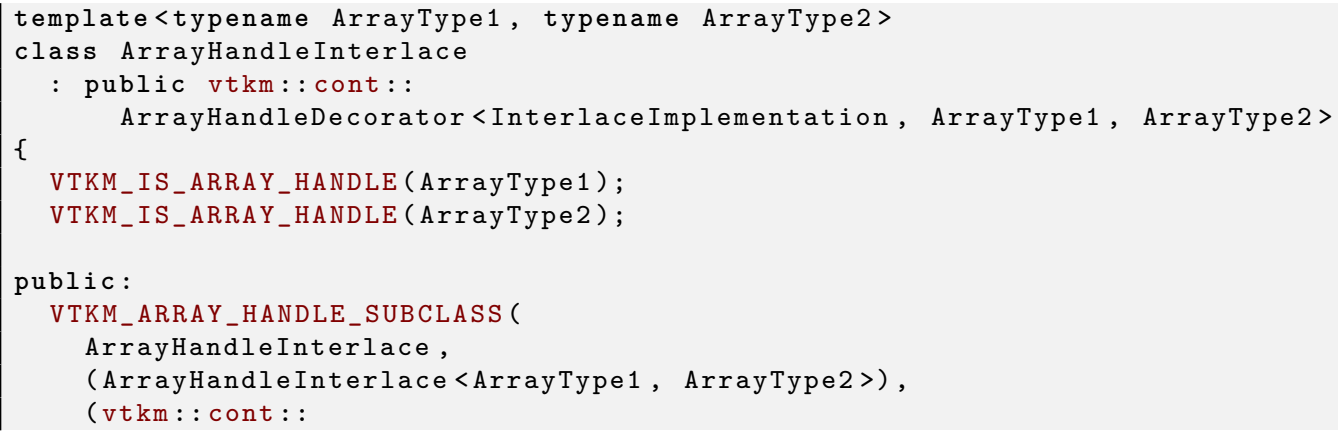




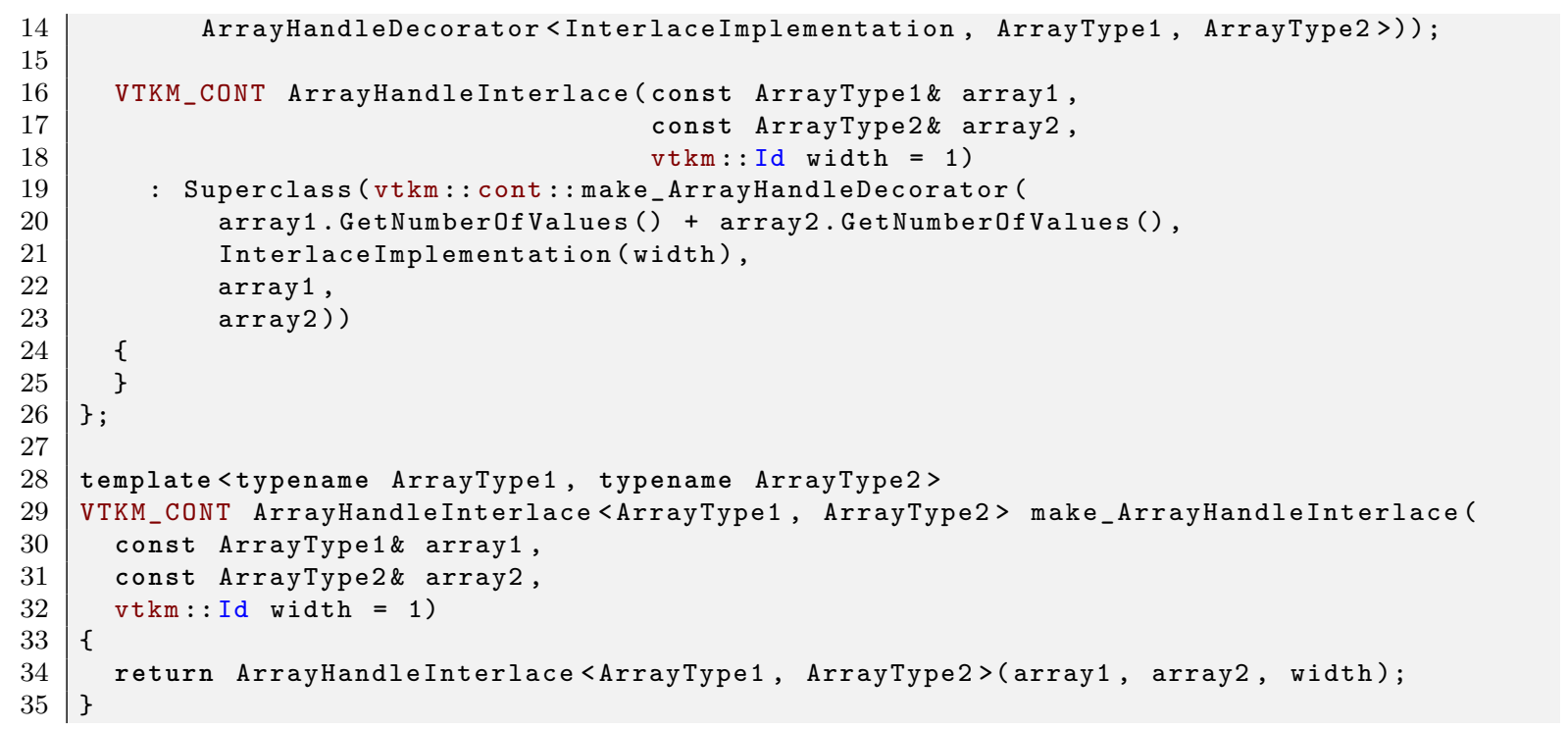

Subclasses of ArrayHandle provide constructors that establish the state of the array handle. All array handle subclasses must also use either the VTKM_ARRAY_HANDLE_SUBCLASS macro or the VTKM_ARRAY_HANDLE_SUBCLASS_NT macro. Both of these macros define the types Superclass, ValueType, and StorageTag as well as a set of constructors and operators expected of all ArrayHandle classes. The difference between these two macros is that VTKM_ARRAY_HANDLE_SUBCLASS is used in templated classes whereas VTKM_ARRAY_HANDLE_SUBCLASS_NT is used in non-templated classes.

The ArrayHandle subclass in Example 35.13 is templated, so it uses the VTKM_ARRAY_HANDLE_SUBCLASS macro. This macro takes three parameters. The first parameter is the name of the subclass where the macro is defined, the second parameter is the type of the subclass including the full template specification, and the third parameter is the immediate superclass including the full template specification. The second and third parameters of the macro must be enclosed in parentheses so that the $\mathrm{C}$ pre-processor correctly handles commas in the template specification. (The other macro is described in Section 35.1 on page 310 ).

\subsection{Derived Storage}

A derived storage is a type of fancy array that takes one or more other arrays and changes their behavior in some way. A transformed array (Section 35.2) is a specific type of derived array with a simple mapping. In this section we will demonstrate the steps required to create a more general derived storage. When applicable, it is much easier to create a derived array as a transformed array or using the other fancy arrays than to create your own derived storage. However, if these pre-existing fancy arrays do not work work, for example if your derivation uses multiple arrays or requires general lookups, you can do so by creating your own derived storage. For the purposes of the example in this section, let us say we want 2 array handles to behave as one array with the contents interlaced together. That is, the first $w$ items are the same as the first $w$ of the first array, the second $w$ items are the same as the first $w$ of the second array, the third $w$ items are the same as the second $w$ of the first array, the fourth $w$ items are the same as the second $w$ of the second array, and so on. We could of course actually copy the data, but we can also do it in place. 


\subsubsection{Array Portal}

The first step to creating a derived storage is to build an array portal that will take portals from arrays being derived. The portal must work in both the control and execution environment.

Because the intention of our custom ArrayHandle is to augment the behavior of other ArrayHandle, it is typical for an ArrayPortal of this nature to reference other ArrayPortals.

Example 35.14: Derived array portal for concatenated arrays.

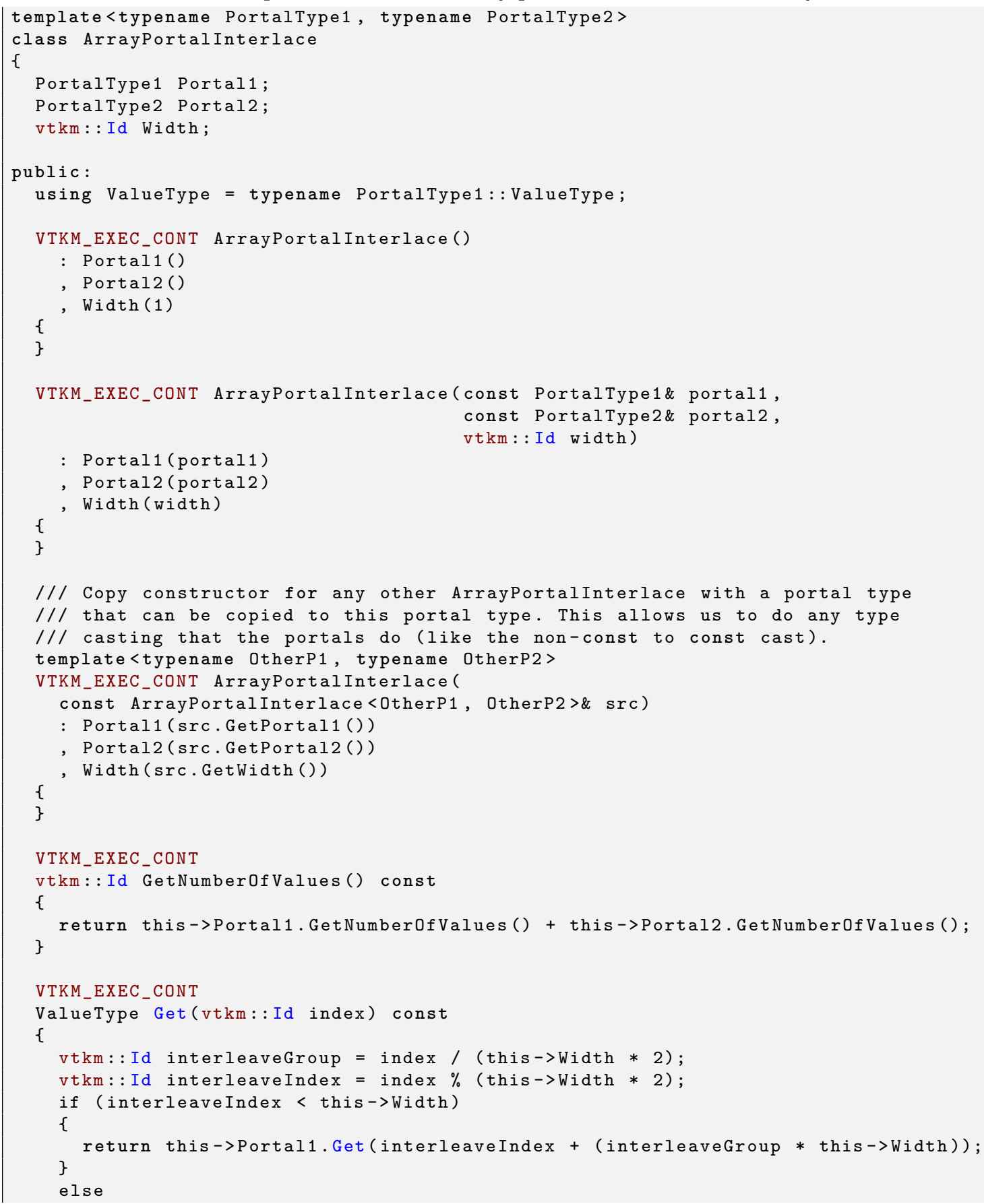




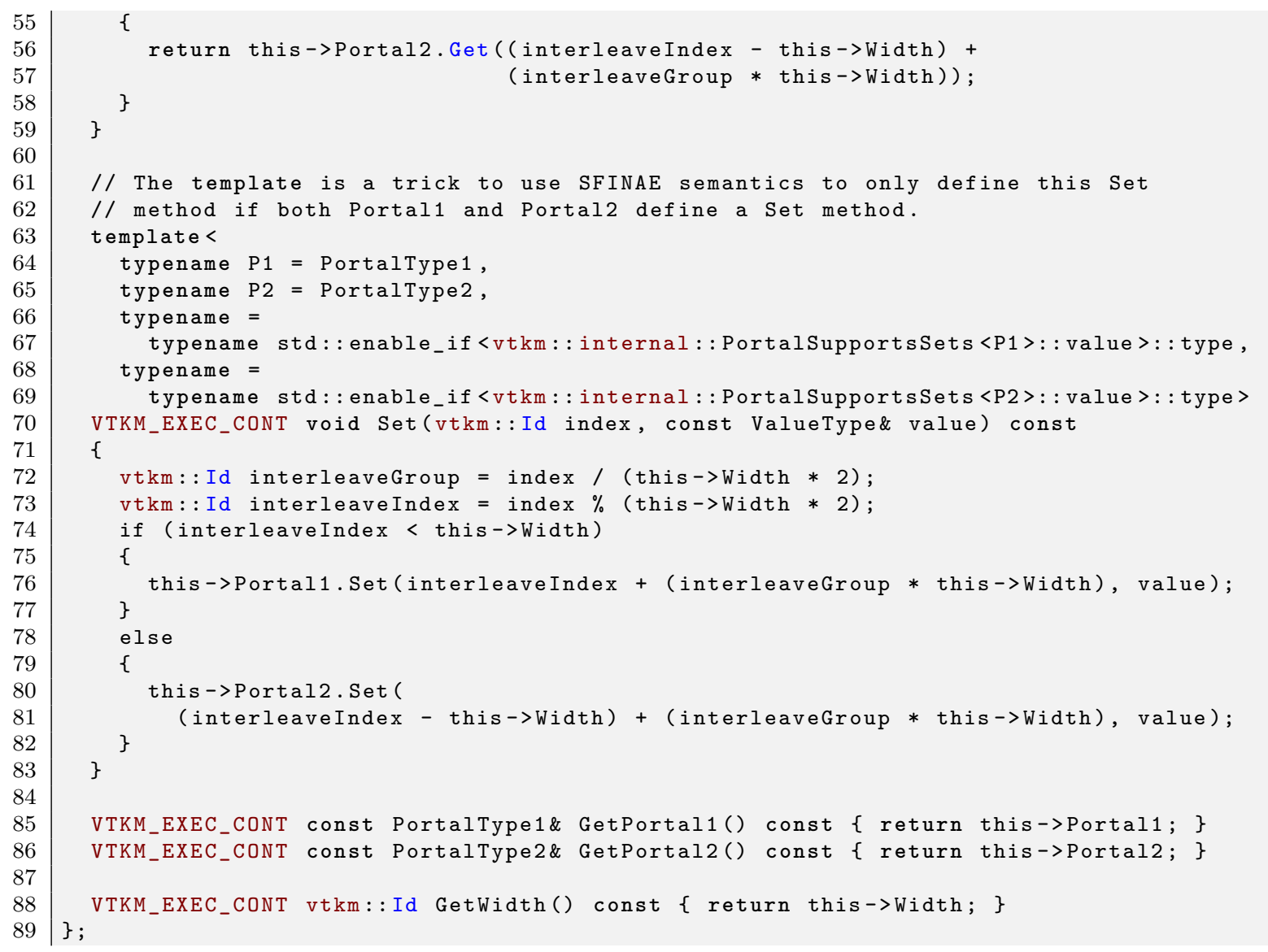

\subsubsection{Storage}

The next step in creating a custom storage is to define a tag for the adapter. We shall call ours StorageTagInterlace and it will be templated on the two array handle types that we are deriving. Then, we need to create a specialization of the templated vtkm: : cont: : internal: :Storage class. The ArrayHandle will instantiate an object using the array container tag we give it, and we define our own specialization so that it runs our interface into the code.

vtkm: : cont: : internal: : Storage has two template arguments: the base type of the array and the storage tag.

Example 35.15: Prototype for vtkm: : cont: : internal: :Storage.

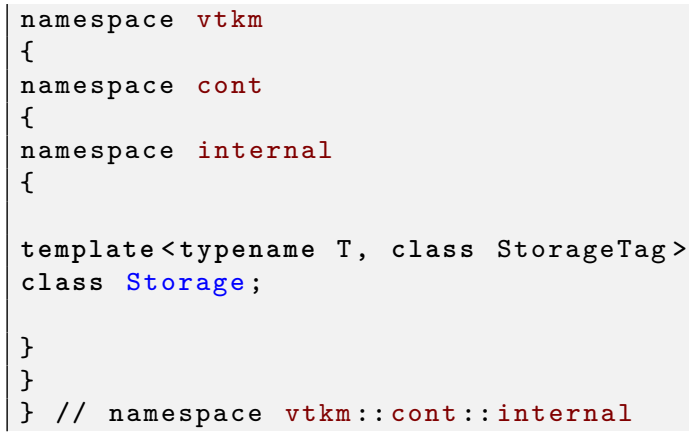


The vtkm: :cont: : internal: :Storage must define the following items.

ReadPortalType The type of an array portal that can be used to access the underlying data. This array portal needs only be read-only. That is, the Set method is optional.

WritePortalType The type of an array portal that can be used to access the underlying data. This array portal should be both read and write capable. If the storage is intended to be read-only, then WritePortalType should be left out and VTKM_STORAGE_NO_WRITE_PORTAL should be declared in the storage class instead.

GetNumberOfBuffers The actual data of an ArrayHandle is stored in a collection of vtkm: :cont: : internal: :Buffer objects. The Storage declares how many Buffer objects are needed by returning the count from the GetNumberOfBuffers method. This method must be declared constexpr and static. A derived array usually contains all the Buffers from the ArrayHandles it is referencing plus an additional Buffer for its own metadata. See Section 35.5.1 for details on Buffer.

GetNumberOfValues This is a static method that takes an array of vtkm::cont::internal::Buffer objects and returns the number of values in the represented array. The number of values is either derived from the size of the Buffers, the size of the arrays being referenced, or stored in the metadata.

ResizeBuffers This is a static method that takes a number of values for the arrays, an array of vtkm: :cont: :internal::Buffer objects, a vtkm: :CopyFlag, and a vtkm: :cont::Token. It then resizes the memory in the Buffer objects to match the requested number of values. If the storage cannot be resized after it is created, then this method should be left out and the VTKM_STORAGE_NO_RESIZE macro should be declared in the storage class instead.

CreateReadPortal This is a static method that takes an array of vtkm::cont::internal::Buffer objects, a vtkm: : cont: :DeviceAdapterId, and a vtkm: :cont::Token. It returns a ReadPortalType that can be used to read the data on the given device.

CreateWritePortal This is a static method that takes an array of vtkm: :cont::internal::Buffer objects, a vtkm: : cont: :DeviceAdapterId, and a vtkm: :cont: :Token. It returns a WritePortalType that can be used to read the data on the given device. If the storage is intended to be read-only, then CreateWritePortal should be left out and VTKM_STORAGE_NO_WRITE_PORTAL should be declared in the storage class instead.

Although it is not necessary, it is often convenient to declare a method in the Storage object to create the array of vtkm: :cont::internal::Buffer objects used for the storage. The vtkm::cont::internal::CreateBuffers function is often helpful for this task.

If the storage is working directly with data in memory, then the function of these items can be implemented through the vtkm::cont::internal::Buffer objects. However, if the storage is modifying the functionality of other array types, then the implementation is mostly performed using the storage for those respective array types. The following example shows the implementation of Storage for our example derived array type.

Example 35.16: Storage for derived container of interlaced arrays.

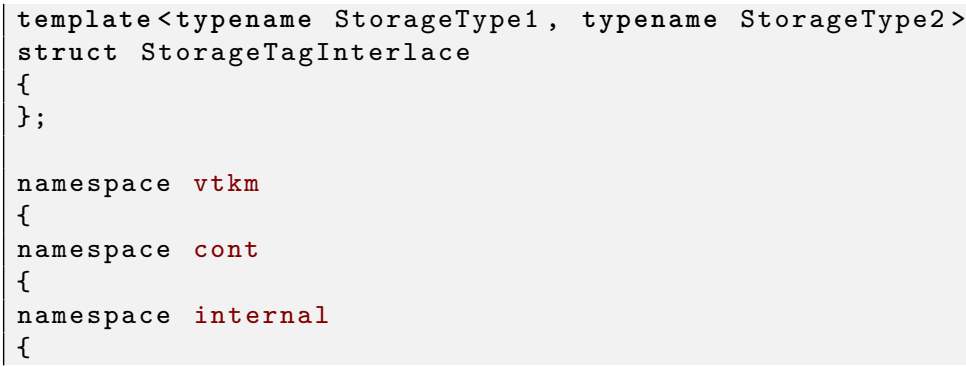




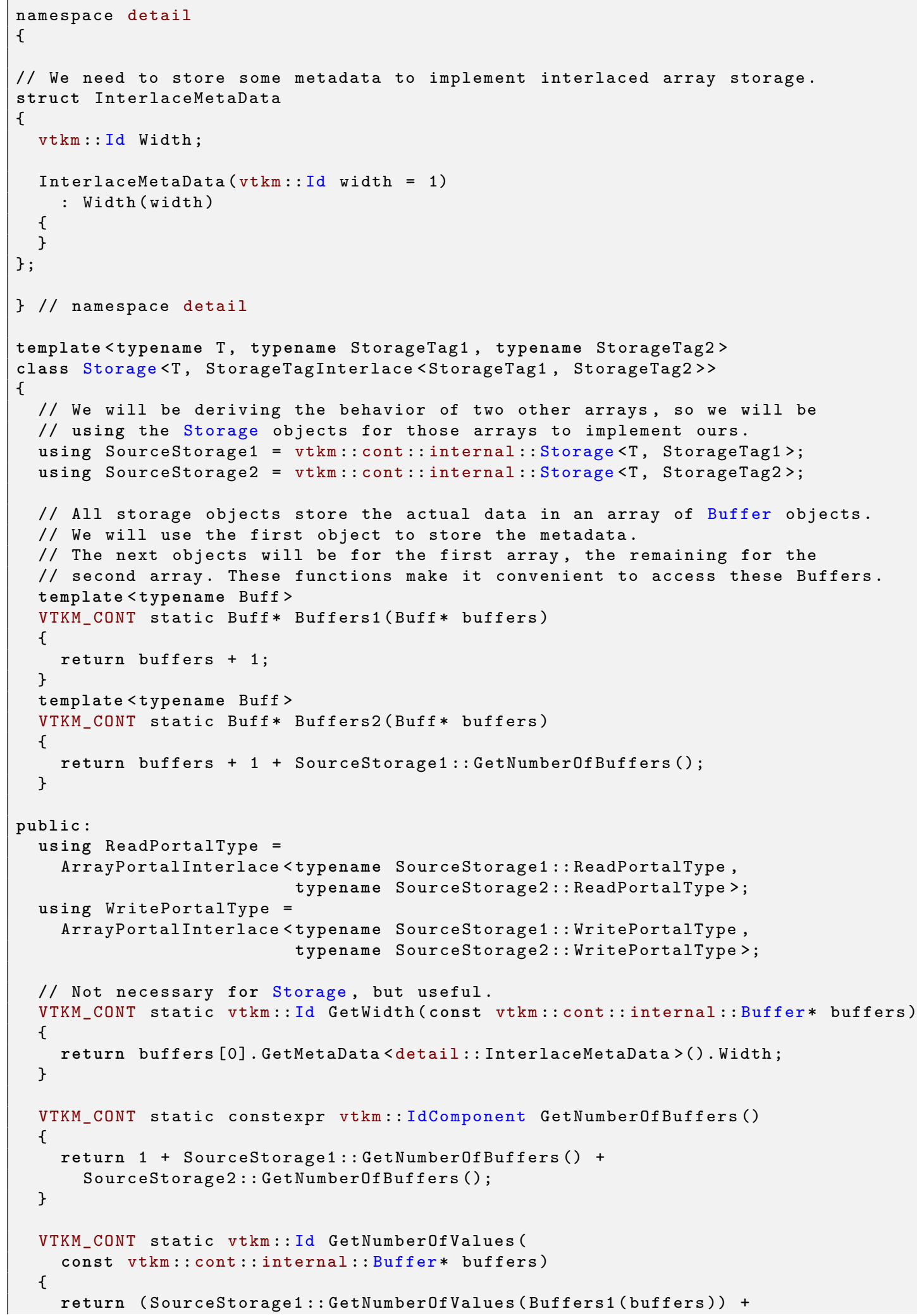




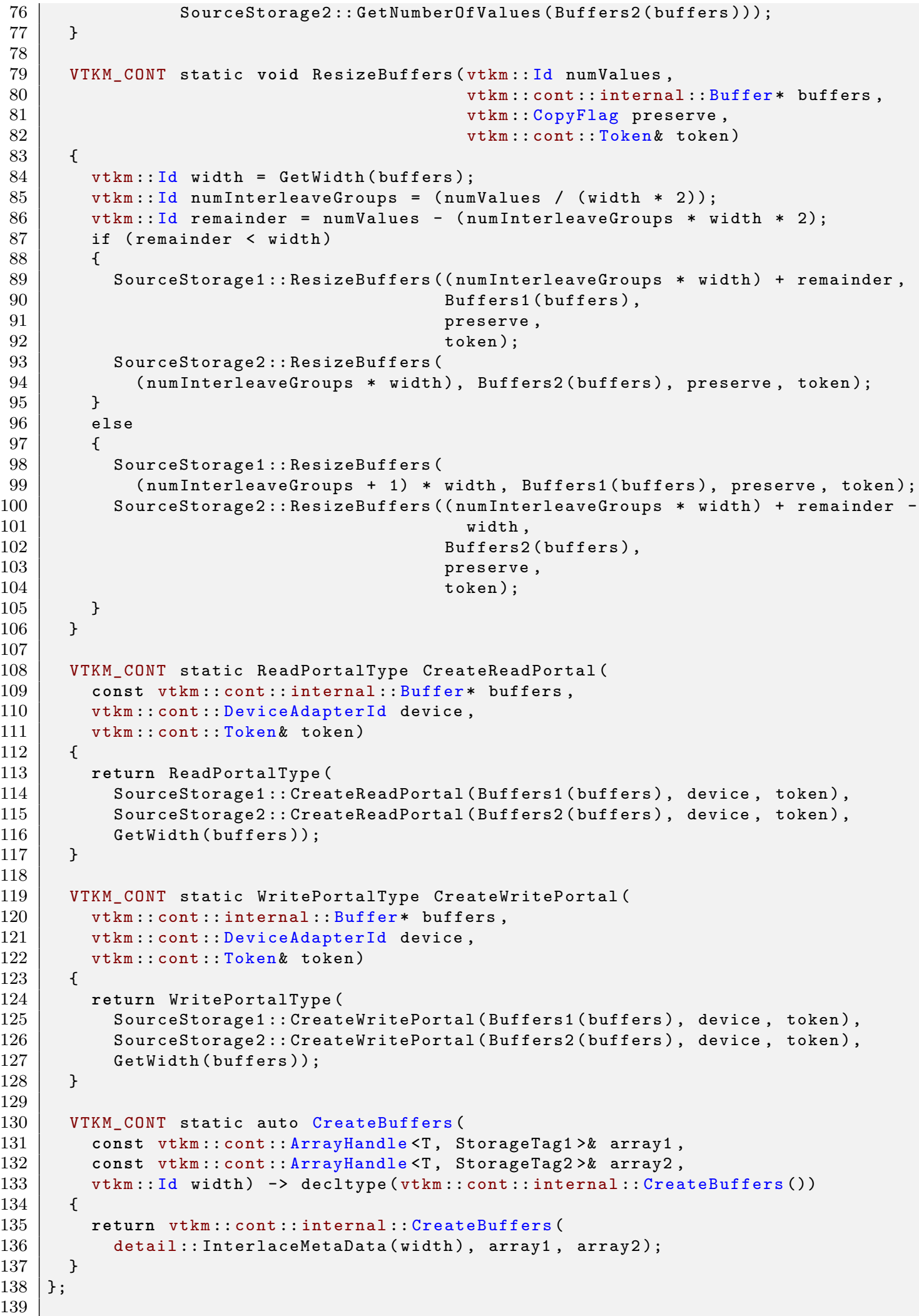


$140 \mid$ \} // namespace internal

141 \} // namespace cont

142 \} // namespace vtkm

\subsubsection{Subclass}

The final step to make a derived storage is to create a mechanism to construct an ArrayHandle with a storage derived from the desired arrays. This can be done by creating a trivial subclass of vtkm: :cont: :ArrayHandle that simply constructs the array handle to the state of an existing storage. It uses a protected constructor of vtkm: : cont: : ArrayHandle that accepts a constructed storage.

Example 35.17: ArrayHandle for derived storage of concatenated arrays.

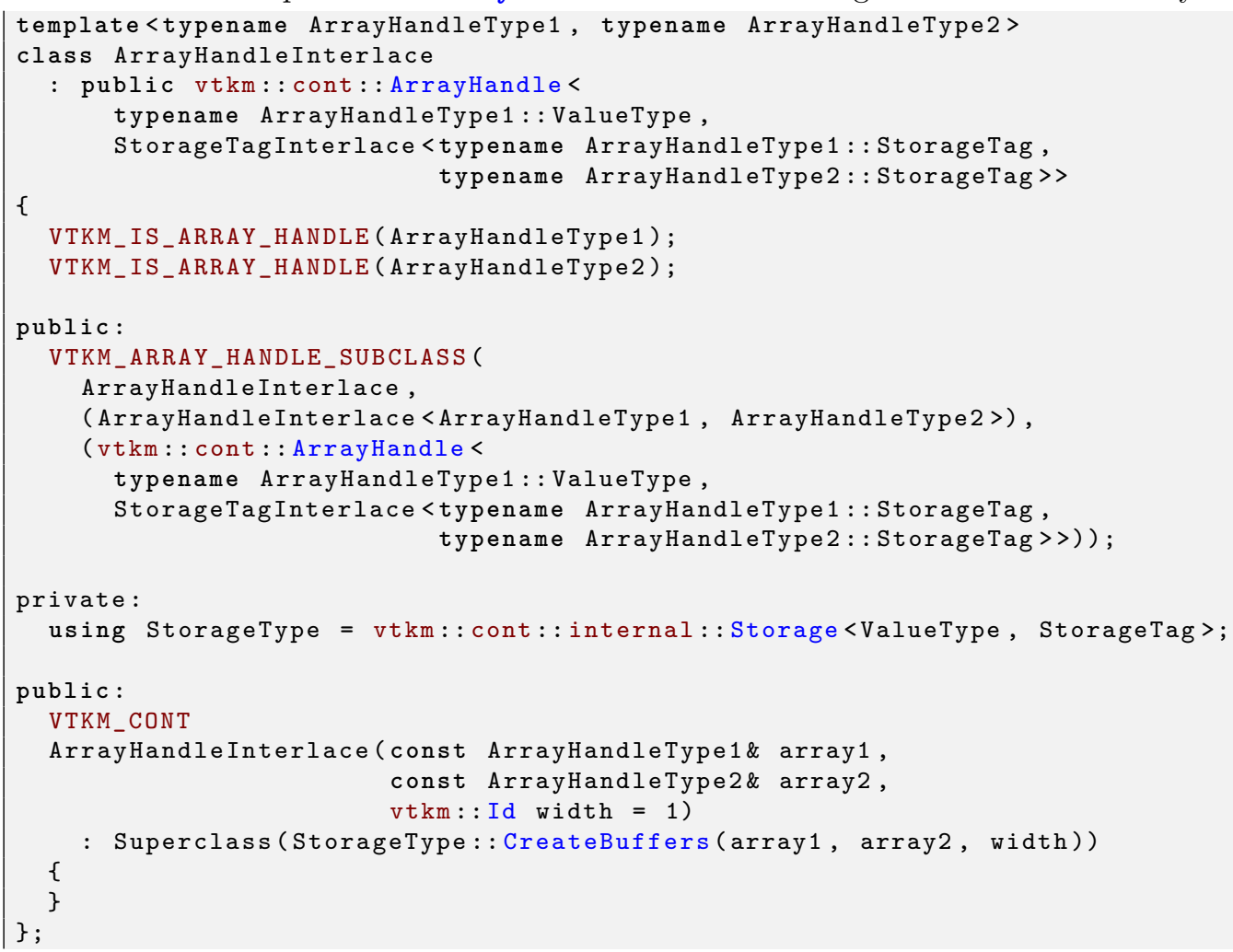

Subclasses of ArrayHandle provide constructors that establish the state of the array handle. All array handle subclasses must also use either the VTKM_ARRAY_HANDLE_SUBCLASS macro or the VTKM_ARRAY_HANDLE_SUBCLASS_NT macro. Both of these macros define the types Superclass, ValueType, and StorageTag as well as a set of constructors and operators expected of all ArrayHandle classes. The difference between these two macros is that VTKM_ARRAY_HANDLE_SUBCLASS is used in templated classes whereas VTKM_ARRAY_HANDLE_SUBCLASS_NT is used in non-templated classes.

The ArrayHandle subclass in Example 35.17 is templated, so it uses the VTKM_ARRAY_HANDLE_SUBCLASS macro. (The other macro is described in Section 35.1 on page 310). This macro takes three parameters. The first parameter is the name of the subclass where the macro is defined, the second parameter is the type of the subclass including the full template specification, and the third parameter is the immediate superclass including the full template specification. The second and third parameters of the macro must be enclosed in parentheses so that the $\mathrm{C}$ pre-processor correctly handles commas in the template specification. 
It is also customary to create helper functions for creating ArrayHandles. This makes it simpler than creating matching template interfaces.

Example 35.18: Helper function for creating a custom derived ArrayHandle.

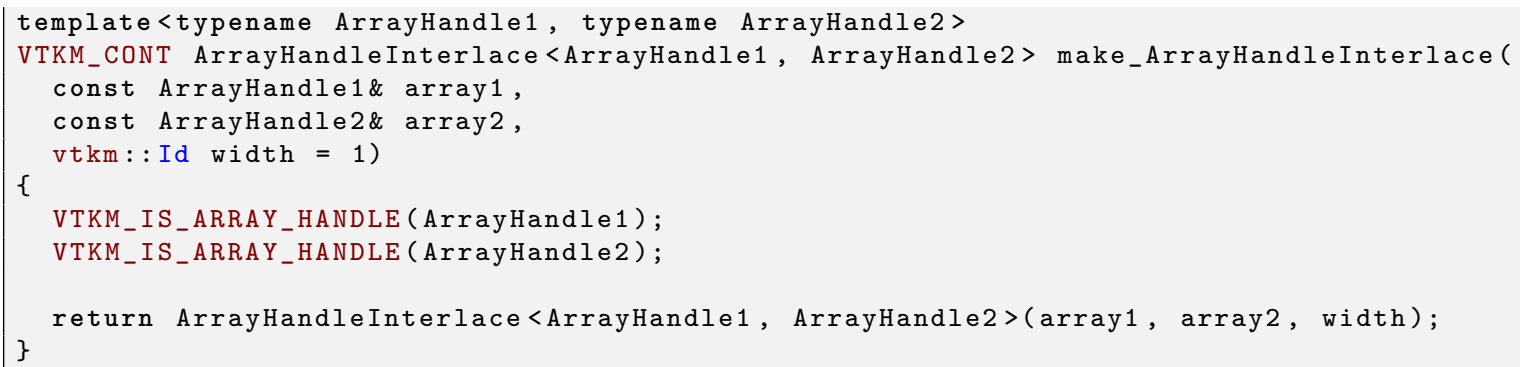

vtkm: : cont: :ArrayHandleCompositeVector is an example of a derived array handle provided by VTK-m. It references some fixed number of other arrays, pulls a specified component out of each, and produces a new component that is a tuple of these retrieved components.

\subsection{Adapting Data Structures}

The intention of the storage parameter for vtkm: :cont: :ArrayHandle is to implement the strategy design pattern to enable VTK-m to interface directly with the data of any third party code source. VTK-m is designed to work with data originating in other libraries or applications. By creating a new type of storage, VTK-m can be entirely adapted to new kinds of data structures.

\section{(0) Did you know?}

3 VTK- $m$ comes with several types of ArrayHandle that can adapt memory in different ways. In practice, it is rare to have to write a custom ArrayHandle to adapt to a data structure, and this example is particularly contrived. However, we document it here for completeness.

\section{Common Errors \\ 3 Keep in mind that memory layout used can have an effect on the running time of algorithms in VTK-m. Different data layouts and memory access can change cache performance and introduce memory affinity problems. The example code given in this section will likely have poorer cache performance than the basic storage provided by VTK-m. However, that might be an acceptable penalty to avoid data copies.}

In this section we demonstrate the steps required to adapt the array handle to a data structure provided by a third party. For the purposes of the example, let us say that some fictitious library named "foo" has a simple structure named FooAttributes that holds the field values for a particular part of a mesh, and then maintain the field values for all locations in a mesh in a FooFields object.

Example 35.19: Fictitious field storage used in custom array storage examples.

1 struct FooAttributes 


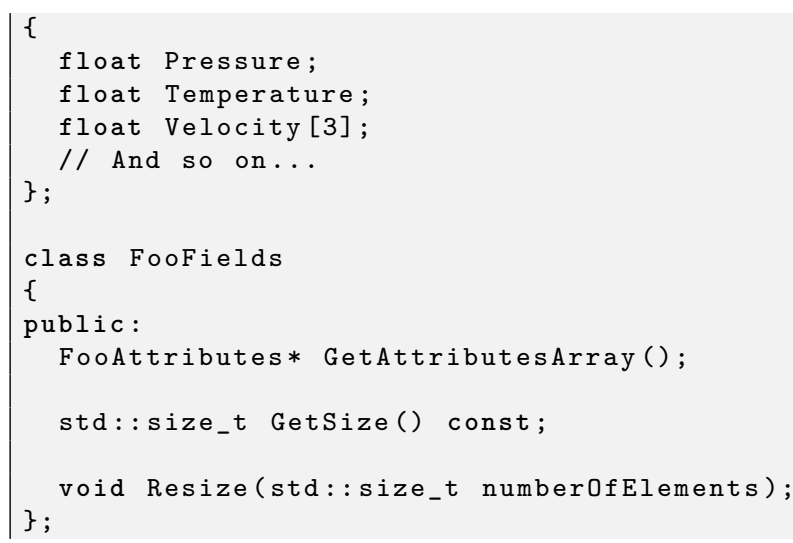

There are few restrictions on the structure of the data. The only real restriction is that you must be able to get the data in buffers of raw $\mathrm{C}$ pointers containing trivially copiable objects. In this example, we note that FooFields can return an array of FooAttributes, which satisfies this requirement.

VTK-m expects separate arrays for each of the fields rather than a single array containing a structure holding all of the fields. However, rather than copy each field to its own array, we can create a storage for each field that points directly to the data in a FooFields object.

\subsubsection{Buffer Objects}

VTK-m manages data across devices using the vtkm: :cont: : internal: :Buffer object. As its name implies, Buffer manages a buffer in memory. This block of bytes can be allocated on different devices and the data it contains will be transferred across them. The Buffer object operates by specifying its size and then requesting pointers to the reserved memory on different devices.

Buffer contains the following methods.

GetNumberOfBytes Returns the number of bytes held by the buffer. Buffer actually allocates memory lazily, so there might not actually be any memory allocated anywhere. It is also possible that memory is simultaneously allocated on multiple devices. The number of bytes is returned as a vtkm: :BufferSizeType, which might be a larger integer than vtkm: :Id.

SetNumberOfBytes Changes the size of the buffer. SetNumberOfBytes has three arguments. The first argument is the number of bytes to allocate. The second argument is a vtkm: CopyFlag that indicates whether any existing data in the buffer should be preserved. The third argument is a vtkm: : cont: : Token that ensures that the resize will not interfere with other operations happening on the Buffer's data.

IsAllocatedOnHost Returns true if the Buffer has memory allocated on the host (for the control environment).

IsAllocatedOnDevice Returns true if the Buffer has memory allocated on the device specified by a given device adapter tag. If vtkm: : cont: : DeviceAdapterTagAny is given as the device, then this returns true if the Buffer is allocated on any device. If vtkm: : cont: : DeviceAdapterTagUnknown is given as the device, then this returns true if the Buffer is allocated on the host (same as IsAllocatedOnHost).

ReadPointerHost Returns a readable host (control environment) pointer to the buffer. Memory will be allocated and data will be copied as necessary. A vtkm: :cont: :Token object is passed to ReadPointerHost, and the memory at the pointer will be valid as long as the Token is still in scope. Any write operation to this buffer will be blocked until then. 
ReadPointerDevice Returns a readable device pointer to the buffer. The first argument to ReadPointerDevice is a vtkm: :cont: :DeviceAdapterId, and the pointer returned will only be valid for this device. If the device is vtkm: : cont: :DeviceAdapterTagUnknown, then this method has the same behavior as ReadPointerHost. Memory will be allocated and data will be copied as necessary. A vtkm::cont::Token object is passed to ReadPointerDevice, and the memory at the pointer will be valid as long as the Token is still in scope. Any write operation to this buffer will be blocked until then.

WritePointerHost Returns a writable host (control environment) pointer to the buffer. Memory will be allocated and data will be copied as necessary. A vtkm: : cont: :Token object is passed to WritePointerHost, and the memory at the pointer will be valid as long as the Token is still in scope. Any read or write operation to this buffer will be blocked until then.

WritePointerDevice Returns a writable device pointer to the buffer. The first argument to WritePointerDevice is a vtkm: : cont: :DeviceAdapterId, and the pointer returned will only be valid for this device. If the device is vtkm: :cont: :DeviceAdapterTagUnknown, then this method has the same behavior as WritePointerHost. Memory will be allocated and data will be copied as necessary. A vtkm::cont::Token object is passed to WritePointerDevice, and the memory at the pointer will be valid as long as the Token is still in scope. Any read or write operation to this buffer will be blocked until then.

DeepCopyFrom Copies the data from the provided buffer into this buffer. If a device is given, then the copy will be preferred for that device.

ReleaseDeviceResources Unallocates the buffer from all devices. This method preserves the data on the host even if the data must be transferred there.

SetMetaData Takes an arbitrary object and copies it to the metadata of this buffer. Any existing metadata is deleted. Any object can be used as metadata as long as the object has a default constructor and is copiable. Holding metadata in a Buffer is optional, but it can be helpful for storing additional information or objects that cannot be implied by the buffer itself.

GetMetaData Gets the metadata for the buffer. When you call this method, you have to specify a template parameter for the type of the metadata. If the metadata has not yet been set in this buffer, a new metadata object is created, set to this buffer, and returned. If metadata of a different type has already been set, then an exception is thrown. The returned value is a reference that can be manipulated to alter the metadata of the buffer.

HasMetaData Returns whether the Buffer holds metadata.

MetaDataIsType Determines if the metadata for the buffer is set to a particular type. Specify the type of metadata as a template argument.

In addition to using Buffer to allocate data on devices and the host, you can wrap a Buffer around data that is already allocated. This is done by using the vtkm: :cont: :internal::MakeBuffer function. This method takes 6 arguments: the vtkm::cont::DeviceAdapterId of where the memory is allocated (vtkm::cont::DeviceAdapterTagUnknown if on the host), the pointer to the memory buffer, a pointer to a container managing the buffer, the size of the buffer in bytes, a function used to delete the buffer, and a function used to deallocate the buffer. An example of using MakeBuffer is given later.

\subsubsection{Array Portal}

The first step in creating an adapter storage is to create an array portal to the data. This is described in more detail in Section 27.1 and is generally straightforward for simple containers like this, which simple set and get values in a $\mathrm{C}$ array. Here is an example implementation for our FooFields container. 
Example 35.20: Array portal to adapt a third-party container to VTK-m.

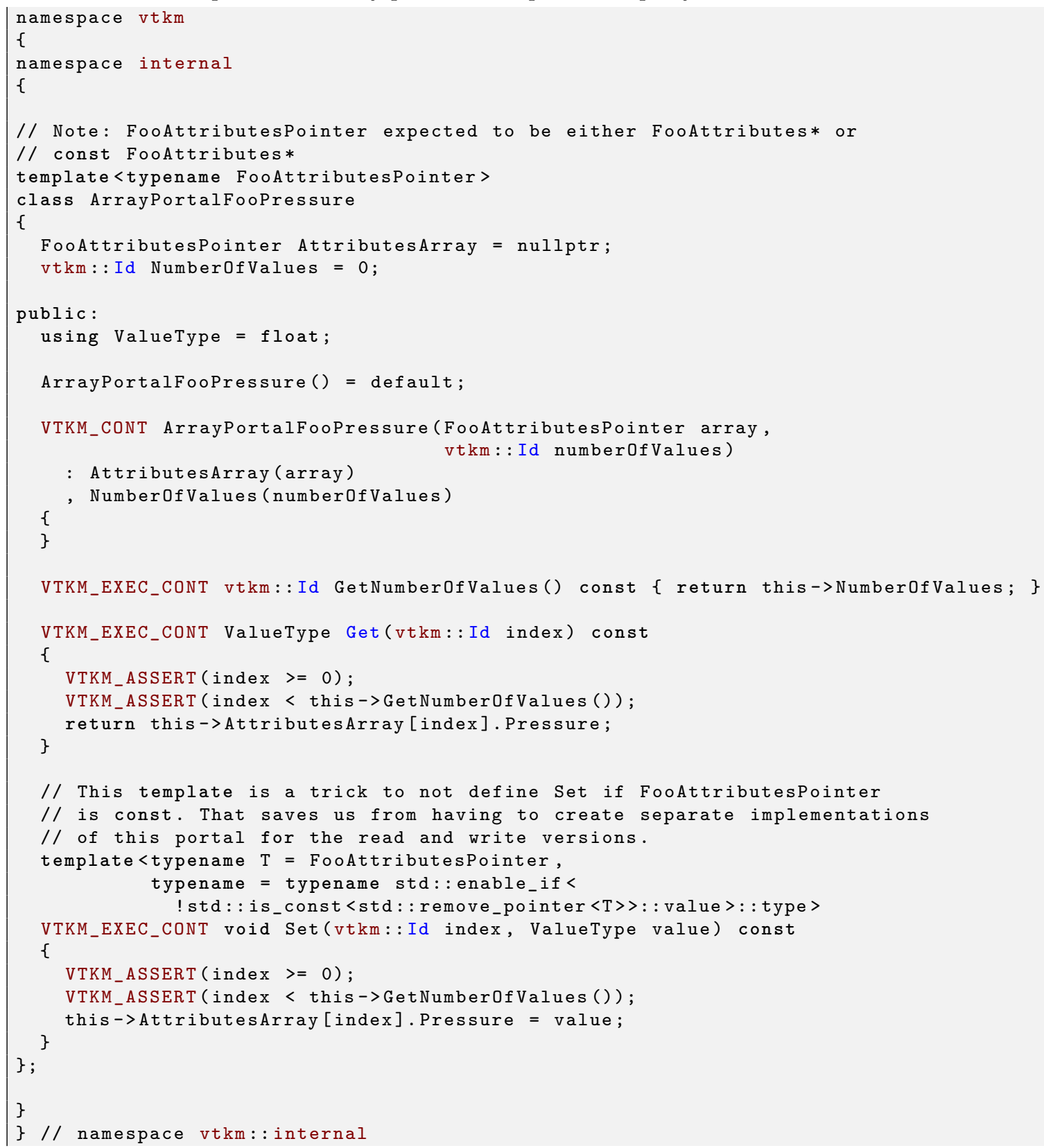

\subsubsection{Storage}

The next step in creating an adapter storage is to define a tag for the adapter. We shall call ours StorageTagFooPressure. Then, we need to create a specialization of the templated vtkm: : cont: :internal: :Storage class. The ArrayHandle will instantiate an object using the array container tag we give it, and we define our own specialization so that it runs our interface into the code.

vtkm: : cont: : internal: :Storage has two template arguments: the base type of the array and the storage tag. 


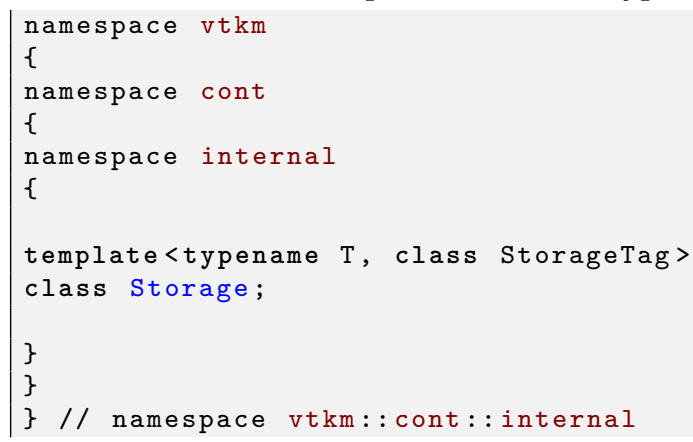

The vtkm: : cont: : internal: :Storage must define the following items.

ReadPortalType The type of an array portal that can be used to access the underlying data. This array portal needs only be read-only. That is, the Set method is optional.

WritePortalType The type of an array portal that can be used to access the underlying data. This array portal should be both read and write capable. If the storage is intended to be read-only, then WritePortalType should be left out and VTKM_STORAGE_NO_WRITE_PORTAL should be declared in the storage class instead.

GetNumberOfBuffers The actual data of an ArrayHandle is stored in a collection of vtkm: :cont: : internal: :Buffer objects. The Storage declares how many Buffer objects are needed by returning the count from the GetNumberOfBuffers method. This method must be declared constexpr and static. A derived array usually contains all the Buffers from the ArrayHandles it is referencing plus an additional Buffer for its own metadata. See Section 35.5.1 for details on Buffer.

GetNumberOfValues This is a static method that takes an array of vtkm: :cont::internal::Buffer objects and returns the number of values in the represented array. The number of values is either derived from the size of the Buffers, the size of the arrays being referenced, or stored in the metadata.

ResizeBuffers This is a static method that takes a number of values for the arrays, an array of vtkm: :cont: :internal::Buffer objects, a vtkm: CopyFlag, and a vtkm: :cont::Token. It then resizes the memory in the Buffer objects to match the requested number of values. If the storage cannot be resized after it is created, then this method should be left out and the VTKM_STORAGE_NO_RESIZE macro should be declared in the storage class instead.

CreateReadPortal This is a static method that takes an array of vtkm::cont::internal::Buffer objects, a vtkm: :cont::DeviceAdapterId, and a vtkm::cont::Token. It returns a ReadPortalType that can be used to read the data on the given device.

CreateWritePortal This is a static method that takes an array of vtkm: :cont: :internal: :Buffer objects, a vtkm: : cont: :DeviceAdapterId, and a vtkm: :cont::Token. It returns a WritePortalType that can be used to read the data on the given device. If the storage is intended to be read-only, then CreateWritePortal should be left out and VTKM_STORAGE_NO_WRITE_PORTAL should be declared in the storage class instead.

The following provides an example implementation of our adapter to FooFields. It relies on the ArrayPortalFooPressure provided in Example 35.20.

Example 35.22: Storage to adapt a third-party container to VTK-m.

\begin{tabular}{l|}
1 \\
2
\end{tabular}

// Includes or definition for ArrayPortalFoopressure 


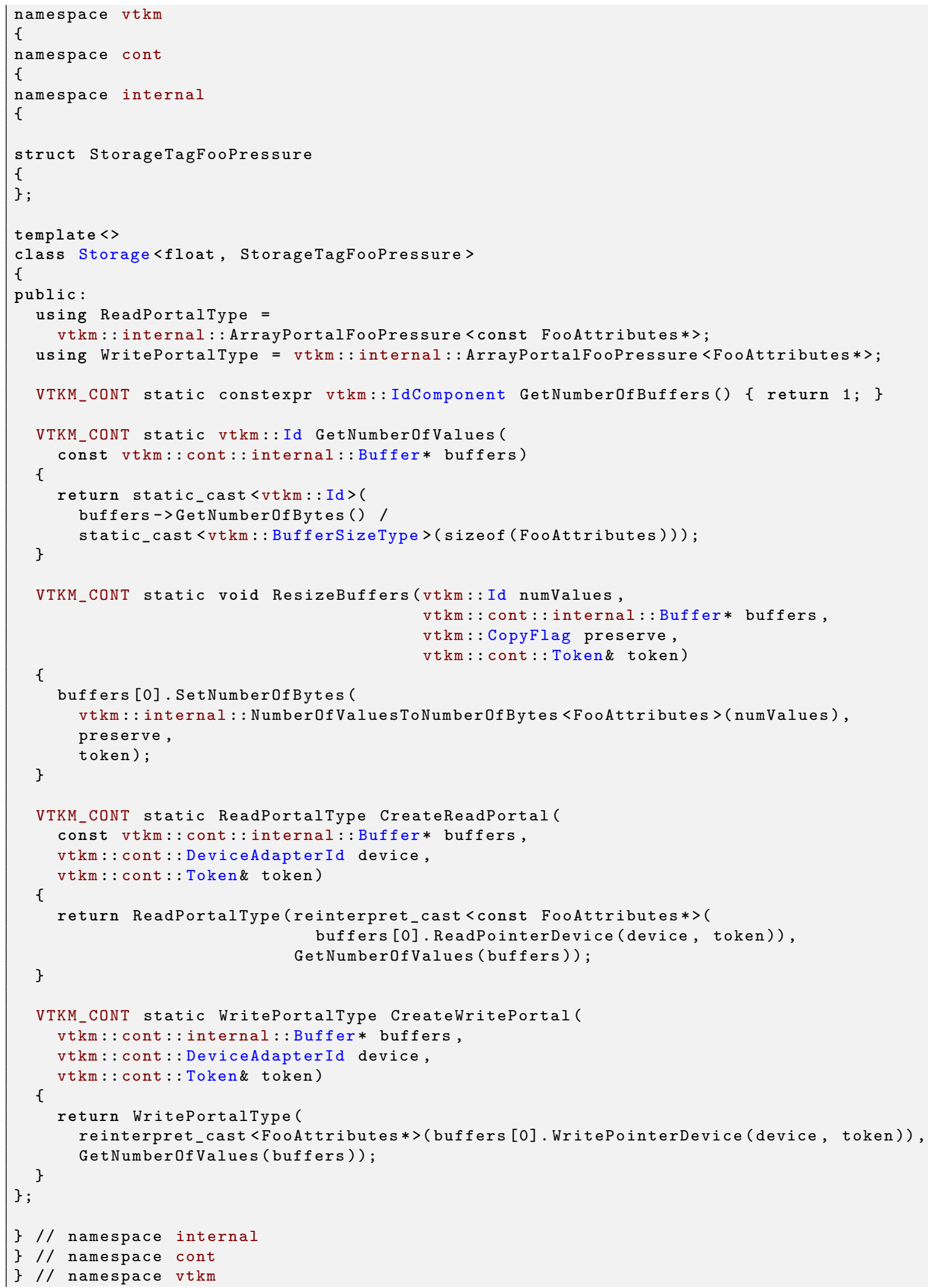




\subsubsection{Subclass}

The final step to make a storage adapter is to make a mechanism to construct an ArrayHandle that points to a particular storage. This can be done by creating a trivial subclass of vtkm: : cont: : ArrayHandle that simply constructs the array handle to the state of an existing container.

Example 35.23: Array handle to adapt a third-party container to VTK-m.

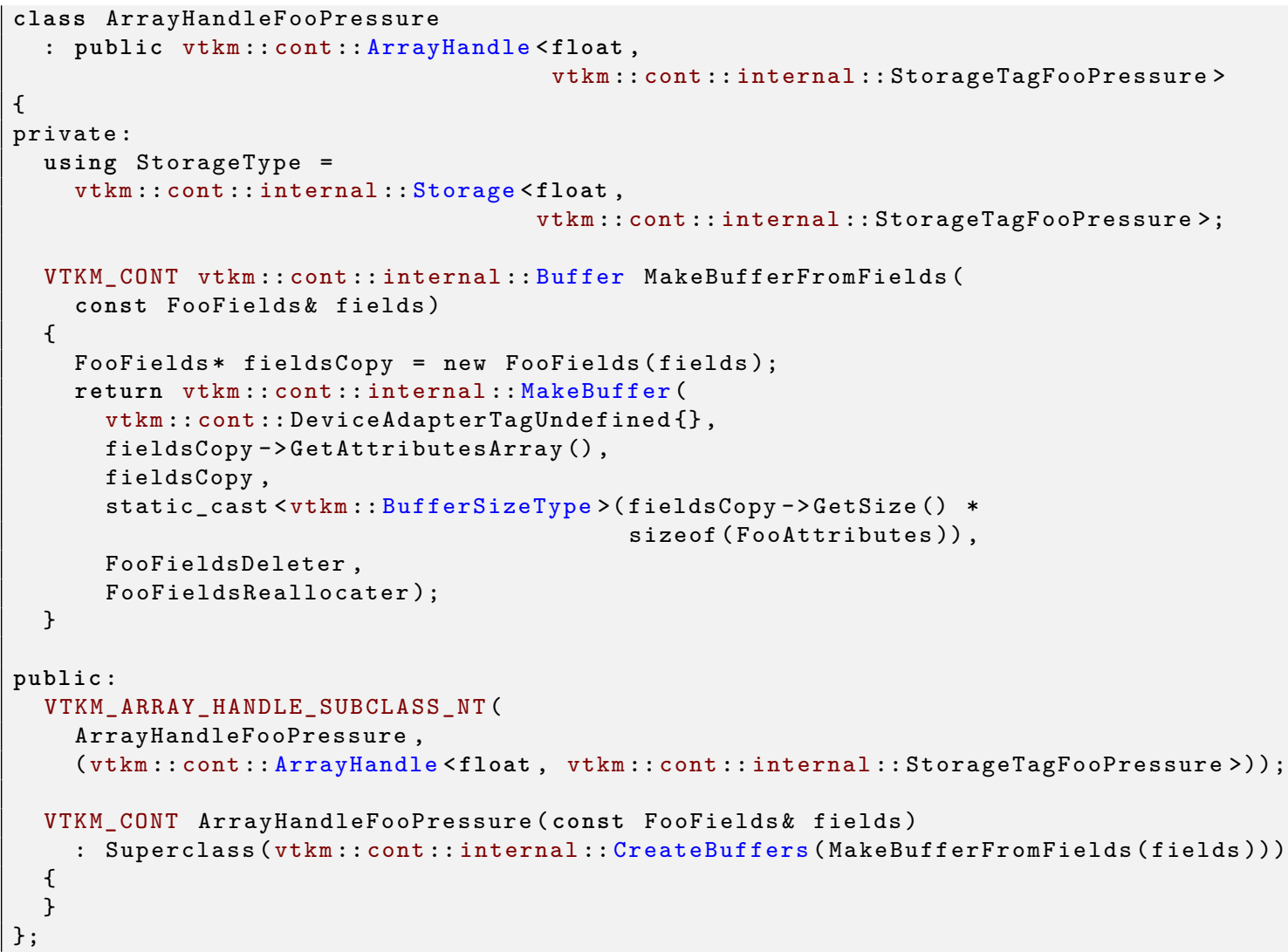

One interesting feature of this example is the constructor on line 29 that wraps the ArrayHandle around an existing FooFields object. This allows VTK-m to read and write directly to and from the memory shared with the FooFields. To make this work, ArrayHandleFooPressure creates a new vtkm: :cont: :internal: :Buffer object using the vtkm: : cont: : internal: :MakeBuffer function (lines 14-21). The first 4 arguments are straightforward: device holding the memory, the actual memory, the object containing the memory, and the number of bytes in the buffer. The fifth and sixth arguments are function pointers that handle deleting and reallocating the buffer (in the control environment). These functions can be defined as follows.

Example 35.24: Memory handling functions to adapt a third-party data structure to ArrayHandle.

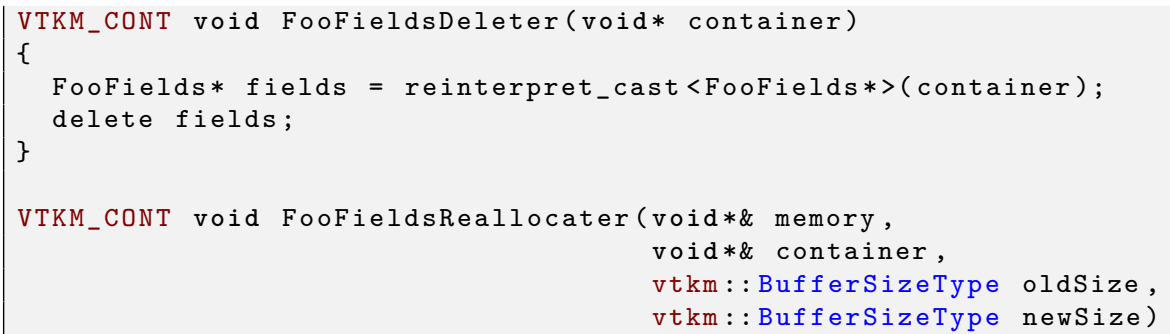




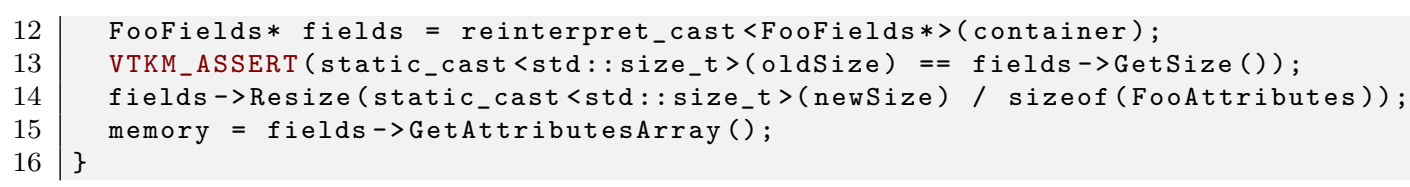

With this new version of ArrayHandle, VTK-m can now read to and write from the FooFields structure directly.

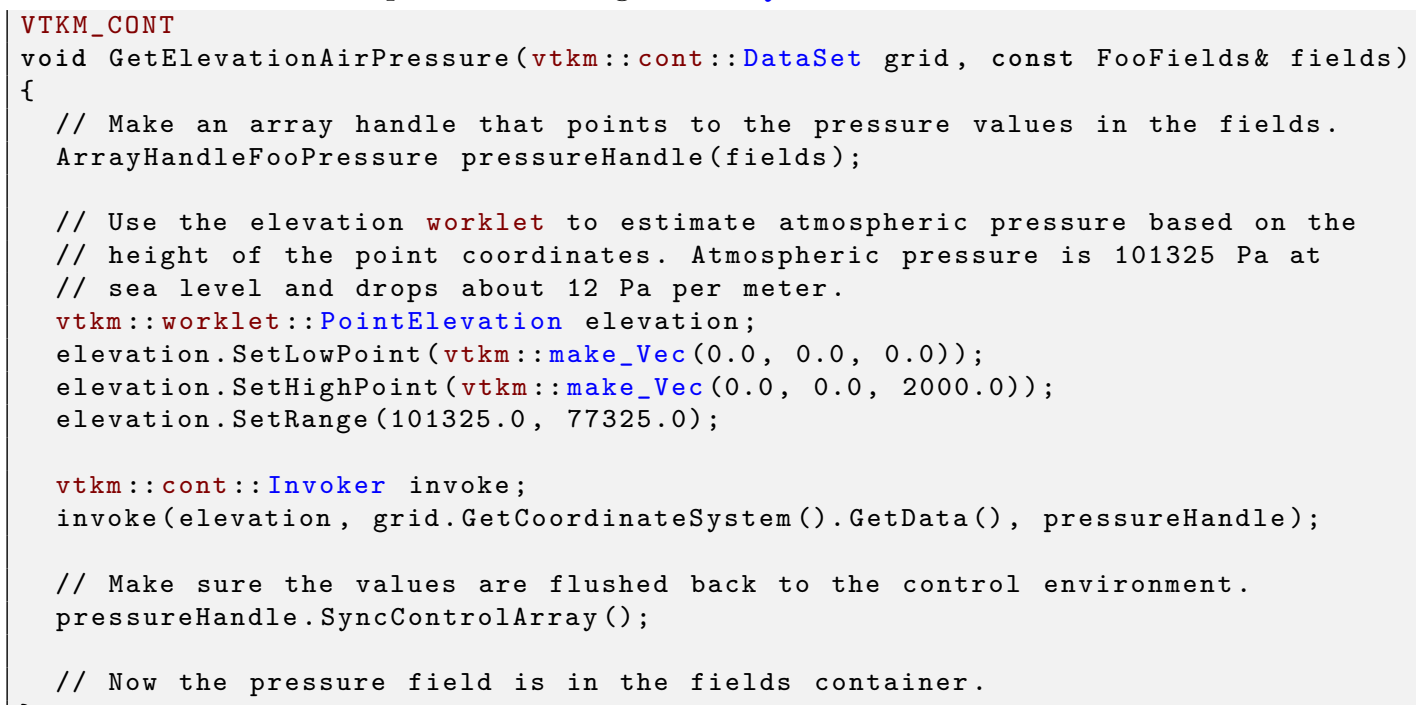

\section{Common Errors}

When using an ArrayHandle in VTK-m some code may be executed in an execution environment with a different memory space. In these cases data written to an ArrayHandle with a custom storage will not be written directly to the storage system you defined. Rather, they will be written to a separate array in the execution environment. If you need to access data in your custom data structure, make sure you call SyncControlArray on the ArrayHandle, as is demonstrated in Example 35.25.

\section{Common Errors}

One of the challenges of introducing a new storage for ArrayHandle is that if the rest of the VTK-m does not recognize the new ArrayHandle, they will not be built with compile support for it. Thus, if you do make \& a custom data adapter for ArrayHandle, you will likely need to define a new set of default types that adds the new storage to VTKM_DEFAULT_STORAGE_LIST. 


\section{REGRESSION TESTING}

VTK-m has hundreds of regression tests built-in, to test the functionality of the entire VTK-m infrastructure on new platforms. In this chapter we will discuss how to run regression tests in VTK-m, as well as how to create new regression tests.

0 Did you know?

¿VTK-m's regression test infrastructure is enabled by default. If you don't need regression tests and are looking for a faster compile time, you can disable it using the CMake configuration variable described in Section 2.2.

\subsection{Running Regression Testing}

This section details how to run VTK-m's regression tests. First will explore how to use ctest to run these tests. ctest is the easiest option for running regression tests, as it sets a number of required arguments to the testing infrastructure automatically. Second, we will give an overview of how to run the regression tests without using ctest, and list the primary command line arguments for doing so.

\subsubsection{Regression Testing Using ctest}

The following code examples show how to run the regression tests in VTK-m using ctest. Example 36.1 shows how to run all of the enabled regression tests in VTK-m.

Example 36.1: Running all regression tests (Unix commands).

1 cd vtkm-build

2 ctest

You can get a list of all the available tests by giving ctest the $-\mathrm{N}$ option, which suppresses actually running the tests (see Example 36.2).

Example 36.2: List all available regression tests (Unix commands).

1 cd vtkm-build

2 ctest $-\mathrm{N}$

Tests can be selected by using the $-\mathrm{R}$ option to ctest. The $-\mathrm{R}$ option is followed by a string or regular expression to match the names of tests to run (see Example 36.3). 
Example 36.3: Running a single regression test (Unix commands).

1 cd vtkm-build

2 ctest -R SystemInformation

Verbose testing output can be selected by using the $-\mathrm{V}$ option to ctest. The $-\mathrm{V}$ options causes the tests to print the underlying run command used to launch each test, along with detailed test progression information (see Example 36.4).

Example 36.4: Running a single regression test with verbose output (Unix commands). The verbose output will first give the exact command used to run the regression test, along with detailed test progression information.

1 cd vtkm-build

2 ctest $-R-V$ SystemInformation

\subsubsection{Regression Testing Without ctest}

It is also possible to run VTK-m regression tests without using ctest. This can be accomplished by running individual unit test wrappers that are located in the $<$ path/to/vtk-m/build $>/$ bin directory. These tests require specific command line options in order for tests to run correctly.

Example 36.5 shows how to run a specific rendering test by passing in the location of the VTK-m data-dir and the baseline-dir

Example 36.5: Running a single regression test without calling ctest (Unix commands).

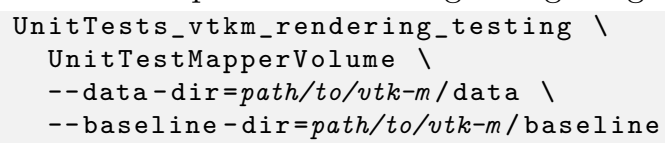

\subsection{Creating Regression Tests}

This section will detail the process and expectations for new regression tests in VTK-m.

\subsubsection{How to Add Data to VTK-m}

VTK-m uses Git LFS for all regression test data. In order to download or add test data to VTK-m you will need to have Git LFS installed. Once installed, you will add unit test data to the data directory in the VTK-m repository. Data in this directory is classified according to its type: structured or unstructured.

Example 36.6: Adding test data to the VTK-m repository (Unix commands).

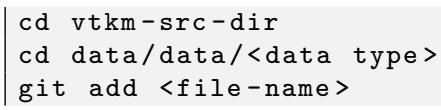




\section{Part V}

\section{Core Development}



Most operations in VTK-m do not require specifying on which device to run. For example, you may have noticed that when using vtkm: : cont: : Invoker to execute a worklet, you do not need to specify a device; it chooses a device for you. Internally, the Invoker has a mechanism to automatically select a device, try it, and fall back to other devices if the first one fails. We saw this at work in the implementation of filters in Chapter 22.

The Invoker is internally using a function named vtkm: : cont : :TryExecute to choose a device. This TryExecute function can be also be used in other instances where a specific device needs to be chosen.

TryExecute is a simple, generic mechanism to run an algorithm that requires a device adapter without directly specifying a device adapter. vtkm: : cont: :TryExecute is a templated function. The first argument is a functor object whose parenthesis operator takes a device adapter tag and returns a bool that is true if the call succeeds on the given device. If any further arguments are given to TryExecute, they are passed on to the functor. Thus, the parenthesis operator on the functor should take a device adapter tag as its first argument and any remaining arguments must match those passed to TryExecute.

To demonstrate the operation of TryExecute, consider an operation to find the average value of an array. Doing so with a given device adapter is a straightforward use of the reduction operator.

Example 37.1: A function to find the average value of an array in parallel.

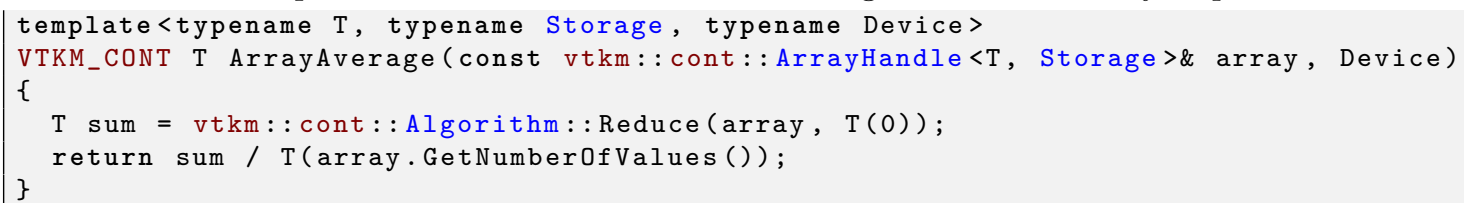

The function in Example 37.1 requires a device adapter. We want to make an alternate version of this function that does not need a specific device adapter but rather finds one to use. To do this, we first make a functor as described earlier. It takes a device adapter tag as an argument, calls the version of the function shown in Example 37.1, and returns true when the operation succeeds. We then create a new version of the array average function that does not need a specific device adapter tag and calls TryExecute with the aforementioned functor.

Example 37.2: Using TryExecute.

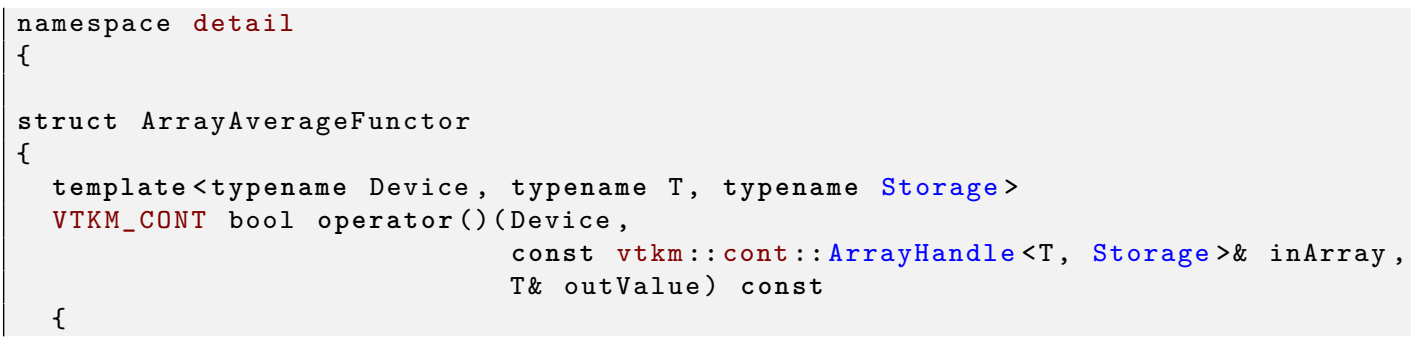




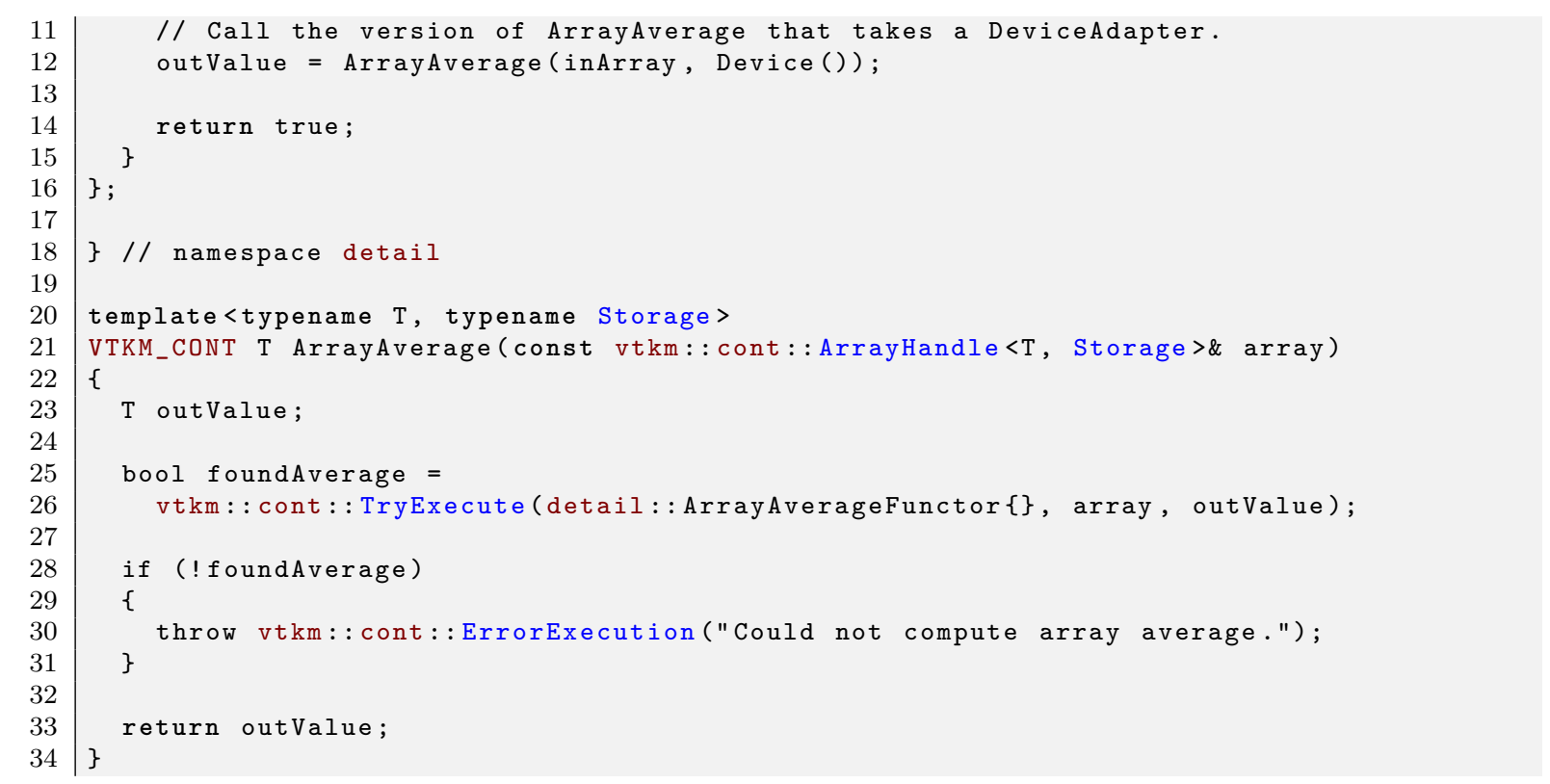

When TryExecute calls the operation of your functor, it will catch any exceptions that the functor might throw. TryExecute will interpret any thrown exception as a failure on that device and try another device. If all devices fail, TryExecute will return a false value rather than throw its own exception. This means if you want to have an exception thrown from a call to TryExecute, you will need to check the return value and throw the exception yourself. 


\section{IMPLEMENTING DEVICE ADAPTERS}

VTK-m comes with several implementations of device adapters so that it may be ported to a variety of platforms. It is also possible to provide new device adapters to support yet more devices, compilers, and libraries. A new device adapter provides a tag, a class to manage arrays in the execution environment, a class to establish virtual objects in the execution environment, a collection of algorithms that run in the execution environment, and (optionally) a timer.

Most device adapters are associated with some type of device or library, and all source code related directly to that device is placed in a subdirectory of vtkm/cont. For example, files associated with CUDA are in vtkm/cont/cuda, files associated with the Intel Threading Building Blocks (TBB) are located in vtkm/cont/tbb, and files associated with OpenMP are in vtkm/cont/openmp. The documentation here assumes that you are adding a device adapter to the VTK-m source code and following these file conventions.

For the purposes of discussion in this section, we will give a simple example of implementing a device adapter using the std: : thread class provided by $\mathrm{C}++11$. We will call our device Cxx11Thread and place it in the directory vtkm/cont/cxx11.

By convention the implementation of device adapters within VTK-m are divided into 6 header files with the names DeviceAdapterTag*.h, DeviceAdapterRuntimeDetector*.h, DeviceAdapterMemoryManager*.h, RuntimeDeviceConfiguration*.h, VirtualObjectTransfer*.h, and DeviceAdapterAlgorithm*.h, which are hidden in internal directories. The DeviceAdapter*.h that most code includes is a trivial header that simply includes these other 7 files. For our example std: :thread device, we will create the base header at vtkm/cont/cxx11/DeviceAdapterCxx11Thread.h. The contents are the following (with minutia like include guards removed).

Example 38.1: Contents of the base header for a device adapter.

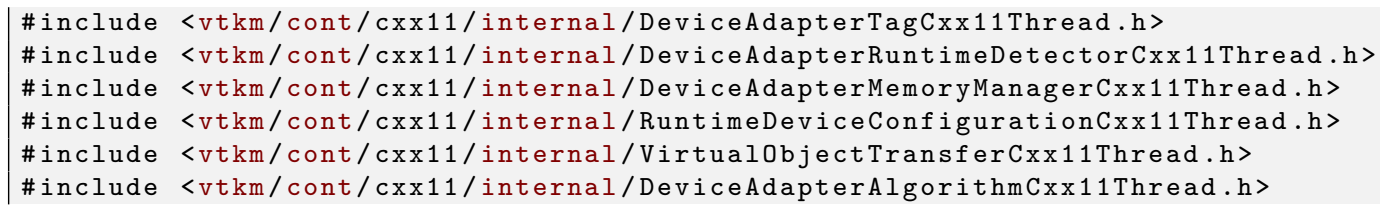

The reason VTK-m breaks up the code for its device adapters this way is that there is an interdependence between the implementation of each device adapter and the mechanism to pick a default device adapter. Breaking up the device adapter code in this way maintains an acyclic dependence among header files.

\subsection{Tag}

The device adapter tag, as described in Section 12.1 is a simple empty type that is used as a template parameter to identify the device adapter. Every device adapter implementation provides one. The device adapter tag is 
typically defined in an internal header file with a prefix of DeviceAdapterTag.

The device adapter tag should be created with the macro VTKM_VALID_DEVICE_ADAPTER. This adapter takes an abbreviated name that it will append to DeviceAdapterTag to make the tag structure. It will also create some support classes that allow VTK-m to introspect the device adapter. The macro also expects a unique integer identifier that is usually stored in a macro prefixed with VTKM_DEVICE_ADAPTER_. These identifiers for the device adapters provided by the core VTK-m are declared in vtkm/cont/internal/DeviceAdapterTag.h.

The following example gives the implementation of our custom device adapter, which by convention would be placed in the vtkm/cont/cxx11/internal/DeviceAdapterTagCxx11Thread.h header file.

Example 38.2: Implementation of a device adapter tag.

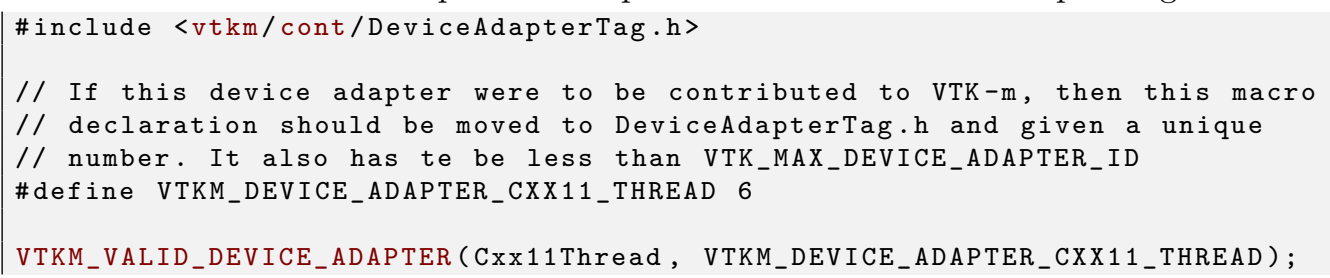

This new device adapter tag needs to be added to vtkm: : cont: : DeviceAdapterListCommon, which is defined in vtkm/cont/DeviceAdapterList.h. Other components of VTK-m will use this list to write code for the device. If you do not add the device tag to this list, then the device will not be tried when things are invoked in the execution environment, and directly specifying execution on this device will likely fail.

Example 38.3: Modification of DeviceAdapterListCommon in DeviceAdapterList.h

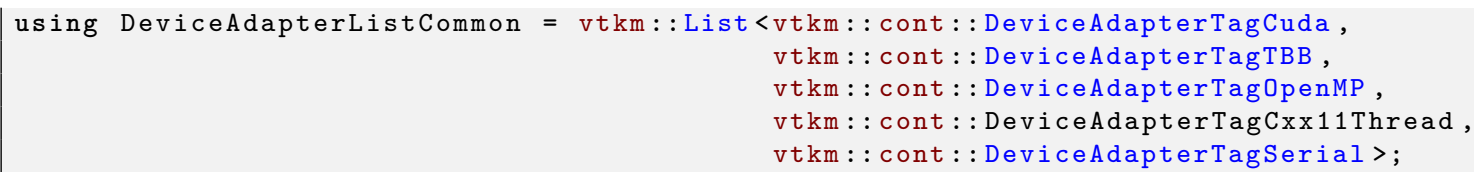

\section{Did you know?}

The order of device adapter tags in vtkm::cont::DeviceAdapterListCommon matters. Devices will be tried in the order listed in this list. Thus, the most "preferred" devices should be listed first. In Example 38.3, our new $C++11$ thread device will be used before the serial device but after the other parallel devices.

It is OK for vtkm: : cont: :DeviceAdapterListCommon to contain device adapter tags for devices that are not being compiled for. These devices will be registered as inactive and be skipped.

\subsection{Runtime Detector}

VTK-m defines a template named vtkm: :cont: :DeviceAdapterRuntimeDetector that provides the ability to detect whether a given device is available on the current system. DeviceAdapterRuntimeDetector has a single template argument that is the device adapter tag.

Example 38.4: Prototype for DeviceAdapterRuntimeDetector.

\begin{tabular}{l|ll}
1 & namespace & vtkm \\
2 & \{ & \\
3 & namespace & cont
\end{tabular} 


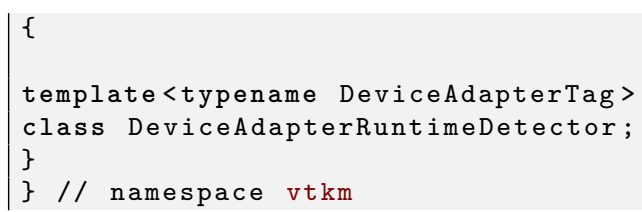

All device adapter implementations must create a specialization of DeviceAdapterRuntimeDetector. They must contain a method named DeviceAdapterRuntimeDetector: :Exists that returns a true or false value to indicate whether the device is available on the current runtime system. For our simple $\mathrm{C}++$ threading example, the $\mathrm{C}++$ threading is always available (even if only one such processing element exists) so our implementation simply returns true if the device has been compiled.

Example 38.5: Implementation of DeviceAdapterRuntimeDetector specialization

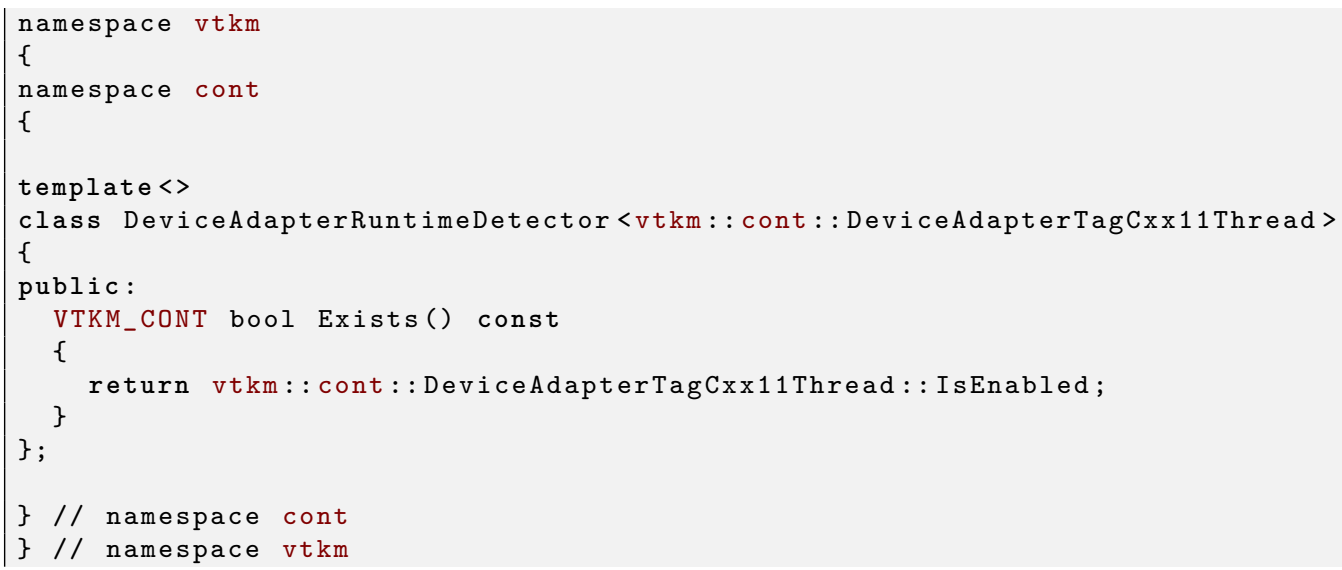

\subsection{Memory Manager}

VTK-m defines a template named vtkm::cont::internal::DeviceAdapterMemoryManager that provides the ability to allocate memory on the device and copy data. DeviceAdapterMemoryManager has a single template argument that is the device adapter tag.

Example 38.6: Prototype for DeviceAdapterMemoryManager.

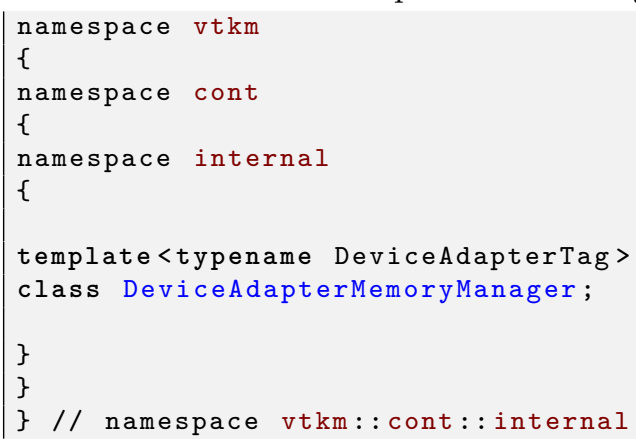

All device adapter implementations must create a specialization of DeviceAdapterMemoryManager. This specialization of DeviceAdapterMemoryManager must inherit from vtkm: : cont: : internal: :DeviceAdapterMemoryManagerBase. The DeviceAdapterMemoryManager allocates memory and returns it wrapped in a vtkm: : cont: :internal: :BufferInfo object. The superclass provides the DeviceAdapterMemoryManagerBase : :ManageArray 
method to take a raw pointer for the device (captured as a void *) along with some metadata and management functions and returns that pointer wrapped in a BufferInfo management object.

A specialization of DeviceAdapterMemoryManager must override the following pure virtual methods (which are defined in the DeviceAdapterMemoryManagerBase superclass).

GetDevice Return a vtkm: :cont: :DeviceAdapterId for the device that this memory manager allocates and deallocates for.

Allocate Given a buffer size in bytes, allocates the buffer on the device and returns it in a BufferInfo object.

CopyHostToDevice Copies a BufferInfo object for memory allocated on the host to the device. DeviceAdapterMemoryManager must implement two forms of CopyHostToDevice. The first form takes just a source BufferInfo and returns a new BufferInfo containing a copy of the data on the device. If the device supports shared or unified memory, this can be a shallow copy. The second form takes both a source BufferInfo and a pre-allocated destination BufferInfo.

CopyDeviceToHost Copies a BufferInfo object for memory allocated on the device to the host. DeviceAdapterMemoryManager must implement two forms of CopyDeviceToHost. The first form takes just a source BufferInfo and returns a new BufferInfo containing a copy of the data on the host. If the device supports shared or unified memory, this can be a shallow copy. The second form takes both a source BufferInfo and a pre-allocated destination BufferInfo.

CopyDeviceToDevice Copies a BufferInfo object for memory allocated on the device to another buffer on the device. DeviceAdapterMemoryManager must implement two forms of CopyDeviceToDevice. The first form takes just a source BufferInfo and returns a new BufferInfo containing a copy of the data on the device. The second form takes both a source BufferInfo and a pre-allocated destination BufferInfo.

If the control and execution environments share the same memory space, the execution array manager can, and should, share buffers among host and "device" and shallow copy data when possible. VTK-m comes with a class called vtkm::cont::internal: :DeviceAdapterMemoryManagerShared that provides the implementation for a device memory manager that shares a memory space with the control environment. In this case, the DeviceAdapterMemoryManager specialization need only override the GetDevice method. (DeviceAdapterMemoryManagerShared will provide all other necessary overrides.)

Continuing our example of a device adapter based on C++11's std::thread class, here is the implementation of DeviceAdapterMemoryManager, which by convention would be placed in the vtkm/cont/cxx11/internal/DeviceAdapterMemoryManagerCxx11Thread.h header file.

Example 38.7: Specialization of DeviceAdapterMemoryManager.

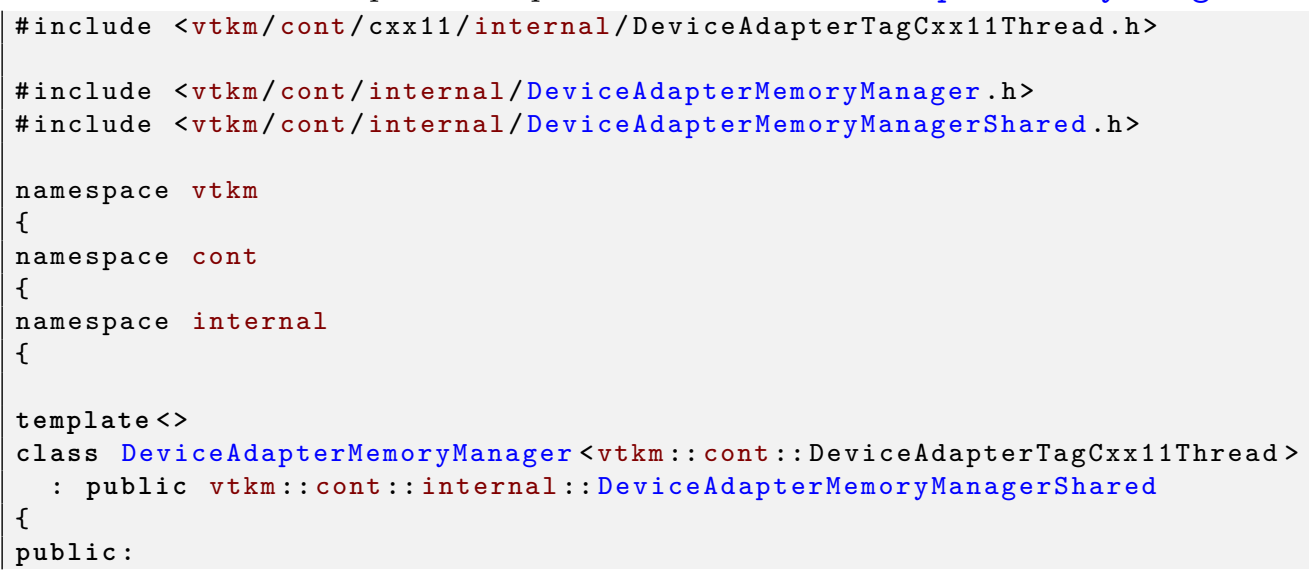




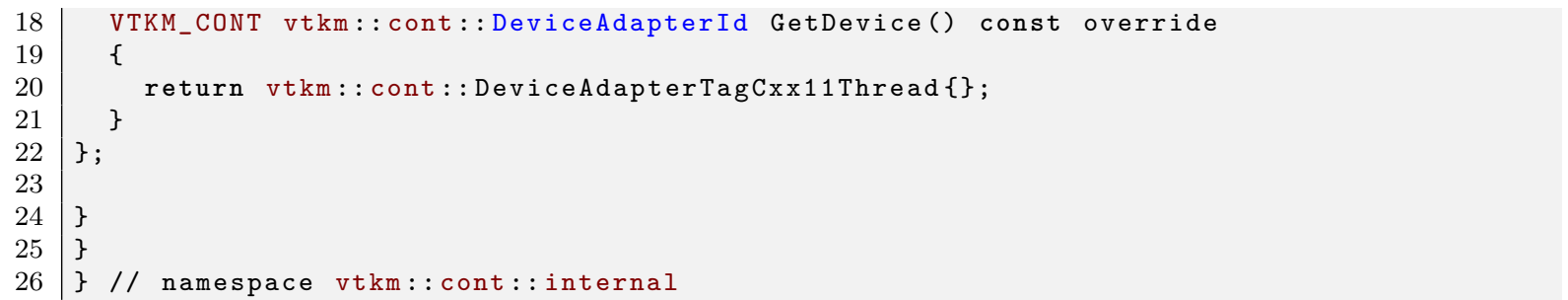

\section{Did you know?}

You may notice that although vtkm: :cont: :internal::DeviceAdapterMemoryManager requires methods to allocate memory, it has no methods to delete memory. This is because all memory created by a vtkm::cont::internal::DeviceAdapterMemoryManager is wrapped in a vtkm::cont::internal::BufferInfo object. Responsibility for the memory management is taken over by BufferInfo and the memory will be automatically deleted once it is no longer used.

\subsection{Runtime Device Configuration}

VTK-m defines a template named vtkm: : cont: :internal: :RuntimeDeviceConfiguration that makes it possible to initialize various runtime configuration parameters of the underlying devices. RuntimeDeviceConfiguration has a single template argument that is the device adapter tag.

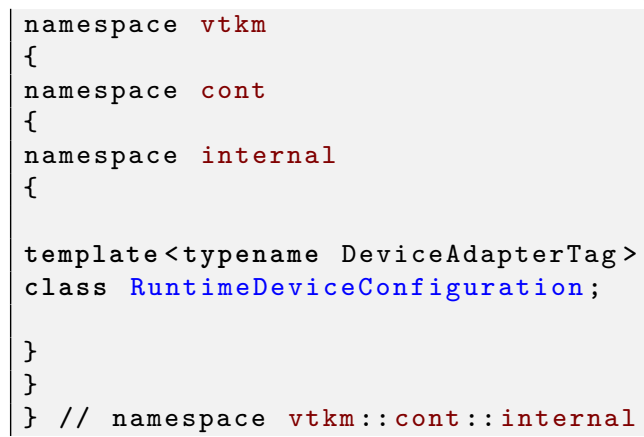

All device adapter implementations must create a specialization of RuntimeDeviceConfiguration. This specialization of RuntimeDeviceConfiguration must inherit from vtkm: :cont: :internal: :RuntimeDeviceConfigurationBase. The RuntimeDeviceConfiguration provides various RuntimeDeviceConfigurationBase: : Set* and RuntimeDeviceConfigurationBase::Get* methods for setting and accessing device specific runtime parameters. The superclass provides the RuntimeDeviceConfigurationBase: Initialize method that takes in a RuntimeDeviceConfigurationOptions argument used to set various device parameters when VTK-m is initialized.

Specializations of RuntimeDeviceConfiguration must override the GetDevice virtual method, which returns a vtkm: :cont: :DeviceAdapterId for the device that this runtime device configuration is overseeing. Specializations of RuntimeDeviceConfiguration are not required to override the following methods defined in RuntimeDeviceConfigurationBase. These methods should be overridden only if suitable device specific runtime parameters can be set or queried. 
SetThreads Takes the provided vtkm::Id and attempts to set the number of threads to use for this specific device. Return a vtkm: :cont: :internal : :RuntimeDeviceConfigReturnCode representing the success/failure of the device operation.

SetNumaRegions Takes the provided vtkm::Id and attempts to set the number of numa regions to use for this specific device Return a vtkm: : cont : : internal : :RuntimeDeviceConfigReturnCode representing the success/failure of the device operation.

SetDeviceInstance Takes the provided vtkm::Id and attempts to set the specific device instance to use for this device Return a vtkm::cont::internal::RuntimeDeviceConfigReturnCode representing the success/failure of the device operation.

GetThreads Takes the provided vtkm: : Id and attempts to set it to the number of threads this device is currently specified to use. Return a vtkm: :cont: :internal: :RuntimeDeviceConfigReturnCode representing the success/failure of the device operation.

GetNumaRegions Takes the provided vtkm::Id and attempts to set it to the number of numa regions this device is currently specified to use. Return a vtkm: : cont: : internal : :RuntimeDeviceConfigReturnCode representing the success/failure of the device operation.

GetDeviceInstance Takes the provided vtkm::Id and attempts to set it to the specific device instance this device is currently set to use. Return a vtkm: : cont: : internal : :RuntimeDeviceConfigReturnCode representing the success/failure of the device operation.

GetMaxThreads Takes the provided vtkm: :Id and attempts to set it to the maximum number of threads allowed by this device. Return a vtkm::cont: internal::RuntimeDeviceConfigReturnCode representing the success/failure of the device operation.

GetMaxDevices Takes the provided vtkm: :Id and attempts to set it to the mximum number of devices currently allowed by this device. Return a vtkm: : cont: : internal : :RuntimeDeviceConfigReturnCode representing the success/failure of the device operation.

ParseExtraArguments Called before RuntimeDeviceConfigurationBase: : Initialize, used to perform extra command line argument parsing specific for a given device. Currently only overriden by the vtkm: : cont : :internal: :RuntimeDeviceConfigurationKokkos device.

InitializeSubsystem Called at the very end of RuntimeDeviceConfigurationBase: : Initialize, and is used to perform additional subystem initialize for a given device. Currently over overriden by the vtkm: : cont : :internal: : RuntimeDeviceConfigurationKokkos device.

Continuing our example of a device adapter based on $\mathrm{C}++11$ 's std::thread class, here is the implementation of RuntimeDeviceConfiguration, which by convention would be placed in the vtkm/cont/cxx11/internal/RuntimeDeviceConfigurationCxx11Thread.h header file.

Example 38.9: Specialization of RuntimeDeviceConfiguration.

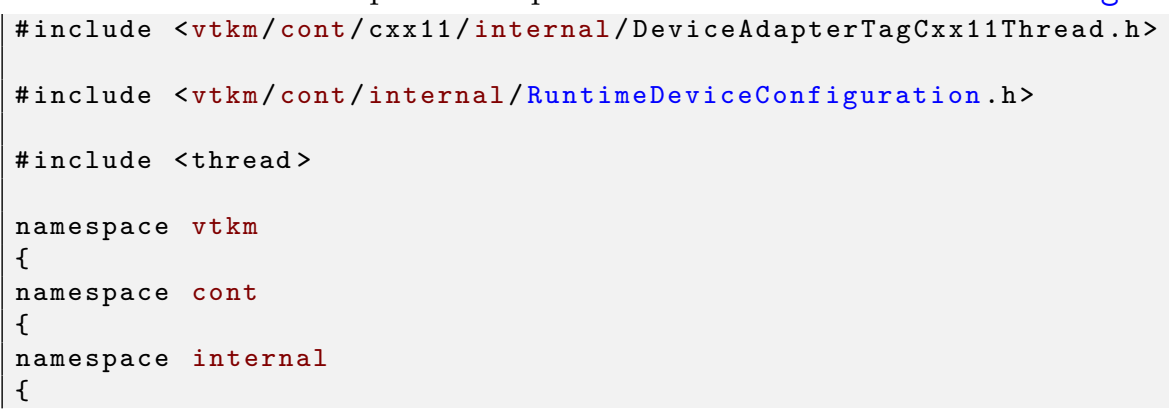




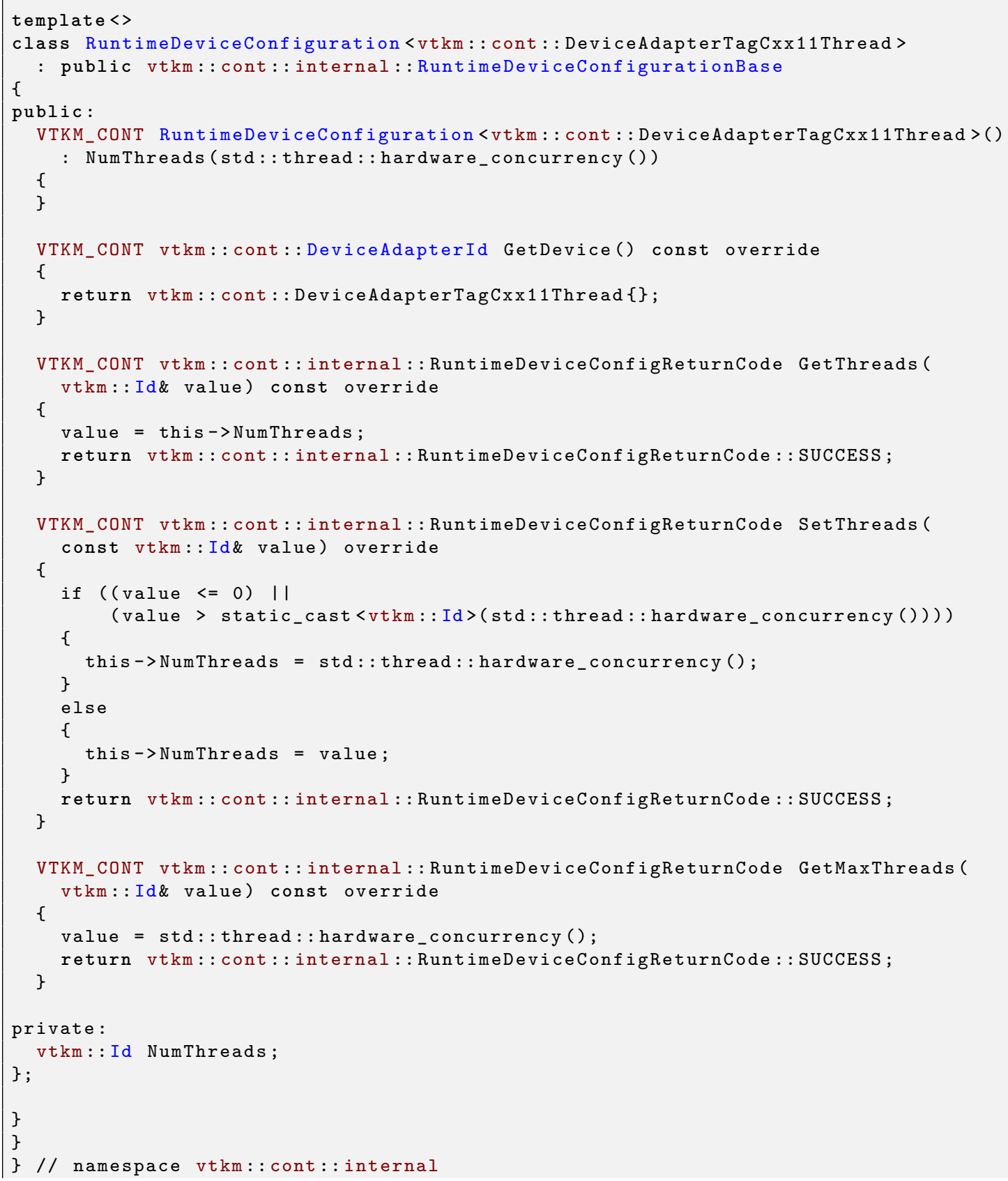

\section{Common Errors}

vtkm: : cont: : Initialize automatically initializes the vtkm: :cont: :internal: :RuntimeDeviceConfiguration for all available devices using parse VTK-m command line arguments. These device runtime configurations are statically managed through the vtkm: :cont: :RuntimeDeviceInformation class, which ensures that there is exactly one initialized instance of each vtkm: : cont: : internal : RuntimeDeviceCon- 


\begin{abstract}
figuration available for each device. This guarantees that vtkm: : cont: : internal: :RuntimeDeviceConfiguration device classes cannot be initialized more than once, but may lead to device initialization inconsistencies when attempting to access a vtkm: :cont: : internal: :RuntimeDeviceConfiguration before calling vtkm: :cont::Initialize. When creating a new vtkm::cont::internal: :RuntimeDeviceConfiguration it is important to add an include for the new DeviceAdapterRuntimeDetector: :header to vtkm: : cont: :RuntimeDeviceInformation so that the new device is compiled correctly. Additionally, it is important to note that accessing a vtkm: :cont: : internal: :RuntimeDeviceConfiguration via RuntimeDeviceInformation: :GetRuntimeConfiguration inside the DeviceAdapterRuntimeDetector: :Exists method will initialize the underlying device incorrectly since VTK-m performs device existence checks while parsing command line arguments.
\end{abstract}

\title{
38.5 Virtual Object Transfer
}

VTK-m defines a template named vtkm::cont::internal::VirtualObjectTransfer that is responsible for instantiating virtual objects in the execution environment. Virtual objects are a deprecated feature that will be removed in a future version of VTK-m. The VirtualObjectTransfer class is the internal mechanism that allocates space for the object and sets up the virtual method tables for them. This class has two template parameters. The first parameter is the concrete derived type of the virtual object to be transferred to the execution environment. It is assumed that after the object is copied to the execution environment, a pointer to a base superclass of this concrete derived type will be used. The second template argument is the device adapter on which to put the object.

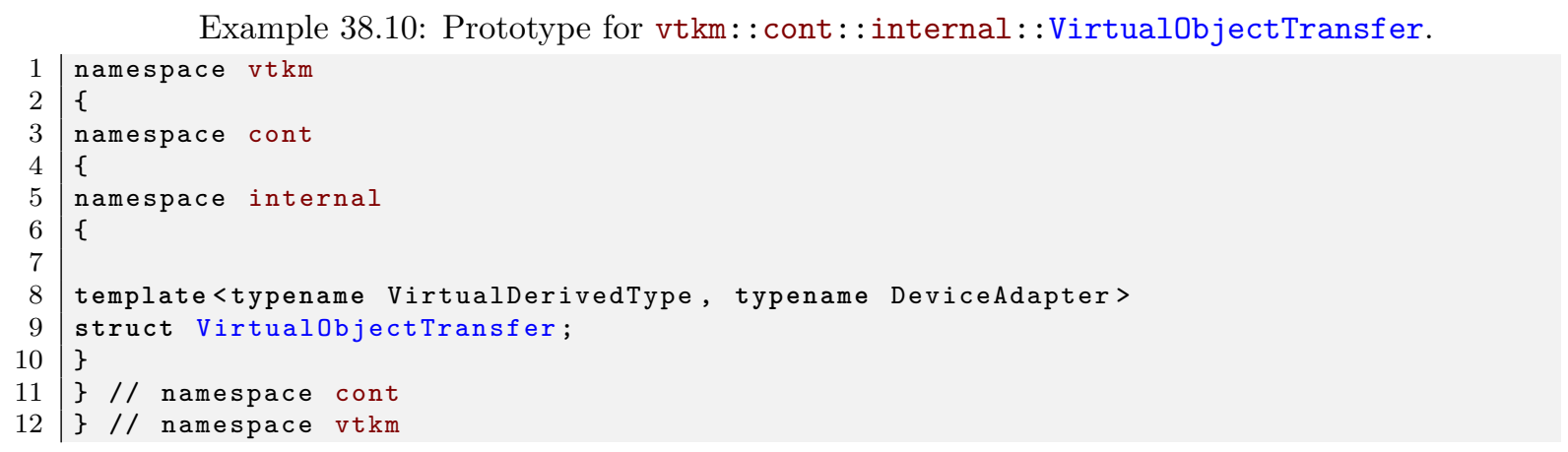

A device adapter must provide a partial specialization of VirtualobjectTransfer for its device adapter tag. This partial specialization is typically defined in an internal header file with a prefix of VirtualObjectTransfer. The implementation for VirtualObjectTransfer can establish a virtual object in the execution environment based on an object in the control environment, update the state of said object, and release all the resources for the object. VirtualobjectTransfer must provide the following methods.

VirtualObjectTransfer (constructor) A VirtualObjectTransfer has a constructor that takes a pointer to the derived type that (eventually) gets transferred to the execution environment of the given device adapter. The object provided must stay valid for the lifespan of the VirtualobjectTransfer object.

PrepareForExecution Transfers the virtual object (given in the constructor) to the execution environment and returns a pointer to the object that can be used in the execution environment. The returned object may not be valid in the control environment and should not be used there. PrepareForExecution takes a single bool argument. If the argument is false and PrepareForExecution was called previously, then the method can return the same data as the last call without any updates. If the argument is true, then the data in 
the execution environment is always updated regardless of whether data was copied in a previous call to PrepareForExecution. This argument is used to tell the VirtualobjectTransfer whether the object in the control environment has changed and has to be updated in the execution environment.

ReleaseResources Frees up any resources in the execution environment. Any previously returned virtual object from PrepareForExecution becomes invalid. (The destructor for VirtualObjectTransfer should also release the resources.)

Specializations of this template typically take on one of two forms. If the control and execution environments have separate memory spaces, then this class behaves by copying the concrete control object to the execution environment (where the virtual table will be invalid) and a new object is created in the execution environment by copying the object from the control environment. It can be assumed that the object can be trivially copied (with the exception of the virtual method table).

\section{Did you know?}

For some devices, like CUDA, it is either only possible or more efficient to allocate data from the host (the control environment). To avoid having to allocate data from the device (the execution environment), implement PrepareForExecution by first allocating data from the host and then running code on the device that does a "placement new" to create and copy the object in the pre-allocated space.

However, if the control and execution environments share the same memory space, the virtual object transfer can, and should, just bind directly with the target concrete object. VTK-m comes with a class called vtkm: :cont: : internal::VirtualObjectTransferShareWithControl that provides the implementation for a virtual object transfer that shares a memory space with the control environment. In this case, making the VirtualObjectTransfer specialization be a trivial subclass is sufficient. Continuing our example of a device adapter based on C++11's std: : thread class, here is the implementation of VirtualobjectTransfer, which by convention would be placed in the vtkm/cont/cxx11/internal/VirtualObjectTransferCxx11Thread.h header file.

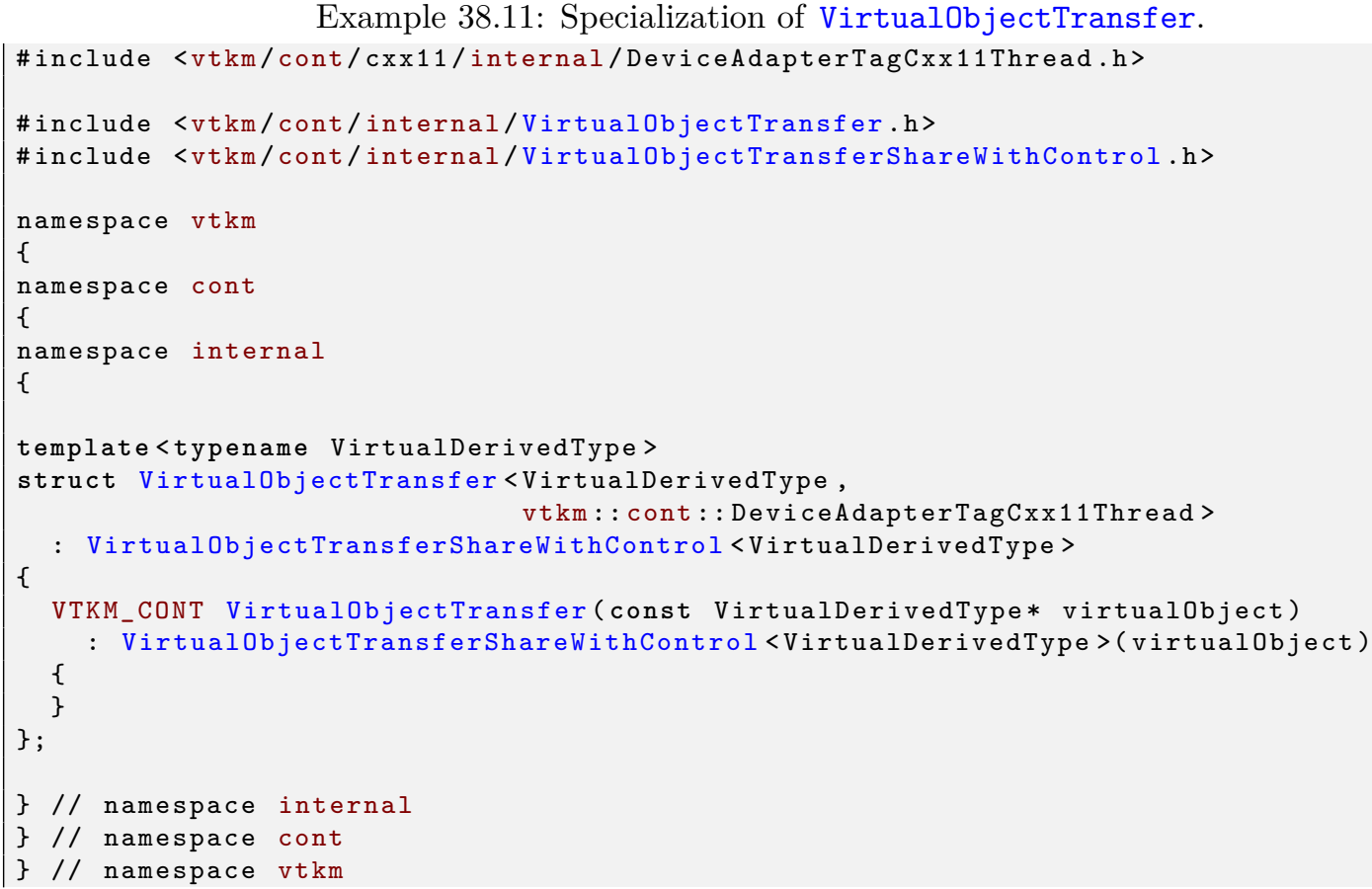




\subsection{Algorithms}

A device adapter implementation must also provide a specialization of vtkm: : cont: : DeviceAdapterAlgorithm, which provides the underlying implementation of the algorithms described in Chapter 34. The implementation for the device adapter algorithms is typically placed in a header file with a prefix of DeviceAdapterAlgorithm.

Although there are many methods in DeviceAdapterAlgorithm, it is seldom necessary to implement them all. Instead, VTK-m comes with vtkm: : cont: : internal: :DeviceAdapterAlgorithmGeneral that provides generic implementation for most of the required algorithms. By deriving the specialization of DeviceAdapterAlgorithm from DeviceAdapterAlgorithmGeneral, only the implementations for Schedule and Synchronize need to be implemented. All other algorithms can be derived from those.

That said, not all of the algorithms implemented in DeviceAdapterAlgorithmGeneral are optimized for all types of devices. Thus, it is worthwhile to provide algorithms optimized for the specific device when possible. In particular, it is best to provide specializations for the sort, scan, and reduce algorithms.

It is standard practice to implement a specialization of DeviceAdapterAlgorithm by having it inherit from vtkm::cont::internal::DeviceAdapterAlgorithmGeneral and specializing those methods that are optimized for a particular system. DeviceAdapterAlgorithmGeneral is a templated class that takes as its single template parameter the type of the subclass. For example, a device adapter algorithm structure named DeviceAdapterAlgorithm<DeviceAdapterTagFoo> will subclass DeviceAdapterAlgorithmGeneral<DeviceAdapterAlgorithm<DeviceAdapterTagFoo> >.

\section{id you know?}

The convention of having a subclass be templated on the derived class' type is known as the Curiously Recurring Template Pattern (CRTP). In the case of DeviceAdapterAlgorithmGeneral, VTK-m uses this CRTP behavior to allow the general implementation of these algorithms to run Schedule and other specialized algorithms in the subclass.

One point to note when implementing the Schedule methods is to make sure that errors handled in the execution environment are handled correctly. As described in Chapter 23, errors are signaled in the execution environment by calling RaiseError on a functor or worklet object. This is handled internally by the vtkm: :exec: :internal: :ErrorMessageBuffer class. ErrorMessageBuffer really just holds a small string buffer, which must be provided by the device adapter's Schedule method.

So, before Schedule executes the functor it is given, it should allocate a small string array in the execution environment, initialize it to the empty string, encapsulate the array in an ErrorMessageBuffer object, and set this buffer object in the functor. When the execution completes, Schedule should check to see if an error exists in this buffer and throw a vtkm: :cont: :ErrorExecution if an error has been reported.

\section{Common Errors}

Exceptions are generally not supposed to be thrown in the execution environment, but it could happen on devices that support them. Nevertheless, few thread schedulers work well when an exception is thrown in them. Thus, when implementing adapters for devices that do support exceptions, it is good practice to catch them within the thread and report them through the ErrorMessageBuffer. 
The following example is a minimal implementation of device adapter algorithms using C++11's std: : thread class. Note that no attempt at providing optimizations has been attempted (and many are possible). By convention this code would be placed in the vtkm/cont/cxx11/internal/DeviceAdapterAlgorithmCxx11Thread.h header file.

Example 38.12: Minimal specialization of DeviceAdapterAlgorithm.

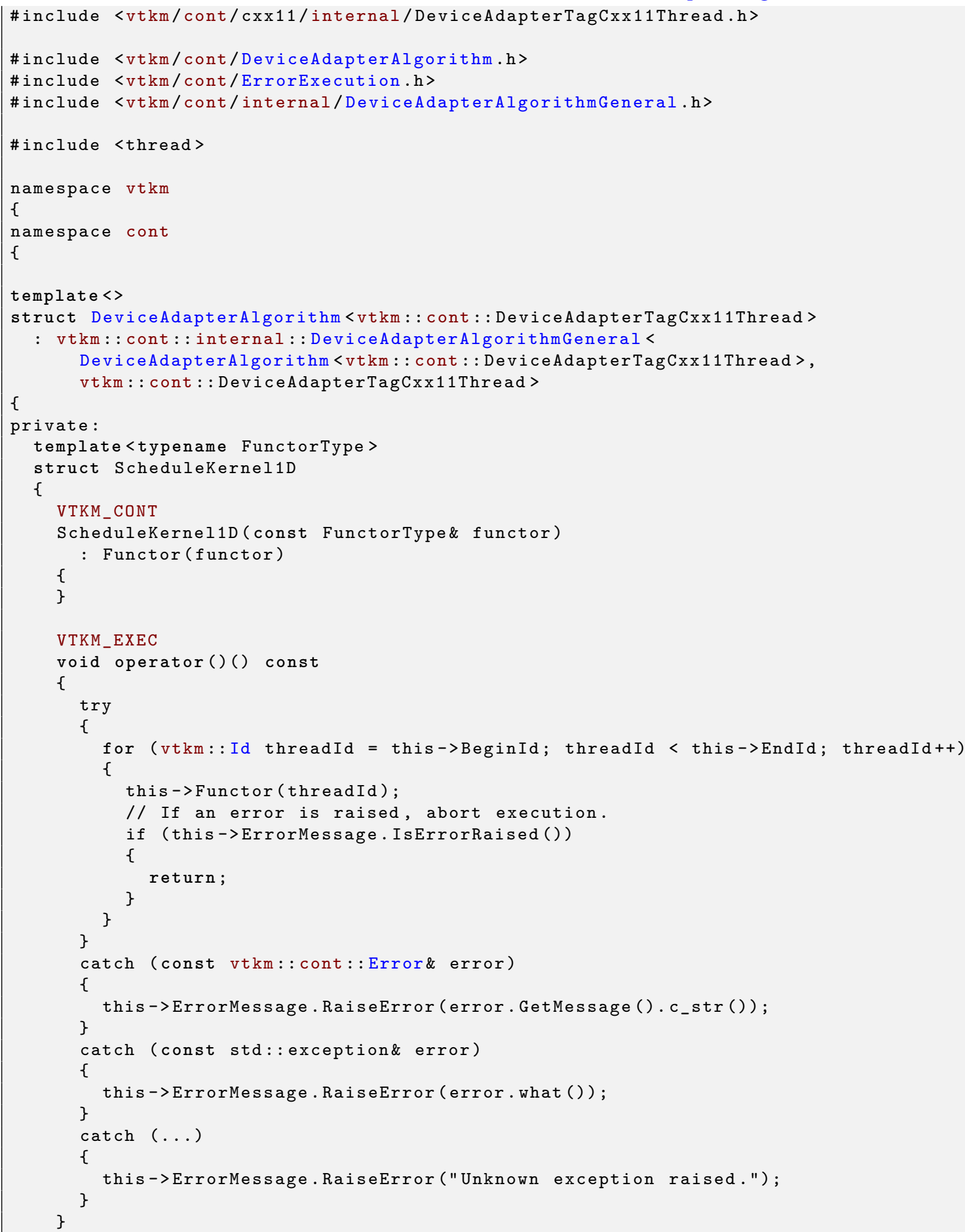




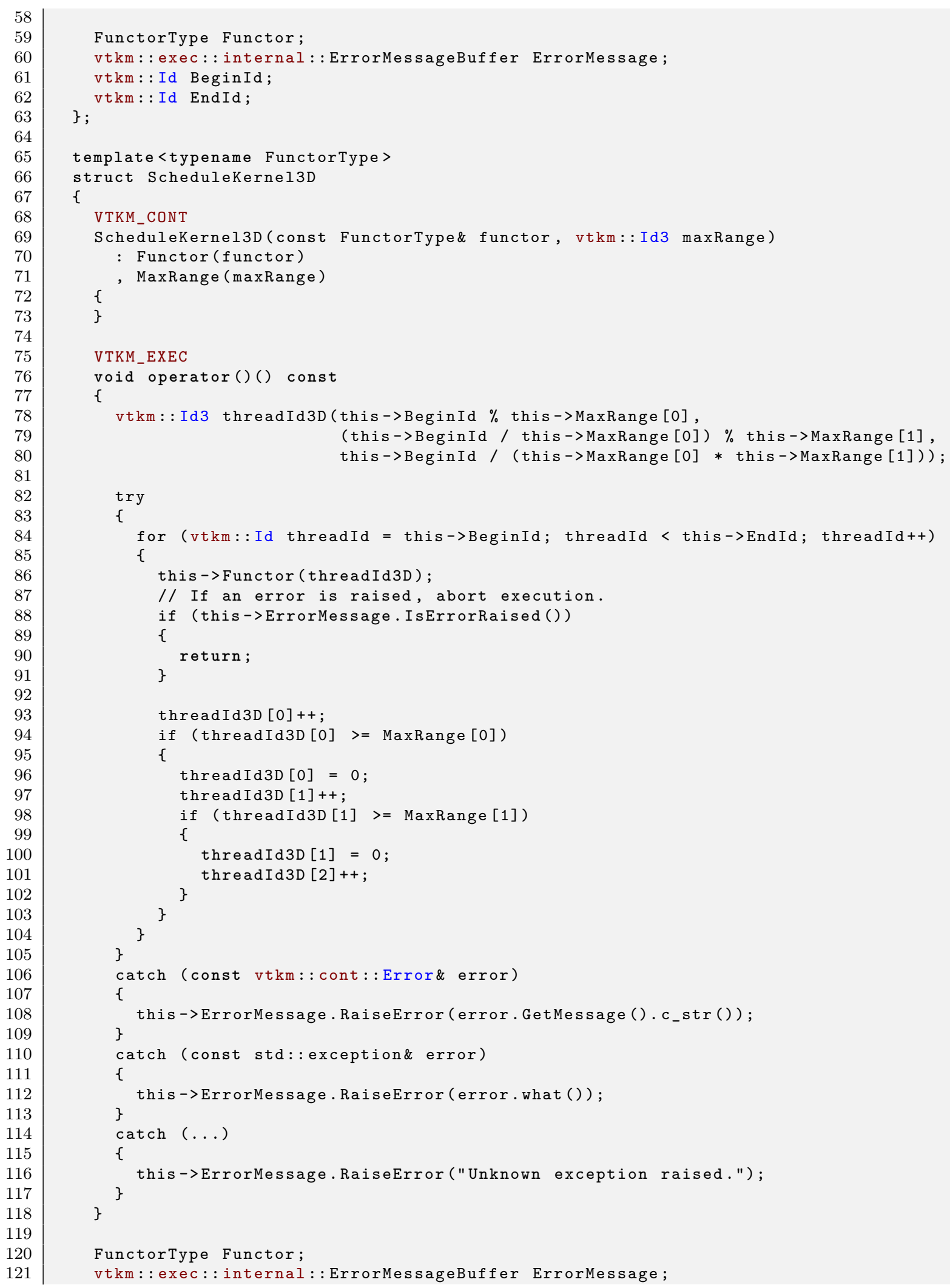

\section{FunctorType Functor;}

vtkm: : exec: : internal : ErrorMessageBuffer ErrorMessage; vtkm:: Id BeginId; 


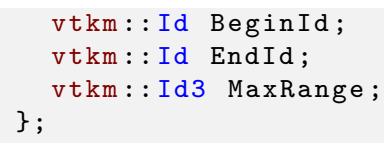




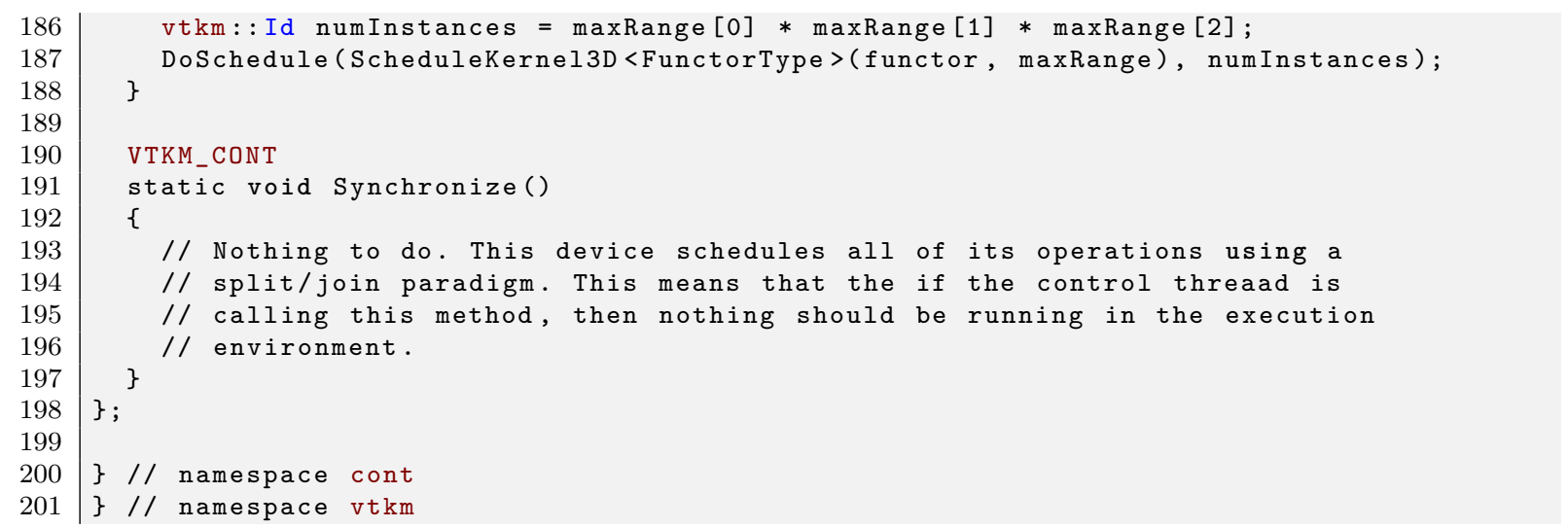

\subsection{Timer Implementation}

The VTK-m timer, described in Chapter 13, delegates to an internal class named vtkm: : cont: :DeviceAdapterTimerImplementation. The interface for this class is the same as that for vtkm: :cont::Timer. A default implementation of this templated class uses the system timer and the Synchronize method in the device adapter algorithms.

However, some devices might provide alternate or better methods for implementing timers. For example, the TBB and CUDA libraries come with high resolution timers that have better accuracy than the standard system timers. Thus, the device adapter can optionally provide a specialization of DeviceAdapterTimerImplementation, which is typically placed in the same header file as the device adapter algorithms.

Continuing our example of a custom device adapter using $\mathrm{C}++11$ 's std: :thread class, we could use the default timer and it would work fine. But $\mathrm{C}++11$ also comes with a std: :chrono package that contains some portable time functions. The following code demonstrates creating a custom timer for our device adapter using this package. By convention, DeviceAdapterTimerImplementation is placed in the same header file as DeviceAdapterAlgorithm.

Example 38.13: Specialization of DeviceAdapterTimerImplementation.

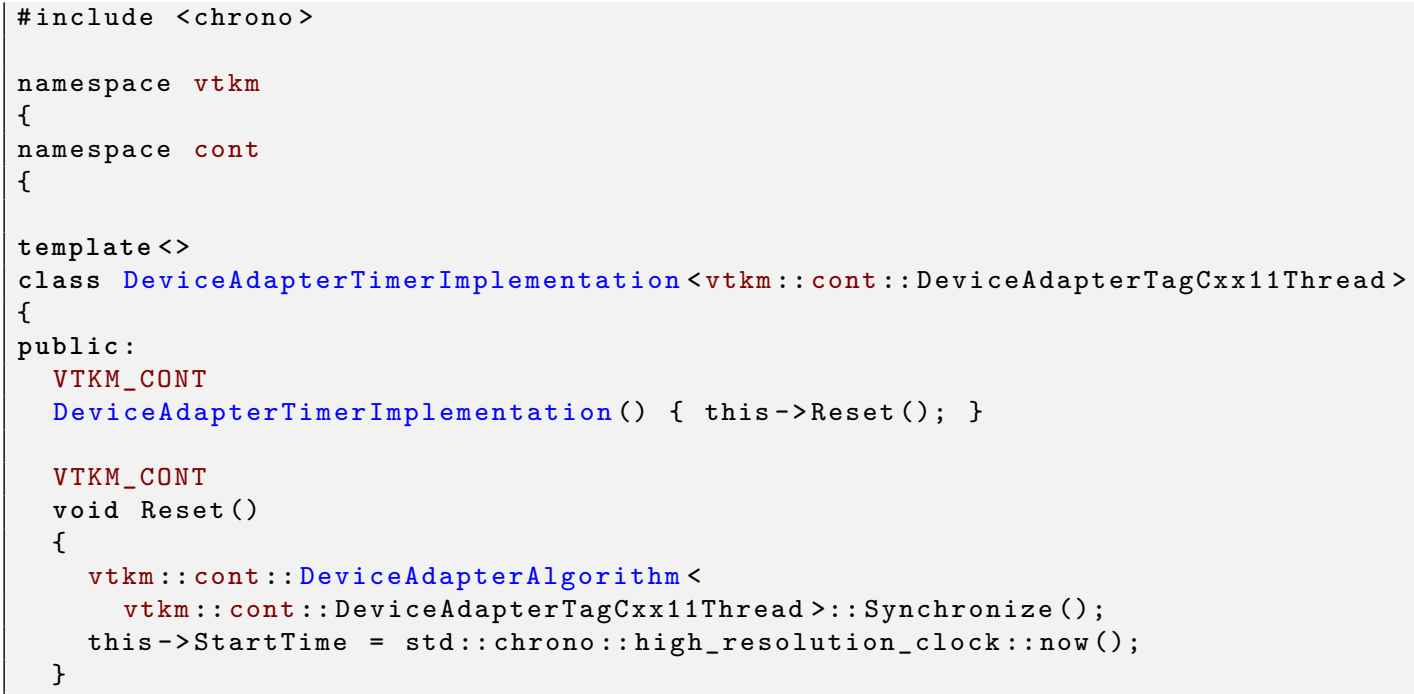




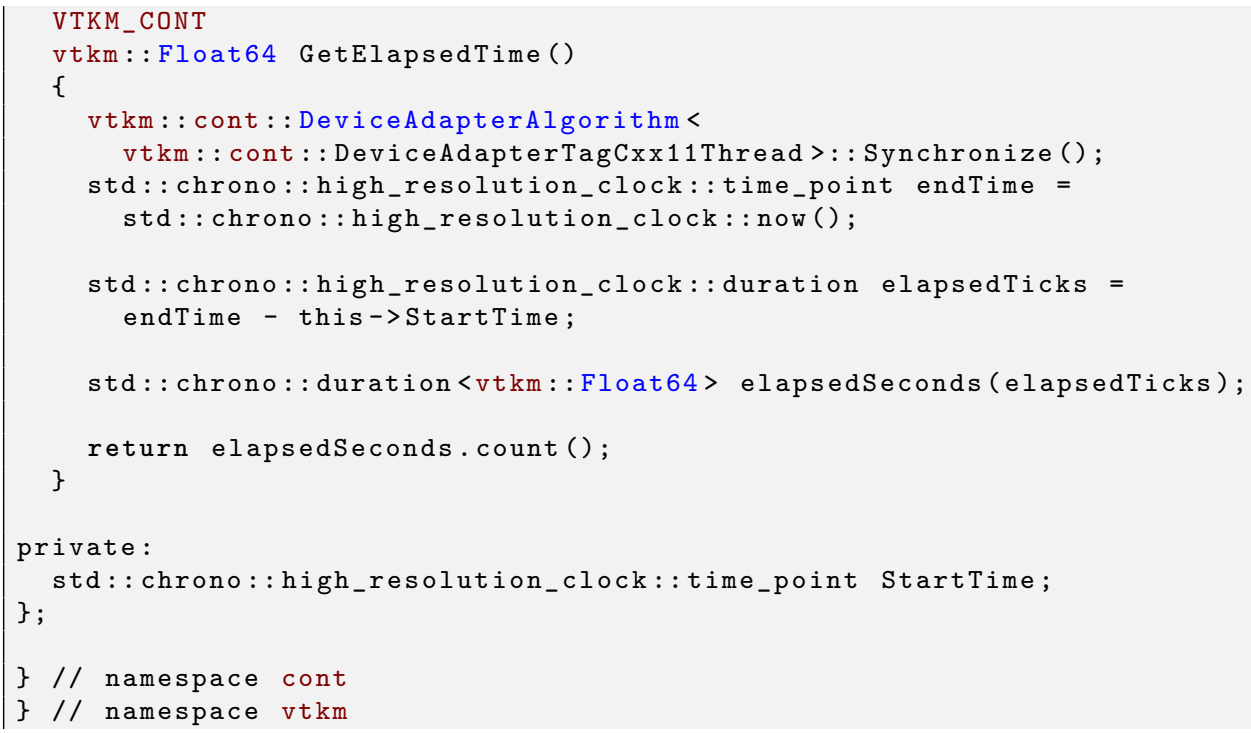





\section{FUNCTION INTERFACE OBJECTS}

For flexibility's sake a worklet is free to declare a ControlSignature with whatever number of arguments are sensible for its operation. The Invoker is expected to support arguments that match these arguments, and part of the invocation operation may require these arguments to be augmented before the worklet is scheduled. This leaves the invoker with the tricky task of managing some collection of arguments of unknown size and unknown types.

To simplify this management, VTK-m has the vtkm: :internal: :FunctionInterface class. FunctionInterface is a templated class that manages a generic set of arguments and return value from a function. An instance of FunctionInterface holds an instance of each argument. You can apply the arguments in a FunctionInterface object to a functor of a compatible prototype, and the resulting value of the function call is saved in the FunctionInterface.

\subsection{Declaring and Creating}

vtkm: internal::FunctionInterface is a templated class with a single parameter. The parameter is the signature of the function. A signature is a function type. The syntax in $\mathrm{C}++$ is the return type followed by the argument types encased in parentheses.

Example 39.1: Declaring vtkm: : internal: :FunctionInterface.

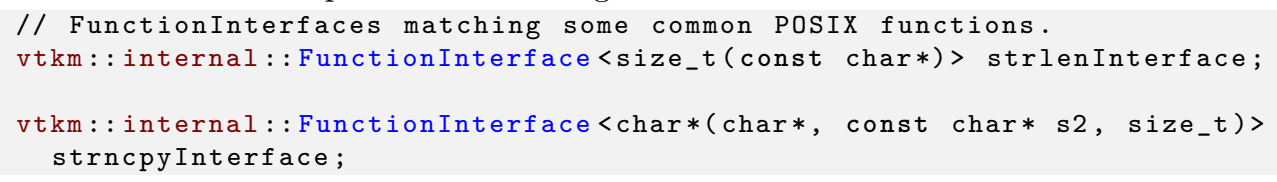

The vtkm: : internal: :make_FunctionInterface function provides an easy way to create a FunctionInterface and initialize the state of all the parameters. make_FunctionInterface takes a variable number of arguments, one for each parameter. Since the return type is not specified as an argument, you must always specify it as a template parameter.

Example 39.2: Using vtkm: : internal : :make_FunctionInterface.

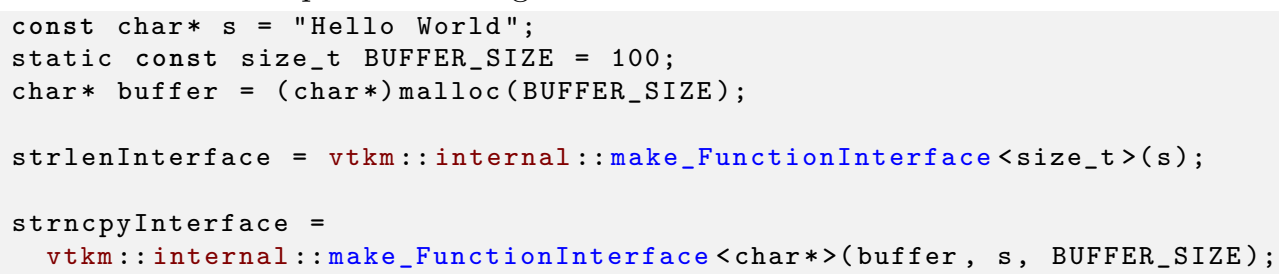




\subsection{Parameters}

Once created, FunctionInterface contains methods to query and manage the parameters and objects associated with them. The number of parameters can be retrieved either with the constant field ARITY or with the GetArity method.

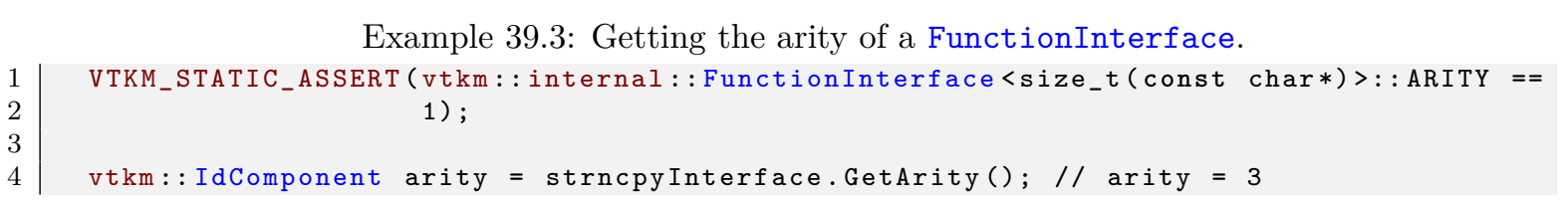

You can use the vtkm: : internal: : ParameterGet function to retrieve a parameter from a FunctionInterface. When using ParameterGet, you have to specify the index of the parameter using a template argument. Note that the parameters in FunctionInterface start at index 1. Although this is uncommon in $\mathrm{C}++$, it is customary to number function arguments starting at 1 .

Example 39.4: Using ParameterGet.

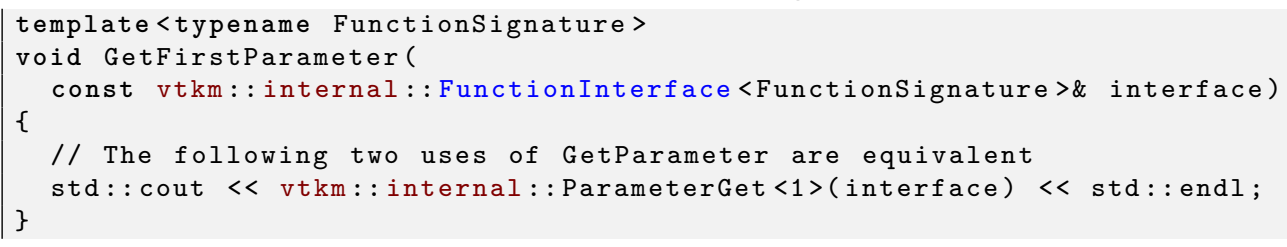

\subsection{Transformations}

Rather than replace a single item in a FunctionInterface, it is desirable to change them all in a similar way. FunctionInterface supports a "static transform" that will replace all of the arguments with new types defined at compile time.

The static transform method (named StaticTransformCont) operates by accepting a functor that defines a function with two arguments. The first argument is the FunctionInterface parameter to transform. The second argument is an instance of the vtkm: : internal: : IndexTag templated class that statically identifies the parameter index being transformed. An IndexTag object has no state, but the class contains a static integer named INDEX. The function returns the transformed argument.

The functor must also contain a templated class named ReturnType with an internal type named type that defines the return type of the transform for a given parameter type. ReturnType must have two template parameters. The first template parameter is the type of the FunctionInterface parameter to transform. It is the same type as passed to the operator. The second template parameter is a vtkm: : IdComponent specifying the index.

The transformation is only applied to the parameters of the function. The return argument is unaffected.

The return type can be determined with the StaticTransformType template in the FunctionInterface class. StaticTransformType has a single parameter that is the transform functor and contains a type named type that is the transformed FunctionInterface.

In the following example, a static transform is used to convert a FunctionInterface to a new object that has the pointers to the parameters rather than the values themselves. The parameter index is always ignored as all parameters are uniformly transformed. 
Example 39.5: Using a static transform of function interface class.

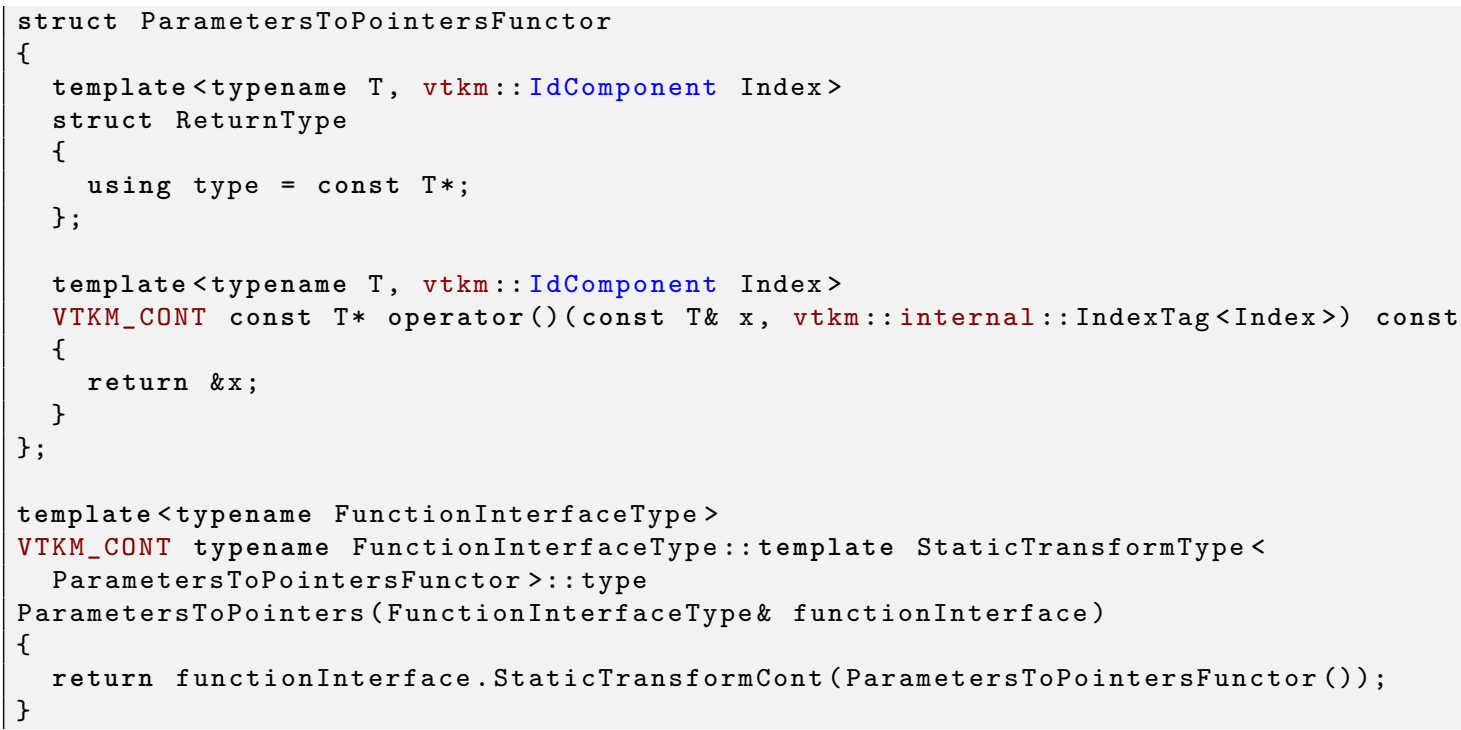





\section{WORKLET ARGUMENTS}

From the ControlSignature and ExecutionSignature defined in worklets, VTK-m uses template metaprogramming to build the code required to manage data from the control to the execution environment. These signatures contain tags that define the meaning of each argument and control how the argument data are transferred from the control to execution environments and broken up for each worklet instance.

Chapter 21 documents the many ControlSignature and ExecutionSignature tags that come with the worklet types. This chapter discusses the internals of these tags and how they control data management. Defining new worklet argument types can allow you to define new data structures in VTK-m. New worklet arguments are also usually a critical components for making new worklet types, as described in Chapter 41.

The management of data in worklet arguments is handled by three classes that provide type checking, transportation, and fetching, respectively. This chapter will first describe these type checking, transportation, and fetching classes and then describe how ControlSignature and ExecutionSignature tags specify these classes.

Throughout this chapter we demonstrate the definition of worklet arguments using an example of a worklet argument that represents line segments in 2D. The input for such an argument expects an ArrayHandle containing floating point vtkm: :Vec s of size 2 to represent coordinates in the plane. The values in the array are paired up to define the two endpoints of each segment, and the worklet instance will receive a Vec-2 of Vec-2's representing the two endpoints. In practice, it is generally easier to use a vtkm: : cont: : ArrayHandleGroupVec (see Section 26.13), but this is a simple example for demonstration purposes. Plus, we will use this special worklet argument for our example of a custom worklet type in Chapter 41.

\subsection{Type Checks}

Before attempting to move data from the control to the execution environment, the VTK-m invokers check the input types to ensure that they are compatible with the associated ControlSignature concept. This is done with the vtkm: : cont: :arg: :TypeCheck struct.

The TypeCheck struct is templated with two parameters. The first parameter is a tag that identifies which check to perform. The second parameter is the type of the control argument (after any dynamic casts). The TypeCheck class contains a static constant Boolean named value that is true if the type in the second parameter is compatible with the tag in the first or false otherwise.

Type checks are implemented with a defined type check tag (which, by convention, is defined in the vtkm: :cont: :arg namespace and starts with TypeCheckTag) and a partial specialization of the vtkm: :cont: :arg: :TypeCheck structure. The following type checks (identified by their tags) are provided in VTK-m.

vtkm: :cont: :arg: :TypeCheckTagExecObject True if the type is an execution object. All execution objects 
must derive from vtkm: : cont: :ExecutionObjectBase and follow the conventions of that class.

vtkm: : cont: :arg: :TypeCheckTagArrayIn True if the type is a vtkm: : cont: : ArrayHandle that is capable of reading.

vtkm: : cont: :arg: :TypeCheckTagArrayOut True if the type is a vtkm: :cont: : ArrayHandle that is capable of writing.

vtkm: : cont: :arg: :TypeCheckTagArrayInOut True if the type is a vtkm: : cont: : ArrayHandle that is capable of reading and writing.

vtkm: :cont: :arg: :TypeCheckTagAtomicArray Similar to TypeCheckTagArrayInOut except it only returns true for array types with values that are supported for atomic arrays.

vtkm: : cont: :arg: :TypeCheckTagBitField True if the type is a vtkm: :cont: :BitField.

vtkm: : cont: : arg: :TypeCheckTagCellSet True if and only if the object is a vtkm: : cont: : CellSet or one of its subclasses.

vtkm: :cont: :arg: :TypeCheckTagCellSetStructured True if the object is a vtkm: :cont::CellSetStructured.

vtkm: : cont: :arg: :TypeCheckTagExecObject True if the type is a subclass of vtkm: :cont::ExecutionObjectBase. See Chapter 29 for more information on execution objects for worklets.

vtkm: : cont: :arg: :TypeCheckTagKeys True if and only if the object is a vtkm: : worklet: :Keys class.

Here are some trivial examples of using TypeCheck. Typically these checks are done internally in the base VTK-m invoker code, so these examples are for demonstration only.

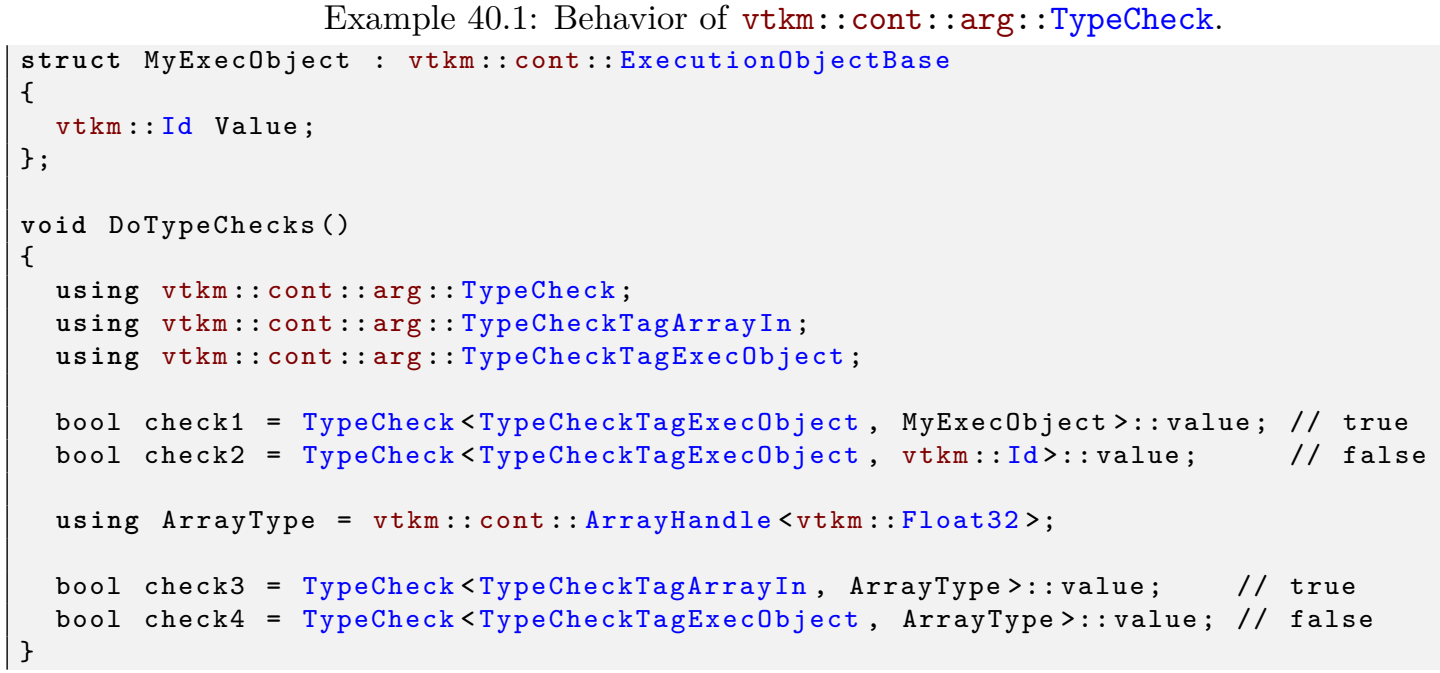

Example 40.1: Behavior of vtkm: : cont: :arg: :TypeCheck.

A type check is created by first defining a type check tag object, which by convention is placed in the vtkm: :cont: :arg namespace and whose name starts with TypeCheckTag. Then, create a specialization of the vtkm: :cont: : arg: : TypeCheck template class with the first template argument matching the aforementioned tag. As stated previously, the TypeCheck class must contain a value static constant Boolean representing whether the type is acceptable for the corresponding Invoker argument.

This example of a TypeCheck returns true for control objects that are ArrayHandles with a value type that is a floating point vtkm: :Vec of size 2 . 
Example 40.2: Defining a custom TypeCheck.

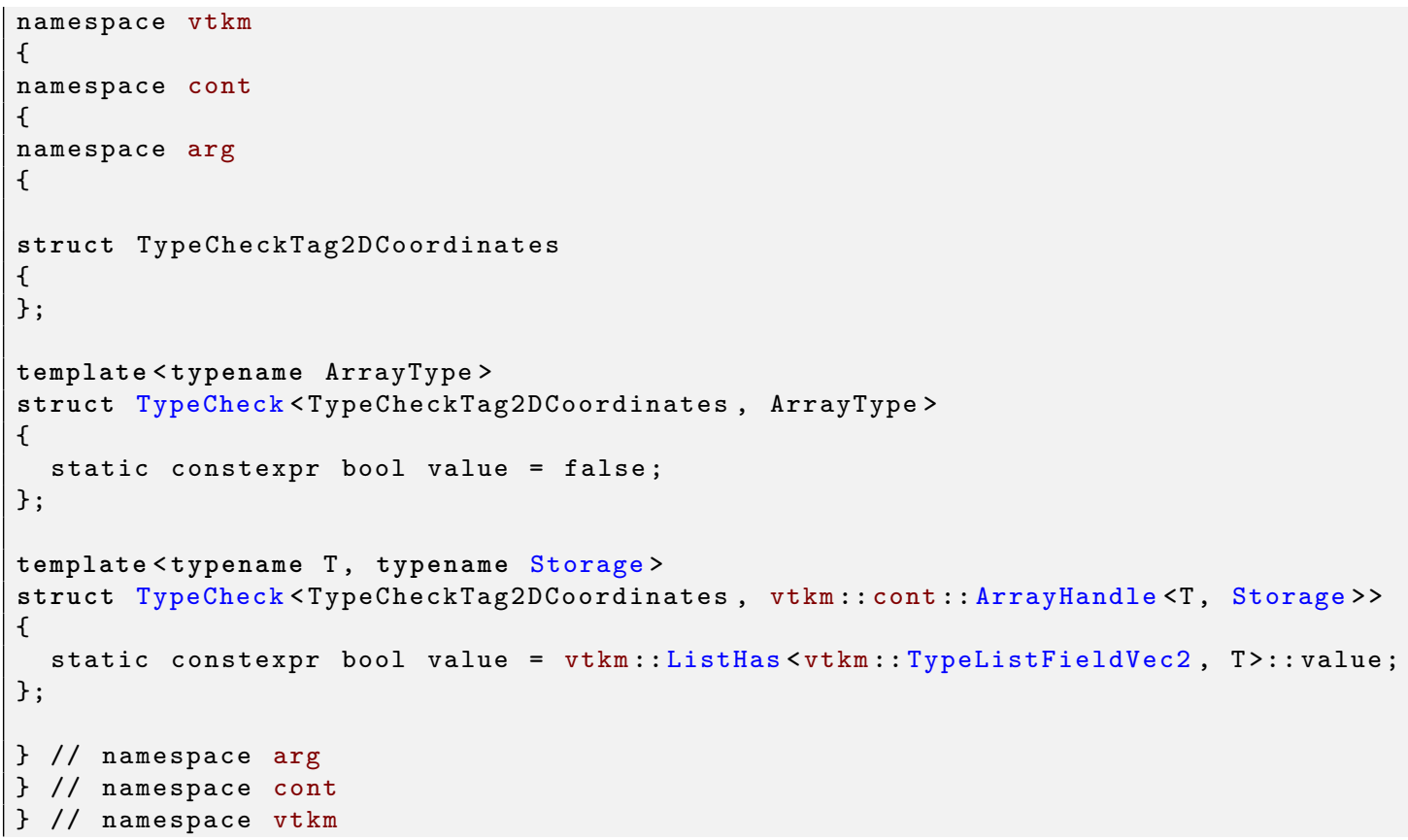

\subsection{Transport}

After all the argument types are checked, the VTK-m dispatch mechanism must load the data into the execution environment before scheduling a job to run there. This is done with the vtkm: : cont: : arg: :Transport struct.

The Transport struct is templated with three parameters. The first parameter is a tag that identifies which transport to perform. The second parameter is the type of the control parameter (after any dynamic casts). The third parameter is a device adapter tag for the device on which the data will be loaded.

A Transport contains a type named ExecObjectType that is the type used after data is moved to the execution environment. A Transport also has a const parenthesis operator that takes 5 arguments: the control-side object that is to be transported to the execution environment, the control-side object that represents the input domain, the size of the input domain, the size of the output domain, and a reference to a vtkm: : cont: : Token object that is used to define the scope of any generated execution environment objects. The parenthesis operator returns an execution-side object. This operator is called in the control environment, and the operator returns an object that is ready to be used in the execution environment.

Transports are implemented with a defined transport tag (which, by convention, is defined in the vtkm: : cont: :arg namespace and starts with TransportTag) and a partial specialization of the vtkm: : cont: : arg: : Transport structure. The following transports (identified by their tags) are provided in VTK-m.

vtkm: : cont: :arg: :TransportTagExecObject Calls PrepareForInput on the provided object. The returned execution object is what is provided by PrepareForInput. See Chapter 29 for more information on execution objects for worklets.

vtkm: : cont: :arg: :TransportTagArrayIn Loads data from a vtkm::cont::ArrayHandle onto the specified device using the array handle's PrepareForInput method. The size of the array must be the same as the input domain. The returned execution object is an array portal. 
vtkm: :cont: :arg: :TransportTagArrayOut Allocates data onto the specified device for a vtkm::cont::ArrayHandle using the array handle's PrepareForOutput method. The array is allocated to the size of the output domain. The returned execution object is an array portal.

vtkm: : cont: :arg: :TransportTagArrayInOut Loads data from a vtkm: :cont: :ArrayHandle onto the specified device using the array handle's PrepareForInPlace method. The size of the array must be the same size as the output domain (which is not necessarily the same size as the input domain). The returned execution object is an array portal.

vtkm: : cont: : arg: :TransportTagWholeArrayIn Loads data from a vtkm: : cont: : ArrayHandle onto the specified device using the array handle's PrepareForInput method. This transport is designed to be used with random access whole arrays, so unlike TransportTagArrayIn the array size can be unassociated with the input domain. The returned execution object is an array portal.

vtkm: :cont: :arg: :TransportTagWholeArrayOut Readies data from a vtkm::cont: :ArrayHandle onto the specified device using the array handle's PrepareForOutput method. This transport is designed to be used with random access whole arrays, so unlike TransportTagArrayOut the array size can be unassociated with the input domain. Thus, the array must be pre-allocated and its size is not changed. The returned execution object is an array portal.

vtkm: : cont: :arg: :TransportTagWholeArrayInOut Loads data from a vtkm: :cont: :ArrayHandle onto the specified device using the array handle's PrepareForInPlace method. This transport is designed to be used with random access whole arrays, so unlike TransportTagArrayInOut the array size can be unassociated with the input domain. The returned execution object is an array portal.

vtkm: :cont: :arg: :TransportTagAtomicArray Loads data from a vtkm::cont: ArrayHandle and creates a vtkm: :exec: :AtomicArray.

vtkm: : cont: :arg: :TransportTagBitFieldIn Loads data from a vtkm: :cont: :BitField onto the specified device using the PrepareForInput method.

vtkm: : cont: :arg: :TransportTagBitFieldInOut Loads data from a vtkm: :cont: :BitField onto the specified device using the PrepareForInPlace method.

vtkm::cont::arg::TransportTagBitFieldInOut This is essentially the same as TransportTagBitFieldInOut. Because a bit field does not follow the size of the domain, the array must be sized before passed to an invoke.

vtkm: :cont: :arg: :TransportTagCellSetIn Loads data from a vtkm::cont::Cellset object. The TransportTagCellSetIn it a templated class with two parameters: the "from" topology and the "to" topology. (See Section 21.2.3 for a description of "from" and "to" topologies.) The returned execution object is a connectivity object (as described in Section 28.3).

vtkm: : cont: :arg: :TransportTagTopologyFieldIn Similar to TransportTagArrayIn except that the size is checked against the "from" topology of a cell set for the input domain. The input domain object is assumed to be a vtkm: : cont: : Cellset.

vtkm: : cont: :arg: :TransportTagKeysIn Loads data from a vtkm::worklet::Keys object. This transport is intended to be used for the input domain of a vtkm: :worklet::WorkletReduceByKey. The returned execution object is of type vtkm: :exec: : internal: :ReduceByKeyLookup.

vtkm::cont::arg::TransportTagKeyedValuesIn Loads data from a vtkm::cont::ArrayHandle onto the specified device using the array handle's PrepareForInput method. This transport uses the input domain object, which is expected to be a vtkm: :worklet: :Keys object, and groups the entries in the array by unique keys. The returned execution object is an array portal of grouped values. 
vtkm: :cont: :arg: :TransportTagKeyedValuesOut Loads data from a vtkm::cont::ArrayHandle onto the specified device using the array handle's PrepareForOutput method. This transport uses the input domain object, which is expected to be a vtkm: : worklet: :Keys object, and groups the entries in the array by unique keys. The returned execution object is an array portal of grouped values.

vtkm: : cont: :arg: :TransportTagKeyedValuesInOut Loads data from a vtkm: : cont: :ArrayHandle onto the specified device using the array handle's PrepareForInPlace method. This transport uses the input domain object, which is expected to be a vtkm::worklet::Keys object, and groups the entries in the array by unique keys. The returned execution object is an array portal of grouped values.

Here are some trivial examples of using Transport. Typically this movement is done internally in the VTK-m dispatching code, so these examples are for demonstration only.

Example 40.3: Behavior of vtkm: : cont: : arg: :Transport.

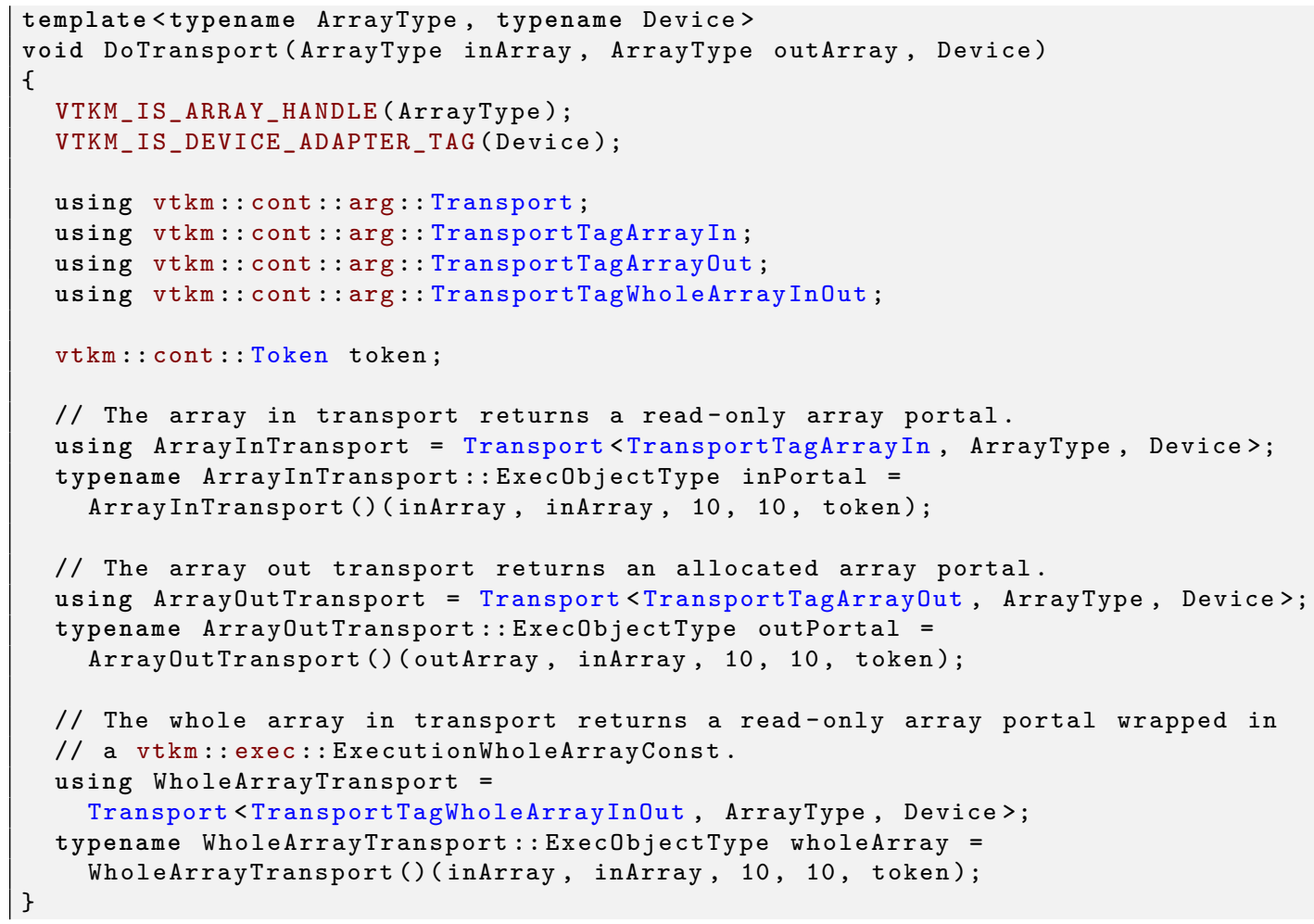

A transport is created by first defining a transport tag object, which by convention is placed in the vtkm: :cont: : arg namespace and whose name starts with TransportTag. Then, create a specialization of the vtkm: :cont: :arg: :Transport template class with the first template argument matching the aforementioned tag. As stated previously, the Transport class must contain an ExecObjectType type and a parenthesis operator turning the associated control argument into an execution environment object.

This example internally uses a vtkm: : cont: :ArrayHandleGroupVec to take values from an input ArrayHandle and pair them up to represent line segments. The resulting execution object is an array portal containing Vec-2 values of Vec-2's.

Example 40.4: Defining a custom Transport.

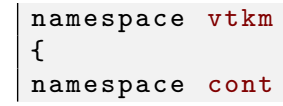




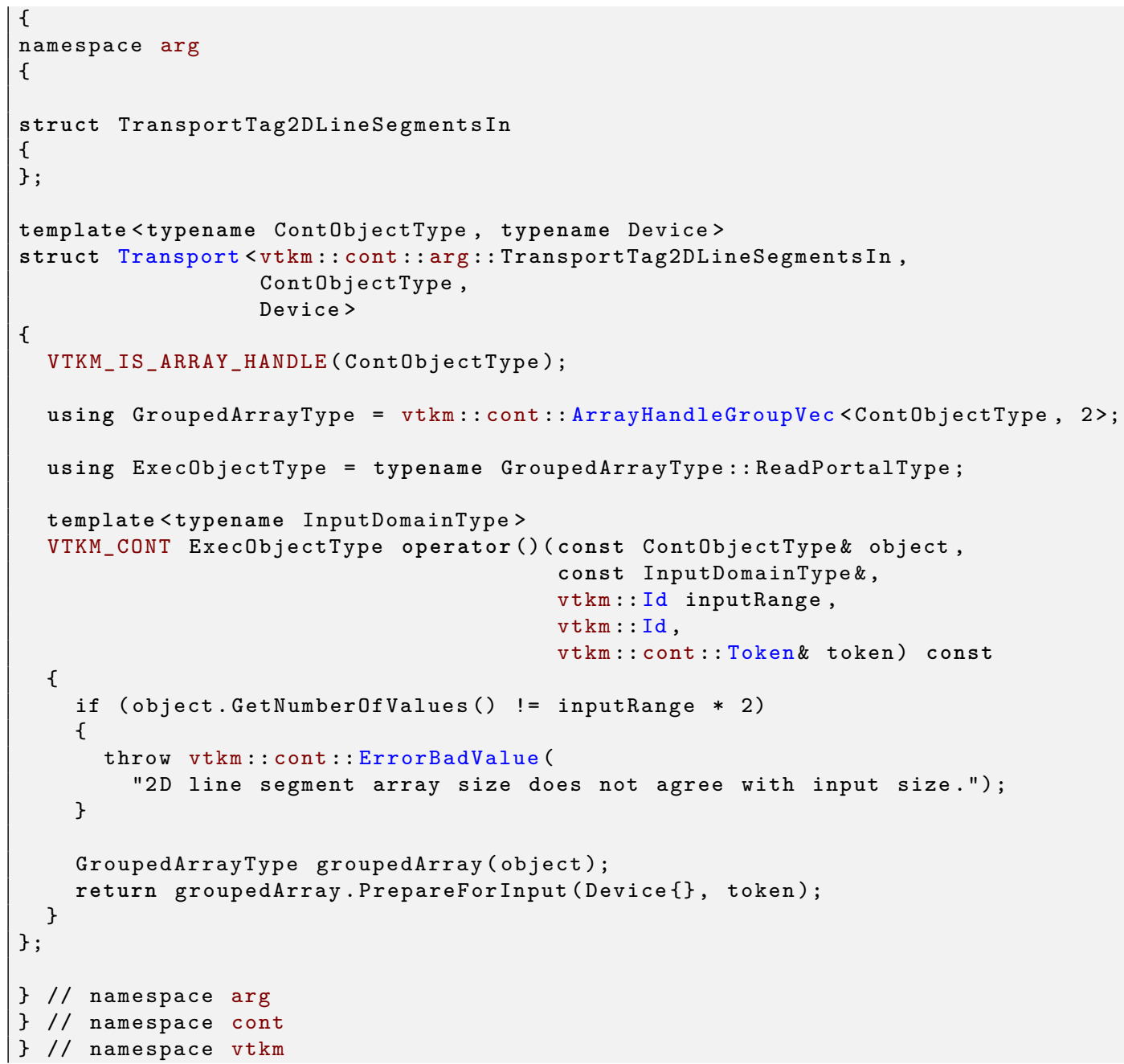

\section{Common Errors}

It is fair to assume that the Transport's control object type matches whatever the associated TypeCheck allows. However, it is good practice to provide a secondary compile-time check in the Transport class, like the one on line 17 in Example 40.4, for debugging purposes in case there is a problem with the TypeCheck or this Transport is used with an unexpected TypeCheck.

\subsection{Fetch}

Before the function of a worklet is invoked, the VTK-m internals pull the appropriate data out of the execution object and pass it to the worklet function. A class named vtkm: :exec: :arg: :Fetch is responsible for pulling this data out and putting computed data in to the execution objects. 
The Fetch struct is templated with three parameters. The first parameter is a tag that identifies which type of fetch to perform. The second parameter is a different tag that identifies the aspect of the data to fetch.

The third template parameter to a Fetch struct is the type of the execution object that is created by the Transport (as described in Section 40.2). This is generally where the data are fetched from.

A Fetch also has a pair of methods named Load and Store that get data from and add data to the execution object at a given domain or thread index.

In both Load and Store methods, its first parameter is a thread indices object which manages the multiple indices that are relevant to the worklet invocation including the input index, output index, and visit index, all of which can be different.

The specific type of the thread indices object depends on the type of worklet begin invoked, but all thread indices classes implement methods named GetInputIndex, GetOutputIndex, and GetVisitIndex to get those respective indices. The thread indices object may also contain other methods to get information pertinent to the associated worklet's execution. For example a thread indices object associated with a topology map has methods to get the shape identifier and incident from indices of the current input object. Thread indices objects are discussed in more detail in Section 41.2.

Fetches are specified with a pair of fetch and aspect tags. Fetch tags are by convention defined in the vtkm: :exec: :arg namespace and start with FetchTag. Likewise, aspect tags are also defined in the vtkm: : exec: : arg namespace and start with AspectTag. The Fetch class is partially specialized on these two tags.

The most common aspect tag is vtkm: :exec: :arg: :AspectTagDefault, and all fetch tags should have a specialization of vtkm: :exec: :arg: :Fetch with this tag. The following list of fetch tags describes the execution objects they work with and the data they pull for each aspect tag they support.

vtkm: :exec: :arg: :FetchTagExecObject Simply returns an execution object. This fetch only supports the AspectTagDefault aspect. The Load returns the executive object in the associated parameter. The Store does nothing.

vtkm: :exec: :arg: :FetchTagWholeCellSetIn Loads data from a cell set. The Load simply returns the execution object created with a TransportTagCellsetIn and the Store does nothing.

vtkm: :exec: :arg::FetchTagArrayDirectIn Loads data from an array portal. This fetch only supports the AspectTagDefault aspect. The Load gets data directly from the domain (thread) index. The Store does nothing.

vtkm: :exec: :arg: :FetchTagArrayDirectOut Stores data to an array portal. This fetch only supports the AspectTagDefault aspect. The Store sets data directly to the domain (thread) index. The Load does nothing.

vtkm: :exec: :arg: :FetchTagCellSetIn Load data from a cell set. This fetch is used with the worklet topology maps to pull topology information from a cell set. The Load simply returns the cell shape of the given input cells and the Store method does nothing. This tag is typically used with the input domain object, and aspects like vtkm: :exec: :arg: :AspectTagIncidentElementCount and vtkm: :exec: :arg: : AspectTagIncidentElementIndices are used to get more detailed information.

vtkm: :exec: :arg: :FetchTagArrayTopologyMapIn Loads data from the "from" topology in a topology map. For example, in a point to cell topology map, this fetch will get the field values for all points attached to the cell being visited. The Load returns a Vec-like object containing all the incident field values whereas the Store method does nothing. This fetch is designed for use in topology maps and expects the input domain to be a cell set. 
A fetch is created by first defining a fetch tag object, which by convention is placed in the vtkm: :exec: : arg namespace and whose name starts with FetchTag. Then, create a specialization of the vtkm: :exec: :arg: :Fetch template class with the first template argument matching the aforementioned tag. As stated previously, the Fetch class must contain a pair of Load and Store methods that get a value out of the data and store a value in the data, respectively.

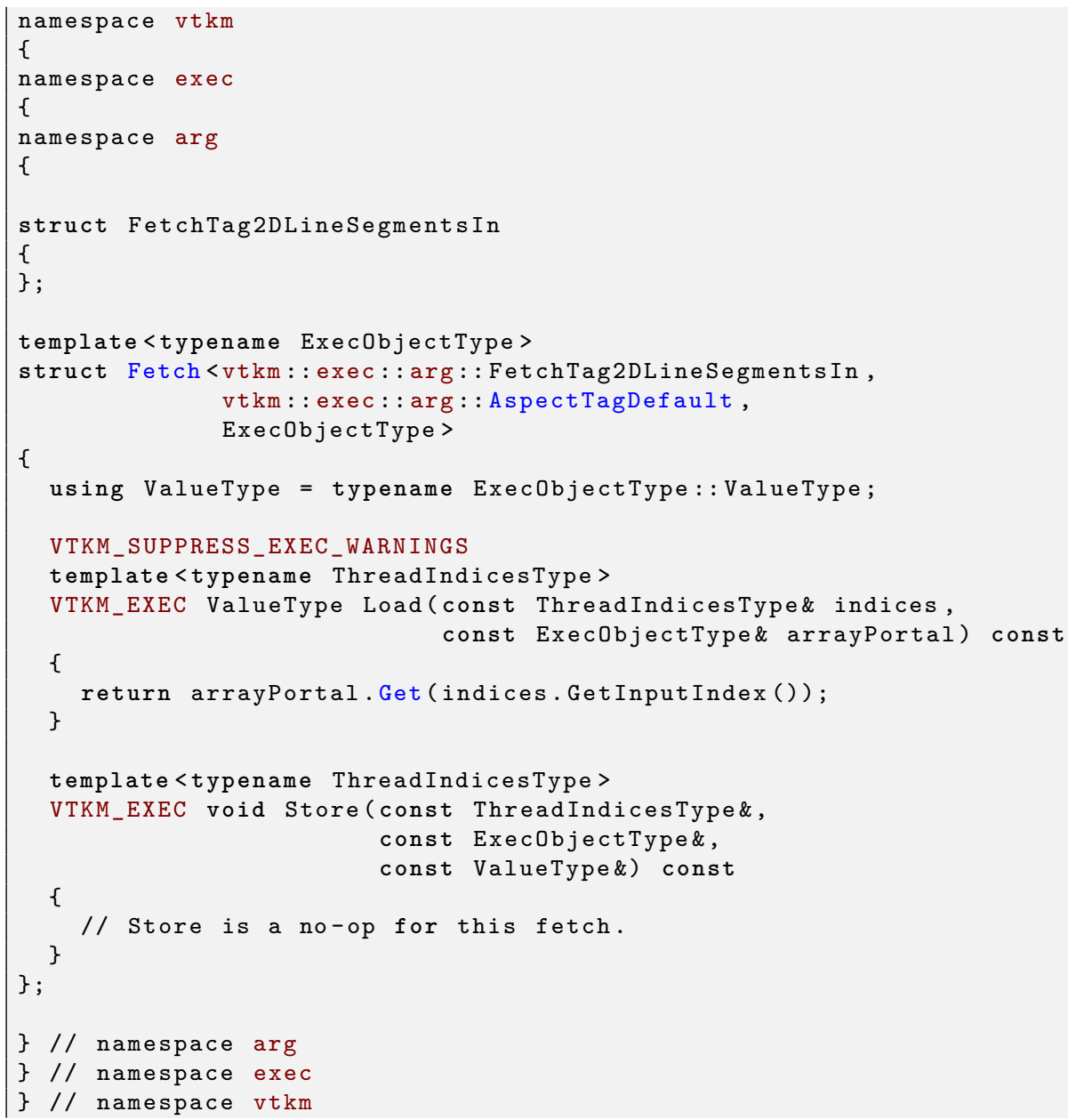

0 Did you know?

3 The fetch defined in Example 40.5 could actually be replaced by the more general FetchTagArrayDirectIn that already comes with VTK-m. This example is mostly provided for demonstrative purposes.

In addition to the aforementioned aspect tags that are explicitly paired with fetch tags, VTK-m also provides some aspect tags that either modify the behavior of a general fetch or simply ignore the type of fetch.

vtkm::exec: :arg: :AspectTagDefault Performs the "default" fetch. Every fetch tag should have an implementation of vtkm: :exec: :arg: :Fetch with that tag and AspectTagDefault. 
vtkm: : exec: : arg: : AspectTagWorkIndex Simply returns the domain (or thread) index ignoring any associated data. This aspect is used to implement the WorkIndex execution signature tag.

vtkm: :exec: :arg: :AspectTagInputIndex Returns the index of the element being used from the input domain. This is often the same as the work index but can be different if a scatter is being used. (See Section 31.1 for information on scatters in worklets.)

vtkm::exec: :arg::AspectTagOutputIndex Returns the index of the element being written to the output. This is generally the same as the work index.

vtkm: :exec: :arg: :AspectTagVisitIndex Returns the visit index corresponding to the current input. Together the pair of input index and visit index are unique.

vtkm::exec::arg::AspectTagCellShape Returns the cell shape from the input domain. This aspect is designed to be used with topology maps.

vtkm: :exec: :arg: :AspectTagIncidentElementCount Returns the number of elements associated with the "from" topology that are incident to the input element of the "to" topology. This aspect is designed to be used with topology maps.

vtkm: :exec: :arg: :AspectTagIncidentElementIndices Returns a Vec-like object containing the indices to the elements associated with the "from" topology that are incident to the input element of the "to" topology. This aspect is designed to be used with topology maps.

vtkm: :exec: :arg: :AspectTagValueCount Returns the number of times the key associated with the current input. This aspect is designed to be used with reduce by key maps.

An aspect is created by first defining an aspect tag object, which by convention is placed in the vtkm: :exec: : arg namespace and whose name starts with AspectTag. Then, create specializations of the vtkm: :exec: :arg: :Fetch template class where appropriate with the second template argument matching the aforementioned tag.

This example creates a specialization of a Fetch to retrieve the first point of a line segment.

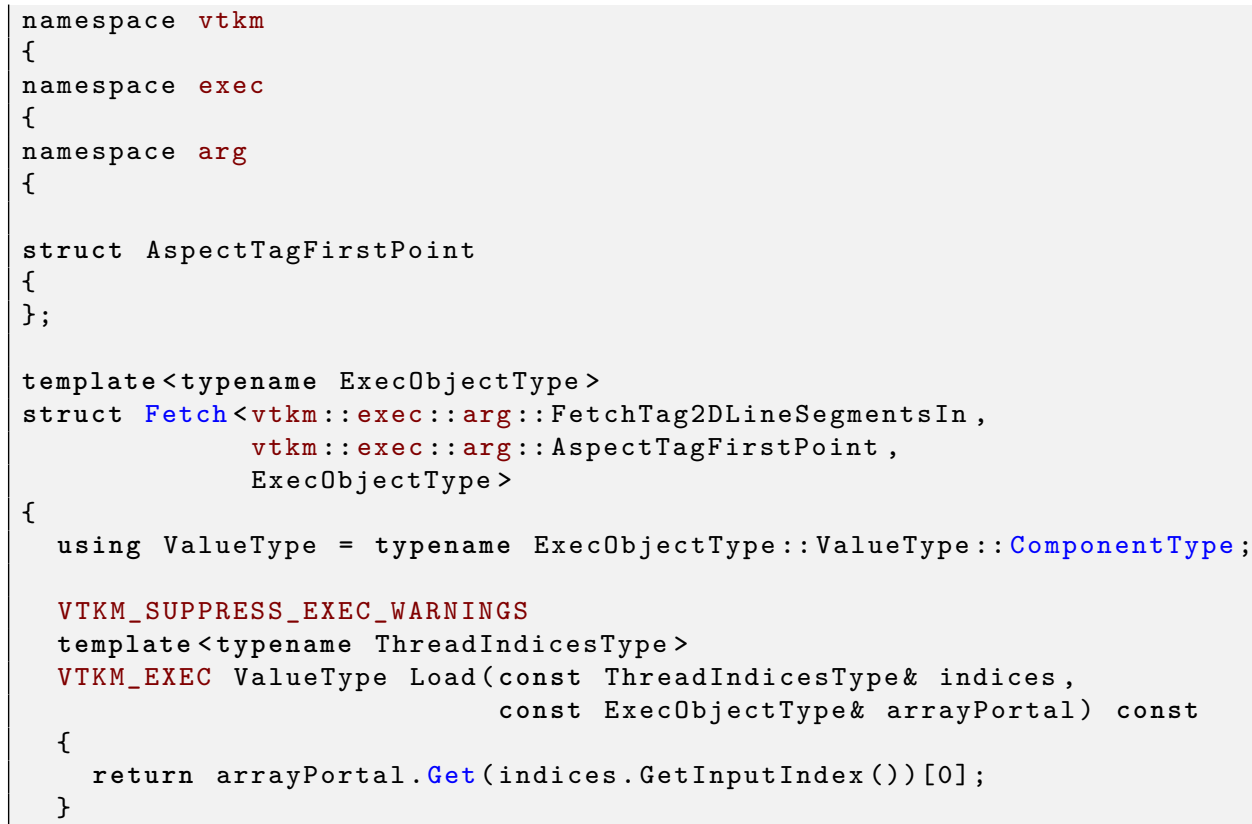




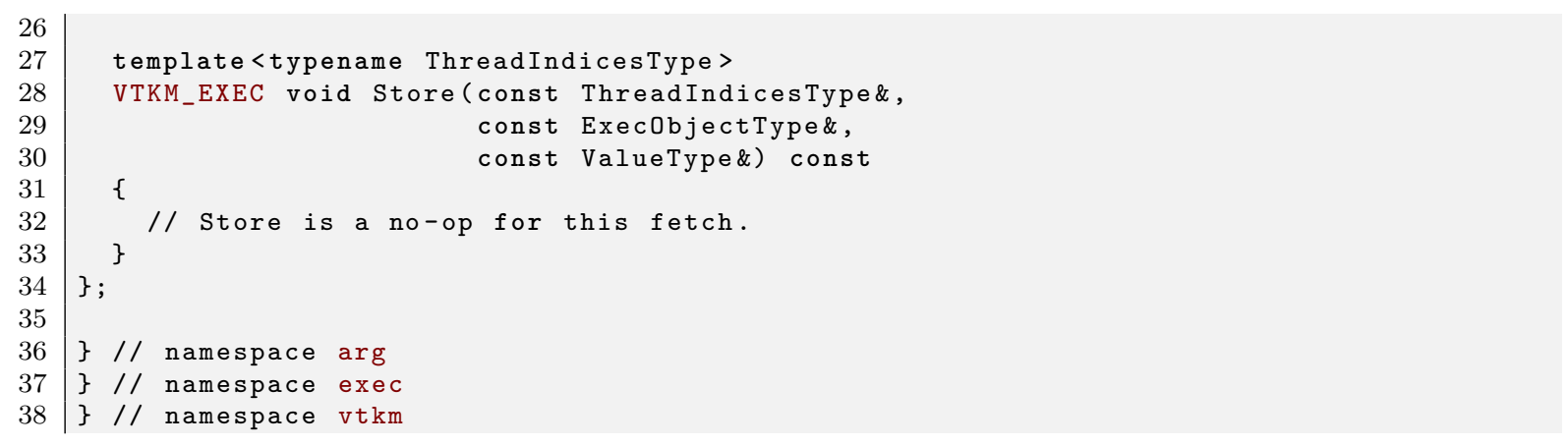

\subsection{Creating New ControlSignature Tags}

The type checks, transports, and fetches defined in the previous sections of this chapter conspire to interpret the arguments given to a Invoker and provide data to an instance of a worklet. What remains to be defined are the tags used in the ControlSignature and ExecutionSignature that bring these three items together. These two types of tags are defined differently. In this section we discuss the Controlsignature tags.

A ControlSignature tag is defined by a struct (or equivocally a class). This struct is typically defined inside a worklet (or, more typically, a worklet superclass) so that it can be used without qualifying its namespace. VTK-m has requirements for every defined Controlsignature tag.

The first requirement of a ControlSignature tag is that it must inherit from vtkm: : cont : : arg: : ControlSignatureTagBase. You will get a compile error if you attempt to use a type that is not a subclass of ControlSignatureTagBase in a ControlSignature.

The second requirement of a ControlSignature tag is that it must contain the following three types: TypeCheckTag, TransportTag, and FetchTag. As the names would imply, these specify tags for TypeCheck, Transport, and Fetch classes, respectively, which were discussed earlier in this chapter.

The following example defines a Controlsignature tag for an array that represents 2D line segments using the classes defined in previous examples.

Example 40.7: Defining a new ControlSignature tag.

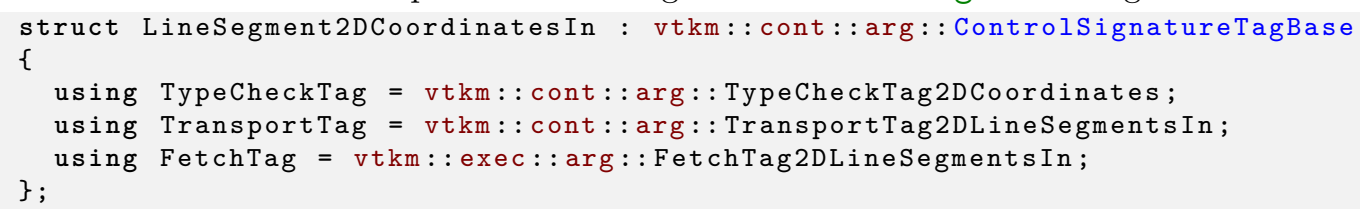

Once defined, this tag can be used like any other ControlSignature tag.

Example 40.8: Using a custom ControlSignature tag.

using Controlsignature = void(LineSegment2DCoordinatesIn coordsIn,

Fieldout vecOut,

FieldIn index);

\subsection{Creating New ExecutionSignature Tags}

An ExecutionSignature tag is defined by a struct (or equivocally a class). This struct is typically defined inside a worklet (or, more typically, a worklet superclass) so that it can be used without qualifying its namespace. 
VTK-m has requirements for every defined ExecutionSignature tag.

The first requirement of an ExecutionSignature tag is that it must inherit from vtkm: :exec: :arg: : ExecutionSignatureTagBase. You will get a compile error if you attempt to use a type that is not a subclass of ExecutionSignatureTagBase in an ExecutionSignature.

The second requirement of an ExecutionSignature tag is that it must contain a type named AspectTag, which is set to an aspect tag. As discussed in Section 40.3, the aspect tag is passed as a template argument to the vtkm: :exec: :arg: :Fetch class to modify the data it loads and stores. The numerical ExecutionSignature tags (i.e. $\_1, \ldots 2$, etc.) operate by setting the AspectTag to vtkm: :exec: : arg: : AspectTagDefault, effectively engaging the default fetch.

The third requirement of an ExecutionSignature tag is that it contains an INDEX member that is a static const vtkm: : IdComponent. The number that INDEX is set to refers to the Controlsignature argument from which that data come from (indexed starting at 1). The numerical ExecutionSignature tags (i.e. _1, _2, etc.) operate by setting their INDEX values to the corresponding number (i.e. 1, 2, etc.). An ExecutionSignature tag might take another tag as a template argument and copy the INDEX from one to another. This allows you to use a tag to modify the aspect of another tag. Most often this is used to apply a particular aspect to a numerical ExecutionSignature tag (i.e. _1, _2, etc.). Still other ExecutionSignature tags might not need direct access to any ControlSignature arguments (such as those that pull information from thread indices). If the INDEX does not matter (because the execution object parameter to the Fetch Load and Store is ignored). In this case, the ExecutionSignature tag can set the INDEX to 1, because there is guaranteed to be at least one control argument.

The following example defines an ExecutionSignature tag to get the coordinates for only the first point in a 2D line segment. The defined tag takes as an argument another tag (generally one of the numeric tags), which is expected to point to a ControlSignature argument with a LineSegment2DCoordinatesIn (as defined in Example 40.7).

Example 40.9: Defining a new ExecutionSignature tag.

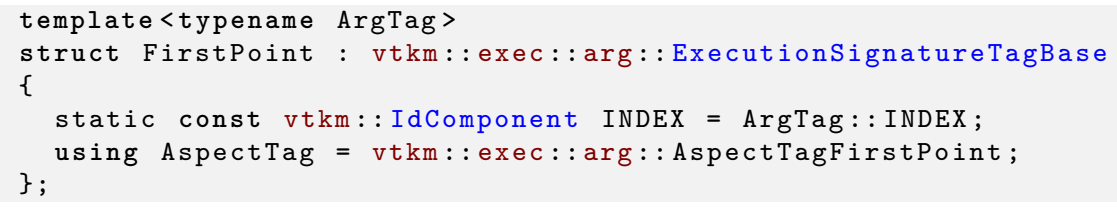

Once defined, this tag can be used like any other ExecutionSignature tag.

Example 40.10: Using a custom ExecutionSignature tag.

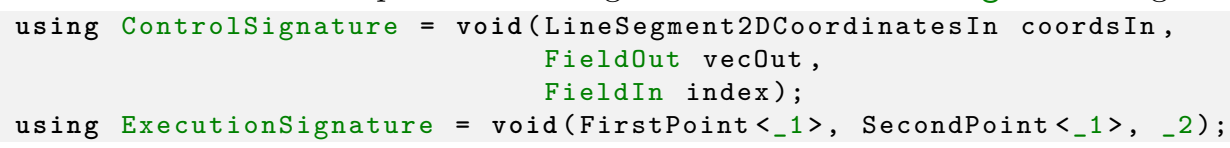



The basic building block for an algorithm in VTK-m is the worklet. Chapter 21 describes the different types of worklet types provided by VTK-m and how to use them to create algorithms. However, it is entirely possible that this set of worklet types does not directly cover what is needed to implement a particular algorithm. One way around this problem is to use some of the numerous back doors provided by VTK-m to provide less restricted access in the execution environment such as using whole arrays for random access.

However, it make come to pass that you encounter a particular pattern of execution that you find useful for implementing several algorithms. If such is the case, it can be worthwhile to create a new worklet type that directly supports such a pattern. Creating a new worklet type can provide two key advantages. First, it makes implementing algorithms of this nature easier, which saves developer time. Second, it can make the implementation of such algorithms safer. By encapsulating the management of structures and regulating the data access, users of the worklet type can be more assured of correct behavior.

This chapter documents the process for creating new worklet types. The operation of a worklet requires the coordination of several different object types such as dispatchers, argument handlers, and thread indices. This chapter will provide examples of all these required components. To tie all these features together, we start this chapter with a motivating example for an implementation of a custom worklet type. The chapter then discusses the individual components of the worklet, which in the end come together for the worklet type that is then demonstrated.

\subsection{Motivating Example}

For our motivation to create a new worklet type, let us consider the use case of building fractals. Fractals are generally not a primary concern of visualization libraries like VTK-m, but building a fractal (or approximations of fractals) has similarities the the computational geometry problems in scientific visualization. In particular, we consider the class of fractals that is generated by replacing each line in a shape with some collection of lines. These types of fractals are interesting because, in addition to other reasons, the right parameters result in a shape that has infinite length confined to a finite area.

A simple but well known example of a line fractal is the Koch Snowflake. The Koch Snowflake starts as a line or triangle that gets replaced with the curve shown in Figure 41.1.

The fractal is formed by iteratively replacing the curve's lines with this basic shape. Figure 41.2 shows the second iteration and then several subsequent iterations that create a "fuzzy" curve. The curve is confined to a limited area regardless of how many iterations are performed, but the length of the curve approaches infinity as the number of iterations approaches infinity.

In our finite world we want to estimate the curve of the Koch Snowflake by performing a finite amount of 


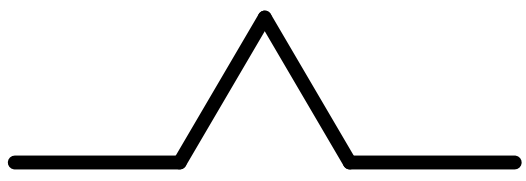

Figure 41.1: Basic shape for the Koch Snowflake.

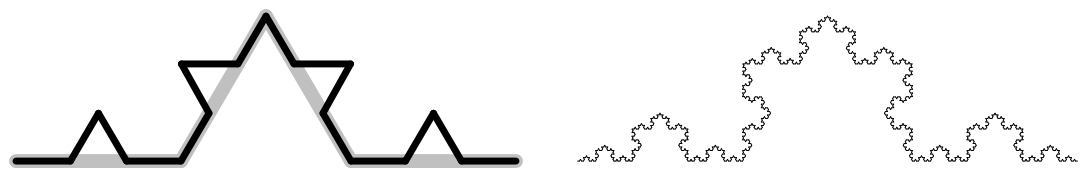

Figure 41.2: The Koch Snowflake after the second iteration (left image) and after several more iterations (right image).

iterations. This is similar to a Lindenmayer system but with less formality. The size of the curve grows quickly and in practice it takes few iterations to make close approximations.

\section{Did you know?}

3 The Koch Snowflake is just one example of many line fractals we can make with this recursive line subStitution, which is why it is fruitful to create a worklet type to implement such fractals. We use the Koch Snowflake to set up the example here. Section 41.6 provides several more examples.

To implement line fractals of this nature, we want to be able to define the lines of the base shape in terms of parametric coordinates and then transform the coordinates to align with a line segment. For example, the Koch Snowflake base shape could be defined with parametric coordinates shown in Figure 41.3.

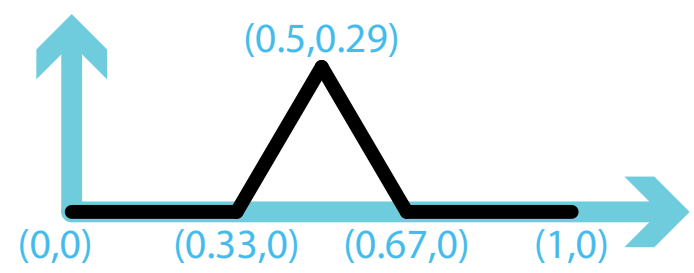

Figure 41.3: Parametric coordinates for the Koch Snowflake shape.

Given these parametric coordinates, for each line we define an axis with the main axis along the line segment and the secondary axis perpendicular to that. Given this definition, we can perform each fractal iteration by applying this transform for each line segment as shown in Figure 41.4.

To implement the application of the line fractal demonstrated in Figure 41.4, let us define a class named LineFractalTransform that takes as its constructor the coordinates of two ends of the original line. As its operator, LineFractalTransform takes a point in parametric space and returns the coordinates in world space in respect to the original line segment. We define this class in the vtkm: :exec namespace because the intended use case is by worklets of the type we are making. A definition of LineFractalTransform is given in Example 41.1

Example 41.1: A support class for a line fractal worklet. 


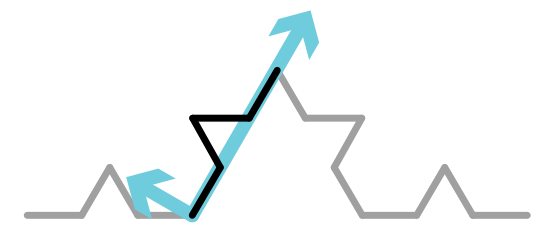

Figure 41.4: Applying the line fractal transform for the Koch Snowflake.

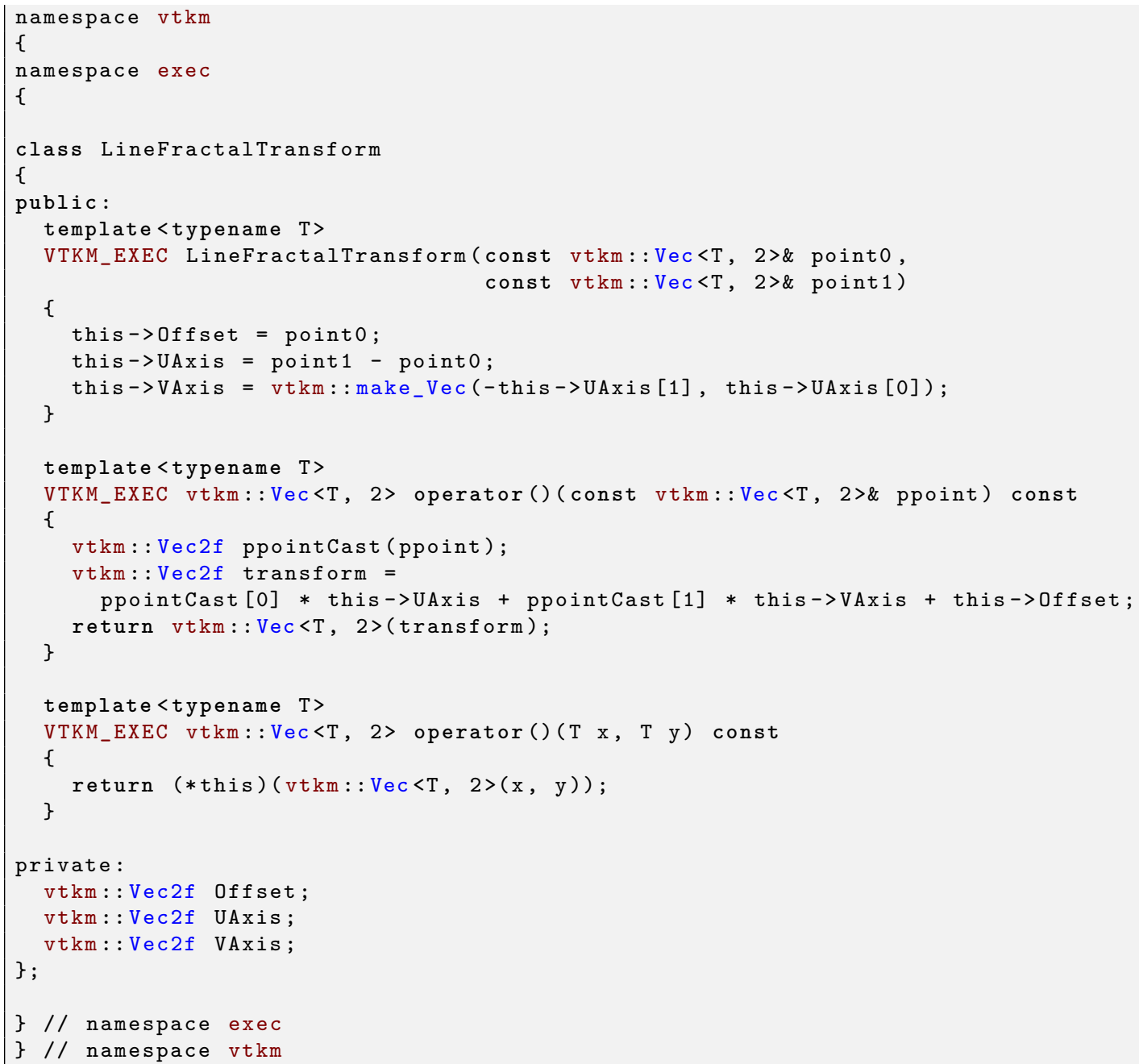

\section{Did you know?}

The definition of LineFractalTransform (or something like it) is not strictly necessary for implementing a worklet type. However, it is common to implement such supporting classes that operate in the execution environment in support of the operations typically applied by the worklet type.

The remainder of this chapter is dedicated to defining a WorkletLineFractal class and supporting objects that 
allow you to easily make line fractals. Example 41.2 demonstrates how we intend to use this worklet type.

Example 41.2: Demonstration of how we want to use the line fractal worklet.

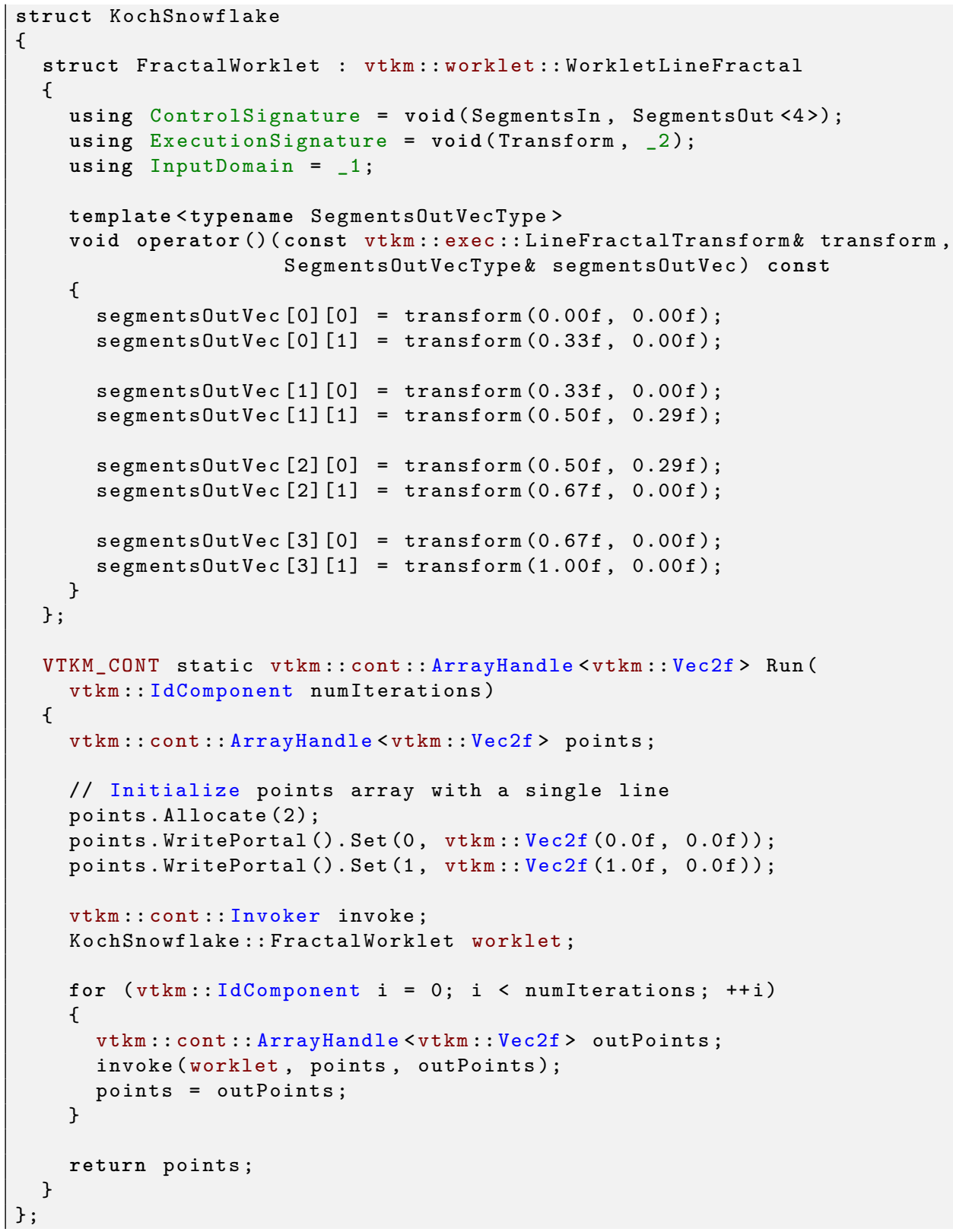

\subsection{Thread Indices}

The first internal support class for implementing a worklet type is a class that manages indices for a thread. As the name would imply, the thread indices class holds a reference to an index identifying work to be done by the current thread. This includes indices to the current input element and the current output element. The thread indices object can also hold other information (that may not strictly be index data) about the 
input and output data. For example, the thread indices object for topology maps (named vtkm: :exec: : arg: :ThreadIndicesTopologyMap) maintains cell shape and connection indices for the current input object.

As is discussed briefly in Section 40.3, a thread indices object is passed to the methods of the class vtkm: :exec: : arg: :Fetch to retrieve data from the execution object. The thread indices object serves two important functions for the Fetch. The first function is to cache information about the current thread that is likely to be used by multiple objects retrieving information. For example, in a point to cell topology map data from point fields must be retrieved by looking up indices in the topology connections. It is more efficient to retrieve the topology connections once and store them in the thread indices than it is to look them up independently for each field.

The second function of thread indices is to make it easier to find information about the input domain when fetching data. Once again, getting point data in a point to cell topology map requires looking up connectivity information in the input domain. However, the Fetch object for the point field does not have direct access to the data for the input domain. Instead, it gets this information from the thread indices.

All worklet classes have a method named GetThreadIndices that constructs a thread indices object for a given thread. GetThreadIndices is called with 5 parameters: a unique index for the thread (i.e. worklet instance), an array portal that maps output indices to input indices (which might not be one-to-one if a scatter is being used), an array portal that gives the visit index for each output index, an array portal that maps each unique thread index to the output index for that thread, and the execution object for the input domain.

The base worklet implementation provides an implementation of GetThreadIndices that creates a vtkm: :exec: : arg: :ThreadIndicesBasic object. This provides the minimum information required in a thread indices object, but non-trivial worklet types are likely to need to provide their own thread indices type. This following example shows the implementation of GetThreadIndices we will use in our worklet type superclass (discussed in more detail in Section 41.4).

Example 41.3: Implementation of GetThreadIndices in a worklet superclass.

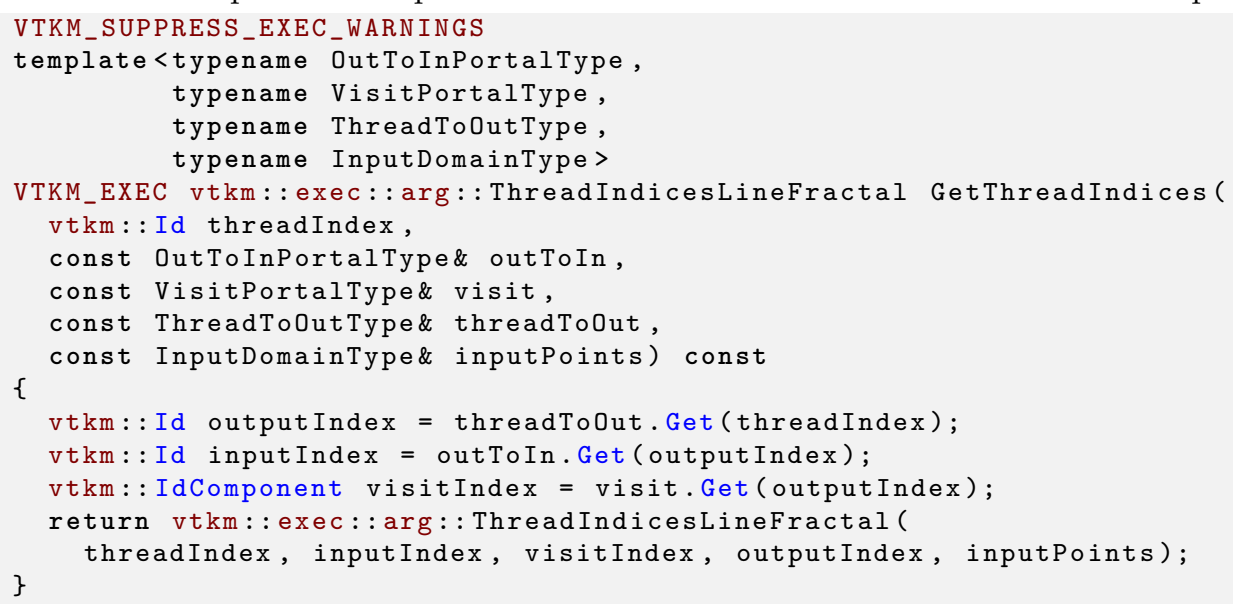

As we can see in Example 41.3, our new worklet type needs a custom thread indices class. Specifically, we want the thread indices class to manage the coordinate information of the input line segment.

Did you know?

\ The implementation of a thread indices object we demonstrate here stores point coordinate information in addition to actual indices. It is acceptable for a thread indices object to store data that are not strictly indices. That said, the thread indices object should only load data (index or not) that is almost certain to 
be used by any worklet implementation. The thread indices object is created before any time that the worklet operator is called. If the thread indices object loads data that is never used by a worklet, that is a waste.

An implementation of a thread indices object usually derives from vtkm: :exec: :arg: :ThreadIndicesBasic (or some other existing thread indices class) and adds to it information specific to a particular worklet type.

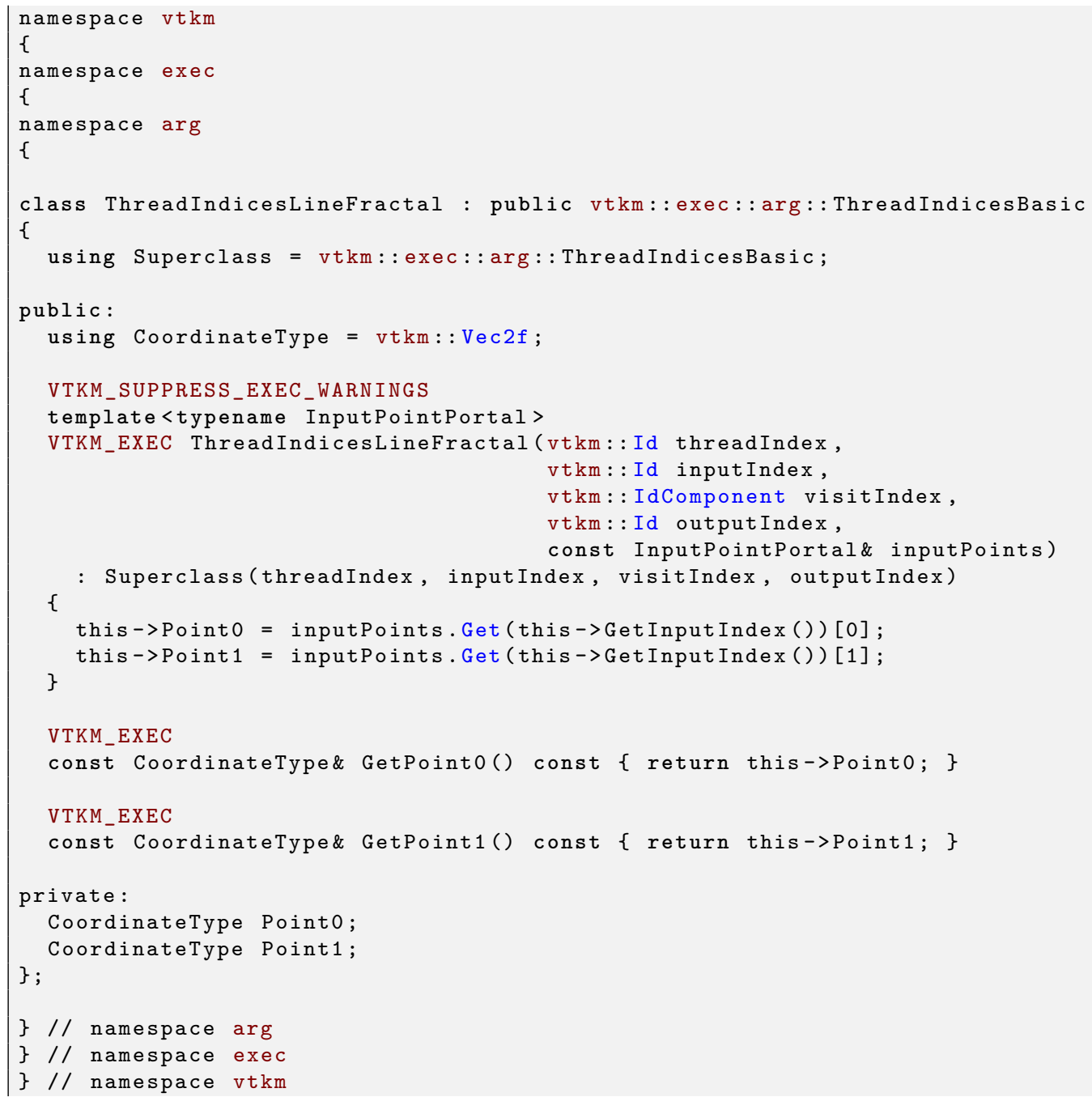

\subsection{Signature Tags}

It is common that when defining a new worklet type, the new worklet type is associated with new types of data. Thus, it is common that implementing new worklet types involves defining custom tags for ControlSignatures and ExecutionSignatures. This in turn typically requires creating custom TypeCheck, Transport, and Fetch classes.

Chapter 40 describes in detail the process of defining new worklet types and the associated code to manage data from a vtkm: : cont: : Invoker argument to the data that are passed to the worklet operator. Rather than repeat 
the discussion, readers should review Chapter 40 for details on how custom arguments are defined for a new worklet type. In particular, we use the code from Examples 40.2 (page 359), 40.4 (page 361), and 40.5 (page 364 ) to implement an argument representing $2 \mathrm{D}$ line segments (which is our input domain). All these examples culminate in the definition of a Controlsignature tag in our worklet superclass.

Example 41.5: Custom ControlSignature tag for the input domain of our example worklet type.

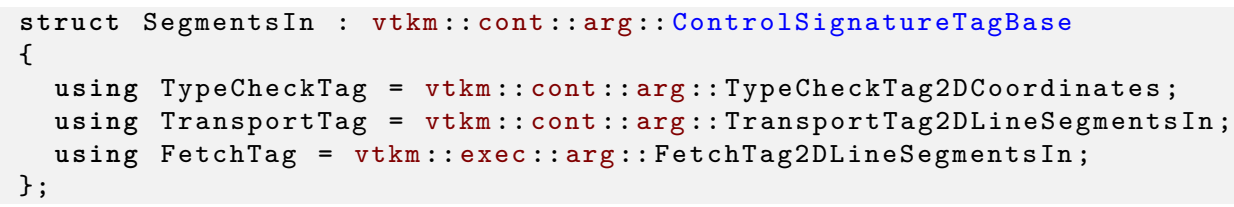

As you have worked with different existing worklet types, you have likely noticed that different worklet types have special ExecutionSignature tags to point to information in the input domain. For example, a point to cell topology map has special ExecutionSignature tags for getting the input cell shape and the indices to all points incident on the current input cell. We described in the beginning of the chapter that we wanted our worklet type to provide worklet implementations an object named LineFractalTransform (Example 41.1), so it makes sense to define our own custom ExecutionSignature tag to provide this object.

Chapter 40 gives an example of a custom ExecutionSignature tag that modifies what information is fetched from an argument (Examples 40.6 and 40.9). However, ExecutionSignature tags that only pull data from input domain behave a little differently because they only get information from the thread indices object and ignore the associated data object. This is done by providing a partial specialization of vtkm: :exec: : arg: :Fetch that specializes on the aspect tag but not on the fetch tag.

Example 41.6: A Fetch for an aspect that does not depend on any control argument.

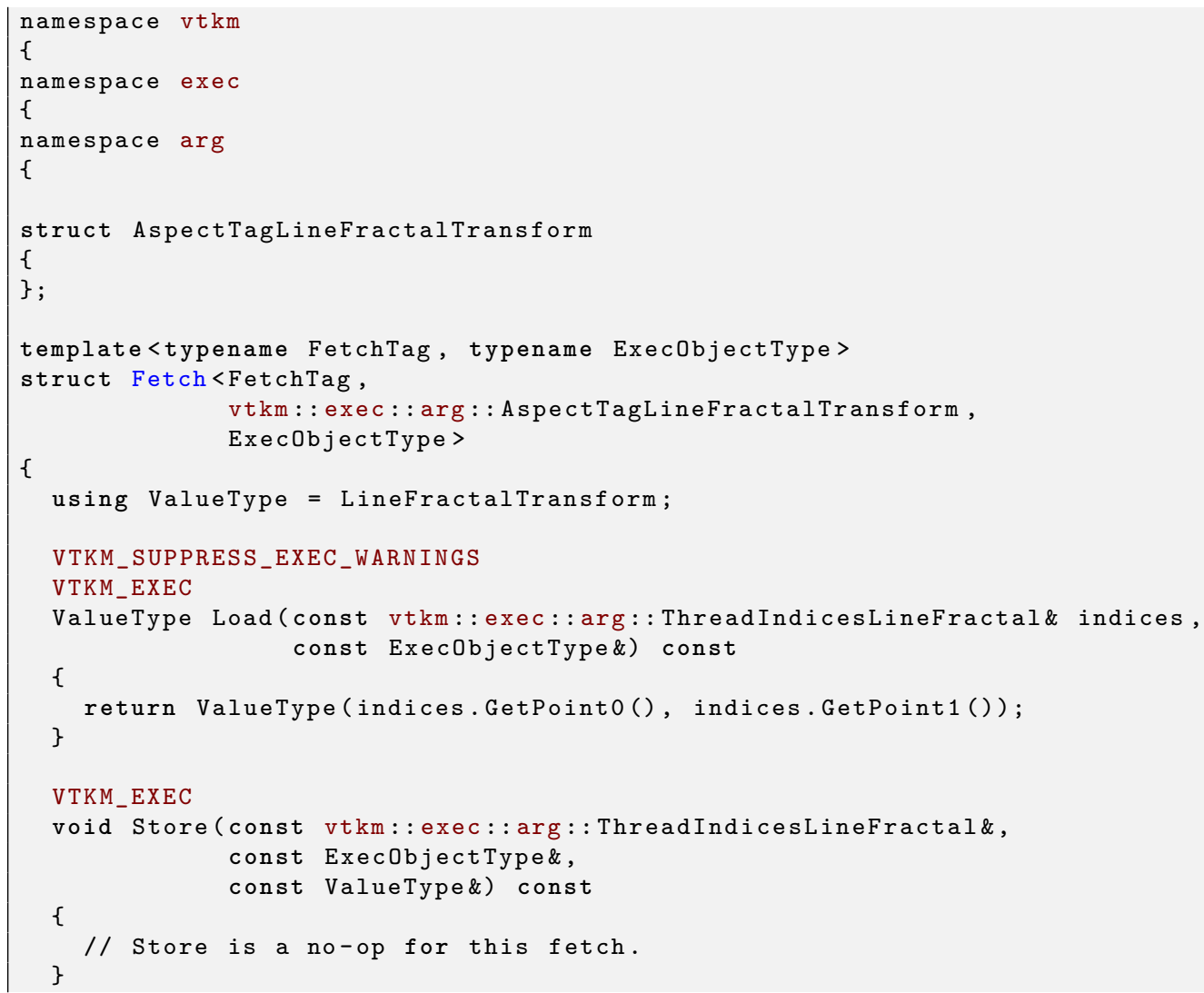




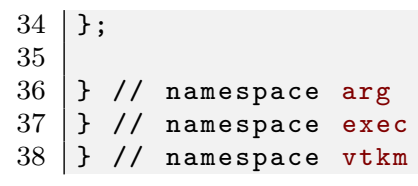

The definition of an associated ExecutionSignature tag simply has to use the define aspect as its AspectTag. The tag also has to define a INDEX member (which is required of all ExecutionSignature tags). This is problematic as this execution argument does not depend on any particular control argument. Thus, it is customary to simply set the INDEX to 1 . There is guaranteed to be at least one ControlSignature argument for any worklet implementation. Thus, the first argument is sure to exist and can then be ignored.

Example 41.7: Custom ExecutionSignature tag that only relies on input domain information in the thread indices.

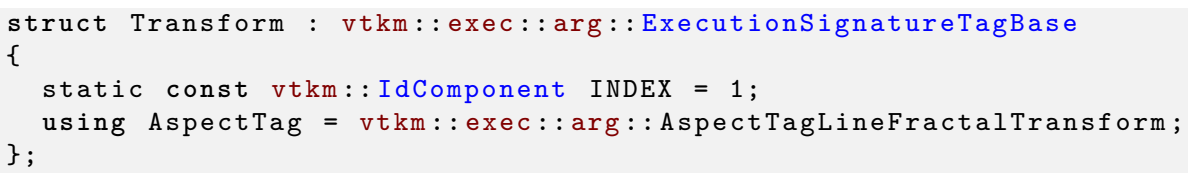

So far we have discussed how to get input line segments into our worklet. We also need a ControlSignature tag to represent the output line segments created by instances of our worklet. The motivating example has each worklet outputting a fixed number (greater than 1) of line segments for each input line segment. To manage this, we will define another ControlSignature tag that outputs these line segments (as two Vec-2 coordinates). This is defined as a Vec of Vec-2's. The tag takes the number of line segments as a template argument.

Example 41.8: Output ControlSignature tag for our motivating example.

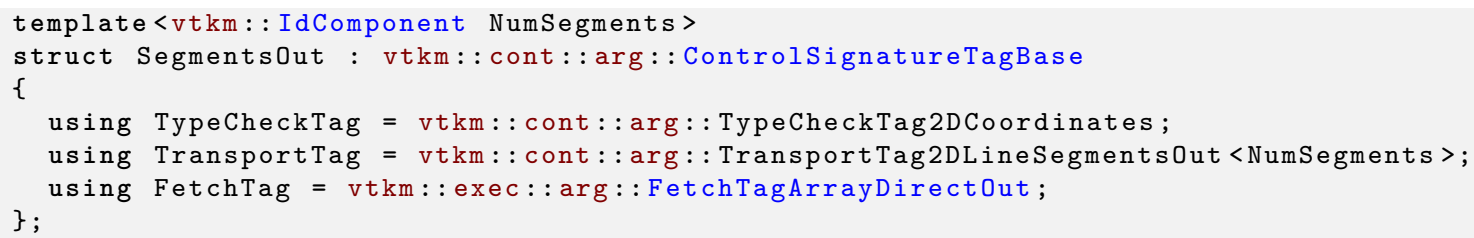

You can see that the tag in Example 41.8 relies on a custom transport named TransportTag2DLineSegmentsOut. There is nothing particularly special about this transport, but we provide the implementation here for completeness.

Example 41.9: Implementation of Transport for the output in our motivating example.

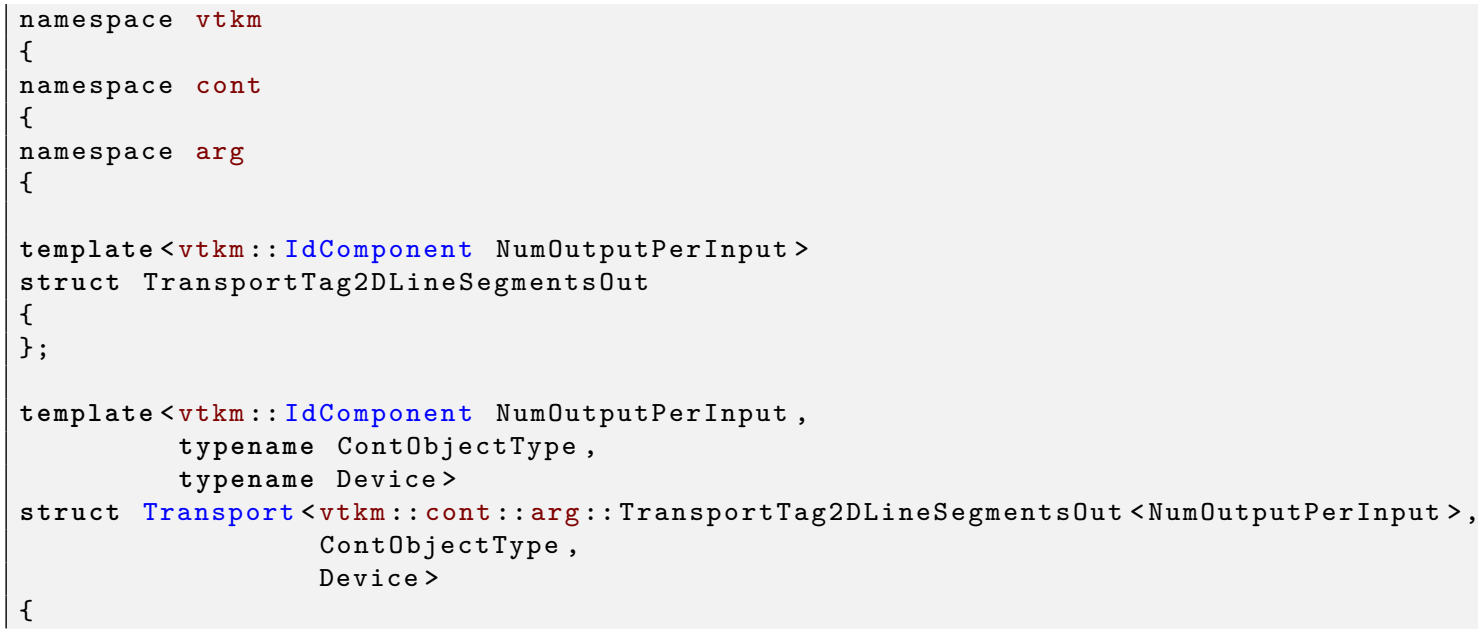




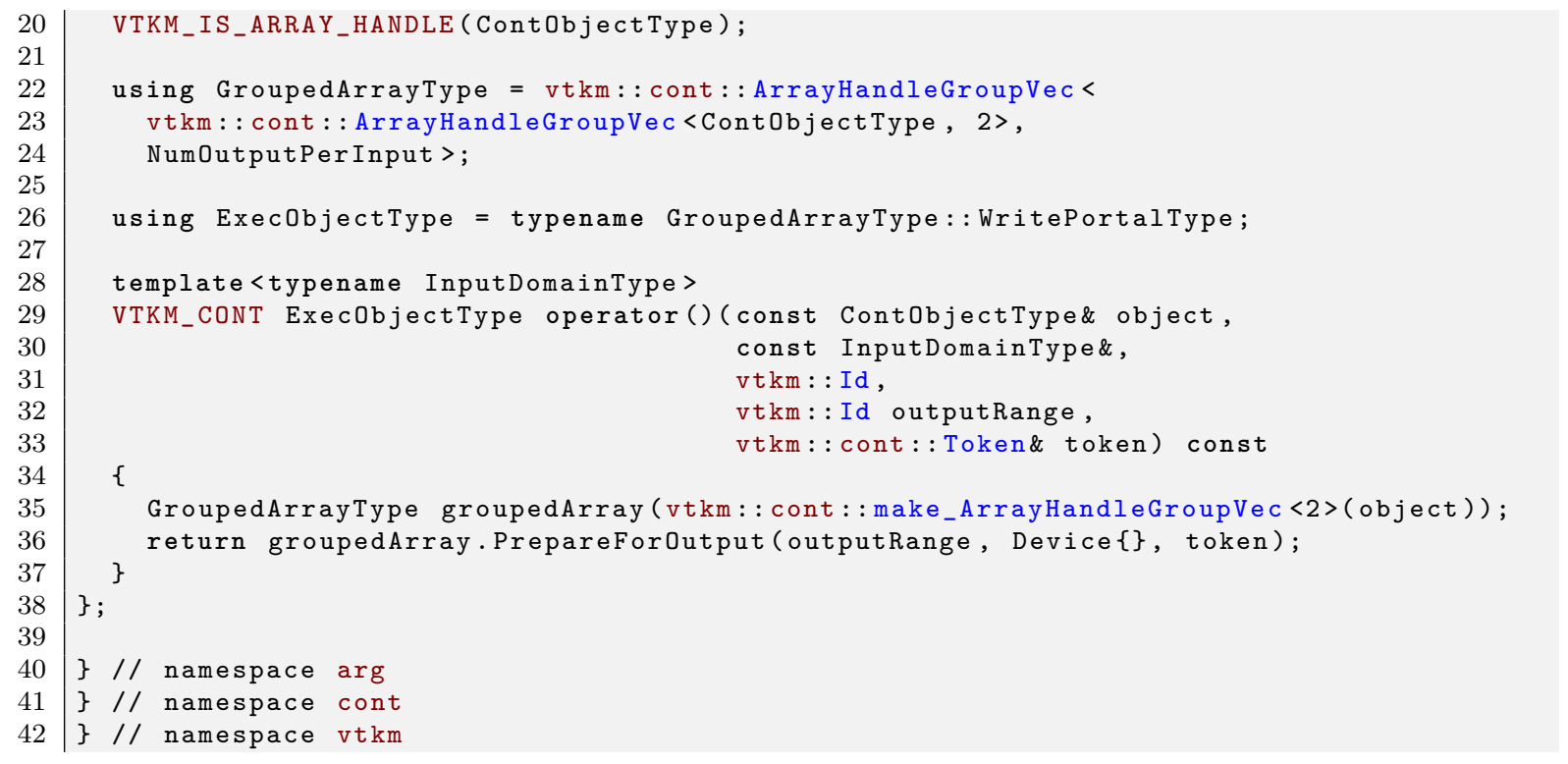

In addition to these special ControlSignature tags that are specific to the nature of our worklet type, it is common to need to replicate some more common or general ControlSignature tags. One such tag, which is appropriate for our worklet type, is a "field" type that takes an array with exactly one value associated with each input or output element. We can build these field tags using existing type checks, transports, and fetches. The following example defines a FieldIn tag for our fractal worklet type. A FieldOut tag can be made in a similar manner.

Example 41.10: Implementing a FieldIn tag.

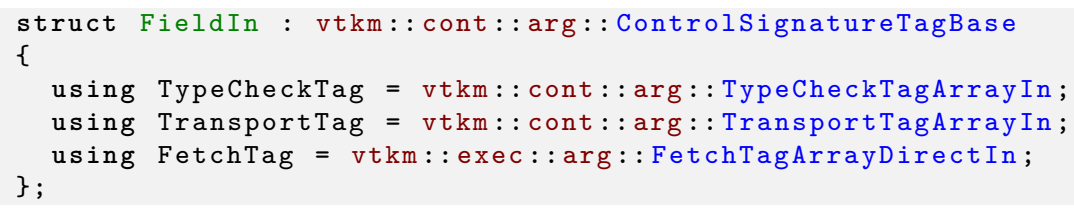

\subsection{Worklet Superclass}

The penultimate step in defining a new worklet type is to define a class that will serve as the superclass of all implementations of worklets of this type. This class itself must inherit from vtkm: : worklet: :internal::WorkletBase. By convention the worklet superclass is placed in the vtkm: :worklet namespace and its name starts with Worklet.

Within the worklet superclass we define the signature tags (as discussed in Section 41.3) and the GetThreadIndices method (as discussed in Section 41.2. The worklet superclass can also override other default behavior of the WorkletBase (such as special scatter). And the worklet superclass can provide other items that might be particularly useful to its subclasses (such as commonly used tags). Also, the worklet superclass must declare a Dispatcher template that points to a dispatcher object used to invoke the worklet. The dispatcher is created in Section 41.5.

Example 41.11: Superclass for a new type of worklet.

1 namespace vtkm

2 \{ 


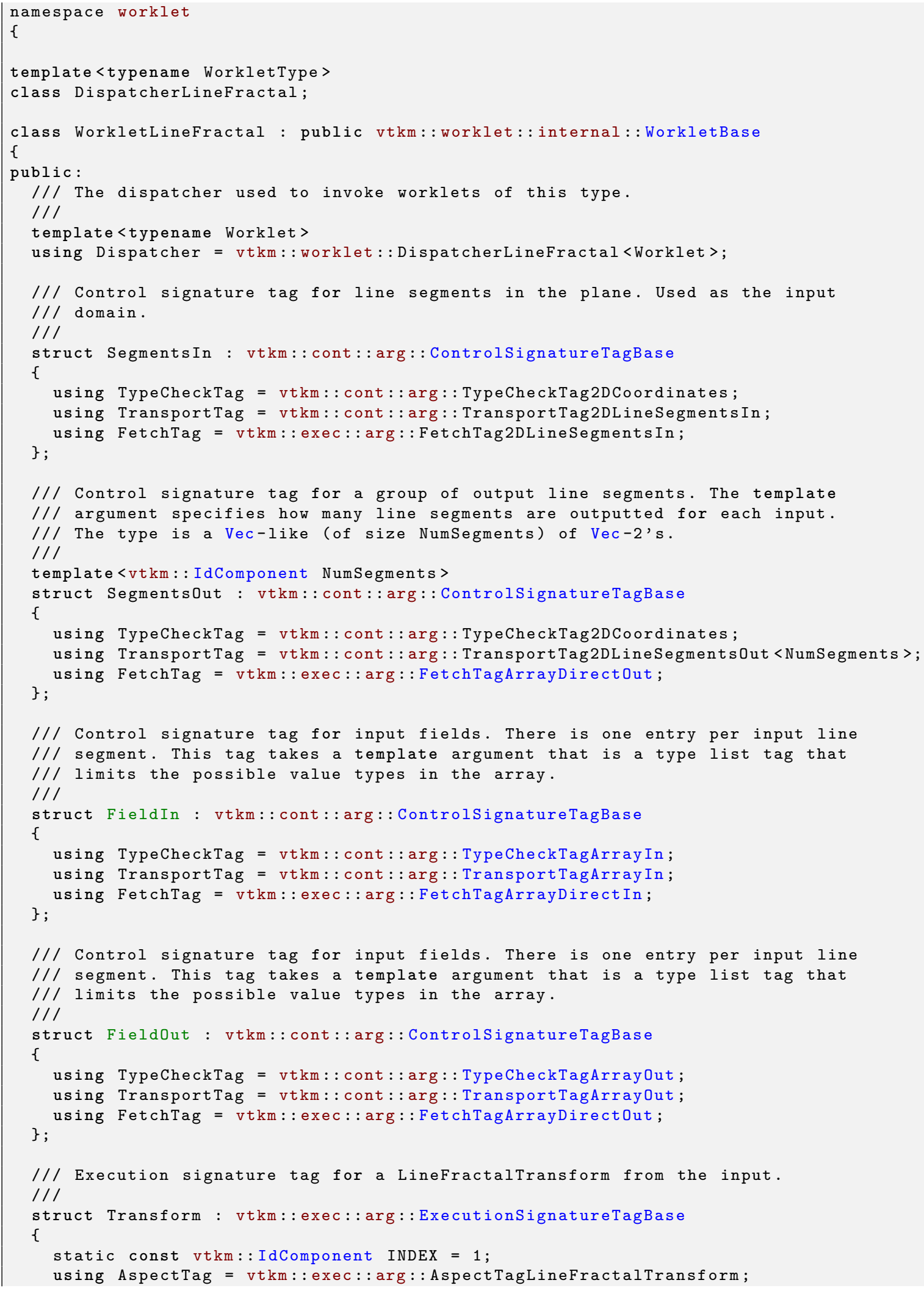




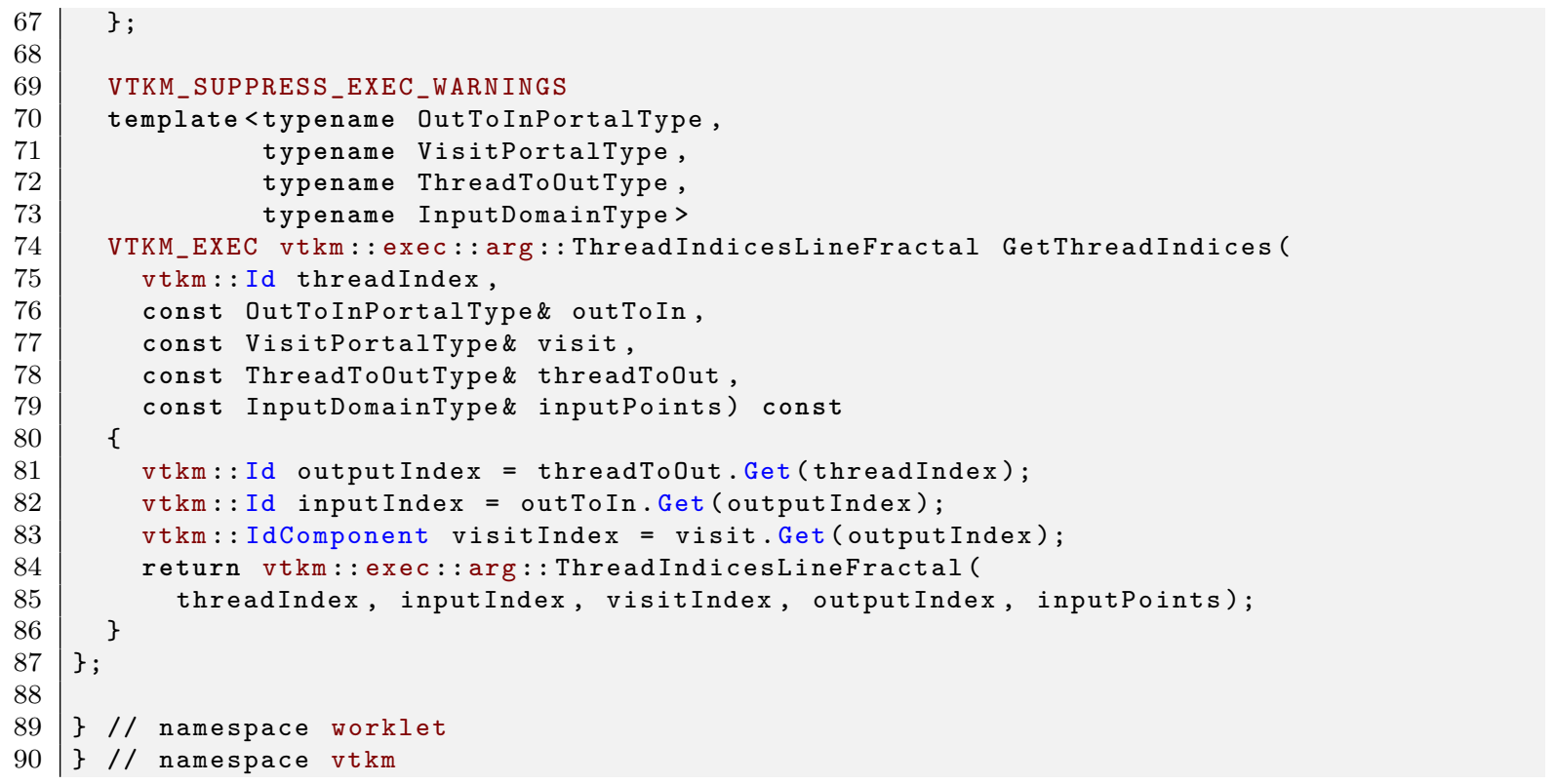

\section{Common Errors}

Be wary of creating worklet superclasses that are templated. The $C++$ compiler rules for superclass templates that are only partially specialized are non-intuitive. If a subclass does not fully resolve the template, features of the superclass such as signature tags will have to be qualified with typename keywords, which reduces the usability of the class.

\subsection{Dispatcher}

Worklets are instantiated in the control environment and run in the execution environment. This means that the control environment must have a means to invoke worklets that start running in the execution environment. This is ostensibly done by the vtkm::cont: : Invoker object, but the Invoker does this through a dispatcher object.

A dispatcher object is an object in the control environment that has an instance of a worklet and can invoke that worklet with a set of arguments. There are multiple types of dispatcher objects, each corresponding to a type of worklet object. All dispatcher objects have at least one template parameter: the worklet class being invoked, which is always the first argument. All dispatcher objects must be constructed with an instance of the worklet they are to invoke.

All dispatcher classes have a method named Invoke that launches the worklet in the execution environment. The arguments to Invoke must match those expected by the worklet, which is specified by something called a control signature.

The following is a list of the dispatchers defined in VTK-m. The dispatcher classes correspond to the list of worklet types specified in Chapter 21 . 
vtkm: :worklet::DispatcherMapField The dispatcher used in conjunction with a worklet that subclasses vtkm: : worklet: :WorkletMapField. The dispatcher class has one template argument: the worklet type.

vtkm: : worklet: :DispatcherMapTopology The dispatcher used in conjunction with a worklet that subclasses vtkm: : worklet: :WorkletMapTopology or one of its sibling classes (such as vtkm: : worklet: :WorkletVisitCellsWithPoints). The dispatcher class has one template argument: the worklet type.

vtkm: : worklet: :DispatcherPointNeighborhood The dispatcher used in conjunction with a worklet that subclasses vtkm: :worklet: :WorkletPointNeighborhood. The dispatcher class has one template argument: the worklet type.

vtkm: : worklet: :DispatcherReduceByKey The dispatcher used in conjunction with a worklet that subclasses vtkm: :worklet::WorkletReduceByKey. The dispatcher class has one template argument: the worklet type.

The final element required for a new worklet type is an associated dispatcher class for invoking the worklet.

Example 41.12: Standard template arguments for a dispatcher class.

1 template<typename WorkletType >

2 class DispatcherLineFractal

A dispatcher implementation inherits from vtkm: : worklet: : internal::DispatcherBase. DispatcherBase is itself a templated class with the following three templated arguments.

1. The dispatcher class that is subclassing DispatcherBase. All template arguments must be given.

2. The type of the worklet being dispatched (which by convention is the first argument of the dispatcher's template).

3. The expected superclass of the worklet, which is associated with the dispatcher implementation. DispatcherBase will check that the worklet has the appropriate superclass and provide a compile error if there is a mismatch.

\section{Q Did you know?}

$\sum$ The convention of having a subclass be templated on the derived class' type is known as the Curiously Recurring Template Pattern (CRTP). In the case of DispatcherBase, VTK-m uses this CRTP behavior to allow the general implementation of Invoke to run DoInvoke in the subclass, which as we see in a moment is itself templated.

Example 41.13: Subclassing DispatcherBase.

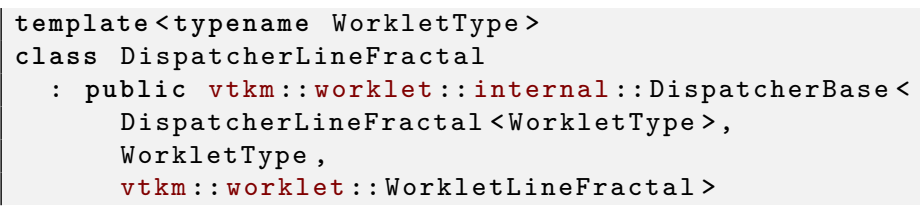

The dispatcher should have two constructors. The first constructor takes a worklet and a dispatcher. Both arguments should have a default value that is a new object created with its default constructor. It is good practice to put a warning on this constructor letting users know if they get a compile error there it is probably because the worklet or dispatcher does not have a default constructor and they need to provide one. The second constructor just takes a dispatcher. 
Example 41.14: Typical constructor for a dispatcher.

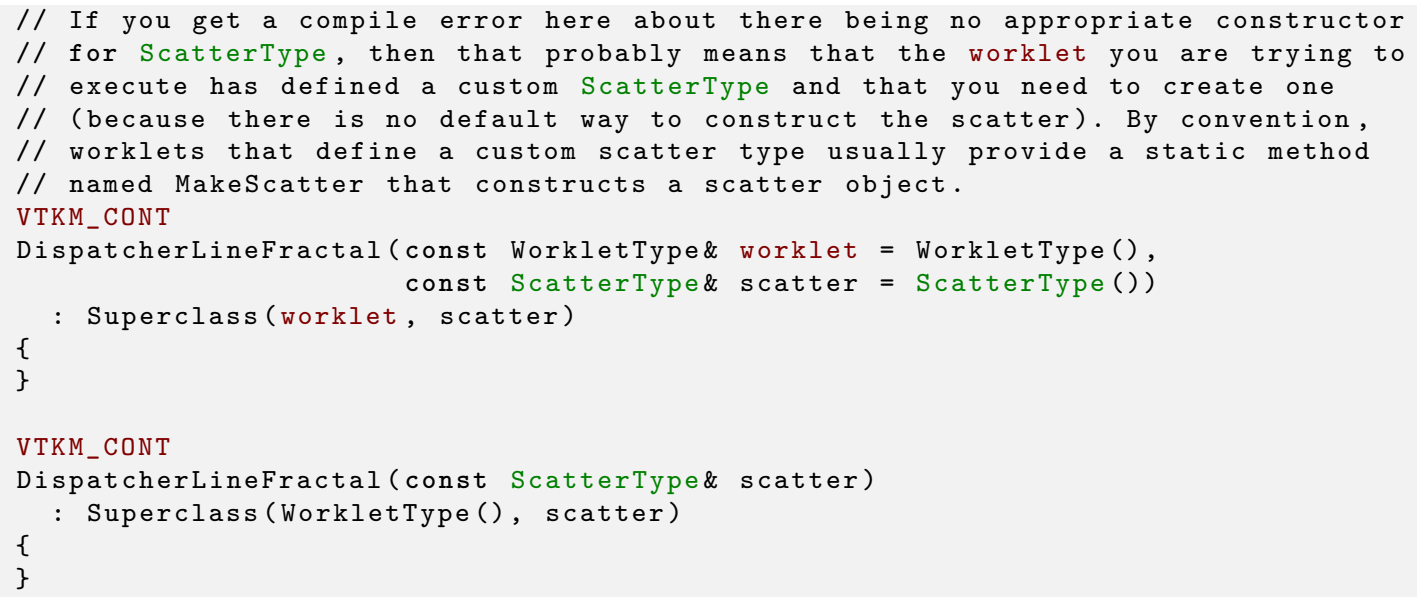

Finally, the dispatcher must implement a const method named DoInvoke. The DoInvoke method should take a single argument. The argument will be an object of type vtkm: internal:: Invocation although it is usually more convenient to just express the argument type as a single template parameter. The Invocation could contain several data items, so it is best to pass this argument as a constant reference.

Example 41.15: Declaration of DoInvoke of a dispatcher.

Invocation is an object that encapsulates the state and data relevant to the invoke. Invocation contains multiple types and data items. For brevity only the ones most likely to be used in a DoInvoke method are documented here. We discuss these briefly before getting back to the implementation of DoInvoke.

vtkm: : internal: : Invocation contains a data member named Parameters that contains the data passed to the Invoke method of the dispatcher (with some possible transformations applied). Parameters is stored in a vtkm: : internal: :FunctionInterface template object. (FunctionInterface is described in Chapter 39.) The specific type of Parameters is defined as type ParameterInterface in the Invoke object.

The Invoke object also contains the types ControlInterface and ExecutionInterface that are FunctionInterface classes built from the ControlSignature and ExecutionSignature of the worklet. These FunctionInterface classes provide a simple mechanism for introspecting the arguments of the worklet's signatures.

All worklets must also define an input domain index, which points to one of the ControlSignature/Invoke arguments. This number is also captured in the vtkm::internal::Invocation object in a field named InputDomainIndex. For convenience, Invocation also has the type InputDomainTag set to be the same as the ControlSignature argument corresponding to the input domain. Likewise, Invocation has the type InputDomainType set to be the same type as the (transformed) input domain argument to Invoke. Invocation also has a method name GetInputDomain that returns the invocation object passed to Invoke.

Getting back to the implementation of a dispatcher, the DoInvoke should first verify that the ControlSignature argument associated with the input domain is of the expected type. This can be done by comparing the Invocation: :InputDomainTag with the expected signature tag using a tool like std::is_same. This step is not strictly necessary, but is invaluable to users diagnosing issues with using the dispatcher. It does not hurt to also check that the Invoke argument for the input domain is also the same as expected (by checking Invocation: : InputDomainType). It is additionally helpful to have a descriptive comment near these checks.

Example 41.16: Checking the input domain tag and type.

1 // Get the control signature tag for the input domain. 


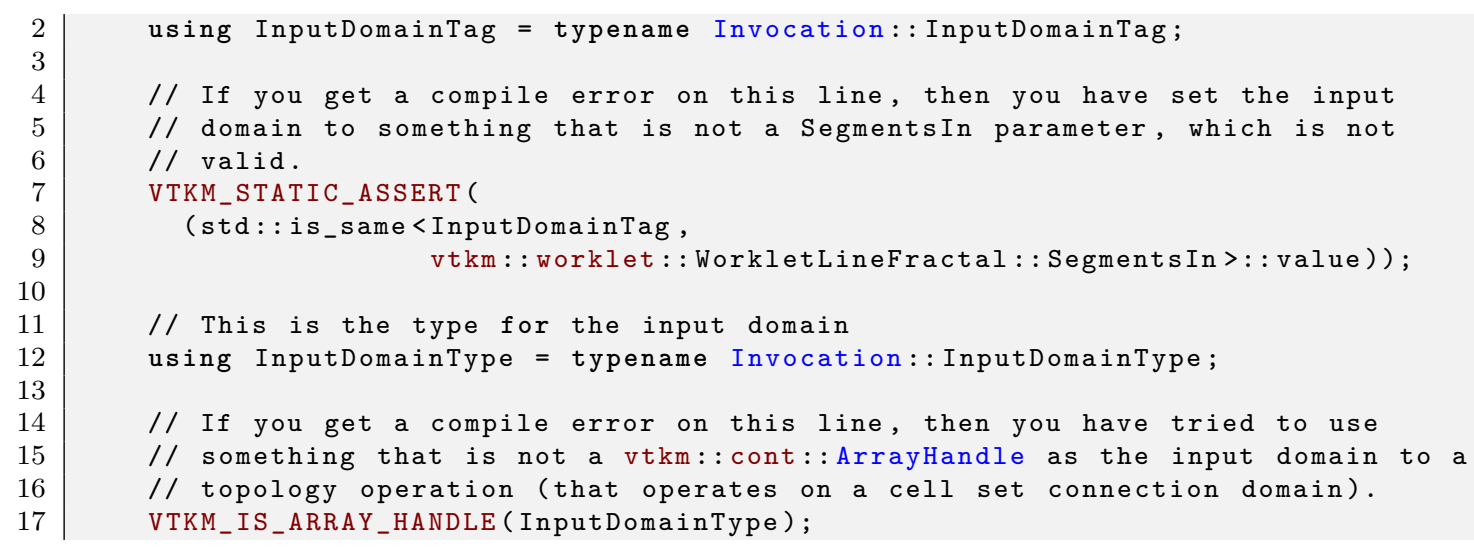

Next, DoInvoke must determine the size in number of elements of the input domain. When the default identity scatter is used, the input domain size corresponds to the number of instances the worklet is executed. (Other scatters will transform the input domain size to an output domain size, and that output domain size will determine the number of instances.) The input domain size is generally determined by using Invocation:: : GetInputDomain and querying the input domain argument. In our motivating example, the input domain is an ArrayHandle and the input domain size is half the size of the array (since array entries are paired up into line segments).

The final thing DoInvoke does is call BasicInvoke on its DispatcherBase superclass. BasicInvoke does the complicated work of transferring arguments, scheduling the parallel job, and calling the worklet's operator. BasicInvoke takes three arguments: the Invocation object, the size of the input domain, and the device adapter tag to run on.

Example 41.17: Calling BasicInvoke from a dispatcher's DoInvoke.

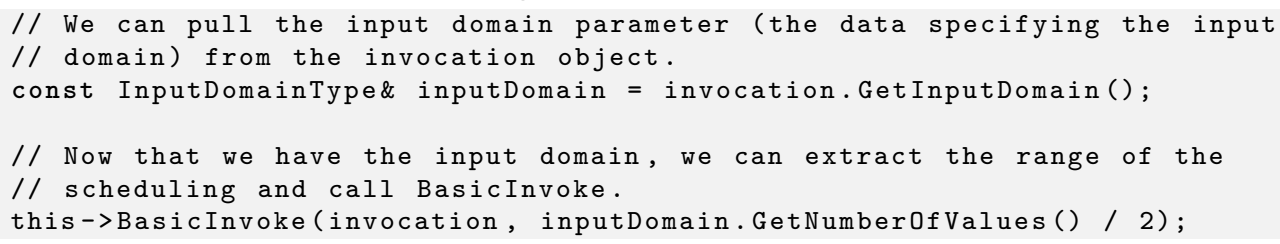

Putting this all together, the following example demonstrates the full implementation of the dispatcher for our motivating example.

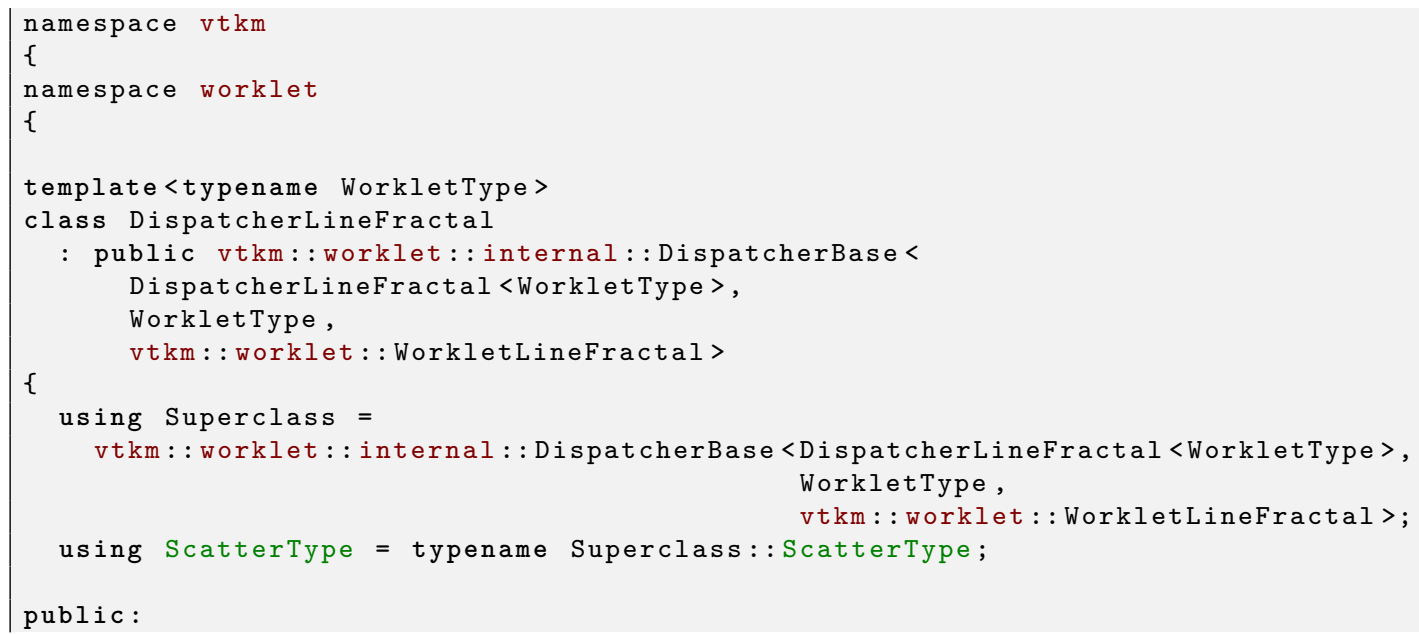




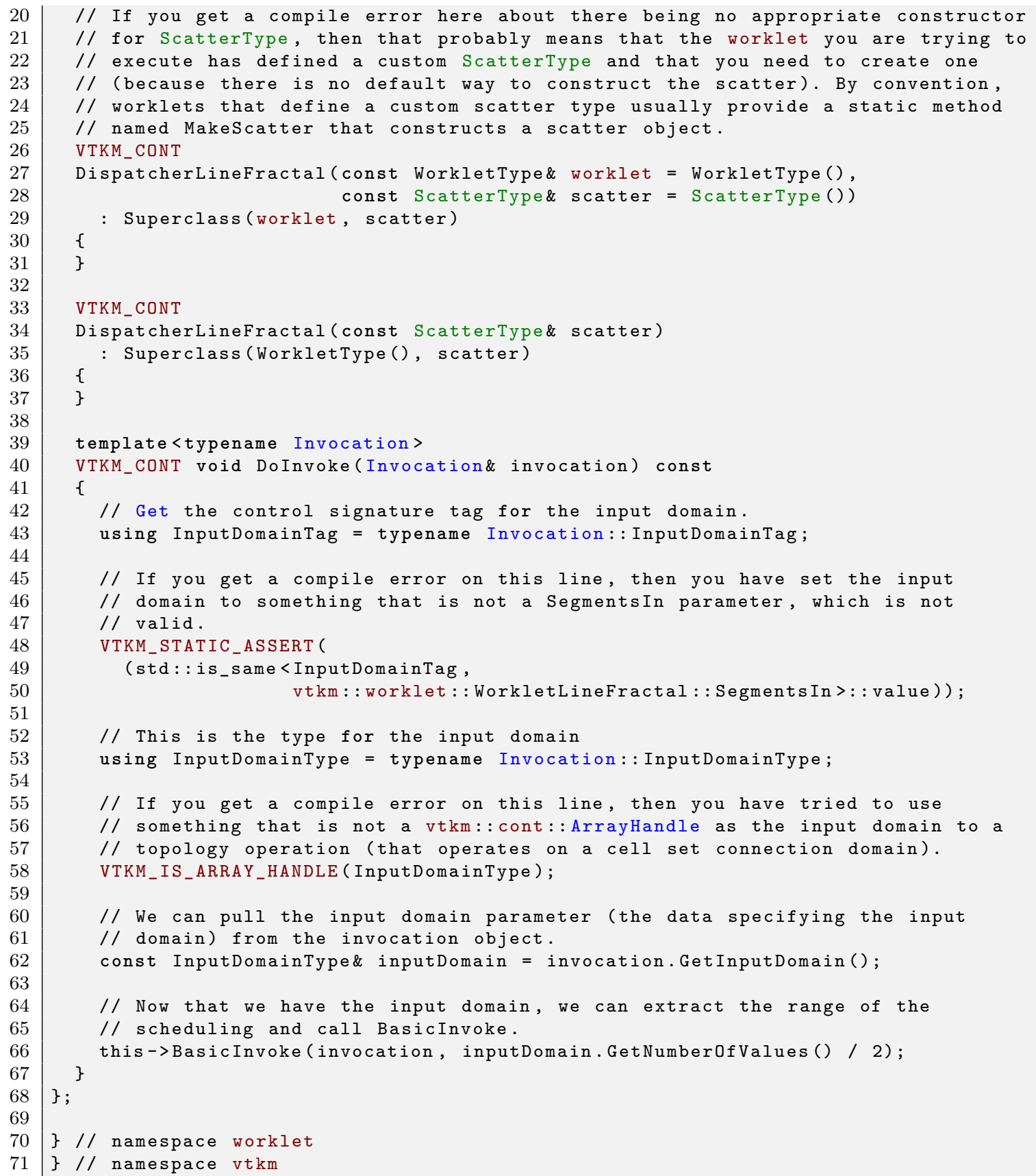

\subsection{Using the Worklet}

Now that we have our full implementation of a worklet type that generates line fractals, let us have some fun with it. The beginning of this chapter shows an implementation of the Koch Snowflake. The remainder of this chapter demonstrates other fractals that are easily implemented with our worklet type. 


\subsubsection{Quadratic Type 2 Curve}

There are multiple variants of the Koch Snowflake. One simple but interesting version is the quadratic type 1 curve. This fractal has a shape similar to what we used for Koch but has right angles and goes both up and down as shown in Figure 41.5.

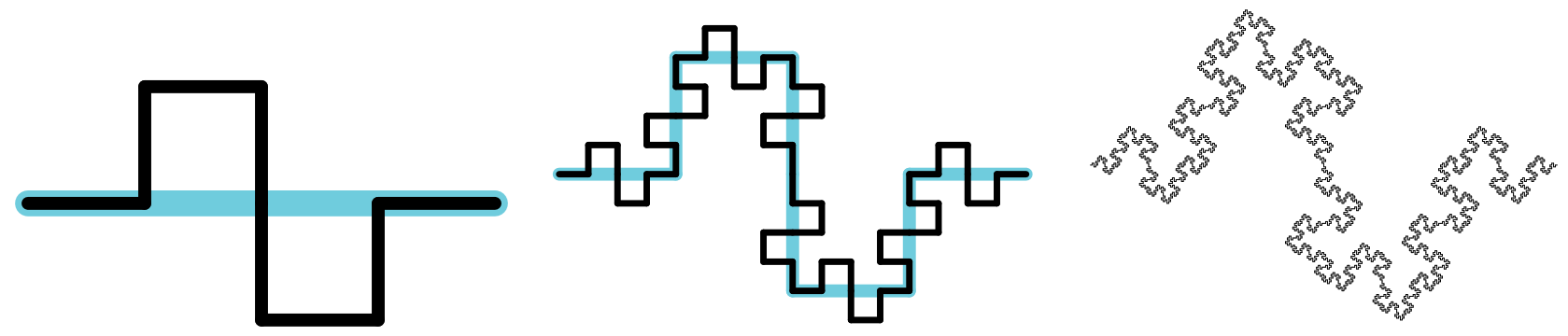

Figure 41.5: The quadratic type 2 curve fractal. The left image gives the first iteration. The middle image gives the second iteration. The right image gives the result after a few iterations.

The quadratic type 2 curve is implemented exactly like the Koch Snowflake except we output 8 lines to every input instead of 4, and, of course, the positions of the lines we generate are different.

Example 41.19: A worklet to generate a quadratic type 2 curve fractal.

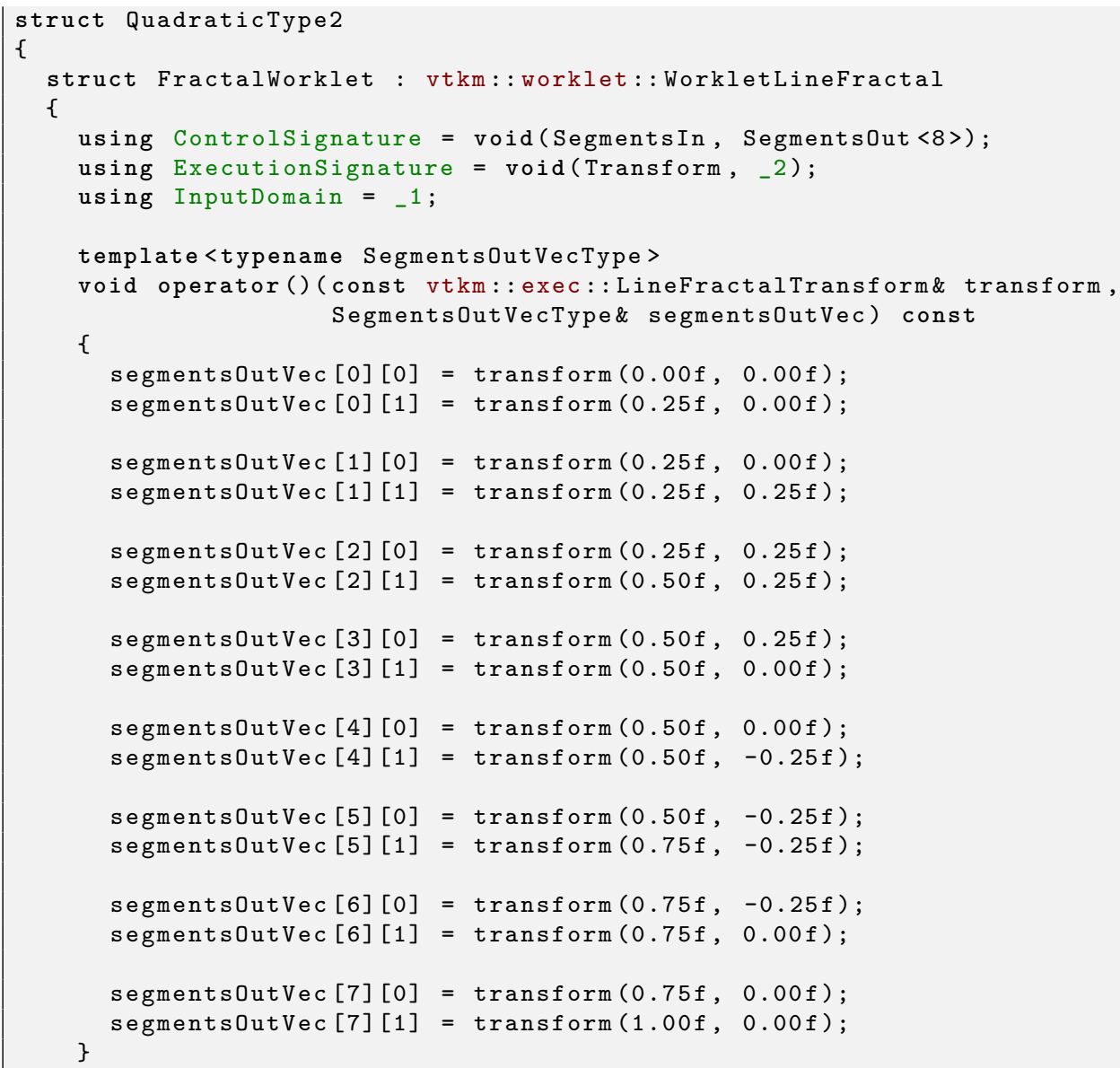




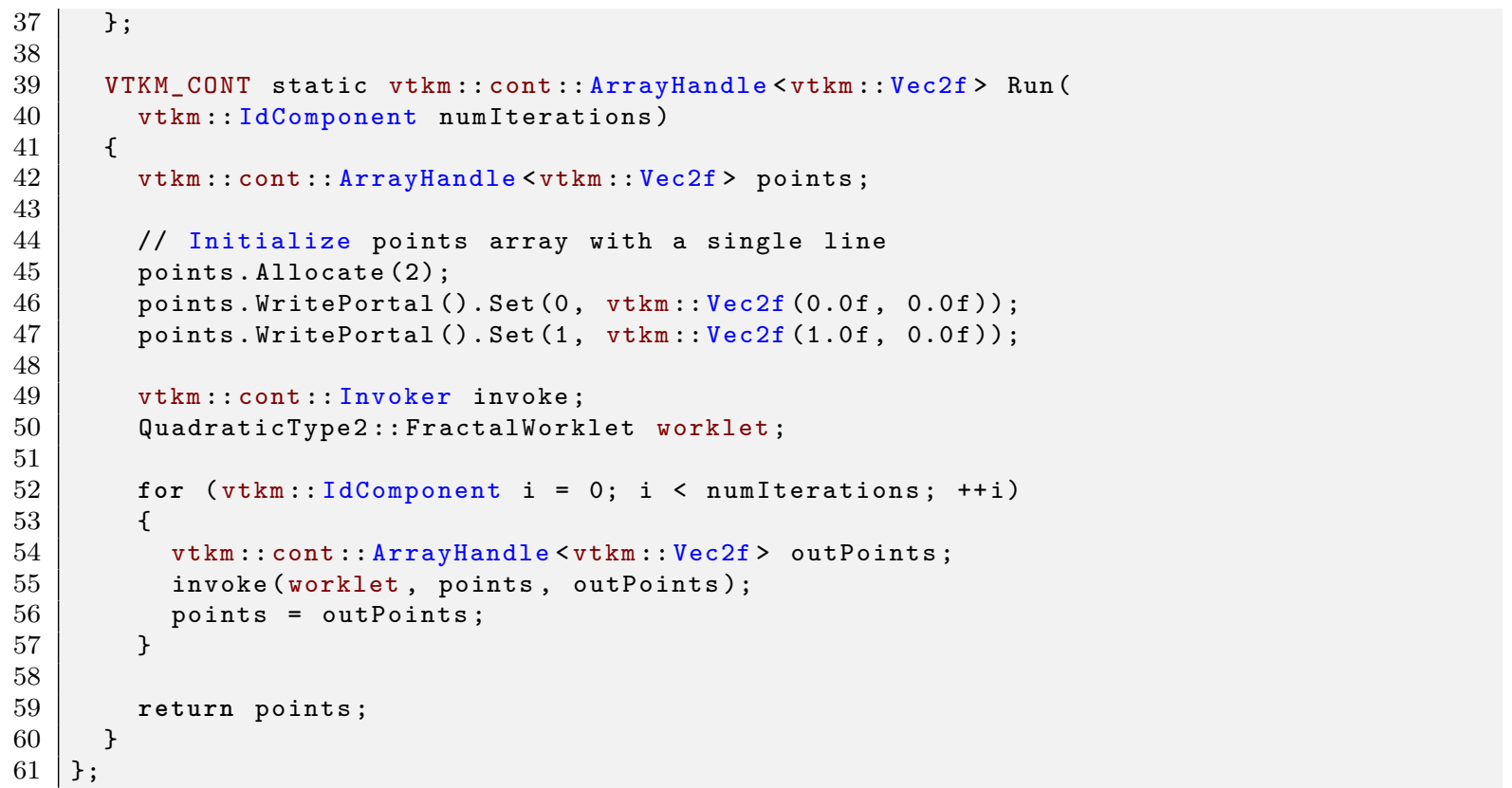

\subsubsection{Tree Fractal}

Another type of fractal we can make is a tree fractal. We will make a fractal similar to a Pythagoras tree except using lines instead of squares. Our fractal will start with a vertical line that will be replaced with the off-center "Y" shape shown in Figure 41.6. Iterative replacing using this "Y" shape produces a bushy tree shape.
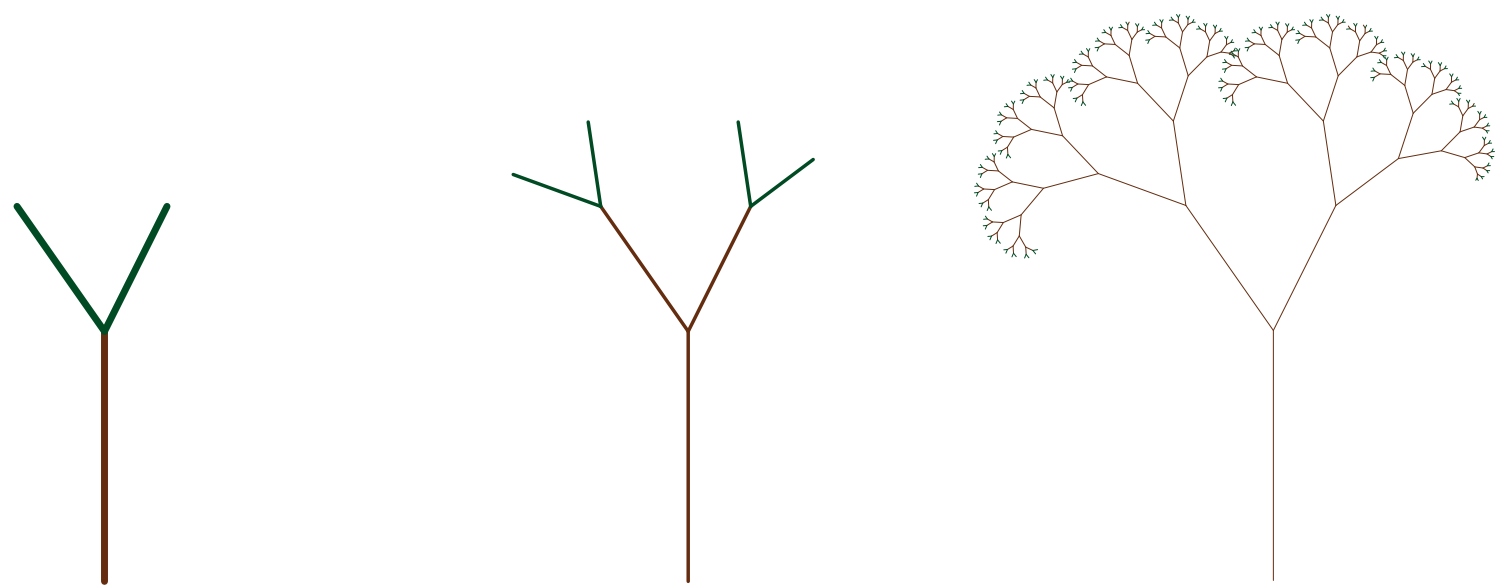

Figure 41.6: The tree fractal replaces each line with the "Y" shape shown at left. An iteration grows branches at the end (middle). After several iterations the tree branches out to the bushy shape at right.

One complication of implementing this tree fractal is that we really only want to apply the "Y" shape to the "leaves" of the tree. For example, once we apply the "Y" to the trunk, we do not want to apply it to the trunk again. If we were to apply it to the trunk again, we would create duplicates of the first layer of branches.

We can implement this feature in our worklet by using a count scatter. (Worklet scatters are described in Section 31.1.) Instead of directing the fractal worklet to generate 3 output line segments for every input line 
segment, we tell the fractal worklet to generate just 1 output line segment. We then use a scatter counting to generate 3 line segments for the leaves and 1 line segment for all other line segments. The count array for the initial iteration is initialized to a single 3 . Each iteration then creates the count array for the next iteration by writing a 1 for the base line segment and a 3 from the other two line segments.

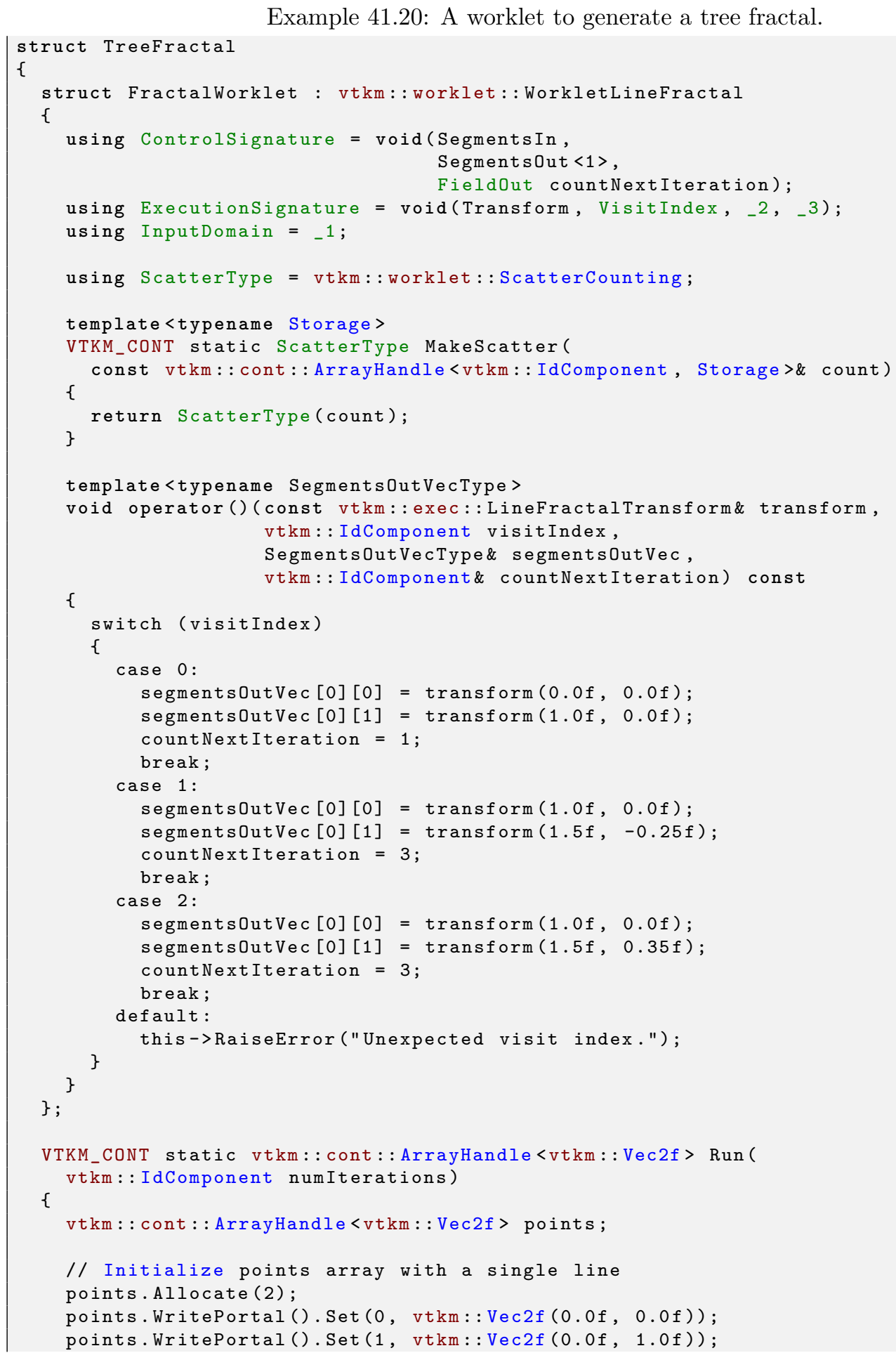




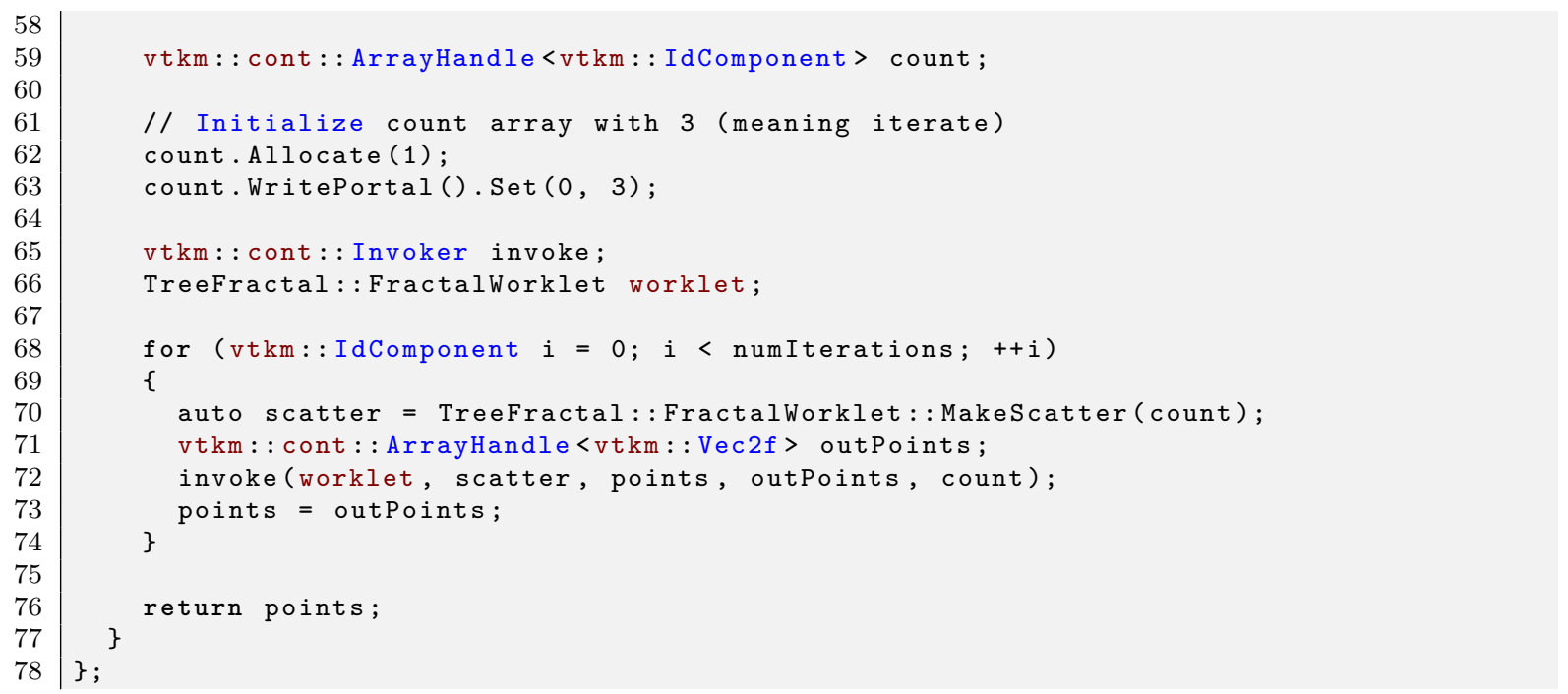

\subsubsection{Dragon Fractal}

The next fractal we will implement is known as the dragon fractal. The dragon fractal is also sometimes known as the Heighway dragon or the Harter-Heighway dragon after creators John Heighway, Bruce Banks, and William Harter. It is also sometimes colloquially referred to as the Jurassic Park dragon as the fractal was prominently featured in the Jurassic Park novel by Michael Crichton.

The basic building block is simple. Each line segment is replaced by two line segments bent at 90 degrees and attached to the original segments endpoints as shown in Figure 41.7. As you can see by the fourth iteration a more complicated pattern starts to emerge. Figure 41.8 shows the twelfth iteration a demonstrates a repeating spiral.
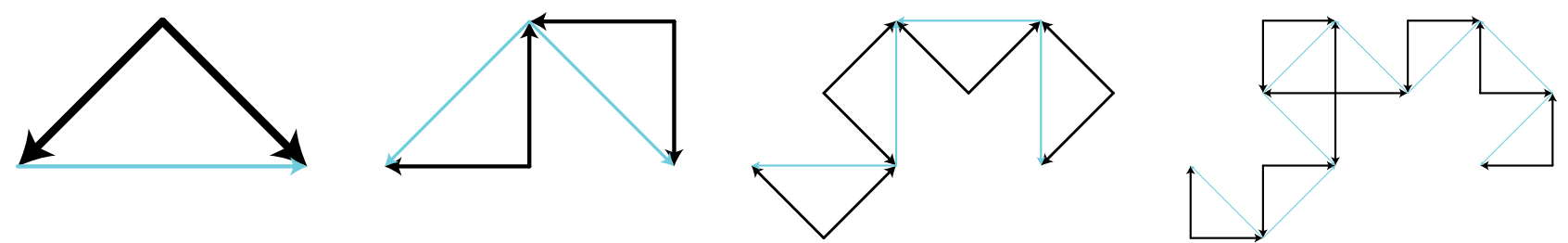

Figure 41.7: The first four iterations of the dragon fractal. The cyan lines give the previous iteration for reference.

What makes the dragon fractal different than the Koch Snowflake and similar fractals like the the quadratic curves implementation-wise is that the direction shape flips from one side to another. Note in the second image of Figure 41.7 the first bend is under the its associated line segment whereas the second is above its line segment. The easiest way for us to control the bend is to alternate the direction of the line segments. In Figure 41.7 each line segment has an arrowhead indicating the orientation of the first and second point with the arrowhead at the second point. Note that the shape is defined such that the first point of both line segments meet at the right angle. With the shape defined this way, each iteration is applied to put the bend to the left of the segment with respect to an observer at the first point looking at the second point.

Other than reversing the direction of half the line segments, the implementation of the dragon fractal is nearly identical to the Koch Snowflake. 


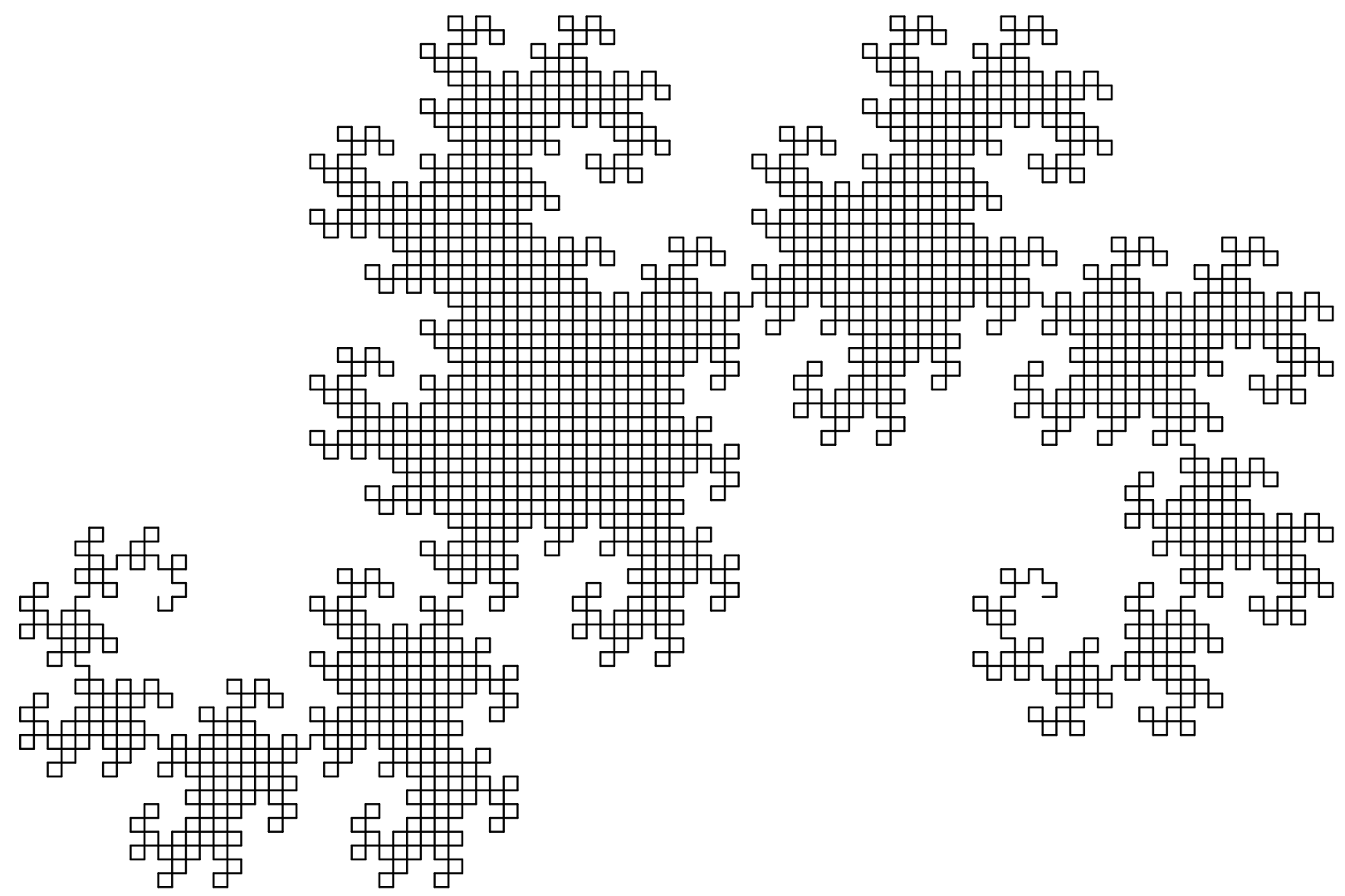

Figure 41.8: The dragon fractal after 12 iterations.

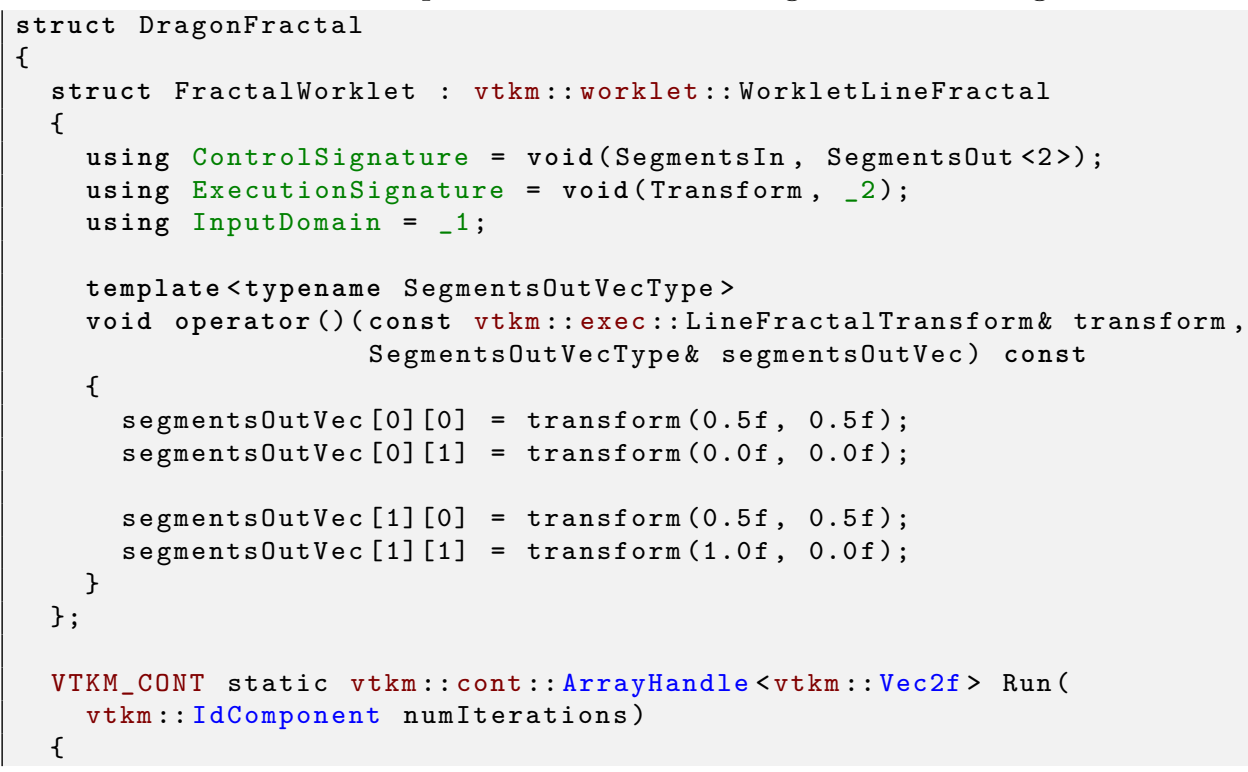




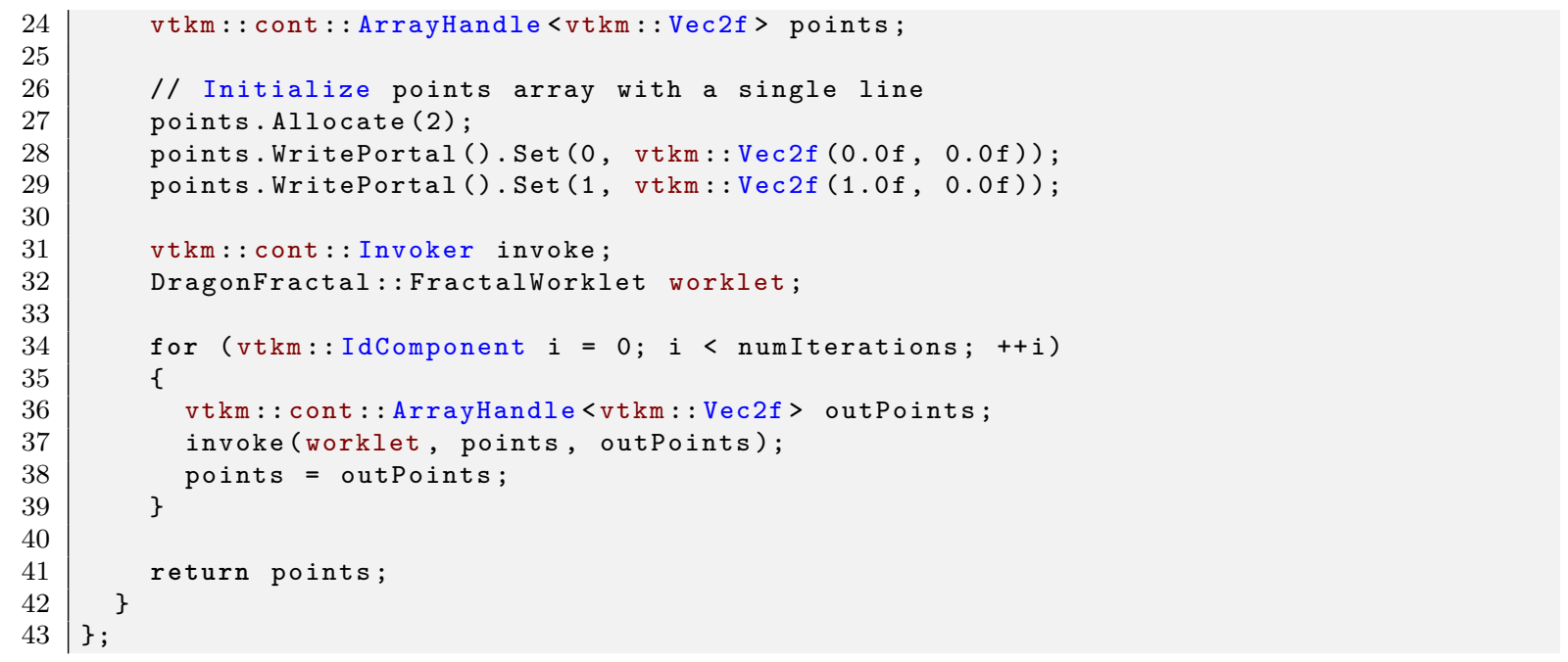

\subsubsection{Hilbert Curve}

For our final example we will look into using our fractal worklet to construct a space-filling curve. A space-filling curve is a type of fractal that defines a curve that, when iterated to its infinite length, completely fills a space. Space-filling curves have several practical uses by allowing you to order points in a 2 dimensional or higher space in a 1 dimensional array in such a way that points close in the higher dimensional space are usually close in the 1 dimensional ordering. For this fractal we will be generating the well-known Hilbert curve. (Specifically, we will be generating the 2D Hilbert curve.)

The 2D Hilbert curve fills in a rectangular region in space. (Our implementation will fill a unit square in the $[0,1]$ range, but a simple scaling can generalize it to any rectangle.) Without loss of generality, we will say that the curve starts in the lower left corner of the region and ends in the lower right corner. The Hilbert curve starts by snaking around the lower-left corner then into the upper-left followed by the upper-right and then lower-right. The curve is typically generated by recursively dividing and orienting these quadrants.

To generate the Hilbert curve in our worklet system, we will define our line segments as the connection from the lower left of (entrance to) the region to the lower right of (exit from) the region. The fractal generation breaks this line to a 4 segment curve that moves up, then right, then back down. Figure 41.9 demonstrates the Hilbert curve. (Readers familiar with the Hilbert curve might notice the shape is a bit different than other representations. Where many derivations derive the Hilbert curve by connecting the center of oriented boxes, our derivation uses a line segment along one edge of these boxes. The result is a more asymmetrical shape in early iterations, but the two approaches are equivalent as the iterations approach infinity.)

Like the dragon fractal, the Hilbert curve needs to flip the shape in different directions. For example, the first iteration, shown at left in Figure 41.9, is drawn to the "left" of the initial line along the horizontal axis. The next iteration, the second image in Figure 41.9, is created by drawing the shape to the "right" of the vertical line segments but to the left of the horizontal segments.

Section 41.6.3 solved this problem for the dragon fractal by flipping the direction of some of the line segments. Such an approach would work for the Hilbert curve, but it results in line segments being listed out of order and with inconsistent directions with respect to the curve. For the dragon fractal, the order and orientation of line segments is of little consequence. But for many applications of a space-filling curve the distance along the curve is the whole point, so we want the order of the line segments to be consistent with the curve.

To support this flipped shape while preserving the line segment order, we will use a data field attached to the 


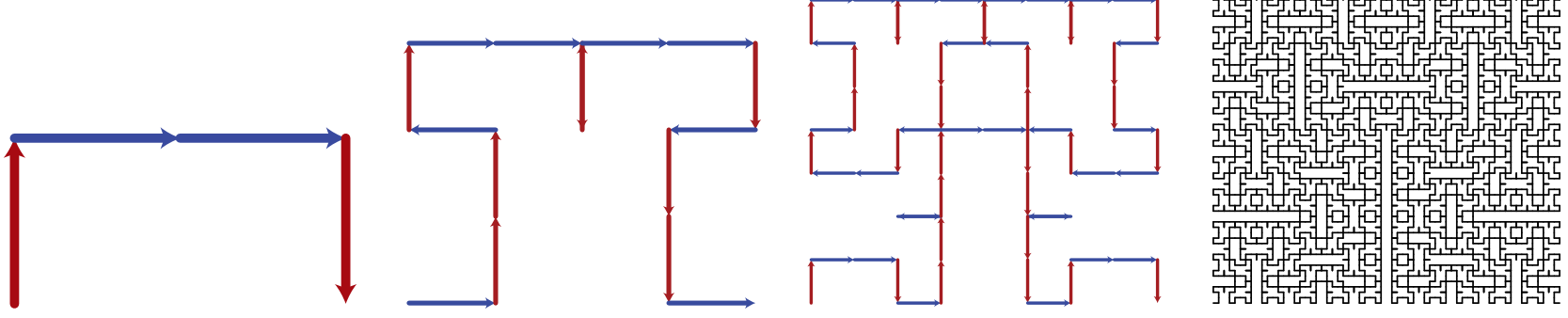

Figure 41.9: The first, second, third, and sixth iterations, respectively, of the Hilbert curve fractal.

line segments. That is, each line segment will have a value to represent which way to draw the shape. If the field value is set to 1 (represented by the blue line segments in Figure 41.9), then the shape is drawn to the "left." If the field value is set to -1 (represented by the red line segments in Figure 41.9), then the shape is inverted and drawn to the "right." This field is passed in and out of the worklet using the FieldIn and FieldOut tags.

Example 41.22: A worklet to generate the Hilbert curve.

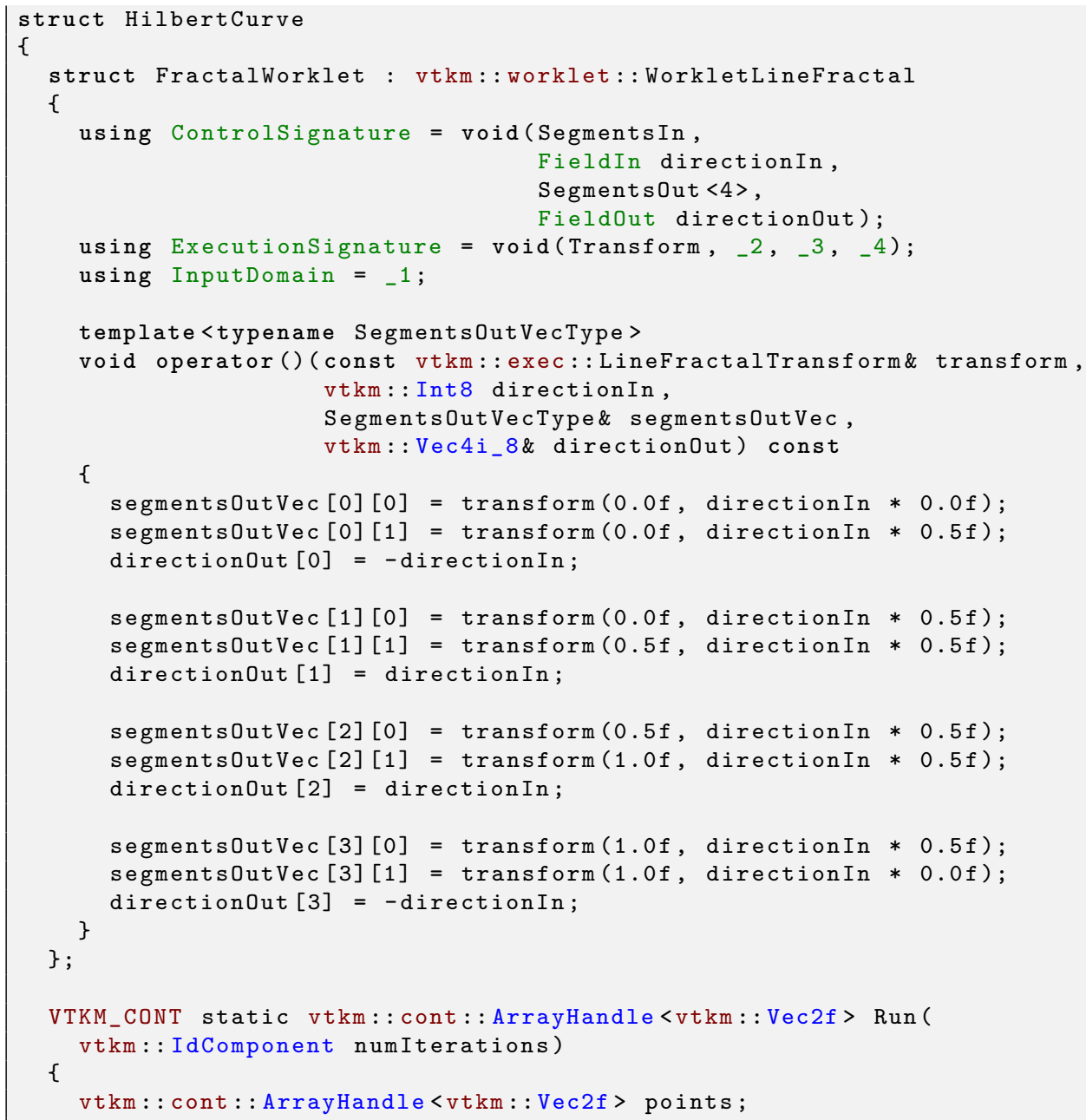




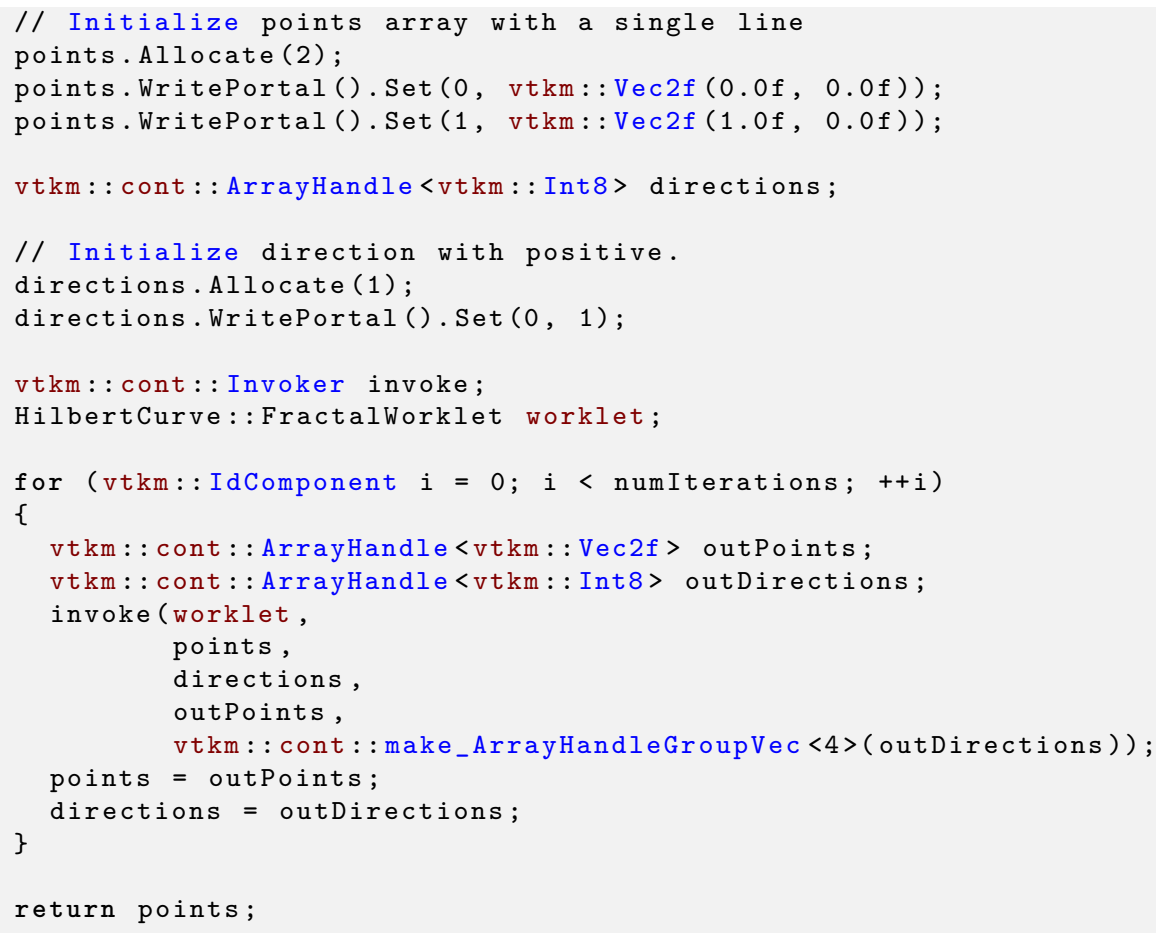





\section{Part VI}

Appendix 

$\pi, 199,200$

_1, 122, 123, 161, 164, 166, 169, 172, 176, 367

_2, 122, 123, 161, 164, 166, 169, 172, 176, 367

__device_-, 114

__host__, 114

Abs, 197

absolute value, 197

ACos, 197

ACosH, 197

Actor, 81, 93

actor, 17, 81

Add, 240

AddActor, 81

AddCell, 34

AddCellField, 35

AddHelp, 27

AddPoint, 34

AddPointField, 35

Algorithm, xxvii, 293, 303, 306

BitFieldToUnorderedSet, 293, 295

Copy, 293, 294

CopyIf, 294

CopySubRange, 295

CountSetBits, 295

Fill, 295

LowerBounds, 296

Reduce, 296, 297

ReduceByKey, 297

ScanExclusive, 298-300

ScanExclusiveByKey, 299

ScanExtended, 299, 300

ScanInclusive, 297-300

ScanInclusiveByKey, 298

Schedule, 300

Sort, 300, 301

SortByKey, 301

Transform, 301

Unique, 302

UpperBounds, 302

algorithm, 293-303, 346-350

bit field to unordered set, 293 copy, 294

copy if, 294

copy sub range, 295

count set bits, 295

fill, 295

lower bounds, 296

reduce, 296

reduce by key, 297

scan

exclusive, 298

exclusive by key, 299

inclusive, 297

inclusive by key, 298

scan extend, 299

schedule, 300

selecting device, 303

sort, 300

by key, 301

stream compact, 294

synchronize, 301

transform, 301

unique, 302

upper bounds, 302

Allocate, 115, 119, 232, 284, 293, 340

AppendPartition, 41

AppendPartitions, 41

Apply, 149, 150

ApplyPolicy, 186

ApplyPolicyCellSet, 193

ApplyPolicyCellSetStructured, 194

ApplyPolicyCellSetUnstructured, 194

ApplyPolicyFieldOfType, 193

arccosine, 197

arcsine, 197

arctangent, 197

AREA, 64

arg namespace, 357-359, 361, 363-365

argc, 27

argv, 27

ARITY, 354

array handle, 115-119, 229-235, 307-330

adapting, 323-330 
allocate, 232

Cartesian product, 222-223

cast, 218-219

composite vector arrays, 223-224

const, 119

constant, 215-216

counting, 217-218

deep copy, 118

derived, 316-323

discard, 219

execution environment, 233-235

extract component, 224-225

fancy, 215-227, 323

group vector, 225-227

implicit, 308-310

index, 217

maximum, 233

minimum, 233

permutation, 219-221

populate, 232

portal, 229-232

random, 216-217

range, 233

rectilinear point coordinates, 222-223

storage, 118-119, 307-330

default, 307, 330

subclassing, 309, 322, 329

swizzle, 225

transform, 310-312

uniform point coordinates, 221-222

unknown, 283-292

view, 216

zip, 221

array of structures, 308

array portal, 229-232

ArrayCopy, 118, 287

ArrayCopy.h, 118

ArrayHandle, xxi, xxvii, 90, 115-118, 126, 182, 186, 189, 190, 193, 229, 232, 233, 307, 308, 322, 323, 329, 358-361

Allocate, 115, 119, 232, 284, 293

array of structures, 308

DeepCopyFrom, 116, 118

GetNumberOfValues, 115

IsOnDevice, 116

IsOnHost, 116

PrepareForInPlace, 116, 234, 360, 361

PrepareForInput, 116, 234, 245, 359, 360

PrepareForOutput, 116, 119, 234, 360, 361

ReadPortal, 116, 231, 232

ReadPortalType, 231, 234

ReleaseResources, 115, 119

ReleaseResourcesExecution, 115

StorageTag, 310, 312, 316, 322

structure of arrays, 308
Superclass, 310, 312, 316, 322

SyncControlArray, 116, 231, 330

ValueType, 224, 225, 310, 312, 316, 322

WritePortal, 116, 119, 231, 232

WritePortalType, 231, 234

ArrayHandleBase, 90

GetReadPointer, 90

ArrayHandleBasic, 308

ArrayHandleCartesianProduct, 222

ArrayHandleCast, 218

ArrayHandleCast.h, 218

ArrayHandleCompositeVector, 223, 323

ArrayHandleCompositeVector.h, 224

ArrayHandleConstant, 215

ArrayHandleConstant.h, 216

ArrayHandleCounting, 217, 258, 309

ArrayHandleCounting.h, 218

ArrayHandleDecorator, 312, 315

ArrayHandleDiscard, 219

ArrayHandleExtractComponent, 224

ArrayHandleExtractComponent.h, 224

ArrayHandleGroupVec, 225, 263, 268, 274, 357, 361

ArrayHandleGroupVec.h, 226

ArrayHandleGroupVecVariable, 226, 278, 300

ArrayHandleGroupVecVariable.h, 227

ArrayHandleImplicit, 308, 309

ArrayHandleImplicit.h, 309

ArrayHandleIndex, 217

ArrayHandlePermutation, 219, 264

ArrayHandlePermutation.h, 220

ArrayHandleRecombineVec, 290

ArrayHandleSOA, 308

ArrayHandleSwizzle, 225

ArrayHandleSwizzle.h, 225

ArrayHandleTransform, 310, 311

ArrayHandleUniformPointCoordinates, 221

ArrayHandleView, 216

ArrayHandleView.h, 216

ArrayHandleZip, 221

ArrayHandleZip.h, 221

ArrayPortal

Get, 229, 237

GetNumberOfValues, 229

Set, 229, 237, 319, 327

ValueType, 229

ArrayPortalFromIterators, 229

ArrayPortalToIteratorBegin, 231

ArrayPortalToIteratorEnd, 231

ArrayPortalToIterators, 230

GetBegin, 230

GetEnd, 230

IteratorType, 230

ArrayPortalToIterators.h, 231

ArrayRangeCompute, 233

ArrayRangeCompute.h, 233 
AsArrayHandle, 285, 287

AsField, 188, 192

ASin, 197

ASinH, 197

aspect, 363-367

cell shape, 365

default, 363, 364, 367

incident element count, 365

incident indices, 365

input index, 365

output index, 365

value count, 365

visit index, 365

work index, 365

ASPECT_GAMMA, 64

ASPECT_RATIO, 64

AspectTag, 367

AspectTagCellShape, 365

AspectTagDefault, 363, 364, 367

AspectTagIncidentElementCount, 363, 365

AspectTagIncidentElementIndices, 363, 365

AspectTagInputIndex, 365

AspectTagOutputIndex, 365

AspectTagValueCount, 365

AspectTagVisitIndex, 365

AspectTagWorkIndex, 365

assert, 96-97, 195 static, 96-97

Assert.h, 96

Association::ANY, 77

Association::CELL_SET, 78

Association::POINTS, 78

Association::WHOLE_MESH, 78

AssociationEnum, 79

ATan, 197

ATan2, 197

ATanH, 198

atomic array, 239-241

AtomicArray, 160, 164, 166, 169, 171, 176, 360

AtomicArrayExecutionObject, 240

Add, 240

CompareAndSwap, 240

AtomicArrayInOut, xxv, 160, 163, 166, 169, 171, 176, 240

average, 48, 66-67

AverageByKey, 269

Azimuth, 88

azimuth, 88

background color, 83

bit field to unordered set, 293

BitField, 358, 360

PrepareForInPlace, 360

PrepareForInput, 360

BitFieldToUnorderedSet, 293, 295

BitwiseAnd, 305

BitwiseOr, 305
BitwiseXor, 305

Boundary, 172

BoundaryState, 172

InBoundary, 173

InXBoundary, 173

InYBoundary, 173

InZBoundary, 173

MaxNeighborIndices, 173

MinNeighborIndices, 172, 173

Bounds, xxii, 41, 42, 85, 107, 135, 136

Center, 136

Contains, 136

Include, 136

IsNonEmpty, 136

Union, 136

$\mathrm{X}, 135,136$

$\mathrm{Y}, 135,136$

Z, 135, 136

BoundsCompute, 42

BoundsGlobalCompute, 42

Box, 107

SetBounds, 107

SetMaxPoint, 107

SetMinPoint, 107

box, 107

Buffer, 319, 324, 327, 329

DeepCopyFrom, 325

GetMetaData, 325

GetNumberOfBytes, 324

HasMetaData, 325

IsAllocatedOnDevice, 324

IsAllocatedOnHost, 324

MetaDataIsType, 325

ReadPointerDevice, 325

ReadPointerHost, 324, 325

ReleaseDeviceResources, 325

SetMetaData, 325

SetNumberOfBytes, 324

WritePointerDevice, 325

WritePointerHost, 325

BufferInfo, 339, 341

BufferSizeType, 324

BUILD_SHARED_LIBS, 9

Camera, xx, 85, 87-89, 91

Azimuth, 88

Dolly, 89

Elevation, 88

Pan, 85, 89, 92

ResetToBounds, 89, 90

Roll, 89

SetClippingRange, 87

SetFieldOfView, 87

SetLookAt, 87

SetModeTo2D, 85

SetModeTo3D, 85 
SetPosition, 86

SetViewRange2D, 85

SetViewUp, 87

TrackballRotate, 91

Zoom, 86, 89, 92

camera, $85-89$

2D, 85-86

3D, 86-89

azimuth, 88

clipping range, 87

elevation, 88

far clip plane, 87

field of view, 87

focal point, 86

interactive, 91-93

look at, 86

mouse, 91-93

near clip plane, 87

pan, 85-86, 89

pinhole, 86

position, 86

reset, 89-90

up, 87

view range, 85

view up, 87

zoom, 86,89

CanConvert, 285, 286

CanRunOn, 101

Canvas, 82

GetColorBuffer, 90

canvas, 17,82

ray tracer, 82

CanvasRayTracer, 82, 83

Cartesian product array handle, 222-223

Cast, 154

cast array handle, 218-219

CastAndCall, 288, 291

CastAndCallForTypes, 287, 288

CastAndCallWithExtractedArray, 291

Cbrt, 198

Ceil, 198

ceiling, 198

Cell, 241

cell, 205-214

derivative, 209-210

edge, 36, 210-212

face, 36, 210, 212-214

gradient, 209-210

interpolation, 209

parametric coordinates, 208-209

point, 36, 210

shape, 210

world coordinates, 208-209

cell average, 48

cell gradients, 60-61 cell locator, 249-252

bounding interval hierarchy, 250

chooser, 250

general, 250

rectilinear grid, 250

two level, 250

uniform grid, 250

cell set, 29, 36

explicit, 37-38

generate, 261-281

permutation, 38-39

shape, 36

single type, 37-38

structured, 36

whole, 241-244

cell shape, 36, 205-208

cell traits, 207-208

CELL_SHAPE_EMPTY, 205

CELL_SHAPE_HEXAHEDRON, 32, 38, 206

CELL_SHAPE_LINE, 32, 38, 206

CELL_SHAPE_POLY_LINE, 32, 38, 206

CELL_SHAPE_POLYGON, 32, 38, 206

CELL_SHAPE_PYRAMID, 32, 38, 206

CELL_SHAPE_QUAD, 32, 38, 206

CELL_SHAPE_TETRA, 32, 38, 206

CELL_SHAPE_TRIANGLE, 32, 38, 206

CELL_SHAPE_VERTEX, 32, 38, 206

CELL_SHAPE_WEDGE, 32, 38, 206

CellAverage, 48

CellClassification, 60

GHOST, 60

GHOST., 60

INVALID, 60

NORMAL, 60

CellCount, 166

CellDerivative, 209

CellDerivative.h, 209

CellEdge.h, 211, 266

CellEdgeCanonicalId, 211, 266, 270

CellEdgeLocalIndex, 211

CellEdgeNumberOfEdges, 211

CellFace.h, 212

CellFaceCanonicalId, 212

CellFaceLocalIndex, 212

CellFaceNumberOfFaces, 212

CellFaceNumberOfPoints, 212

CellIndices, 167

CellInterpolate, 209

CellInterpolate.h, 209

CellLocator, 251

FindCell, 251

GetCellSet, 250

GetCoordinates, 250

SetCellSet, 250

SetCoordinates, 250 
Update, 250

CellLocatorBoundingIntervalHierarchy, 250

SetMaxLeafSize, 250

SetNumberOfPlanes, 250

CellLocatorChooser, 250

CellLocatorGeneral, 250

SetConfigurator, 250

CellLocatorRectilinearGrid, 250

CellLocatorTwoLevel, 250

SetDensityL1, 250

SetDensityL2, 250

CellLocatorUniformGrid, 250

CellMetric, 63

AREA, 64

ASPECT_GAMMA, 64

ASPECT_RATIO, 64

CONDITION, 64

DIAGONAL_RATIO, 64

DIMENSION, 64

JACOBIAN, 64

MAX_ANGLE, 64

MAX_DIAGONAL, 64

MIN_ANGLE, 64

MIN_DIAGONAL, 64

ODDY, 64

RELATIVE_SIZE_SQUARED, 64

SCALED_JACOBIAN, 64

SHAPE, 65

SHAPE_AND_SIZE, 65

SHEAR, 65

SKEW, 65

STRETCH, 65

TAPER, 65

VOLUME, 65

WARPAGE, 65

CellNotFound, 151, 251

CellSet, 36, 161, 164, 166, 169, 171, 176, 184, 193, 245, 261, 358,360

CellSetConnectivity, 52

CellSetExplicit, 37, 40, 241, 277, 300 generate, $277-281$

CellSetExtrude, 39

CellSetIn, 123, 163, 165, 168, 170

CellSetPermutation, 38, 39

CellSetSingleType, 37, 261, 263, 267, 273 generate, $261-277$

CellSetStructured, 36, 170, 241, 358

CellShape, 164, 170

CellShape.h, 205

CellShapeIdToTag, 205

Tag, 206

CellShapeTag, 207, 242

CellShapeTagEmpty, 205

CellShapeTagGeneric, 205, 242

Id, 205
CellShapeTagHexahedron, 32, 38, 206

CellShapeTagLine, 32, 38, 206

CellShapeTagPolygon, 32, 38, 206

CellShapeTagPolyLine, 32, 38, 206

CellShapeTagPyramid, 32, 38, 206

CellShapeTagQuad, 32, 38, 206

CellShapeTagTetra, 32, 38, 206

CellShapeTagTriangle, 32, 38, 206

CellShapeTagVertex, 32, 38, 206

CellShapeTagWedge, 32, 38, 206

CellTopologicalDimensionsTag, 207

CellTraits, 207

IsSizeFixed, 207

NUM_POINTS, 207

TOPOLOGICAL_DIMENSIONS, 207

TopologicalDimensionsTag, 207

CellTraits.h, 207

CellTraitsTagSizeFixed, 207

CellTraitsTagSizeVariable, 207

Center, 135, 136

classify ghost cells, 60

clean grid, 49-50

CleanGrid, 49

GetCompactPointFields, 49

GetFastMerge, 49

GetMergePoints, 49

GetRemoveDegenerateCells, 49

GetTolerance, 49

GetToleranceIsAbsolute, 49

SetCompactPointFields, 49

SetFastMerge, 49

SetMergePoints, 49

SetRemoveDegenerateCells, 49

SetTolerance, 49

SetToleranceIsAbsolute, 49

clip

field, 50-51

implicit function, 51-52

clipping range, 87

ClipWithField, 50

GetClipValue, 50

SetClipValue, 50

SetInvertClip, 50

ClipWithImplicitFunction, 51, 105, 107

GetImplicitFunction, 51

SetImplicitFunction, 51

SetInvertClip, 51

CMake, 7-9, 11-13, 25

configuration, 7-9

BUILD_SHARED_LIBS, 9

CMAKE_BUILD_TYPE, 9, 10

CMAKE_INSTALL_PREFIX, 9

CMAKE_PREFIX_PATH, 11

VTKm_CUDA_Architecture, 9

VTKm_DIR, 11 
VTKm_ENABLE_BENCHMARKS, 9

VTKm_ENABLE_CUDA, 9

VTKm_ENABLE_EXAMPLES, 9

VTKm_ENABLE_LOGGING, 9, 153

VTKm_ENABLE_OPENMP, 9

VTKm_ENABLE_RENDERING, 9, 12

VTKm_ENABLE_TBB, 9

VTKm_ENABLE_TESTING, 9

VTKm_USE_64BIT_IDS, 9, 22

VTKM_USE_DOUBLE_PRECISION, 21

VTKm_USE_DOUBLE_PRECISION, 9, 22

VTKm_VERSION, 25

VTKm_VERSION_FULL, 25

VTKm_VERSION_MAJOR, 25

VTKm_VERSION_MINOR, 25

VTKm_VERSION_PATCH, 25

version, 25

VTK-m library

vtkm_cont, 12

vtkm_filter, 12

vtkm_io, 12

vtkm_rendering, 12

vtkm_source, 12

VTK-m package, 11-13

libraries, 11-12

variables, $12-13$

version, 25

VTKm_ENABLE_CUDA, 12

VTKm_ENABLE_MPI, 12

VTKm_ENABLE_OPENMP, 12

VTKm_ENABLE_RENDERING, 12

VTKm_ENABLE_TBB, 12

VTKm_FOUND, 12

VTKm_VERSION, 12

VTKm_VERSION_FULL, 12

VTKm_VERSION_MAJOR, 12

VTKm_VERSION_MINOR, 12

VTKm_VERSION_PATCH, 12

CMAKE_BUILD_TYPE, 9,10

CMAKE_INSTALL_PREFIX, 9

CMAKE_PREFIX_PATH, 11

CMakeLists.txt, 18

coding conventions, 112

Color, 83

color

background, 83

foreground, 83

color tables, 93-94

default, 93

ColorTable, 58, 93

column, 202

CompareAndSwap, 240

COMPONENT, 58, 59

ComponentType, 138

composite vector array handle, 223-224 compression

zfp, 76-77

ComputePointGradient, 61

CONDITION, 64

connected components, 52-53

cell, 52

field, 52-53

image, 52-53

Connectivity

CellShapeTag, 242

GetCellShape, 242

GetIndices, 242

GetNumberOfElements, 242

GetNumberOfIndices, 242

GetNumberOfIndicices, 242

IndicesType, 242

constant array handle, 215-216

cont namespace, 112, 113

Contains, 135, 136

Contour, 54

GetComputeFastNormalsForStructured, 55

GetComputeFastNormalsForUnstructured, 55

GetGenerateNormals, 55

GetIsoValue, 54

GetMergeDuplicatePoints, 55

GetNormalArrayName, 55

SetComputeFastNormalsForStructured, 55

SetComputeFastNormalsForUnstructured, 55

SetGenerateNormals, 55

SetIsoValue, 54

SetMergeDuplicatePoints, 55

SetNormalArrayName, 55

contour, 54-55

control environment, 111, 112

control signature, xiv, xxi, xxviii, xxix, 121-122, 124, 160, $161,163-166,168-170,172,175,176,178,237$, $240,241,245,253,353,357,366,367,374-377$, 381

atomic array, 239-241

execution object, $245-247$

tags, 366

whole array, 237-239

whole cell set, 241-244

ControlSignatureTagBase, 366

FetchTag, 366

TransportTag, 366

TypeCheckTag, 366

ConvertNumComponentsToOffsets, 37, 226, 278

coordinate system, 29, 41

coordinate system transform, 53-54

cylindrical, 53-54

spherical, 54

CoordinateSystem, 41, 249

GetBounds, 41

Copy, 293, 294 
copy, 294

copy if, 294

copy sub range, 295

CopyDeviceToDevice, 340

CopyDeviceToHost, 340

CopyFlag, 117, 118, 290, 314, 319, 324, 327

Off, 117,290

On, 117, 118, 232

CopyHostToDevice, 340

CopyIf, 294

CopyInto, 133, 139

CopyShallowIfPossible, 286

CopySign, 198

CopySubRange, 295

Cos, 198

CosH, 198

cosine, 198

count set bits, 295

counting array handle, 217-218

CountSetBits, 295

Create, 30, 32

CreateBuffers, 319

CreateFromPoints, 107

CreateReadPortal, 319, 327

CreateResult, 127, 183

CreateResult.h, 183

CreateResultFieldCell, 183

CreateResultFieldPoint, 183

CreateWritePortal, 319, 327

Cross, 200

cross product, 55-56, 200

CrossProduct, 55

GetOutputFieldName, 56

GetPrimaryCoordinateSystemIndex, 56

GetPrimaryFieldName, 56

GetSecondaryCoordinateSystemIndex, 56

GetSecondaryFieldName, 56

GetUseCoordinateSystemAsPrimaryField, 56

GetUseCoordinateSystemAsSecondaryField, 56

SetOutputFieldName, 56

SetPrimaryCoordinateSystem, 56

SetPrimaryField, 56

SetSecondaryCoordinateSystem, 56

SetSecondaryField, 56

SetUseCoordinateSystemAsPrimaryField, 56

SetUseCoordinateSystemAsSecondaryField, 56

ctest, 331-332

cube root, 198

CUDA, 9, 99, 114

cuda namespace, 113

Cylinder, 106

SetAxis, 106

SetCenter, 106

SetRadius, 106

cylinder, 106 cylindrical coordinate system transform, 53-54

CylindricalCoordinateSystemTransform, 53

SetCartesianToCylindrical, 53

SetCartesianToSpherical, 54

SetCylindricalToCartesian, 53

SetSphericalToCartesian, 54

data set, 29-42

building, 29-36

cell set, see cell set

clean, 49-50

coordinate system, see coordinate system

field, see field

generate, 261-281

partitioned, see partitioned data set

data set filter, 186-189

data set with field filter, 189-192

DataSet, 16, 29-31, 41, 43-45, 47, 48, 78, 81, 113, 115, 127, 182-186, 189, 190, 192, 249, 252, 261

AddCellField, 35

AddPointField, 35

GetCellSet, 184, 186, 193

DataSet.h, 113

DataSetBuilderExplicit, 32

Create, 32

DataSetBuilderExplicitIterative, 34

AddCell, 34

AddPoint, 34

DataSetBuilderRectilinear, 30

Create, 30

DataSetBuilderUniform, 30

Create, 30

Debug, 9

decompression

zfp, 77

deep array copy, 118

DeepCopyFrom, 116, 118, 286, 325

DefaultAnyDevice, 27, 28

DegenerateCellDetected, 151

derivative, 209-210

derived storage, 316-323

detail namespace, 113

determinant, 202

Device, 28

device adapter, 99, 337-351

algorithm, 293-303, 346-350

bit field to unordered set, 293

copy, 294

copy if, 294

copy sub range, 295

count set bits, 295

fill, 295

lower bounds, 296

reduce, 296

reduce by key, 297

scan extend, 299 
schedule, 300

sort, 300

stream compact, 294

synchronize, 301

transform, 301

unique, 302

upper bounds, 302

any, 100

id, 100-101

provided, 100

implementing, 337-351

memory manager, 339-341

runtime detector, 338-339

runtime device configuration, 341-344

runtime tracker, 101-102

getting, 101

scoped, 102

tag, 99-100, 337-338

provided, 99-100

timer, 350-351

try execute, 335-336

undefined, 100

virtual object transfer, 344-346

DeviceAdapterAlgorithm, 346

DeviceAdapterAlgorithmGeneral, 346

DeviceAdapterCuda.h, 99

DeviceAdapterId, 28, 100-102, 303, 319, 325, 327, 340, 341

GetId, 100

GetName, 100

Is ValueValid, 100, 101

DeviceAdapterKokkos.h, 99

DeviceAdapterList.h, 338

DeviceAdapterListCommon, 338

DeviceAdapterMemoryManager, 339, 341

Allocate, 340

CopyDeviceToDevice, 340

CopyDeviceToHost, 340

CopyHostToDevice, 340

GetDevice, 340

DeviceAdapterMemoryManagerBase, 339

ManageArray, 339

DeviceAdapterMemoryManagerShared, 340

DeviceAdapterNameType, 100

DeviceAdapterOpenMP.h, 99

DeviceAdapterRuntimeDetector, 338

Exists, 339, 344

h, 344

DeviceAdapterSerial.h, 99

DeviceAdapterTag, 101

DeviceAdapterTag.h, 338

DeviceAdapterTagAny, 28, 100, 104, 324

DeviceAdapterTagCuda, 99

DeviceAdapterTagKokkos, 99

DeviceAdapterTagOpenMP, 99

DeviceAdapterTagSerial, 99
DeviceAdapterTagTBB, 99

DeviceAdapterTagUndefined, 28, 100

DeviceAdapterTagUnknown, 324, 325

DeviceAdapterTBB.h, 99

DeviceAdapterTimerImplementation, 350

DIAGONAL_RATIO, 64

DIMENSION, 64

DimensionalityTag, 137

Disable, 102

DisableDevice, 101

discard array handle, 219

Dispatcher, 377

dispatcher, 379-383

creating new, 380-383

invocation object, 381

DispatcherBase, 380

Invoke, 379

DispatcherMapField, 380

DispatcherMapTopology, 380

DispatcherPointNeighborhood, 380

DispatcherReduceByKey, 380

DoExecute, xxiii, 126, 127, 181-183, 185, 186, 188-193, 263, $264,268,269,274,275,279,281$

Dolly, 89

DoMapField, xxiii, 186, 188-190, 192, 264, 269

Dot, 132

dot product, 56-57

DotProduct, 56

GetOutputFieldName, 57

GetPrimaryCoordinateSystemIndex, 56

GetPrimaryFieldName, 56

GetSecondaryCoordinateSystemIndex, 57

GetSecondaryFieldName, 56

GetUseCoordinateSystemAsPrimaryField, 56

GetUseCoordinateSystemAsSecondaryField, 56

SetOutputFieldName, 57

SetPrimaryCoordinateSystem, 56

SetPrimaryField, 56

SetSecondaryCoordinateSystem, 57

SetSecondaryField, 56

SetUseCoordinateSystemAsPrimaryField, 56

SetUseCoordinateSystemAsSecondaryField, 56

dragon fractal, 387-389

DynamicCellSet, 193

edge, 36, 210-212

Elevation, 88

elevation, 67, 88

Enable, 102

environment, 111, 112

control, 111, 112

execution, 111, 112

Epsilon, 198

Equal, 304

Error, 95, 154

GetMessage, 95 
error codes, 150-151

ErrorBadAllocation, 95, 101

ErrorBadDevice, 102

ErrorBadType, 95, 289

ErrorBadValue, 95, 101

ErrorCode, 150, 208, 209, 211, 212

CellNotFound, 151, 251

DegenerateCellDetected, 151

InvalidEdgeId, 150

InvalidFaceId, 150

InvalidNumberOfPoints, 150

InvalidPointId, 150

InvalidShapeId, 150

MalformedCellDetected, 151

MatrixFactorizationFailed, 150

OperationOnEmptyCell, 151

SolutionDidNotConverge, 150

Success, 150, 151, 251

ErrorExecution, 95, 195, 300, 346

ErrorInternal, 96

ErrorIO, 96

ErrorMessageBuffer, 346

ErrorOnBadArgument, 27

ErrorOnBadOption, 27

errors, 95-97, 195

assert, 96-97, 195

control environment, 95-96

execution environment, 95, 195, 300

worklet, 195

ErrorString, 151

exec namespace, 112, 113, 251, 253, 370

ExecObject, xxv, 161, 162, 164, 166, 169, 171, 176, 245, 249-251, 253

ExecObjectType, 359, 361

Execute, 16, 42, 47, 53, 54, 71, 73, 75, 76, 182, 186, 189, 190, 192

execution environment, 111,112

execution object, 245-247

execution signature, xiv, xxi, xxviii, xxix, 121-123, 161, 164, $166,169,171,172,176,256,357,366,367,374-$ 376,381

tags, 366-367

ExecutionObjectBase, 161, 164, 166, 169, 171, 176, 245, $250,253,358$

PrepareForExecution, 161, 164, 166, 169, 171, 176, 245

PrepareForInput, 359

ExecutionSignatureTagBase, 367

AspectTag, 367

INDEX, 367

Exists, 339, 344

Exp, 198

Exp10, 198

Exp2, 198

explicit cell set, $37-38$

single type, $37-38$ explicit mesh, 31

connectivity, 31

offsets, 31

shapes, 31

ExpM1, 198

exponential, 198

external faces, 57

ExternalFaces, 57

GetCompactPoints, 57

GetPassPolyData, 57

SetCompactPoints, 57

SetPassPolyData, 57

extract, 57-58

extract component array handle, 224-225

extract structured, 57-58

ExtractArrayFromComponents, 290, 291

ExtractComponent, 289, 290

ExtractStructured, 57

GetIncludeBoundary, 58

GetSampleRate, 58

GetVOI, 58

SetIncludeBoundary, 58

SetSampleRate, 58

SetVOI, 58

face, $36,210,212-214$

external, 57

false_type, 97

fancy array handle, 215-227, 323

far clip plane, 87

Fatal, 154

Fetch, 362-365, 367, 373, 375

Load, 363, 364, 367

Store, 363, 364, 367

fetch, 362-366

$$
\text { aspect, see aspect }
$$

cell set, 363

direct input array, 363

direct output array, 363

execution object, 363

topology map array input, 363

whole cell set, 363

FetchTag, 366

FetchTagArrayDirectIn, 363

FetchTagArrayDirectOut, 363

FetchTagArrayTopologyMapIn, 363

FetchTagCellSetIn, 363

FetchTagExecObject, 363

FetchTagWholeCellSetIn, 363

Field, 40-42, 77-79, 188, 192, 193

Association::ANY, 77

Association::CELL_SET, 78

Association::POINTS, 78

Association::WHOLE_MESH, 78

AssociationEnum, 79

GetRange, 40, 42 
field, 29, 40, 48

field filter, 181-186 using cells, 184-186

field map worklet, 159-162

field of view, 87

field to colors, 58-59

FieldIn, 122, 123, 160, 170, 171, 377

FieldInCell, 163, 165

FieldInIncident, 168

FieldInNeighborhood, 171, 172

FieldInOut, 160, 163, 166, 169, 171

FieldInOutCell, 163

FieldInOutPoint, 166

FieldInPoint, 163, 165

FieldInVisit, 168

FieldMetaData

AsField, 192

IsCellField, 192

IsPointField, 192

FieldMetadata, 182, 183, 186, 188-190, 192

AsField, 188

IsCellField, 188

IsPointField, 188

FieldNeighborhood, 171, 172

Get, 172

FieldOut, 122, 160, 163, 166, 169, 171, 377

FieldOutCell, 163

FieldOutPoint, 166

FieldPointIn, 122, 209, 210

FieldRangeCompute, 42

FieldRangeGlobalCompute, 42

FieldSelection, xx, 78, 79

MODE_EXCLUDE, 79

MODE_NONE, 78

FieldToColors, 58, 59

COMPONENT, 58, 59

FieldToColors, 59

GetColorTable, 58

GetMappingComponent, 59

GetMappingMode, 58

GetNumberOfSamplingPoints, 59

GetOutputMode, 59

IsMappingComponent, 59

IsMappingMagnitude, 59

IsMappingScalar, 59

IsOutputRGB, 59

IsOutputRGBA, 59

MAGNITUDE, 58, 59

RGB, 58, 59

RGBA, 58, 59

SCALAR, 58

SetColorTable, 58

SetMappingComponent, 59

SetMappingMode, 58, 59

SetMappingToComponent, 59
SetMappingToMagnitude, 59

SetMappingToScalar, 58

SetNumberOfSamplingPoints, 59

SetOutputMode, 59

SetOutputToRGB, 59

SetOutputToRGBA, 59

file I/O, 43-46

read, 15-16, 43-44

write, 44-45

Fill, 295

fill, 295

Filter, 78

DoExecute, 126, 127, 181-183, 186, 192, 193

Execute, 42, 47, 192

Invoke, 127, 186

SetFieldsToPass, 78, 79, 181, 186, 189

filter, 16, 47-79, 111

contour, 54-55

data set, 186-189

data set with field, 189-192

field, 181-186 using cells, 184-186

fields, $77-79$

input, $77-78$

passing, 78-79

FTLE, 62-63

implementation, 125-127, 181-194

input fields, 77-78

isosurface, $54-55$

Lagrangian coherent structures, 62-63

Marching Cubes, 54-55

passing fields, 78-79

pathlines, 71

policy, 192-194

stream tracing, 69-71

streamline, 69

streamlines, 69

streamsurface, 70

supported types, 182, 185, 191

threshold, 73

filter namespace, 47, 113, 125, 181

FilterDataSet, 186

DoExecute, xxiii, 186, 188, 263, 264, 268, 269, 274, $275,279,281$

DoMapField, xxiii, 186, 188, 264, 269

Execute, 186

Invoke, 263, 268

MapFieldOntoOutput, 186, 187

FilterDataSetWithField, 189

DoExecute, xxiii, 189-191

DoMapField, xxiii, 189, 190, 192

Execute, 189, 190

MapFieldOntoOutput, 189, 190

FilterField, 125, 181, 183, 186, 189

DoExecute, 126, 185 
Execute, 53, 54, 182

GetOutputFieldName, 183

SetActiveCoordinateSystem, 78

SetActiveField, 77

SetOutputFieldName, 48, 53, 54, 183

SetUseCoordinateSystemAsField, 78

FindCell, 251

FindNearestNeighbor, 253

finite time Lyapunov exponent, see FTLE

Float32, xxii, 21, 131, 137, 142, 193, 199, 285

Float64, 21, 131, 135, 136, 142, 199, 285

FloatDefault, 21, 22, 105, 116, 131, 284, 289

FloatDistance, 198

Floor, 198

floor, 198

flow

FTLE, 62-63

Lagrangian coherent structures, 62-63

pathlines, 71

stream tracing, 69-71

streamline, 69

streamlines, 69

streamsurface, 70

FMod, 198

focal point, 86

Force, 102

ForceDevice, 101

ForEach, 148

foreground color, 83

Frustum, 107

CreateFromPoints, 107

SetNormal, 107

SetNormals, 107

SetPlane, 107

SetPlanes, 107

frustum, 107

FTLE, 62-63

function interface, 353-355

static transform, 354-355

function modifier, 113, 114, 123, 245

function signature, 353

function types, 122

functional array, 308-310

FunctionInterface, xxviii, 353, 381

ARITY, 354

GetArity, 354

StaticTransformCont, 354

StaticTransformType, 354

functions

implicit, 105-108

functor, 111, 308

FunctorBase, 195, 300

RaiseError, 151, 195, 300

Get, 147, 172, 229, 237

Get*, 341
GetActiveFieldName, 76, 77

GetAdvectionTime, 63

GetAllInRange, 73

GetArity, 354

GetAutoOrientNormals, 72

GetAuxiliaryGridDimensions, 63

GetBegin, 230

GetBinDelta, 62

GetBounds, 41

GetCamera, 85

GetCellNormalsName, 72

GetCellSet, 184, 186, 193, 250

GetCellShape, 242

GetChangeCoordinateSystem, 68

GetClipValue, 50

GetColorBuffer, 90

GetColorTable, 58

GetCompactPointFields, 49

GetCompactPoints, 57

GetComponent, 139

GetComputeDivergence, 61

GetComputedRange, 62

GetComputeFastNormalsForStructured, 55

GetComputeFastNormalsForUnstructured, 55

GetComputeGradient, 61

GetComputePointGradient, 61

GetComputeQCriterion, 61

GetComputeVorticity, 61

GetConsistency, 72

GetCoordinates, 250, 252

GetDevice, 104, 340, 341

GetDeviceInstance, 342

GetDivergenceName, 61

GetElapsedTime, 103, 104

GetEnd, 230

GetFastMerge, 49

GetField, 41

GetFlipNormals, 72

GetFlowMapOutput, 63

GetGenerateCellNormals, 72

GetGenerateNormals, 55

GetGeneratePointNormals, 72

GetHumanReadableSize, 157

GetId, 100

GetImplicitFunction, 51

GetIncludeBoundary, 58

GetIndices, 242

GetInputIndex, 363

GetIsoValue, 54

GetLogLevelName, 154

GetLowerThreshold, 73

GetMappingComponent, 59

GetMappingMode, 58

GetMaxDevices, 342

GetMaxThreads, 342 
GetMergeDuplicatePoints, 55

GetMergePoints, 49

GetMessage, 95

GetMetaData, 325

GetName, 100

GetNormalArrayName, 55

GetNormalizeCellNormals, 72

GetNumaRegions, 342

GetNumberOfBuffers, 319, 327

GetNumberOfBytes, 324

GetNumberOfComponents, 139

GetNumberOfDimensions, 74

GetNumberOfElements, 242

GetNumberOfIndices, 242

GetNumberOfIndicices, 242

GetNumberOfPartitions, 41

GetNumberOfSamplingPoints, 59

GetNumberOfSteps, 62

GetNumberOfValues, 115, 229, 283, 319, 327

GetOutputFieldName, 56, 57, 63, 183

GetOutputIndex, 363

GetOutputMode, 59

GetOutputToInputMap, 264, 268

GetPartition, 41

GetPartitions, 41

GetPassPolyData, 57

GetPointNormalsName, 72

GetPrimaryCoordinateSystemIndex, 56

GetPrimaryFieldName, 56

GetQCriterionName, 61

GetRange, 40, 42, 62

GetRate, 76

GetReadPointer, 90

GetRemoveDegenerateCells, 49

GetRuntimeConfiguration, 344

GetRuntimeDeviceTracker, 101

GetSampleRate, 58

GetSecondaryCoordinateSystemIndex, 56, 57

GetSecondaryFieldName, 56

GetSizeString, 157

GetSpatialBounds, 89

GetStackTrace, 157

GetStepSize, 62

GetStorageTypeName, 286

GetThreadName, 153

GetThreads, 342

GetTolerance, 49

GetToleranceIsAbsolute, 49

GetUpperThreshold, 73

GetUseAuxiliaryGrid, 63

GetUseCoordinateSystemAsPrimaryField, 56

GetUseCoordinateSystemAsSecondaryField, 56

GetUseFlowMapOutput, 63

GetValueTypeName, 286

GetVisitIndex, 363
GetVOI, 58

GetVorticityName, 61

GHOST, 60

ghost cell

classify, 60

remove, 60

GHOST., 60

GhostCellClassify, 60

GhostCellRemove, 60

RemoveAllGhost, 60

RemoveByType, 60

git, 7

Gradient, 60, 105

ComputePointGradient, 61

GetComputeDivergence, 61

GetComputeGradient, 61

GetComputePointGradient, 61

GetComputeQCriterion, 61

GetComputeVorticity, 61

GetDivergenceName, 61

GetQCriterionName, 61

GetVorticityName, 61

SetColumnMajorOrdering, 61

SetComputeDivergence, 61

SetComputeGradient, 61

SetComputePointGradient, 60, 61

SetComputeQCriterion, 61

SetComputeVorticity, 61

SetDivergenceName, 61

SetOutputFieldName, 60

SetQCriterionName, 61

SetRowMajorOrdering, 61

SetVorticityName, 61

gradient, 209-210

gradients, 60-61

group vector array handle, 225-227

h, 223,344

Harter-Heighway dragon, 387

Hash, 270

Hash.h, 270

HashType, 270

HasMetaData, 325

HasMultipleComponents, 138

Heighway dragon, 387

hexahedron, 32, 38, 206

Hilbert curve, 389-391

Histogram, 62

GetBinDelta, 62

GetComputedRange, 62

GetRange, 62

SetNumberOfBins, 62

SetOutputFieldName, 62

SetRange, 62

histogram, 62, 177, 240

hyperbolic arccossine, 197 
hyperbolic arcsine, 197

hyperbolic cosine, 198

hyperbolic sine, 200

hyperbolic tangent, 198, 200

I/O, 43-46

Id, $22,23,31,52,53,116,131,132,138,141,142,161,164$, $167,170,172,176,177,205,217,226,242,263$, 267, 274, 284, 289, 290, 296, 300, 302, 309, 324, 342

id

device adapter, 100-101

provided, 100

Id $2,23,132,141,142,211,262,266,267,270,273$

Id3, xxii, 23, 74, 132, 138, 139, 141, 142, 212, 221, 233, 284, 290,300

Id4, 23, 132

IdComponent, 22, 131, 132, 142, 143, 161, 164, 166, 167, $170,172,173,176,177,205,207,211,212,224$, $225,242,256,354,367$

IdComponent2, 23, 132

IdComponent3, 23

IdComponent4, 23, 132

identity matrix, 202

image, 30

ImageConnectivity, 52

ImageReaderPNG, 44

ReadDataSet, 44

SetPointFieldName, 44

ImageReaderPNM, 44

PixelDepth, 45

ReadDataSet, 44

SetPixelDepth, 45

SetPointFieldName, 44

WriteDataSet, 45

ImageWriterPNG, 45

PixelDepth, 45

SetPixelDepth, 45

WriteDataSet, 45

ImageWriterPNM, 45

implicit array handle, 308-310

implicit function

clip, 51-52

implicit functions, 105-108

box, 107

cylinder, 106

frustum, 107

general, 107-108

plane, 105

sphere, 105

implicit storage, 308-310

ImplicitFunction, 51, 105

Gradient, 105

Value, 105

ImplicitFunctionGeneral, 107

ImplicitFunctionHandle, 51
InBoundary, 173

IncidentElementCount, 170

IncidentElementIndices, 170

Include, 135, 136

INDEX, 367

index array handle, 217

IndexTag, 354

IndicesType, 242

Infinity, 198

Info, 154

initialization, 15, 27-28

Initialize, 15, 27, 153, 155, 341-344

Initialize.h, 15

InitializeOptions, 27

AddHelp, 27

DefaultAnyDevice, 27, 28

ErrorOnBadArgument, 27

ErrorOnBadOption, 27

None, 27

RequireDevice, 27

Strict, 27

InitializeResult, 27, 28

Device, 28

InitializeSubsystem, 342

input domain, xxi, 121, 123, 160, 163, 165, 168, 170, 175

input index, 256

InputIndex, 161, 164, 167, 170, 172, 177, 256

InsertPartition, 41

int, 21

Int16, 22, 131

Int32, 22, 131, 193, 240

Int64, 22, 131, 240

Int8, 22, 100, 131

Intel Threading Building Blocks, 9, 99

interactive rendering, 90-93

OpenGL, 90-91

internal namespace, 113

interoperability, 113

interpolation, 209

INVALID, 60

InvalidEdgeId, 150

InvalidFaceId, 150

InvalidNumberOfPoints, 150

InvalidPointId, 150

InvalidShapeId, 150

inverse cosine, 197

inverse hyperbolic cosine, 197

inverse hyperbolic sine, 197

inverse hyperbolic tangent, 198

inverse matrix, 202

inverse sine, 197

inverse tangent, 197

Invocation, 381

invocation object, 381

Invoke, 127, 186, 263, 268, 379 
invoke, 379

Invoker, 123, 127, 256, 335, 374, 379

InXBoundary, 173

InYBoundary, 173

InZBoundary, 173

io namespace, 29, 43, 44, 113

is_same, 97

IsAllocatedOnDevice, 324

IsAllocatedOnHost, 324

IsBaseComponentType, 289

IsCellField, 188, 192

IsFinite, 199

IsInf, 199

IsMappingComponent, 59

IsMappingMagnitude, 59

IsMappingScalar, 59

IsNan, 199

IsNegative, 199

IsNonEmpty, 135, 136

IsOnDevice, 116

IsOnHost, 116

isosurface, 54-55

IsOutputRGB, 59

IsOutputRGBA, 59

isovolume, 50-51

IsPointField, 188, 192

IsSizeFixed, 207

IsSizeStatic, 138

IsStorageType, 286

IsType, 286

IsValueType, 286

IsValueValid, 100, 101

IsZeroInitialized, 304

IteratorType, 230

JACOBIAN, 64

Jurassic Park dragon, 387

kernel, 111

Keys, xxiii, 175, 178, 268, 269, 358, 360, 361

KeysIn, 175, 178

Koch Snowflake, 369

Kokkos, 99

Lagrangian coherent structures, 62-63

LagrangianStructures, 62

GetAdvectionTime, 63

GetAuxiliaryGridDimensions, 63

GetFlowMapOutput, 63

GetNumberOfSteps, 62

GetOutputFieldName, 63

GetStepSize, 62

GetUseAuxiliaryGrid, 63

GetUseFlowMapOutput, 63

SetAdvectionTime, 63

SetAuxiliaryGridDimensions, 63
SetFlowMapOutput, 63

SetNumberOfSteps, 62

SetOutputFieldName, 63

SetStepSize, 62

SetUseAuxiliaryGrid, 63

SetUseFlowMapOutput, 63

LCS, see Lagrangian coherent structures

Length, 135

Lerp, 201

less, 140

level of detail, 74

Lindenmayer system, 370

line, 32, 38, 206

linear interpolation, 201

linear system, 202

List, 141, 143-146, 287, 288

List.h, 141, 143, 144

ListAppend, 144

ListApply, 144

ListAt, 143

ListCross, 145

ListEmpty, 141

ListForEach, 146

ListHas, 143

ListIndexOf, 143

ListIntersect, 144

ListRemoveIf, 145

lists, 141-146 types, 141-142

ListSize, 143

ListTransform, 145

ListUniversal, 141, 143-145

Load, 363, 364, 367

locator

cell, 249-252

point, 252-254

LOD, 74

Log, 199

Log10, 199

Log1P, 199

Log2, 199

logarithm, 199

logging, 153-158 initialization, 153

levels, 153-155

Logging.h, 155, 157

LogicalAnd, 304

LogicalNot, 304

LogicalOr, 304

LogLevel, 154

Cast, 154

Error, 154

Fatal, 154

Info, 154

MemCont, 154 
MemExec, 154

MemTransfer, 154

Off, 154

Perf, 154

UserFirst, 154

UserLast, 154

UserVerboseFirst, 154

UserVerboseLast, 154

Warn, 154

loguru, 153

look at, 86

lower bounds, 296

LowerBounds, 296

MAGNITUDE, 58, 59

Magnitude, 201

magnitude, 74

MagnitudeSquared, 201

make_ArrayHandle, 116

make_ArrayHandleCartesianProduct, 223

make_ArrayHandleCast, 218

make_ArrayHandleCompositeVector, 224

make_ArrayHandleConstant, 216

make_ArrayHandleCounting, 218

make_ArrayHandleExtractComponent, 224

make_ArrayHandleGroupVec, 226

make_ArrayHandleGroupVecVariable, 227

make_ArrayHandleImplicit, 309

make_ArrayHandleMove, 118

make_ArrayHandlePermutation, 220

make_ArrayHandleSwizzle, 225

make_ArrayHandleTransform, 311

make_ArrayHandleView, 216

make_ArrayHandleZip, 221

make_FunctionInterface, xxviii, 353

make_ImplicitFunctionHandle, 51

make_Pair, 147

make_Vec, 131

make_VecC, 133

MakeBuffer, 325, 329

MakeTuple, 147

MalformedCellDetected, 151

ManageArray, 339

map, 159

map field, 160-162

map point neighborhood, 170-174

map topology, 168-170

MapFieldOntoOutput, 186, 187, 189, 190

Mapper, 82

mapper, 17, 82, 84

MapperCylinder, 82

MapperPoint, 82, 84

MapperQuad, 82

MapperRayTracer, 82

MapperVolume, 82

MapperWireframer, 82, 84
Marching Cubes, 54-55

math, 197-204

Math.h, 197

Matrix, 201, 202

matrix, 201-202

Matrix.h, 201, 202

MatrixDeterminant, 202

MatrixFactorizationFailed, 150

MatrixGetColumn, 202

MatrixGetRow, 202

MatrixIdentity, 202

MatrixInverse, 202

MatrixMultiply, 202

MatrixRow, 202

MatrixSetColumn, 202

MatrixSetRow, 202

MatrixTranspose, 202

Max, 135, 199, 305

MAX_ANGLE, 64

MAX_DIAGONAL, 64

Maximum, 305

maximum, 199

MaxNeighborIndices, 173

MemCont, 154

MemExec, 154

MemTransfer, 154

mesh quality, 63

MeshQuality, 16, 63

Execute, 16

SetOutputFieldName, 63

MeshQuality.h, 16

MetaDataIsType, 325

metaprogramming, 141

method modifier, 113, 114, 123, 245

Min, 135, 199, 305

MIN_ANGLE, 64

MIN_DIAGONAL, 64

MinAndMax, 305

Minimum, 305

minimum, 199

MinNeighborIndices, 172, 173

MODE_EXCLUDE, 79

MODE_NONE, 78

ModF, 199

modifier

control, 113, 114, 123, 245

execution, 113, 114, 123, 245

mouse rotation, 91-92

namespace, 112

detail, 113

internal, 113

vtkm, 31, 112, 113, 197, 205

vtkm::cont, 112, 113

vtkm::cont::arg, 357-359, 361

vtkm::cont::cuda, 113 
vtkm::cont::tbb, 113

vtkm::exec, 112, 113, 251, 253, 370

vtkm::exec::arg, 363-365

vtkm::filter, 47, 113, 125, 181

vtkm::io, 29, 43, 44, 113

vtkm::opengl, 113

vtkm::rendering, 81, 113

vtkm::worklet, 113, 187, 377

Nan, 199

natural logarithm, 199

NDEBUG, 96

near clip plane, 87

negative, 199

NegativeInfinity, 199

neighborhood worklet, 170-174 radius, 172

NewInstance, 284

NewInstanceBasic, 284

NewInstanceFloatBasic, 284

Newton's method, 202-204

NewtonsMethod, 202

NewtonsMethod.h, 202

NewtonsMethodResult, 203

None, 27

NORMAL, 60

Normal, 201

Normalize, 201

normals, $71-72$

auto orient, 71

consistency, 72

flip, 71

not a number, 199

NotEqual, 304

NotZeroInitialized, 190, 304

NUM_COMPONENTS, 139

NUM_POINTS, 207

NumericTag, 137

ODDY, 64

Off, $117,154,290$

On, 117, 118, 232

OpenGL, 90-91, 113

opengl namespace, 113

OpenMP, 9, 99

OperationOnEmptyCell, 151

output index, 256

OutputIndex, 161, 164, 167, 170, 172, 177, 256

packages, 112-113

Paint, 17, 83, 90

Pair, 79, 147, 221, 223

Pan, 85, 89, 92

ParameterGet, 354

parametric coordinates, 208-209

ParametricCoordinates.h, 208

ParametricCoordinatesCenter, 208
ParametricCoordinatesPoint, 208

ParametricCoordinatesToWorldCoordinates, 209

ParseExtraArguments, 342

Particle, 69-71

particle density, 65

cic, 66

ngp, 66

ParticleDensityCloudInCell, 66

ParticleDensityNearestGridPoint, 66

partitioned data set, 41-42

PartitionedDataSet, 29, 41, 42, 47

AppendPartition, 41

AppendPartitions, 41

GetField, 41

GetNumberOfPartitions, 41

GetPartition, 41

GetPartitions, 41

InsertPartition, 41

ReplacePartition, 41

Pathline, 70

SetNextDataSet, 71

SetNextTime, 71

SetNumberOfSteps, 71

SetPreviousTime, 71

SetSeeds, 71

Pathlines

SetStepSize, 71

pathlines, 70-71

Perf, 154

permutation cell set, 38-39

permuted array handle, 219-221

pervasive parallelism, 111

Pi, 199

Pi_2, 199

Pi_3, 199

Pi_4, 199

pinhole camera, 86

PIXEL_16, 45

PIXEL_8, 45

PixelDepth, 45

PIXEL_16, 45

PIXEL_8, 45

Plane, 105

SetNormal, 105

SetOrigin, 105

plane, 105

Point, 241

point, 36, 210

point average, 66-67

point elevation, 67

point gradients, 60-61

point locator, 249, 252-254

sparse grid, 252

point neighborhood worklet, 159, 170-174

point transform, 68 
PointAverage, 66

SetOutputFieldName, 66

PointCount, 164

PointElevation, 67

SetHighPoint, 67

SetLowPoint, 67

SetOutputFieldName, 67

SetRange, 67

PointIndices, 164, 211, 212

PointLocator, 252, 253

FindNearestNeighbor, 253

GetCoordinates, 252

SetCoordinates, 252

Update, 252

PointLocatorSparseGrid, 253

SetNumberOfBins, 253

SetRange, 253

PointTransform, 68

GetChangeCoordinateSystem, 68

SetChangeCoordinateSystem, 68

SetOutputFieldName, 68

SetRotation, 68

SetRotationX, 68

SetRotationY, 68

SetRotationZ, 68

SetScale, 68

SetTransform, 68

SetTranslation, 68

policy, 186, 192-194

PolicyBase.h, 192

poly line, 32, 38, 206

polygon, 32, 38, 206

Pow, 199

power, 199

predicates and operators, 303-306

binary operators, 305

binary predicates, 304-305

creating custom comparators, 306

unary predicates, 304

PrepareForExecution, 161, 164, 166, 169, 171, 176, 245

PrepareForInPlace, 116, 234, 360, 361

PrepareForInput, 116, 234, 245, 359, 360

PrepareForOutput, 116, 119, 234, 360, 361

Product, 305

pseudocolor, 93

pyramid, 32, 38, 206

quadratic roots, 200

QuadraticRoots, 200

quadrilateral, 32, 38, 206

RaiseError, 151, 195, 300

random bits array, 216-217

Range, xxii, 40, 42, 85, 135, 136, 233

Center, 135

Contains, 135
Include, 135

IsNonEmpty, 135

Length, 135

Max, 135

Min, 135

Union, 135

range

array, 233

field, 40

RCbrt, 200

read file, 15-16, 43-44

ReadDataSet, 16, 43, 44

ReadPointerDevice, 325

ReadPointerHost, 324, 325

ReadPortal, 116, 231, 232

ReadPortalType, 231, 234, 319, 327

Ready, 104

reciprocal cube root, 200

reciprocal square root, 200

rectilinear grid, 30

rectilinear point coordinates array handle, 222-223

Reduce, 296, 297

reduce, 296

reduce by key, 297

reduce by key worklet, 159, 174-180, 265

ReduceByKey, 297

ReduceByKeyLookup, 360

ReducedValuesIn, 175, 176

ReducedValuesInOut, 176

ReducedValuesOut, 175

regular grid, 30

RELATIVE_SIZE_SQUARED, 64

Release, 9

ReleaseDeviceResources, 325

ReleaseResources, 115, 119

ReleaseResourcesExecution, 115

Remainder, 200

remainder, 198, 200

RemainderQuotient, 200

RemoveAllGhost, 60

RemoveByType, 60

rendering, 16-17, 81-94

actor, 81

camera, 85-89

2D, 85-86

3D, 86-89

azimuth, 88

clipping range, 87

elevation, 88

far clip plane, 87

field of view, 87

focal point, 86

look at, 86

mouse, 91-93

near clip plane, 87 
pan, 85-86, 89

position, 86

reset, 89-90

up, 87

view range, 85

view up, 87

zoom, 86,89

canvas, 82

color tables, 93-94

default, 93

interactive, 90-93

mapper, 82, 84

OpenGL, 90-91

scene, 81

view, 83-84

rendering namespace, 81, 113

ReplacePartition, 41

ReportAllocationFailure, 101

ReportBadDeviceFailure, 102

RequireDevice, 27

Reset, 101, 104

ResetDevice, 101

ResetToBounds, 89, 90

ResetTypes, 288, 289

ResizeBuffers, 319, 327

RGB, 58, 59

RGBA, 58, 59

RMagnitude, 201

Roll, 89

Round, 200

round down, see floor

round up, see ceiling

row, 202

RSqrt, 200

runtime device tracker, 101-102

getting, 101

scoped, 102

RuntimeDeviceConfigReturnCode, 342

RuntimeDeviceConfiguration, 341, 343, 344

GetDevice, 341

GetDeviceInstance, 342

GetMaxDevices, 342

GetMaxThreads, 342

GetNumaRegions, 342

GetThreads, 342

InitializeSubsystem, 342

ParseExtraArguments, 342

SetDeviceInstance, 342

SetNumaRegions, 342

SetThreads, 342

RuntimeDeviceConfigurationBase, 341

Get*, 341

Initialize, 341, 342

Set*, 341

RuntimeDeviceConfigurationKokkos, 342
RuntimeDeviceInformation, 343, 344

GetRuntimeConfiguration, 344

RuntimeDeviceTracker, 101

CanRunOn, 101

DisableDevice, 101

ForceDevice, 101

ReportAllocationFailure, 101

ReportBadDeviceFailure, 102

Reset, 101

ResetDevice, 101

RuntimeDeviceTrackerMode, 102

Disable, 102

Enable, 102

Force, 102

SaveAs, 17, 83

SCALAR, 58

scalar, 75

SCALED_JACOBIAN, 64

scan

exclusive, 298

exclusive by key, 299

inclusive, 297

inclusive by key, 298

scan extend, 299

ScanExclusive, 298-300

ScanExclusiveByKey, 299

ScanExtended, 299, 300

ScanInclusive, 297-300

ScanInclusiveByKey, 298

scatter, 255-259

scatter type, 256

ScatterCounting, 255, 257, 259, 262, 265, 270, 275

GetOutputToInputMap, 264, 268

ScatterIdentity, 255

ScatterPermutation, 255, 258, 259

ScatterUniform, 255, 257, 262

Scene, 81, 89

AddActor, 81

GetSpatialBounds, 89

scene, 17, 81

Schedule, 300

schedule, 300

scoped device adapter, 102

ScopedRuntimeDeviceTracker, 102

serial, 99

Set, 229, 237, 319, 327

Set*, 341

SetActiveCoordinateSystem, 78

SetActiveField, 74, 76, 77

SetAdvectionTime, 63

SetAllInRange, 73

SetAutoOrientNormals, 71, 72

SetAuxiliaryGridDimensions, 63

SetAxis, 106

SetBackground, 83 
SetBounds, 107

SetCamera, 85

SetCapping, 73

SetCartesianToCylindrical, 53

SetCartesianToSpherical, 54

SetCellNormalsName, 72

SetCellSet, 250

SetCenter, 105, 106

SetChangeCoordinateSystem, 68

SetClippingRange, 87

SetClipValue, 50

SetColorTable, 58

SetColumnMajorOrdering, 61

SetCompactPointFields, 49

SetCompactPoints, 57

SetComponent, 139

SetComputeDivergence, 61

SetComputeFastNormalsForStructured, 55

SetComputeFastNormalsForUnstructured, 55

SetComputeGradient, 61

SetComputePointGradient, 60, 61

SetComputeQCriterion, 61

SetComputeVorticity, 61

SetConfigurator, 250

SetConsistency, 72

SetCoordinates, 250, 252

SetCylindricalToCartesian, 53

SetDensityL1, 250

SetDensityL2, 250

SetDeviceInstance, 342

SetDivergenceName, 61

SetFastMerge, 49

SetFieldOfView, 87

SetFieldsToPass, 78, 79, 181, 186, 189

SetFlipNormals, 71, 72

SetFlowMapOutput, 63

SetForeground, 83

SetGenerateCellNormals, 72

SetGenerateNormals, 55

SetGeneratePointNormals, 72

SetHighPoint, 67

SetImplicitFunction, 51

SetIncludeBoundary, 58

SetInvertClip, 50, 51

SetIsoValue, 54

SetLogLevelName, 154

SetLookAt, 87

SetLowerThreshold, 73

SetLowPoint, 67

SetMappingComponent, 59

SetMappingMode, 58, 59

SetMappingToComponent, 59

SetMappingToMagnitude, 59

SetMappingToScalar, 58

SetMaxLeafSize, 250
SetMaxPoint, 107

SetMergeDuplicatePoints, 55

SetMergePoints, 49

SetMetaData, 325

SetMinPoint, 107

SetModeTo2D, 85

SetModeTo3D, 85

SetNextDataSet, 71

SetNextTime, 71

SetNormal, 105, 107

SetNormalArrayName, 55

SetNormalField, 75

SetNormalizeCellNormals, 72

SetNormals, 107

SetNumaRegions, 342

SetNumberOfBins, 62, 253

SetNumberOfBytes, 324

SetNumberOfDivisions, 74

SetNumberOfPlanes, 250

SetNumberOfSamplingPoints, 59

SetNumberOfSides, 73

SetNumberOfSteps, 62, 69-71

SetOrigin, 105

SetOutputFieldName, 48, 53, 54, 56, 57, 60, 62, 63, 66-68, $72,74-76,183$

SetOutputMode, 59

SetOutputToRGB, 59

SetOutputToRGBA, 59

SetPassPolyData, 57

SetPixelDepth, 45

SetPlane, 107

SetPlanes, 107

SetPointFieldName, 44

SetPointNormalsName, 72

SetPosition, 86

SetPreviousTime, 71

SetPrimaryCoordinateSystem, 56

SetPrimaryField, 56

SetQCriterionName, 61

SetRadius, 73, 105, 106

SetRange, 62, 67, 253

SetRate, 76, 77

SetRemoveDegenerateCells, 49

SetRotation, 68

SetRotationX, 68

SetRotationY, 68

SetRotationZ, 68

SetRowMajorOrdering, 61

SetSampleRate, 58

SetScalarFactorField, 75

SetScale, 68

SetSecondaryCoordinateSystem, 56, 57

SetSecondaryField, 56

SetSeeds, 69-71

SetSphericalToCartesian, 54 
SetStderrLogLevel, 155

SetStepSize, 62, 69-71

SetThreadName, 153

SetThreads, 342

SetTolerance, 49

SetToleranceIsAbsolute, 49

SetTransform, 68

SetTranslation, 68

SetUpperThreshold, 73

SetUseAuxiliaryGrid, 63

SetUseCoordinateSystemAsField, 78

SetUseCoordinateSystemAsPrimaryField, 56

SetUseCoordinateSystemAsSecondaryField, 56

SetUseFlowMapOutput, 63

SetVectorField, 76

SetViewRange2D, 85

SetViewUp, 87

SetVOI, 58

SetVorticityName, 61

SHAPE, 65

shape, 36, 205-208, 210

edge, 36, 210-212

face, $36,210,212-214$

point, 36, 210

SHAPE_AND_SIZE, 65

SHEAR, 65

signature, 122, 353

control, xiv, xxi, xxviii, xxix, 121-122, 124, 160, 161, 163-166, 168-170, 172, 175, 176, 178, 237, 240, $241,245,253,353,357,366,367,374-377,381$

tags, 366

execution, xiv, xxi, xxviii, xxix, 121-123, 161, 164, 166 , $169,171,172,176,256,357,366,367,374-376$, 381

tags, 366-367

signature tags, 122

$\_1,122,123,161,164,166,169,172,176,367$

$2,122,123,161,164,166,169,172,176,367$

AtomicArrayInOut, xxv, 160, 163, 166, 169, 171, 176, 240

Boundary, 172

Cell, 241

CellCount, 166

CellIndices, 167

CellSetIn, 123, 163, 165, 168, 170

CellShape, 164, 170

ExecObject, xxv, 161, 162, 164, 166, 169, 171, 176, 245, 249-251, 253

FieldIn, 122, 123, 160, 170, 171, 377

FieldInCell, 163, 165

FieldInIncident, 168

FieldInNeighborhood, 171, 172

FieldInOut, 160, 163, 166, 169, 171

FieldInOutCell, 163

FieldInOutPoint, 166
FieldInPoint, 163, 165

FieldInVisit, 168

FieldOut, 122, 160, 163, 166, 169, 171, 377

FieldOutCell, 163

FieldOutPoint, 166

FieldPointIn, 122, 209, 210

IncidentElementCount, 170

IncidentElementIndices, 170

InputIndex, 161, 164, 167, 170, 172, 177, 256

KeysIn, 175, 178

OutputIndex, 161, 164, 167, 170, 172, 177, 256

Point, 241

PointCount, 164

PointIndices, 164, 211, 212

ReducedValuesIn, 175, 176

ReducedValuesInOut, 176

ReducedValuesOut, 175

ThreadIndices, 161, 164, 167, 170, 172, 177

ValueCount, 176, 178

ValuesIn, 175, 176

ValuesInOut, 175

ValuesOut, 175

VisitIndex, 161, 164, 167, 170, 172, 177, 256, 257, 262

WholeArrayIn, xxv, 160, 163, 166, 169, 171, 176, 237, 238

WholeArrayInOut, 160, 163, 166, 169, 171, 176, 237

WholeArrayOut, 160, 162, 163, 166, 169, 171, 176, 237

WholeCellSetIn, xxv, 161, 164, 166, 169, 171, 176, 241, 242, 266, 272

WorkIndex, 122, 123, 161, 162, 164, 167, 170, 172, 176, $177,256,365$

SignBit, 200

Sin, 200

sine, 200

single type cell set, 37-38

SinH, 200

size_t, 22

SKEW, 65

SolutionDidNotConverge, 150

SolveLinearSystem, 202

Sort, 300, 301

sort, 300

by key, 301

SortByKey, 301

SortGreater, 304

SortLess, 304

Sphere, 51, 105, 107

SetCenter, 105

SetRadius, 105

sphere, 105

spherical coordinate system transform, 54

SphericalCoordinateSystem Transform, 54

Sqrt, 200

square root, 200

Start, 103, 104 
Started, 104

static assert, 96-97

StaticAssert.h, 96

StaticTransformCont, 354

StaticTransformType, 354

std::pair, 147

std::tuple, 147

std::vector, 117

Stop, 103, 104

Stopped, 104

Storage, xxvii, 318, 319, 326, 327 CreateReadPortal, 319, 327

CreateWritePortal, 319, 327

GetNumberOfBuffers, 319, 327

GetNumberOfValues, 319, 327

ReadPortalType, 319, 327

ResizeBuffers, 319, 327

WritePortalType, 319, 327

storage, 118-119, 307-330

adapting, 323-330

default, 307, 330

derived, 316-323

implicit, 308-310

StorageTag, 310, 312, 316, 322

StorageTagBasic, 119, 308

StorageTagSOA, 308

Store, 363, 364, 367

stream compact, 294

stream tracing, 69-71

Streamline, 69

SetNumberOfSteps, 69

SetSeeds, 69

SetStepSize, 69

streamlines, 69

StreamSurface, 70

SetNumberOfSteps, 70

SetSeeds, 70

SetStepSize, 70

streamsurface, 70

STRETCH, 65

Strict, 27

structure of arrays, 308

structured cell set, 36

Success, 150, 151, 251

Sum, 305

Superclass, 310, 312, 316, 322

supported types, 182, 185, 191

surface normals, 71-72

auto orient, 71

consistency, 72

flip, 71

surface simplification, 74-75

SurfaceNormals, 71

Execute, 71

GetAutoOrientNormals, 72
GetCellNormalsName, 72

GetConsistency, 72

GetFlipNormals, 72

GetGenerateCellNormals, 72

GetGeneratePointNormals, 72

GetNormalizeCellNormals, 72

GetPointNormalsName, 72

SetAutoOrientNormals, 71, 72

SetCellNormalsName, 72

SetConsistency, 72

SetFlipNormals, 71, 72

SetGenerateCellNormals, 72

SetGeneratePointNormals, 72

SetNormalizeCellNormals, 72

SetOutputFieldName, 72

SetPointNormalsName, 72

swizzle array handle, 225

SyncControlArray, 116, 231, 330

synchronize, 301

Tag, 206

tag, 136

cell shape, 205-207

device adapter, 99-100

provided, 99-100

dimensionality, 137

lists, 141-146

multiple components, 138

numeric, 137

shape, 205-207

single component, 138

static vector size, 138

topology element, 168

type lists, 141-142

type traits, 137-138

variable vector size, 138

vector traits, 138-140

Tan, 200

tangent, 200

TanH, 200

TAPER, 65

TBB, 9, 99

tbb namespace, 113

template metaprogramming, 141

testing, 331-332

creating tests, 332

ctest, 331-332

without ctest, 332

tetrahedron, 32, 38, 206

thread indices, 363, 372-374

thread name, 153

ThreadIndices, 161, 164, 167, 170, 172, 177

ThreadIndicesBasic, 373, 374

GetInputIndex, 363

GetOutputIndex, 363

GetVisitIndex, 363 
ThreadIndicesTopologyMap, 373

Threshold, 73, 190

Execute, 73

GetAllInRange, 73

GetLowerThreshold, 73

GetUpperThreshold, 73

SetAllInRange, 73

SetLowerThreshold, 73

SetUpperThreshold, 73

threshold, 73

Timer, xxi, 103, 104, 350

GetDevice, 104

GetElapsedTime, 103, 104

Ready, 104

Reset, 104

Start, 103, 104

Started, 104

Stop, 103, 104

Stopped, 104

timer, 103-104, 350-351

Token, 234, 245, 314, 319, 324, 325, 327, 359

TOPOLOGICAL_DIMENSIONS, 207

TopologicalDimensionsTag, 207

topology element tag, 168

topology map worklet, 159, 168-170

TopologyElementTag.h, 168

TopologyElementTagCell, 168, 241

TopologyElementTagEdge, 168

TopologyElementTagFace, 168

TopologyElementTagPoint, 168, 241

TrackballRotate, 91

traits, 136-140

type, $137-138$

vector, 138-140

transfer virtual object, 344-346

Transform, 149, 301

transform, 68, 301

transformed array, 310-312

Transport, xxviii, 359, 361

ExecObjectType, 359, 361

transport, 359-362

atomic array, 360

bit field, 360

cell set, 360

execution object, 359

input array, 359

input array keyed values, 360

input/output array, 360

input/output array keyed values, 361

keys, 360

output array, 360

output array keyed values, 361

topology mapped field, 360

whole array input, 360

whole array input/output, 360 whole array output, 360

TransportTag, 366

TransportTagArrayIn, 359

TransportTagArrayInOut, 360

TransportTagArrayOut, 360

TransportTagAtomicArray, 360

TransportTagBitFieldIn, 360

TransportTagBitFieldInOut, 360

TransportTagCellSetIn, 360

TransportTagExecObject, 359

TransportTagKeyedValuesIn, 360

TransportTagKeyedValuesInOut, 361

TransportTagKeyedValuesOut, 361

TransportTagKeysIn, 360

TransportTagTopologyFieldIn, 360

TransportTagWholeArrayIn, 360

TransportTagWholeArrayInOut, 360

TransportTagWholeArrayOut, 360

transpose matrix, 202

triangle, 32, 38, 206

TriangleNormal, 201

true_type, 97

try execute, 335-336

TryExecute, 335

Tube, 73

SetCapping, 73

SetNumberOfSides, 73

SetRadius, 73

tube, 73-74

Tuple, 147, 150

Apply, 149, 150

ForEach, 148

Get, 147

Transform, 149

TupleElement, 147

TupleSize, 147

TwoPi, 200

type check, 357-359

array, 358

atomic array, 358

cell set, 358

execution object, 357,358

keys, 358

type check bit field, 358

type lists, 141-142

type traits, 137-138

type_traits, 97

TypeCheck, xxviii, 357, 358

value, 358

TypeCheckTag, 366

TypeCheckTagArrayIn, 358

TypeCheckTagArrayInOut, 358

TypeCheckTagArrayOut, 358

TypeCheckTagAtomicArray, 358

TypeCheckTagBitField, 358 
TypeCheckTagCellSet, 358

TypeCheckTagCellSetStructured, 358

TypeCheckTagExecObject, 357, 358

TypeCheckTagKeys, 358

TypeList.h, 141, 142

TypeListAll, 142

TypeListCommon, 142

TypeListField, 142

TypeListFieldScalar, 142

TypeListFieldVec2, 142

TypeListFieldVec3, 142

TypeListFieldVec4, 142

TypeListId, 141

TypeListId2, 141

TypeListId3, 141

TypeListIdComponent, 142

TypeListIndex, 142

TypeListScalarAll, 142

TypeListVecAll, 142

TypeListVecCommon, 142

Types.h, 21, 113, 133, 142

TypeToString, 157

TypeTraits, xxii, 137, 294

DimensionalityTag, 137

NumericTag, 137

ZeroInitialization, 137, 294

TypeTraitsIntegerTag, 137

TypeTraitsRealTag, 137

TypeTraitsScalarTag, 137

TypeTraitsVectorTag, 137

UInt16, 22, 131

UInt32, 22, 131

UInt64, 22, 131, 217

UInt8, 22, 31, 60, 131

UncertainArrayHandle, 288

CastAndCall, 288, 291

ResetTypes, 288

UncertainArrayHandle.h, 289

uniform grid, 30

uniform point coordinates array handle, 221-222

Union, 135, 136

Unique, 302

unique, 302

Unit64, 216

unknown array handle, 283-292

allocation, 283

cast, 285-289

const, 291-292

construct, 283

copy, 286-287

query type, 285-286

UnknownArrayHandle, 146, 283

Allocate, 284

AsArrayHandle, 285, 287

CanConvert, 285, 286
CastAndCallForTypes, 287, 288

CastAndCallWithExtractedArray, 291

CopyShallowIfPossible, 286

DeepCopyFrom, 286

ExtractArrayFromComponents, 290, 291

ExtractComponent, 289, 290

GetNumberOfValues, 283

GetStorageTypeName, 286

GetValueTypeName, 286

IsBaseComponentType, 289

IsStorageType, 286

IsType, 286

IsValueType, 286

NewInstance, 284

NewInstanceBasic, 284

NewInstanceFloatBasic, 284

ResetTypes, 288, 289

unstructured grid, 31

Update, 250, 252

upper bounds, 302

UpperBounds, 302

UserFirst, 154

UserLast, 154

UserVerboseFirst, 154

UserVerboseLast, 154

Value, 105

value, 358

ValueCount, 176, 178

ValuesIn, 175, 176

ValuesInOut, 175

ValuesOut, 175

ValueType, 224, 225, 229, 310, 312, 316, 322

Vec, xxii, xxiv, 131-133, 136, 138, 139, 142, 172, 173, 200203, 208-210, 218, 223-225, 227, 233, 289, 290, $305,308,357,358$

CopyInto, 133

Vec-like, 133-134, 138

Vec2f, 22, 132

Vec2f_32, 132

Vec2f_64, 132

Vec2i, 23, 132

Vec2i_16, 132

Vec2i_32, 132

Vec2i_64, 132

Vec2i_8, 132

Vec2ui, 23, 132

Vec2ui_16, 132

Vec2ui_32, 132

Vec2ui_64, 132

Vec2ui_8, 132

Vec3f, 22, 105, 107, 132, 284, 289

Vec3f_32, 22, 132

Vec3f_64, 22, 132

Vec3i, 23, 132

Vec3i_16, 132 
Vec3i_32, 132

Vec3i_64, 132

Vec3i_8, 132

Vec3ui, 23, 132

Vec3ui_16, 132

Vec3ui_32, 132

Vec3ui_64, 132

Vec3ui_8, 58, 132

Vec4f, 22, 132

Vec4f_32, 132

Vec4f_64, 132

Vec4i, 23, 132

Vec4i_16, 132

Vec4i_32, 132

Vec4i_64, 132

Vec4i_8, 132

Vec4ui, 23, 132

Vec4ui_16, 132

Vec4ui_32, 132

Vec4ui_64, 132

Vec4ui_8, 23, 58, 132

VeciUint32, 1¿, 216

VecC, 133, 134

VecCConst, xxii, 133, 134

VecFromPortal, 134

VecFromPortalPermute, 134

VecRectilinearPointCoordinates, 134

vector, 76,117

vector analysis, 200-201

vector magnitude, 74

vector traits, 138-140

Vector Analysis.h, 200

VectorMagnitude, 74

SetActiveField, 74

SetOutputFieldName, 74

VecTraits, xxii, 138, 139

ComponentType, 138

CopyInto, 139

GetComponent, 139

GetNumberOfComponents, 139

HasMultipleComponents, 138

IsSizeStatic, 138

NUM_COMPONENTS, 139

SetComponent, 139

VecTraitsTagMultipleComponents, 138

VecTraitsTagSingleComponent, 138

VecTraitsTagSizeStatic, 138

VecTraitsTagSizeVariable, 138

VecVariable, xxii, 134

version, 25-26

CMake, 25

macro, 25-26

Version.h, 25, 26

vertex, 32, 38, 206

vertex clustering, 74-75
VertexClustering, 74

GetNumberOfDimensions, 74

SetNumberOfDivisions, 74

View, 83, 85, 89

GetCamera, 85

Paint, 17, 83, 90

SaveAs, 17, 83

SetBackground, 83

SetCamera, 85

SetForeground, 83

view, 17, 83-84

view array handle, 216

view up, 87

View2D, 83

View3D, 83

virtual object transfer, 344-346

VirtualObjectTransfer, xxviii, 344

VirtualObjectTransferShareWithControl, 345

visit cells worklet, 159, 162-165

visit index, 256

visit points worklet, 159, 165-168

VisitIndex, 161, 164, 167, 170, 172, 177, 256, 257, 262

VOLUME, 65

VTK-m CMake package, 11-13

libraries, 11-12

vtkm_cont, 12

vtkm_filter, 12

vtkm_io, 12

vtkm_rendering, 12

vtkm_source, 12

variables, $12-13$

VTKm_ENABLE_CUDA, 12

VTKm_ENABLE_MPI, 12

VTKm_ENABLE_OPENMP, 12

VTKm_ENABLE_RENDERING, 12

VTKm_ENABLE_TBB, 12

VTKm_FOUND, 12

VTKm_VERSION, 12

VTKm_VERSION_FULL, 12

VTKm_VERSION_MAJOR, 12

VTKm_VERSION_MINOR, 12

VTKm_VERSION_PATCH, 12

version, 25

VTKDataSetReader, 16, 43

ReadDataSet, 16, 43

VTKDataSetReader.h, 16

VTKDataSetWriter, 45

WriteDataSet, 45

vtkm namespace, 31, 112, 113, 197, 205

vtkm/cont/ArrayHandleCartesianProduct.h/h, 223

vtkm/cont/cuda/DeviceAdapterCuda.h, 99

vtkm/cont/internal/DeviceAdapterTag.h, 338

vtkm/cont/kokkos/DeviceAdapterKokkos.h, 99

vtkm/cont/openmp/DeviceAdapterOpenMP.h, 99

vtkm/cont/tbb/DeviceAdapterTBB.h, 99 
vtkm/cont/ArrayCopy.h, 118

vtkm/cont/ArrayHandleCast.h, 218

vtkm/cont/ArrayHandleCompositeVector.h, 224

vtkm/cont/ArrayHandleConstant.h, 216

vtkm/cont/ArrayHandleCounting.h, 218

vtkm/cont/ArrayHandleExtractComponent.h, 224

vtkm/cont/ArrayHandleGroupVec.h, 226

vtkm/cont/ArrayHandleGroupVecVariable.h, 227

vtkm/cont/ArrayHandleImplicit.h, 309

vtkm/cont/ArrayHandlePermutation.h, 220

vtkm/cont/ArrayHandleSwizzle.h, 225

vtkm/cont/ArrayHandleView.h, 216

vtkm/cont/ArrayHandleZip.h, 221

vtkm/cont/ArrayPortalToIterators.h, 231

vtkm/cont/ArrayRangeCompute.h, 233

vtkm/cont/DataSet.h, 113

vtkm/cont/DeviceAdapterList.h, 338

vtkm/cont/DeviceAdapterSerial.h, 99

vtkm/cont/Initialize.h, 15

vtkm/cont/Logging.h, 155, 157

vtkm/cont/UncertainArrayHandle.h, 289

vtkm/exec/CellDerivative.h, 209

vtkm/exec/CellEdge.h, 211, 266

vtkm/exec/CellFace.h, 212

vtkm/exec/CellInterpolate.h, 209

vtkm/exec/ParametricCoordinates.h, 208

vtkm/filter/CreateResult.h, 183

vtkm/filter/MeshQuality.h, 16

vtkm/filter/PolicyBase.h, 192

vtkm/io/VTKDataSetReader.h, 16

vtkm::cont, 112, 113

vtkm::cont::arg, 357-359, 361

vtkm::cont::cuda, 113

vtkm::cont::tbb, 113

vtkm::exec, 112, 113, 251, 253, 370

vtkm::exec::arg, 363-365

vtkm::filter, 47, 113, 125, 181

vtkm::io, 29, 43, 44, 113

vtkm::opengl, 113

vtkm::rendering, 81, 113

vtkm::worklet, 113, 187, 377

VTKM_ARRAY_HANDLE_SUBCLASS，310，312，316, 322

VTKM_ARRAY_HANDLE_SUBCLASS_NT，310，312, 316,322

VTKM_ASSERT, xxi, 96, 195

VTKM_CONT, 113, 114, 245

vtkm_cont, 12

VTKm_CUDA_Architecture, 9

VTKM_DEFAULT_STORAGE_LIST, 287, 330

VTKM_DEFAULT_STORAGE_TAG, 308

VTKM_DEFAULT_TYPE_LIST, 142, 287

VTKm_DIR, 11

VTKm_ENABLE_BENCHMARKS, 9

VTKm_ENABLE_CUDA, 9, 12
VTKm_ENABLE_EXAMPLES, 9

VTKm_ENABLE_LOGGING, 9, 153

VTKm_ENABLE_MPI, 12

VTKm_ENABLE_OPENMP, 9, 12

VTKm_ENABLE_RENDERING, 9, 12

VTKm_ENABLE_TBB, 9, 12

VTKm_ENABLE_TESTING, 9

VTKM_EXEC, 113, 114, 123, 245

VTKM_EXEC_CONT, 113, 114, 123, 245

vtkm_filter, 12

VTKm_FOUND, 12

vtkm_io, 12

VTKM_IS_ARRAY_HANDLE, 119, 127

VTKM_IS_CELL_SHAPE_TAG, 205

VTKM_IS_DEVICE_ADAPTER_TAG, 100

VTKM_IS_LIST, 143

VTKM_LOG_ERROR_CONTEXT, 157

VTKM_LOG_F, 155, 156

VTKM_LOG_IF_F, 156

VTKM_LOG_IF_S, 156

VTKM_LOG_S, 155, 156

VTKM_LOG_SCOPE, 156

VTKM_LOG_SCOPE_FUNCTION, 156

vtkm_rendering, 12

vtkm_source, 12

VTKM_STATIC_ASSERT, xxi, 96, 97

VTKM_STATIC_ASSERT_MSG, 96

VTKM_STORAGE_NO_RESIZE, 319, 327

VTKM_STORAGE_NO_WRITE_PORTAL, 319, 327

VTKM_SUPPRESS_EXEC_WARNINGS, 114

VTKm_USE_64BIT_IDS, 9, 22

VTKM_USE_DOUBLE_PRECISION, 21

VTKm_USE_DOUBLE_PRECISION, 9, 22

VTKM_VALID_DEVICE_ADAPTER, 338

VTKM_VERSION, 25

VTKm_VERSION, 12, 25

VTKM_VERSION_FULL, 25

VTKm_VERSION_FULL, 12, 25

VTKM_VERSION_MAJOR, 25

VTKm_VERSION_MAJOR, 12, 25

VTKM_VERSION_MINOR, 25

VTKm_VERSION_MINOR, 12, 25

VTKM_VERSION_PATCH, 25

VTKm_VERSION_PATCH, 12, 25

vtkm/Assert.h, 96

vtkm/CellShape.h, 205

vtkm/CellTraits.h, 207

vtkm/Hash.h, 270

vtkm/List.h, 141, 143, 144

vtkm/Math.h, 197

vtkm/Matrix.h, 201, 202

vtkm/NewtonsMethod.h, 202

vtkm/StaticAssert.h, 96

vtkm/TopologyElementTag.h, 168

vtkm/TypeList.h, 141, 142 
vtkm/Types.h, 21, 113, 133, 142

vtkm/VectorAnalysis.h, 200

vtkm/Version.h, 25, 26

vtkmGenericCellShapeMacro, 207

Warn, 154

warp scalar, 75

warp vector, 76

WARPAGE, 65

WarpScalar, 75

Execute, 75

SetNormalField, 75

SetOutputFieldName, 75

SetScalarFactorField, 75

WarpVector, 76

Execute, 76

SetOutputFieldName, 76

SetVectorField, 76

wedge, $32,38,206$

whole array, 237-239

whole cell set, 241-244

WholeArrayIn, xxv, 160, 163, 166, 169, 171, 176, 237, 238

WholeArrayInOut, 160, 163, 166, 169, 171, 176, 237

WholeArrayOut, 160, 162, 163, 166, 169, 171, 176, 237

WholeCellSetIn, xxv, 161, 164, 166, 169, 171, 176, 241, 242, 266,272

wireframe, 84

WorkIndex, 122, 123, 161, 162, 164, 167, 170, 172, 176, 177, 256,365

worklet, 111, 121-124, 159-180, 195, 237-247, 255-259

atomic array, 239-241

control signature, 122

creating, 121-123, 159-180, 195, 237-247, 255-259

error handling, 195

execution object, 245-247

execution signature, 122-123

input domain, 123

invoke, $123-124$

scatter, 255-259

whole array, 237-239

whole cell set, 241-244

worklet namespace, 113, 187, 377

worklet types, 159-180

creating new, 369-391

field map, 159-162

point neighborhood, 159, 170-174

reduce by key, 159, 174-180, 265

topology map, 159, 168-170

visit cells, 159, 162-165

visit points, $159,165-168$

WorkletBase, 377

Dispatcher, 377

WorkletMapField, 159, 160, 255, 380

WorkletMapTopology, 159, 168, 380

WorkletPointNeighborhood, 159, 170, 380

WorkletReduceByKey, 159, 174, 266, 360, 380
WorkletVisitCellsWithPoints, 159, 162, 168, 255, 380

WorkletVisitPointsWithCells, 159, 165

world coordinates, 208-209

WorldCoordinatesToParametricCoordinates, 209

write file, 44-45

WriteDataSet, 45

WritePointerDevice, 325

WritePointerHost, 325

WritePortal, 116, 119, 231, 232

WritePortalType, 231, 234, 319, 327

$\mathrm{X}, 135,136$

Y, 135,136

Z, 135, 136

ZeroInitialization, 137, 294

zfp

compression, 76-77

decompression, 77

ZFPCompressor, 76

GetActiveFieldName, 76

GetRate, 76

SetActiveField, 76

SetRate, 76

ZFPDecompressor, 77

GetActiveFieldName, 77

SetActiveField, 77

SetRate, 77

zipped array handles, 221

Zoom, 86, 89, 92 